\title{
NATIONAL TRENDS IN DRUG USE AND RELATED FACTORS AMONG AMERICAN HIGH SCHOOL STUDENTS AND YOUNG ADULTS, 1975-1986
}




\title{
NATIONAL TRENDS IN DRUG USE AND RELATED FACTORS AMONG AMERICAN HIGH SCHOOL STUDENTS AND YOUNG ADULTS, 1975-1986
}

\author{
by \\ Lloyd D. Johnston, Ph.D. \\ Patrick M. O'Malley, Ph.D. \\ Jerald G. Bachman, Ph.D. \\ The University of Michigan \\ Institute for Social Research
}

\author{
National Institute on Drug Abuse \\ 5600 Flshers Lane \\ Rockville, Maryland 20857
}

\author{
U.S. DEPARTMENT OF HEALTH AND HUMAN SERVICES \\ Publlc Health Service \\ Alcohol, Drug Abuse, and Mental Health Administration
}


This publication was written by the principal investigators and staff of The Monitoring the Future project, at the Institute for Social Research, The University of Michigan, under Research Grant No. R01 DA01411 from the National Institute on Drug Abuse.

\section{Public Domain Notice}

All material appearing in this volume is in the public domain and may be reproduced or copied without permission from the Institute or the authors. Citation of the source is appreciated.

DHHS Publication No. (ADM) 87-1535

Printed 1987 


\section{CONTENTS}

$\underline{\text { Page }}$

List of Tables $\ldots \ldots \ldots \ldots \ldots \ldots \ldots \ldots \ldots \ldots$

List of Figures $\ldots \ldots \ldots \ldots \ldots \ldots \ldots \ldots \ldots \ldots$ viii

Introduction $\ldots \ldots \ldots \ldots \ldots \ldots \ldots \ldots \ldots \ldots \ldots$

The Inclusion of College Students and Young Adults

Generally . . . . . . . . . . . . . . . . . 1

Content Areas Covered in This Report ........... 2

Purposes and Rationale for This Research . . . . . . . . . 3

Research Design and Procedures for the Surveys of

Seniors ....................... 4

Research Design for the Follow-Up Surveys after High

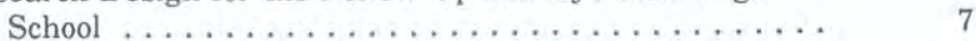

Follow-Up Procedures $\ldots \ldots \ldots \ldots \ldots \ldots \ldots \ldots$

Panel Retention Rates .................. 8

Representativeness and Validity ............. 8

Validity of the Measures of Self-Reported Drug Use . . . . . 10

A Note about the Stimulant Results for $1979-1982 \ldots \ldots$. . 12

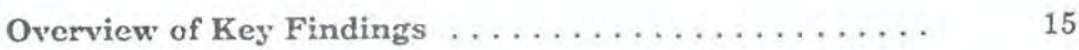

\section{HIGH SCHOOL SENIORS}

Prevalence of Drug Use Among High School Seniors ... 26

Prevalence of Drug Use in 1986: All Seniors ... . . . . 26

Lifetime, Annual, and Monthly Prevalence . . . . . . . 26

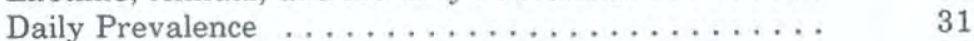

Noncontinuation Rates .................... 33

Prevalence Comparisons for Important Subgroups . . . . . . $\quad 35$

Sex Differences .................... 35

Differences Related to College Plans .......... 39

Regional Differences ................... 42

Differences Related to Population Density ... . . . . 44

"Crack" Cocaine: Prevalence Rates and Subgroup

Differences...................... 45

Trends in Drug Use Among High School Seniors . . . . . 46

Trends in Prevalence 1975-1986: All Seniors . . . . . . . 46

Trends in Noncontinuation Rates .............. 69

Comparisons Among Subgroups in Trends in Prevalence . $\quad 72$ 
Sex Differences in Trends ................

Trend Differences Related to College Plans . . . . . . . $\quad 75$

Regional Differences in Trends ............ 77

Trend Differences Related to Population Density .... 80

Use at Earlier Grade Levels $\ldots \ldots \ldots \ldots \ldots \ldots \ldots \ldots \quad 85$

Incidence of Use by Grade Level . . . . . . . . . . . . $\quad 85$

Trends in Use at Earlier Grade Levels . . . . . . . . . 87

Degree and Duration of Drug Highs $\ldots \ldots \ldots \ldots \ldots \ldots \quad 111$

Trends in Degree and Duration of Drug Highs ........ 115

Attitudes and Beliefs about Drugs . . . . . . . . . 118

Perceived Harmfulness of Drugs . . . . . . . . . . 119

Beliefs in 1986 about Harmfulness . . . . . . . . 119

Trends in Perceived Harmfulness . . . . . . . . . . 121

Personal Disapproval of Drug Use .............. 125

Extent of Disapproval in $1986 \ldots \ldots \ldots \ldots \ldots \ldots . .125$

Trends in Disapproval ................ 127

Attitudes Regarding the Legality of Drug Use . . . . . . 127

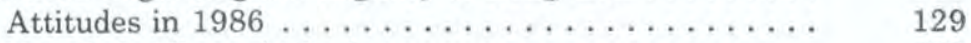

Trends in These Attitudes . . . . . . . . . . . . 129

The Legal Status of Marijuana .............. $\quad 130$

Attitudes and Predicted Response to Legalization .... 130

Trends in Attitudes and Predicted Responses ...... 130

The Social Milieu . . . . . . . . 133

Perceived Attitudes of Parents and Friends . . . . . . . 133

Perceptions of Parental Attitudes ............ 133

Current Perceptions of Friends' Attitudes . . . . . . 135

A Comparison of the Attitudes of Parents, Peers, and Respondents ................ 136

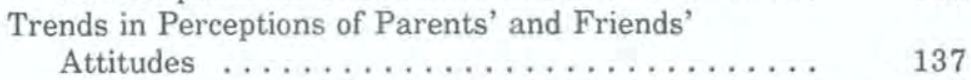

Exposure to Drug Use by Friends and Others ........ 142

Exposure to Drug Use in $1986 \ldots \ldots \ldots \ldots \ldots . .142$

Recent Trends in Exposure to Drug Use . . . . . . . 146

Implications for Validity of Self-Reported Usage
Questions $\ldots \ldots \ldots \ldots \ldots \ldots \ldots \ldots \ldots \ldots \ldots \ldots$

Perceived Availability of Drugs . . . . . . . . . . . $\quad 150$

Perceived Availability in $1986 \ldots \ldots \ldots \ldots \ldots \ldots . \quad 150$

Trends in Perceived Availability $\ldots \ldots \ldots \ldots \ldots \ldots \quad 150$ 


\section{YOUNG ADULTS POST-HIGH SCHOOL}

Prevalence of Drug Use Among Young Adults Post-High

School . . . . . . . . . . . . . . . . . . . .

A Note on Lifetime Prevalence Estimates . . . . . . . . .

Prevalence of Drug Use in 1986 Among Young Adults

as a Function of Age . . . . . . . . . . . . . .

Sex Differences in Prevalence Among Young Adults .... 161

Trends in Drug Use Among Young Adults Post-High

School . . . . . . . . . . . . . . . . . . . .

Trends in Prevalence Through 1986: Young Adults ....

\section{COLLEGE STUDENTS}

Prevalence of Drug Use Among College Students ..... .

Prevalence of Drug Use in 1986: College Students .... .

Sex Differences in Prevalence Among College Students .. 204

Trends in Drug Use Among College Students . . . . . . . 228

Trends in Prevalence 1980-1986: College Students . . . . 228

Sex Differences in Trends Among College Students . . . . 231

\section{OTHER FINDINGS FROM THE STUDY}

The Use of Nonprescription Stimulants $\ldots \ldots \ldots \ldots \ldots$

Prevalence of Use in $1986 \ldots \ldots \ldots \ldots \ldots \ldots$

Subgroup Differences . . . . . . . . . . . . .

Trends in Use ..................... 238

The Use of Marijuana on a Daily Basis . . . . . . . 240

Lifetime Prevalence of Daily Use ... . . . . . . . . . . 240

Grade of First Daily Use . . . . . . . . . . . . . . . . . 241

Recency of Daily Use . . . . . . . . . . . . . . . . . . 241

Duration of Daily Use . . . . . . . . . . . . . . . . . 243

Subgroup Differences . . . . . . . . . . . . . . . . . . 243

Trends in the Use of Marijuana on a Daily Basis . . . . . . 244 
Medically Supervised Use of Psychotherapeutic Drugs . 246

Some Correlates of Cigarette Smoking $\ldots \ldots \ldots \ldots \ldots \quad 248$

Other Data on Correlates and Trends ........... 250

\section{APPENDIX}

Estimates Adjusted for Absentees and Dropouts . . . . . 258

The Effects of Missing Absentees . . . . . . . . . . . $\quad 259$

The Effects of Missing Dropouts ............... 259

Summary and Conclusions ................ 265

Examples of Revised Estimates for Two Drugs ....... 265 


\section{LIST OF TABLES}

\section{HIGH SCHOOL STUDENTS}

1. Lifetime Prevalence (Percent Ever Used) of Sixteen Types of Drugs: Observed Estimates and 95\% Confidence Limits, Class of $1986 \ldots \ldots \ldots \ldots \ldots \ldots$

2. Lifetime Prevalence (Percent Ever Used) and Recency of Use of Sixteen Types of Drugs, Class of $1986 \ldots \ldots$

3. Lifetime Prevalence of Use of Sixteen Types of Drugs by Subgroups, Class of $1986 \ldots \ldots \ldots \ldots \ldots$

4. Annual Prevalence of Use of Seventeen Types of Drugs by Subgroups, Class of $1986 \ldots \ldots \ldots \ldots \ldots$

5. Thirty-Day Prevalence of Use of Sixteen Types of Drugs by Subgroups, Class of $1986 \ldots \ldots \ldots \ldots$

6. Thirty-Day Prevalence of Daily Use of Marijuana, Alcohol, and Cigarettes by Subgroups, Class of $1986 \ldots$

7. Trends in Lifetime Prevalence of Sixteen Types of

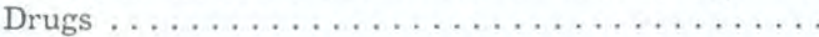

8. Trends in Annual Prevalence of Seventeen Types of Drugs .......................

9. Trends in Thirty-Day Prevalence of Sixteen Types of

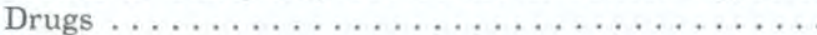

10. Trends in Thirty-Day Prevalence of Daily Use of Six-

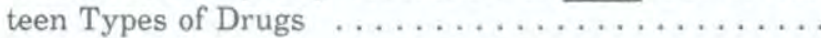

11. Trends in Lifetime, Annual, and Thirty-Day Prevalence in an Index of Illicit Drug Use $\ldots \ldots \ldots \ldots \ldots$

12. Trends in Noncontinuation Rates Among Seniors Who Ever Used Drug in Lifetime . . . . . . . . . .

13. Trends in Noncontinuation Rates Among Seniors Who Used Drug Ten or More Times in Lifetime . . . . . . .

14. Grade of First Use for Sixteen Types of Drugs, Class of

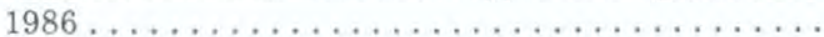

15. Trends in Perceived Harmfulness of Drugs ........ 
16. Trends in Proportions Disapproving of Drug Use .....

17. Trends in Attitudes Regarding Legality of Drug Use . .

18. Trends in Attitudes Regarding Marijuana Laws .....

19. Trends in Proportion of Friends Disapproving of Drug

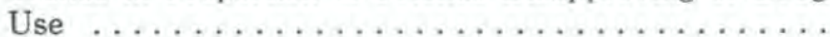

20. Trends in Proportion of Friends Using Drugs . . . . . .

21. Trends in Exposure to Drug Use ..............

22. Trends in Reported Availability of Drugs $\ldots \ldots \ldots \ldots$

\section{YOUNG ADULTS POST-HIGH SCHOOL}

23. Prevalence of Use of Thirteen Types of Drugs, 1986: Among Follow-Up Respondents 1-9 Years Beyond High School by Sex ......................

24. Trends in Annual Prevalence of Thirteen Types of Drugs: Among Follow-Up Respondents 1-9 Years Beyond High School ..................

25. Trends in Thirty-Day Prevalence of Twelve Types of Drugs: Among Follow-Up Respondents 1-9 Years Beyond High School . . . . . . . . . . . . . . . .

26. Trends in Thirty-Day Prevalence of Daily Use of Twelve Types of Drugs: Among Follow-Up Respondents 1-9 Years Beyond High School ............

27. Trends in Annual and Thirty-Day Prevalence of an Illicit Drug Use Index: Among Follow-Up Respondents 1-9 Years Beyond High School by Sex ..........

\section{COLLEGE STUDENTS}

28. Trends in Annual Prevalence of Thirteen Types of Drugs: Among College Students 1-4 Years Beyond High School . . . . . . . . . . . . . . . . .

29. Trends in Thirty-Day Prevalence of Twelve Types of Drugs: Among College Students 1-4 Years Beyond High School . . . . . . . . . . . . . . . . . . 
30. Trends in Thirty-Day Prevalence of Daily Use of Twelve Types of Drugs: Among College Students 1-4 Years Beyond High School .................

31. Trends in Annual and Thirty-Day Prevalence of an Illicit Drug Use Index: Among College Students 1-4 Years Beyond High School by Sex ...........

\section{OTHER FINDINGS FROM THE STUDY}

32. Various Stimulants: Trends in Lifetime, Annual, and Thirty-day Prevalence by Sex $\ldots \ldots \ldots \ldots \ldots \ldots$

33. Percent of Respondents in Each Category of an Illicit Drug Use Index Who Have Tried Various Over-theCounter Stimulants, Class of $1986 \ldots \ldots \ldots \ldots \ldots \ldots$

34. Daily Marijuana Use: Responses to Selected Questions by Subgroups, 1986 Seniors . . . . . . . . . . . .

35. Trends in Daily Use of Marijuana in Lifetime by Sub-

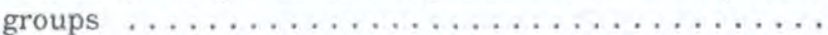

36. Smoking as Related to Average Academic Grades in High School, Class of $1985 \ldots \ldots \ldots \ldots \ldots \ldots \ldots$

37. Lifetime Use of Drugs by Five Categories of Smoking Behavior, Class of $1985 \ldots \ldots \ldots \ldots \ldots \ldots \ldots$

38. Annual Use of Drugs by Five Categories of Smoking Behavior, Class of $1985 \ldots \ldots \ldots \ldots \ldots \ldots \ldots$.

39. Thirty-Day Use of Drugs by Five Categories of Smoking Behavior, Class of $1985 \ldots \ldots \ldots \ldots \ldots \ldots$.

40. Daily Use of Drugs During the Last Thirty-Days by Five Categories of Smoking Behavior, Class of 1985 .. 


\section{LIST OF FIGURES}

$\underline{\text { Page }}$

1. Location of Schools Surveyed in $1986 \ldots \ldots \ldots \ldots \ldots$

\section{HIGH SCHOOL STUDENTS}

2. Prevalence and Recency of Use: Eleven Types of

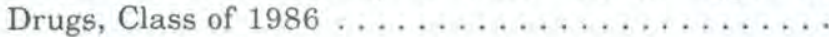

3. Thirty-Day Prevalence of Daily Use: Eleven Types of Drugs, Class of $1986 \ldots \ldots \ldots \ldots \ldots \ldots \ldots$

4. Noncontinuation Rates: Percent of Seniors Who Used Drug Once or More in Lifetime but Did Not Use in Past

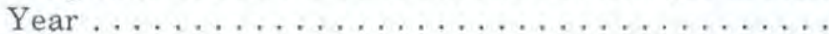

5. States Included in the Four Regions of the Country ...

6. Trends in Lifetime Prevalence of an Illicit Drug Use Index: All Seniors . . . . . . . . . . . . . . .

7. Trends in Annual Prevalence of an Hlicit Drug Use Index: All Seniors . . . . . . . . . . . . . . .

8. Trends in Thirty-Day Prevalence of an Illicit Drug Use Index: All Seniors . . . . . . . . . . . . . . . .

9. Trends in Lifetime, Annual, and Thirty-Day Prevalence of Sixteen Drugs

a. Marijuana and Stimulants $\ldots \ldots \ldots \ldots \ldots \ldots$

b. Tranquilizers, Inhalants, and Nitrites . . . . . .

c. Sedatives, Barbiturates, and Methaqualone .....

d. Hallucinogens, LSD, and PCP . . . . . . . . .

e. Cocaine, Other Opiates, and Heroin . . . . . . . .

f. Alcohol and Cigarettes ............... 64

10. Trends in Thirty-Day Prevalence of Daily Use of Marijuana, Alcohol, and Cigarettes by Sex .......

11. Trends in Two-Week Prevalence of Heavy Drinking

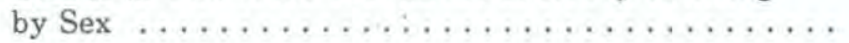

12. Trends in Annual Prevalence of an Illicit Drug Use Index by $\operatorname{Sex} \ldots \ldots \ldots \ldots \ldots \ldots \ldots \ldots$ 
13. Trends in Annual Prevalence of an Illicit Drug Use Index by College Plans . . . . . . . . . . . .

14. Trends in Annual Prevalence of an Illicit Drug Use Index by Region of the Country $\ldots \ldots \ldots \ldots \ldots$

15. Trends in Lifetime Prevalence of Cocaine Use by Region of the Country ...............

16. Trends in Annual Prevalence of an Illicit Drug Use Index by Population Density . . . . . . . . . .

17. Trends in Lifetime Prevalence for Earlier Grade Levels

a. Use of Any Illicit Drug . . . . . . . . . . . . .

b. Use of Any Illicit Drug Other than Marijuana ... 93

c. Use of Any Illicit Drug Other than Marijuana or

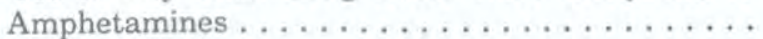

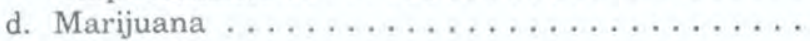

e. Cocaine .................... 96

f. Stimulants . . . . . . . . . . . . . . 97

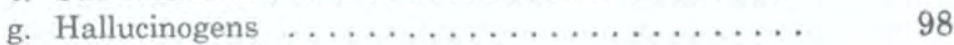

h. $\operatorname{LSD}_{\text {. }} \ldots \ldots \ldots \ldots \ldots \ldots \ldots \ldots \ldots \ldots \ldots \ldots$

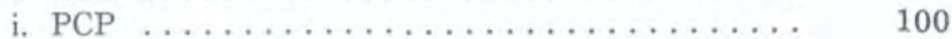

j. Inhalants . . . . . . . . . . . . . . 101

k. Nitrites . . . . . . . . . . . . . . . . . 102

1. Sedatives ................... 103

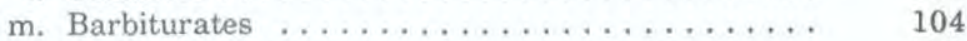

n. Methaqualone ................... 105

o. Tranquilizers ................... 106

p. Heroin .......................... 107

q. Other Opiates ................... 108

r. Cigarette Smoking on a Daily Basis ... . . . . . 109

s. Alcohol ....................... 110

18. Degree of Drug High Attained by Recent Users . . . . . 112

19. Duration of Drug High Attained by Recent Users ... 113

20. Trends in Perceived Harmfulness: Marijuana and Cigarettes . . . . . . . . . . . . . . .

21. Trends in Perceived Harmfulness: Other Drugs .....

22. Trends in Disapproval of Illicit Drug Use: Seniors,

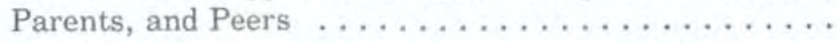

23. Trends in Disapproval of Licit Drug Use: Seniors,

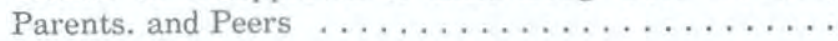


24. Proportion of Friends Using Each Drug as Estimated by Seniors, in $1986 \ldots \ldots \ldots \ldots \ldots \ldots \ldots \ldots \ldots \ldots \ldots \ldots \ldots$

25. Trends in Perceived Availability of Drugs ........

\section{YOUNG ADULTS POST-HIGH SCHOOL}

26-38. Lifetime, Annual, and Thirty-Day Prevalence Among Young Adults, 1986, by Age Group

26. Any Illicit Drug $\ldots \ldots \ldots \ldots \ldots \ldots \ldots$

27. Any Illicit Drug Other than Marijuana ......

28. Any Illicit Drug Other than Marijuana or

29. Marijuana (and Daily) .............

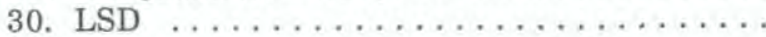

31. Cocaine ................... 168

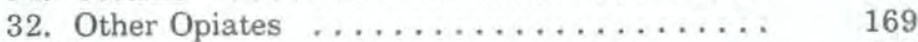

33. Stimulants . . . . . . . . . . . . . . . 170

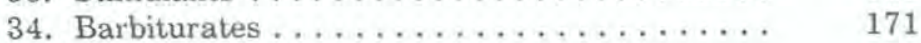

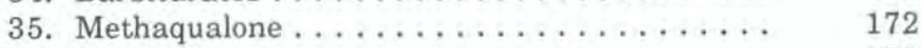

36. Tranquilizers ................. 173

37. Alcohol (Various Prevalence Rates) ........ 174

38. Cigarettes (Thirty-Day, Daily, and Half-Pack) 175

39-51. Trends in Annual Prevalence Among Young Adults by Age Group

39. Any Illicit Drug . . . . . . . . . . . . . 183

40. Any Illicit Drug Other than Marijuana . . . . . . 184

41. Any Illicit Drug Other than Marijuana or Stimulants ................

42. Marijuana

a. Annual . . . . . . . . . . . . . . . 186

b. Thirty-Day and Daily .......... 187

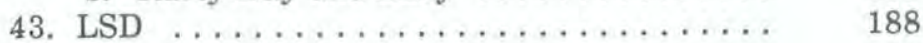

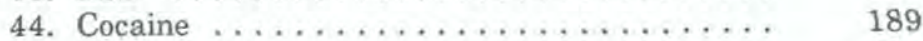

45. Other Opiates ................. 190

46. Stimulants . . . . . . . . . . . . . . . . 191

47. Barbiturates ... . . . . . . . . . . . . . . 192

48. Methaqualone .................. 193

49. Tranquilizers . . . . . . . . . . . . . . . 194

50. Alcohol

a. Annual . . . . . . . . . . . . . . . . . 195

b. Thirty-Day and Daily ... . . . . . . 196

c. Two-Week Prevalence of Five or More Drinks in a Row ............. 
51. Cigarettes

a. Thirty-Day and Daily ...........

b. Thirty-Day Use of Half-Pack-a-Day or

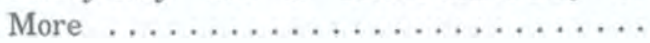

\section{COLLEGE STUDENTS}

52-64. Trends in Annual Prevalence Among College Students Vs. Others and Among Male and Female College Students 1-4 Years Beyond High School

52. Any Illicit Drug $\ldots \ldots \ldots \ldots \ldots \ldots \ldots$

53. Any Illicit Drug Other than Marijuana . . . . .

54. Any Illicit Drug Other than Marijuana or Stimulants ................ 212

55. Marijuana

a. Annual .................... 213

b. Daily ....................... 214

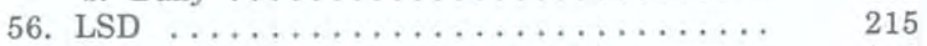

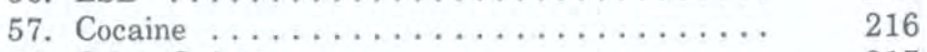

58. Other Opiates $\ldots \ldots \ldots \ldots \ldots \ldots \ldots \ldots \ldots \ldots$

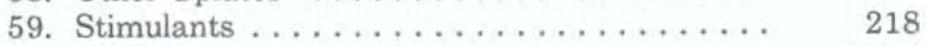

60. Barbiturates ................... 219

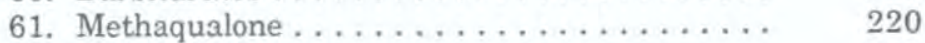

62. Tranquilizers . . . . . . . . . . . 221

63. Alcohol

a. Annual .................... 222

b. Daily ...................... 223

c. Two Week Prevalence of 5 or More Drinks

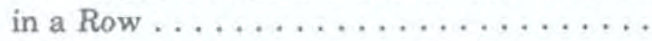

64. Cigarettes

a. Thirty-Day . . . . . . . . . . . . 225

b. Daily .................. 226

c. Thirty-Day Use of Half-Pack-a-Day or

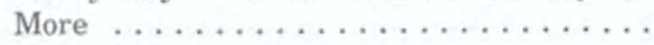

\section{OTHER FINDINGS FROM THE STUDY}

65. Prevalence and Recency of Use, by Sex: Amphetamines and Non-Prescription Stimulants, Class of $1986 \ldots \ldots \ldots \ldots \ldots \ldots \ldots \ldots \ldots \ldots \ldots \ldots \ldots \ldots \ldots \ldots \ldots \ldots$ 


\section{APPENDIX}

A-1. High School Completion by Persons 20-24 Years Old,

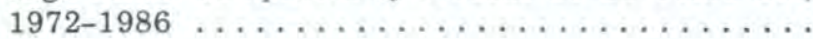

A-2. Estimates of Prevalence and Trends for the Entire Age/Class Cohort, Adjusting for Absentees and

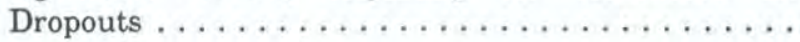




\section{INTRODUCTION}

This report is the tenth in an annual series reporting the drug use and related attitudes of America's high school seniors. The findings, which cover the high school classes of 1975 through 1986, come from an ongoing national research and reporting program entitled Monitoring the Future: A Continuing Study of the Lifestyles and Values of Youth. The program is conducted by the University of Michigan's Institute for Social Research, and is funded primarily by the National Institute on Drug Abuse. The study is sometimes referred to as the High School Senior Survey, since each year a representative sample of all seniors in public and private high schools in the coterminous United States is surveyed. However, the study also includes representative samples of young adults from previous graduating classes who are administered follow-up surveys by mail.

Published on a less frequent interval is a series of larger, more detailed volumes. The most recent was published by the National Institute on Drug Abuse in 1984 under the title Drugs and American High School Students: 1975-1983. In addition to presenting a full chapter of descriptive information for each of the various classes of drugs, each larger volume contains chapters on attitudes and beliefs about drugs and various relevant aspects of the social milieu, as well as several appendices dealing with validity, sampling error estimation, and survey instrumentation.

Two of the major topics which continue to be included in this present series of annual reports are the current prevalence of drug use among American high school seniors, and trends in use by seniors since the study began in 1975. Also reported are data on grade of first use, trends in use at earlier grade levels, intensity of drug use, attitudes and beliefs among seniors concerning various types of drug use, and their perceptions of certain relevant aspects of the social environment.

\section{THE INCLUSION OF COLLEGE STUDENTS AND YOUNG ADULTS GENERALLY}

Data on the prevalence and trends in drug use among young adults who have completed high school are also incorporated into this report. The period of young adulthood (late teens to the late twenties) is particularly important because this tends to be a time of peak levels of use for many 
drugs. The continuing epidemic of cocaine use among young adults also makes this an age group of particular policy importance.

The Monitoring the Future study design includes continuing follow-up panel studies of a subsample of the participants in each participating senior class, beginning with the class of 1976. Thus, data were gathered in 1986 on representative samples of the graduating classes of 1976 through 1985 , corresponding to modal ages of 19 to 28 .

Separate data are presented on college students specifically. This segment of the young adult population has not been well represented in national surveys to date, because many college students live on campus, in dormitories, fraternities, and sororities, and these group dwellings are not included in the national household survey population.

\section{CONTENT AREAS COVERED IN THIS REPORT}

Eleven separate classes of drugs are distinguished in this report: marijuana (including hashish), inhalants, hallucinogens, cocaine (including crack), heroin, natural and synthetic opiates other than heroin, stimulants (more specifically, amphetamines), sedatives, tranquilizers, alcohol, and cigarettes. (This particular organization of drug use classes was chosen to heighten comparability with a parallel series of publications based on the National Institute on Drug Abuse's national household surveys on drug abuse.) Separate statistics are also presented here for several sub-classes of drugs: PCP and LSD (both hallucinogens), barbiturates and methaqualone (both sedatives), and the amyl and butyl nitrites (both inhalants). PCP and the nitrites were added to our measurements for the first time in 1979 because of increasing concern over their rising popularity and possibly deleterious effects; trend data are thus only available for them since 1979. For similar reasons, "crack" cocaine was added to the 1986 survey and is included in this report for the first time. ${ }^{1}$ Barbiturates and methaqualone, which constitute the two components of the "sedatives" class as used here, have been separately measured from the outset. They have been presented separately because their trend lines are substantially different.

Except for the findings on alcohol, cigarettes, and nonprescription stimulants, practically all of the information reported here deals with illicit drug use. Respondents are asked to exclude any occasions on which they used any of the psychotherapeutic drugs under medical supervision. (Some data on the medically supervised use of such drugs are contained in the full 1977, 1978, 1981, and 1983 volumes.)

${ }^{1}$ See last section in the chapter entitled "Prevalence of Drug Use Among High School Seniors". 
Throughout this report we have chosen to focus considerable attention on drug use at the higher frequency levels rather than simply reporting proportions who have ever used various drugs. This is done to help differentiate levels of seriousness, or extent, of drug involvement. While there still is no public consensus of what levels or patterns of use constitute "abuse," there is surely a consensus that higher levels of use are more likely to have detrimental effects for the user and society than are lower levels. We have also introduced indirect measures of dosage per occasion, by asking respondents the duration and intensity of the highs they usually experience with each type of drug. One section of this report deals with those results.

For both licit and illicit drugs, separate sections of this report are devoted to age of first use; the seniors' own attitudes and beliefs; the attitudes, beliefs, and behaviors of others in the seniors' social environment; and perceived drug availability.

In 1982 we added a special section, under "Other Findings from the Study," dealing with the use of nonprescription stimulants, including diet pills, stay-awake pills, and the "look-alike" pseudo-amphetamines. Questions on these substances were placed in the survey beginning in 1982 because the use of such substances appeared to be on the rise, and also because their inappropriate inclusion by some respondents in their answers about amphetamine use were affecting the observed trends. The "Other Findings from the Study" section continues to present trend results on those nonprescription substances.

That section also presents trend results from a set of questions on the use of marijuana at a daily or near-daily level. These questions were added to enable us to develop a more complete individual history of daily use over a period of years, and they reveal some very interesting facts about the frequent users of this drug. The "Other Findings" section also presents a synopsis of results from the study reported in a recent journal article on trends in the medically supervised use of the psychotherapeutic drugs such as amphetamines, tranquilizers, sedatives, and narcotics. Finally, it includes a synopsis of original findings on some correlates of cigarette smoking contained in Congressional Testimony dealing with cigarette advertising which was delivered during the past year. Specifically, findings are presented relevant to the age of initiation of daily smoking, the addictive nature of smoking, its strong negative relationship with academic performance, and its strong positive relationship with the use of illicit drugs and alcohol.

\section{PURPOSES AND RATIONALE FOR THIS RESEARCH}

Perhaps no area has proven more clearly appropriate for the application of systematic research and reporting than the drug field, given its rapid rate of change, its importance for the well-being of the nation, and 
the amount of legislative and administrative intervention addressed to it. Young people are often at the leading edge of social change; and this has been particularly true in the case of drug use. The massive upsurge in illicit drug use during the last twenty-five years has proven to be primarily a youth phenomenon, with onset of use most likely to occur during adolescence. From one year to the next particular drugs rise or fall in popularity, and related problems occur for youth, for their families, for governmental agencies, and for society as a whole. This year's findings show that considerable change is still taking place.

One of the major purposes of the Monitoring the Future series is to develop an accurate picture of the current drug use situation and trends. Having a reasonably accurate assessment of the basic size and contours of the problem of illicit drug use among young Americans is a prerequisite for rational public debate and policy making. In the absence of reliable prevalence data, substantial misconceptions can develop and resources can be misallocated. In the absence of reliable data on trends, early detection and localization of emerging problems are more difficult, and assessments of the impact of major historical and policy-induced events are much more conjectural.

The Monitoring the Future study has a number of purposes in addition to prevalence and trend estimation-purposes which are not addressed in any detail in this volume. Among them are: gaining a better understanding of the lifestyles and value orientations associated with various patterns of drug use, and monitoring how those orientations are shifting over time; determining the immediate and more general aspects of the social environment which are associated with drug use and abuse; determining how drug use is affected by major transitions in social environment (such as entry into military service, civilian employment, college, unemployment) or in social roles (marriage, parenthood); distinguishing age effects from cohort and period effects in determining drug use; determining the effects of social legislation on all types of substance use; and determining the changing connotations of drug use and changing patterns of multiple drug use among youth. Readers interested in publications dealing with any of these other areas should write the authors at the Institute for Social Research, Room 2030, The University of Michigan, Ann Arbor, Michigan, 48106-1248.

\section{RESEARCH DESIGN AND PROCEDURES FOR THE SURVEYS OF SENIORS}

The data from high school seniors are collected during the spring of each year, beginning with the class of 1975. Each data collection takes place in approximately 125 to 135 public and private high schools selected to provide an accurate cross-section of high school seniors throughout the coterminous United States. (See Figure 1.) 


\section{FIGURE 1}

Location of Schools Surveyed in 1986

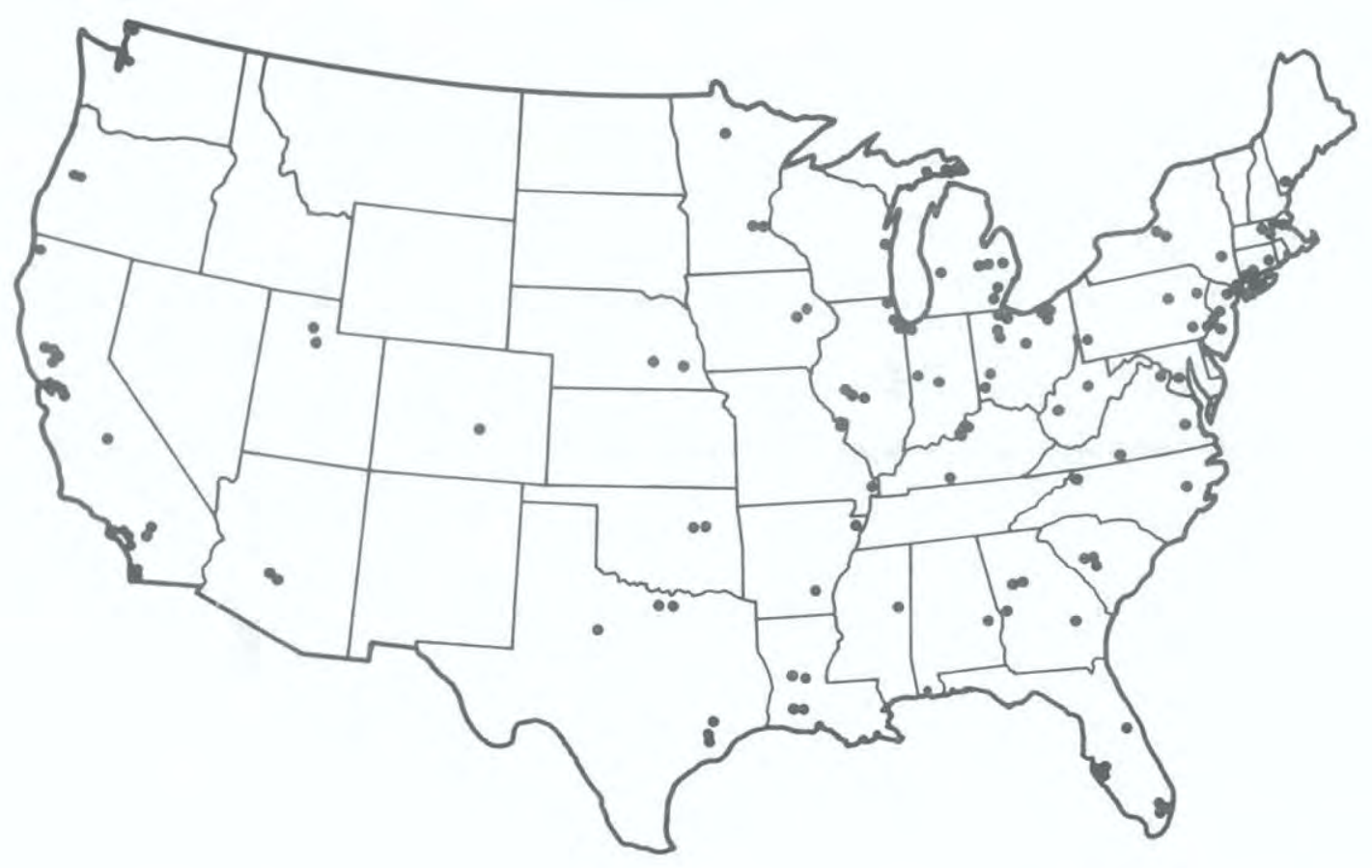


There are several reasons for choosing the senior year of high school as an optimal point for monitoring the drug use and related attitudes of youth. First, the completion of high school represents the end of an important developmental stage in this society, since it demarcates both the end of universal public education and, for many, the end of living in the parental home. Therefore, it is a logical point at which to take stock of the cumulated influences of these two environments on American youth. Further, the completion of high school represents the jumping-off point from which young people diverge into widely differing social environments and experiences. Finally, there are some important practical advantages to building a system of data collections around samples of high school seniors. The need for systematically repeated, large-scale samples from which to make reliable estimates of change requires that considerable stress be laid on efficiency as well as feasibility. The last year of high school constitutes the final point at which a reasonably good national sample of an age-specific cohort can be drawn and studied economically.

One limitation in the design is that it does not include in the target population those young men and women who drop out of high school before graduation-between 15 and 20 percent of each age cohort. The omission of high school dropouts does introduce biases in the estimation of certain characteristics of the entire age group; however, for most purposes, the small proportion of dropouts sets outer limits on the bias. Further, since the bias from missing dropouts should remain just about constant from year to year, their omission should introduce little or no bias into the various types of change being estimated for the majority of the population. ${ }^{2}$ Indeed, we believe the changes observed over time for those who finish high school are likely to parallel the changes for dropouts in most instances.

Sampling procedures. A multi-stage procedure is used for securing the nationwide sample of high school seniors each year. Stage 1 is the selection of particular geographic areas, Stage 2 the selection of one or more high schools in each area, and Stage 3 the selection of seniors within each high school.

This three-stage sampling procedure yielded the following numbers of participating schools and students:

${ }^{2}$ See the Appendix for a detailed discussion of the likely effects of the exclusion of dropouts on estimates of prevalence of drug use and trends in drug use among the entire age cohort. 


\begin{tabular}{|c|c|c|c|c|c|c|c|c|c|c|c|c|}
\hline & $\begin{array}{c}\text { Clnke } \\
\text { of } \\
1075\end{array}$ & $\begin{array}{c}\text { Claks } \\
\text { of } \\
1976\end{array}$ & $\begin{array}{c}\text { Clnks } \\
\text { of } \\
1977\end{array}$ & $\begin{array}{c}\text { Cinss } \\
\text { of } \\
\text { in78 }\end{array}$ & $\begin{array}{c}\text { Cines } \\
\text { of } \\
\text { ig79 }\end{array}$ & $\begin{array}{c}\text { Clnk5 } \\
\text { of } \\
\text { In80 }\end{array}$ & $\begin{array}{c}\text { Clnke } \\
\text { of } \\
1081\end{array}$ & $\begin{array}{c}\text { Clnsk } \\
\text { of } \\
\text { tn82 }\end{array}$ & $\begin{array}{c}\text { Clnes } \\
\text { of } \\
1083\end{array}$ & $\begin{array}{c}\text { Clnss } \\
\text { of } \\
1984\end{array}$ & $\begin{array}{c}\text { Class } \\
\text { of } \\
1085\end{array}$ & $\begin{array}{c}\text { Clnss } \\
\text { of } \\
1986 \\
\end{array}$ \\
\hline Number public nehonis & 111 & 108 & 108 & 111 & 111 & 107 & 109 & 116 & 112 & 117 & 115 & 113 \\
\hline Number peivate scliools & 14 & is & 16 & 20 & 20 & 20 & 10 & 21 & 22 & 17 & 17 & 16 \\
\hline ToLal number schools & 125 & 123 & 124 & 131 & 131 & 127 & 128 & 137 & 134 & 134 & 132 & 129 \\
\hline Totnl number ntudents & 15,791 & 16,678 & 18,430 & 18,924 & 16,602 & 10,524 & 18,267 & 18,348 & 16,947 & 16,499 & 16,502 & 15,713 \\
\hline Student responne rate & $78 \%$ & $77 \%$ & $79 \%$ & $83 \%$ & 825 & 825 & 813 & $83 \%$ & $84 \%$ & $83 \%$ & $84 \%$ & $83 \%$ \\
\hline
\end{tabular}

Questionnaire administration. About ten days before the administration students are given flyers explaining the study. The actual questionnaire administrations are conducted by the local Institute for Social Research representatives and their assistants, following standardized procedures detailed in a project instruction manual. The questionnaires are administered in classrooms during a normal class period whenever possible; however, circumstances in some schools require the use of larger group administrations.

Questionnaire format. Because many questions are needed to cover all of the topic areas in the study, much of the questionnaire content is divided into five different questionnaire forms (which are distributed to participants in an ordered sequence that ensures five virtually identical subsamples). About one-third of each questionnaire form consists of key or "core" variables which are common to all forms. All demographic variables, and nearly all of the drug use variables included in this report, are included in this "core" set of measures. Many of the questions dealing with attitudes, beliefs, and perceptions of relevant features of the social milieu are contained in only a single form, however, and are thus based on one-fifth as many cases (i.e., approximately 3,100 respondents in 1986 ).

\section{RESEARCH DESIGN FOR THE FOLLOW-UP SURVEYS AFTER HIGH SCHOOL}

Beginning with the graduating class of 1976, each class is being followed up annually after high school. From the approximately 17,000 seniors originally participating in a given class, a representative sample of 2,400 individuals is chosen for follow-up. In order to ensure sufficient numbers of drug users in the follow-up surveys, those fitting certain criteria of current drug use (that is, those reporting current daily marijuana use in senior year or use of any of the other illicit drugs in the previous 30 days) are selected with higher probability (by a factor of 3.0) than the remaining seniors. Differential weighting is used in all follow-up analyses to compensate for the differential sampling probabilities. 
The 2,400 selected respondents from each class are randomly assigned to one of two matching groups of 1,200 each; one group is surveyed on even-numbered calendar years, while the other group is surveyed on odd-numbered years. This two-year cycle is intended to reduce respondent burden.

\section{FOLLOW-UP PROCEDURES}

Using information provided by respondents at the time of the senior survey (name, address, phone number, and the name and address of someone who would always know how to reach them), we contact the students selected for the panels by mail. Newsletters are sent each year and name and address corrections are requested. Questionnaires are sent by certified mail in the spring of each year. A check for $\$ 5.00$, made out to the respondent, is attached to the front. Reminder letters and post cards go out at fixed intervals thereafter; finally, those not responding receive a prompting phone call from the Survey Research Center's phone interviewing facility in Ann Arbor. If requested, a second copy of the questionnaire is sent.

\section{PANEL RETENTION RATES}

To date the panel retention rates have remained quite high. In the first follow-up after high school, about $85 \%$ of the original panel returned questionnaires. The retention rate reduces ordinally with time, as might be expected; however, the 1986 panel retention from the Class of 1976-the oldest of the panels, now aged 28 and 10 years past high school-remains at $73.4 \%$.

Since attrition is to a modest degree associated with drug use, we have introduced corrections into the prevalence estimates presented here for the follow-up panels. These raise the prevalence estimates from what they would be uncorrected, but only slightly. We believe the resulting estimates to be the most accurate obtainable, but still low for the age group as a whole due to the omission of dropouts and absentees from the population covered by the original panels.

\section{REPRESENTATIVENESS AND VALIDITY}

School participation. Schools are invited to participate in the study for a two-year period. With only very few exceptions, each school in the original sample, after participating for one year of the study, has agreed to participate for a second year. Each year thus far, from 66 percent to 80 percent of the schools invited to participate initially have agreed to do so; for each school refusal, a similar school (in terms of size, geographic area, urbanicity, etc.) is recruited as a replacement. 
The selection of replacement schools almost entirely removes problems of bias in region, urbanicity, and the like, that might result from certain schools refusing to participate. Other potential biases are more subtle, however. If, for example, it turned out that most schools with "drug problems" refused to participate, that would seriously bias the sample. And if any other single factor were dominant in most refusals, that also might suggest a source of serious bias. In fact, however, the reasons for a school refusing to participate are varied and are often a function of happenstance events; only a small proportion specifically object to the drug content of the survey. Thus we feel quite confident that school refusals have not seriously biased the surveys.

Schools are selected in such a way that half of each year's sample is comprised of schools which participated the previous year, and half is comprised of schools which will participate the next year. This staggered half-sample design is used to check on possible biases in the yearto-year trend estimates derived from the full samples. Specifically, separate sets of one-year trends are computed using first that halfsample of schools which participated in both 1975 and 1976, then the half-sample which participated in both 1976 and 1977, and so on. Thus, each one-year trend estimate derived in this way is based on a set of about 65 schools. When the resulting trend data (examined separately for each class of drugs) are compared with trends based on the total sample of schools, the results are highly similar, indicating that the trend estimates are little affected by turnover or shifting refusal rates in the school samples. (The absolute prevalence estimates for a given year are not as accurate using just the half-sample.)

Student participation. Completed questionnaires are obtained from $77 \%$ to $84 \%$ of all sampled students in participating schools each year. The single most important reason that students are missed is absence from class at the time of data collection; in most cases it is not workable to schedule a special follow-up data collection for absent students. Students with fairly high rates of absenteeism also report above-average rates of drug use; therefore, there is some degree of bias introduced into the prevalence estimates by our missing the absentees. Much of that bias could be corrected through the use of special weighting; however, we decided not to do so because the bias in overall drug use estimates was determined to be quite small, and because the necessary weighting procedures would have introduced undesirable complications. (Appendix $\mathrm{A}$ of the most recent detailed report ${ }^{3}$ provides a discussion of this point and the Appendix to this report shows trend and prevalence estimates which would result with corrections for absentees included.)

${ }^{3}$ Johnston, L.D., O'Malley, P.M., \& Bachman, J.G. (1984). Highlights from drugs and American high school students: 1975-1983. (DHHS Publication No. ADM 85-1374.) Washington, D.C.: U.S. Government Printing Office. 
Of course, some students are not absent from class, but simply refuse when asked to complete a questionnaire. However, the proportion of explicit refusals amounts to less than 1 percent of the target sample.

Sampling accuracy of the estimates. For purposes of this introduction, it is sufficient to note that drug use estimates based on the total sample of seniors each year have confidence intervals that average about $\pm 1 \%$ (as shown in Table 1, confidence intervals vary from $\pm 2.2 \%$ to smaller than $\pm 0.3 \%$, depending on the drug). This means that had we been able to invite all schools and all seniors in the 48 coterminous states to participate, the results from such a massive survey should be within about one percentage point of our present findings for most drugs at least 95 times out of 100 . We consider this to be a high level of accuracy, and one that permits the detection of fairly small changes from one year to the next.

\section{VALIDITY OF THE MEASURES OF SELF-REPORTED DRUG USE}

A question which always arises in the study of sensitive behaviors like drug use is whether honest reporting can be secured. Like most studies dealing with sensitive behaviors, we have no direct, objective validation of the present measures; however, the considerable amount of inferential evidence that exists strongly suggests that the self-report questions produce largely valid data. A more complete discussion of the contributing evidence which leads to this conclusion may be found in other publications; here we will only briefly summarize the evidence. ${ }^{4}$

First, using a three wave panel design, we established that the various measures of self-reported drug use have a high degree of reliability-a necessary condition for validity. ${ }^{5}$ In essence, this means that respondents were highly consistent in their self-reported behaviors over a three- to four-year time interval. Second, we found a high degree of consistency among logically related measures of use within the same questionnaire administration. Third, the proportion of seniors reporting some illicit drug use by senior year has reached two-thirds of all

${ }^{4}$ Johnston, L.D., \& O'Malley, P.M. (1985). Issues of validity and population coverage in student surveys of drug use. In B.A. Rouse, N.J. Kozel, \& L.G. Richards (Eds.), Self-report methods of estimating drug use: Meeting current challenges to validity (NIDA Research Monograph No. 57; (ADM) 851402). Washington, D.C.: U.S. Government Printing Office; Johnston, L.D., O'Malley, P.M., \& Bachman, J.G. (1984). Drugs and American high school students: 1975-1983 (NIDA (ADM) 80-976). Washington, D.C.: U.S. Government Printing Office.

${ }^{5}$ O'Malley, P.M., Bachman, J.G., \& Johnston, L.D. (1983). Reliability and consistency in self-reports of drug use. International Journal of the Addictions, $18,805-824$. 
respondents in peak years and nearly as high as $80 \%$ in some follow-up years, which constitutes prima facie evidence that the degree of underreporting must be very limited. Fourth, the seniors' reports of use by their friends about which they would presumably have less reason to distort-has been highly consistent with self-reported use in terms of both prevalence and trends in prevalence, as will be discussed later in this report. Fifth, we have found self-reported drug use to relate in consistent and expected ways to a number of other attitudes, behaviors, beliefs, and social situations-in other words, there is strong evidence of "construct validity." Sixth, the missing data rates for the self-reported use questions are only very slightly higher than for the preceding nonsensitive questions, in spite of the instruction to respondents to leave blank those drug use questions they felt they could not answer honestly. And seventh, the great majority of respondents, when asked, say they would answer such questions honestly if they were users.

This is not to argue that self-reported measures of drug use are valid in all cases. In the present study we have gone to great lengths to create a situation and set of procedures in which students feel that their confidentiality will be protected. We have also tried to present a convincing case as to why such research is needed. We think the evidence suggests that a high level of validity has been obtained. Nevertheless, insofar as there exists any remaining reporting bias, we believe it to be in the direction of underreporting. Thus, we believe our estimates to be lower than their true values, even for the obtained samples, but not substantially so.

Consistency and the measurement of trends. One further point is worth noting in a discussion of the validity of the findings. The Monitoring the Future project is designed to be sensitive to changes from one time to another. Accordingly, the measures and procedures have been standardized and applied consistently across each data collection. To the extent that any biases remain because of limits in school and/or student participation, and to the extent that there are distortions (lack of validity) in the responses of some students, it seems very likely that such problems will exist in much the same way from one year to the next. In other words, biases in the survey estimates will tend to be consistent from one year to another, which means that our measurement of trends should be affected very little by any such biases. The smooth and consistent nature of most trend curves reported for the various drugs provides rather compelling empirical support for this assertion.

\section{A NOTE ABOUT THE STIMULANT RESULTS FOR 1979-1982}

In reporting their psychotherapeutic drug use, respondents are instructed to exclude not only medically-supervised use, but also any use of over-the-counter (i.e., nonprescription) drugs. However, begin- 
ning in about 1979 we believe that some of those reporting stimulant (amphetamine) use were erroneously including the use of over-thecounter stay-awake and diet pills, as well as other pills intentionally manufactured to look like amphetamines, and sold under names which sound like them, but which contain no controlled substances. The advertising and sale of over-the-counter diet pills (most of which contain the mild stimulant phenylpropanolamine) burgeoned at about that time, as was also true for the "sound-alike, look-alike" pills (most of which contain caffeine). We believe that the inappropriate inclusion of these noncontrolled stimulants in the responses to our surveys accounted for much of the observed sharp rise in reported "amphetamine" use in 1980 and 1981. Therefore, the reader is advised to view the unadjusted amphetamine-use statistics for those years with some caution.

In the 1982 survey, some new questions were introduced on the use of both controlled and noncontrolled stimulants. (We also kept the old version of the question in two questionnaire forms in the high school surveys so that it would be possible to "splice" the trend lines resulting from the old and new questions.) Since 1982 we have included statistics on "amphetamines, adjusted" - which are based on these new questions contained in three questionnaires in 1982 and 1983 and then in all five questionnaires in 1984 and thereafter. We believe these questions have been successful at getting respondents to exclude over-the-counter stimulants and those "look-alike" stimulants which the user knows are look-alikes. However, as is true with several other drug classes, the user may at times be ingesting a substance other than the one he or she thinks it to be. Thus, some erroneous self-reports of "amphetamine" use may remain.

An upward bias from the inclusion of over-the-counter and look-alike stimulants would have affected not only the stimulant (amphetamine) trend statistics in the years in question, but also trend statistics for the composite indexes entitled "use of any illicit drug" and "use of any illicit drug other than marijuana." Since these indexes had been used consistently in this monograph series to compare important subgroups (such as those defined by sex, region, college plans, etc.) we decided to keep them, but to include an adjusted value based on calculations in which amphetamines have been excluded. In other words, this adjusted statistic reflects "use of any illicit drugs other than marijuana or amphetamines," and is included to show what happens when amphetamine use-and any upward biases in trends it might containis excluded entirely from the trend statistics since 1975.

A second adjusted statistic has also been included since 1982, when the revised amphetamine questions were introduced. It gives our best estimate of overall illicit drug use, including the use of real amphetamines as measured by the revised amphetamine questions. A $\triangleleft$ symbol is used to denote this estimate in any figures presenting data on these two illicit drug use indexes, whereas a 4 symbol is used to 
denote estimates in which amphetamines are excluded entirely. (See Figure 6 for an example.)

It is worth noting that these two classes of drug use (over-the-counter and look-alike stimulants) which are not actually amphetamine use, but which are sometimes inadvertently reported as amphetamine use, reflect two quite different types of behavior. Presumably most users of over-the-counter diet and stay-awake pills are using them for functional reasons and not for recreational purposes. On the other hand, it seems likely that most users of the look-alike pseudo-amphetamines are using them for recreational purposes. (In fact, in many cases the user who purchased them on the street may think he or she has the real thing.) Thus, the inclusion of the look-alikes may have introduced a bias in the estimates of true amphetamine use, but not in the estimates of a class of behavior-namely, trying to use controlled stimulants for recreational purposes. Some would argue that the latter is the more important factor to be monitoring in any case. 


\section{OVERVIEW OF KEY FINDINGS}

This monograph reports findings from the ongoing research and reporting project entitled Monitoring the Future: A Continuing Study of the Lifestyles and Values of Youth. Each year since 1975, in-school surveys of nationally representative samples of high school seniors have been conducted. In addition, in each year since 1976, representative subsamples of the participants from each previously graduating class have been surveyed by mail.

Findings on the prevalence and trends in drug use and related factors are reported for high school seniors and also for young adult high school graduates 19-28 years old. Trend data are presented for varying time intervals, ranging from eleven years for the high school senior surveys (18 year olds over the interval 1975-1986) to one year for the oldest age band (27-28 year olds over the interval 1985-1986). Results are given separately for college students, a particularly important subset of this young adult population for which there currently exist no other nationally representative data.

A number of important findings emerge from these three national subpopulations-high school seniors, young adults through age 28 , and college students.

- One very encouraging finding from the 1986 survey is that the stall observed in 1985 in the longer-term downward trend in the use of any illicit drugs, the use of marijuana, and the use of any illicit drugs other than marijuana turned out to be just that-a stall. The downturn resumed again in 1986 among all three sub-populations: that is, high school seniors, college students, and young adults generally. The fact that the stall occurred in all three groups leads us to conclude that it was real, though we have no ready explanation for why it occurred.

- The annual prevalence of marijuana use among seniors fell to the lowest level since the study began (39\%, down $2 \%$ from 1985). Among young adults one to nine years past high school, it fell by $3 \%$ to $37 \%$. The two groups have identical rates of daily marijuana use $(4.0 \%)$, and both are down significantly from 1985. For seniors this represents a nearly two-thirds overall drop in daily use from the peak level of $10.7 \%$, observed in 1978 . College students reached a daily 
use rate of only $2.1 \%$ in 1986 (down from our first reading in 1980 of $7.2 \%$ ).

- Less encouraging is the fact that cocaine use has shown little sign of dropping from its peak levels. Annual prevalence is $6 \%$ among high school students, $17 \%$ among college students, and 20\% among all young adults (including college students) one to nine years past high school. While all of these rates dropped a fraction of a percent in 1986, none was down significantly. Although a growing proportion of seniors are reaching the conclusion that regular cocaine use entails "great risk" for the user (up from $69 \%$ in 1980 to $82 \%$ in 1986), there has been little change in the perceived risk of experimenting with the drug (up only $2 \%$ from 1980 to $34 \%$ in 1986). Only $54 \%$ think there is much risk associated with occasional use.

As with all the illicit drugs, lifetime cocaine prevalence climbs with age, actually reaching $40 \%$ by age 27 to 28 . Unlike all of the other illicit drugs, active use-i.e., use during the past year or past month-also climbs substantially after high school, as the annual prevalence figures mentioned above indicate.

- Another troublesome part of the cocaine story is to be found in a dangerous shift in the mode of administration being used, undoubtedly in large part due to the advent of crack cocainean inexpensive, purified, smokeable form of the drug. We have found: (a) that the proportion of seniors who say that they smoked cocaine more than doubled between 1983 and 1986 , from $2.5 \%$ to $6.0 \%$; (b) that the proportion of seniors reporting daily cocaine use in the month prior to the survey doubled between 1984 and 1986 , from $0.2 \%$ to $0.4 \%$; and (c) that the proportion of seniors who said that they had both used cocaine in the past year and had been unable to stop using at some time also doubled between 1983 and 1986, from $0.4 \%$ to $0.8 \%$,

For the first time this year we asked specifically about crack use. Seniors who reported any cocaine use in the prior twelve months were asked if they had ever used crack. Some $4.1 \%$ indicated that they had. (A more extensive set of questions about crack will be included in future surveys.) An examination of subgroup differences showed crack use to be particularly concentrated among the noncollege-bound and, as is true for cocaine generally, considerably more prevalent in the Northeast and West, than in the South or North Central. It also tends to be more concentrated in the most urban areas, but its use is by no means confined to a few major cities. 
(See Table 4.) In fact, we find that about half of the high schools in the study showed some reported crack use. Among all young adults who are high school graduates, the annual prevalence of crack is $3.2 \%$, slightly lower than among high school seniors. College students are much less likely to report use of crack compared to other high school graduates of the same age; $1.3 \%$ of college students repcrted use in the past twelve months versus $4.3 \%$ of their noncollege age peers.

- Another widely used class of illicit drugs showing an important shift in 1986 is stimulants (or more specifically, amphetamines). There were sizeable declines in use among all three sub-populations in 1986, which continued a longerterm trend that began in 1982. Since 1982, annual prevalence has fallen from $20 \%$ to $13 \%$ among seniors and from $21 \%$ to $10 \%$ among college students. In general, the decline has been sharper among young adults, including college students, than among high school seniors. (This has also been true for marijuana, LSD, and methaqualone.)

Concurrent with this drop in amphetamine use is a sharp increase in the use of over-the-counter stay-awake pills, which usually contain caffeine as their active ingredient. Their annual prevalence has risen from $12 \%$ in 1982 to $22 \%$ in 1986 .

The other two classes of nonprescription stimulants-the "look alikes" and the over-the-counter diet pills-have actually shown some fall-off in recent years. Still, $40 \%$ of young women have tried diet pills by the end of senior year, $23 \%$ have used them in the past year, and $10 \%$ in just the past month.

- $L S D$ use has been fairly constant over the last several years in all three sub-populations, following a period of some decline. (While the annual college prevalence rose significantly in 1986, this merely offset a dip in 1985: usage levels are still the same as in 1983 and 1984.)

- PCP use also has been constant for several years among high school seniors at quite a low level (annual prevalence of $2.4 \%$ in 1986), having fallen earlier from a high of $7.0 \%$ in 1979 . (PCP is not reported for the follow-up surveys, because it is included in only one questionnaire form, yielding too few cases.)

- The annual prevalence of heroin use has been very steady since 1979 among seniors at $0.5 \%$ to $0.6 \%$. (It had earlier fallen from $1.0 \%$ in 1975.) The heroin statistics for young 
adults and college students have also remained quite stable in recent years at low rates (about $0.2 \%$ ).

- The use of opiates other than heroin has been quite level over the life of the study. Seniors have had an annual prevalence rate of $5 \%$ or $6 \%$ since 1975 . Young adults in their twenties have generally shown a similar cross-time pattern.

- After a long and substantial decline which began in 1977, tranquilizer use among high school seniors appears to have stabilized in the last several years at around $6 \%$ annual prevalence (compared to $11 \%$ in 1977), at about $5 \%$ for the young adult sample, and at about $4 \%$ for the college student sample.

- Similarly, the long-term decline in barbiturate use, which began at least as early as 1975 , appears to have pretty much run its course, with the annual prevalence among seniors at $4.2 \%$ in 1986 (compared to $10.7 \%$ in 1975). Annual prevalence of this class of sedative drugs is even lower among the young adult sample $(2.4 \%)$, and among college students specifically $(2.1 \%)$. None of these groups showed a significant change in 1986.

- Methaqualone, another sedative drug, has shown quite a different trend pattern. Its use rose steadily among seniors from 1975 to 1981 , when annual prevalence reached $8 \%$. It then fell rather sharply to $2 \%$ by 1986 , including a significant drop in 1986. In recent years, shrinking availability apparently played a role in this drop, as legal manufacture and distribution of the drug ceased.

- The reported use of inhalants (adjusted) has risen some over the past several years among seniors, with annual prevalence going from $6.2 \%$ in 1983 to $8.9 \%$ in 1986 . This is due in part to a slight rise in the use of the amyl and butyl nitrites, for which the annual prevalence rose from $3.6 \%$ in 1983 to $4.7 \%$ in 1986.

- As a result of these various changes, the three classes of illicitly used drugs which now impact on appreciable proportions of young Americans in their late teens and twenties are marijuana, cocaine, and stimulants. Among high school seniors they show annual prevalence rates in 1986 of $39 \%$, $13 \%$, and $13 \%$ respectively. Among college students the comparable annual prevalence rates in 1986 are $41 \%, 17 \%$, and $10 \%$; and for all high school graduates one to nine years post high school (the "young adult" sample) they are 37\%, 20\%, and $11 \%$. 
- A number of additional interesting findings emerge from the sections in this report dealing with age-related changes in use. One is that the already high proportion of young people who by senior year have at least tried any illicit drug (58\% in 1986) grows substantially larger up through the midtwenties (where it reaches nearly $80 \%$ in 1986). There is a similar rise in the proportion using any illicit drug other than marijuana (38\% among seniors in 1986 vs. about $60 \%$ among those in their mid-twenties). Lifetime prevalence for marijuana reaches about $75 \%$ by the mid-twenties (vs. $51 \%$ among 1986 seniors) and for cocaine about $40 \%$ (vs. $17 \%$ among 1986 seniors).

On the other hand, active illicit drug use among the older age groups has tended to approximate the levels observed among seniors. This has been true for the annual prevalence of any illicit drug, marijuana, and tranquilizers. It has also been true for daily marijuana use. In fact, the young adult sample actually has lower rates of annual prevalence than high school seniors on five drugs-LSD, methaqualone, barbiturates, stimulants and opiates other than heroin. Cocaine, of course, is the exception in that active use rises until about age 22 , where it reaches a plateau.

- American college students (one to four years past high school), when compared to all high school graduates their age, show annual usage rates for a number of drugs which are about average, including any illicit drug, any illicit drug other than marijuana, marijuana specifically (although their rate of daily marijuana use is below average for their age group), cocaine, methaqualone, and opiates other than heroin. For several drugs, however, they have rates of use which are below average for their age group, including LSD, stimulants, barbiturates, and tranquilizers (although differences between the two groups on the last two drugs have just about been eliminated).

Since college-bound seniors in high school had tended to have lower rates of use on all of these illicit drugs, their eventually attaining parity on some of them reflects a "catching up" to some degree. As some results from the study published elsewhere have shown, the "catching up" may be explainable more in terms of differential rates of leaving the parental home and of getting married than in terms of any direct effects of college per se. (College students are more likely to leave the parental home and less likely to get married than their age peers.) 
- In general, the trends since 1980 in illicit substance use among American college students are found to parallel those for their age group as a whole. That means that for most drugs there has been a decline in use over the five-year interval. Further, all young adult high school graduates through age 27 , as well as college students taken separately, show trends which are highly parallel for the most part to the trends among high school seniors, although declines in the active use of many of the drugs over the past half decade have been proportionately larger in these two older populations than among high school seniors (particularly the declines in LSD and stimulant use).

- Regarding sex differences in the three populations, males are more likely to use most illicit drugs, and the differences tend to be largest at the higher frequency levels. Daily marijuana use among high school seniors in 1986, for example, is reported by $5.7 \%$ of males vs. $2.3 \%$ of females; among all young adults by $5.3 \%$ of males vs. $2.9 \%$ of females; and among college students, specifically, by $2.8 \%$ of males vs. $1.5 \%$ of females. The only exceptions to the rule that males are more frequently users of illicit drugs than females occur for stimulant use in high school, where females are slightly higher. The sexes attain near parity on tranquilizer use, as well, in all three sub-groups.

Insofar as there have been differential trends for the two sexes among any of these populations, they have been in the direction of a diminution of differences between the sexes. For college students, previous differences in the usage rates for methaqualone, LSD and daily marijuana use are disappearing as the prevalence rates for both sexes converge toward zero (which means that use by males has fallen more). The same is happening for daily marijuana use use among young adults generally, as well as high school seniors. There is also some convergence between the sexes in stimulant use among all three sub-populations. The convergence is again due to a faster drop in use among males.

- Regarding alcohol use in these age groups, several findings are noteworthy. First, during the period of recent decline in the use of marijuana and other drugs there appears not to have been any "displacement effect" in terms of any increase in alcohol use among seniors. (It was not uncommon to hear such a displacement hypothesis asserted.) In fact, the opposite seems to be true. Since 1980, the monthly prevalence of alcohol use among seniors has gradually declined, from $72 \%$ in 1980 to $65 \%$ in 1986 . Daily use declined from a peak of $6.9 \%$ in 1979 to $4.8 \%$ in 1984 (with no further 
decline though 1986); and the prevalence of drinking five or more drinks in a row during the prior two-week interval fell from $41 \%$ in 1983 to $37 \%$ in 1985 (with no further drop in 1986).

There remains a quite substantial sex difference among high school seniors in the prevalence of occasions of heavy drinking ( $28 \%$ for females vs. $46 \%$ for males in 1986), but this difference has been diminishing very gradually since the study began over a decade ago.

The data from college students, however, show a somewhat different pattern in relation to alcohol use. They show very little drop off in monthly prevalence since 1980 (about $2.0 \%$ ), about the same drop in daily use as among seniors (from $6.5 \%$ in 1980 to $4.6 \%$ in 1986 ) and roughly a $1 \%$ to $2 \%$ increase in the prevalence of occasions of heavy drinking, which is at $45 \%$ in 1986 -appreciably higher than the $37 \%$ among high school seniors.

The $45 \%$ figure is also higher than the rate observed among their age peers (i.e., those one to four years past high school) not in college $(38 \%)$, which means that college students are well above average on occasions of heavy drinking. Since the college-bound seniors in high school are consistently less likely to report occasions of heavy drinking than the noncollegebound, this reflects a "catching up and passing" their peers after high school.

A more detailed analysis shows that the divergent trends between high school students and college students in occasions of heavy drinking is due to an increase (since 1982) among male college students specifically. (The proportion of them reporting five or more drinks in a row rose from $52 \%$ in 1982 to $58 \%$ in 1986.) Female college students, if anything, showed some decline in such behavior over the same time interval (from $37 \%$ in 1982 to $34 \%$ in 1986). Thus an already large sex difference at the college level has become even larger. (There has not been a comparable increase among noncollege males to that observed among college males.)

College students overall have a daily drinking rate $(4.6 \%)$ which is below that of their age peers $(6.6 \%)$, suggesting that they are somewhat more likely to confine their drinking to weekends, on which occasions they tend to drink a lot. (Again, college men have much higher rates of daily drinking than college women: $6.4 \%$ vs. $3.1 \%$.) The rate of daily drinking has fallen among college students from $6.5 \%$ in 1980 to 
$4.6 \%$ in 1986. A similar drop has been occurring among their noncollege peers.

In sum, heavy party drinking among males in college is common and is becoming more common. Among high school students, however, there has been a recent decline in such behavior. Sex differences in occasions of heavy drinking appear to be diminishing somewhat at the high school level at the same time that they are enlarging at the college level.

- A number of important findings have emerged from the study concerning cigarette smoking among American adolescents and young adults. Of greatest importance is the fact that by late adolescence sizeable proportions of young people still are establishing regular cigarette habits, despite the demonstrated health risks associated with smoking. In fact, since the study began in 1975 , cigarettes have comprised the class of substance most frequently used on a daily basis by high school students. While their daily smoking rate did drop considerably between 1977 and 1981 (from 29\% to 20\%), it has dropped very little in the five years since (by another $1.6 \%$ ), despite the appreciable downturn which has occurred in most other forms of drug use (including alcohol) during this period. And, despite all the adverse publicity and restrictive legislation addressed to the subject during the eighties, the proportion of seniors who perceive "great risk" to the user of suffering physical (or other) harm from pack-a-day smoking has risen only $2 \%$ since 1980 (to $66 \%$ in 1986). That means that a third of American adolescents still do not feel there is a great risk associated with smoking.

Initiation of daily smoking most often occurs in grades 7 through 9 (i.e., at modal ages 12 to 14), with rather little further initiation after high school (although a number of light smokers make the transition to heavy smoking in the first two years after high school). Analyses presented in this volume and elsewhere have shown that cigarette smoking shows a dramatic "cohort effect." That is, if a class (or birth) cohort establishes an unusually high rate of srnoking at an early age relative to other cohorts, it is likely to remain high throughout the life cycle.

As we report in the section on "Other Findings from the Study," some $53 \%$ of the half-pack-a-day (or more) smokers in senior year say that they have tried to quit smoking and found they could not. Of those who are daily smokers in high school, nearly three-quarters are daily smokers 7 to 9 years later (based on the 1985 survey), despite the fact that in high school only $5 \%$ of them thought they would "definitely" be 
smoking 5 years hence. Clearly, the smoking habit is established at an early age and is difficult to break for those young people who have it.

Smoking bears a strong negative relationship with academic performance and a strong positive relationship with the use of the illicit drugs (marijuana in particular) and with alcohol use. For example, of the current pack-a-day smokers in high school, $95 \%$ have used an illicit drug, $81 \%$ have used an illicit drug other than marijuana, and $26 \%$ are current daily users of some illicit drug (mostly marijuana). The college-bound in high school, and those in college, are much less likely to smoke than their age peers. Females are a little more likely than their male counterparts to smoke in high school and in young adulthood for those not in college; among college students, females are considerably more likely to be smokers than males.

- To summarize, over the last six years there has been an appreciable decline in the use of a number of the illicit drugs among seniors, and even larger declines in their use among American college students and young adults more generally. However, in 1985 there was a stall in these favorable trends in all three populations, as well as an increase in active cocaine use. In 1986 we saw the general decline resume and the prevalence of cocaine level off, albeit at peak levels. However, there have been some worrisome trends since 1983 among those using cocaine in mode of administration, rates of daily use, and an indicator of dependence.

- While the overall picture has improved considerably in the past six years, the amount of illicit as well as licit drug use among America's younger age groups is still striking when one takes into account the following facts:

By their mid-twenties, nearly $80 \%$ of today's young adults have tried an illicit drug, including some $60 \%$ who have tried some illicit drug other than (usually in addition to) marijuana. Even for high school seniors these proportions still stand at $58 \%$ and $38 \%$, respectively.

By age 27 , roughly $40 \%$ have tried cocaine. As early as the senior year of high school, some $17 \%$ have done so.

One in twenty-five high school seniors in 1986 smokes marijuana daily, and roughly the same proportion of young adults aged 19 to $27 \mathrm{do}$, as well. Of the 
seniors $15 \%$ had been daily marijuana smokers at some time, and of the young adults, $20 \%$.

About one in twenty seniors drinks alcohol daily, and some $37 \%$ have had five or more drinks in a row at least once in the prior two weeks. Even more young adults one to four years past high school report such recent occasions of heavy drinking, and the prevalence of such behavior among male college students reaches $58 \%$.

Some $30 \%$ of seniors have smoked cigarettes in the month prior to the survey and $19 \%$ are daily smokers. In addition, many of the lighter smokers will convert to heavy smoking after high school. For example, one in every four young adults aged 19 to 27 are daily smokers $(25 \%)$, and one in five $(20 \%)$ smoke a halfpack-a-day or more. (Further, the inclusion of high school dropouts would raise those proportions considerably.)

- This nation's high school students and other young adults still show a level of involvement with illicit drugs which is greater than can be found in any other industrialized nation in the world. Even by historical standards in this country, these rates still remain extremely high. Their occasions of heavy drinking are also very high and of public health concern, as is the continuing initiation of large proportions of young people to cigarette smoking. 


\section{HIGH SCHOOL SENIORS}




\section{PREVALENCE OF DRUG USE AMONG HIGH SCHOOL SENIORS}

This section summarizes the levels of drug use reported by the high school class of 1986. Data are included for lifetime use, use during the past year, use during the past month, and daily use. There is also a comparison of key subgroups in the population (based on sex, college plans, region of the country, and population density or urbanicity).

Because we think that the revised questions on amphetamine use, introduced in 1982, give a more accurate picture of the actual use of that controlled substance, all references to amphetamine prevalence rates in this section will be based on that revised version (including references to proportions using "any illicit drug" or "any illicit drug other than marijuana").

It should be noted that all of the prevalence statistics given in this section are based on participating seniors only. Prevalence rate estimates reflecting adjustments for absentees and dropouts may be found in the Appendix to this report.

\section{PREVALENCE OF DRUG USE IN 1986: ALL SENIORS}

\section{Lifetime, Annual, and Monthly Prevalence}

- Nearly three-fifths of all seniors (58\%) report illicit drug use (using the revised definition of amphetamines) at some time in their lives. However, a substantial proportion of them have used only marijuana ( $20 \%$ of the sample or $34 \%$ of all illicit users).

- Nearly four in every ten seniors (38\%) report using an illicit drug other than marijuana at some time. ${ }^{6}$

- Table 1 provides the $95 \%$ confidence interval around the lifetime prevalence estimate for each drug, and Figure 2 gives a ranking of the various drug classes on the basis of their lifetime prevalence figures.

${ }^{6}$ Use of "other illicit drugs" includes any use of hallucinogens, cocaine, or heroin or any use of other opiates, stimulants, sedatives, or tranquilizers that is not under a doctor's orders. 
TABLE 1

\section{Lifetime Prevalence (Percent Ever}

Used) of Sixteen Types of Drugs:

Observed Estimates and $\mathbf{9 5 \%}$ Confidence Limits

Class of 1986

(Approx. $\mathrm{N}=15200$ )

\begin{tabular}{|c|c|c|c|}
\hline & $\begin{array}{l}\text { Lower } \\
\text { limit }\end{array}$ & $\begin{array}{l}\text { Observed } \\
\text { estimate }\end{array}$ & $\begin{array}{l}\text { Upper } \\
\text { limit }\end{array}$ \\
\hline Marijuana/Hashish & 48.7 & 50.9 & 53.1 \\
\hline $\begin{array}{l}\text { Inhalants }{ }^{\mathrm{a}} \\
\text { Inhalants Adjusted }{ }^{b}\end{array}$ & $\begin{array}{l}14.8 \\
18.7\end{array}$ & $\begin{array}{l}15.9 \\
20.1\end{array}$ & $\begin{array}{l}17.1 \\
21.6\end{array}$ \\
\hline Amyl \& Butyl Nitrites ${ }^{C}$ & 7.3 & 8.6 & 10.1 \\
\hline $\begin{array}{l}\text { Hallucinogens } \\
\text { Hallucinogens Adjusted }{ }^{d}\end{array}$ & $\begin{array}{r}8.6 \\
10.8\end{array}$ & $\begin{array}{r}9.7 \\
11.9\end{array}$ & $\begin{array}{l}10.9 \\
13.0\end{array}$ \\
\hline $\begin{array}{l}\text { LSD } \\
\text { PCPC }\end{array}$ & $\begin{array}{l}6.3 \\
3.8\end{array}$ & $\begin{array}{l}7.2 \\
4.8\end{array}$ & $\begin{array}{l}8.2 \\
6.0\end{array}$ \\
\hline Cocaine & 15.5 & 16.9 & 18.4 \\
\hline Heroin & 0.8 & 1.1 & 1,4 \\
\hline Other opiates ${ }^{\mathrm{e}}$ & 8.2 & 9.0 & 9.8 \\
\hline Stimulants Adjusted ${ }^{e, f}$ & 21.8 & 23.4 & 25.0 \\
\hline Sedatives ${ }^{e}$ & 9.3 & 10.4 & 11.6 \\
\hline $\begin{array}{l}\text { Barbiturates }{ }^{\mathrm{e}} \\
\text { Methaqualone }^{\mathrm{e}}\end{array}$ & $\begin{array}{l}7.4 \\
4.4\end{array}$ & $\begin{array}{l}8.4 \\
5.2\end{array}$ & $\begin{array}{l}9.5 \\
6.1\end{array}$ \\
\hline Tranquilizers $^{e}$ & 9.8 & 10.9 & 12.1 \\
\hline Alcohol & 89.7 & 91.3 & 92.7 \\
\hline Cigarettes & 65.8 & 67.6 & 69.3 \\
\hline
\end{tabular}

aData based on four questionnaire forms. $\mathrm{N}$ is four-fifths of $\mathrm{N}$ indicated.

${ }^{b}$ Adjusted for underreporting of amyl and butyl nitrites. See text for details.

${ }^{\mathrm{C}}$ Data based on a single questionnaire form. $\mathrm{N}$ is one-fifth of $\mathrm{N}$ indicated.

dAdjusted for underreporting of PCP. See text for details.

'Only drug use which was not under a doctor's orders is included here.

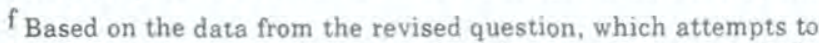
exclude the inappropriate reporting of non-prescription stimulants. 
- Marijuana is by far the most widely used illicit drug with $51 \%$ reporting some use in their lifetime, $39 \%$ reporting some use in the past year, and $23 \%$ reporting some use in the past month.

- The most widely used class of other illicit drugs is stimulants ( $23 \%$ lifetime prevalence, adjusted). ${ }^{7}$ Next come inhalants (adjusted) at $20 \%$ and cocaine at $17 \%$. These are followed closely by hallucinogens (adjusted) at $12 \%$, tranquilizers at $11 \%$, and sedatives at $10 \% .^{8}$

- The inhalant estimates have been adjusted upward because we observed that not all users of one subclass of inhalantsamyl and butyl nitrites (described below)-report themselves as inhalant users. Because we included questions specifically about nitrite use for the first time in one 1979 questionnaire form, we were able to discover this problem and make estimates of the degree to which inhalant use was being underreported in the overall estimates. As a result, all prevalence estimates for inhalants have been increased, with the proportional increase being greater for the more recent time intervals (i.e., last month, last year) because use of the other common inhalants, such as glue and aerosols, is more likely to have been discontinued prior to senior year, making nitrite use proportionally more important in later years.

- The specific classes of inhalants known as amyl and butyl nitrites, which are sold legally and go by the street names of "poppers" or "snappers" and such brand names as Locker Room and Rush, have been tried by one in every twelve seniors $(8.6 \%)$.

- We also discovered in 1979 , by adding questions specifically about PCP use, that some users of PCP do not report themselves as users of hallucinogens-even though PCP is explicitly included as an example in the questions about hallucinogens. Thus, since 1979 the hallucinogen prevalence

\footnotetext{
${ }^{7}$ See note at the end of the introductory section concerning the interpretation of stimulant statistics.

${ }^{8}$ Only use which was not medically supervised is included in the figures cited in the main body of this report.
} 
TABLE 2

\section{Lifetime Prevalence (Percent Ever Used) and Recency of Use of Sixteen Types of Drugs Class of 1986}

$$
\text { (Approx. } \mathrm{N}=15200 \text { ) }
$$

\begin{tabular}{|c|c|c|c|c|c|}
\hline & $\begin{array}{l}\text { Ever } \\
\text { used }\end{array}$ & $\begin{array}{c}\text { Past } \\
\text { month }\end{array}$ & $\begin{array}{c}\text { Past } \\
\text { year, } \\
\text { not } \\
\text { past } \\
\text { month } \\
\end{array}$ & $\begin{array}{l}\text { Not } \\
\text { past } \\
\text { year } \\
\end{array}$ & $\begin{array}{c}\text { Never } \\
\text { used }\end{array}$ \\
\hline Marijuana/Hashish & 50.9 & 23.4 & 15.4 & 12.1 & 49.1 \\
\hline $\begin{array}{l}\text { Inhalants }{ }^{\mathrm{a}} \\
\text { Inhalants Adjusted }{ }^{b}\end{array}$ & $\begin{array}{l}15.9 \\
20.1\end{array}$ & $\begin{array}{l}2.5 \\
3.2\end{array}$ & $\begin{array}{l}3.6 \\
5.7\end{array}$ & $\begin{array}{r}9.8 \\
11.2\end{array}$ & $\begin{array}{l}84.1 \\
79.9\end{array}$ \\
\hline Amyl \& Butyl Nitrites ${ }^{c}$ & 8.6 & 1.3 & 3.4 & 3.9 & 91.4 \\
\hline $\begin{array}{l}\text { Hallucinogens } \\
\text { Hallucinogens Adjusted }\end{array}$ & $\begin{array}{r}9.7 \\
11.9\end{array}$ & $\begin{array}{l}2.5 \\
3.5\end{array}$ & $\begin{array}{l}3.5 \\
4.1\end{array}$ & $\begin{array}{l}3.7 \\
4.3\end{array}$ & $\begin{array}{l}90.3 \\
88.1\end{array}$ \\
\hline $\begin{array}{l}\text { LSD } \\
\text { PCPC }\end{array}$ & $\begin{array}{l}7.2 \\
4.8\end{array}$ & $\begin{array}{l}1.7 \\
1.3\end{array}$ & $\begin{array}{l}2.8 \\
1.1\end{array}$ & $\begin{array}{l}2.7 \\
2.4\end{array}$ & $\begin{array}{l}92.8 \\
95.2\end{array}$ \\
\hline Cocaine & 16.9 & 6.2 & 6.5 & 4.2 & 83.1 \\
\hline Heroin & 1.1 & 0.2 & 0.3 & 0.6 & 98.9 \\
\hline Other opiates ${ }^{e}$ & 9.0 & 2.0 & 3.2 & 3.8 & 91.0 \\
\hline Stimulants Adjusted ${ }^{e} f$ & 23.4 & 5.5 & 7.9 & 10.0 & 76.6 \\
\hline Sedatives ${ }^{\mathrm{e}}$ & 10.4 & 2.2 & 3.0 & 5.2 & 89.6 \\
\hline $\begin{array}{l}\text { Barbiturates }{ }^{\mathrm{e}} \\
\text { Methaqualone }^{\mathrm{e}}\end{array}$ & $\begin{array}{l}8.4 \\
5.2\end{array}$ & $\begin{array}{l}1.8 \\
0.8\end{array}$ & $\begin{array}{l}2.4 \\
1.3\end{array}$ & $\begin{array}{l}4.2 \\
3.1\end{array}$ & $\begin{array}{l}91.6 \\
94.8\end{array}$ \\
\hline Tranquilizers ${ }^{e}$ & 10.9 & 2.1 & 3.7 & 5.1 & 89.1 \\
\hline Alcohol & 91.3 & 65.3 & 19.2 & 6.8 & 8.7 \\
\hline Cigarettes & 67.6 & 29.6 & \multicolumn{2}{|c|}{$(38.0) \mathrm{g}$} & 32.4 \\
\hline
\end{tabular}

${ }^{a}$ Data based on four questionnaire forms. $\mathrm{N}$ is four-fifths of $\mathrm{N}$ indicated.

${ }^{b}$ Adjusted for underreporting of amyl and butyl nitrites. See text for details.

${ }^{\mathrm{c}}$ Data based on a single questionnaire form. $\mathrm{N}$ is one-fifth of $\mathrm{N}$ indicated.

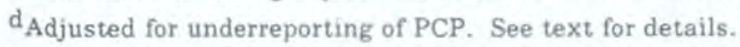

'Only drug use which was not under a doctor's orders is included here.

${ }^{f}$ Based on the data from the revised question, which attempts to exclude the inappropriate reporting of non-prescription stimulants.

gThe combined total for the two columns is shown because the question asked did not discriminate between the two answer categories. 
FIGURE 2

Prevalence and Recency of Use

Eleven Types of Drugs, Class of 1986

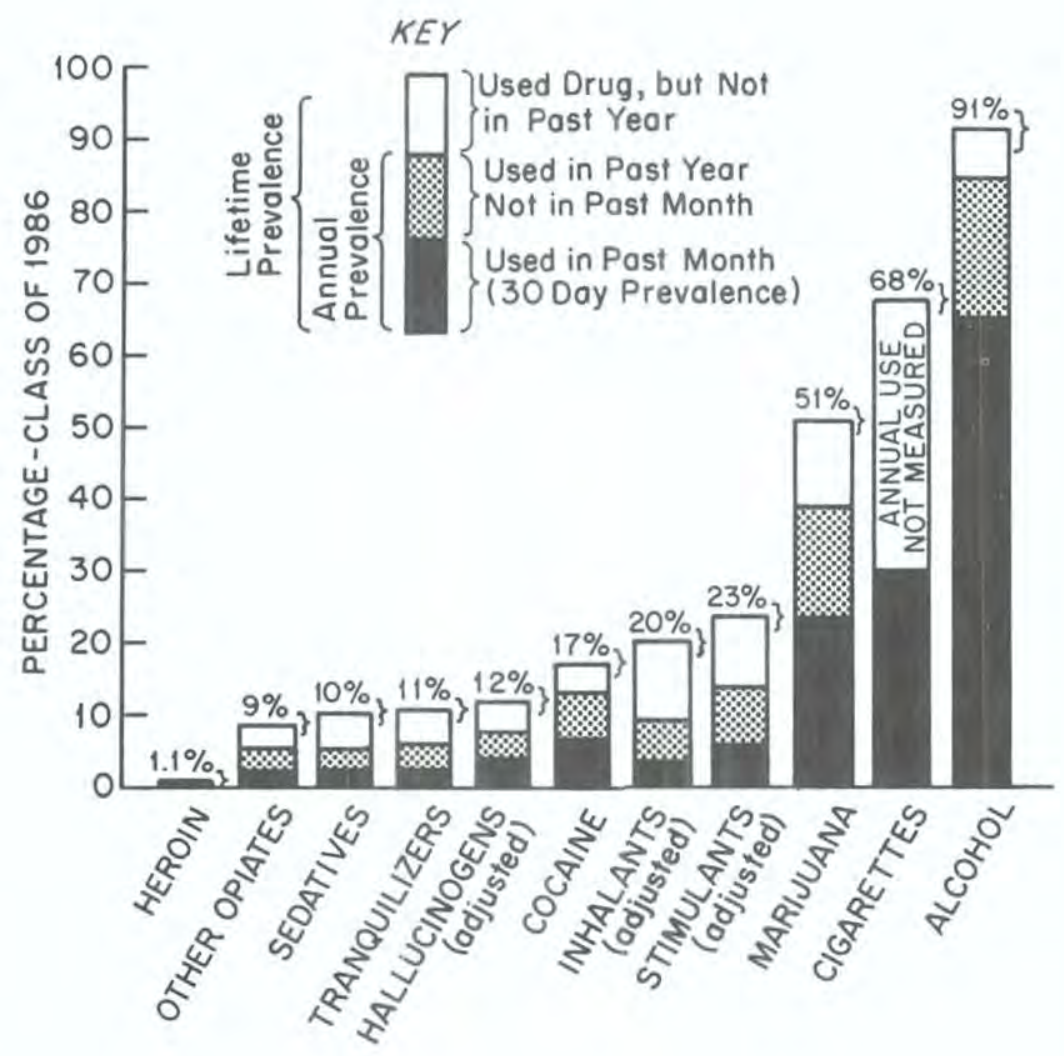

NOTES: The bracket near the top of a bar indicates the lower and upper limits of the $95 \%$ confidence interval. 
and trend estimates also have been adjusted upward to correct for this known underreporting. ${ }^{9}$

- Lifetime prevalence for the specific hallucinogenic drug $\boldsymbol{P C P}$ now stands at $5 \%$, somewhat lower than that of the other most widely used hallucinogen, LSD (lifetime prevalence, $7 \%)$.

- Opiates other than heroin have been used by about one in eleven seniors $(9 \%)$.

- Only $1.1 \%$ of the sample admitted to ever using any heroin, the most infrequently used drug. But given the highly illicit nature of this drug, we deem it the most likely to be underreported.

- Within the general class "sedatives," the specific drug metha. qualone has been used by nearly as many seniors (5\% lifetime prevalence) as the other, much broader subclass of sedatives, barbiturates (8\%).

- The illicit drug classes remain in roughly the same order whether ranked by lifetime, annual, or monthly prevalence, as the data in Figure 2 illustrate. The only important change in ranking occurs for inhalants, because use of certain of them, like glues and aerosols, tends to be discontinued at a relatively early age.

- Use of either of the two major licit drugs, alcohol and cigarettes, remains more widespread than use of any of the illicit drugs. Nearly all students have tried alcohol $(91 \%)$ and the great majority $(65 \%)$ have used it in just the past month.

- Some two-thirds $(68 \%)$ report having tried cigarettes at some time, and nearly one-third $(30 \%)$ smoked at least some in the past month.

\section{Daily Prevalence}

- Frequent use of any of these drugs is of greatest concern from a health and safety standpoint. Tables 6 and 10 and Figure 3 show the prevalence of daily or near-daily use of the various classes of drugs. For all drugs except cigarettes,

${ }^{9}$ Because the data to adjust inhalant and hallucinogen use are available from only a single questionnaire form in a given year, the original uncorrected variables will be used in most relational analyses. We believe relational analyses will be least affected by these underestimates and that the most serious impact is on prevalence estimates, which are adjusted appropriately. 


\section{FIGURE 3}

Thirty-Day Prevalence of Daily Use

Eleven Types of Drugs, Class of 1986

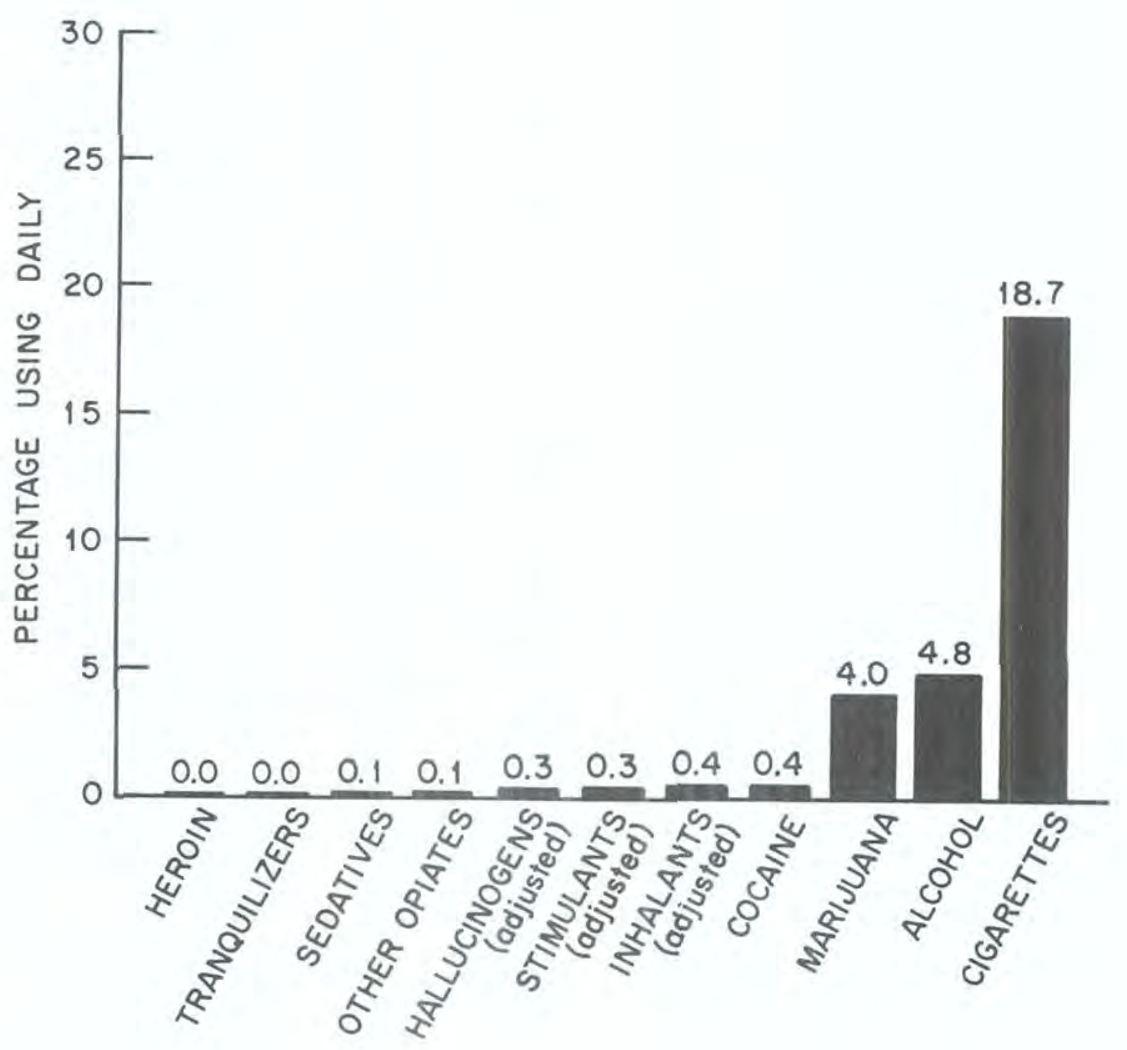


respondents are considered daily users if they indicate that they had used the drug on twenty or more occasions in the preceding 30 days. In the case of cigarettes, respondents explicitly state the use of one or more cigarettes per day.

- The displays show that cigarettes are used daily by more of the respondents $(19 \%)$ than any of the other drug classes. In fact, $11.4 \%$ say they smoke half-a-pack or more per day.

- Another important fact is that marijuana is still used on a daily or near-daily basis by a substantial fraction of the age group $(4.0 \%)$, or about one in every twenty-five seniors. A slightly larger proportion (4.8\%) drink alcohol that often.

- Less than $1 \%$ of the respondents report daily use of any one of the illicit drugs other than marijuana. Still, $0.5 \%$ report daily use of nitrites, $0.4 \%$ report daily use of cocaine, and of inhalants (adjusted). The next highest daily-use figure is for amphetamines (adjusted version which excludes the nonprescription stimulants) and hallucinogens (adjusted), both at $0.3 \%, P C P$, specifically, is used daily by $0.2 \%$ of all seniors. While very low, these figures are not inconsequential, given that $1 \%$ of each high school class represents roughly 30,000 individuals.

- Sedatives and opiates other than heroin are used daily by only about $0.1 \%$.

- While daily alcohol use stands at $4.8 \%$ for this age group, a substantially greater proportion report occasional heavy drinking. In fact, $37 \%$ state that on at least one occasion during the prior two-week interval they had five or more drinks in a row.

\section{NONCONTINUATION RATES}

An indication of the extent to which people who try a drug do not continue to use it can be derived from calculating the percentage, based on those who ever used a drug (once or more), who did not use it the 12 months preceding the survey. ${ }^{10}$ These "noncontinuation rates" are provided for all drug classes in Figure 4 for the class of 1986. We use the word "noncontinuation" rather than "discontinuation," since the lat-

${ }^{10}$ This operationalization of noncontinuation has an inherent problem in that users of a given drug who initiate use in senior year by definition cannot be noncontinuers. Thus, the definition tends to understate the noncontinuation rate, particularly for drugs that tend to be initiated late in high school rather than in earlier years. 


\section{FIGURE 4}

Noncontinuation Rates: Percent of Seniors Who Used Drug Once or More in Lifetime but Did Not Use in Past Year

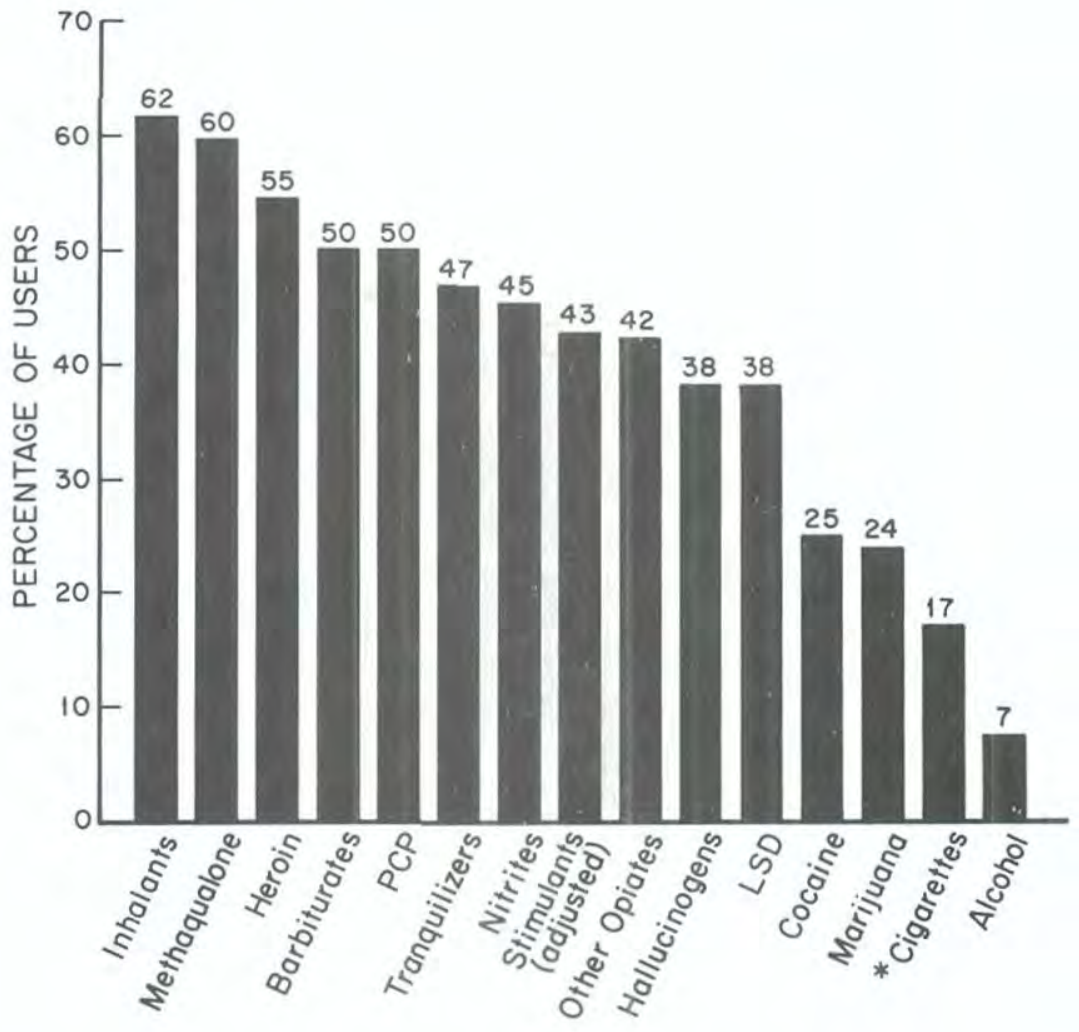

*Percent of regular smokers (ever) who did not smoke at all in the last thirty days. 
ter might imply discontinuing an established pattern of use, and our current operational definition includes experimental users as well as established users.

- It may be seen in Figure 4 that noncontinuation rates vary widely among the different drugs.

- The highest noncontinuation rate by senior year $(62 \%)$ is found for inhalants, most of which tend to be used at younger ages. The nitrites specifically, however, are used somewhat later as the $45 \%$ noncontinuation rate illustrates.

- Marijuana has the lowest noncontinuation rate $(24 \%)$ in senior year of any of the illicit drugs; this occurs because a relatively high proportion of users continue to use at some level over an extended period.

- Cocaine also has a low noncontinuation rate $(25 \%)$ but this is partly because of its relatively late age of onset.

- Methaqualone currently shows a relatively high noncontinuation rate $(60 \%)$, which accounts in part for the recent dramatic decline in overall use.

- The remaining illicit drugs have noncontinuation rates ranging from $38 \%$ to $55 \%$.

- Noncontinuation rates for the two licit drugs are extremely low. Alcohol, which has been tried by nearly all seniors $(91 \%)$, is used in senior year by nearly all of those who have ever tried it (93\% of the $91 \%$ ).

- For cigarettes the definition of continuation is a little different; it is the percentage of those who say they ever smoked "regularly" who also reported smoking at least one cigarette during the past month. Hardly any of these regular smokers (only $17 \%$ of them) have ceased active use. (A comparable definition of noncontinuation to that used for other drugs is not possible, since cigarette use in the past year is not asked of respondents.)

\section{PREVALENCE COMPARISONS FOR IMPORTANT SUBGROUPS}

\section{Sex Differences}

- In general, higher proportions of males than females are involved in illicit drug use, especially heavy drug use; 
TABLE 3

Lifetime Prevalence of Use of Sixteen Types of Drugs

by Subgroups, Class of 1986

(Entries are percentsges)

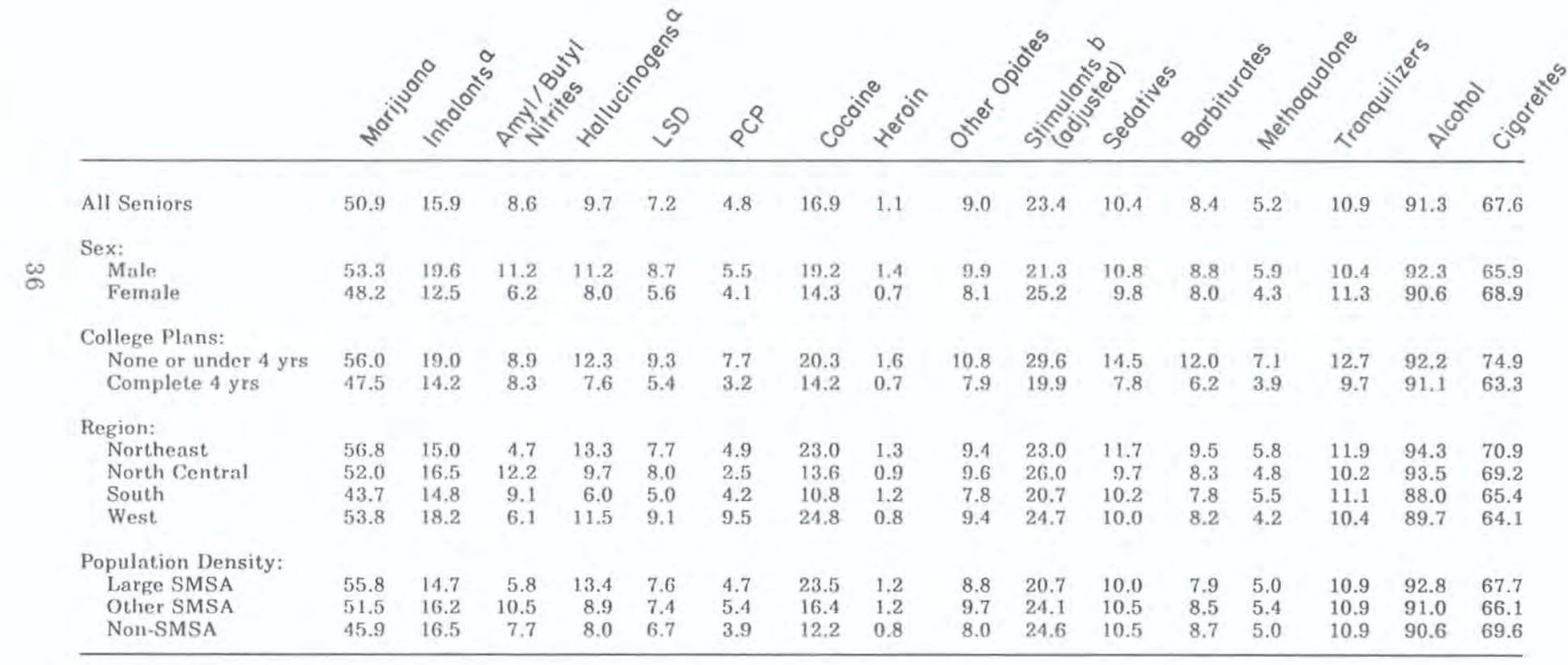

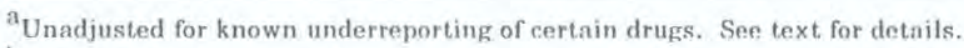

${ }^{b}$ Based on the data from the revised question, which attempts to exclude the inappropriate reporting of non-prescription stimulants. 
however, this picture is a complicated one (see Tables 3 through 6).

- Overall the proportion using marijuana is only slightly higher among males, but daily use of marijuana is more than twice as frequent among males (5.7\% vs. $2.3 \%$ for females).

- Males also have considerably higher prevalence rates on most other illicit drugs. The annual prevalence (Table 4) for inhalants (unadjusted and adjusted), hallucinogens (unadjusted and adjusted), heroin, methaqualone, and the specific drugs $L S D$ and the nitrites tend to be one and onehalf to three and one-half times as high among males as among females. Males also report somewhat higher annual rates of use than females for cocaine, opiates other than heroin, and barbiturates. Further, males account for an even greater share of the frequent or heavy users of these various classes of drugs.

- Only in the case of stimulants do the annual prevalence rates (as well as frequent usage patterns) for females exceed those for males-and then only by small amounts. Annual prevalence for stimulants (adjusted) is $13.8 \%$ for females vs. $12.7 \%$ for males. This reversal in sex differences is due to the fact that substantially more females than males use stimulants for purposes of weight loss-an instrumental, as opposed to social/recreational, use of the drug. ${ }^{11}$

- Despite the fact that all but one of the individual classes of illicit drugs are used more by males than by females, the proportions of both sexes who report using some illicit drug other than marijuana during the last year are not substantially different $(26 \%$ for males vs. $25 \%$ for females; see Figure 12). Even if amphetamine use is excluded from the comparisons altogether, fairly comparable proportions of both sexes ( $22 \%$ for males vs. $19 \%$ for females) report using some illicit drug other than marijuana during the year. If one thinks of going beyond marijuana as an important threshold point in the sequence of illicit drug use, then nearly equal proportions of both sexes were willing to cross that threshold at least once during the year. However, on the average the female "users" take fewer types of drugs and use them with less frequency than their male counterparts.

${ }^{11} J o h n s t o n$, L.D. \& O'Malley, P.M. (1986). Why do the nation's students use drugs and alcohol? Self-reported reasons from nine national surveys. Journal of Drug Issues, 16, 29-66. 


\section{TABLE 4}

\section{Annual Prevalence of Use of Seventeen Types of Drugs}

by Subgroups, Class of 1986

(Entries are percentages)
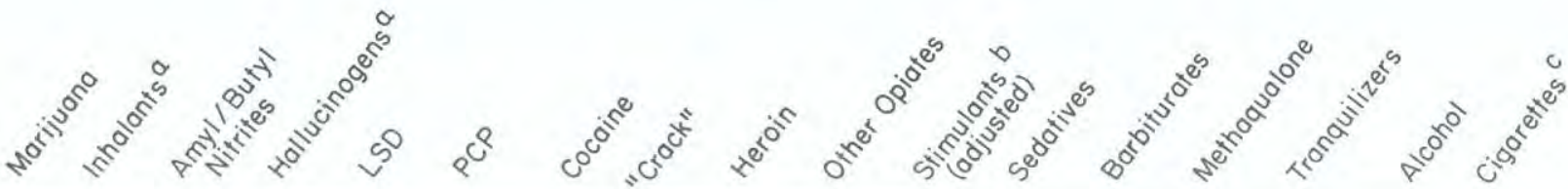

\begin{tabular}{|c|c|c|c|c|c|c|c|c|c|c|c|c|c|c|c|c|c|}
\hline All Seniors & 38.8 & 6.1 & 4.7 & 6.0 & 4.5 & 2.4 & 12.7 & 4,1 & 0.5 & 5.2 & 13,4 & 5.2 & 4.2 & 2.1 & 5.8 & 84.5 & - \\
\hline \multicolumn{18}{|l|}{ Sex: } \\
\hline Male & 41.2 & 7.8 & 6.9 & 7.2 & 5.5 & 2.8 & 14.3 & 4.2 & 0.7 & 5.9 & 12.7 & 5.7 & 4.7 & 2.7 & 5.9 & 85.8 & - \\
\hline Female & 36.0 & 4.7 & 2.9 & 4.7 & 3.4 & 2,0 & 10.9 & 3.6 & 0.2 & 4.6 & 13.8 & 4.4 & 3.8 & 1.4 & 5.8 & 83.4 & - \\
\hline \multicolumn{18}{|l|}{ College Plans; } \\
\hline None or under 4 yrs & 42.7 & 7.7 & 5.3 & 7.4 & 5.9 & 3.1 & 15.7 & 5.2 & 0.8 & 6.7 & 17.7 & 7.5 & 6,1 & 3.2 & 7.2 & 85.1 & - \\
\hline Complete 4 yrs & 36.1 & 5.2 & 4.4 & 4.7 & 3.3 & 2.1 & 10.4 & 2.8 & 0.4 & 4.3 & 10.9 & 3.6 & 3.0 & 1.4 & 5.1 & 84.6 & - \\
\hline \multicolumn{18}{|l|}{ Region: } \\
\hline Northeast & 44.6 & 5.6 & 2.2 & 7.9 & 5.1 & 1.6 & 17.9 & 6.0 & 0.7 & 5.7 & 12.6 & 6.4 & 5.2 & 2.9 & 6.4 & 88.7 & - \\
\hline North Central & 40.2 & 6.7 & 6.6 & 6.6 & 5.3 & 1.2 & 10.1 & 3.1 & 0.4 & 5.8 & 15.2 & 5.0 & 4.2 & 2.0 & 5.5 & 88.4 & - \\
\hline South & 31.7 & 5.7 & 5.8 & 3.3 & 2.6 & 2.0 & 7.1 & 1.6 & 0.5 & 4.2 & 11.5 & 5.1 & 4.1 & 2.0 & 6.3 & 78.4 & - \\
\hline West & 41.2 & 6.6 & 2.8 & 7.2 & 5.9 & 6.3 & 20.0 & 7.5 & 0.5 & 5.4 & 15.0 & 3.9 & 3.3 & 1.4 & 4.8 & 82.9 & - \\
\hline \multicolumn{18}{|l|}{ Population Density: } \\
\hline Large SMSA & 42.6 & 5.2 & 2.2 & 7.6 & 4.4 & 2.1 & 18.8 & 5.9 & 0.7 & 4.8 & 11.2 & 4.7 & 3.7 & 2.0 & 5.3 & 86.8 & - \\
\hline Other SMSA & 39.4 & 6.3 & 5.9 & 5.9 & 4.9 & 3.0 & 12.0 & 3.5 & 0.4 & 5.6 & 14.2 & 5.2 & 4.4 & 2.0 & 5.7 & 84.1 & - \\
\hline Non-SMSA & 34.7 & 6.6 & 4.8 & 4.9 & 4.0 & 1.7 & 9.0 & 3.5 & 0.5 & 5.0 & 14.1 & 5.5 & 4.5 & 2.3 & 6.4 & 83.0 & - \\
\hline
\end{tabular}

${ }^{\mathrm{a}}$ Unadjusted for known underreporting of certain drugs. See text for details.

${ }^{b}$ Based on the data from the revised question, which attempts to exclude the inappropriate reporting of non-prescription stimulants.

${ }^{\mathrm{C}}$ Annual prevalence is not available. 
- Frequent use of alcohol tends to be disproportionately concentrated among males. Daily use, for example, is reported by $6.7 \%$ of the males vs. only $2.8 \%$ of the females. Also, males are more likely than females to drink large quantities of alcohol in a single sitting (i.e., $46 \%$ of males report taking five or more drinks in a row in the prior two weeks, vs. $28 \%$ of females).

- Finally, while there is not at present an appreciable sex difference in cigarette smoking, what difference there is shows females smoking more. For example, at the level of smoking a half-a-pack or more daily: $11.6 \%$ of the females smoke this heavily vs. $10.7 \%$ of the males. There is a larger difference in proportions reporting any use during the past month; $31 \%$ of the females vs. $28 \%$ of the males.

\section{Differences Related to College Plans}

- Overall, seniors who are expecting to complete four years of college (referred to here as the "college-bound") have lower rates of illicit drug use than those not expecting to do so (see Tables 3 through 6 and Figure 13).

- Annual marijuana use is reported by $36 \%$ of the collegebound vs. $43 \%$ of the noncollege-bound.

- There is a substantial difference in the proportion of these two groups using any illicit drug(s) other than marijuana (adjusted). In 1986, 22\% of the college-bound reported any such behavior in the prior year vs. $31 \%$ of the noncollege-bound. (If amphetamine use is excluded from these "other illicit drugs," the figures are $18 \%$ vs. $25 \%$, respectively.)

- For most of the specific illicit drugs other than marijuana, annual prevalence is higher-sometimes substantially higher-among the noncollege-bound, as Table 4 illustrates. In fact, current (30-day) prevalence is roughly one and onehalf to two times as high among the noncollege-bound as among the college-bound for all of the illicit drugs, with the exception of marijuana and the nitrites.

- Frequent use of many of these illicit drugs shows even larger contrasts related to college plans (see Table 6). Daily marijuana use, for example, is more than twice as high among those not planning four years of college $(6.2 \%)$ as among the college-bound (2.3\%). 
TABLE 5

Thirty-Day Prevalence of Use of Sixteen Types of Drugs

by Subgroups, Class of 1986

(Entries are percentages)

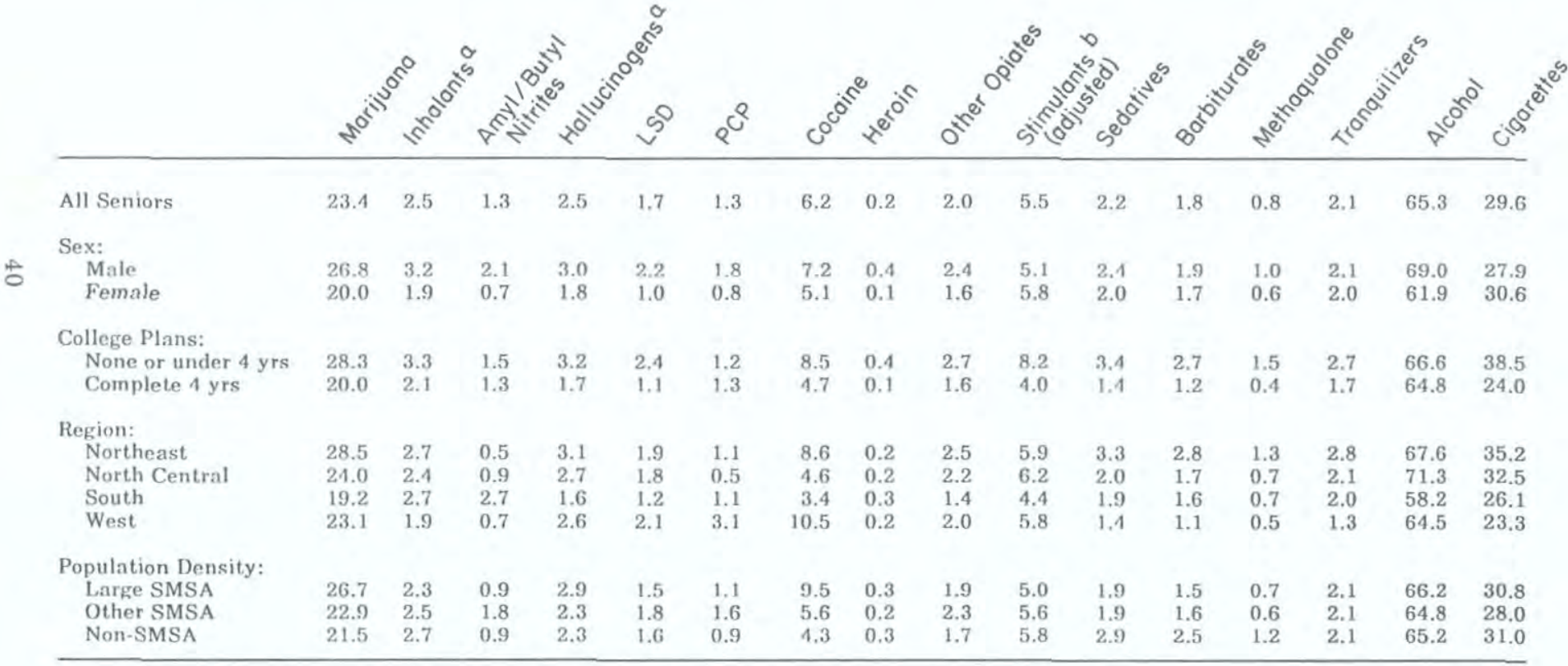

${ }^{a}$ Unadjusted for known underreporting of certain drugs. See text for details.

${ }^{b}$ Based on the data from the revised question, which attempts to exclude the inappropriate reporting of non-prescription stimulants. 
TABLE 6

Thirty-Day Prevalence of Daily Use of Marijuana, Alcohol, and Cigarettes by Subgroups, Class of 1986

\begin{tabular}{|c|c|c|c|c|c|}
\hline & \multirow[b]{3}{*}{$\begin{array}{c}\mathrm{N} \\
\text { (Approx.) } \\
\end{array}$} & \multicolumn{4}{|c|}{ Percent who used daily in last thirty days } \\
\hline & & \multirow[b]{2}{*}{ Marijuana } & \multirow[b]{2}{*}{ Alcohol } & \multicolumn{2}{|c|}{ Cigarettes } \\
\hline & & & & $\begin{array}{c}\text { One } \\
\text { or more } \\
\end{array}$ & $\begin{array}{c}\text { Half-pack } \\
\text { or more }\end{array}$ \\
\hline All Seniors & 15200 & 4.0 & 4.8 & 18.7 & 11.4 \\
\hline \multicolumn{6}{|l|}{ Sex: } \\
\hline $\begin{array}{l}\text { Male } \\
\text { Female }\end{array}$ & $\begin{array}{l}7100 \\
7700\end{array}$ & $\begin{array}{l}5.7 \\
2.3\end{array}$ & $\begin{array}{l}6.7 \\
2.8\end{array}$ & $\begin{array}{l}16.9 \\
19.8\end{array}$ & $\begin{array}{l}10.7 \\
11.6\end{array}$ \\
\hline \multicolumn{6}{|l|}{ College Plans: } \\
\hline $\begin{array}{l}\text { None or under } 4 \text { yrs } \\
\text { Complete } 4 \text { yrs }\end{array}$ & $\begin{array}{l}5100 \\
9100\end{array}$ & $\begin{array}{l}6.2 \\
2.3\end{array}$ & $\begin{array}{l}6.8 \\
3.5\end{array}$ & $\begin{array}{l}28.2 \\
12.8\end{array}$ & $\begin{array}{r}19.2 \\
6.4\end{array}$ \\
\hline \multicolumn{6}{|l|}{ Region: } \\
\hline $\begin{array}{l}\text { Northeast } \\
\text { North Central } \\
\text { South } \\
\text { West }\end{array}$ & $\begin{array}{l}3600 \\
4300 \\
4700 \\
2600\end{array}$ & $\begin{array}{l}5.0 \\
4.0 \\
2.8 \\
4.6\end{array}$ & $\begin{array}{l}4.9 \\
4.9 \\
4.9 \\
4.2\end{array}$ & $\begin{array}{l}24.9 \\
19.9 \\
15.8 \\
13.4\end{array}$ & $\begin{array}{r}15.6 \\
12.3 \\
10.0 \\
6.5\end{array}$ \\
\hline \multicolumn{6}{|l|}{ Population Density: } \\
\hline $\begin{array}{l}\text { Large SMSA } \\
\text { Other SMSA } \\
\text { Non-SMSA }\end{array}$ & $\begin{array}{l}3700 \\
7000 \\
4500\end{array}$ & $\begin{array}{l}4.6 \\
3.9 \\
3.6\end{array}$ & $\begin{array}{l}4.0 \\
4.5 \\
5.8\end{array}$ & $\begin{array}{l}20.6 \\
17.0 \\
19.8\end{array}$ & $\begin{array}{r}12.2 \\
9.6 \\
13.3\end{array}$ \\
\hline
\end{tabular}


- Frequent alcohol use is also more prevalent among the noncollege-bound. For example, drinking on a daily basis is reported by $6.8 \%$ of the noncollege-bound vs. only $3.5 \%$ of the college-bound. Instances of heavy drinking are also related to college plans: $34 \%$ of the college-bound report having five or more drinks in a row at least once during the preceding two weeks, vs. $41 \%$ of the noncollege-bound. Drinking that heavily on six or more occasions in the last two weeks is reported by $3.4 \%$ of the college-bound vs. $6.8 \%$ of the noncollege-bound. On the other hand, there are practically no differences between these groups in lifetime, annual, or monthly prevalence of alcohol use.

- By far the largest difference in substance use between the college and noncollege-bound involves cigarette smoking. There is a dramatic difference here, with only $6.4 \%$ of the college-bound smoking a half-a-pack or more daily compared with $19.2 \%$ of the noncollege-bound.

\section{Regional Differences}

- There are now some fair-sized regional differences in rates of illicit drug use among high school seniors. (See Figure 5 for a regional division map of the states included in the four regions of the country.) The highest (adjusted) rate is in the Northeast, where $50 \%$ say they have used a drug illicitly in the past year, followed closely by the West at $48 \%$, and the North Central with $45 \%$. The South is by far the lowest, with $37 \%$ having used any illicit drug during the year (see Figure 14).

- There are comparable regional variations in terms of the percentage using some illicit drug other than marijuana (adjusted) in the past year (although the West leads the Northeast for this measure): $32 \%$ in the West, $30 \%$ in the Northeast, $25 \%$ in the North Central, and $21 \%$ in the South.

- The Northeast and West rank relatively high in the use of some illicit drug other than marijuana, due in part to their high level of cocaine use. In fact, the regional differences in cocaine have been the largest observed. For example, annual prevalence is nearly three times as high in the West $(20.0 \%)$ and Northeast $(17.9 \%)$ as in the South $(7.1 \%)$. The North Central also has a relatively low annual prevalence rate $(10.1 \%)$.

- Other specific illicit substances vary in the extent to which they show regional variation, as Table 4 illustrates for the annual prevalence measure. 


\section{FIGURE 5}

States Included in the Four Regions of the Country

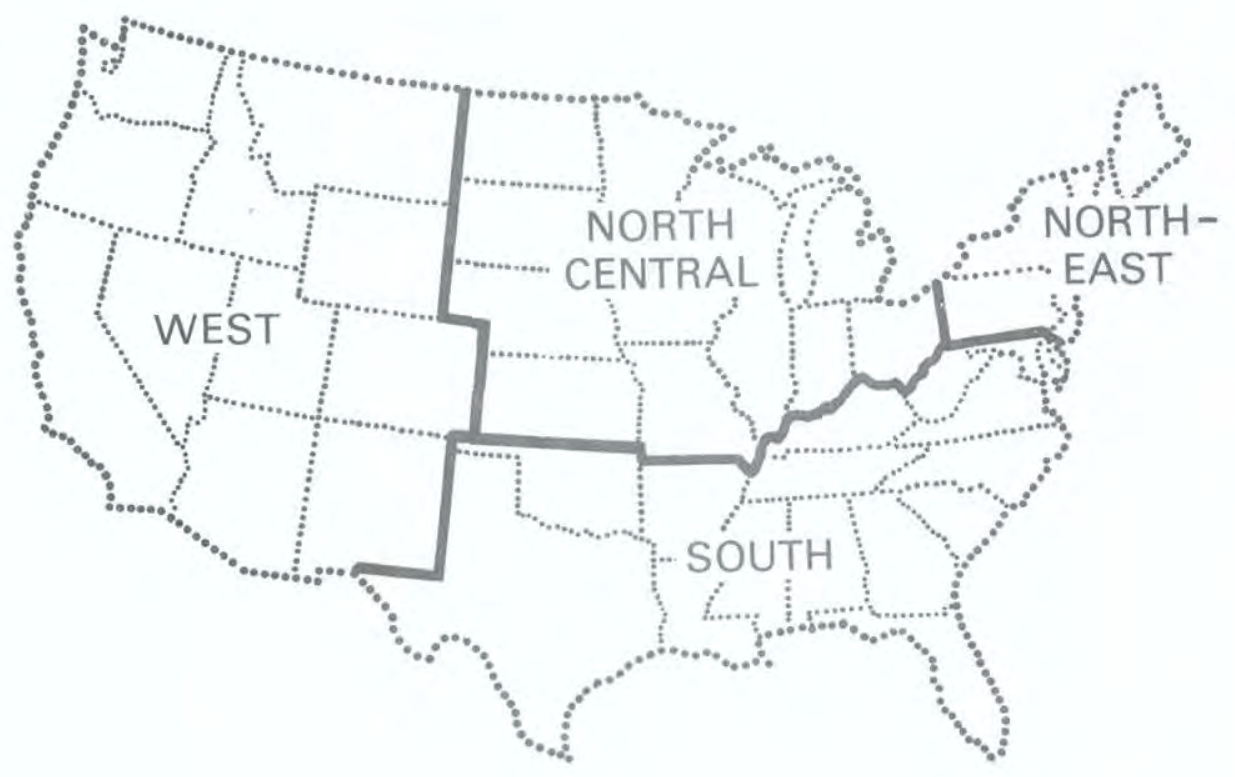

These are the four major regions of the country as defined by the U.S. Bureau of the Census. 
Two drugs are highest in the Northeast and lowest in the South with the West and North Central in between: marijuana and hallucinogens (unadjusted). The West ranks first on three of the drugs which show the largest proportional variation among the regions: cocaine, PCP, and $L S D$; but despite its quite high rate of use of these drugs, it is the West that shows the lowest levels of use for barbiturates, methaqualone, and tranquilizers (all central nervous system depressants). For all of these the Northeast shows the highest rate of use. Stimulants show still a third pattern, with the highest use in the North Central and West and lowest in the South.

- Alcohol use-in particular, the rate of occasional heavy drinking - tends to be somewhat lower in the South and West than it is in the Northeast and North Central.

- A similar, though much larger, regional difference occurs for regular cigarette smoking. Smoking half-a-pack or more a day occurs most often in the Northeast ( $16 \%$ of seniors), with the North Central (12\%) and the South (10\%) somewhat lower, and the West (7\%) lower still.

\section{Differences Related to Population Density}

- Three levels of population density (or urbanicity) have been distinguished for analytical purposes: (1) large SMSA's, which are the twelve largest Standard Metropolitan Statistical Areas in the 1980 Census; (2) other SMSA's, which are the remaining Standard Metropolitan Statistical Areas; and (3) non-SMSA's, which are the sampling areas not designated as metropolitan by the Census.

- Overall illicit drug use is highest in the largest metropolitan areas ( $48 \%$ annual prevalence, adjusted), slightly lower in the other metropolitan areas $(45 \%)$, and lowest in the nonmetropolitan areas (40\%) (see Figure 16).

- The same ranking occurs for the use of illicit drugs other than marijuana: $28 \%$ annual prevalence (adjusted) in the largest cities, $26 \%$ in the other cities, and $23 \%$ in the nonmetropolitan areas. (With amphetamine use excluded, these numbers drop-to $25 \%, 21 \%$, and $17 \%$, respectively-but still retain the same rank order.)

- For specific drugs, one of the largest absolute differences associated with urbanicity occurs for marijuana, which has an annual prevalence of $43 \%$ in the large cities but only $35 \%$ in the nonmetropolitan areas (Table 4). 
- However, by far the greatest proportional difference, as well as the greatest absolute difference, occurs for cocaine, where there is more than twice as much use in the large metropolitan areas $(19 \%)$ as in the nonmetropolitan areas $(9 \%)$.

- There has been some tendency for a few other drugs to be associated positively with urbanicity; however, the relationships have not been strong, nor have they remained consistent from one year to another.

\section{"CRACK" COCAINE: PREVALENCE RATES AND SUBGROUP DIFFERENCES}

A single question concerning the use of cocaine in crack form was included in the 1986 survey for the first time. It was included on a single questionnaire form $(\mathrm{N}=3100)$ in a section which was answered only by those who reported use of cocaine in the prior twelve months (thus providing annual prevalence but not lifetime or thirty-day prevalence). It asked whether they had used cocaine in crack form but not how often they had used it. (More detailed questions are being included in future surveys.) The results from this question are provided in Table 4 and are summarized below.

- Approximately one in twenty-five seniors $(4.1 \%)$ reported some experience with crack in the past year. This amounts to about one-third of the number reporting any cocaine use in the past year $(12.7 \%)$.

- Usage rates were only slightly higher among males $(4.2 \%)$ than females (3.6\%), but were substantially higher among the noncollege-bound $(5.2 \%)$ than the college-bound $(2.8 \%)$.

- Regional difference follow the same pattern as for cocaine generally: annual prevalence is highest in the West $(7.5 \%)$ and Northeast $(6.0 \%)$, lower in the North Central $(3.1 \%)$, and lowest in the South (1.6\%).

- The larger cities have a higher rate of use $(5.9 \%)$ than the smaller cities or the non-urban areas (both $3.5 \%$ ), but clearly crack has moved beyond the confines of a few large cities. 


\section{TRENDS IN DRUG USE AMONG HIGH SCHOOL SENIORS}

This section summarizes trends in drug use, comparing the twelve graduating classes of 1975 through 1986. As in the previous section, the outcomes discussed include measures of lifetime use, use during the past year, use during the past month, and daily use. Also, trends are compared among the key subgroups.

\section{TRENDS IN PREVALENCE 1975-1986: ALL SENIORS}

- The years 1978 and 1979 marked the crest of a long and dramatic rise in marijuana use among American high school students. As Tables 7 through 10 illustrate, annual and 30day prevalence of marijuana use levelled between 1978 and 1979 , following a steady rise in the preceding years. In 1980 both statistics dropped for the first time and continued to decline every year, except in 1985 when there was a brief pause. In 1986, they stand at $12-13 \%$ below their all-time highs. Lifetime prevalence, which had remained unchanged in 1980, finally began to drop in 1981, though more gradually. It decreased significantly in 1986, but still is only $9.5 \%$ below its all time high. As we will discuss later, there have been some significant changes in the attitudes and beliefs that young people hold in relation to marijuana.

- Of greater importance is the even sharper downward trend which has been continuing to occur for daily marijuana use. Between 1975 and 1978 there was an almost two-fold increase in daily use. The proportion reporting daily use in the class of $1975(6.0 \%)$ came as a surprise to many; and then that proportion rose rapidly, so that by 1978 one in every nine high school seniors $(10.7 \%)$ indicated that he or she used the drug on a daily or nearly daily basis (defined as use on 20 or more occasions in the last 30 days). In 1979 we reported that this rapid and troublesome increase had come to a halt, with a $0.4 \%$ drop occurring that year. By 1986 the daily usage rate has dropped to $4.0 \%$-or one in every twenty-five seniors-well below the $6 \%$ level we first observed in 1975. As later sections of this report document, much of this dramatic reversal appears to be due to a continuing increase in concerns about possible adverse effects from 
TABLE 7

Trends in Lifetime Prevalence of Sixteen Types of Drugs

Percent ever used

\begin{tabular}{|c|c|c|c|c|c|c|c|c|c|c|c|c|}
\hline $\begin{array}{c}\text { Clnss } \\
\text { of } \\
1975 \\
\end{array}$ & $\begin{array}{c}\text { Class } \\
\text { of } \\
1976 \\
\end{array}$ & $\begin{array}{c}\text { Class } \\
\text { of } \\
1977 \\
\end{array}$ & $\begin{array}{c}\text { Class } \\
\text { of } \\
1978 \\
\end{array}$ & $\begin{array}{c}\text { Class } \\
\text { of } \\
1979 \\
\end{array}$ & $\begin{array}{c}\text { Class } \\
\text { of } \\
1980 \\
\end{array}$ & $\begin{array}{c}\begin{array}{c}\text { Class } \\
\text { of } \\
1981 \\
\end{array} \\
\end{array}$ & $\begin{array}{c}\begin{array}{c}\text { Class } \\
\text { of } \\
1982 \\
\end{array} \\
\end{array}$ & $\begin{array}{c}\text { Class } \\
\text { of } \\
1983 \\
\end{array}$ & $\begin{array}{c}\text { Class } \\
\text { of } \\
1984 \\
\end{array}$ & $\begin{array}{c}\text { Class } \\
\text { of } \\
1985 \\
\end{array}$ & $\begin{array}{c}\begin{array}{c}\text { Class } \\
\text { of } \\
1986 \\
\end{array} \\
\end{array}$ & $\begin{array}{l}\text { '85-'86 } \\
\text { change } \\
\end{array}$ \\
\hline$(9400)$ & $(15400)$ & $(17100)$ & $(17800)$ & (15500) & (15900) & $(17500)$ & $(17700)$ & $(16300)$ & $(15900)$ & $(16000)$ & (15200) & \\
\hline 47.3 & 52.8 & 56.4 & 59.2 & 60.4 & 60.3 & 59.5 & 58.7 & 57.0 & 54.9 & 54.2 & 50.9 & $-3.3 \mathrm{ss}$ \\
\hline $\begin{array}{l}\text { NA } \\
\text { NA }\end{array}$ & $\begin{array}{r}10.3 \\
N A\end{array}$ & $\begin{array}{r}11.1 \\
N A\end{array}$ & $\begin{array}{r}12.0 \\
N A\end{array}$ & $\begin{array}{l}12.7 \\
18.2\end{array}$ & $\begin{array}{l}11.9 \\
17.3\end{array}$ & $\begin{array}{l}12.3 \\
17.2\end{array}$ & $\begin{array}{l}12.8 \\
17.7\end{array}$ & $\begin{array}{l}13.6 \\
18.2\end{array}$ & $\begin{array}{l}14.4 \\
18.0\end{array}$ & $\begin{array}{l}15.4 \\
18.1\end{array}$ & $\begin{array}{l}15.9 \\
20.1\end{array}$ & $\begin{array}{l}+0.5 \\
+2.0 \mathrm{~s}\end{array}$ \\
\hline NA & $N A$ & NA & NA & 11,1 & 11.1 & 10.1 & 9.8 & 8.4 & 8.1 & 7.9 & 8.6 & +0.7 \\
\hline $\begin{array}{r}16.3 \\
N A\end{array}$ & $\begin{array}{r}15.1 \\
N A\end{array}$ & $\begin{array}{r}13.9 \\
N A\end{array}$ & $\begin{array}{r}14.3 \\
N A\end{array}$ & $\begin{array}{l}14.1 \\
17.7\end{array}$ & $\begin{array}{l}13.3 \\
15.6\end{array}$ & $\begin{array}{l}13.3 \\
15.3\end{array}$ & $\begin{array}{l}12.5 \\
14.3\end{array}$ & $\begin{array}{l}11.9 \\
13.6\end{array}$ & $\begin{array}{l}10.7 \\
12.3\end{array}$ & $\begin{array}{l}10.3 \\
12.1\end{array}$ & $\begin{array}{r}9.7 \\
11.9\end{array}$ & $\begin{array}{l}-0.6 \\
-0.2\end{array}$ \\
\hline $\begin{array}{r}11.3 \\
\text { NA }\end{array}$ & $\begin{array}{r}11.0 \\
\text { NAA }\end{array}$ & $\begin{array}{l}9.8 \\
\text { NA }\end{array}$ & $\begin{array}{l}9.7 \\
\text { NA }\end{array}$ & $\begin{array}{r}9.5 \\
12.8\end{array}$ & $\begin{array}{l}9.3 \\
9.6\end{array}$ & $\begin{array}{l}9.8 \\
7.8\end{array}$ & $\begin{array}{l}9.6 \\
6.0\end{array}$ & $\begin{array}{l}8.9 \\
5.6\end{array}$ & $\begin{array}{l}8.0 \\
5.0\end{array}$ & $\begin{array}{l}7.5 \\
4.9\end{array}$ & $\begin{array}{l}7.2 \\
4.8\end{array}$ & $\begin{array}{l}-0.3 \\
-0.1\end{array}$ \\
\hline 9.0 & 9.7 & 10.8 & 12.9 & 15.4 & 15.7 & 16.5 & 16.0 & 16.2 & 16.1 & 17.3 & 16.9 & -0.4 \\
\hline 2.2 & 1.8 & 1.8 & 1.6 & 1.1 & 1.1 & 1.1 & 1.2 & 1.2 & 1.3 & 1.2 & 1.1 & -0.1 \\
\hline 9.0 & 9.6 & 10.3 & 9.9 & 10.1 & 9.8 & 10.1 & 9.6 & 9.4 & 9.7 & 10.2 & 9.0 & $-1.2 \mathrm{~s}$ \\
\hline $\begin{array}{r}22.3 \\
N A\end{array}$ & $\begin{array}{r}22.6 \\
N A\end{array}$ & $\begin{array}{r}23.0 \\
N A\end{array}$ & $\begin{array}{r}22.9 \\
N A\end{array}$ & $\begin{array}{r}24.2 \\
N A\end{array}$ & $\begin{array}{r}26.4 \\
N A\end{array}$ & $\begin{array}{r}32.2 \\
N A\end{array}$ & $\begin{array}{l}35.6 \\
27.9\end{array}$ & $\begin{array}{l}35.4 \\
26.9\end{array}$ & $\begin{array}{r}\text { NA } \\
27.9\end{array}$ & $\begin{array}{r}\text { NA } \\
26.2\end{array}$ & $\begin{array}{r}\text { NA } \\
23.4\end{array}$ & $\begin{array}{c}\text { NA } \\
-2.8 s s\end{array}$ \\
\hline 18.2 & 17.7 & 17.4 & 16.0 & 14.6 & 14.9 & 16.0 & 15.2 & 14.4 & 13.3 & 11.8 & 10.4 & $-1.4 \mathrm{~s}$ \\
\hline $\begin{array}{r}16.9 \\
8.1\end{array}$ & $\begin{array}{r}16.2 \\
7.8\end{array}$ & $\begin{array}{r}15.6 \\
8.5\end{array}$ & $\begin{array}{r}13.7 \\
7.9\end{array}$ & $\begin{array}{r}11.8 \\
8.3\end{array}$ & $\begin{array}{r}11.0 \\
9.5\end{array}$ & $\begin{array}{l}11.3 \\
10.6\end{array}$ & $\begin{array}{l}10.3 \\
10.7\end{array}$ & $\begin{array}{r}9.9 \\
10.1\end{array}$ & $\begin{array}{l}9.9 \\
8.3\end{array}$ & $\begin{array}{l}9.2 \\
6.7\end{array}$ & $\begin{array}{l}8.4 \\
5.2\end{array}$ & $\begin{array}{l}-0.8 \\
-1.5 \mathrm{ss}\end{array}$ \\
\hline 17.0 & 16.8 & 18.0 & 17.0 & 16.3 & 15.2 & 14.7 & 14.0 & 13.3 & 12.4 & 11.9 & 10.9 & -1.0 \\
\hline 90.4 & 91.9 & 92.5 & 93.1 & 93.0 & 93.2 & 92.6 & 92.8 & 92,6 & 92.6 & 92.2 & 91.3 & -0.9 \\
\hline 73.6 & 75.4 & 75.7 & 75.3 & 74.0 & 71.0 & 71.0 & 70.1 & 70.6 & 69.7 & 68.8 & 67.6 & -1.2 \\
\hline
\end{tabular}

NOTES: Level of significance of difference between the two most recent classes: $\mathrm{s}=.05, \mathrm{ss}=.01, \mathrm{sss}=, 001$, NA indicates data not available.

Data based on four questionnaire forms. $\mathrm{N}$ is four-fifths of $\mathrm{N}$ indicated.

Adjusted for underreporting of amyl and butyl nitrites. See text for details.

Data based on a single questionnaire form. $\mathrm{N}$ is one-fifth of $\mathrm{N}$ indicated.

Adjusted for underreporting of PCP. See text for details.

Only drug use which was not under a doctor's orders is included here.

Based on the data from the revised question, which attempts to exclude the inappropriate reporting of non-prescription stimulants. 
TABLE 8

Trends in Annual Prevalence of Seventeen Types of Drugs

Percent who used in last twelve months

\begin{tabular}{|c|c|c|c|c|c|c|c|c|c|c|c|c|}
\hline $\begin{array}{c}\text { Class } \\
\text { of } \\
1975 \\
\end{array}$ & $\begin{array}{c}\text { Class } \\
\text { of } \\
1976 \\
\end{array}$ & $\begin{array}{c}\text { Class } \\
\text { of } \\
1977 \\
\end{array}$ & $\begin{array}{c}\text { Class } \\
\text { of } \\
1978 \\
\end{array}$ & $\begin{array}{c}\text { Class } \\
\text { of } \\
1979 \\
\end{array}$ & $\begin{array}{c}\text { Class } \\
\text { of } \\
1980 \\
\end{array}$ & $\begin{array}{c}\text { Class } \\
\text { of } \\
1981 \\
\end{array}$ & $\begin{array}{c}\text { Class } \\
\text { of } \\
1982 \\
\end{array}$ & $\begin{array}{c}\text { Class } \\
\text { of } \\
1983 \\
\end{array}$ & $\begin{array}{c}\text { Class } \\
\text { of } \\
1984 \\
\end{array}$ & $\begin{array}{c}\text { Class } \\
\text { of } \\
1985 \\
\end{array}$ & $\begin{array}{c}\text { Class } \\
\text { of } \\
1986 \\
\end{array}$ & $\begin{array}{l}' 85-' 86 \\
\text { change } \\
\end{array}$ \\
\hline$(9400)$ & $(15400)$ & $(17100)$ & $(17800)$ & (15500) & (15900) & $(17500)$ & $(17700)$ & $(16300)$ & (15900) & $(16000)$ & (15200) & \\
\hline 40.0 & 44.5 & 47,6 & 50.2 & 50.8 & 48.8 & 46.1 & 44.3 & 42.3 & 40.0 & 40.6 & 38.8 & -1.8 \\
\hline $\begin{array}{l}\text { NA } \\
\text { NA }\end{array}$ & $\begin{array}{l}3.0 \\
N A\end{array}$ & $\begin{array}{l}3.7 \\
N A\end{array}$ & $\begin{array}{l}4.1 \\
N A\end{array}$ & $\begin{array}{l}5.4 \\
8.9\end{array}$ & $\begin{array}{l}4.6 \\
7.9\end{array}$ & $\begin{array}{l}4.1 \\
6.1\end{array}$ & $\begin{array}{l}4.5 \\
6.6\end{array}$ & $\begin{array}{l}4.3 \\
6.2\end{array}$ & $\begin{array}{l}5.1 \\
7.2\end{array}$ & $\begin{array}{l}5.7 \\
7.5\end{array}$ & $\begin{array}{l}6.1 \\
8.9\end{array}$ & $\begin{array}{l}+0.4 \\
+1.4 \mathrm{~s}\end{array}$ \\
\hline NA & $\mathrm{NA}$ & NA & NA & 6.5 & 5.7 & 3.7 & 3.6 & 3.6 & 4.0 & 4.0 & 4.7 & +0.7 \\
\hline $\begin{array}{r}11.2 \\
N A\end{array}$ & $\begin{array}{r}9.4 \\
N A\end{array}$ & $\begin{array}{l}8.8 \\
N A\end{array}$ & $\begin{array}{r}9.6 \\
N A\end{array}$ & $\begin{array}{r}9.9 \\
11.8\end{array}$ & $\begin{array}{r}9.3 \\
10.4\end{array}$ & $\begin{array}{r}9.0 \\
10.1\end{array}$ & $\begin{array}{l}8.1 \\
9.0\end{array}$ & $\begin{array}{l}7.3 \\
8.3\end{array}$ & $\begin{array}{l}6.5 \\
7.3\end{array}$ & $\begin{array}{l}6.3 \\
7.6\end{array}$ & $\begin{array}{l}6.0 \\
7.6\end{array}$ & $\begin{array}{r}-0.3 \\
0.0\end{array}$ \\
\hline $\begin{array}{l}7.2 \\
\mathrm{NA}\end{array}$ & $\begin{array}{l}6.4 \\
\text { NA }\end{array}$ & $\begin{array}{l}5.5 \\
\text { NA }\end{array}$ & $\begin{array}{l}6.3 \\
\text { NA }\end{array}$ & $\begin{array}{l}6.6 \\
7.0\end{array}$ & $\begin{array}{l}6.5 \\
4.4\end{array}$ & $\begin{array}{l}6.5 \\
3.2\end{array}$ & $\begin{array}{l}6.1 \\
2.2\end{array}$ & $\begin{array}{l}5.4 \\
2.6\end{array}$ & $\begin{array}{l}4.7 \\
2.3\end{array}$ & $\begin{array}{l}4.4 \\
2.9\end{array}$ & $\begin{array}{l}4.5 \\
2.4\end{array}$ & $\begin{array}{l}+0.1 \\
-0.5\end{array}$ \\
\hline $\begin{array}{l}5.6 \\
\text { NA }\end{array}$ & $\begin{array}{l}6.0 \\
\text { NA }\end{array}$ & $\begin{array}{l}7.2 \\
\text { NA }\end{array}$ & $\begin{array}{l}9.0 \\
\text { NA }\end{array}$ & $\begin{array}{r}12.0 \\
\text { NA }\end{array}$ & $\begin{array}{r}12.3 \\
\text { NA }\end{array}$ & $\begin{array}{r}12.4 \\
\text { NA }\end{array}$ & $\begin{array}{r}11.5 \\
\text { NA }\end{array}$ & $\begin{array}{r}11.4 \\
\text { NA }\end{array}$ & $\begin{array}{r}11.6 \\
\text { NA }\end{array}$ & $\begin{array}{r}13.1 \\
\text { NA }\end{array}$ & $\begin{array}{r}12.7 \\
4.1\end{array}$ & $\begin{array}{r}-0.4 \\
\text { NA }\end{array}$ \\
\hline 1.0 & 0.8 & 0.8 & 0.8 & 0.5 & 0.5 & 0.5 & 0.6 & 0.6 & 0.5 & 0.6 & 0.5 & -0.1 \\
\hline 5.7 & 5.7 & 6.4 & 6.0 & 6.2 & 6.3 & 5.9 & 5.3 & 5.1 & 5.2 & 5.9 & 5.2 & $-0.7 \mathrm{~s}$ \\
\hline $\begin{array}{r}16.2 \\
N A\end{array}$ & $\begin{array}{r}15.8 \\
N A\end{array}$ & $\begin{array}{r}16.3 \\
N A\end{array}$ & $\begin{array}{r}17.1 \\
N A\end{array}$ & $\begin{array}{r}18.3 \\
N A\end{array}$ & $\begin{array}{r}20.8 \\
N A\end{array}$ & $\begin{array}{r}26.0 \\
N A\end{array}$ & $\begin{array}{l}26.1 \\
20.3\end{array}$ & $\begin{array}{l}24.6 \\
17.9\end{array}$ & $\begin{array}{r}\text { NA } \\
17.7\end{array}$ & $\begin{array}{r}\mathrm{NA} \\
15.8\end{array}$ & $\begin{array}{r}\text { NA } \\
13.4\end{array}$ & $\begin{array}{c}\text { NA } \\
-2.4 s s s\end{array}$ \\
\hline 11.7 & 10.7 & 10.8 & 9.9 & 9.9 & 10.3 & 10.5 & 9.1 & 7.9 & 6.6 & 5.8 & 5.2 & -0.6 \\
\hline $\begin{array}{r}10.7 \\
5.1\end{array}$ & $\begin{array}{l}9.6 \\
4.7\end{array}$ & $\begin{array}{l}9.3 \\
5.2\end{array}$ & $\begin{array}{l}8.1 \\
4.9\end{array}$ & $\begin{array}{l}7.5 \\
5.9\end{array}$ & $\begin{array}{l}6.8 \\
7.2\end{array}$ & $\begin{array}{l}6.6 \\
7.6\end{array}$ & $\begin{array}{l}5.5 \\
6.8\end{array}$ & $\begin{array}{l}5.2 \\
5.4\end{array}$ & $\begin{array}{l}4.9 \\
3.8\end{array}$ & $\begin{array}{l}4.6 \\
2.8\end{array}$ & $\begin{array}{l}4.2 \\
2.1\end{array}$ & $\begin{array}{l}-0.4 \\
-0.7 \mathrm{~s}\end{array}$ \\
\hline 10.6 & 10.3 & 10.8 & 9.9 & 9.6 & 8.7 & 8.0 & 7.0 & 6.9 & 6.1 & 6.1 & 5.8 & -0.3 \\
\hline 84.8 & 85.7 & 87.0 & 87.7 & 88.1 & 87.9 & 87.0 & 86.8 & 87.3 & 86.0 & 85.6 & 84.5 & -1.1 \\
\hline NA & NA & NA & NA & NA & NA & NA & NA & $\mathrm{NA}$ & $\mathrm{NA}$ & NA & NA & NA \\
\hline
\end{tabular}

NOTES: Level of significance of difference between the two most recent classes: $\mathrm{s}=.05, \mathrm{ss}=.01, \mathrm{sss}=.001$. NA indicates data not available,

${ }^{\mathrm{D}}$ Data based on four questionnaire forms. $\mathrm{N}$ is four-fifths of $\mathrm{N}$ indicated.

Adjusted for underreporting of amyl and butyl nitrites. See text for details.

Data based on a single questionnaire form. $\mathrm{N}$ is one-finh of $\mathrm{N}$ indicated.

Adjusted for underreporting of PCP. See text for details.

Only drug use which was not under a doctor's orders is included here.

Based on the data from the revised question, which attempts to exclude the inappropriate reporting of non-prescription stimulants. 
TABLE 9

Trends in Thirty-Day Prevalence of Sixteen Types of Drugs

Percent who used in last thirty days

\begin{tabular}{|c|c|c|c|c|c|c|c|c|c|c|c|c|}
\hline $\begin{array}{c}\text { Class } \\
\text { of } \\
1975 \\
\end{array}$ & $\begin{array}{c}\text { Class } \\
\text { of } \\
1976 \\
\end{array}$ & $\begin{array}{c}\text { Class } \\
\text { of } \\
1977 \\
\end{array}$ & $\begin{array}{c}\text { Class } \\
\text { of } \\
1978 \\
\end{array}$ & $\begin{array}{c}\text { Class } \\
\text { of } \\
1979 \\
\end{array}$ & $\begin{array}{c}\text { Class } \\
\text { of } \\
1980 \\
\end{array}$ & $\begin{array}{c}\text { Class } \\
\text { of } \\
1981 \\
\end{array}$ & $\begin{array}{c}\text { Class } \\
\text { of } \\
1982 \\
\end{array}$ & $\begin{array}{c}\text { Class } \\
\text { of } \\
1983 \\
\end{array}$ & $\begin{array}{c}\text { Class } \\
\text { of } \\
1984 \\
\end{array}$ & $\begin{array}{c}\text { Class } \\
\text { of } \\
1985 \\
\end{array}$ & $\begin{array}{c}\text { Class } \\
\text { of } \\
1986 \\
\end{array}$ & $\begin{array}{l}' 85-' 86 \\
\text { change }\end{array}$ \\
\hline$(9400)$ & $(15400)$ & $(17100)$ & $(17800)$ & $(15500)$ & (15900) & $(17500)$ & $(17700)$ & $(16300)$ & $(15900)$ & $(16000)$ & (15200) & \\
\hline 27.1 & 32.2 & 35.4 & 37.1 & 36.5 & 33.7 & 31.6 & 28.5 & 27.0 & 25.2 & 25.7 & 23.4 & $-2.3 \mathrm{~s}$ \\
\hline $\begin{array}{l}\text { NA } \\
N A\end{array}$ & $\begin{array}{r}0.9 \\
N A\end{array}$ & $\begin{array}{r}1.3 \\
N A\end{array}$ & $\begin{array}{r}1.5 \\
N A\end{array}$ & $\begin{array}{l}1.7 \\
3.2\end{array}$ & $\begin{array}{l}1.4 \\
2.7\end{array}$ & $\begin{array}{l}1.5 \\
2.5\end{array}$ & $\begin{array}{l}1.5 \\
2.5\end{array}$ & $\begin{array}{l}1.7 \\
2.5\end{array}$ & $\begin{array}{l}1.9 \\
2.6\end{array}$ & $\begin{array}{l}2.2 \\
3.0\end{array}$ & $\begin{array}{l}2.5 \\
3.2\end{array}$ & $\begin{array}{l}+0.3 \\
+0.2\end{array}$ \\
\hline NA & NA & NA & NA & 2.4 & 1.8 & 1.4 & 1.1 & 1.4 & 1.4 & 1.6 & 1.3 & -0.3 \\
\hline $\begin{array}{r}1.7 \\
N A\end{array}$ & $\begin{array}{l}3.4 \\
N A\end{array}$ & $\begin{array}{l}4.1 \\
N A\end{array}$ & $\begin{array}{r}3.9 \\
N A\end{array}$ & $\begin{array}{l}4.0 \\
5.3\end{array}$ & $\begin{array}{l}3.7 \\
4.4\end{array}$ & $\begin{array}{l}3.7 \\
4.5\end{array}$ & $\begin{array}{l}3.4 \\
4.1\end{array}$ & $\begin{array}{l}2.8 \\
3.5\end{array}$ & $\begin{array}{l}2.6 \\
3.2\end{array}$ & $\begin{array}{l}2.5 \\
3.8\end{array}$ & $\begin{array}{l}2.5 \\
3.5\end{array}$ & $\begin{array}{r}0.0 \\
-0.3\end{array}$ \\
\hline $\begin{array}{l}2.3 \\
\text { NA }\end{array}$ & $\begin{array}{r}1.9 \\
\text { NA }\end{array}$ & $\begin{array}{l}2.1 \\
\mathrm{NA}\end{array}$ & $\begin{array}{l}2.1 \\
\mathrm{NA}\end{array}$ & $\begin{array}{l}2.4 \\
2.4\end{array}$ & $\begin{array}{l}2.3 \\
1.4\end{array}$ & $\begin{array}{l}2.5 \\
1.4\end{array}$ & $\begin{array}{l}2.4 \\
1.0\end{array}$ & $\begin{array}{l}1.9 \\
1.3\end{array}$ & $\begin{array}{l}1.5 \\
1.0\end{array}$ & $\begin{array}{l}1.6 \\
1.6\end{array}$ & $\begin{array}{l}1.7 \\
1.3\end{array}$ & $\begin{array}{l}+0.1 \\
-0.3\end{array}$ \\
\hline 1.9 & 2.0 & 2.9 & 3.9 & 5.7 & 5.2 & 5.8 & 5.0 & 4.9 & 5.8 & 6.7 & 6.2 & -0.5 \\
\hline 0.4 & 0.2 & 0.3 & 0.3 & 0.2 & 0.2 & 0.2 & 0.2 & 0.2 & 0.3 & 0.3 & 0.2 & -0.1 \\
\hline 2.1 & 2.0 & 2.8 & 2.1 & 2.4 & 2.4 & 2.1 & 1,8 & 1.8 & 1.8 & 2.3 & 2.0 & -0.3 \\
\hline $\begin{array}{r}8.5 \\
N A\end{array}$ & $\begin{array}{r}7.7 \\
N A\end{array}$ & $\begin{array}{l}8.8 \\
N A\end{array}$ & $\begin{array}{l}8.7 \\
N A\end{array}$ & $\begin{array}{l}9.9 \\
N A\end{array}$ & $\begin{array}{r}12.1 \\
N A\end{array}$ & $\begin{array}{c}15.8 \\
N A\end{array}$ & $\begin{array}{l}13.7 \\
10.7\end{array}$ & $\begin{array}{r}12.4 \\
8.9\end{array}$ & $\begin{array}{l}\text { NA } \\
8.3\end{array}$ & $\begin{array}{l}\text { NA } \\
6.8\end{array}$ & $\begin{array}{l}\text { NA } \\
5.5\end{array}$ & $\begin{array}{c}\mathrm{NA} \\
-1.3 \mathrm{ss}\end{array}$ \\
\hline 5.4 & 4.5 & 5.1 & 4.2 & 4.4 & 4.8 & 4.6 & 3.4 & 3.0 & 2.3 & 2.4 & 2.2 & -0.2 \\
\hline $\begin{array}{l}4.7 \\
2.1\end{array}$ & $\begin{array}{l}3.9 \\
1.6\end{array}$ & $\begin{array}{l}4.3 \\
2.3\end{array}$ & $\begin{array}{l}3.2 \\
1.9\end{array}$ & $\begin{array}{l}3.2 \\
2.3\end{array}$ & $\begin{array}{l}2.9 \\
3.3\end{array}$ & $\begin{array}{l}2.6 \\
3.1\end{array}$ & $\begin{array}{l}2.0 \\
2.4\end{array}$ & $\begin{array}{l}2.1 \\
1.8\end{array}$ & $\begin{array}{l}1.7 \\
1.1\end{array}$ & $\begin{array}{l}2.0 \\
1.0\end{array}$ & $\begin{array}{l}1.8 \\
0.8\end{array}$ & $\begin{array}{l}-0.2 \\
-0.2\end{array}$ \\
\hline 4.1 & 4.0 & 4.6 & 3.4 & 3.7 & 3.1 & 2.7 & 2.4 & 2.5 & 2.1 & 2.1 & 2.1 & 0.0 \\
\hline 68.2 & 68.3 & 71.2 & 72.1 & 71.8 & 72.0 & 70.7 & 69.7 & 69.4 & 67.2 & 65.9 & 65.3 & -0.6 \\
\hline 36.7 & 38.8 & 38.4 & 36.7 & 34.4 & 30.5 & 29.4 & 30.0 & 30.3 & 29.3 & 30.1 & 29.6 & -0.5 \\
\hline
\end{tabular}

Marijuana/Hashish

Approx. $\mathrm{N}=$

Inhalants ${ }^{\mathrm{a}}$

Inhalants Adjusted ${ }^{b}$

Amyl \& Butyl Nitrites ${ }^{c}$

Hallucinogens

Hallucinogens Adjusted

LSD

$\mathrm{PCP}^{\mathrm{C}}$

Cocaine

Heroin

Other opiates ${ }^{\mathrm{e}}$

Stimulants ${ }^{e}$

Stimulants Adjusted ${ }^{c, f}$

Sedatives $^{\mathrm{e}}$

Barbiturates ${ }^{e}$

Methaqualone

Tranquilizers ${ }^{\mathrm{e}}$

Alcohol

Cigarettes

pprox. $\mathrm{N}=$

Class

NOTES: Level of significance of difference between the two most recent classes: $\mathrm{s}=.05, \mathrm{ss}=.01, \mathrm{sss}=.001$. NA indicates data not available.

Data based on four questionnaire forms. $\mathrm{N}$ is four-fifths of $\mathrm{N}$ indicated.

$\mathrm{b}$ Adjusted for underreporting of amyl and butyl nitrites. See text for details.

Data based on a single questionnaire form. $\mathrm{N}$ is one-fifth of $\mathrm{N}$ indicated.

Adjusted for underreporting of PCP. See text for details.

Only drug use which was not under a doctor's orders is included here.

Based on the data from the revised question, which attempts to exclude the inappropriate reporting of non-prescription stimulants. 
TABLE 10

Trends in Thirty-Day Prevalence of Daily Use of Sixteen Types of Drugs

Percent who used daily in last thirty days

\begin{tabular}{|c|c|c|c|c|c|c|c|c|c|c|c|c|}
\hline $\begin{array}{c}\text { Class } \\
\text { of } \\
1975 \\
\end{array}$ & $\begin{array}{c}\text { Class } \\
\text { of } \\
1976 \\
\end{array}$ & $\begin{array}{c}\begin{array}{c}\text { Class } \\
\text { of } \\
1977 \\
\end{array} \\
\end{array}$ & $\begin{array}{c}\text { Class } \\
\text { of } \\
1978 \\
\end{array}$ & $\begin{array}{c}\text { Class } \\
\text { of } \\
1979 \\
\end{array}$ & $\begin{array}{c}\begin{array}{c}\text { Class } \\
\text { of } \\
1980 \\
\end{array} \\
\end{array}$ & $\begin{array}{c}\text { Class } \\
\text { of } \\
1981 \\
\end{array}$ & $\begin{array}{c}\text { Class } \\
\text { of } \\
1982 \\
\end{array}$ & $\begin{array}{c}\text { Class } \\
\text { of } \\
1983 \\
\end{array}$ & $\begin{array}{c}\text { Class } \\
\text { of } \\
1984 \\
\end{array}$ & $\begin{array}{c}\text { Class } \\
\text { of } \\
1985 \\
\end{array}$ & $\begin{array}{c}\text { Class } \\
\text { of } \\
1986 \\
\end{array}$ & $\begin{array}{l}' 85-' 86 \\
\text { change } \\
\end{array}$ \\
\hline (9400) & (15400) & $(17100)$ & $(17800)$ & $(15500)$ & (15900) & $(17500)$ & $(17700)$ & $(16300)$ & $(15900)$ & $(16000)$ & $(15200)$ & \\
\hline 6.0 & 8.2 & 9.1 & 10.7 & 10.3 & 9.1 & 7.0 & 6.3 & 5.5 & 5.0 & 4.9 & 4.0 & $-0.9 \mathrm{~s}$ \\
\hline $\begin{array}{l}\text { NA } \\
N A\end{array}$ & $\begin{array}{r}0.0 \\
N A\end{array}$ & $\begin{array}{r}0.0 \\
N A\end{array}$ & $\begin{array}{l}0.1 \\
N A\end{array}$ & $\begin{array}{l}0.0 \\
0.1\end{array}$ & $\begin{array}{l}0.1 \\
0.2\end{array}$ & $\begin{array}{l}0.1 \\
0.2\end{array}$ & $\begin{array}{l}0.1 \\
0.2\end{array}$ & $\begin{array}{l}0.1 \\
0.2\end{array}$ & $\begin{array}{l}0.1 \\
0.2\end{array}$ & $\begin{array}{l}0.2 \\
0.4\end{array}$ & $\begin{array}{l}0.2 \\
0.4\end{array}$ & $\begin{array}{l}0.0 \\
0.0\end{array}$ \\
\hline NA & NA & NA & NA & 0.0 & 0.1 & 0.1 & 0.0 & 0.2 & 0.1 & 0.3 & 0.5 & +0.2 \\
\hline $\begin{array}{r}0.1 \\
N A\end{array}$ & $\begin{array}{r}0.1 \\
N A\end{array}$ & $\begin{array}{r}0.1 \\
N A\end{array}$ & $\begin{array}{l}0.1 \\
N A\end{array}$ & $\begin{array}{l}0.1 \\
0.2\end{array}$ & $\begin{array}{l}0.1 \\
0.2\end{array}$ & $\begin{array}{l}0.1 \\
0.1\end{array}$ & $\begin{array}{l}0.1 \\
0.2\end{array}$ & $\begin{array}{l}0.1 \\
0.2\end{array}$ & $\begin{array}{l}0.1 \\
0.2\end{array}$ & $\begin{array}{l}0.1 \\
0.3\end{array}$ & $\begin{array}{l}0.1 \\
0.3\end{array}$ & $\begin{array}{l}0.0 \\
0.0\end{array}$ \\
\hline $\begin{array}{l}0.0 \\
\text { NA }\end{array}$ & $\begin{array}{l}0.0 \\
\text { NA }\end{array}$ & $\begin{array}{l}0.0 \\
\mathrm{NA}\end{array}$ & $\begin{array}{l}0.0 \\
\text { NA }\end{array}$ & $\begin{array}{l}0.0 \\
0.1\end{array}$ & $\begin{array}{l}0.0 \\
0.1\end{array}$ & $\begin{array}{l}0.1 \\
0.1\end{array}$ & $\begin{array}{l}0.0 \\
0.1\end{array}$ & $\begin{array}{l}0.1 \\
0.1\end{array}$ & $\begin{array}{l}0.1 \\
0.1\end{array}$ & $\begin{array}{l}0.1 \\
0.3\end{array}$ & $\begin{array}{l}0.0 \\
0.2\end{array}$ & $\begin{array}{r}0.0 \\
-0.1\end{array}$ \\
\hline 0.1 & 0.1 & 0.1 & 0.1 & 0.2 & 0.2 & 0.3 & 0.2 & 0.2 & 0.2 & 0.4 & 0.4 & +0.1 \\
\hline 0.1 & 0.0 & 0.0 & 0.0 & 0.0 & 0.0 & 0.0 & 0.0 & 0.1 & 0.0 & 0.0 & 0.0 & 0.0 \\
\hline 0.1 & 0.1 & 0.2 & 0.1 & 0.0 & 0.1 & 0.1 & 0.1 & 0.1 & 0.1 & 0.1 & 0.1 & 0.0 \\
\hline $\begin{array}{r}0.5 \\
N A\end{array}$ & $\begin{array}{c}0.4 \\
N A\end{array}$ & $\begin{array}{r}0.5 \\
N A\end{array}$ & $\begin{array}{l}0.5 \\
N A\end{array}$ & $\begin{array}{l}0.6 \\
N A\end{array}$ & $\begin{array}{r}0.7 \\
N A\end{array}$ & $\begin{array}{l}1.2 \\
N A\end{array}$ & $\begin{array}{l}1.1 \\
0.7\end{array}$ & $\begin{array}{l}1.1 \\
0.8\end{array}$ & $\begin{array}{l}\text { NA } \\
0.6\end{array}$ & $\begin{array}{l}\mathrm{NA} \\
0.4\end{array}$ & $\begin{array}{l}\text { NA } \\
0.3\end{array}$ & $\begin{array}{r}\mathrm{NA} \\
-0.1\end{array}$ \\
\hline 0.3 & 0.2 & 0.2 & 0.2 & 0.1 & 0.2 & 0.2 & 0.2 & 0.2 & 0.1 & 0,1 & 0.1 & 0.0 \\
\hline $\begin{array}{l}0.1 \\
0.0\end{array}$ & $\begin{array}{l}0.1 \\
0.0\end{array}$ & $\begin{array}{l}0.2 \\
0.0\end{array}$ & $\begin{array}{l}0.1 \\
0.0\end{array}$ & $\begin{array}{l}0.0 \\
0.0\end{array}$ & $\begin{array}{l}0.1 \\
0.1\end{array}$ & $\begin{array}{l}0.1 \\
0.1\end{array}$ & $\begin{array}{l}0.1 \\
0.1\end{array}$ & $\begin{array}{l}0.1 \\
0.0\end{array}$ & $\begin{array}{l}0.0 \\
0.0\end{array}$ & $\begin{array}{l}0.1 \\
0.0\end{array}$ & $\begin{array}{l}0.1 \\
0.0\end{array}$ & $\begin{array}{l}0.0 \\
0.0\end{array}$ \\
\hline 0.1 & 0.2 & 0.3 & 0.1 & 0.1 & 0.1 & 0.1 & 0.1 & 0.1 & 0.1 & 0.0 & 0.0 & 0.0 \\
\hline 5.7 & 5.6 & 6.1 & 5.7 & 6.9 & 6.0 & 6.0 & 5.7 & 5.5 & 4.8 & 5.0 & 4.8 & -0.3 \\
\hline 26.9 & 28.8 & 28.8 & 27.5 & 25.4 & 21.3 & 20.3 & 21.1 & 21.2 & 18.7 & 19.5 & 18.7 & -0.8 \\
\hline
\end{tabular}

NOTES: Level of significance of difference between the two most recent classes: $\mathrm{s}=.05, \mathrm{ss}=.01, \mathrm{sss}=.001$. NA indicates data not available.

Data based on four questionnaire forms. $\mathrm{N}$ is four-fifths of $\mathrm{N}$ indicated.

Adjusted for underreporting of amyl and butyl nitrites. See text for details.

Data based on a single questionnaire form. $\mathrm{N}$ is one-fifth of $\mathrm{N}$ indicated.

Adjusted for underreporting of PCP. See text for details.

'Only drug use which was not under a doctor's orders is included here.

Based on the data from the revised question, which attempts to exclude the inappropriate reporting of non-prescription stimulants.

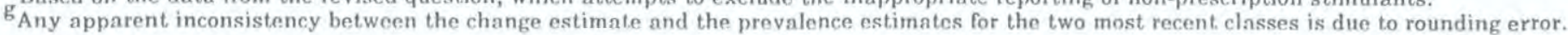


regular use, and a growing perception that peers would disapprove of regular marijuana use.

- Until 1978, the proportion of seniors involved in any illicit drug use had increased steadily, primarily because of the increase in marijuana use. About $54 \%$ of the classes of 1978 and 1979 reported having tried at least one illicit drug during the last. year, up from $45 \%$ in the class of 1975 . Between 1979 and 1984, however, the proportion reporting using any illicit drug during the prior year dropped by 1 or $2 \%$ annually until 1985, when no further decline was observed: in fact, there had been a slight increase in the proportion reporting use of any illicit drug in the previous year from $45.8 \%$ in 1984 to $46.3 \%$ in 1985 (revised version). This year the decline resumed, to $44.3 \%$ (the drop between 1985 and 1986 is significant at the .05 level). The overall decline in the proportion of students having any involvement with illicit drugs appears to be due primarily to the change in marijuana use.

- As Figure 6 and Table 11 illustrate, between 1976 and 1982 there had been a very gradual, steady increase in the proportion who have ever used some illicit drug other than marijuana. The proportion going beyond marijuana in their lifetime had risen from $35 \%$ to $45 \%$ between 1976 and 1982 . The revised statistic remained stable between 1983 and 1984 , decreased slightly in 1985 , and then decreased by another $2 \%$ in 1986. The annual prevalence of such behaviors (Figure 7), which had risen 9\% between 1976 and 1981 , leveled in 1982, and then dropped back slightly in each subsequent year to $26 \%$ in 1986 . But the current (or 30-day) prevalence figures actually began to drop a year earlier-in 1982-and have shown the largest proportional drop (as may be seen in Figure 8 and in Table 11).

- Most of the earlier rise in other illicit drug use appeared to be due to the increasing popularity of cocaine with this age group between 1976 and 1979, and then due to the increasing use of stimulants between 1979 and 1982. However, as stated earlier, we believe that this upward shift was exaggerated because some respondents included instances of using over-the-counter stimulants in their reports of amphetamine use. (See discussion at the end of the introductory section.) A rather different picture of what trends have been occurring in the proportions using illicit drugs other than marijuana emerges when self-reported amphetamine use is excluded from the calculations altogether. (This obviously understates the percentage using illicits other than marijuana in any given year, but it might yield a more accurate picture of 
TABLE 11

Trends in Lifetime, Annual, and Thirty-Day Prevalence in an Index of Illicit Drug Use (Based on Original and Adjusted Amphetamine Questions) ${ }^{\mathrm{a}}$

\begin{tabular}{|c|c|c|c|c|c|c|c|c|c|c|c|c|c|}
\hline & $\begin{array}{c}\text { Class } \\
\text { of } \\
1975 \\
\end{array}$ & $\begin{array}{c}\text { Class } \\
\text { of } \\
1976 \\
\end{array}$ & $\begin{array}{c}\text { Class } \\
\text { of } \\
1977 \\
\end{array}$ & $\begin{array}{c}\text { Class } \\
\text { of } \\
1978 \\
\end{array}$ & $\begin{array}{c}\text { Class } \\
\text { of } \\
1979 \\
\end{array}$ & $\begin{array}{c}\begin{array}{c}\text { Class } \\
\text { of } \\
1980 \\
\end{array} \\
\end{array}$ & $\begin{array}{c}\text { Class } \\
\text { of } \\
1981 \\
\end{array}$ & $\begin{array}{c}\text { Class } \\
\text { of } \\
1982 \\
\end{array}$ & $\begin{array}{c}\text { Clnss } \\
\text { of } \\
1983 \\
\end{array}$ & 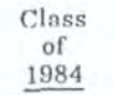 & $\begin{array}{c}\text { Class } \\
\text { of } \\
1985 \\
\end{array}$ & $\begin{array}{c}\text { Class } \\
\text { of } \\
1986 \\
\end{array}$ & $\begin{array}{l}' 85-' 86 \\
\text { change }\end{array}$ \\
\hline Approx. $N=$ & $(9400)$ & $(15400)$ & $(17100)$ & $(17800)$ & $(15500)$ & (15900) & $(17500)$ & (17700) & $(16300)$ & (15900) & $(16000)$ & (15200) & \\
\hline
\end{tabular}

Percent reporting use in lifetime

\section{Marijuana Only Adjusted Version \\ Any Illicit Drug Ofher Than Marijuana Adjusted Version}

Total: Any Illicit Drug Use
Adjusted Version

,

$\begin{array}{cr}19.0 & 22.9 \\ - & \\ 36.2 & 35 \\ - & \end{array}$

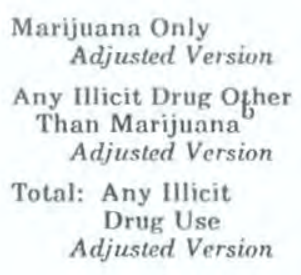

$$
\begin{aligned}
& \text { Any Illicit } \\
& \text { Drug Use } \\
& \text { Adjusted Version }
\end{aligned}
$$

20.8
23.3

22.5

21.3

20.9

$19.9-1.0$

$-$

-

\begin{tabular}{|c|c|c|c|c|c|c|c|c|c|c|c|c|c|}
\hline Marijuana Only & 18.8 & 22.7 & 25.1 & 26.7 & 26.0 & 22.7 & 18.1 & 17.0 & 16.6 & - & - & - & \\
\hline Adjusted Version & - & - & - & - & - & - & - & 19.3 & 19.0 & 17.8 & 18.9 & 18.4 & -0.5 \\
\hline $\begin{array}{l}\text { Any Illicit Drug Ofhe } \\
\text { Than Marijuana }\end{array}$ & 26.2 & 25.4 & 26.0 & 27.1 & 28.2 & 30.4 & 34.0 & 33.8 & 32.5 & - & - & - & \\
\hline Adjusted Version & - & - & - & - & - & - & - & 30.1 & 28.4 & 28.0 & 27.4 & 25.9 & -1.5 \\
\hline $\begin{array}{l}\text { Total: Any Illicit } \\
\text { Drug Use }\end{array}$ & 45.0 & 48.1 & 51.1 & 53.8 & 51.2 & 53.1 & 52.1 & 50.8 & 49.1 & - & - & - & \\
\hline \multirow[t]{2}{*}{ Adjusted Version } & - & - & - & - & - & - & - & 49.4 & 47.4 & 45.8 & 46.3 & 44.3 & $-2.0 \mathrm{~s}$ \\
\hline & \multicolumn{12}{|c|}{ Percent reporting use in last thirty days } & \\
\hline Marijuana Only & 15.3 & 20.3 & 22.4 & 23.8 & 22.2 & 18.8 & 15.2 & 14.3 & 14.0 & - & - & - & \\
\hline Adjusted Version & - & - & - & - & - & - & - & 15.5 & 15.1 & 14.1 & 14.8 & 13.9 & -0.9 \\
\hline $\begin{array}{c}\text { Any Illicit Drug Othe } \\
\text { Than Marijuana }\end{array}$ & 15.4 & 13.9 & 15.2 & 15.1 & 16.8 & 18.4 & 21.7 & 19.2 & 18.1 & - & - & - & \\
\hline Adjusted Version & - & - & - & - & - & - & - & 17.0 & 15.4 & 15.1 & 14.9 & 13.2 & $-1.7 \mathrm{ss}$ \\
\hline Total: Any Illicit & & & & & & & & & & & & & \\
\hline Drug Use & 30.7 & 34.2 & 37.6 & 38.9 & 38.9 & 37.2 & 36.9 & 33.5 & 32.4 & - & - & - & \\
\hline Adjusted Version & - & - & - & - & - & - & - & 32.5 & 30.5 & 29.2 & 29.7 & 27.1 & $-2.6 s s$ \\
\hline
\end{tabular}

$$
1
$$

$\begin{array}{cccc}- & - & - & - \\ 35.8 & 36.5 & 37.4 & 38.7 \\ - & - & - & -\end{array}$

$\begin{array}{ccc}42.8 & 45.0 & 44.4 \\ - & 41.1 & 40.4\end{array}$

$40 . \overline{3}$

39.7

37.7

$-2.0 \mathrm{~s}$

$\begin{array}{rr}65.1 & 65.4 \\ - & -\end{array}$

65,6

65.8
64.4

64.1
62.9

$61 . \overline{6}$

60.

$57 . \overline{6}-3.0 \mathrm{ss}$

Percent reporting use in last twelve months

NOTES: Level of significance of difference between the two most recent classes: $\mathrm{s}=.05, \mathrm{ss}=.01, \mathrm{sss}=.001$.

Adjusted questions about stimulant use were introduced in 1982 to exclude more completely the inappropriate reporting of non-prescription stimulants.

b Use of "other illicit drugs" includes any use of hallucinogens, cocaine, and heroin, or any use of other opiates, stimulants, sedatives, or tranquilizers not under a doctor's orders. 
trends in proportions up through 1982, when new questions were introduced to deal with the problem directly.) Figures 6-8 (and other figures to follow) have been annotated with small markings ( ) next to each year's bar, showing where the shaded area would stop if amphetamine (stimulant) use were excluded entirely. The cross-time trend in these markings shows that the proportion going beyond marijuana to illicits other than amphetamines during the prior year was almost constant between 1975 and 1981. However, this figure began to drop gradually from $24 \%$ in 1981 to $21 \%$ in 1986, where it has since levelled.

- Thus, with stimulants excluded from the calculations entirely, we are able to see a gradual drop between 1981 and 1984 in the proportion of seniors using illicit drugs other than marijuana, following a considerable period of virtually level use. With stimulants (including the incorrectly reported ones) included in the definition, we also see a downturn in recent years, but following a period of considerable increase. Finally, using the corrected stimulant statistics for 1982 and thereafter (marked with the symbol $(\triangleleft)$ in Figures $6-8$ ), we still see the downturn in recent years, but it follows a period of what we deduce to have been a modest increase in use from the mid-seventies to 1982 . Note that the use of illicits other than marijuana continued to drop in 1985 and 1986 (because amphetamine use has been dropping); however, the use of illicits other than marijuana or amphetamines has not dropped in the past two years.

- Although the overall proportion using illicit drugs other than marijuana has changed rather gradually during recent years, more varied and turbulent changes have been occurring for specific drugs within the class. (See Tables 7, 8, and 9 for trends in lifetime, annual, and monthly prevalence figures for each class of drugs.)

- From 1976 to 1979 cocaine exhibited a substantial increase in popularity, with annual prevalence going from $6 \%$ in the class of 1976 to $12 \%$ in the class of $1979-$ a two-fold increase in just three years. For the nation as a whole, we judge there to have been little or no change in any of the cocaine prevalence statistics between 1979 and 1984. (Some possible regional changes will be discussed below.) In 1985, however, we reported statistically significant increases in annual and monthly use. While these measures did not show further increase in 1986, it is noteworthy that they did not drop by a statistically significant amount either, considering the amount of adverse publicity cocaine use was receiving by then. 
FIGURE 6

Trends in Lifetime Prevalence of an Mllicit Drug Use Index

All Seniors

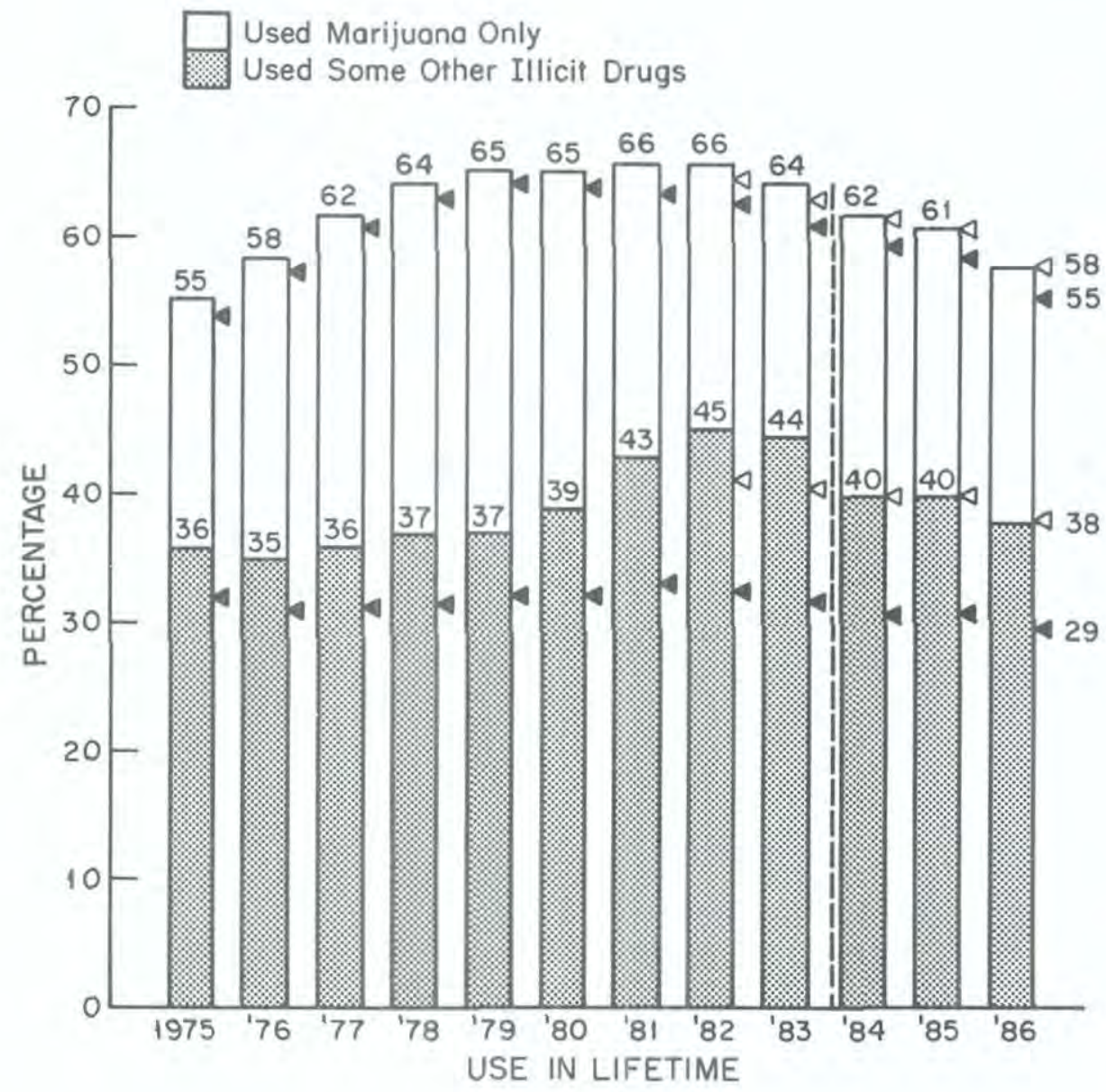

NOTES: Use of "some other illicit drugs" includes any use of hallucinogens, cocaine, and heroin, or any use which is not under a doctor's orders of other opiates, stimulants, sedatives, or tranquilizers.

4 indicates the percentage which results if all stimulants are excluded from the definition of "illicit drugs." $\triangleleft$ shows the percentage which results if only non-prescription stimulants are excluded.

The dashed vertical line indicates that after 1983 the shaded and open bars are defined by using the amphetamine questions which were revised to exclude non-prescription stimulants from the definition of "illicit drugs." 
Some special analyses conducted this year to explore the role of crack in these statistics reveals some adverse developments not captured in the overall prevalence statistics. For example, we find that (a) the proportion of seniors reporting that they smoked cocaine (as well as having used in the past year) doubled between 1983 and 1986 from $2.5 \%$ to $6.0 \%$, (b) there was also a doubling in the same period (from $0.4 \%$ to $0.8 \%$ ) in the proportion of all seniors who said that they both had used cocaine during the prior year and had at some time been unable to stop using when they tried to stop, and (c) there was a doubling between 1984 and 1986 in the proportion of seniors reporting active daily use (from $0.2 \%$ to $0.4 \%$ ). We think it likely that the advent of crack use during this period contributed to these developments. (Recall that the annual prevalence of crack use stands at $4.1 \%$ in 1986 , the first year in which such use was measured directly.)

- Like cocaine use, inhalant use had been rising steadily in the mid-1970's, though more slowly and from a lower overall level. Annual prevalence (in the unadjusted version) rose from $3.0 \%$ in 1976 and reached a peak of $5.4 \%$ in 1979 . Then, between 1979 and 1983, there was an overall declinein part due to a substantial drop in the use of the amyl and butyl nitrites, for which annual prevalence declined from $6.5 \%$ in 1979 to $3.6 \%$ in 1983 . Both measures increased slightly between 1983 and 1985 , with annual use for inhalants (adjusted for use of nitrites) increasing from $6.2 \%$ in 1983 to $7.5 \%$ in 1985 , and the nitrites increasing from $3.6 \%$ to $4.0 \%$. In 1986 annual inhalant use jumped sig. nificantly to $8.9 \%$, and nitrites use also increased, though not significantly, to $4.7 \%$. Current (30-day) use of inhalants also increased by $0.2 \%$, while nitrite use decreased by $0.3 \%$.

- Stimulant (amphetamine) use, which had remained relatively unchanged between 1975 and 1978, began to show evidence of a gradual increase in use in 1979, with even greater increases to occur in 1980 and 1981. Between 1976 and 1981 , reported annual prevalence rose by a full $10.2 \%$ (from $15.8 \%$ in 1976 to $26.0 \%$ in 1981 ); and daily use tripled, from $0.4 \%$ in 1976 to $1.2 \%$ in 1981 . As stated earlier, we think these increases were exaggerated-perhaps sharply exaggerated-by respondents in the 1980 and 1981 surveys in particular including nonamphetamine, over-the-counter diet pills (as well as "look-alike" and "sound-alike" pills) in their answers. In 1982, we added new versions of the questions on amphetamine use, which were more explicit in instructing respondents not to include such nonprescription pills. (These were added to only three of the five forms of the questionnaire being used; the amphetamine questions were left 
FIGURE 7

Trends in Annual Prevalence of an Illicit Drug Use Index

All Seniors

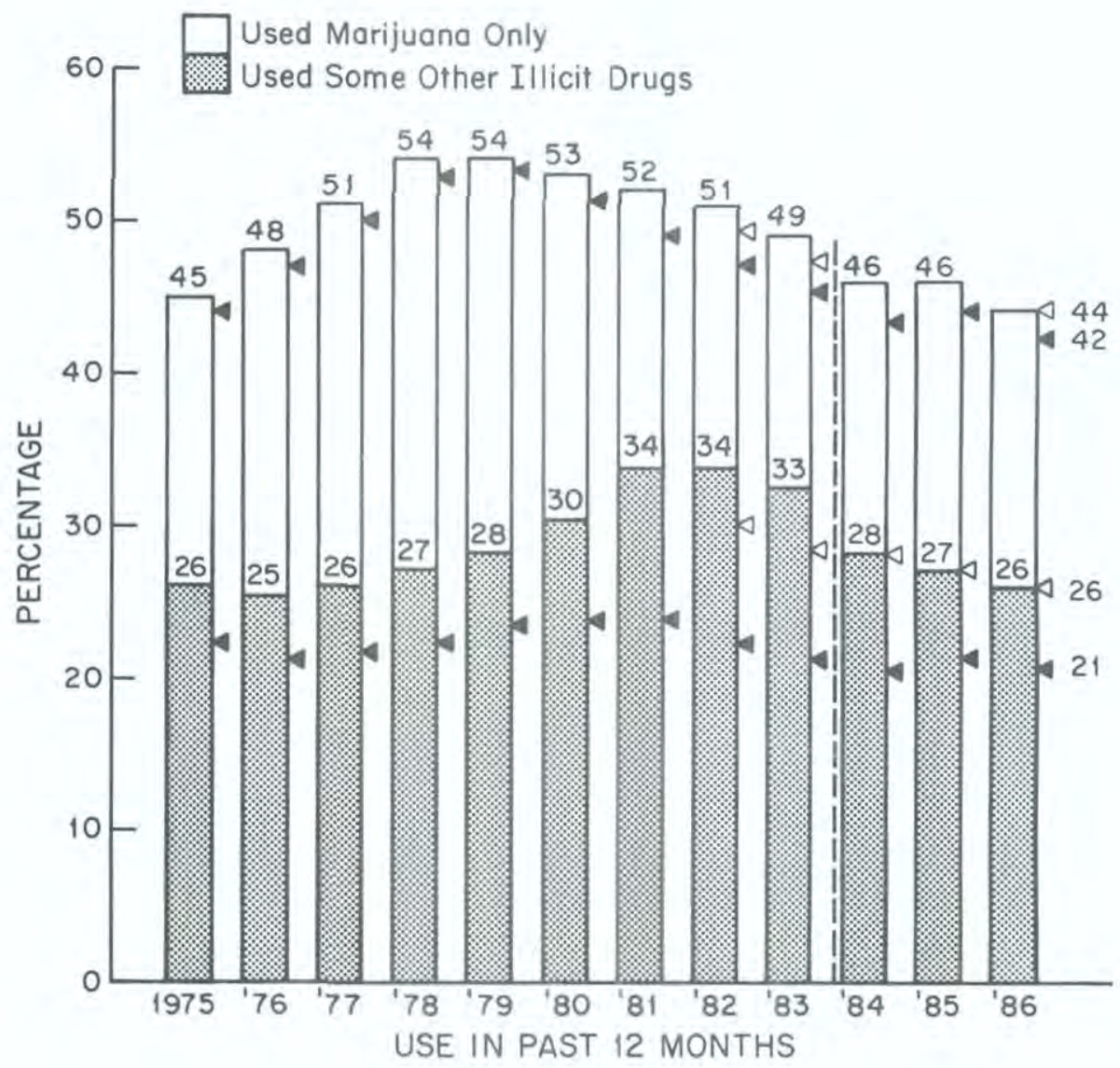

NOTES: Use of "some other illicit drugs" includes any use of hallucinogens, cocaine, and heroin, or any use which is not under a doctor's orders of other opiates, stimulants, sedatives, or tranquilizers.

4 indicates the percentage which results if all stimulants are excluded from the definition of "illicit drugs." $\triangleleft$ shows the percentage which results if only non-prescription stimulants are excluded.

The dashed vertical line indicates that after 1983 the shaded and open bars are defined by using the amphetamine questions which were revised to exclude non-prescription stimulants from the definition of "illicit drugs." 
unchanged in the other two forms until 1984.) As a result, Tables 7 through 11 give two estimates for amphetamines: one is based on the unchanged questions, which provides comparable data across time for longer-term trend estimates; the second (adjusted) estimate, based on the revised questions, provides our best assessments of current prevalence and recent trends in true amphetamine use. ${ }^{12}$

As can be seen in 1982 and 1983, the two years for which both adjusted and unadjusted statistics are available, the unadjusted showed a modest amount of overreporting. Both types of statistics, however, suggest that a downturn in the current use of stimulants began to occur in 1982 and has continued since. For example, between 1982 and 1986 the annual prevalence for amphetamines (adjusted) fell by roughly one-third, from $20 \%$ to $13 \%$. Current use fell by an even larger proportion. Still, in the class of 1986 nearly a quarter of all seniors $(23.4 \%)$ have tried amphetamines (adjusted).

- For sedatives the sustained, gradual decline between 1975 and 1979 halted in 1980 and 1981. For example, annual prevalence, which dropped steadily from $11.7 \%$ in 1975 to $9.9 \%$ in 1979 , increased slightly to $10.5 \%$ by 1981 . In 1982 , though, the longer-term decline resumed again and annual prevalence has now fallen to $5.2 \%$. In sum, annual sedative use has dropped by more than one-half since the study began in 1975. But, the overall trend lines for sedatives mask differential trends occurring for the two components of the measure (see Figure 9c). Barbiturate use has declined rather steadily since 1975 , and now stands at below half its 1975 level in terms of annual prevalence (i.e., at $4.2 \%$ vs. $10.7 \%$ in 1975). Methaqualone use, on the other hand, rose sharply from 1976 until 1981. (In fact, it was the only drug other than stimulants that was still rising in 1981.) But in 1982 , the use of methaqualone also began to decline, which accounted for the overall sedative category resuming its decline. Annual use now stands at less than one-third of its peak level observed by 1981 (2.1\% in 1986 vs. $7.6 \%$ in 1981).

- The usage statistics for tranquilizers peaked in 1977, and have declined since then. Lifetime prevalence has dropped from $18 \%$ in 1977 to $11 \%$ in 1986 , annual prevalence from $11 \%$ to $6 \%$, and 30 -day prevalence from $4.6 \%$ to $2.1 \%$.

${ }^{12}$ We think the unadjusted estimates for the earliest years of the survey were probably little affected by the improper inclusion of nonprescription stimulants, since sales of the latter did not burgeon until after the 1979 data collection. 
FIGURE 8

Trends in Thirty-Day Prevalence of an Illicit Drug Use Index All Seniors

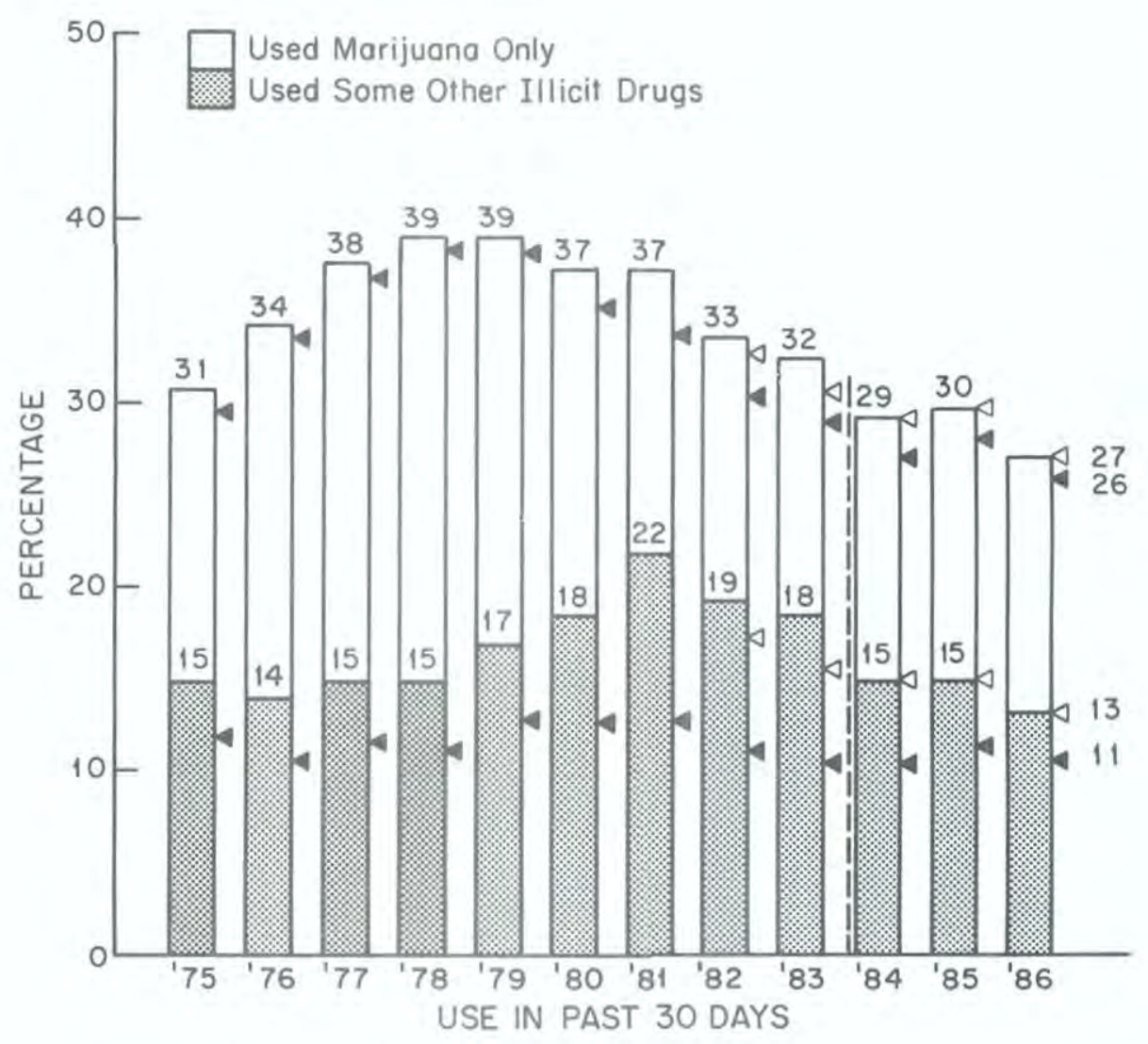

NOTES: Use of "some other illicit drugs" includes any use of hallucinogens, cocaine, and heroin, or any use which is not under a doctor's orders of other opiates, stimulants, sedatives, or tranquilizers.

indicates the percentage which results if all stimulants are excluded from the definition of "illicit drugs." $\triangleleft$ shows the percentage which results if only non-prescription stimulants are excluded.

The dashed vertical line indicates that after 1983 the shaded and open bars are defined by using the amphetamine questions which were revised to exclude non-prescription stimulants from the definition of "illicit drugs." 
FIGURE 9a

Trends in Lifetime, Annual, and Thirty-Day Prevalence of Sixteen Drugs
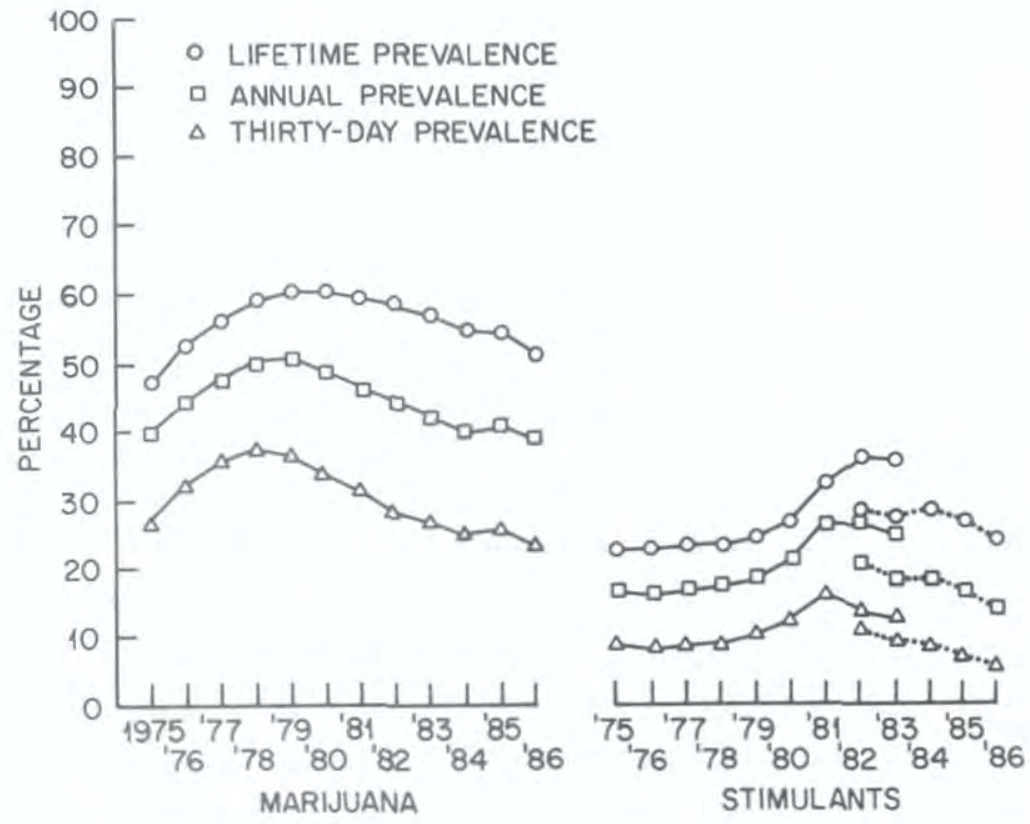

NOTE: The dotted lines connect percentages which result if non-prescription stimulants are excluded. 


\section{FIGURE $9 \mathrm{~b}$}

Trends in Lifetime, Annual, and Thirty-Day Prevalence of Sixteen Drugs
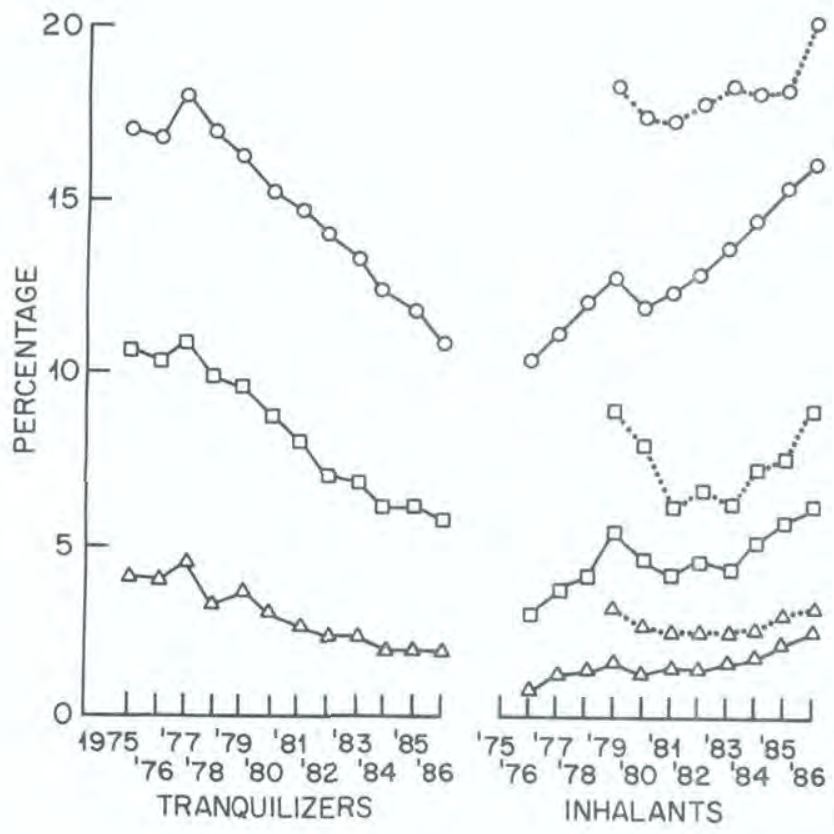

- LIFETIME PREVALENCE

- ANNUAL PREVALENCE

$\triangle$ THIRTY-DAY PREVALENCE

TRANQUILIZERS

INHALANTS
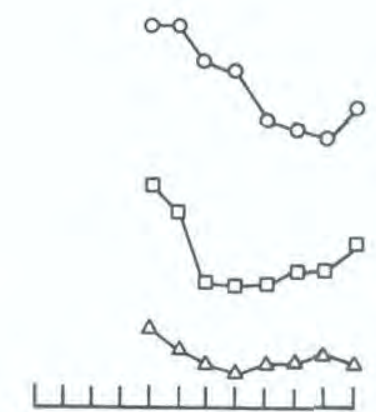



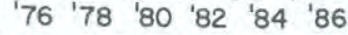

AMYL \& BUTYL NITRITES

NOTE: The dotted lines connect percentages which are adjusted for underreporting of amyl and butyl nitrites. 


\section{FIGURE $9 c$}

Trends in Lifetime, Annual, and Thirty-Day Prevalence of Sixteen Drugs
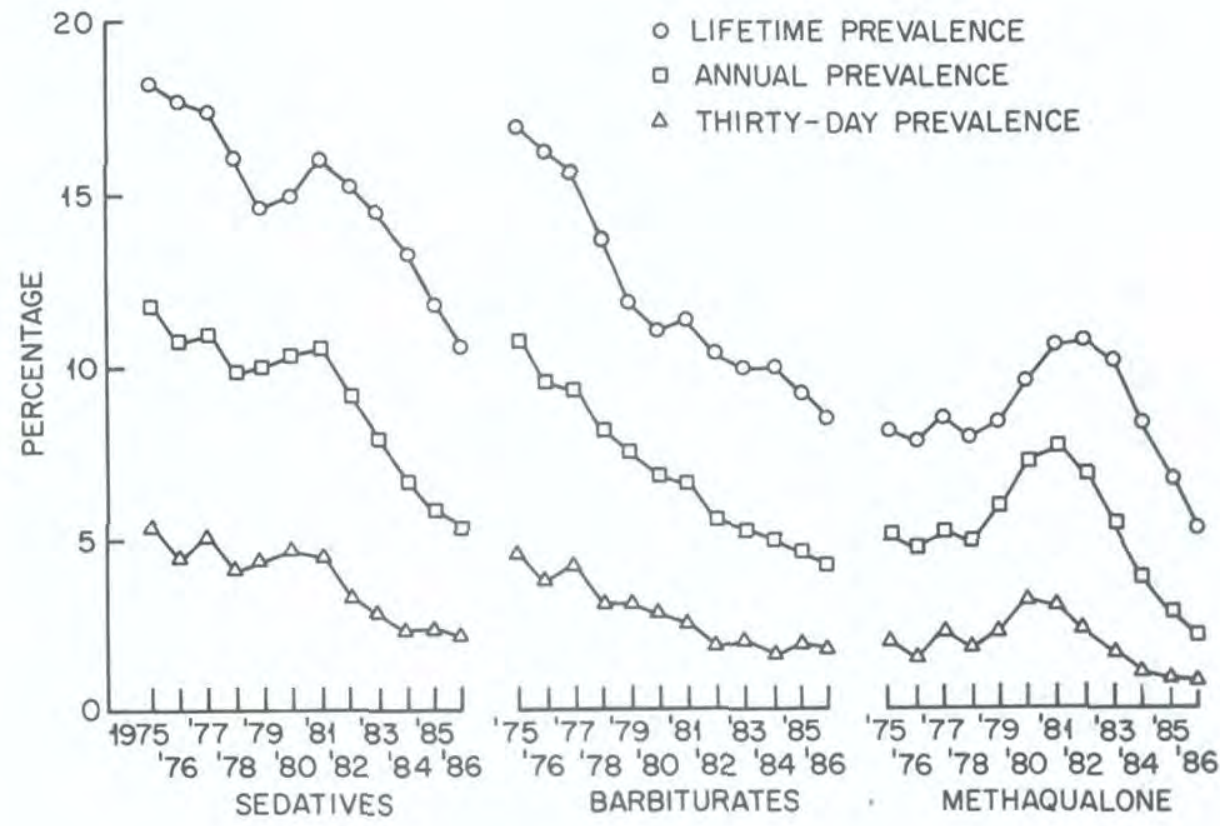
FIGURE 9d

Trends in Lifetime, Annual, and Thirty-Day Prevalence of Sixteen Drugs

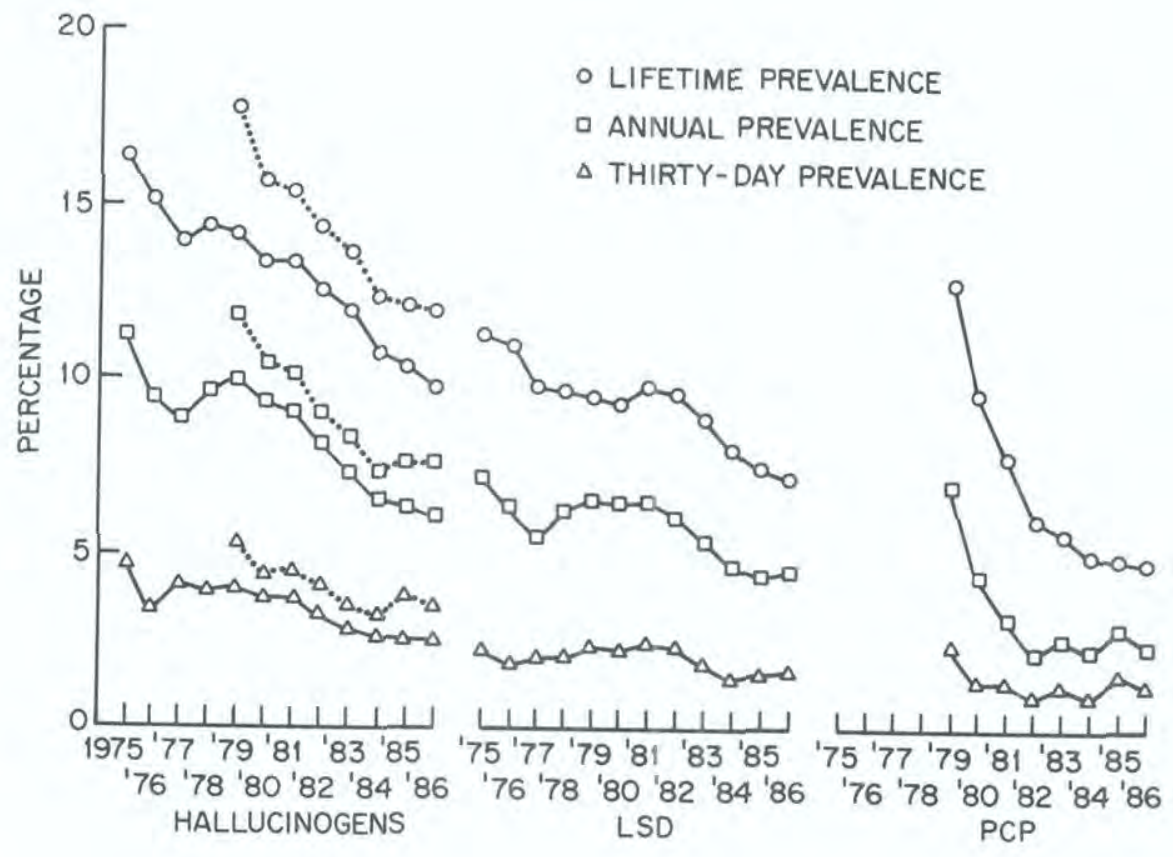

NOTE: The dotted lines connect percentages which are adjusted for underreporting of PCP. 
FIGURE 9e

Trends in Lifetime, Annual, and Thirty-Day Prevalence of Sixteen Drugs
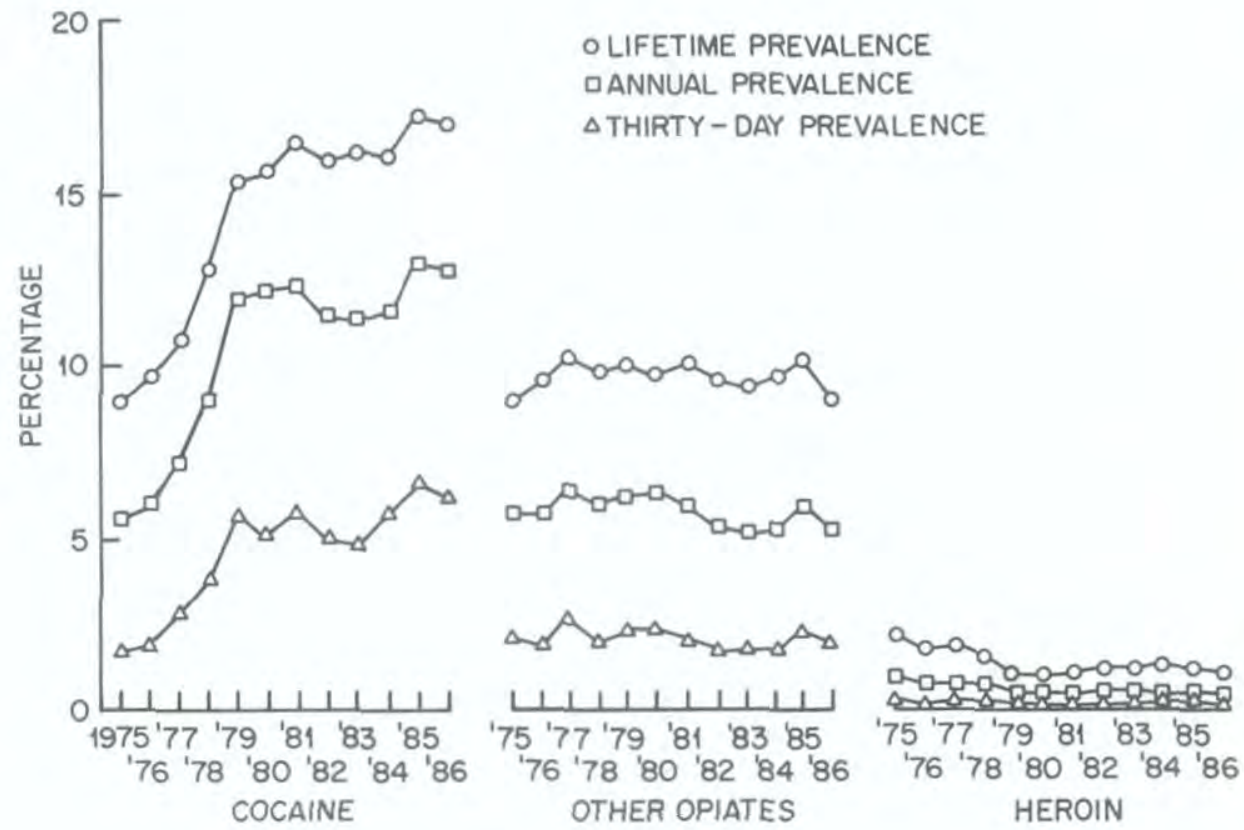


\section{FIGURE $9 f$}

Trends in Lifetime, Annual, and Thirty-Day Prevalence of Sixteen Drugs

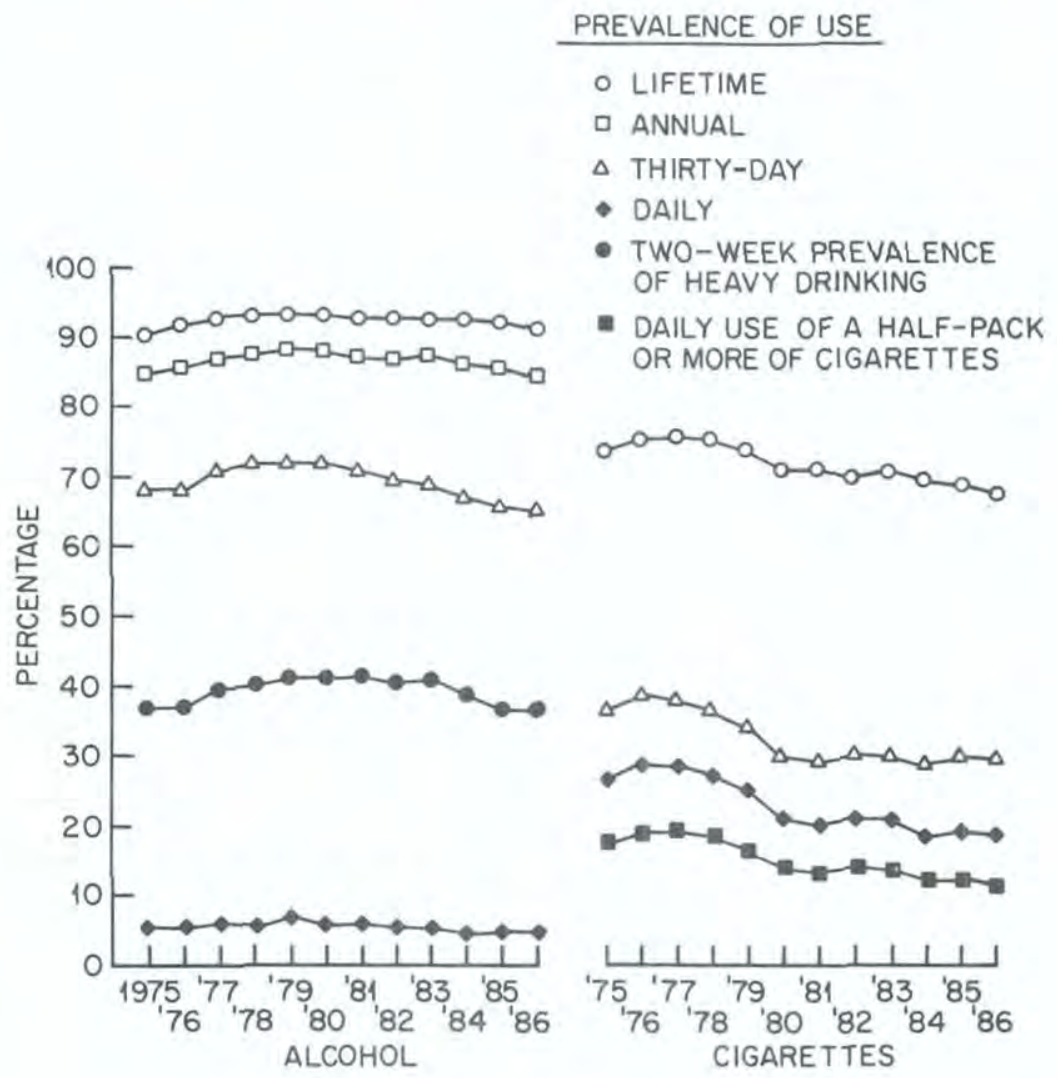


FIGURE 10

Trends in Thirty-Day Prevalence of Daily Use of Marijuana, Alcohol, and Cigarettes

by Sex
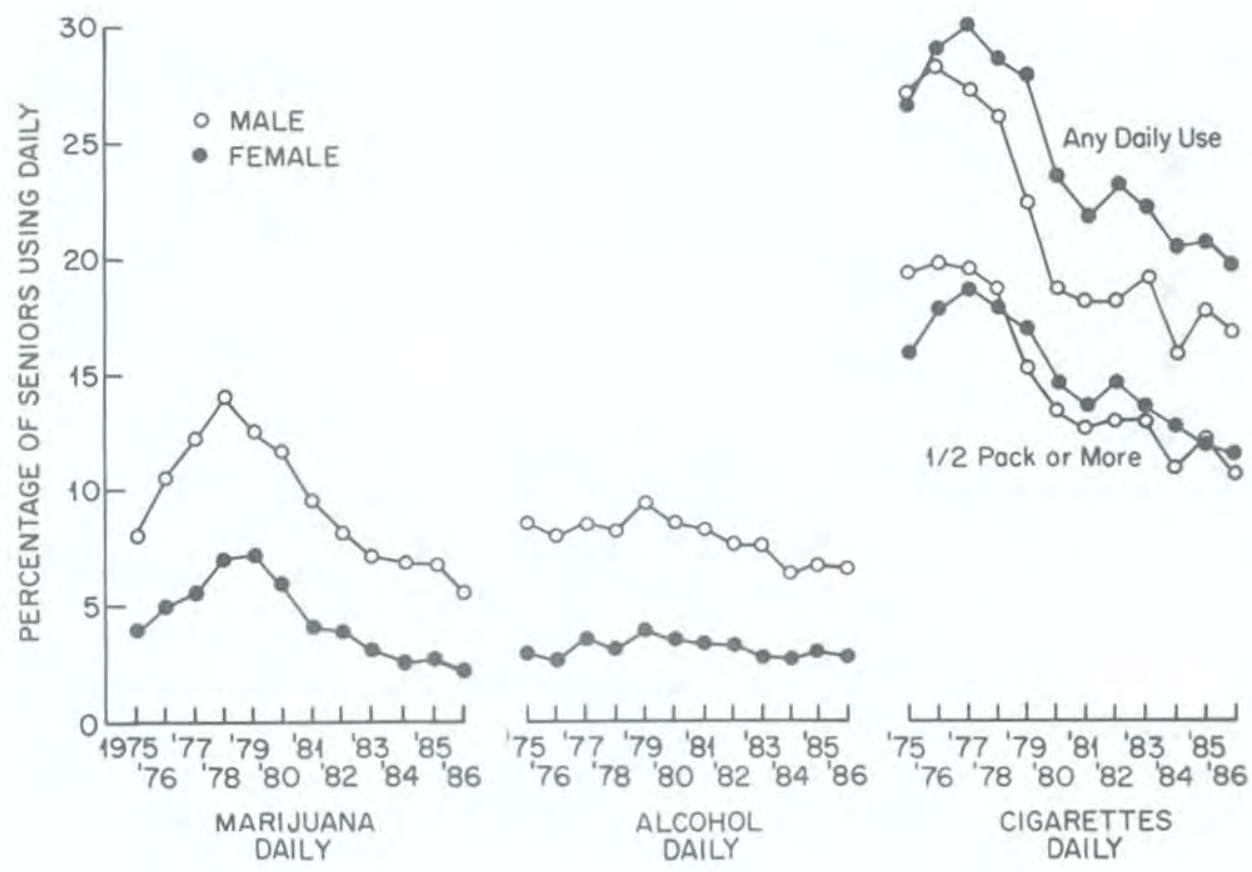

NOTE: Daily use for alcohol and marijuana is defined as use on 20 or more occasions in the past thirty days. Daily use of cigarettes is defined as smoking one or more cigarettes per day in the past thirty days. 


\section{FIGURE 11}

Trends in Two-Week Prevalence of Heavy Drinking by Sex

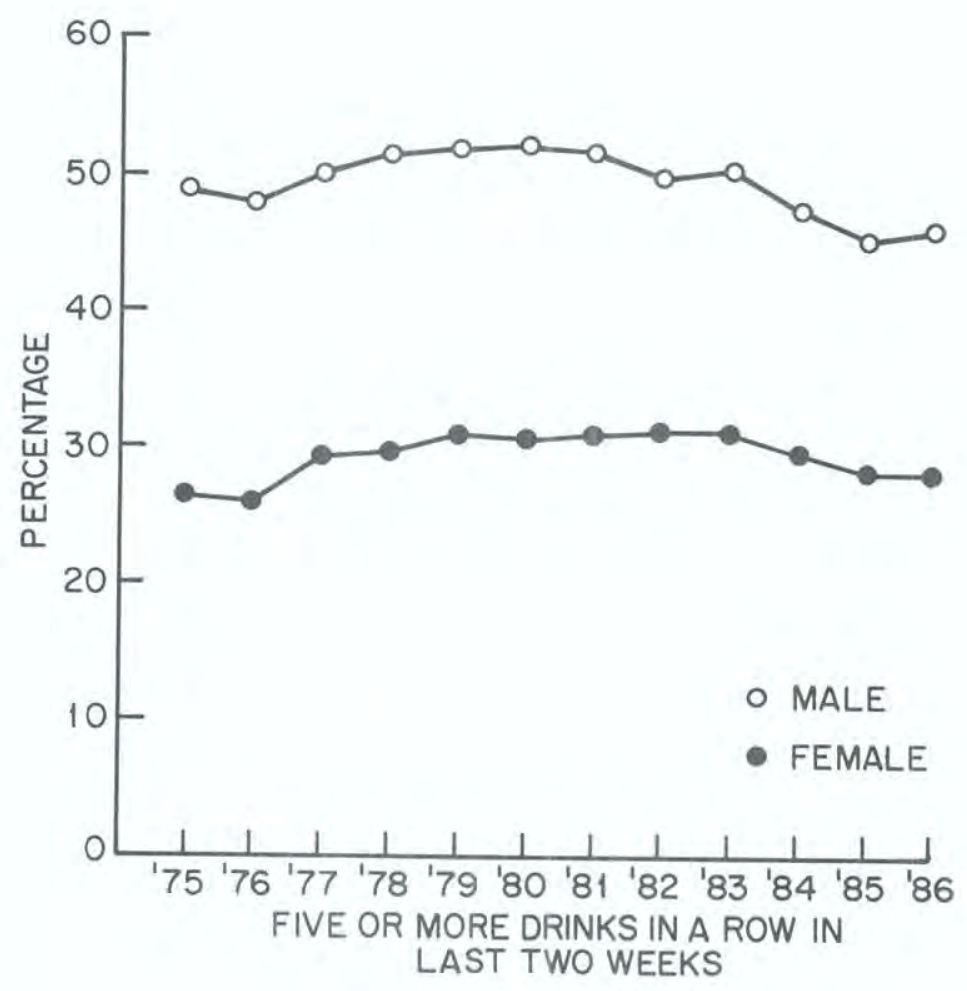


(Annual and 30-day rates in 1986 are unchanged from 1985 and 1984, but lifetime prevalence continued to decline.)

- Between 1975 and 1979 the prevalence of heroin use had been dropping rather steadily. Lifetime prevalence dropped from $2.2 \%$ in 1975 to $1.1 \%$ in 1979 and annual prevalence had also dropped by half, from $1.0 \%$ in 1975 to $0.5 \%$ in 1979 . This decline halted in 1980 and the statistics have remained almost constant since then.

- From 1975 to 1981 the use of opiates other than heroin remained fairly stable, with annual prevalence at or near $6 \%$. Annual prevalence then declined to $5.3 \%$ in 1982 , and has remained relatively stable since.

- Hallucinogen use (unadjusted for underreporting of PCP) declined some in the middle of the seventies (from $11.2 \%$ in 1975 to $9.6 \%$ in 1978 on annual prevalence). It then leveled for several years before beginning another sustained decline. Between 1979, when the first adjusted figures were available, and 1984 , there was a steady decline, with adjusted annual prevalence dropping from $11.8 \%$ in 1979 to $7.3 \%$ in 1984 . These rates have since remained level at $7.6 \%$.

- $L S D$, one of the major drugs comprising the hallucinogen class, showed a modest decline from 1975 to 1977 , followed by considerable stability through 1981. Between 1981 and 1985 , however, there has been a second period of decline, with annual prevalence falling from $6.5 \%$ in 1981 to $4.4 \%$ in 1985. This decline seems to have halted in 1986, with annual prevalence still at $4.5 \%$.

- The lifetime prevalence statistic for the specific hallucinogen $P C P$ showed a continuation of the steady and very substantial decrease which began in 1979 when we first measured the use of this drug (lifetime prevalence has dropped from $12.8 \%$ in the class of 1979 to $5.0 \%$ in the class of 1984$)$. It has since inched downward to $4.8 \%$ in 1986 . The annual and 30-day statistics for PCP, after declining sharply from 1979 to 1984 , have shown little systematic change since then.

- As can be seen from these varied patterns for the several classes of illicit drugs, while the overall proportion of seniors using any illicit drugs in their lifetime other than marijuana or amphetamines has changed rather little over the years, the mix of drugs they are using has changed quite substantially. 
- Turning to the licit drugs, between 1975 and 1978 or 1979 there was a small upward shift in the prevalence of alcohol use among seniors. To illustrate, between 1975 and 1979 the annual prevalence rate rose steadily from $85 \%$ to $88 \%$, the monthly prevalence rose from $68 \%$ to $72 \%$, and the daily prevalence rose from $5.7 \%$ to $6.9 \%$. Since 1979 , there has been virtually no drop in lifetime prevalence, but some drop for the more recent prevalence intervals: between 1979 and 1986 , annual prevalence fell from $88 \%$ to $85 \%$, monthly prevalence from $72 \%$ to $65 \%$, and daily prevalence from $6.9 \%$ to $4.8 \%$. Clearly the change in daily use is the most important of these shifts.

- There was a similar pattern observed in the frequency of occasional heavy drinking. When asked whether they had taken five or more drinks in a row during the prior two weeks, 37\% of the seniors in 1975 said they had. This proportion rose gradually to $41 \%$ by 1979 , where it remained through 1983. In both 1984 and 1985, we observed drops of $2 \%$ in this troublesome statistic, to $37 \%$, exactly where it was in 1975; there was no further change in 1986. Thus, to answer a frequently asked question, there is no evidence that the currently observed drop in marijuana use is leading to a concomitant increase in alcohol use. If anything, there has been some parallel decline in daily alcohol use as well as in occasional heavy drinking.

All of these alcohol statistics (except the one for occasional heavy drinking) continued to decline slightly in 1986, but none of them by a statistically significant amount.

- As for cigarette use, 1976 and 1977 appear to have been the peak years of smoking in this age group, as measured by lifetime, thirty-day, and daily prevalence. (Annual prevalence is not asked.) Over the four subsequent graduating classes, thirty-day prevalence dropped substantially from $38 \%$ in the class of 1977 to $29 \%$ in the class of 1981 . More importantly, daily cigarette use dropped over that same interval from $29 \%$ to $20 \%$, and daily use of half-pack-a-day or more from $19.4 \%$ to $13.5 \%$ between 1977 and 1981 (nearly a onethird decrease). In 1981 we reported that the decline appeared to be decelerating; in 1982 and 1983 it clearly had halted. There was a brief resumption of the earlier decline in 1984 , with daily use falling from $21 \%$ to $19 \%$, and daily use of half-pack-a-day dropping from $13.8 \%$ to $12.3 \%$. Since 1984 , there has been practically no change in most of these statistics, with the exception that smoking at the half-pack-aday level fell by under $1.0 \%$, from $12.3 \%$ in 1984 to $11.4 \%$ in 1986. What seems most noteworthy is the lack of appreci- 
able decline in the smoking rates since 1981, despite (a) the general decline which has occurred for most other drugs (including alcohol), (b) some rise in the perceived harmfulness and personal disapproval associated with smoking, and (c) a considerable amount of restrictive legislation which has been debated and enacted at state and local levels in the past several years.

\section{TRENDS IN NONCONTINUATION RATES}

Table 12 shows how the user noncontinuation rates observed for the various classes of drugs have changed over time. Recall that the noncontinuation rate, as used here, is defined as the percentage of those who ever used the drug who did not use in the year prior to the survey.

- For most drugs there has been relatively little change in noncontinuation rates among those who have tried the drug at least once. There are some noticeable exceptions, however.

- Marijuana has shown some increase in the noncontinuation rates between 1979 (when it was 16\%) and 1984 (when it was $27 \%$ ). This is what gave rise to the greater drop in annual use than in lifetime use described earlier. Since 1984, there has been no further increase in the noncontinuation rate for marijuana.

- The noncontinuation rate for cocaine decreased from 1976 (when it was 38\%) to 1979 (when it was 22\%), corresponding to the period of increase in the overall prevalence of use.

- There was considerably more noncontinuation of stimulant use in $1986(43 \%)$ than in 1982 (when it was 27\%), based on the revised question. Earlier data (based on the unrevised question), suggest that the change began after 1981 .

- Much of the recent decline in sedative use is also accounted for by a changing rate of noncontinuation. For example, in the case of barbiturates the noncontinuation rate rose between 1979 , when it was around $36 \%$, to 1984 when it was around $50 \%$-where it has remained since.

Similarly, in $198024 \%$ of the seniors who ever used methaqualone did not use in the prior year, whereas the comparable statistic by 1986 was more than twice as high, at $60 \%$,

- Tranquilizer users showed a steady, gradual increase in noncontinuation between 1975 and 1982 , as the rate rose 
TABLE 12

Trends in Noncontinuation Rates

Among Seniors Who Ever Used Drug in Lifetime

Percent who did not use in last twelve months

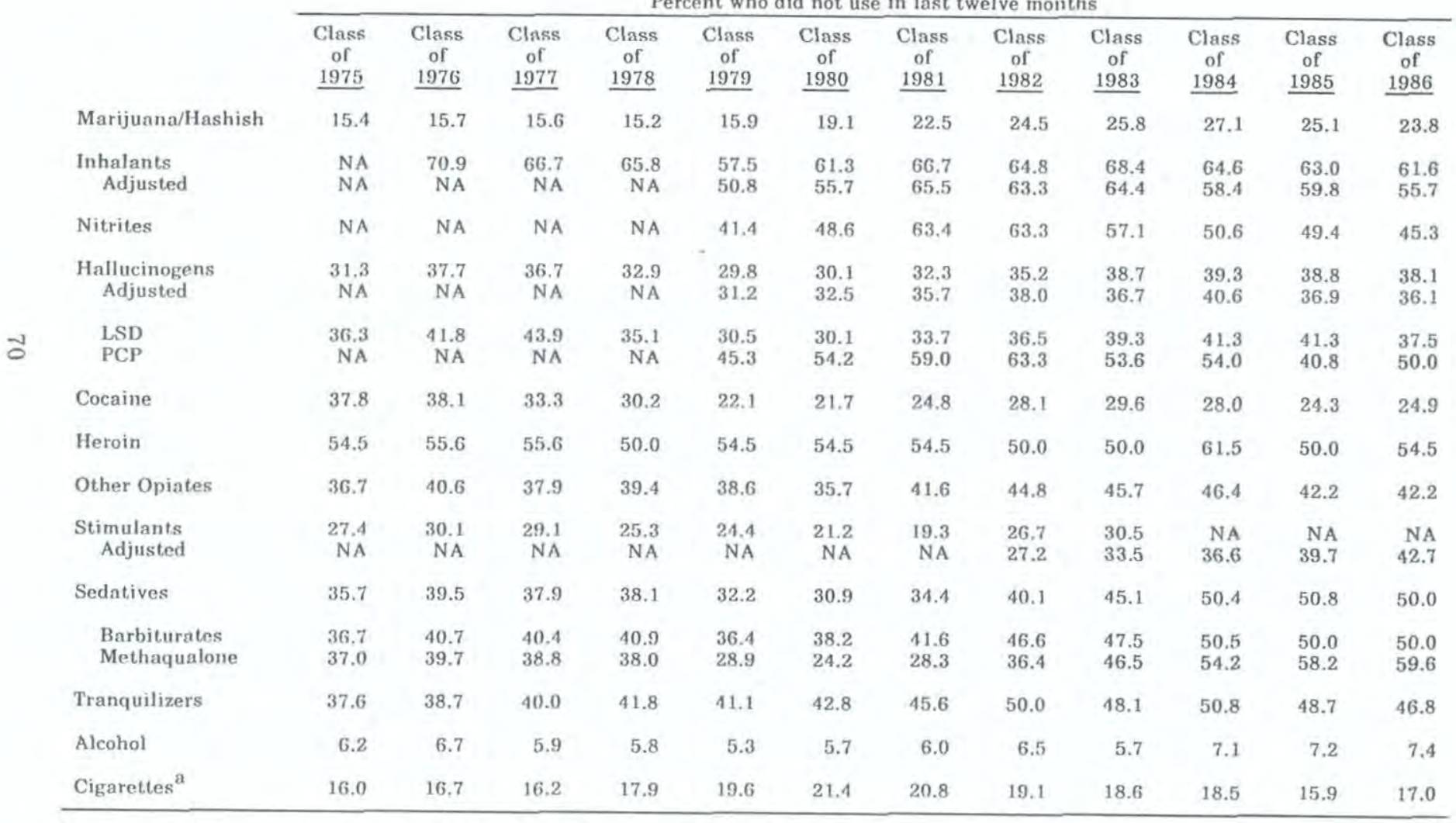

a Percentage of regular smokers (ever) who did not smoke at all in the lnst thirty days. 
TABLE 13

Trends in Noncontinuation Rates Among Seniors Who Used Drug Ten or More Times in Lifetime

Percent who did not use in last twelve months

\begin{tabular}{|c|c|c|c|c|c|c|c|c|c|c|c|c|}
\hline & \multicolumn{12}{|c|}{ Percent who did not use in last twelve months } \\
\hline & $\begin{array}{c}\text { Class } \\
\text { of } \\
1975 \\
\end{array}$ & $\begin{array}{c}\text { Class } \\
\text { of } \\
1976 \\
\end{array}$ & $\begin{array}{c}\text { Class } \\
\text { of } \\
1977 \\
\end{array}$ & $\begin{array}{c}\text { Class } \\
\text { of } \\
1978 \\
\end{array}$ & $\begin{array}{c}\text { Class } \\
\text { of } \\
1979 \\
\end{array}$ & $\begin{array}{c}\text { Class } \\
\text { of } \\
1980 \\
\end{array}$ & $\begin{array}{c}\text { Class } \\
\text { of } \\
1981 \\
\end{array}$ & $\begin{array}{c}\text { Class } \\
\text { of } \\
1982 \\
\end{array}$ & $\begin{array}{c}\text { Class } \\
\text { of } \\
1983 \\
\end{array}$ & $\begin{array}{c}\text { Class } \\
\text { of } \\
1984 \\
\end{array}$ & $\begin{array}{c}\text { Class } \\
\text { of } \\
1985 \\
\end{array}$ & $\begin{array}{c}\begin{array}{c}\text { Class } \\
\text { of } \\
1986 \\
\end{array} \\
\end{array}$ \\
\hline Marijuana/Hashish & 4.0 & 4.0 & 4.1 & 3.7 & 4.6 & 5.4 & 7.2 & 7.6 & 8.3 & 8.8 & 7.8 & 7.9 \\
\hline Inhalants & NA & 48.9 & 42.6 & 34.6 & 23.8 & 25.2 & 23.8 & 27.2 & 23.1 & 23.4 & 25.8 & 15.3 \\
\hline \multicolumn{13}{|l|}{ Nitrites ${ }^{*}$} \\
\hline Hallucinogens & 10.8 & 16.1 & 15.2 & 10.8 & 8.1 & 8.4 & 7.7 & 7.5 & 13.0 & 14.1 & 12.2 & 11.1 \\
\hline $\begin{array}{l}\text { LSD } \\
\mathrm{PCP}^{*}\end{array}$ & 15.2 & 17.3 & 18.0 & 12.2 & 7.4 & 6.4 & 7.1 & 7.5 & 15.3 & 12.1 & 12.6 & 12.2 \\
\hline Cocaine & 7.7 & 8.2 & 6.2 & 3.8 & 3.1 & 3.1 & 3.1 & 2.9 & 6.2 & 3.1 & 2.5 & 3.5 \\
\hline \multicolumn{13}{|l|}{ Heroin ${ }^{2}$} \\
\hline Other Opiates & 9.6 & 11.6 & 9.7 & 9.9 & 8.7 & 10.8 & 10.1 & 13.5 & 16.4 & 15.4 & 12.2 & 13.8 \\
\hline $\begin{array}{l}\text { Stimulants } \\
\text { Adjusted }\end{array}$ & $\begin{array}{l}8.0 \\
\text { NA }\end{array}$ & $\begin{array}{l}9.8 \\
\text { NA }\end{array}$ & $\begin{array}{l}7.6 \\
\text { NA }\end{array}$ & $\begin{array}{l}7.4 \\
\text { NA }\end{array}$ & $\begin{array}{l}6.1 \\
\text { NA }\end{array}$ & $\begin{array}{l}4.1 \\
\text { NA }\end{array}$ & $\begin{array}{r}4.4 \\
\mathrm{NA}\end{array}$ & $\begin{array}{l}6.4 \\
8.4\end{array}$ & $\begin{array}{r}7.5 \\
10.7\end{array}$ & $\begin{array}{r}\text { NA } \\
12.7\end{array}$ & $\begin{array}{r}\text { NA } \\
17.5\end{array}$ & $\begin{array}{r}\text { NA } \\
17.6\end{array}$ \\
\hline Sedatives & 13.6 & 16.2 & 12.4 & 12.8 & 8.6 & 10.5 & 7.6 & 8.6 & 16.4 & 20.8 & 23.6 & 19.7 \\
\hline Barbiturates & 13.4 & 16.5 & 12.9 & 13.5 & 11.2 & 11.7 & 8.9 & 12.6 & 17.7 & 22.8 & 20.6 & 19.7 \\
\hline Methaqualone & 13.5 & 15.9 & 11.9 & 13.1 & 6.1 & 6.0 & 4.9 & 8.0 & 16.3 & 23.3 & 26.7 & 24.9 \\
\hline Tranquilizers & 12.0 & 13.0 & 11.1 & 14.4 & 14.1 & 14.3 & 16.3 & 16.0 & 14.8 & 18.8 & 19.2 & 15.0 \\
\hline Alcohol & 0.6 & 0.8 & 0.6 & 0.9 & 0.7 & 0.8 & 1.0 & 0.9 & 0.9 & 1.1 & 1.2 & 1.0 \\
\hline
\end{tabular}

*The cell entries in these rows were omitted because they were based on fewer than 100 seniors who used ten or more times. All other cells contain more than 100 cases. 
from $38 \%$ to $50 \%$. Since 1982 there has not been any further systematic change, however.

- Table 13 provides noncontinuation rates for seniors who were more established users-that is, for those who report having used the drug ten or more times in their life. It shows that noncontinuation is far less likely among such heavier users than among all users of a given drug. Further, while the trends in noncontinuation mentioned above for marijuana, stimulants, barbiturates, methaqualone, and tranquilizers are all similar to trends observed in the noncontinuation rates for heavier users of those same drugs, the percentage fluctuations tend to be considerably smaller among the heavier users.

\section{COMPARISONS AMONG SUBGROUPS IN TRENDS IN PREVA. LENCE}

\section{Sex Differences in Trends}

- Most of the sex differences mentioned earlier for individual classes of drugs have remained relatively unchanged over the past eleven years-that is, any trends in overall use have occurred about equally among males and females. There are, however, a few exceptions (data not shown).

- Since 1977 , the small sex difference involving tranquilizer use (males this age had used them less frequently than females) have virtually disappeared.

- The ratio of male-female prevalence rates in cocaine use, which was rather large in the mid-1970's, diminished somewhat in the early 1980's. Although the differences have lessened, males still use more frequently than females.

- Regarding stimulant use, a sex difference emerged in 1981 and 1982 using the original version of the question; but the revised question introduced in 1982 showed no sex difference, suggesting that over-the-counter diet pills accounted for females showing higher use in those two years. In 1986, with the revised version of the question, females show slightly higher rates of use of stimulants due to their more frequent use of amphetamines for the purpose of weight loss. Both sexes have shown significant declines in use of stimulants since 1984 .

- An examination of the trends in the proportion of each sex using any illicit drug in the prior year (see Figure 12) 
shows that use among males rose between 1975 and 1978, and then declined steadily until 1986 (from 59\% in 1978 to $46 \%$ in 1986). Use among females increased from 1975 $(41 \%)$ until $1981(51 \%)$ and then dropped through 1986 $(42 \%)$. However, if amphetamine use is deleted from the statistics (see notations in Figure 12), female use peaked earlier (in 1979) and then declined as well. (Note that the declines for both males and females were attributable to the declining marijuana use rates.)

- Regarding the apparent parity between the sexes in the levels and trends in the use of illicit drugs other than marijuana, it can be seen in Figure 12 that, when amphetamine use is excluded from the calculations, somewhat differential levels emerge for males vs. females but the trends tend to remain fairly parallel. In 1986, males' use decreased by $1.4 \%$ but females' use remained the same as in 1985.

- The sex differences in alcohol use have narrowed slightly since 1975. For example, the thirty-day prevalence rates for males and females differed by $12.8 \%$ in $1975(75.0 \%$ vs. $62.2 \%$, respectively), but that difference was down to $7.1 \%$ by $1986(69.0 \%$ vs. $61.9 \%)$. And, although there still remain substantial sex differences in daily use and occasions of heavy drinking, there has been some narrowing of the differences there, as well (Figure 11). For example, between 1975 and 1985 the proportion of males admitting to having five drinks in a row during the prior two weeks showed a net decrease of $3.7 \%$ (from $49.0 \%$ to $45.3 \%$ ), whereas a net increase of $1.8 \%$ occurred for females (from $26.4 \%$ to $28.2 \%) .{ }^{13}$

Although males are far more likely than females to have five or more drinks in a row during the prior two weeks $(46 \%$ vs. $28 \%$ ), there is practically no difference in the proportion of them who had at least one drink during that same interval (41.6\% vs. $42.1 \%$ ). Thus, it is the propensity to drink a lot per occasion that differs between male and female high school seniors, not the propensity to drink at all.

- On one of the five questionnaire forms used in the study, respondents are asked separately about their use of beer,

\footnotetext{
${ }^{13}$ It is worth noting that the same number of drinks produces substantially greater impact on the blood alcohol level of the average female than the average male, because of sex differences in body weight. Thus, sex differences in frequency of actually getting drunk may not be as great as the binge drinking statistics would indicate, since they are based on a fixed number of drinks.
} 


\section{FIGURE 12}

Trends in Annual Prevalence of an Illicit Drug Use Index by Sex
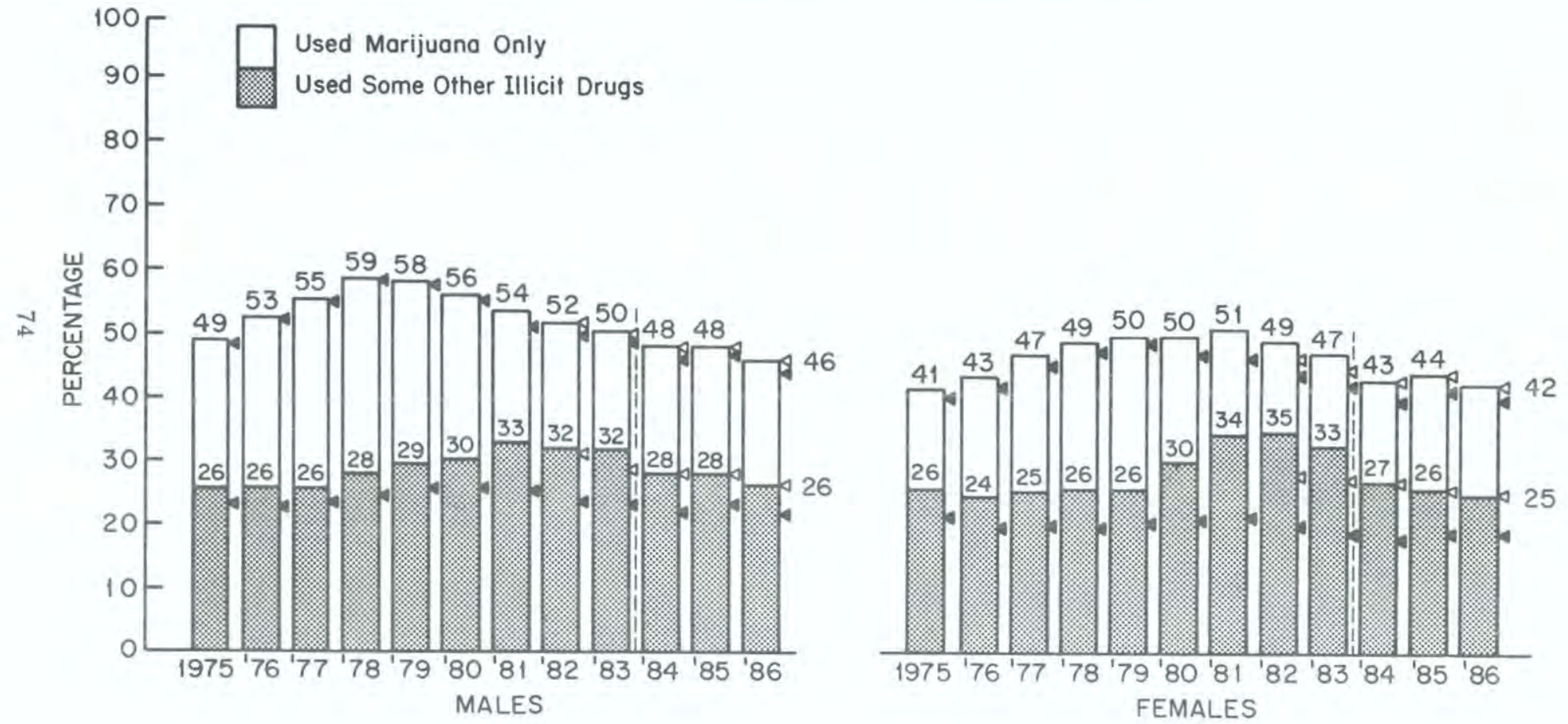

NOTE: See Figure 8 for relevant footnotes. 
wine, and hard liquor. The answers to these questions reveal that it is primarily a differential rate of beer consumption that accounts for the large sex differences in occasions of heavy drinking; $45 \%$ of 1986 senior males report having five or more beers in a row during the prior two weeks vs. $22 \%$ of the females. In contrast, males are only somewhat more likely than fernales to report having 5 or more drinks of hard liquor (25\% for males vs. $19 \%$ for females) and females are a bit more apt to drink wine that heavily $(14.6 \%$ for females vs. $12.4 \%$ for males). This pattern-a large sex difference in heavy use of beer, a much smaller difference in heavy use of hard liquor, and very little difference in heavy use of winehas been present throughout the study, with little systematic change over time.

- Regarding cigarette smoking, we observed in 1977 that females for the first time caught up to males at the half-apack per day smoking level (Figure 10). Then, between 1977 and 1981, both sexes showed a decline in the prevalence of such smoking; but use among males dropped more, resulting in a reversal of the sex differences. As of 1986, females lead males in smoking at least a half-pack per day $(11.6 \%$ vs. $10.7 \%$ ), but the percentages smoking a pack or more are equal in both sexes $(5.8 \%)$. However, at less frequent levels of smoking, there is a somewhat larger sex difference, since there are more occasional smokers among females than among males. For example, in 1986, 31\% of the females report smoking at least once in the prior 30 days, vs. only $28 \%$ of the males.

\section{Trend Differences Related to College Plans}

- Both college-bound and noncollege-bound students have been showing fairly parallel trends in overall illicit drug use over the last several years (see Figure 13). ${ }^{14}$

- Changes in use of the specific drug classes have also been generally quite parallel for the two groups since 1976, with only minor exceptions. (Data not shown). Over the last three years (1983-1986) annual cocaine use has increased very little among the college-bound, but has risen by about one-quarter among the noncollege-bound.

- Increases in annual and 30-day use of inhalants (adjusted) in 1986 occurred primarily among those not planning to complete four years of college.

${ }^{14}$ Because of excessive missing data in 1975 on the variable measuring college plans, group comparisons are not presented for that year. 
FIGURE 13

Trends in Annual Prevalence of an Illicit Drug Use Index

by College Plans
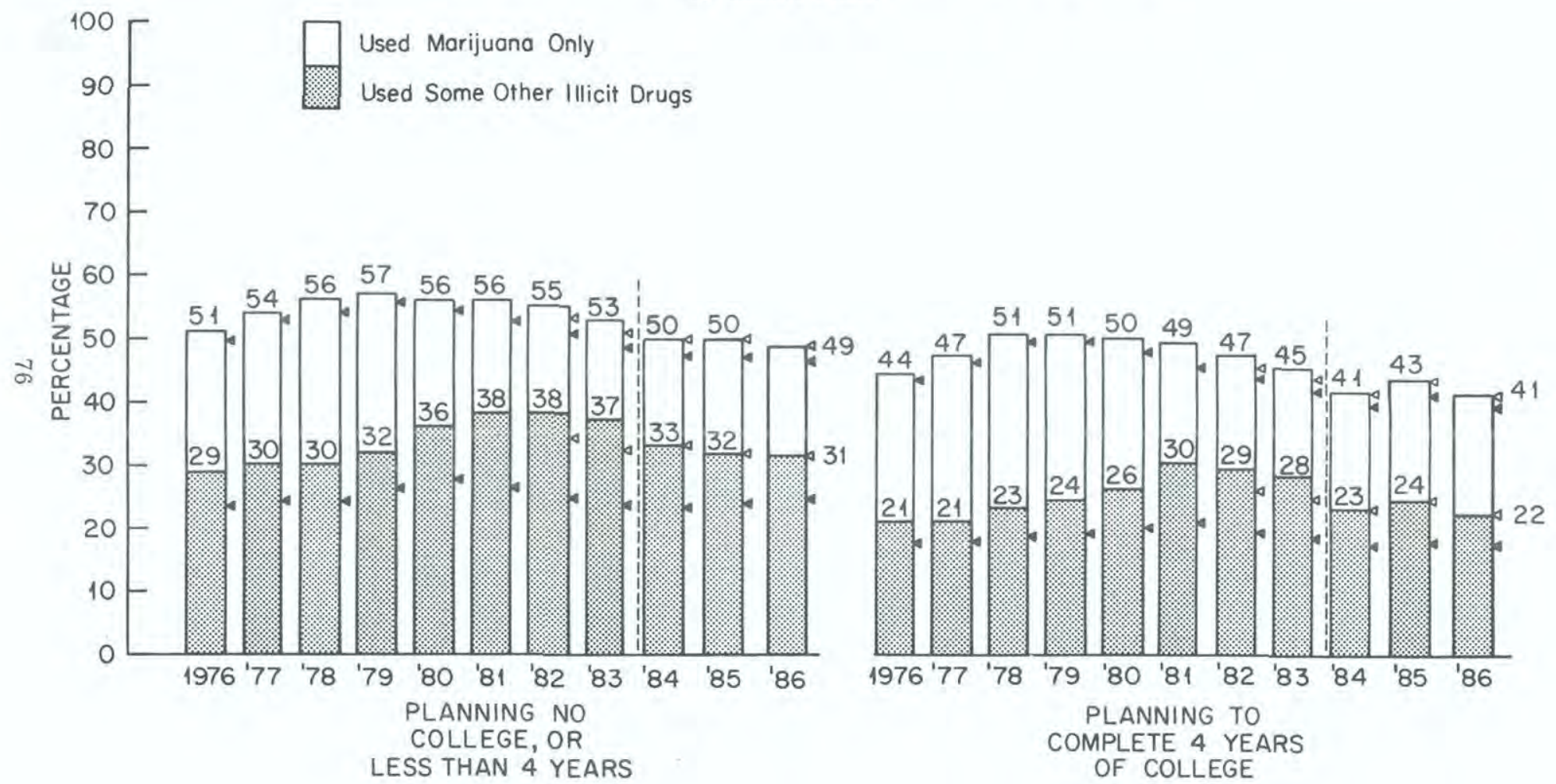

NOTE: See Figure 8 for relevant footnotes. 


\section{Regional Differences in Trends}

- In terms of the proportion of seniors using any illicit drug during the year, all four regions of the country reached their peaks in 1978 or 1979 (Figure 14), and generally have been falling since then.

- As noted earlier, a major factor in the rise of illicit drug use other than marijuana had been an increase in reported amphetamine use. Such a rise appeared in all four regions; however, the rise in lifetime prevalence from 1978 to 1981 was only $6 \%$ in the South, whereas in the other regions the percentages all had risen between $9 \%$ and $12 \%$. In essence, the South has been least affected by both the rise and the fall in reported amphetamine use.

- When amphetamine use is excluded, as shown by the arrow (4) in Figure 14, a rather different picture appears for regional trends during the late seventies and early eighties than the picture given by the shaded bars (which include all reported amphetamine use). Use of illicits other than marijuana or amphetamines actually started to decline in the South and North Central in 1981-both regions having had fairly level rates of use prior to that. Rates in the West and the Northeast did not begin their decline until a year later (1982), after a period of some increase in student involvement with such drugs (but not as great an increase as the unadjusted figures would suggest). Since 1983 this statistic has been fairly level in all four regions.

- Over the longer term cocaine use has shown very different trends in the four regions of the country (see Figure 15 for differences in lifetime prevalence trends). In the midseventies, there was relatively little regional variation in cocaine use. Then, large regional differences emerged so that by 1981 annual use had roughly tripled in the West and Northeast, nearly doubled in the North Central, and increased "only" by about $30 \%$ in the South. Since 1981, there has been some further increase in the Northeast (occurring primarily in 1984). The West has remained relatively stable since 1982. Use in the North Central declined a little from 1981-1984, but in the last two years has given some evidence of increasing. There has been little change in the South since 1979. The net effect has been that there have remained very substantial regional differences in cocaine use since around 1980, with the West and Northeast now showing annual prevalence rates near $20 \%$ vs. $7 \%$ to $10 \%$ for the South and North Central. 
FIGURE 14

Trends in Annual Prevalence of an Illicit Drug Use Index by Region of the Country
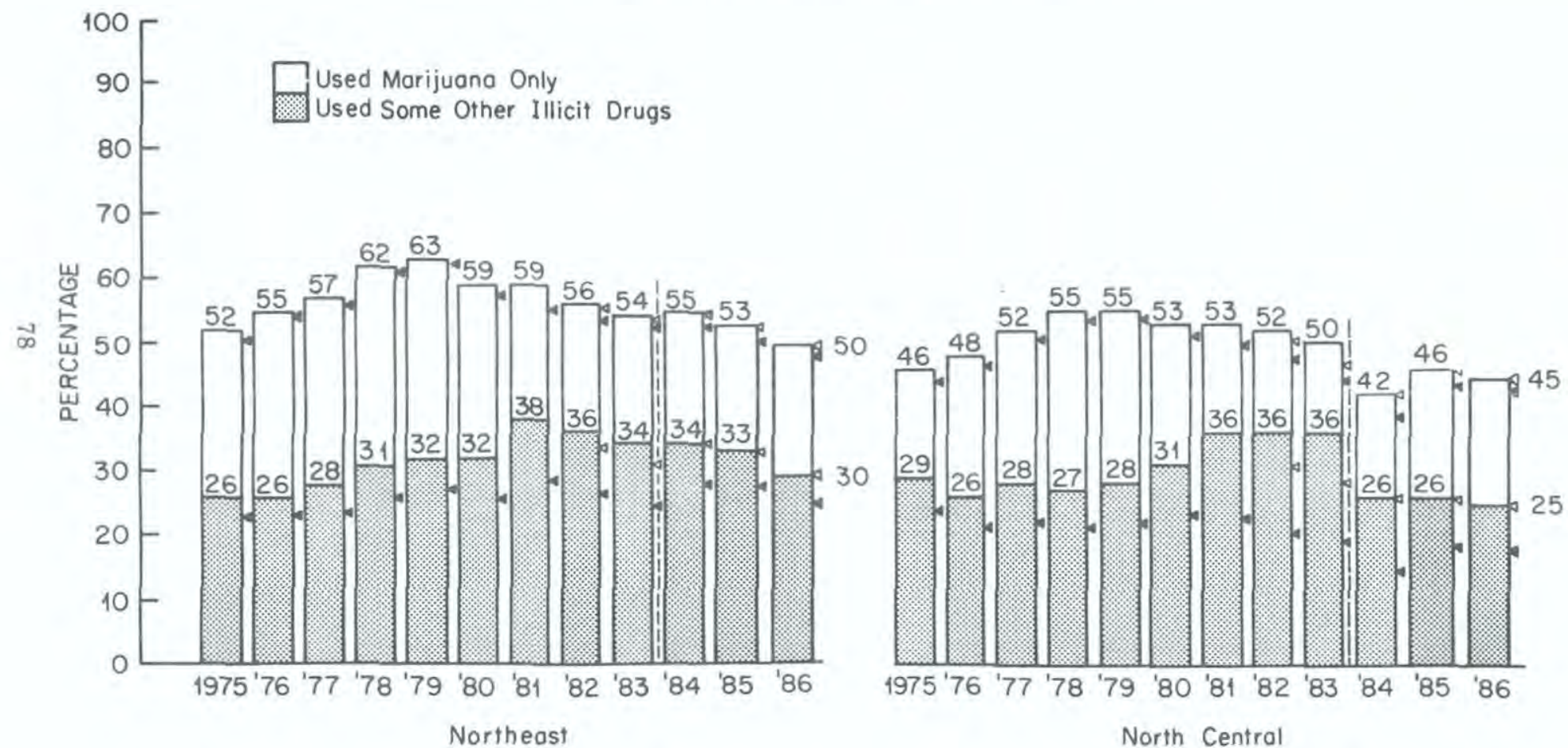

NOTE: See Figure 8 for relevant footnotes. 
Trends in Annual Prevalence of an Illicit Drug Use Index by Region of the Country
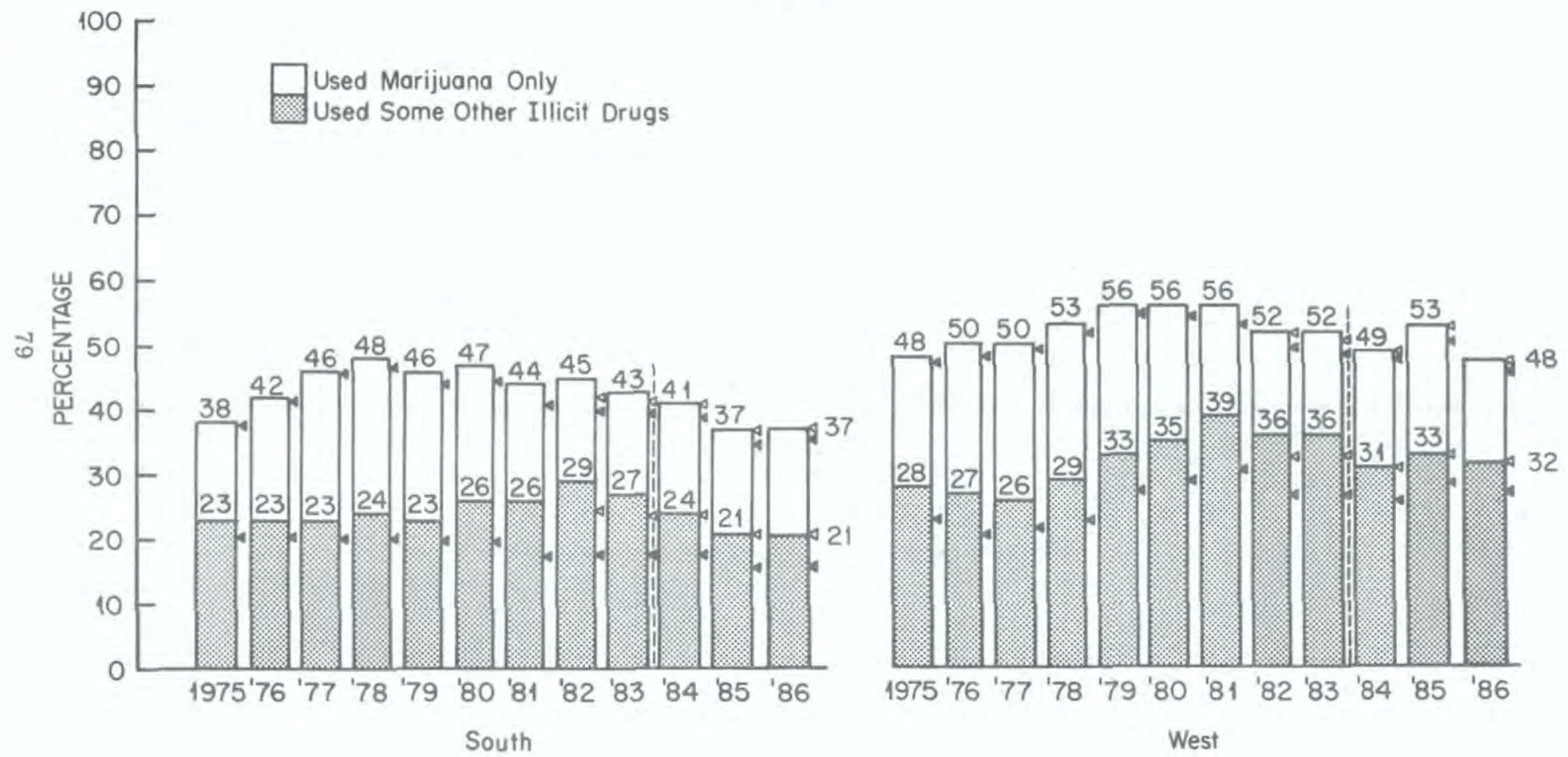

NOTE: See Figure 8 for relevant footnotes. 
- Between 1975 and 1981, sizeable regional differences in hallucinogen use emerged, as use in the South dropped appreciably. In 1981, both the North Central and the West had annual rates that were about two and one-half times higher than the South $(10.3 \%, 10.4 \%$, and $4.1 \%$, respectively), and the Northeast was three times as high (12.9\%). After 1981, hallucinogen use dropped appreciably in all three non-Southern regions (by $3-4 \%$ ), narrowing these differences in absolute terms, though the North Central and West now have annual rates twice that of the South, with the Northeast still two-and-one-half times as high. (Data not shown.)

- Between 1980 and 1982 PCP use dropped precipitously in all regions, though the drop was greatest in the Northeast which in 1980 had a usage rate roughly double that of all the other regions. In general, PCP use has remained low, although there is some evidence of an increase in the West over the past two years.

- The use of nitrite inhalants fell sharply in all regions between 1979 and 1981, and use generally stayed low for several years. Since 1984, however, there is some indication of an increase in use in the North Central. The same is true for inhalants, both unadjusted and adjusted.

- The remaining drugs (i.e,, alcohol, cigarettes, marijuana, heroin, other opiates, barbiturates, methaqualone, and tranquilizers) have shown rather little regional variation in trends.

\section{Trend Differences Related to Population Density}

- There was a peaking in 1979 in the proportions using any illicit drug in all three levels of community size (Figure 16). Although the smaller metropolitan areas and the nonmetropolitan areas never caught up completely with their larger counterparts, they did narrow the gap some between 1975 and 1979. Most of that narrowing was due to changing levels of marijuana use, and most of it occurred prior to 1978.

Since 1979 , there had been a fairly steady decrease in all three groupings on community size-until 1985, when the metropolitan areas remained level and the nonmetropolitan areas showed a slight rise. In 1986 all three showed the resumption of a gradual decline,

- The overall proportion involved in illicit drugs other than marijuana also has peaked in communities of all sizes, but not until 1981 or 1982 . Up to 1981 , the proportions report- 


\section{FIGURE 15}

Trends in Lifetime Prevalence of Cocaine Use by Region of the Country

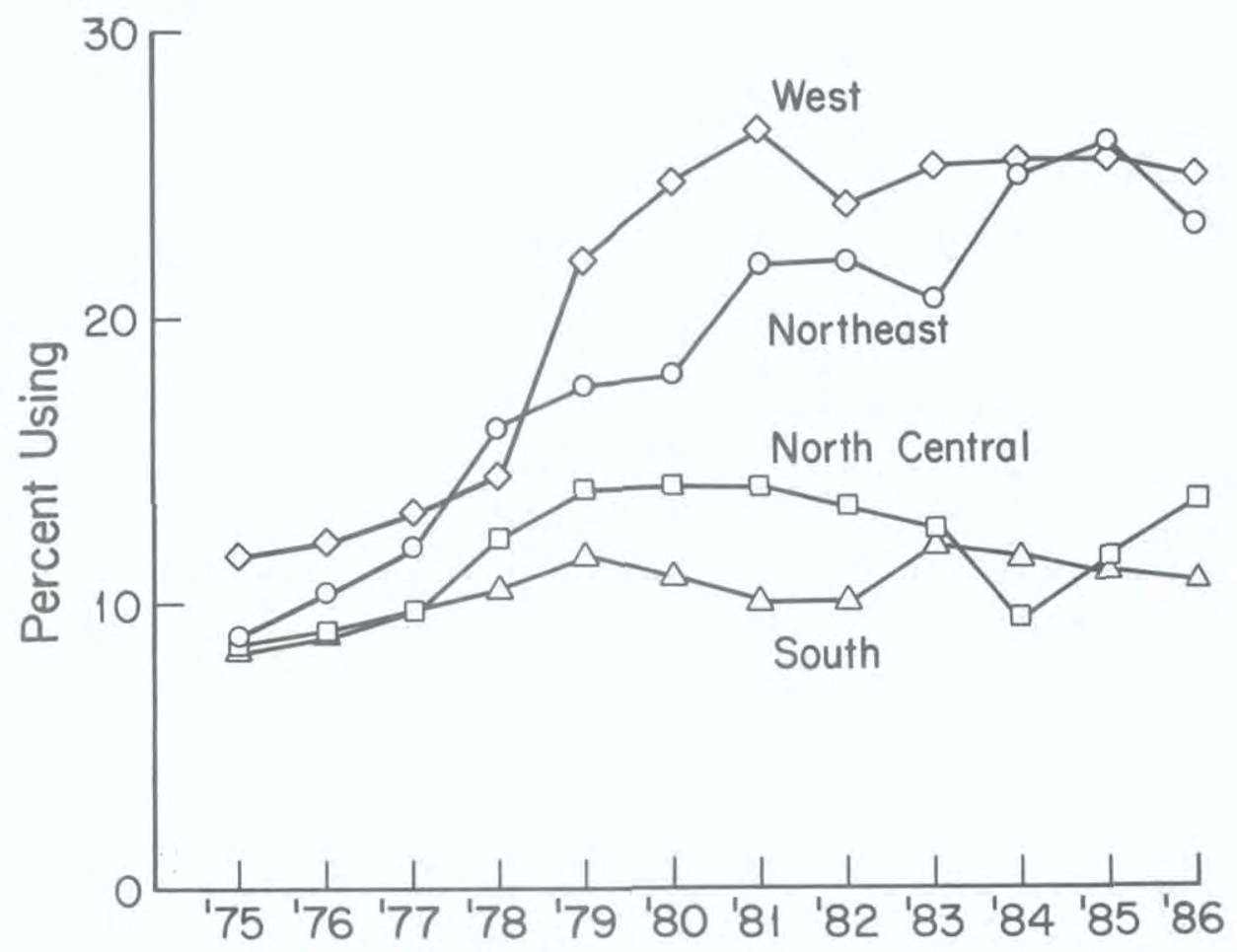


ing the use of some illicit drug other than marijuana in the last 12 months had been increasing continuously (over a fouryear period in the very large cities, and over a three-year period in the smaller metropolitan and nonmetropolitan areas). As can be seen by the special notations in Figure 16, almost all of this increase is attributable to the rise in reported amphetamine use (which likely is artifactual in part). Since 1983 there has been a fair-sized decline in all three groups in the use of illicit drugs other than marijuanaagain largely attributable to changes in amphetamine use. (Trend data not shown for individual drugs.)

- The increase in cocaine use, although dramatic at all levels of urbanicity between 1976 and 1979 , was clearly greatest in the large cities. Between 1980 and 1984 , use was fairly stable in all groupings, and in 1985 they all showed a rise in annual prevalence and in 1986 they all stabilized again.

- There is evidence of a decline in current alcohol use in the large cities in recent years. For example, thirty-day prevalence in the large cities is down by $11 \%$, from $78 \%$ in 1980 to $66 \%$ in 1986; during the same interval, the smaller metropolitan areas decreased $6 \%$ (from $71 \%$ to $65 \%$ ), and the nonmetropolitan areas dropped $4 \%$ (from $69 \%$ to $65 \%$ ). Similarly, daily use decreased between 1980 and 1986 by $3.1 \%$ in the large cities $(7.1 \%$ to $4.0 \%)$, while the smaller cities decreased by $0.9 \%(5.4 \%$ to $4.5 \%)$ and nonmetropolitan areas decreased by $0.3 \%(6.1 \%$ to $5.8 \%)$. And occasional heavy drinking decreased by $8.4 \%$ (from $44.8 \%$ to $36.4 \%$ ) in the large cities, compared to a $3.4 \%$ decrease in other cities $(38.9 \%$ to $35.5 \%)$ and a $2.3 \%$ drop in nonmetropolitan areas $(41.4 \%$ to $39.1 \%)$. These differential shifts result in less variation among the three levels of urbanicity in 1986 than there had been six to ten years earlier.

- Differences related to community size have also narrowed in the cases of $L S D$ (since 1981) due to a greater amount of decrease in the large cities and other cities than in the nonmetropolitan areas (which started out considerably lower). A similar thing appeared to be happening for $P C P$, as well.

- In the last half of the seventies, the use of opiates other than heroin was consistently highest in the large metropolitan areas and lowest in the nonmetropolitan areas. However, in the first six years of the eighties, there has been no consistent difference among these groups.

- The remaining drugs show little variation in trends related to population density. 
FIGURE 16

Trends in Annual Prevalence of an IIlicit Drug Use Index

by Population Density

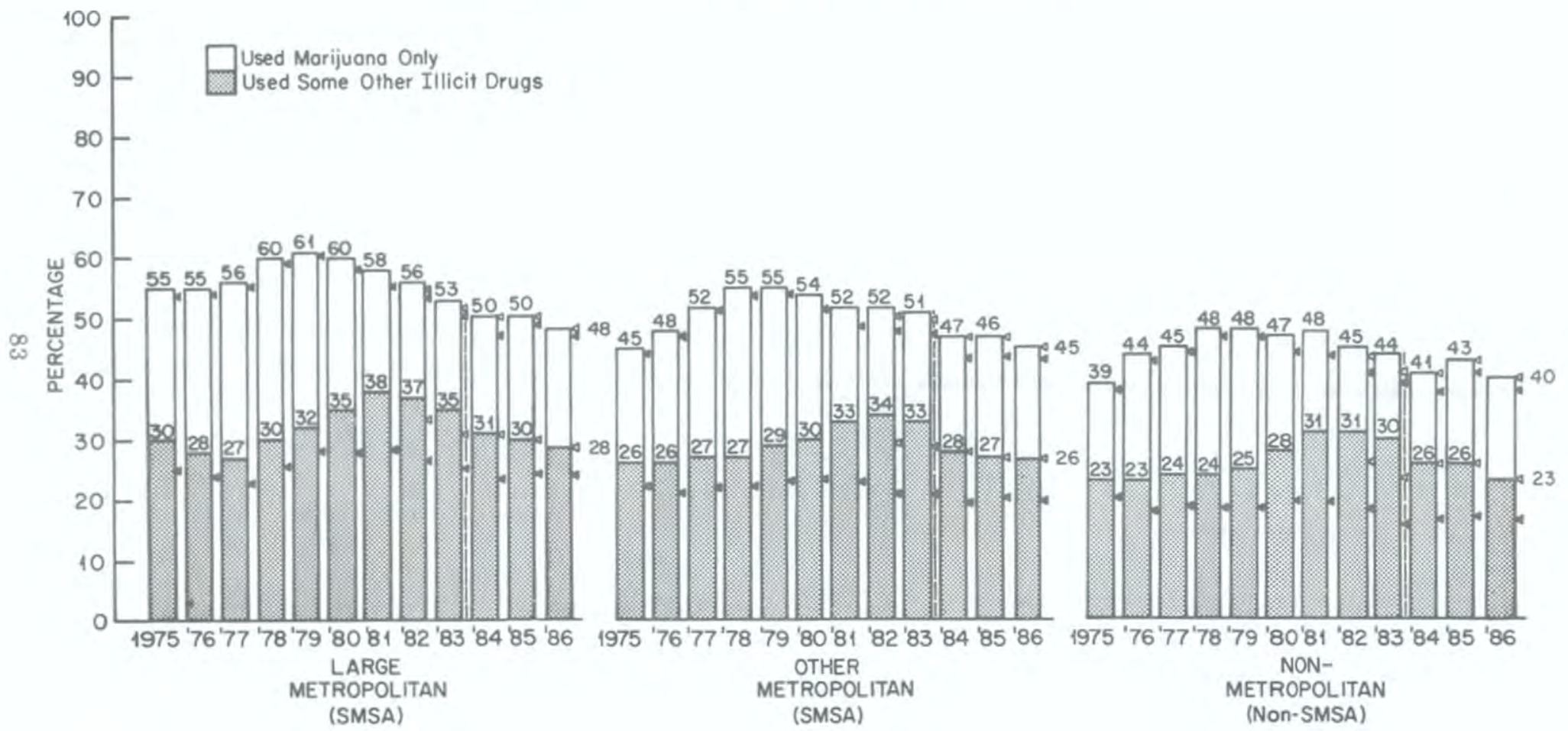

NOTE: See Figure 8 for relevant footnotes. 


\section{USE AT EARLIER GRADE LEVELS}

In two of the five questionnaire forms used in the study, respondents are asked to indicate the grade in which they were enrolled when they first tried each class of drugs. Graphic presentations on a drug-by-drug basis of the trends for earlier grade levels and of the changing age-atonset curves for the various graduating classes are contained in the large 1978, 1981, and 1983 reports from the study (cited earlier). In the present report, most but not all of these figures are included. Table 14 gives the percentage of the 1986 seniors who first tried each drug at each of the earlier grade levels.

\section{INCIDENCE OF USE BY GRADE LEVEL}

- For cigarettes, alcohol, and marijuana, most of the initial experiences took place before high school. For example, regular daily cigarette smoking was begun by $11 \%$ prior to tenth grade vs. $10 \%$ in high school (i.e., in grades 10 through 12). The figures for initial use of alcohol are $55 \%$ prior to and $36 \%$ during high school; and for marijuana, $26 \%$ prior to and $25 \%$ during high school (see Table 14). Also, for the use of inhalants (unadjusted) more than half $(8.4 \%)$ was initiated before tenth grade (vs. $7.5 \%$ after).

For most of the illicit drugs, between $40 \%$ and $56 \%$ of the eventual users (i.e., those who had used by the end of twelfth grade) initiated use prior to tenth grade; methaqualone, barbiturates, inhalants (unadjusted), PCP, amphetamines, and tranquilizers fall in this category.

Among eventual users of hallucinogens, LSD (specifically), nitrites, heroin, and opiates other than heroin, still a substantial minority-between one-quarter and one-thirdinitiate use prior to tenth grade.

- Cocaine presents a contrasting picture to nearly all other drugs in that initiation rates are highest in the last two years of high school; less than $17 \%$ of eventual users in the class of 1986 initiated use prior to tenth grade. Furthermore, followups of earlier graduating classes show that initiation rates remain very high in the years after high school. 


\section{TABLE 14}

\section{Grade of First Use for Sixteen Types of Drugs, Class of 1986}

(Entries are percentages)

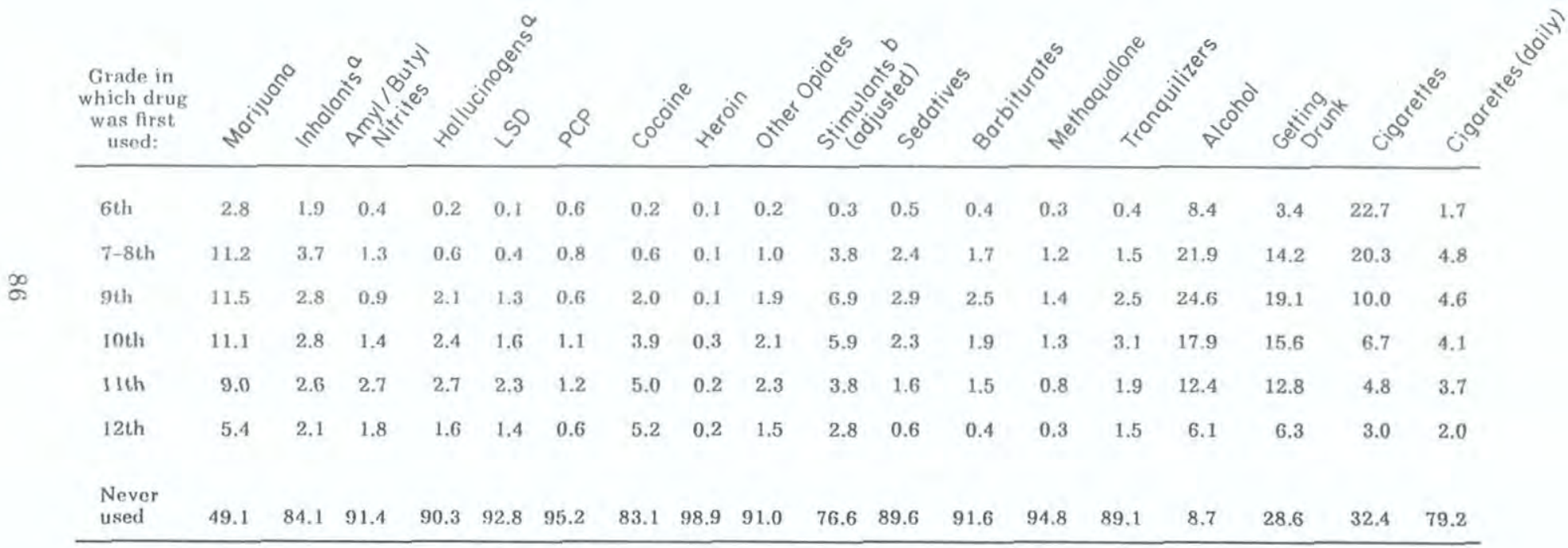

NOTE: This question was asked in two of the five forms $(\mathrm{N}=$ approximately 5600$)$, except for inhalants, PCP, and the nitrites which were asked about in only one form $(\mathrm{N}=$ approximately 2800$)$.

${ }^{\mathrm{a}}$ Unadjusted for known underreporting of certain drugs. See text for details.

${ }^{b}$ Based on the data from the revised question, which attempts to exclude the inappropriate reporting of non-prescription stimulants. 


\section{TRENDS IN USE AT EARLIER GRADE LEVELS}

- Using the retrospective data provided by members of each senior class concerning their grade at first use, it is possible to reconstruct lifetime prevalence curves at lower grade levels during the years when each class was at those various grade levels. Obviously, data from dropouts from school are not included in any of the curves. Figures $17 \mathrm{a}$ through $17 \mathrm{~s}$ show the reconstructed lifetime prevalence curves for earlier grade levels for a number of drugs.

- Figure $17 \mathrm{a}$ provides the trends at each grade level for lifetime use of any illicit drug. It shows that for all grade levels there was a continuous increase in illicit drug involvement through the seventies. The increase is fortunately quite small for use prior to seventh grade; only $1.1 \%$ of the class of 1975 reported having used an illicit drug in 6th grade or below (which was in 1969 for that class), but the figure has increased modestly, and for the class of 1986 is at $3.4 \%$ (which was in 1980 for that class). The lines for the other grade levels all show much steeper upward slopes. For example, about $42 \%$ of the class of 1986 had used some illicit drug by the end of grade 10 , compared to $37 \%$ of the class of 1975.

- Beginning in 1980 , though, there was a leveling off at the high school level (grades 10,11, and 12) in the proportion becoming involved in illicit drugs. The leveling in the lower grades came about a year earlier.

- Most of the increase in any illicit drug use was due to increasing proportions using marijuana. We know this from the results in Figure 17b showing trends for each grade level in the proportion having used any illicit drug other than marijuana in their lifetime. Compared to Figure $17 \mathrm{~d}$ for marijuana use, these trend lines are relatively flat throughout the seventies and, if anything, began to taper off among ninth and tenth graders between 1975 and 1977. The biggest cause of the increases in these curves from 1978 to 1981 was the rise in reports of amphetamine use. As noted earlier, we suspect that at least some of this rise is artifactual. If amphetamine use is removed from the calculations, even greater stability is shown in the proportion using illicits other than marijuana or amphetamines. (See Figure 17c.)

- As can be seen in Figure 17d, for the years covered across the decade of the 70's, marijuana use had been rising steadily at all grade levels down through the seventh-eighth 
grades. Beginning in 1980, marijuana involvement began to decline for grades 9 through 12 . Junior high school use reached an asymptote by the end of the seventies, as well.

There was also some small increase in marijuana use during the 1970's at the elementary level (that is, prior to seventh grade). Use by sixth grade or lower rose gradually from $0.6 \%$ for the class of 1975 (who were sixth graders in 196869 ) to a peak of $4.3 \%$ in the class of 1984 (who were sixth graders in 1977-78). (It began dropping thereafter.) Results from the three most recent national household surveys published by NIDA suggest that this relatively low level of use among this age group continues to hold true: the proportion of 12 to 13 year olds reporting any experience with marijuana was $6 \%$ in 1971 , and was constant at $8 \%$ in 1977 , 1979 , and 1982 . Presumably sixth graders would have even lower absolute rates, since the average age of sixth graders is less than twelve. ${ }^{15}$

- Cocaine use at earlier grade levels is given in Figure 17e. One clear contrast to the marijuana pattern is that most initiation into cocaine use takes place in the last two years of high school (rather than earlier, as is the case for marijuana). Further, most of the increase in cocaine experience between 1976 and 1980 occurred in the 11th and 12th grades, not below. After 1980, experience with cocaine generally remained level until 1985 (for seniors), when a modest upturn was observed.

- The lifetime prevalence statistics for stimulants peaked briefly for grade levels 9 through 12 during the mid-70's. (See Figure 17f.) However, it showed a sharp rise in the late 70 's at virtually all grade levels. As has been stated repeatedly, we believe that some-perhaps most-of this recent upturn is artifactual in the sense that nonprescription stimulants account for much of it. However, regardless of what accounts for it, there was a clear upward secular trend-that is, one derived across all cohorts and grade levels-beginning in 1979. The unadjusted data from the class of 1983 give the first indication of a reversal of this trend. The adjusted data from the classes of 1982 through 1986 suggest that the use of stimulants leveled around 1982 and has fallen appreciably since.

${ }^{15}$ See Miller, J.D., Cisin, I.H., Gardner-Keaton, H., Harrell, A.V., Wirtz, P.W., Abelson, H.I., Fishburne, P.M. (1983). National survey on drug abuse: Main findings 1982. Rockville, MD: National Institute on Drug Abuse. 
- Lifetime prevalence of hallucinogen use (unadjusted for underreporting of $\mathrm{PCP}$ ) began declining among students at most grade levels in the mid-1970's (Figure 17g), and this gradual decline continued in the upper grades. However, it appears that a leveling occurred after 1979 in the lower grades, due almost entirely to the trends in LSD use. (The trend curves for $\mathbf{L S D}$ (Figure $17 \mathrm{~h}$ ) are extremely similar in shape, though lower in level, of course.) This year's data from the class of 1986 suggest that hallucinogen use began declining in the lower grade levels in the early 1980's.

- While there is less trend data for $\boldsymbol{P C P}$, since questions about grade of first use of PCP were not included until 1979, some interesting results emerge. A sharp downturn began around 1979 (see Figure 17i), but decelerated and actually ended by 1986. If the hallucinogen figure (17g) were adjusted for underreporting of PCP use, it would be showing even more downturn in recent years.

- Questions about age at first use for inhalants (unadjusted for the nitrites) have been asked only since 1978. The retrospective trend curves (Figure 17j) suggest that during the mid-1970's, experience with inhalants decreased slightly for most grade levels and then began to rise again. For the upper grade levels there has been a continued gradual rise since 1980 in lifetime prevalence, whereas the curves have been more uneven in the lower grades.

- Since grade-at-first-use data have been gathered for the nitrites beginning in 1979, only limited retrospective data exist (Figure 17k). These do not show the recent increase observed for the overall inhalant category, although the gradual decline in experience with the nitrites, which began around 1980, ended by 1985 .

- Figure 171 shows that the lifetime prevalence of sedative use, like stimulant use, began declining for all grade levels in the mid-70's, then showed some reversal in the late 70's. (Recall that annual prevalence observed for seniors had been declining steadily from 1975 to 1979 .) As the graphs for the two subclasses of sedatives-barbiturates and methaqualone-show, the trend lines have been quite different for them at earlier grade levels as well as in twelfth grade (see Figures $17 \mathrm{~m}$ and $17 \mathrm{n}$ ). Since about 1974 or 1975 , lifetime prevalence of barbiturate use had fallen off sharply at all grade levels for all classes until the late 70's; since then there has been little change (although current use continued to decline among seniors until 1984). 
During the mid-70's methaqualone use started to fall off at about the same time as barbiturate use in nearly all grade levels, but dropped rather little and then flattened. Between 1978 and 1981 there was a fair resurgence in use in nearly all grade levels; but since 1982 there has been a sharp decline.

- Lifetime prevalence of tranquilizer use (Figure 170) also began to decline at all grade levels in the mid-70's. It is noteworthy that the overall decline in tranquilizer use has been considerably greater in the upper grade levels than the lower ones. Overall, it would appear that the tranquilizer trend lines have been following a similar course to that of barbiturates. So far, the curves are different only in that tranquilizer use continued a steady decline among eleventh and twelfth graders, while barbiturate use did not.

- Though a little difficult to see, the heroin lifetime prevalence figures for grades 9 through 12 all began declining in the mid1970's, then leveled, and show no evidence of reversal as yet (Figure 17p).

- The lifetime prevalence of use of opiates other than heroin has remained quite flat at all grade levels since the mid-70's (Figure 17q).

- Figure $17 \mathrm{r}$ presents the lifetime prevalence curves for cigarette smoking on a daily basis. It shows dramatically that initiation to daily smoking was beginning to peak at the lower grade levels in the early to mid-1970's. This peaking did not become apparent among high school seniors until a few years later. In essence, these changes reflect in large part cohort effects-changes which show up consistently across the age band for certain class cohorts. Because of the highly addictive nature of nicotine, this is a type of drugusing behavior in which one would expect to observe enduring differences between cohorts if any are observed at a formative age. The classes of 1982 and 1983 showed some leveling of the previous decline, but the classes of 1984 through 1986 showed an encouraging resumption of the decline while they were in earlier grade levels.

- The curves for lifetime prevalence of alcohol at grades 11 and 12 (Figure 17s) are very flat, reflecting little change over a decade. At the 7-10th grade levels, the curves show slight upward slopes in the early 1970's, indicating that compared to the older cohorts (prior to the class of 1978), more recent classes initiated use at earlier ages. For example, $50 \%$ of the class of 1975 first used alcohol in ninth grade or earlier, com- 
pared to 55 or $56 \%$ for all classes since 1978. These changes are relatively small, however. (Females account for most of the change; $42 \%$ of females in the class of 1975 first used alcohol prior to tenth grade, compared to 51 to $52 \%$ for all classes since 1981.) 


\section{FIGURE 17a}

Use of Any Illicit Drug: Trends in Lifetime

Prevalence for Earlier Grade Levels

Based on Retrospective Reports from Seniors

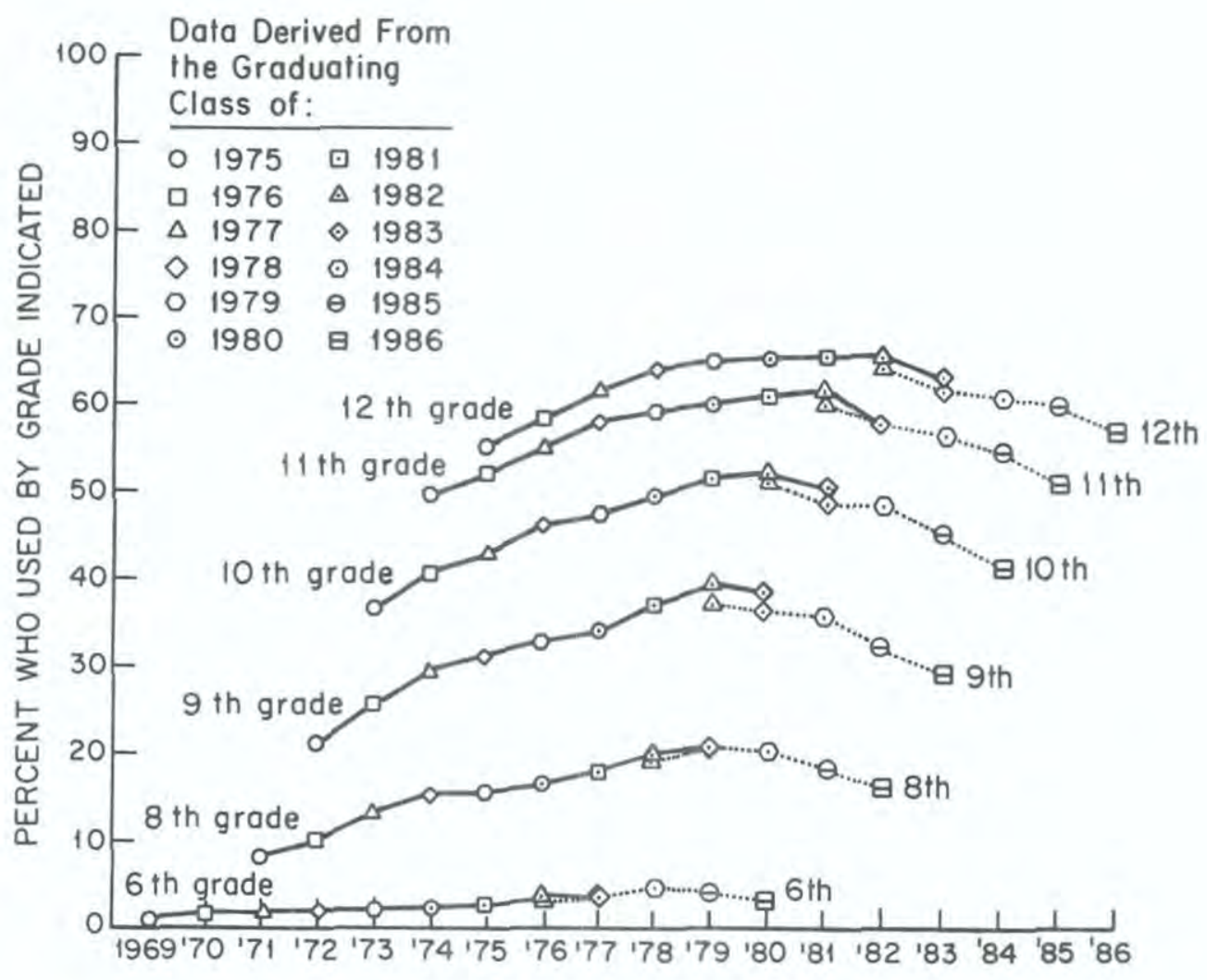

NOTE: The dotted lines connect percentages which result if non-prescription stimulants are excluded. 
FIGURE 17b

\section{Use of Any Illicit Drug Other Than Marijuana: Trends in Lifetime Prevalence for Earlier Grade Levels \\ Based on Retrospective Reports from Seniors}

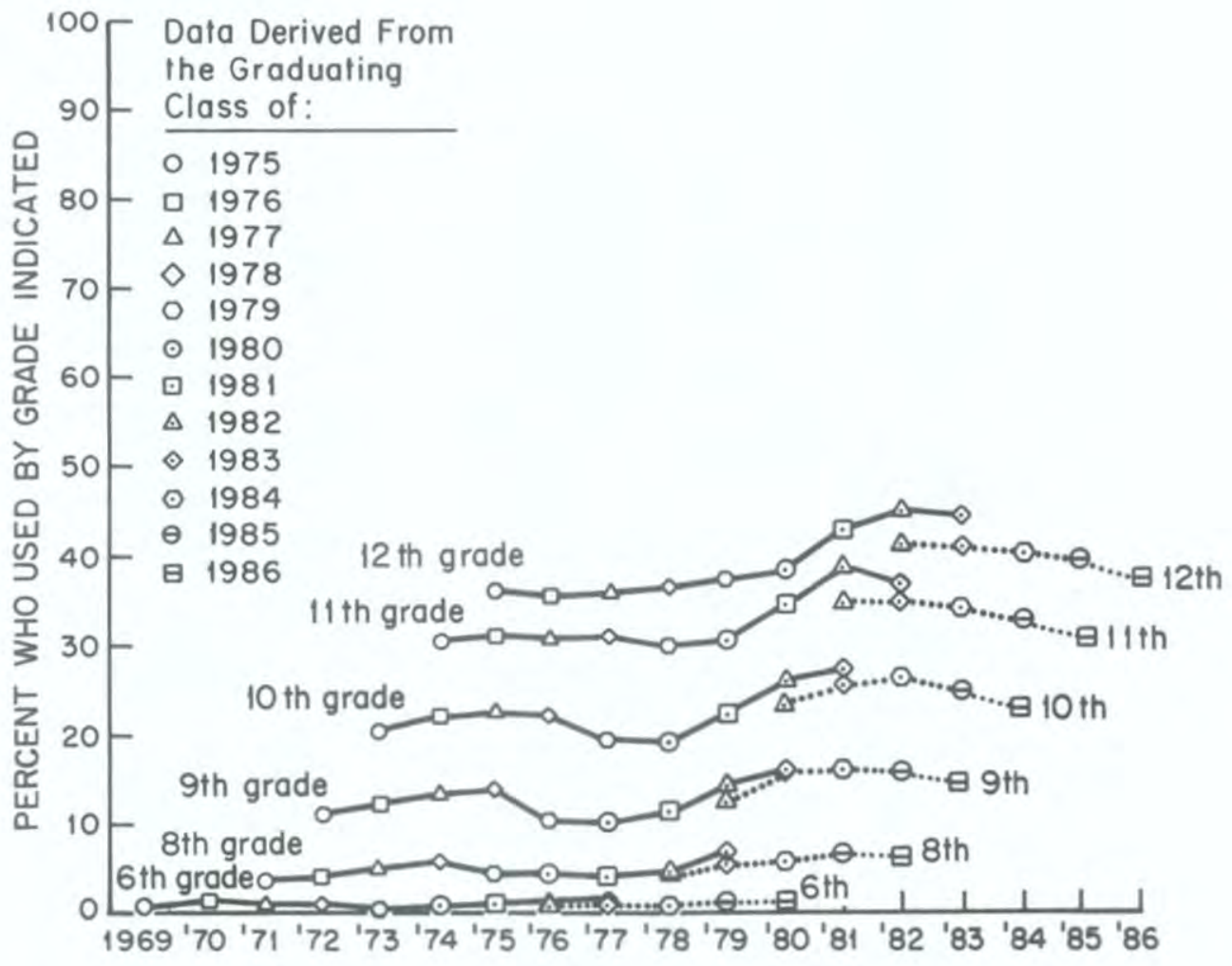

NOTE: The dotted lines connect percentages which result if non-prescription stimulants are excluded. 


\section{FIGURE $17 \mathrm{c}$}

Use of Any Illicit Drug Other Than Marijuana or Amphetamines: Trends in Lifetime Prevalence for Earlier Grade Levels Based on Retrospective Reports from Seniors

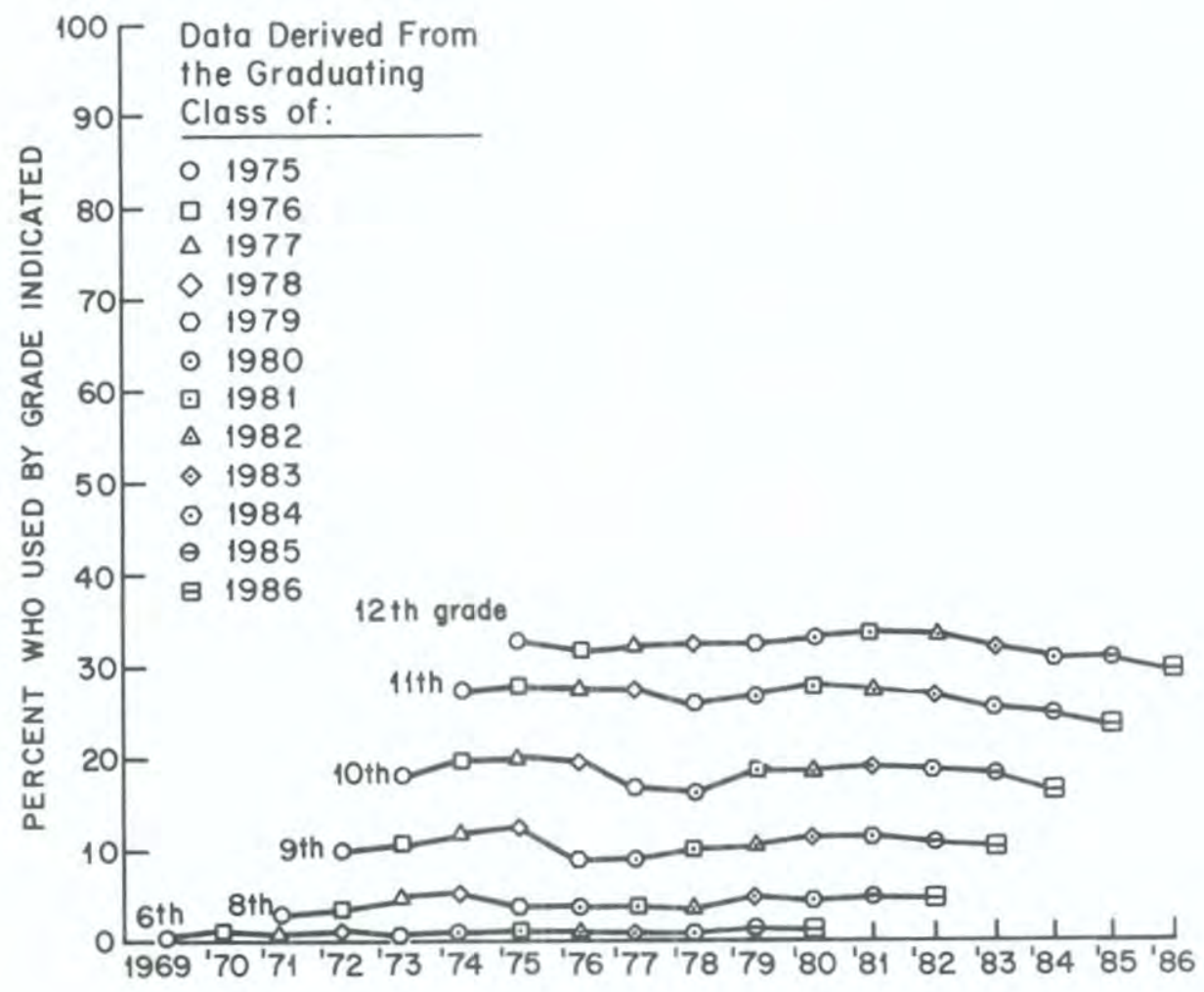




\section{FIGURE 17d}

Marijuana: Trends in Lifetime Prevalence for Earlier Grade Levels Based on Retrospective Reports from Seniors

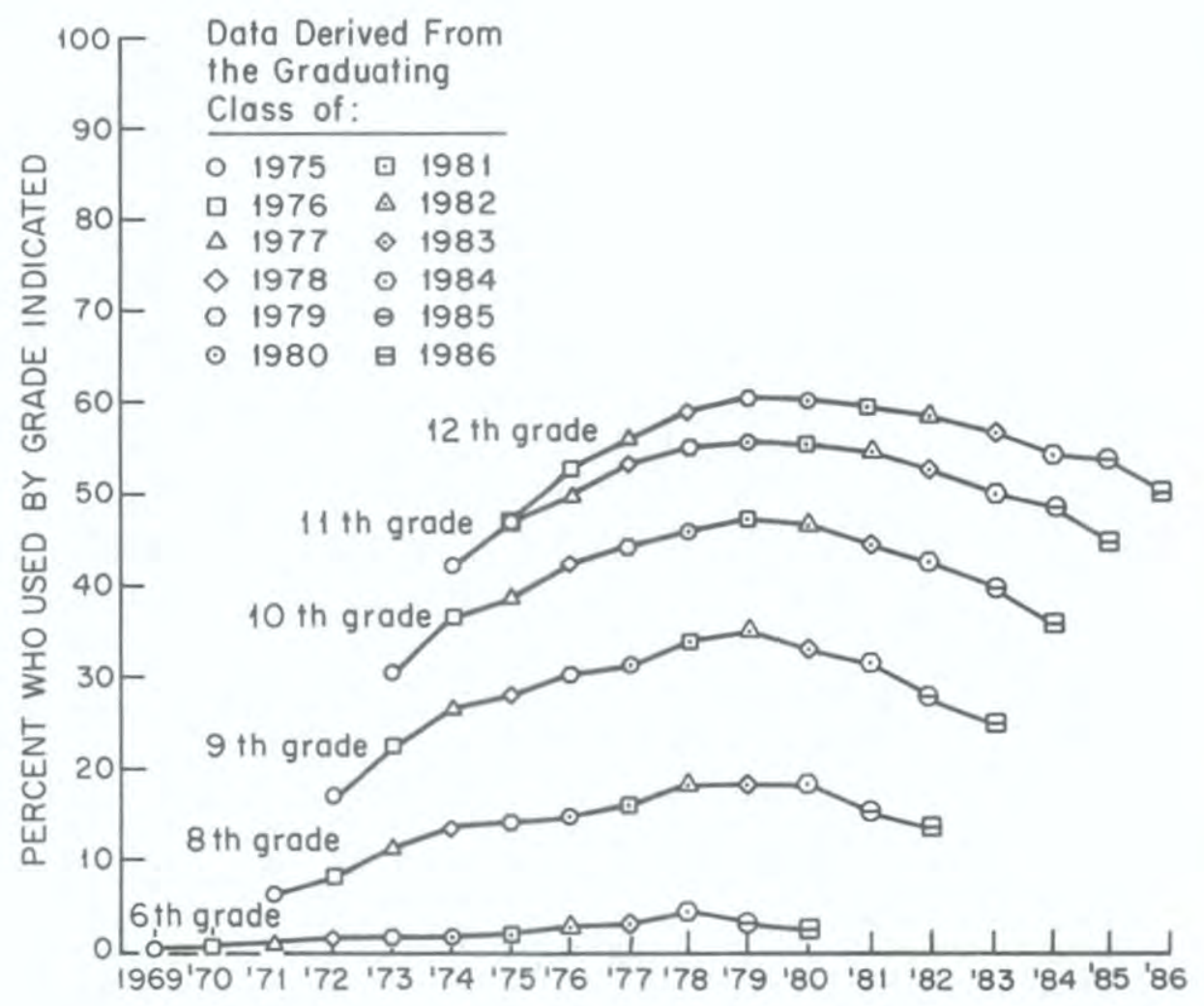




\section{FIGURE 17e}

Cocaine: Trends in Lifetime Prevalence for Earlier Grade Levels Based on Retrospective Reports from Seniors

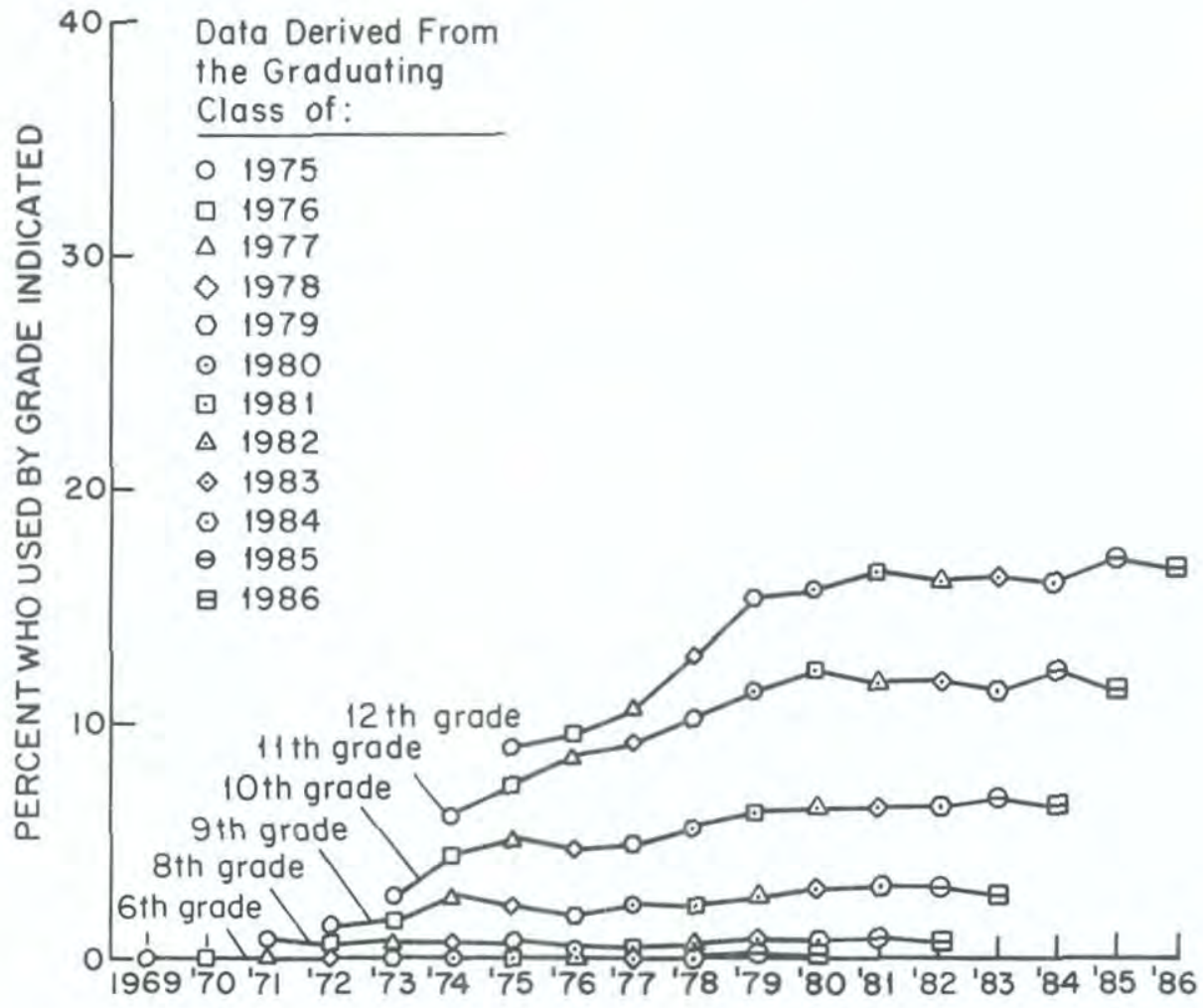




\section{FIGURE 17f}

Stimulants: Trends in Lifetime Prevalence for Earlier Grade Levels Based on Retrospective Reports from Seniors

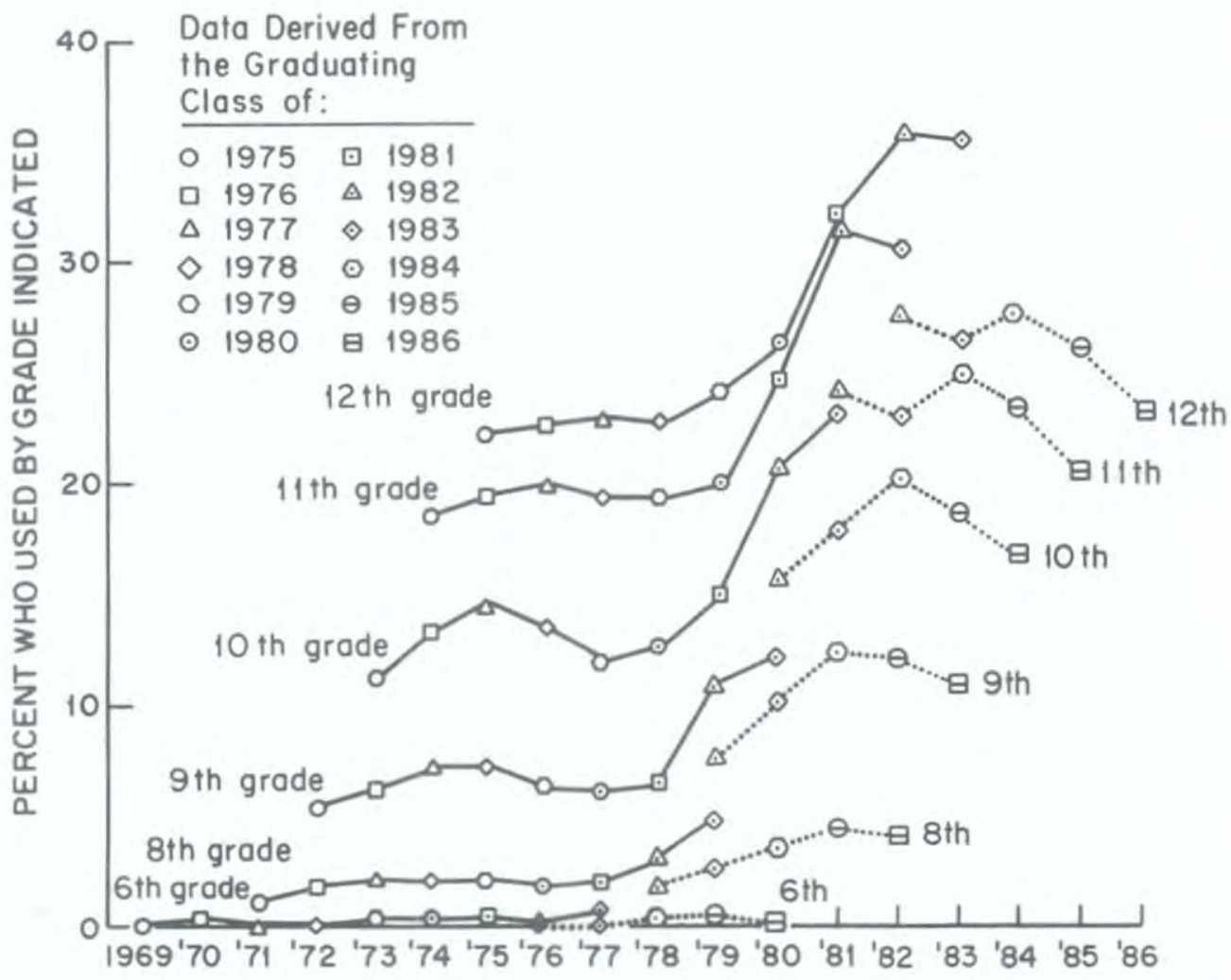

NOTE: The dotted lines connect percentages which result if non-prescription stimulants are excluded. 


\section{FIGURE $17 \mathrm{~g}$}

Hallucinogens: Trends in Lifetime Prevalence for Earlier Grade Levels Based on Retrospective Reports from Seniors

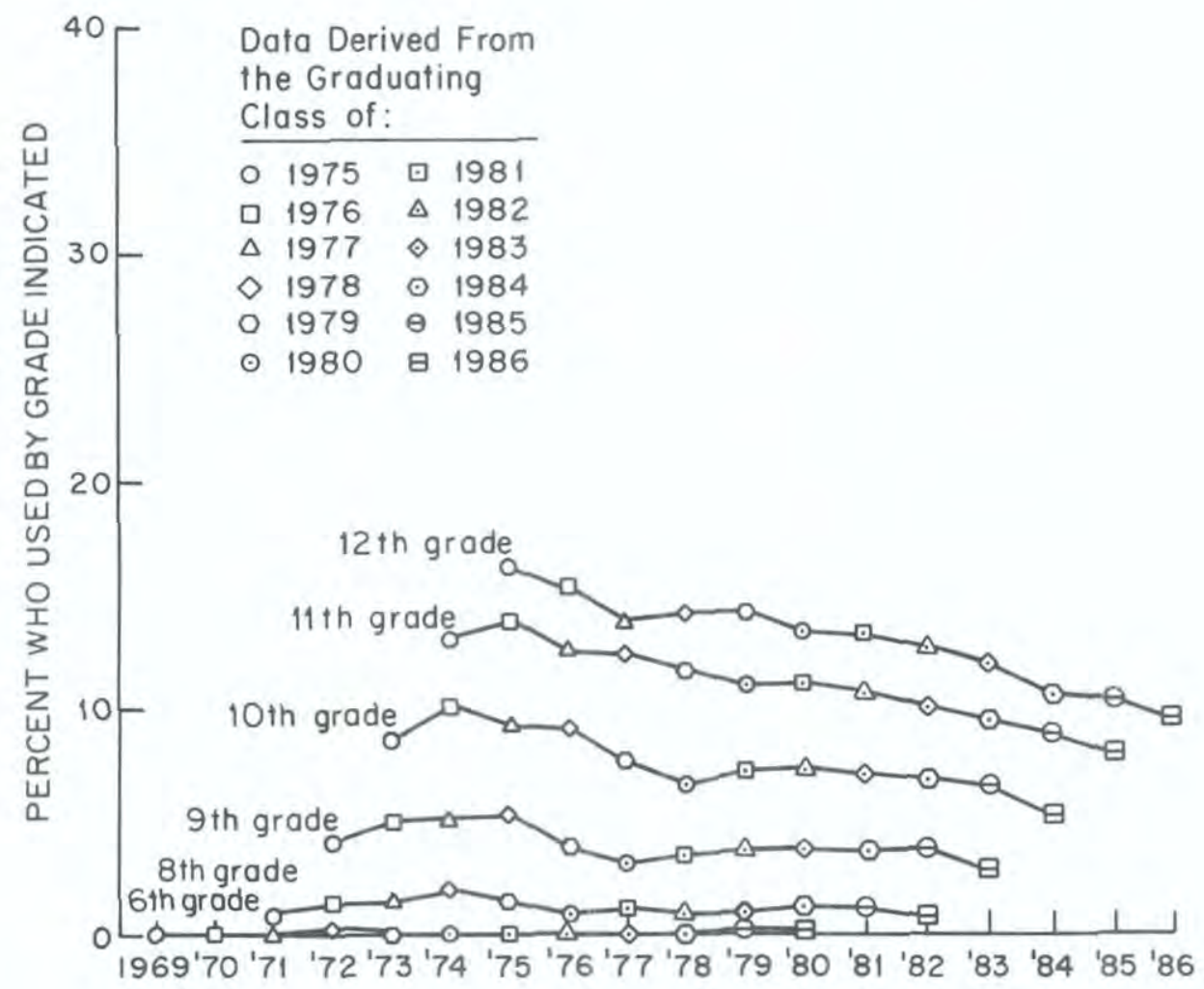




\section{FIGURE $17 \mathrm{~h}$}

LSD: Trends in Lifetime Prevalence for Earlier Grade Levels

Based on Retrospective Reports from Seniors

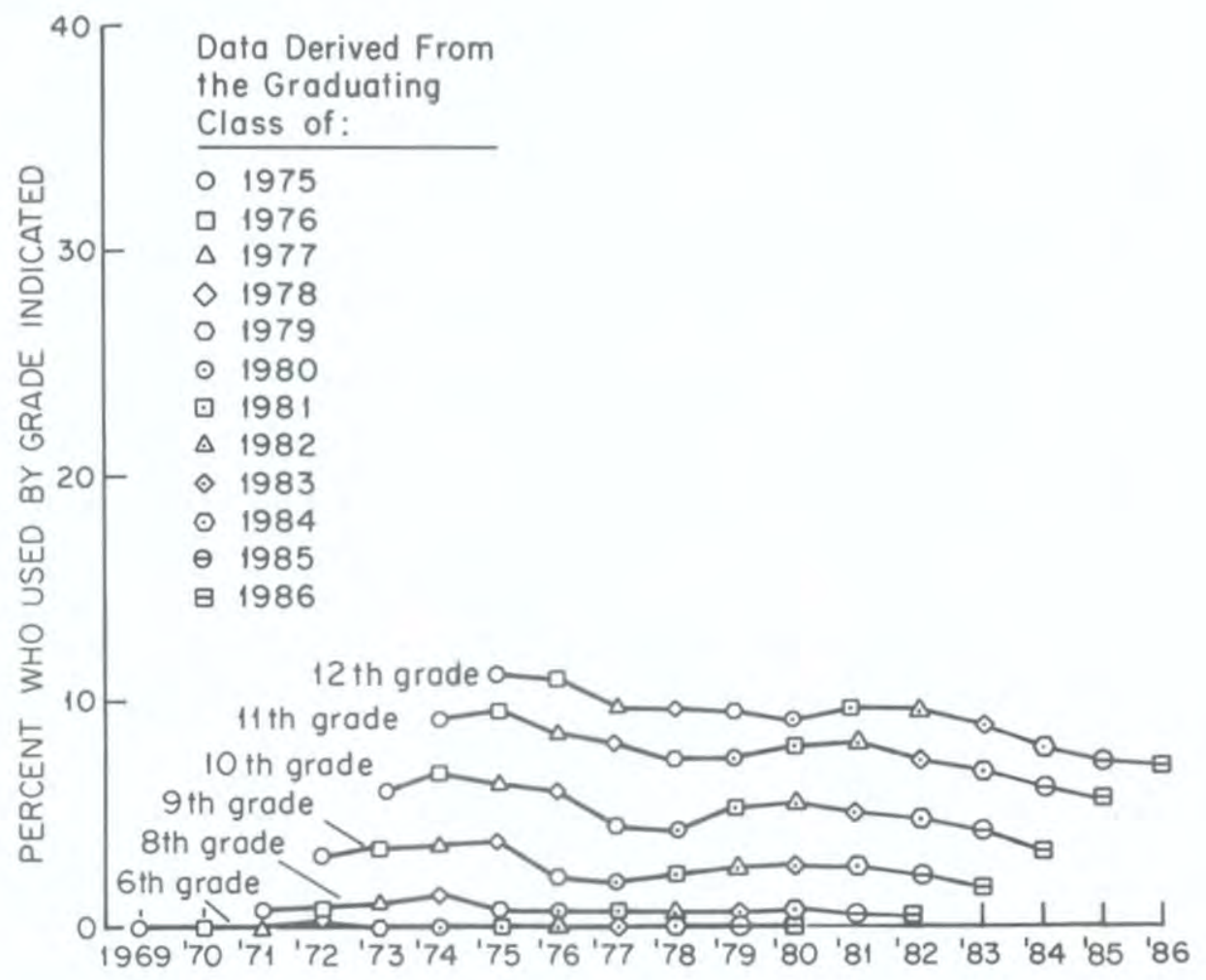




\section{FIGURE 17i}

PCP: Trends in Lifetime Prevalence for Earlier Grade Levels Based on Retrospective Reports from Seniors

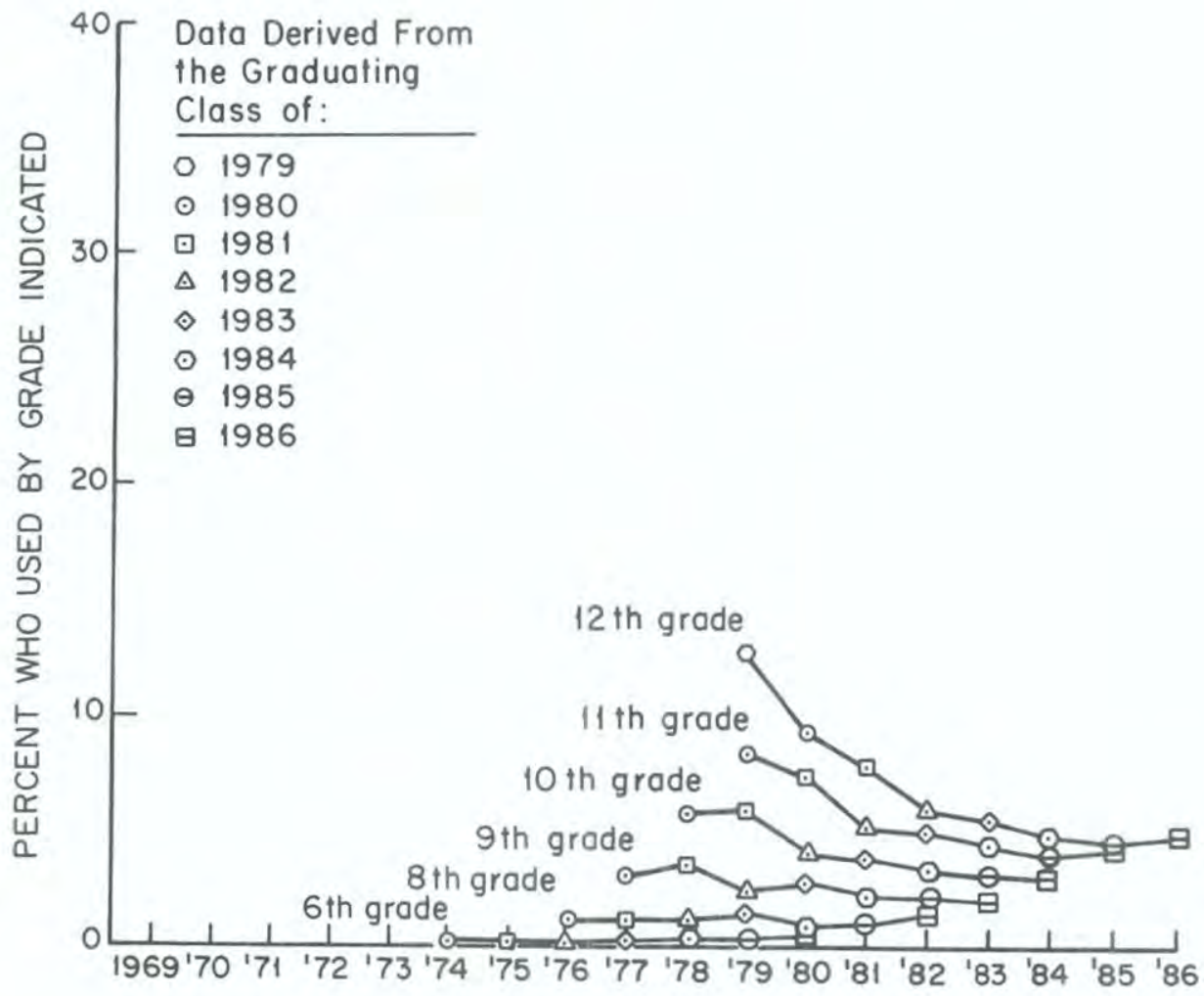




\section{FIGURE $17 \mathrm{j}$}

Inhalants: Trends in Lifetime Prevalence for Earlier Grade Levels Based on Retrospective Reports from Seniors

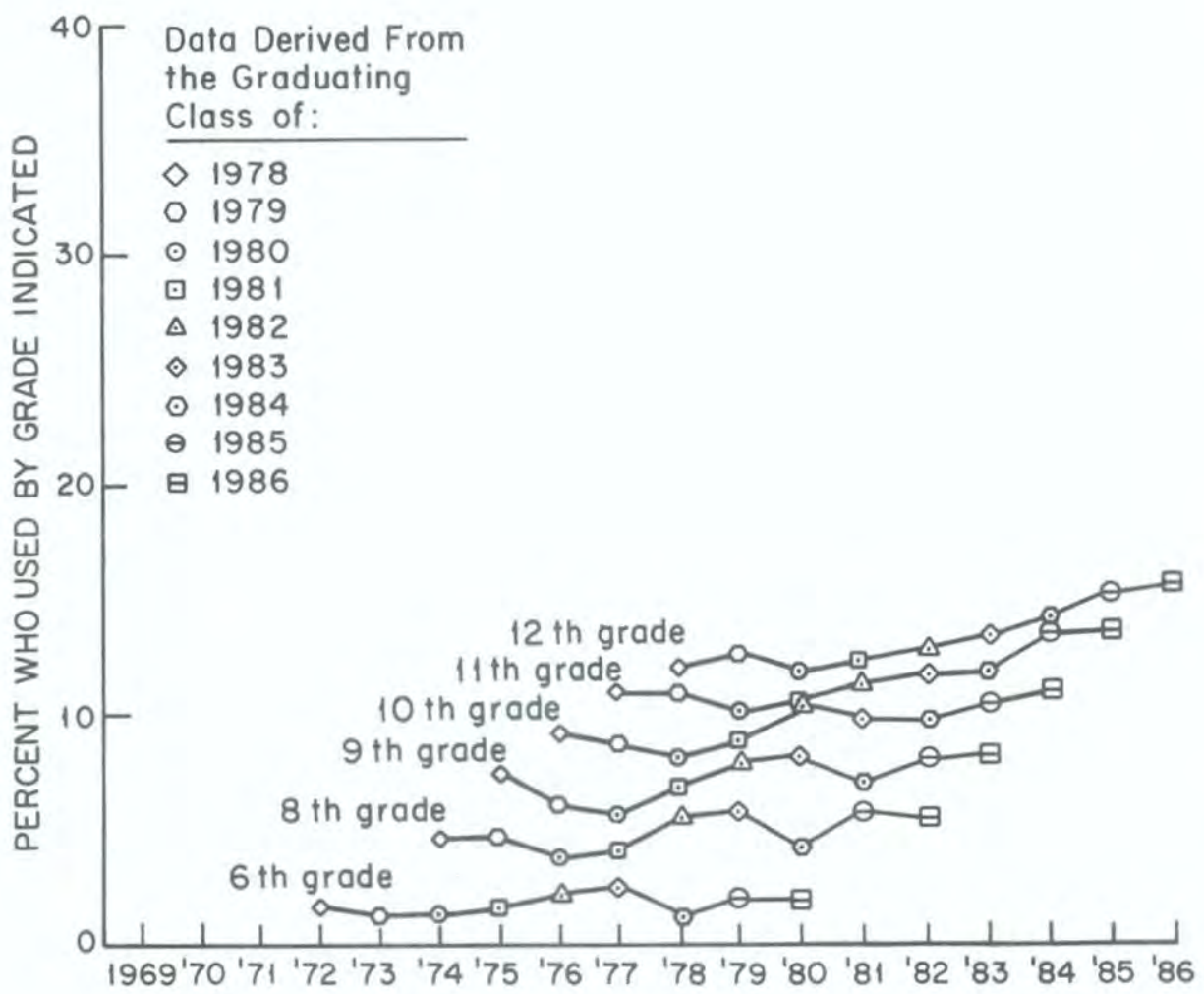




\section{FIGURE 17k}

Nitrites: Trends in Lifetime Prevalence for Earlier Grade Levels

Based on Retrospective Reports from Seniors

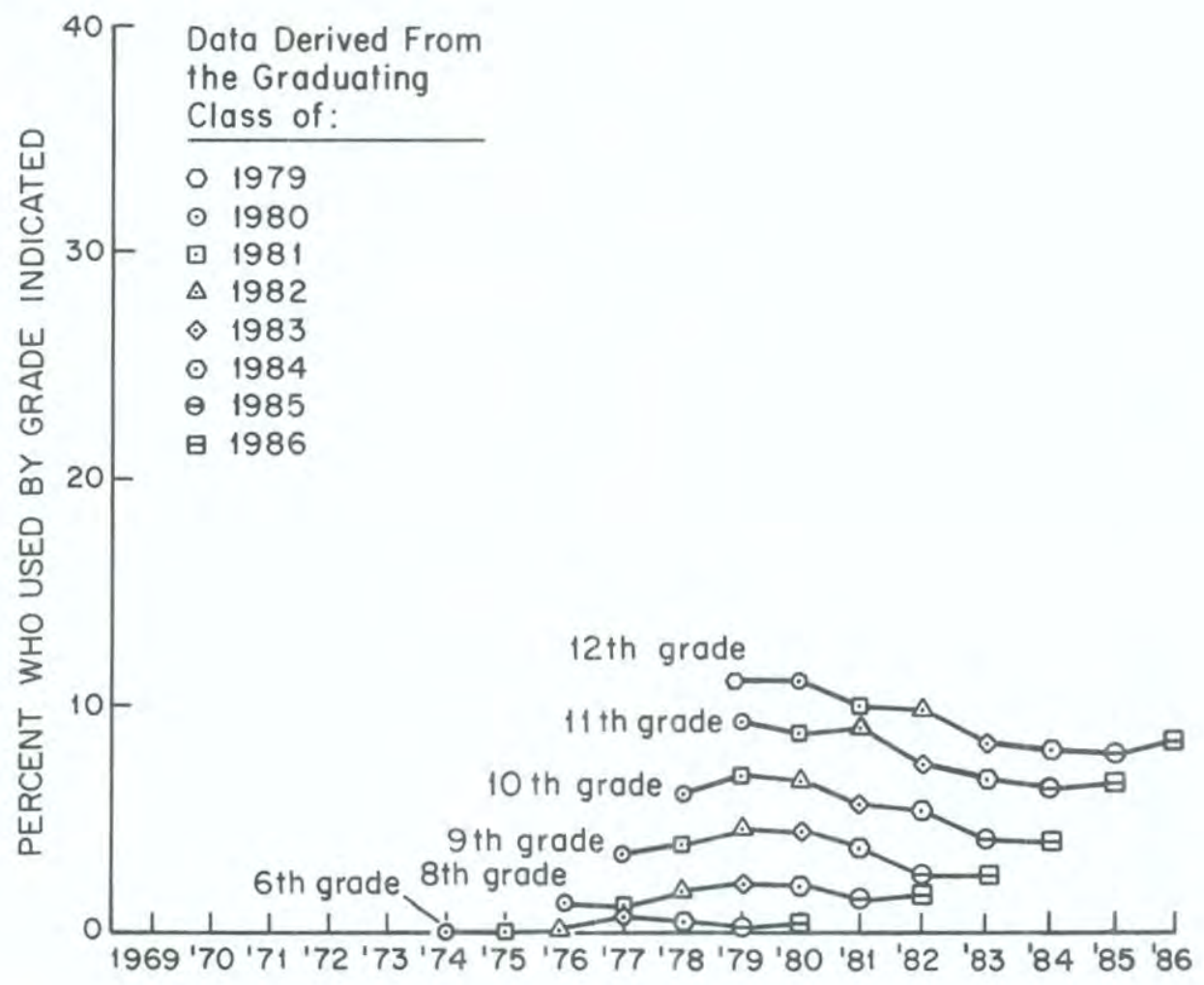




\section{FIGURE 171}

Sedatives: Trends in Lifetime Prevalence for Earlier Grade Levels Based on Retrospective Reports from Seniors

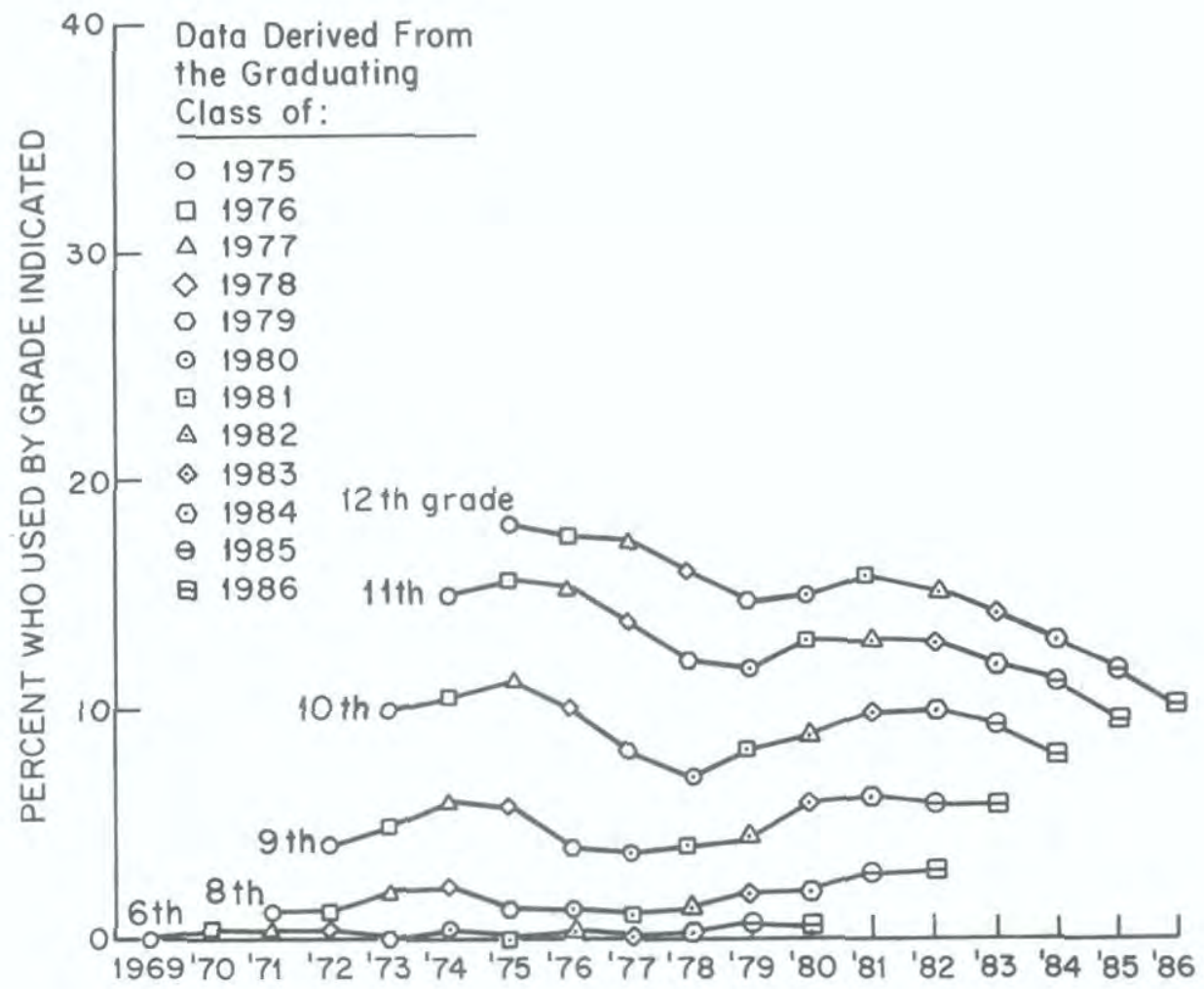




\section{FIGURE $17 \mathrm{~m}$}

Barbiturates: Trends in Lifetime Prevalence for Earlier Grade Levels Based on Retrospective Reports from Seniors

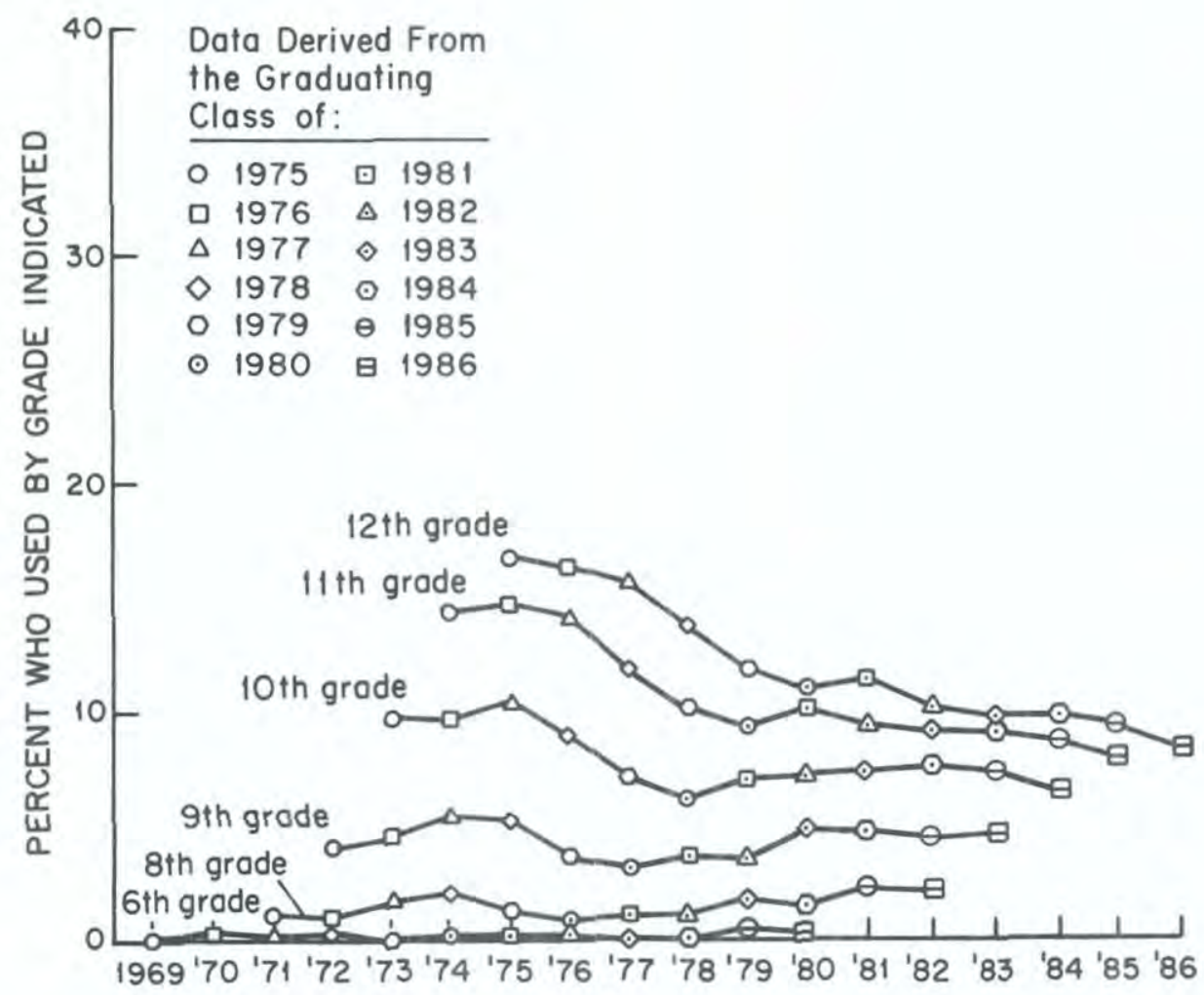




\section{FIGURE $17 \mathrm{n}$}

Methaqualone: Trends in Lifetime Prevalence for Earlier Grade Levels Based on Retrospective Reports from Seniors

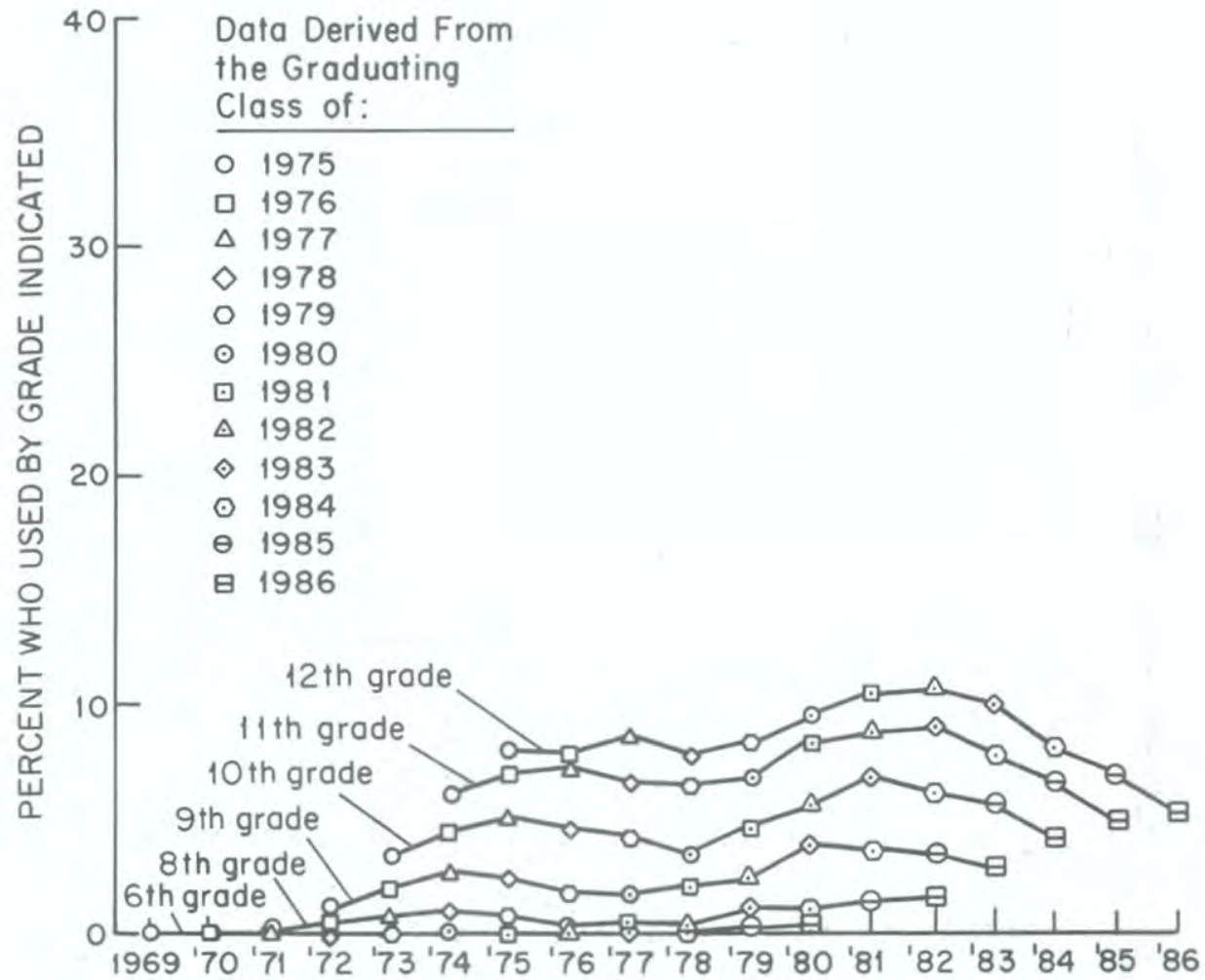




\section{FIGURE 170}

Tranquilizers: Trends in Lifetime Prevalence for Earlier Grade Levels Based on Retrospective Reports from Seniors

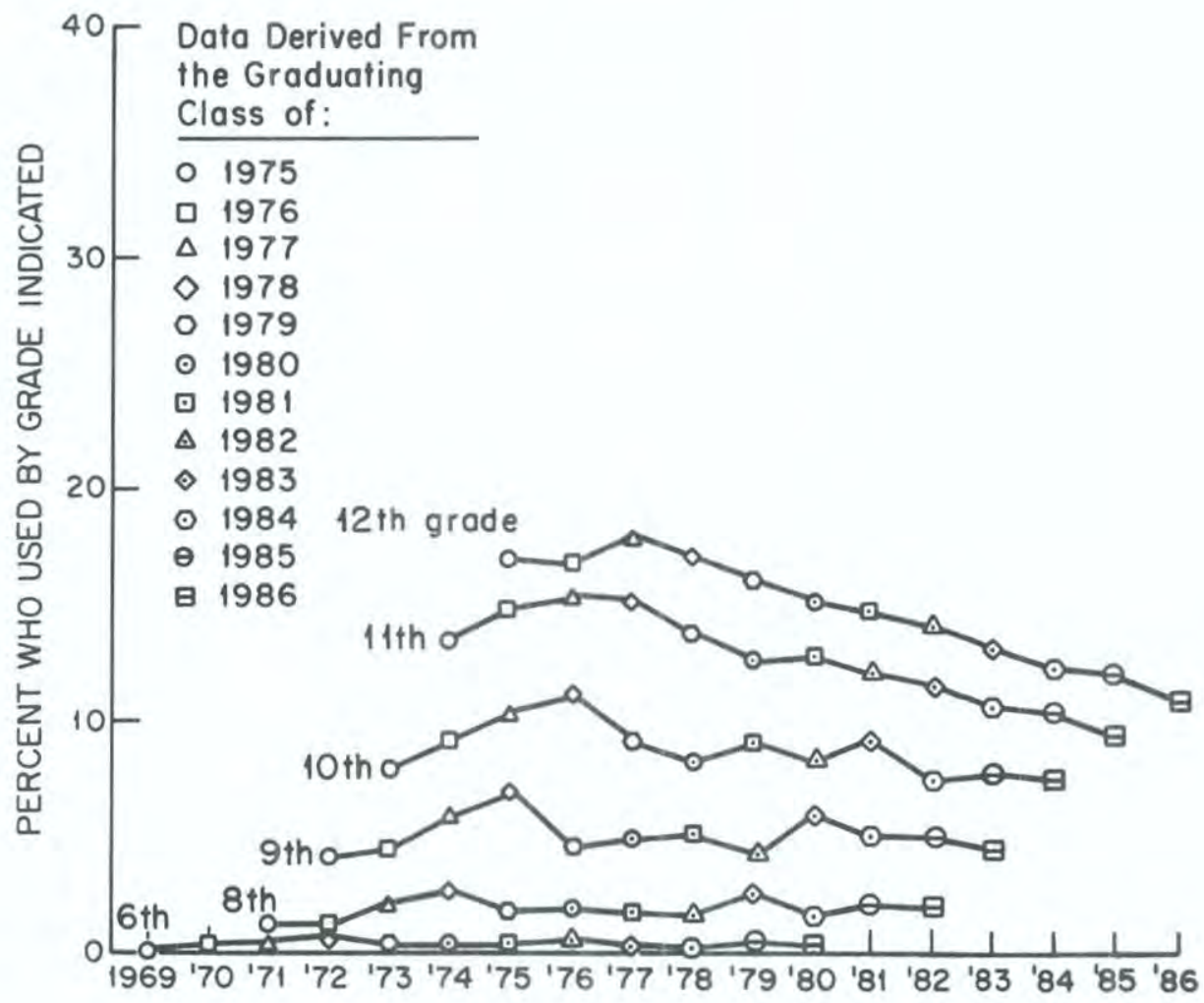


FIGURE $17 \mathrm{p}$

Heroin: Trends in Lifetime Prevalence for Earlier Grade Levels Based on Retrospective Reports from Seniors

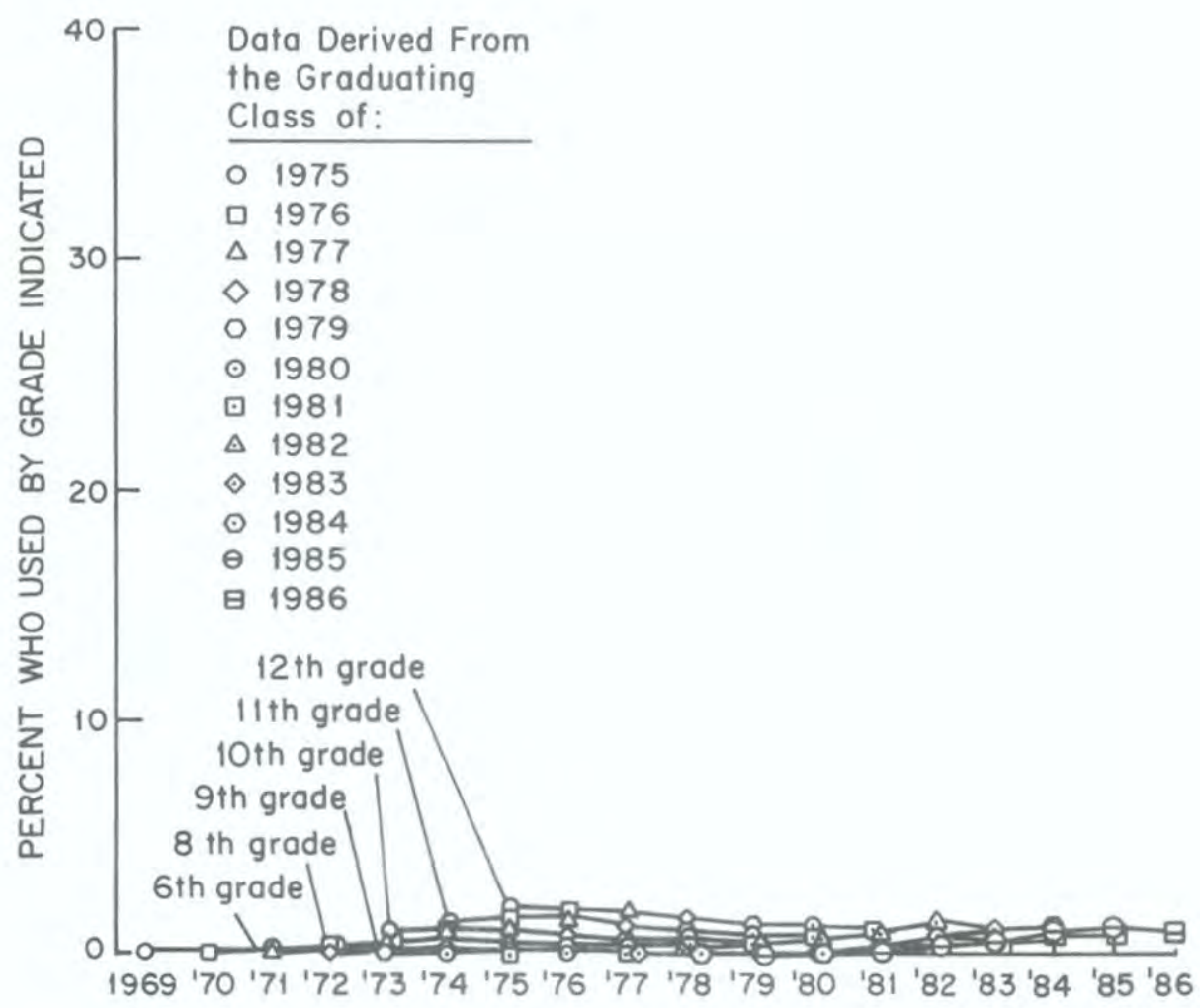




\section{FIGURE $17 q$}

Other Opiates: Trends in Lifetime Prevalence for Earlier Grade Levels Based on Retrospective Reports from Seniors

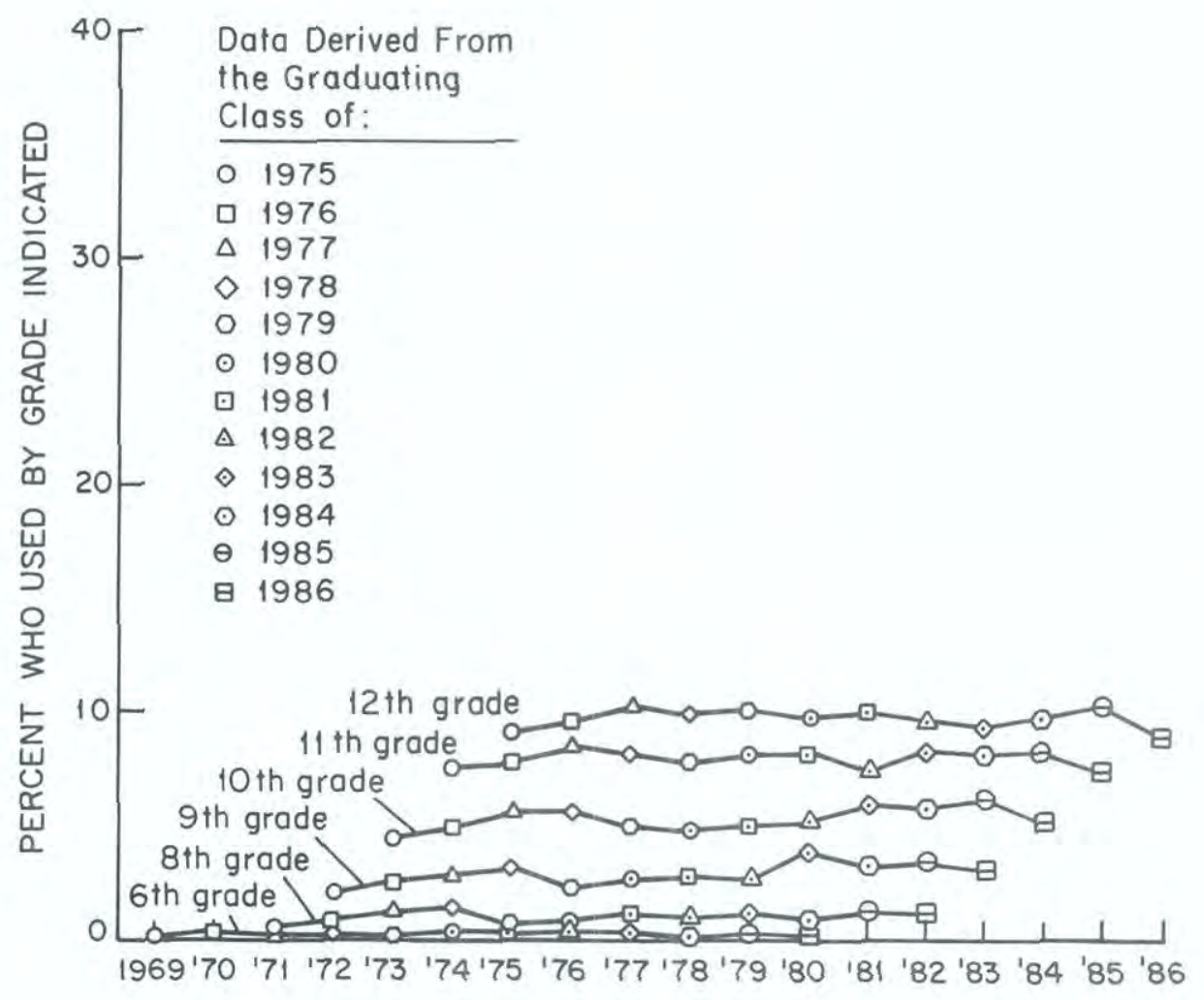




\section{FIGURE $17 \mathrm{r}$}

Cigarette Smoking on a Daily Basis: Trends in Lifetime Prevalence for Earlier Grade Levels

Based on Retrospective Reports from Seniors

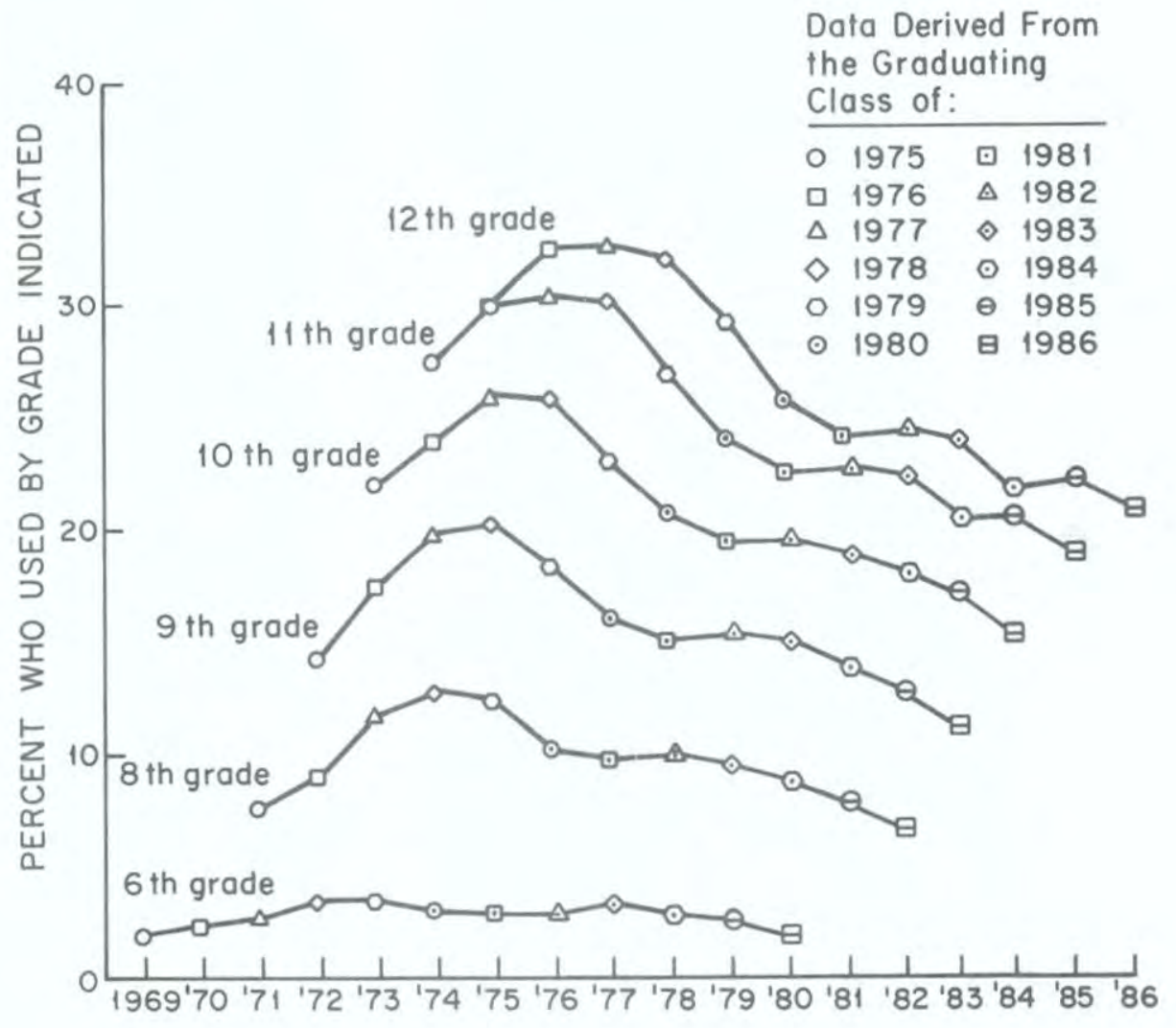




\section{FIGURE 17s}

Alcohol: Trends in Lifetime Prevalence for Earlier Grade Levels Based on Retrospective Reports from Seniors

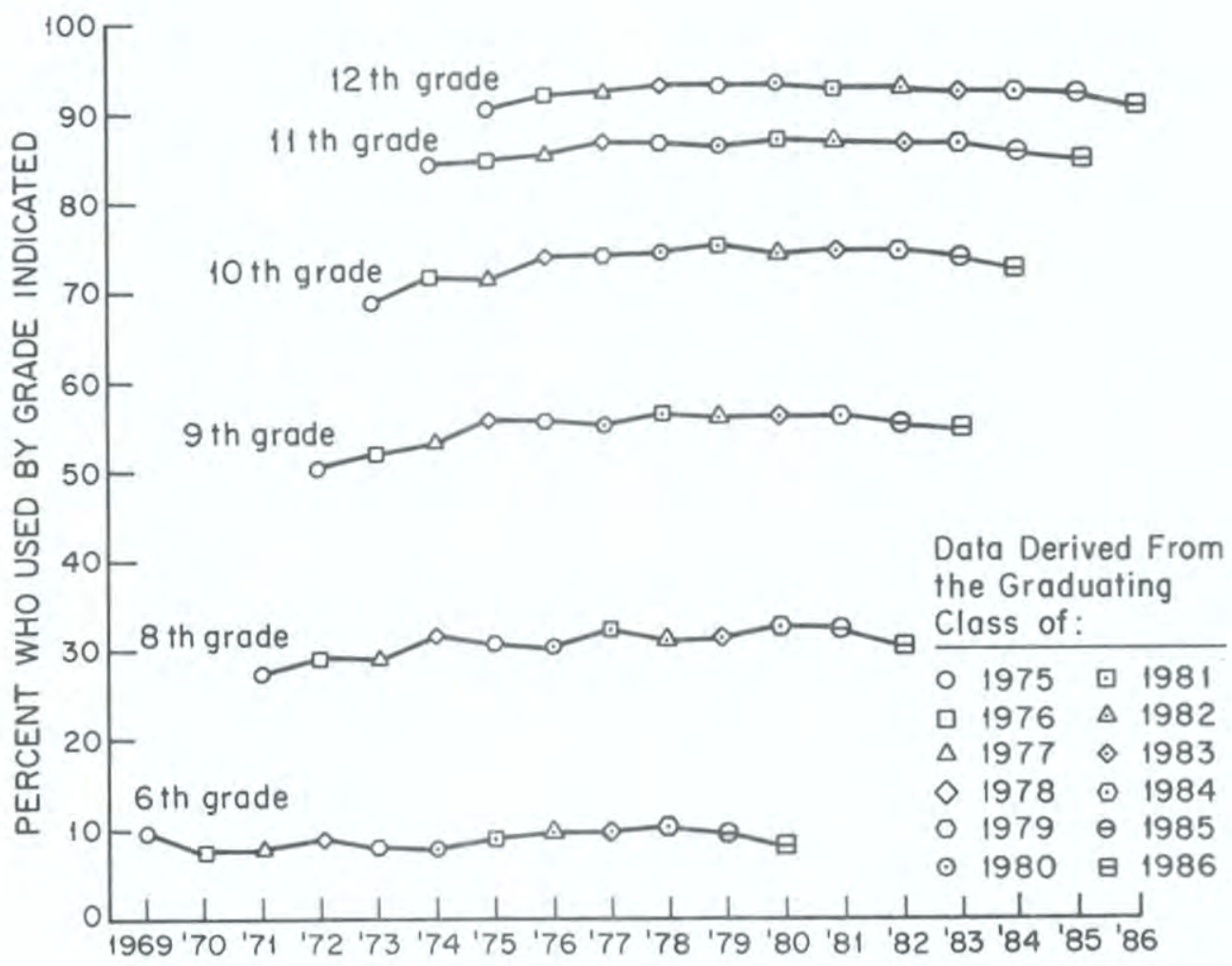




\section{DEGREE AND DURATION OF DRUG HIGHS}

On one of the five questionnaire forms, seniors who report use of a drug during the prior twelve months are asked how long they usually stay high on that drug and how high they usually get. These measures were developed both to help characterize the drug-using event and to provide indirect measures of dose or quantity of drugs consumed.

- Figure 18 shows the proportion of 1986 seniors who say that they usually get "not at all" high, "a little" high, "moderately" high, or "very" high when they use a given type of drug. The percentages are based on all respondents who report use of the given drug class in the previous twelve months, and therefore each bar cumulates to $100 \%$. The ordering from left to right is based on the percentage of users of each drug who report that they usually get "very" high. (The width of each bar is proportional to the percentage of all seniors having used the drug class in the previous year; this should serve as a reminder that even though a large percentage of users of a drug may get very high, they may represent only a small proportion of all seniors.)

- The drugs which usually result in intense highs are the hallucinogens (LSD and other hallucinogens), heroin, and methaqualone (Quaaludes). (Actually, this question was omitted for heroin beginning in 1982, due to small numbers of cases available each year; by an averaging across earlier years indicated that it would rank very close to LSD.)

- Following closely are cocaine and marijuana, with roughly two-thirds of the users of each saying they usually get moderately high or very high when using the drug.

- The four major psychotbrerapeutic drug classesbarbiturates, opiates otherthan heroin, tranquilizers, and stimulants - are less often used to get high; but substantial proportions of users (from 26\% for tranquilizers to $43 \%$ for other opiates) still say they usually get moderately or very high after taking these drugs.

- Relatively few of the many seniors using alcohol say that they usually get very high when drinking, although nearly half usually get at least moderately high. However, for a 
FIGURE 18

Degree of Drug High Attained by Recent Users

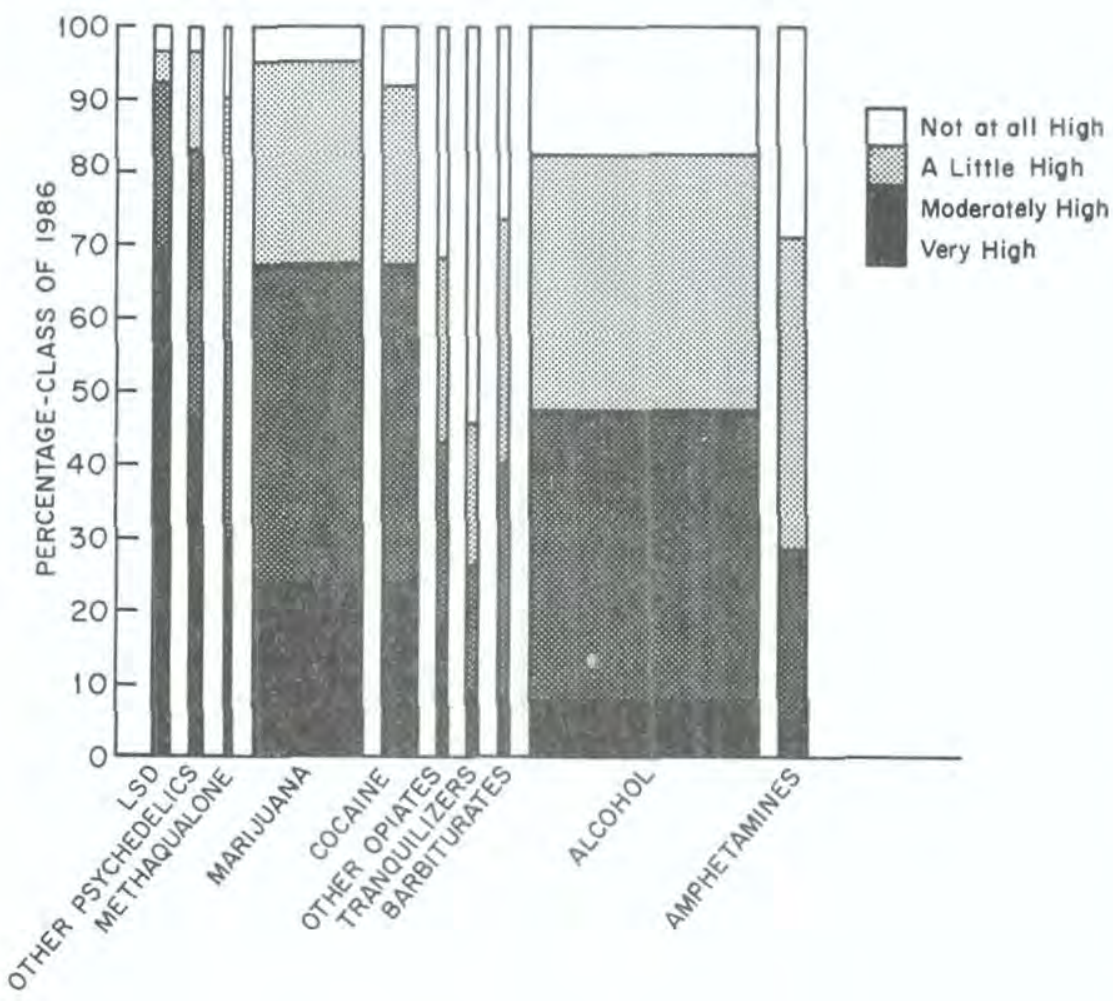

NOTE: The width of each bar is proportionate to the number of seniors reporting any use of each drug in the prior twelve months. Heroin is not included in this figure because these particular questions are not asked of the small number of heroin users. 


\section{FIGURE 19}

\section{Duration of Drug High Attained by Recent Users}

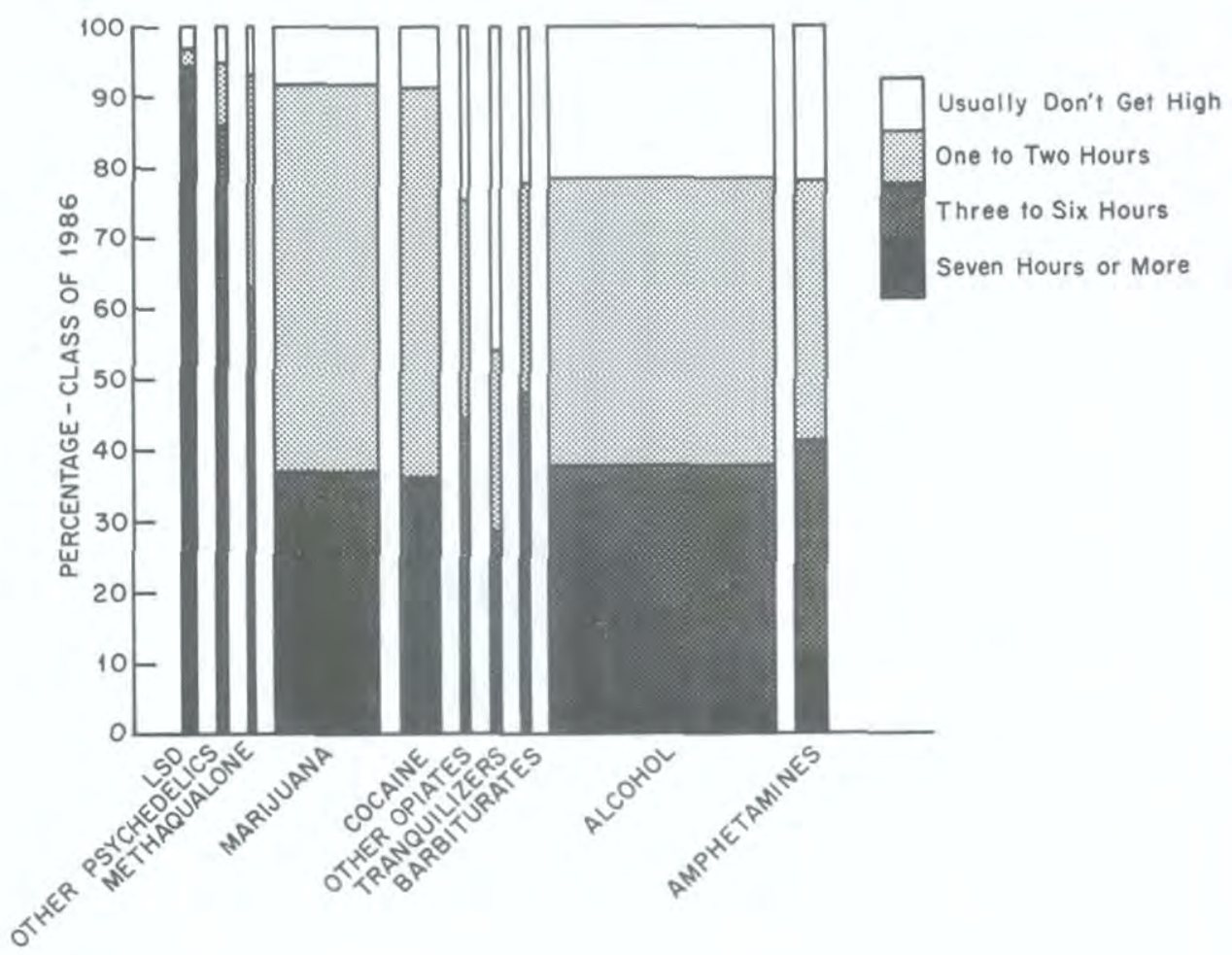

NOTE: The width of each bar is proportionate to the number of seniors reporting any use of each drug in the prior twelve months. Heroin is not included in this figure because these particular questions are not asked of the small number of heroin users. 
given individual we would expect more variability from occasion to occasion in the degree of intoxication achieved with alcohol than with most of the other drugs. Therefore, many drinkers surely get very high at least sometimes, even if that is not "usually" the case.

- Figure 19 presents the data on the duration of the highs usually obtained by users of each class of drugs. The drugs are arranged in the same order as for intensity of highs to permit an examination of the amount of correspondence between the degree and duration of highs.

- As can be seen in Figure 19, those drugs which result in the most intense highs generally tend to result in the longest highs. For example, $L S D$ and other hallucinogens rank one and two respectively on both dimensions, with substantial proportions ( $62 \%$ and $43 \%$ ) of the users of these drugs saying they usually stay high for seven hours or more.

- However, there is not a perfect correspondence between degree and duration of highs. The highs achieved with marijuana, although intense for many users, tend to be relatively short-lived in comparison with most other drugs. The majority of users usually stay high two hours or less, and the modal time is one to two hours $(55 \%)$, but one-third (33\%) report usual highs lasting 3-6 hours.

- For cocaine users the modal high is one to two hours (55\%), though over one-third $(36 \%)$ stay high three or more hours.

- The modal and median duration of highs for methaqualone is three to six hours. Users of barbiturates, opiates other than heroin, stimulants, and tranquilizers report highs of slightly shorter duration.

- In sum, the drugs vary considerably in both the duration and degree of the highs usually obtained with them, though most have a median duration of one to two hours. (These data obviously do not address the qualitative differences in the experiences of being "high.") Sizeable proportions of the users of all of these drugs report that they usually get high for at least three hours per occasion, and for a number of drugs-particularly the hallucinogens-appreciable proportions usually stay high for seven hours or more. 


\section{TRENDS IN DEGREE AND DURATION OF DRUG HIGHS}

- There have been several important shifts over the last several years in the degree or duration of highs usually experienced by users of the various drugs.

- For cocaine the proportion who say they usually get high for only two hours or less increased from $36 \%$ in 1977 to $54 \%$ in 1981 , where it remained level for several years. However, in 1985 this statistic rose to $58 \%$ and in 1986 to $64 \%$ reflecting a substantial shortening in the average duration of highs. There was also some modest decline in the average degree of high attained between 1977 and 1981 , but with little change since.

- For opiates other than heroin, there has been a fairly steady decline since 1975 in both the intensity of the highs usually experienced and in the duration of those highs. In 1975 , 39\% said they usually got "very high" vs. $19 \%$ in 1986. The proportion usually staying high for seven or more hours dropped from $28 \%$ in 1975 to $9 \%$ in 1986 . This substantial shift has occurred in part because an increasing proportion of the users say they do not take these drugs "to get high" ( $4 \%$ in 1975 vs. $20 \%$ in 1986).

- Stimulants showed a substantial decrease between 1975 and 1981 in the proportion of recent users usually getting very high or moderately high (down from $60 \%$ in 1975 to $37 \%$ in 1981). Consistent with this, the proportion of users saying they simply "don't take them to get high" increased from 9\% in 1975 to $20 \%$ by 1981 . In addition, the average reported duration of stimulant highs was declining; $41 \%$ of the 1975 users said they usually stayed high seven or more hours vs. only $17 \%$ of the 1981 users. ${ }^{16}$ In 1982 the revised version of the question about stimulant use was introduced into the form containing subsequent questions on the degree and duration of highs. Based on this revised form, there has been some continued drop in the duration of highs obtained, and (to a lesser extent) in the degree of highs obtained.

${ }^{16}$ The questionnaire form containing the questions on degree and duration of highs is one on which the amphetamine questions were clarified in 1982 , to eliminate the inappropriate inclusion of nonprescription stimulants. One might have expected this change to have increased the degree and duration of highs reported, given that real amphetamines would be expected to have greater psychological impact on the average; but the trends still continued downward that year. 
These substantial decreases in both the degree and duration of highs strongly suggest that there has been some shift in the purpose for which stimulants are being used. An examination of data on self-reported reasons for use tends to confirm this conclusion. In essence, between 1979 and 1984 there was a relative decline in the frequency with which recent users mention "social/recreational" reasons for use, and between 1976 and 1984 there was an increase in mentions of use for instrumental purposes. More recently, since 1984, the shifts have been slight, and tend not to be continuing the pre-1984 trends.

With respect to the social/recreational shifts from 1979 to 1984 , the percent of recent users citing "to feel good or get high" as a reason for stimulant use declined from 58\% to $45 \%$; in 1986 there was a slight reversal, back to $50 \%$. Similarly, "to have a good time with my friends" declined from $38 \%$ to $30 \%$ between 1979 and 1984 ; there was little further change, with the 1986 figure being $29 \%$. There were shifts toward more instrumental use between 1976 and 1984; to lose weight increased by $15 \%$ (to $41 \%$ ); to get more energy increased $13 \%$ (to $69 \%$ ); to stay awake increased by $10 \%$ (to $62 \%$ ) and to get through the day increased by $8 \%$ (to $30 \%$ ). All four measures declined between 1984 and 1986, by approximately $5 \%$ in each case.

In addition to the relative decline seen earlier in recreational reasons for use of stimulants, it also appears that there was at least some increase in the absolute level of recreational use, though clearly not as steep an increase as the trends through 1981 in overall use might have suggested. The data on exposure to people using amphetamines "to get high or for kicks," which will be discussed further in a section below, show a definite increase between 1976 and 1981 (there was a rise of $8 \%$ just between 1979 and 1981). There was no further increase in exposure to people using for those purposes in 1982, however, suggesting that recreational use, as well as overall use, had leveled off; since 1982 there has been a decrease in such exposure.

- In the last few years the degree and duration of highs usually achieved by the shrinking number of barbiturate users and methaqualone users also has been decreasing. The highs achieved by tranquilizer users also have been decreasing since about 1980 .

- For marijuana there had been some general downward trending since 1978 in the degree of the highs usually obtained. In 1978, $73 \%$ of users said they usually got 
"moderately high" or "very high"-a figure which dropped to $64 \%$ by 1983 , and stands at $67 \%$ in 1986 . Some interesting changes also took place in the duration figures between 1978 and 1983. Recall that most marijuana users say they usually stay high either one to two hours or three to six hours. Between 1975 and 1983 there was a steady decline in the proportions saying they stayed high three or more hours (from $52 \%$ in 1975 to $35 \%$ in 1983 ); the proportion stands at $37 \%$ in 1986 . Until 1979 this shift could have been due almost entirely to the fact that progressively more seniors were using marijuana; and the users in more recent classes, who would not have been users in earlier classes, probably tended to be relatively light users. (We deduce this from the fact that the percentage of all seniors reporting three to six hour highs remained relatively unchanged from 1975 to 1979 , while the percentage of all seniors reporting only one to two hour highs increased steadily (from $16 \%$ in 1975 to $25 \%$ in 1979).

However, the overall prevalence rate did not increase over the past seven years (annual prevalence actually dropped by $12 \%)$, but the shift toward shorter average highs continued. Thus we must attribute this shift to another factor, and the one which seems most likely is a general shift (even among the most marijuana-prone segment) toward a less frequent (or less intense) use of the drug. The drop in daily prevalence since 1979, which certainly is disproportionate to the drop in overall prevalence, is consistent with this interpretation. Also consistent is the fact that the average number of "joints" smoked per day (among those who reported any use in the prior month) has been dropping. In $1976,49 \%$ of the recent users of marijuana indicated that they averaged less than one "joint" per day in the prior 30 days, but by 1986 this proportion had risen to $66 \%$. In sum, not only are fewer high school students now using marijuana, but those who are using seem to be using less frequently and to be taking smaller amounts (and doses of the active ingredient) per occasion.

- There are no clearly discernible patterns in the intensity or duration of the highs being experienced with LSD or hallucinogens other than LSD. (Data have not been collected for highs experienced in the use of inhalants, the nitrites specifically, or PCP specifically; and the number of admitted heroin users on a single questionnaire form is inadequate to estimate trends reliably.)

- The intensity and duration of highs associated with alcohol use have been very stable throughout the study period. 


\section{ATTITUDES AND BELIEFS ABOUT DRUGS}

This section presents the cross-time results for three sets of attitude and belief questions. One set concerns seniors' views about how harmful various kinds of drug use would be for the user, the second asks how much seniors personally disapprove of various kinds of drug use, and the third deals with attitudes on the legality of using various drugs under different conditions. (The next section covers the closely related topics of parents' and friends' attitudes about drugs, as the seniors perceive them.)

As the data below show, overall percentages disapproving various drugs, and the percentages believing their use to involve serious risk, both tend to parallel the percentages of actual users. Thus, for example, of the illicit drugs marijuana is the most frequently used and the least likely to be seen as risky to use. This and many other such parallels suggest that the individuals who use a drug are less likely to disapprove use of it or to view its use as involving risk. A series of individual-level analyses of these data confirms this conclusion: strong correlations exist between individual use of drugs and the various attitudes and beliefs about those drugs. Those seniors who use a given drug also are more likely to approve its use, see it as less dangerous, and report their own parents and friends as being at least somewhat more accepting of its use.

The attitudes and beliefs about drug use reported below have been changing during recent years, along with actual behavior. In particular, views about marijuana use, and legal sanctions against use, have shown important trends.

Beginning in 1979, scientists, policy makers, and in particular the electronic and printed media, have given considerable attention to the increasing levels of regular marijuana use among young people, and to the potential hazards associated with such use. As will be seen below, attitudes and beliefs about regular use of marijuana have shifted dramatically since 1979 in a more conservative direction-a shift which coincides with a reversal in the previous rapid rise of daily use, and which very likely reflects the impact of this increased public attention. 


\section{PERCEIVED HARMFULNESS OF DRUGS}

\section{Beliefs in 1986 about Harmfulness}

- A substantial majority of high school seniors perceive regular use of any of the illicit drugs as entailing "great risk" of harm for the user (see Table 15). Some $87 \%$ of the sample feel this way about heroin-the highest proportion for any of these drugs-while $83 \%$ associate great risk with using $L S D$. The proportions attributing great risk to cocaine, barbiturates, and amphetamines are $82 \%, 67 \%$, and $67 \%$, respectively.

- Regular use of cigarettes (i.e., one or more packs a day) is judged by two-thirds of all seniors $(66 \%)$ as entailing a great risk of harm for the user.

- Regular use of marijuana is judged to involve great risk by $71 \%$ of the sample, slightly more than judge cigarette smoking to involve great risk, perhaps in part because marijuana can have dramatic short-term impacts on mood, behavior, self-control, etc., in addition to any long-term physiological impacts.

- Regular use of alcohol was more explicitly defined in several questions. Relatively few $(25 \%)$ associate much risk of harm with having one or two drinks almost daily. Only four in every ten $(39 \%)$ think there is great risk involved in having five or more drinks once or twice each weekend. Fully twothirds $(67 \%)$ think the user takes a great risk in consuming four or five drinks nearly every day, but this means that about a third of the students do not view this pattern of regular heavy drinking as entailing great risk.

- Compared with the above perceptions about the risks of regular use of each drug, many fewer respondents feel that a person runs a "great risk" of harm by simply trying the drug once or twice.

- Very few think there is much risk in using marijuana experimentally $(15 \%)$ or even occasionally $(25 \%)$.

- Experimental use of the other illicit drugs, however, is still viewed as risky by substantial proportions. The percentages associating great risk with experimental use range from about $25 \%$ for amphetamines and barbiturates to $46 \%$ for heroin. Despite the amount of negative publicity cocaine use has received recently, only about a third (34\%) see great risk involved in experimenting with it, and only a little more 
TABLE 15

Trends in Perceived Harmfulness of Drugs

Percentage saying "great risk" ${ }^{a}$

Q. How much do you think people risk harming themselves

(physically or in other

(ways), if they...

Try marijuana once or twice

Smoke marijuana occasionally

Smoke marijuana regularly

Try LSD once or twice

Take LSD regularly

Try cocaine once or twice

Take cocaine occasionally

Take cocaine regularly

Try heroin once or twice

Take heroin occasionally

Take heroin regularly

Try amphetamines once or twice

Take amphetamines regularly

Try barbiturates once or twice

Take barbiturates regularly

Try one or two drinks of an alcoholic beverage (beer, wine, liquor)

Take one or two drinks nearly every day

Take four or five drinks nearly every day

Have five or more drinks once or twice each weekend

Smoke one or more packs of cigarettes per day

\footnotetext{
Approx. $\mathrm{N}=$
}

\begin{tabular}{|c|c|c|c|c|c|c|c|c|c|c|c|c|}
\hline $\begin{array}{c}\text { Class } \\
\text { of } \\
1975 \\
\end{array}$ & $\begin{array}{c}\text { Class } \\
\text { of } \\
1976 \\
\end{array}$ & $\begin{array}{c}\text { Class } \\
\text { of } \\
1977 \\
\end{array}$ & $\begin{array}{c}\text { Class } \\
\text { of } \\
1978 \\
\end{array}$ & $\begin{array}{c}\text { Class } \\
\text { of } \\
1979 \\
\end{array}$ & $\begin{array}{c}\text { Class } \\
\text { of } \\
1980 \\
\end{array}$ & $\begin{array}{c}\text { Class } \\
\text { of } \\
1981 \\
\end{array}$ & $\begin{array}{c}\text { Class } \\
\text { of } \\
1982 \\
\end{array}$ & $\begin{array}{c}\text { Class } \\
\text { of } \\
1983 \\
\end{array}$ & $\begin{array}{c}\text { Class } \\
\text { of } \\
1984 \\
\end{array}$ & $\begin{array}{c}\text { Class } \\
\text { of } \\
1985 \\
\end{array}$ & $\begin{array}{c}\text { Class } \\
\text { of } \\
1986 \\
\end{array}$ & $\begin{array}{l}\text { '85-'86 } \\
\text { change }\end{array}$ \\
\hline $\begin{array}{l}15.1 \\
18.1 \\
43.3\end{array}$ & $\begin{array}{l}11.4 \\
15.0 \\
38.6\end{array}$ & $\begin{array}{r}9.5 \\
13.4 \\
36.4\end{array}$ & $\begin{array}{r}8.1 \\
12.4 \\
34.9\end{array}$ & $\begin{array}{r}9.4 \\
13.5 \\
42.0\end{array}$ & $\begin{array}{l}10.0 \\
14.7 \\
50.4\end{array}$ & $\begin{array}{l}13.0 \\
19.1 \\
57.6\end{array}$ & $\begin{array}{l}11.5 \\
18.3 \\
60.4\end{array}$ & $\begin{array}{l}12.7 \\
20.6 \\
62.8\end{array}$ & $\begin{array}{l}14.7 \\
22.6 \\
66.9\end{array}$ & $\begin{array}{l}14.8 \\
24.5 \\
70.4\end{array}$ & $\begin{array}{l}15.1 \\
25.0 \\
71.3\end{array}$ & $\begin{array}{l}+0.3 \\
+0.5 \\
+0.9\end{array}$ \\
\hline $\begin{array}{l}49.4 \\
81.4\end{array}$ & $\begin{array}{l}45.7 \\
80.8\end{array}$ & $\begin{array}{l}43.2 \\
79.1\end{array}$ & $\begin{array}{l}42.7 \\
81.1\end{array}$ & $\begin{array}{l}41.6 \\
82.4\end{array}$ & $\begin{array}{l}43.9 \\
83.0\end{array}$ & $\begin{array}{l}45.5 \\
83.5\end{array}$ & $\begin{array}{l}44.9 \\
83.5\end{array}$ & $\begin{array}{l}44.7 \\
83.2\end{array}$ & $\begin{array}{l}45.4 \\
83.8\end{array}$ & $\begin{array}{l}43.5 \\
82.9\end{array}$ & $\begin{array}{l}42.0 \\
82.6\end{array}$ & $\begin{array}{l}-1.5 \\
-0.3\end{array}$ \\
\hline $\begin{array}{r}42.6 \\
\text { NA } \\
73.1\end{array}$ & $\begin{array}{r}39.1 \\
\text { NA } \\
72.3\end{array}$ & $\begin{array}{r}35.6 \\
\mathrm{NA} \\
68.2\end{array}$ & $\begin{array}{r}33.2 \\
\mathrm{NA} \\
68.2\end{array}$ & $\begin{array}{r}31.5 \\
\mathrm{NA} \\
69.5\end{array}$ & $\begin{array}{r}31.3 \\
\text { NA } \\
69.2\end{array}$ & $\begin{array}{r}32.1 \\
\text { NA } \\
71.2\end{array}$ & $\begin{array}{r}32.8 \\
\text { NAA } \\
73.0\end{array}$ & $\begin{array}{r}33.0 \\
\text { NA } \\
74.3\end{array}$ & $\begin{array}{r}35.7 \\
\text { NA } \\
78.8\end{array}$ & $\begin{array}{r}34.0 \\
\text { NA } \\
79.0\end{array}$ & $\begin{array}{l}33.5 \\
54.2 \\
82.2\end{array}$ & $\begin{array}{c}-0.5 \\
\text { NA } \\
+3.2 \mathrm{~s}\end{array}$ \\
\hline $\begin{array}{l}60.1 \\
75.6 \\
87.2\end{array}$ & $\begin{array}{l}58.9 \\
75.6 \\
88.6\end{array}$ & $\begin{array}{l}55.8 \\
71.9 \\
86.1\end{array}$ & $\begin{array}{l}52.9 \\
71.4 \\
86.6\end{array}$ & $\begin{array}{l}50.4 \\
70.9 \\
87.5\end{array}$ & $\begin{array}{l}52.1 \\
70.9 \\
86.2\end{array}$ & $\begin{array}{l}52.9 \\
72.2 \\
87.5\end{array}$ & $\begin{array}{l}51.1 \\
69.8 \\
86.0\end{array}$ & $\begin{array}{l}50.8 \\
71.8 \\
86.1\end{array}$ & $\begin{array}{l}49.8 \\
70.7 \\
87.2\end{array}$ & $\begin{array}{l}47.3 \\
69.8 \\
86.0\end{array}$ & $\begin{array}{l}45.8 \\
68.2 \\
87.1\end{array}$ & $\begin{array}{l}-1.5 \\
-1.6 \\
+1.1\end{array}$ \\
\hline $\begin{array}{l}35.4 \\
69.0\end{array}$ & $\begin{array}{l}33.4 \\
67.3\end{array}$ & $\begin{array}{l}30.8 \\
66.6\end{array}$ & $\begin{array}{l}29.9 \\
67.1\end{array}$ & $\begin{array}{l}29.7 \\
69.9\end{array}$ & $\begin{array}{l}29.7 \\
69.1\end{array}$ & $\begin{array}{l}26.4 \\
66.1\end{array}$ & $\begin{array}{l}25.3 \\
64.7\end{array}$ & $\begin{array}{l}24.7 \\
64.8\end{array}$ & $\begin{array}{l}25.4 \\
67.1\end{array}$ & $\begin{array}{l}25.2 \\
67.2\end{array}$ & $\begin{array}{l}25.1 \\
67.3\end{array}$ & $\begin{array}{l}-0.1 \\
+0.1\end{array}$ \\
\hline $\begin{array}{l}34.8 \\
69.1\end{array}$ & $\begin{array}{l}32.5 \\
67.7\end{array}$ & $\begin{array}{l}31.2 \\
68.6\end{array}$ & $\begin{array}{l}31.3 \\
68.4\end{array}$ & $\begin{array}{l}30.7 \\
71.6\end{array}$ & $\begin{array}{l}30.9 \\
72.2\end{array}$ & $\begin{array}{l}28.4 \\
69.9\end{array}$ & $\begin{array}{l}27.5 \\
67.6\end{array}$ & $\begin{array}{l}27.0 \\
67.7\end{array}$ & $\begin{array}{l}27.4 \\
68.5\end{array}$ & $\begin{array}{l}26.1 \\
68.3\end{array}$ & $\begin{array}{l}25.4 \\
67.2\end{array}$ & $\begin{array}{l}-0.7 \\
-1.1\end{array}$ \\
\hline 5.3 & 4.8 & 4.1 & 3.4 & 4.1 & 3.8 & 4.6 & 3.5 & 4.2 & 4.6 & 5.0 & 4.6 & -0.4 \\
\hline 21.5 & 21.2 & 18.5 & 19.6 & 22.6 & 20.3 & 21.6 & 21.6 & 21.6 & 23.0 & 24.4 & 25.1 & +0.7 \\
\hline 63.5 & 61.0 & 62.9 & 63.1 & 66.2 & 65.7 & 64.5 & 65.5 & 66.8 & 68.4 & 69.8 & 66.5 & $-3.3 \mathrm{~s}$ \\
\hline 37.8 & 37.0 & 34.7 & 34.5 & 34.9 & 35.9 & 36.3 & 36.0 & 38.6 & 41.7 & 43.0 & 39.1 & $-3.9 \mathrm{~s}$ \\
\hline 51.3 & 56.4 & 58.4 & 59.0 & 63.0 & 63.7 & 63.3 & 60.5 & 61.2 & 63.8 & 66.5 & 66.0 & -0.5 \\
\hline (2804) & (2918) & (3052) & $(3770)$ & (3250) & (3234) & $(3604)$ & (3557) & (3305) & (3262) & $(3250)$ & (3020) & \\
\hline
\end{tabular}

NOTE: Level of significance of difference between the two most recent classes: $\mathrm{s}=.05, \mathrm{ss}=.01, \mathrm{sss}=.001$.

Answer alternatives were: (1) No risk, (2) Slight risk, (3) Moderate risk, (4) Great risk, and (5) Can'l say, drug unfamiliar. 
than half $(54 \%)$ see great risk in occasional use. These figures suggest one reason why so many young people have eventually gotten into trouble with this extremely dependence-producing drug.

- Practically no one $(5 \%)$ believes there is much risk involved in trying an alcoholic beverage once or twice.

\section{Trends in Perceived Harmfulness}

- Several very important trends have been taking place in recent years in these beliefs about the dangers associated with using various drugs (see Table 15 and Figures 20 and 21).

- One of the most important trends involves marijuana (Figure 20). From 1975 through 1978 there had been a decline in the harmfulness perceived to be associated with all levels of marijuana use; but in 1979, for the first time, there was an increase in these proportions-an increase which preceded any appreciable downturn in use and which has continued fairly steadily since then. By far the most impressive increase has occurred for regular marijuana use, where the proportion perceiving it as involving a great risk has doubled in eight years-from $35 \%$ in 1978 to $71 \%$ in 1986 . This dramatic change occurred during a period in which a substantial amount of scientific and media attention was being devoted to the potential dangers of heavy marijuana use. While there have been some upward shifts in concerns about the harmfulness of occasional, and even experimental, use, they have been nowhere nearly as large. While all of these shifts continued in 1986, they have decelerated considerably.

- There also had been an important increase over a longer period in the number who think pack-a-day cigarette smoking involves great risk to the user (from $51 \%$ in 1975 to $64 \%$ in 1980). This shift corresponded with, and to some degree preceded, the downturn in regular smoking found in this age group (compare Figures $9 f$ and 20). But between 1980 and 1984 this statistic showed no further increase (presaging the end of the decline in use). Since 1984, the percent perceiving great risk in regular smoking has risen a couple of percent. What may be most important is that about a third $(34 \%)$ of these young people do not believe there is a great risk in smoking a pack or more of cigarettes per day, despite all that is known today about the health consequences of cigarette smoking. 


\section{FIGURE 20}

\section{Trends in Perceived Harmfulness: Marijuana and Cigarettes}

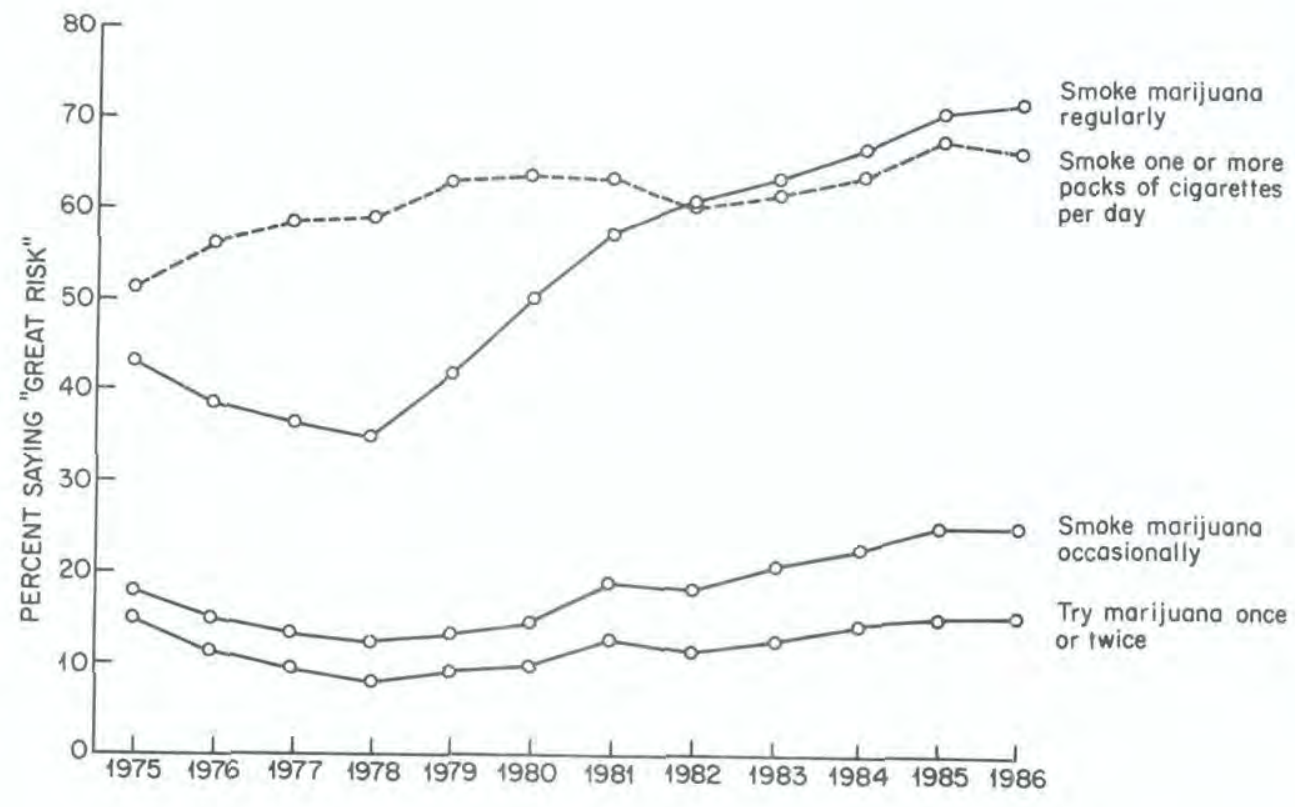


- For most of the other illicit drugs, the period from 1975 to 1979 marked a modest but consistent trend in the direction of fewer students associating much risk with experimental or occasional use of them (Table 15 and Figure 21). Only for amphetamines and barbiturates has this trend continued beyond 1979, until about 1982 in both cases. Over the last several years there has been little change, although perceived risk of harm in experimental or occasional use of the illicit drugs other than marijuana all dropped slightly in 1985 and 1986.

- The percentage who perceived great risk in trying cocaine once or twice dropped from $43 \%$ in 1975 to $31 \%$ in 1980 , which generally corresponds to a period of rapidly increasing use. But perceived risk then began to inch upward over the next four years, to $36 \%$ in 1984 ; a slight decrease since 1984 leaves the current figure at $34 \%$. The proportion seeing great risk in regular cocaine use also dropped somewhat from 1975 to 1977 from $(73 \%$ to $68 \%$ ), and remained fairly level until 1980. Since 1980 there has been an appreciable increase in the risk perceived to be associated with regular use, with the proportion seeing "great risk" rising steadily from $68 \%$ to $82 \%$ in 1986 .

- In sum, between 1975 and 1979 there was a distinct decline in perceived harmfulness associated with use of all the illicit drugs. Since 1979, there has been a dramatic increase in concerns about regular marijuana use, and a more modest increase in concerns about use of that drug at less frequent levels. In general, concerns about use of other illicit drugs have changed rather little over the last several years, although perceived risk in regular use of cocaine has increased appreciably, and risks associated with amphetamine and barbiturate use have dropped slightly.

- Beliefs concerning the risk associated with alcohol use at various levels have remained largely unchanged over the past eight years. The one exception occurred with occasional heavy drinking, where the proportion perceiving great risk rose from a low of $35 \%$ in 1979 to $43 \%$ in 1985 . Almost half (3\%) of this $8 \%$ change occurred in 1984 alone, the first year in which the reported prevalence of this type of drinking actually declined. Thus the gradual change in beliefs about the riskiness of this behavior preceded a change in use by several years-once again suggesting the importance of these beliefs in determining behavior. Unfortunately, there was a significant $4 \%$ drop in this statistic in 1986, coincident with an end to the decline in occasional heavy use. 


\section{FIGURE 21}

\section{Trends in Perceived Harmfulness: Other Drugs}

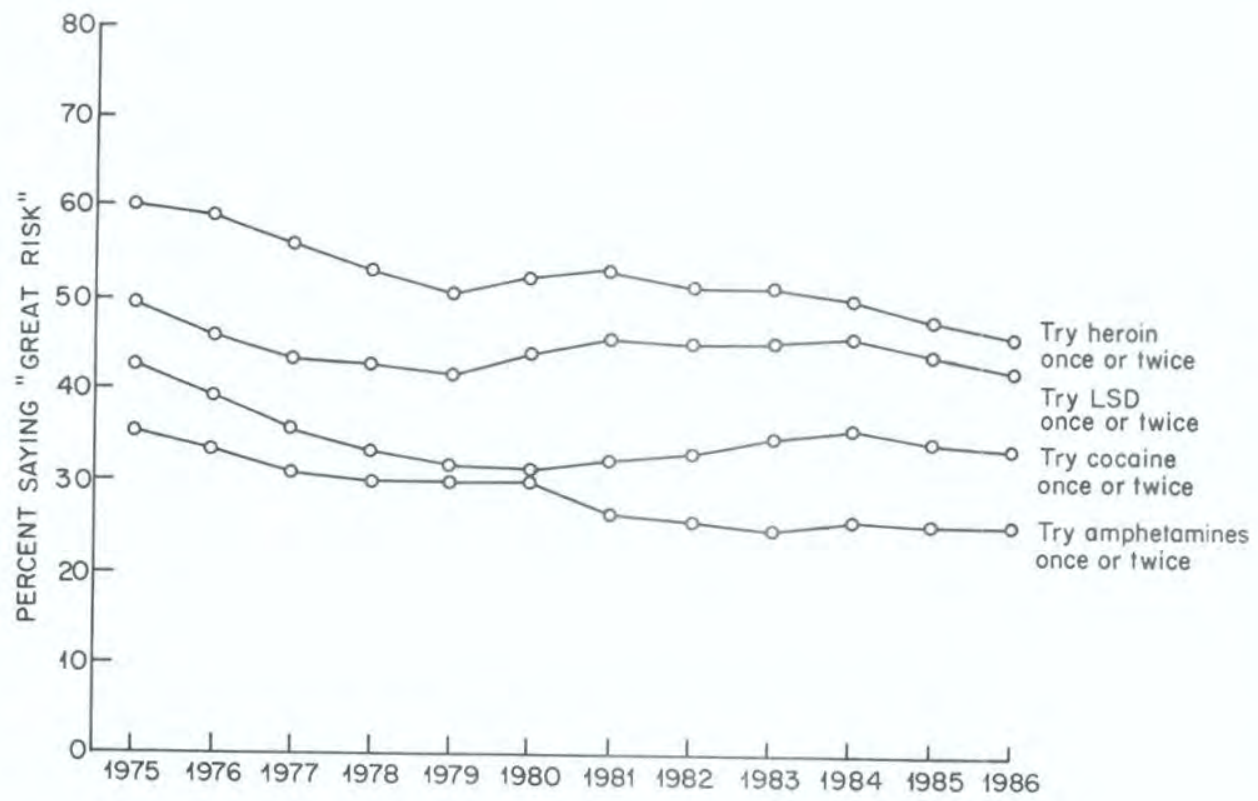




\section{PERSONAL DISAPPROVAL OF DRUG USE}

A different set of questions was developed to try to measure the moral sentiment respondents attach to various types of drug use. The phrasing, "Do you disapprove of people (who are 18 or older) doing each of the following" was adopted.

\section{Extent of Disapproval in 1986}

- The vast majority of these students do not condone regular use of any of the illicit drugs (see Table 16). Even regular marijuana use is disapproved by $87 \%$, and regular use of each of the other illicits receives disapproval from between $94 \%$ and $98 \%$ of today's high school seniors.

- Smoking a pack (or more) of cigarettes per day receives the disapproval of $75 \%$ of the age group.

- Drinking at the rate of one or two drinks daily is disapproved by $73 \%$ of the seniors. A curious finding is that weekend binge drinking (five or more drinks once or twice each weekend) is acceptable to more seniors than is moderate daily drinking; only $62 \%$ disapprove of having five or more drinks once or twice a weekend. This is in spite of the fact that more seniors associate great risk with weekend binge drinking $(39 \%)$ than with the daily drinking (25\%). One likely explanation for these seemingly inconsistent findings may be the fact that a greater proportion of this age group are themselves weekend binge drinkers rather than moderate daily drinkers. They thus express attitudes accepting of their own behavior, even though such attitudes may be somewhat inconsistent with their beliefs about possible consequences.

- For each of the drugs included in the question, fewer people indicate disapproval of experimental or occasional use than of regular use, as would be expected. The differences are not great, however, for the illicit drugs other than marijuana. For example, $80 \%$ disapprove experimenting with cocaire vs. $94 \%$ who disapprove its regular use.

- For marijuana, however, the rate of disapproval varies substantially for different usage habits although not as much as it did in the past. The great majority $(87 \%)$ now disapprove regular use, and only a little more than half (55\%) disapprove trying it. 
TABLE 16

Trends in Proportions Disapproving of Drug Use

Percentage "disapproving"a

Q. Do you disapprove of people (who are 18 or older) doing each of the following? $b$

Try marijuana once or twice

Smoke marijuana occasionally

Smoke marijuana regularly

Try LSD once or twice

Take LSD regularly

Try cocaine once or twice

Take cocaine regularly

Try heroin once or twice

Take heroin occasionally

Take heroin regularly

$\vdash$ Try amphetamines once or twice

S Take amphetamines regularly

Try barbiturates once or twice

Take barbiturates regularly

Tty one or two drinks of an alcoholic beverage (beer, wine, liquor)

Take one or two

every day

Take four or five drinks nearly every day

Have five or more drinks once

or twice each weekend

\begin{tabular}{|c|c|c|c|c|c|c|c|c|c|c|c|c|}
\hline $\begin{array}{c}\text { Class } \\
\text { of } \\
1975 \\
\end{array}$ & $\begin{array}{c}\text { Class } \\
\text { of } \\
1976 \\
\end{array}$ & $\begin{array}{c}\text { Class } \\
\text { of } \\
1977 \\
\end{array}$ & $\begin{array}{c}\text { Class } \\
\text { of } \\
1978 \\
\end{array}$ & $\begin{array}{c}\text { Class } \\
\text { of } \\
1979 \\
\end{array}$ & $\begin{array}{c}\text { Class } \\
\text { of } \\
1980 \\
\end{array}$ & $\begin{array}{c}\text { Class } \\
\text { of } \\
1981 \\
\end{array}$ & $\begin{array}{c}\text { Class } \\
\text { of } \\
1982 \\
\end{array}$ & $\begin{array}{c}\text { Class } \\
\text { of } \\
1983 \\
\end{array}$ & $\begin{array}{c}\text { Class } \\
\text { of } \\
1984 \\
\end{array}$ & $\begin{array}{c}\text { Class } \\
\text { of } \\
1985 \\
\end{array}$ & $\begin{array}{c}\text { Class } \\
\text { of } \\
1986 \\
\end{array}$ & $\begin{array}{l}\text { '85-'86 } \\
\text { change }\end{array}$ \\
\hline $\begin{array}{l}47.0 \\
54.8 \\
71.9\end{array}$ & $\begin{array}{l}38.4 \\
47.8 \\
69.5\end{array}$ & $\begin{array}{l}33.4 \\
44.3 \\
65.5\end{array}$ & $\begin{array}{l}33.4 \\
43.5 \\
67.5\end{array}$ & $\begin{array}{l}34.2 \\
45.3 \\
69.2\end{array}$ & $\begin{array}{l}39.0 \\
49.7 \\
74.6\end{array}$ & $\begin{array}{l}40.0 \\
52.6 \\
77.4\end{array}$ & $\begin{array}{l}45.5 \\
59.1 \\
80.6\end{array}$ & $\begin{array}{l}46.3 \\
60.7 \\
82.5\end{array}$ & $\begin{array}{l}49.3 \\
63.5 \\
84.7\end{array}$ & $\begin{array}{l}51.4 \\
65.8 \\
85.5\end{array}$ & $\begin{array}{l}54.6 \\
69.0 \\
86.6\end{array}$ & $\begin{array}{l}+3.2 \mathrm{~s} \\
+3.2 \mathrm{~s} \\
+1.1\end{array}$ \\
\hline $\begin{array}{l}82.8 \\
94.1\end{array}$ & $\begin{array}{l}84.6 \\
95.3\end{array}$ & $\begin{array}{l}83.9 \\
95.8\end{array}$ & $\begin{array}{l}85.4 \\
96.4\end{array}$ & $\begin{array}{l}86.6 \\
96.9\end{array}$ & $\begin{array}{l}87.3 \\
96.7\end{array}$ & $\begin{array}{l}86.4 \\
96.8\end{array}$ & $\begin{array}{l}88.8 \\
96.7\end{array}$ & $\begin{array}{l}89.1 \\
97.0\end{array}$ & $\begin{array}{l}88.9 \\
96.8\end{array}$ & $\begin{array}{l}89.5 \\
97.0\end{array}$ & $\begin{array}{l}89.2 \\
96.6\end{array}$ & $\begin{array}{l}-0.3 \\
-0.4\end{array}$ \\
\hline $\begin{array}{l}81.3 \\
93.3\end{array}$ & $\begin{array}{l}82.4 \\
93.9\end{array}$ & $\begin{array}{l}79.1 \\
92.1\end{array}$ & $\begin{array}{l}77.0 \\
91.9\end{array}$ & $\begin{array}{l}74.7 \\
90.8\end{array}$ & $\begin{array}{l}76.3 \\
91.1\end{array}$ & $\begin{array}{l}74.6 \\
90.7\end{array}$ & $\begin{array}{r}76.6 \\
91.5\end{array}$ & $\begin{array}{l}77.0 \\
93.2\end{array}$ & $\begin{array}{l}79.7 \\
94.5\end{array}$ & $\begin{array}{l}79.3 \\
93.8\end{array}$ & $\begin{array}{l}80.2 \\
94.3\end{array}$ & $\begin{array}{l}+0.9 \\
+0.5\end{array}$ \\
\hline $\begin{array}{l}91.5 \\
94.8 \\
96.7\end{array}$ & $\begin{array}{l}92.6 \\
96.0 \\
97.5\end{array}$ & $\begin{array}{l}92.5 \\
96.0 \\
97.2\end{array}$ & $\begin{array}{l}92.0 \\
96.4 \\
97.8\end{array}$ & $\begin{array}{l}93.4 \\
96.8 \\
97.9\end{array}$ & $\begin{array}{l}93.5 \\
96.7 \\
97.6\end{array}$ & $\begin{array}{l}93.5 \\
97.2 \\
97.8\end{array}$ & $\begin{array}{l}94.6 \\
96.9 \\
97.5\end{array}$ & $\begin{array}{l}94.3 \\
96.9 \\
97.7\end{array}$ & $\begin{array}{l}94.0 \\
97.1 \\
98.0\end{array}$ & $\begin{array}{l}94.0 \\
96.8 \\
97.6\end{array}$ & $\begin{array}{l}93.3 \\
96.6 \\
97.6\end{array}$ & $\begin{array}{r}-0.7 \\
-0.2 \\
0.0\end{array}$ \\
\hline $\begin{array}{l}74.8 \\
92.1\end{array}$ & $\begin{array}{l}75.1 \\
92.8\end{array}$ & $\begin{array}{l}74.2 \\
92.5\end{array}$ & $\begin{array}{l}74.8 \\
93.5\end{array}$ & $\begin{array}{l}75.1 \\
94.4\end{array}$ & $\begin{array}{l}75.4 \\
93.0\end{array}$ & $\begin{array}{l}71.1 \\
91.7\end{array}$ & $\begin{array}{l}72.6 \\
92.0\end{array}$ & $\begin{array}{l}72.3 \\
92.6\end{array}$ & $\begin{array}{l}72.8 \\
93.6\end{array}$ & $\begin{array}{l}74.9 \\
93.3\end{array}$ & $\begin{array}{l}76.5 \\
93.5\end{array}$ & $\begin{array}{l}+1.6 \\
+0.2\end{array}$ \\
\hline $\begin{array}{l}77.7 \\
93.3\end{array}$ & $\begin{array}{l}81.3 \\
93.6\end{array}$ & $\begin{array}{l}81.1 \\
93.0\end{array}$ & $\begin{array}{l}82.4 \\
94.3\end{array}$ & $\begin{array}{l}84.0 \\
95.2\end{array}$ & $\begin{array}{l}83.9 \\
95.4\end{array}$ & $\begin{array}{l}82.4 \\
94.2\end{array}$ & $\begin{array}{l}84.4 \\
94.4\end{array}$ & $\begin{array}{l}83.1 \\
95.1\end{array}$ & $\begin{array}{l}84.1 \\
95.1\end{array}$ & $\begin{array}{l}84.9 \\
95.5\end{array}$ & $\begin{array}{l}86.8 \\
94.9\end{array}$ & $\begin{array}{l}+1.9 \\
-0.6\end{array}$ \\
\hline 21.6 & 18.2 & 15.6 & 15.6 & 15.8 & 16.0 & 17.2 & 18.2 & 18.4 & 17.4 & 20.3 & 20.9 & +0.6 \\
\hline 67.6 & 68.9 & 66.8 & 67.7 & 68.3 & 69.0 & 69.1 & 69.9 & 68.9 & 72.9 & 70.9 & 72.8 & +1.9 \\
\hline 88.7 & 90.7 & 88.4 & 90.2 & 91.7 & 90.8 & 91.8 & 90.9 & 90.0 & 91.0 & 92.0 & 91.4 & -0.6 \\
\hline 60.3 & 58.6 & 57.4 & 56.2 & 56.7 & 55.6 & 55.5 & 58.8 & 56.6 & 59.6 & 60.4 & 62.4 & +2.0 \\
\hline 67.5 & 65.9 & 66.4 & 67.0 & 70.3 & 70.8 & 69.9 & 69.4 & 70.8 & 73.0 & 72.3 & 75.4 & $+3.1 \mathrm{~s}$ \\
\hline$(2677)$ & (2957) & $(3085)$ & $(3686)$ & (3221) & (3261) & $(3610)$ & $(3651)$ & (3341) & (3254) & (3265) & (3113) & \\
\hline
\end{tabular}

Smoke one or more packs of

cigarettes per day

Approx. $\mathrm{N}=$

NOTE: Level of significance of difference between the two most recent classes: $\mathrm{s}=.05, \mathrm{ss}=.01, \mathrm{sss}=.001$.

a Answer alternatives were: (1) Don't disapprove, (2) Disapprove, and (3) Strongly disapprove. Percentages are shown for

categories (2) and (3) combined.

bThe 1975 question asked about people who are "20 or older." 


\section{Trends in Disapproval}

- Between 1975 and 1977 there occurred a substantial decrease in disapproval of marijuana use at any level of frequency (see Table 16 and Figure 22). About 14\% fewer seniors in the class of 1977 (compared with the class of 1975) disapproved of experimenting, $11 \%$ fewer disapproved of occasional use, and $6 \%$ fewer disapproved of regular use. Since 1977, however, there has been a substantial reversal of that trend, with disapproval of experimental use having risen by $21 \%$, disapproval of occasional use by $25 \%$, and disapproval of regular use by $21 \%$. (These trends continued in 1986.)

- Until 1980 the proportion of seniors who disapproved trying amphetamines had remained extremely stable (at $75 \%$ ). This proportion dropped slightly in 1981 (to $71 \%$ ), but increased thereafter and reached $77 \%$ in 1986.

- During the late 1970 's personal disapproval of experimenting with barbiturates had been increasing (from $78 \%$ in 1975 to $84 \%$ in 1979). Since then it has remained relatively stable.

- Concurrent with the years of increase in actual cocaine use, disapproval of experimental use of cocaine had declined somewhat, from a high of $82 \%$ in 1976 down to $75 \%$ in 1979 . It then leveled for four years, and has since edged up about $3 \%$, so that $80 \%$ of seniors now disapprove of trying cocaine.

- In earlier years disapproval of regular cigarette smoking had been increasing modestly (from $66 \%$ in 1976 to $71 \%$ in 1980 ). It then remained fairly stable through 1983. There has been a modest increase since 1983 , with $75 \%$ of seniors now saying they disapprove of regular cigarette smoking.

- There has been relatively little change in attitudes regarding alcohol use, with one exception. There was a slight softening of attitudes regarding weekend binge drinking, with disapproval dropping from $60 \%$ in 1975 to $56 \%$ in 1978 ; recently, disapproval has been increasing, to a high of $62 \%$ in 1986 .

\section{ATTITUDES REGARDING THE LEGALITY OF DRUG USE}

Since the legal restraints on drug use appeared likely to be in a state of flux for some time, we decided at the beginning of the study to measure attitudes about legal sanctions. Table 17 presents a statement of one set of general questions on this subject along with the answers provided by each senior class. The set lists a sampling of illicit and licit drugs 
TABLE 17

Trends in Attitudes Regarding Legality of Drug Use

Percentage saying "yes"a
Q. Do you think that people (woho are 18 or older) should be prohibiled by law from doing cach of the following? $b$

Smoke marijuana in private

Smoke marijuana in public places

Take LSD in private

Take LSD in public places

N Take heroin in private

Take heroin in public places

Take amphetamines or

$$
\text { barbiturates in private }
$$

Take amphetamines or

$$
\text { barbiturates in public places }
$$

Get drunk in private

Get drunk in public places

Smoke cigarettes in certain specified public places

\begin{tabular}{|c|c|c|c|c|c|c|c|c|c|c|c|c|}
\hline \multicolumn{12}{|c|}{ Percentage saying "yes"a } & \multirow[b]{2}{*}{$\begin{array}{l}\text { '85-'86 } \\
\text { change } \\
\end{array}$} \\
\hline $\begin{array}{c}\text { Class } \\
\text { of } \\
1975 \\
\end{array}$ & $\begin{array}{c}\text { Class } \\
\text { of } \\
1976 \\
\end{array}$ & $\begin{array}{c}\text { Class } \\
\text { of } \\
1977 \\
\end{array}$ & $\begin{array}{c}\text { Class } \\
\text { of } \\
1978 \\
\end{array}$ & $\begin{array}{c}\text { Class } \\
\text { of } \\
1979 \\
\end{array}$ & $\begin{array}{c}\text { Clnss } \\
\text { of } \\
1980 \\
\end{array}$ & $\begin{array}{c}\text { Class } \\
\text { of } \\
1981 \\
\end{array}$ & $\begin{array}{c}\text { Class } \\
\text { of } \\
1982 \\
\end{array}$ & $\begin{array}{c}\text { Class } \\
\text { of } \\
1983 \\
\end{array}$ & $\begin{array}{c}\text { Class } \\
\text { of } \\
1984 \\
\end{array}$ & $\begin{array}{c}\text { Class } \\
\text { of } \\
1985 \\
\end{array}$ & $\begin{array}{c}\text { Class } \\
\text { of } \\
1986 \\
\end{array}$ & \\
\hline 32.8 & 27.5 & 26.8 & 25.4 & 28.0 & 28.9 & 35.4 & 36.6 & 37.8 & 41.6 & 44.7 & 43.8 & -0.9 \\
\hline 63.1 & 59.1 & 58.7 & 59.5 & 61.8 & 66.1 & 67.4 & 72.8 & 73.6 & 75.2 & 78.2 & 78.9 & +0.7 \\
\hline 67.2 & 65.1 & 63.3 & 62.7 & 62.4 & 65.8 & 62.6 & 67.1 & 66.7 & 67.9 & 70.6 & 69.0 & -1.6 \\
\hline 85.8 & 81.9 & 79.3 & 80.7 & 81.5 & 82.8 & 80.7 & 82.1 & 82,8 & 82.4 & 84.8 & 84.9 & +0.1 \\
\hline 76.3 & 72.4 & 69.2 & 68.8 & 68.5 & 70.3 & 68.8 & 69.3 & 69.7 & 69.8 & 73.3 & 71.7 & -1.6 \\
\hline 90.1 & 84.8 & 81.0 & 82.5 & 84.0 & 83.8 & 82.4 & 82.5 & 83.7 & 83.4 & 85.8 & 85.0 & -0.8 \\
\hline 57.2 & 53.5 & 52.8 & 52.2 & 53.4 & 54.1 & 52.0 & 53.5 & 52.8 & 54,4 & 56.3 & 56.8 & +0.5 \\
\hline 79.6 & 76.1 & 73.7 & 75.8 & 77.3 & 76.1 & 74.2 & 75.5 & 76.7 & 76.8 & 78.3 & 79.1 & +0.8 \\
\hline 14.1 & 15.6 & 18.6 & 17.4 & 16.8 & 16.7 & 19.6 & 19.4 & 19.9 & 19.7 & 19.8 & 18.5 & -1.3 \\
\hline 55.7 & 50.7 & 49.0 & 50.3 & 50.4 & 48.3 & 49.1 & 50.7 & 52.2 & 51.1 & 53.1 & 52.2 & -0.9 \\
\hline $\mathrm{NA}$ & NA & 42.0 & 42.2 & 43,1 & 42.8 & 43.0 & 42.0 & 40.5 & 39.2 & 42.8 & 45.1 & +2.3 \\
\hline (2620) & (2959) & $(3113)$ & (3783) & (3288) & (3224) & (36II) & (3627) & (3315) & $(3236)$ & (3254) & $(3074)$ & \\
\hline
\end{tabular}

Approx. $\mathrm{N}=$
NOTE: Level of significance of difference between the two most recent classes: $\mathrm{s}=.05, \mathrm{ss}=.01, \mathrm{sss}=.001$. NA indicates data not available. a Answer alternatives were: (1) No, (2) Not sure, and (3) Yes.

b'The 1975 question asked about people who are " 20 or older." 
and asks whether their use should be prohibited by law. A distinction is consistently made between use in public and use in private-a distinction which proved quite important in the results.

\section{Attitudes in 1986}

- Most seniors (79\%) favor legally prohibiting marijuana use in public places, despite the fact that the majority have used marijuana themselves; but considerably fewer (44\%) feel that way about marijuana use in private.

- In addition, the great majority believe that the use in public of other illicit drugs than marijuana should be prohibited by law (e.g., $79 \%$ in the case of amphetamines and barbiturates, $85 \%$ for heroin).

- Fully $45 \%$ believe that cigarette smoking in public places should be prohibited by law. More think getting drunk in such places should be prohibited (52\%).

- For all drugs, fewer students believe that use in private settings should be illegal, though in the cases of LSD and heroin, the differences are not very substantial.

\section{Trends in These Attitudes}

- From 1975 through 1977 there was a modest decline (from $4 \%$ to $9 \%$, depending on the substance) in the proportion of seniors who favored legal prohibition of private use of any of the illicit drugs. By 1986, however, these proportions have all increased.

- Over the past seven years (from 1979 to 1986) there has been a sharp jump in the proportion favoring legal prohibition of marijuana use, either in private (up from $28 \%$ to $44 \%$ ) or in public (up from $62 \%$ to $79 \%$ ).

- For other illicit drugs, the changes are more modest, but between 1981 and 1986 all showed increased proportions favoring prohibition.

- Smoking cigarettes in public showed the largest increase in the proportion favoring prohibition, while getting drunk met with slightly less disapproval this year than last. 


\section{THE LEGAL STATUS OF MARIJUANA}

Another set of questions goes into more detail about what legal sanctions, if any, students think should be attached to the use and sale of marijuana. Respondents also are asked to guess how they would be likely to react to legalized use and sale of the drug. While the answers to such a question must be interpreted cautiously, a special study of the effects of marijuana decriminalization at the state level, conducted as part of the Monitoring the Future series, suggests that in the aggregate their predictions about how they would react proved relatively accurate. ${ }^{17}$

\section{Attitudes and Predicted Response to Legalization}

- As shown in Table 18, less than one-sixth of all seniors believe marijuana use should be entirely legal (15\%). About one out of four $(26 \%)$ feel it should be treated as a minor violation-like a parking ticket-but not as a crime. Another $17 \%$ indicate no opinion, leaving more than two-fifths $(43 \%)$ who feel it still should be treated as a crime.

- Asked whether they thought it should be legal to sell marijuana if it were legal to use it, a majority (53\%) said "yes." However, nearly all of these respondents would permit sale only to adults, thus suggesting more conservatism on this subject than might generally be supposed.

- High school seniors predict that they would be little affected by the legalization of either the sale or the use of marijuana. Fully $62 \%$ of the respondents say that they would not use the drug even if it were legal to buy and use, and another $19 \%$ indicate they would use it about as often as they do now, or less. Only $5 \%$ say they would use it more often than at present and only another $8 \%$ think they would try it. Some $6 \%$ say they do not know how they would react. The special study of the effects of decriminalization at the state level (which falls short of the hypothetical situation posited in this question) revealed no evidence of any impact on the use of marijuana, nor even on attitudes and beliefs concerning its use.

\section{Trends in Attitudes and Predicted Responses}

- Between 1976 and 1979 seniors' preferences for decriminalization or legalization remained fairly constant; but

${ }^{17}$ See Johnston, L.D., O'Malley, P.M., \& Bachman, J.G. (1981). Marijuana decriminalization: The impact on youth, 1975-1980 (Monitoring the Future Occasional Paper No. 13). Ann Arbor: Institute for Social Research. 
Trends in Attitudes Regarding Marijuana Laws

(Entries are percentages)

Q. There has been a great deal of public debate about whether marijuana use should be legal. Which of the following policies would you favor?

Using marijuana should be entirely legal

It should be a minor violation like a parking ticket but not a crime

It should be a crime

Don't know

Q. If it were legal for people to USE marijuang, should it also be legal to SELL marijuana?

No

Yes, but only to adults

Yes, to anyone

Don't know

Q. If marijuana were legal to usc and legally available, which of the following would you be most likely to do?

Not use it, even if it were legal and available

Try it

Use it about as often as I do now

Use it more often than I do now

Use it less than I do now

\begin{tabular}{|c|c|c|c|c|c|c|c|c|c|c|c|}
\hline $\begin{array}{c}\text { Class } \\
\text { of } \\
1975 \\
\end{array}$ & $\begin{array}{c}\text { Class } \\
\text { of } \\
1976 \\
\end{array}$ & $\begin{array}{c}\text { Class } \\
\text { of } \\
1977 \\
\end{array}$ & $\begin{array}{c}\text { Class } \\
\text { of } \\
1978 \\
\end{array}$ & $\begin{array}{c}\text { Class } \\
\text { of } \\
1979 \\
\end{array}$ & $\begin{array}{c}\text { Class } \\
\text { of } \\
1980 \\
\end{array}$ & $\begin{array}{c}\text { Class } \\
\text { of } \\
1981 \\
\end{array}$ & $\begin{array}{c}\text { Class } \\
\text { of } \\
1982 \\
\end{array}$ & $\begin{array}{c}\text { Class } \\
\text { of } \\
1983 \\
\end{array}$ & $\begin{array}{c}\text { Class } \\
\text { of } \\
1984 \\
\end{array}$ & $\begin{array}{c}\text { Class } \\
\text { of } \\
1985 \\
\end{array}$ & $\begin{array}{c}\text { Class } \\
\text { of } \\
1986 \\
\end{array}$ \\
\hline 27.3 & 32.6 & 33.6 & 32.9 & 32.1 & 26.3 & 23.1 & 20.0 & 18.9 & 18.6 & 16.6 & 14.9 \\
\hline 25.3 & 29.0 & 31.4 & 30.2 & 30.1 & 30.9 & 29.3 & 28.2 & 26.3 & 23.6 & 25.7 & 25.9 \\
\hline 30.5 & 25.4 & 21.7 & 22.2 & 24.0 & 26.4 & 32.1 & 34.7 & 36.7 & 40.6 & 40.8 & 42.5 \\
\hline 16.8 & 13.0 & 13.4 & 14.6 & 13.8 & 16.4 & 15.4 & 17.1 & 18.1 & 17.2 & 16.9 & 16.7 \\
\hline
\end{tabular}

27

$\begin{array}{llll}27.8 & 23.0 & 22.5 & 21.8\end{array}$

16.2

18.9

49.8

13.3

52.

12.7

53.6

12.0

22.9

11.3

25.0

51.8

9.6

12.6

13.6

27.7

48.6

10.5

29.3

46.2

10.7

27.4

47.6

10.5

30.9

45.8

32.6

43.2

33.0

13.2

13.8

14.6

12.8

13.1

14.4

Don't know

$\begin{array}{rrrrrrrrrrrr}53.2 & 50.4 & 50.6 & 46.4 & 50.2 & 53.3 & 55.2 & 60.0 & 60.1 & 62.0 & 63.0 & 62.4 \\ 8.2 & 8.1 & 7.0 & 7.1 & 6.1 & 6.8 & 6.0 & 6.3 & 7.2 & 6.6 & 7.5 & 7.6 \\ 22.7 & 24.7 & 26.8 & 30.9 & 29.1 & 27.3 & 24.8 & 21.7 & 19.8 & 19.1 & 17.7 & 16.8 \\ 6.0 & 7.1 & 7.4 & 6.3 & 6.0 & 4.2 & 4.7 & 3.8 & 4.9 & 4.7 & 3.7 & 5.0 \\ 1.3 & 1.5 & 1.5 & 2.7 & 2.5 & 2.6 & 2.5 & 2.2 & 1.5 & 1.6 & 1.6 & 2.0 \\ 8.5 & 8.1 & 6.6 & 6.7 & 6.1 & 5.9 & 6.9 & 6.0 & 6.4 & 6.0 & 6.5 & 6.1 \\ (2600) & (2970) & (3110) & (3710) & (3280) & (3210) & (3600) & (3620) & (3300) & (3220) & (3230) & (3080)\end{array}$

Approx. N 
in the past six years there has been a sharp drop in the proportion favoring outright legalization (down from $32 \%$ in 1979 to $15 \%$ in 1986), while there was a corresponding increase in the proportion saying marijuana use should be a crime (from $24 \%$ to $43 \%$ ).

- Also reflecting the recent increased conservatism about marijuana, somewhat fewer now would support legalized sale even if use were to be made legal (down from $65 \%$ in 1979 to $53 \%$ in 1986$)$.

- The predictions about personal marijuana use, if sale and use were legalized, have been quite similar for all high school classes. The slight shifts being observed are mostly attributable to the changing proportions of seniors who actually use marijuana.

- In sum, in recent years American young people have become more supportive of legal prohibitions on the use of illegal drugs, whether used in private or in public. The fairly tolerant attitudes of students in the late 70's toward marijuana use have eroded considerably as substantially more think it should be treated as a criminal offense and correspondingly fewer think it should be entirely legal to use. 


\section{THE SOCIAL MILIEU}

The preceding section dealt with seniors' own attitudes about various forms of drug use. Attitudes about drugs, as well as drug-related behaviors, obviously do not occur in a social vacuum. Drugs are discussed in the media; they are a topic of considerable interest and conversation among young people; they are also a matter of much concern to parents, concern which often is strongly communicated to their children. Young people are known to be affected by the actual drugtaking behaviors of their friends and acquaintances, as well as by the availability of the various drugs. This section presents data on several of these relevant aspects of the social milieu.

We begin with two sets of questions about parental and peer attitudes, questions which closely parallel the questions about respondents' own attitudes about drug use, discussed in the preceding section. Since parental attitudes are now included in the survey only intermittently, those discussed here are based on the 1979 results.

\section{PERCEIVED ATTITUDES OF PARENTS AND FRIENDS}

\section{Perceptions of Parental Attitudes}

- A large majority of seniors in 1979 felt that their parents would disapprove or strongly disapprove of their exhibiting any of the drug use behaviors shown in Table 19. (The data for the perceived parental attitudes are not given in tabular form, but are displayed in Figures 22 and 23.)

- Drug use appears to constitute one area in which the position of parents approaches complete unanimity. Over $97 \%$ of seniors said that their parents would disapprove or strongly disapprove of their smoking marijuana regularly, even trying $L S D$ or amphetamines, or having four or five drinks every day. (Although the questions did not include more frequent use of LSD or amphetamines, or any use of heroin, it is obvious that if such behaviors had been included in the list virtually all seniors would have indicated parental disapproval.)

- Even experimental use of marijuana was seen as a parentally disapproved activity by the great majority of the seniors 
TABLE 19

\section{Trends in Proportion of Friends Disapproving of Drug Use}

Q. Hotv do you think your close friends feel (or would feel) about you... .

Trying marijuana once or twice

Sinoking inarijuana occasionally

Smoking marijuana regularly

Trying LSD once or twice

Trying an amphetamine once or twice

Taking one or two drinks nearly every day

Taking four or five drinks

every day

Having five or more drinks once or twice every weekend

Smoking one or more packs of cigarettes per day
Percentage saying friends disapprove ${ }^{\mathrm{a}}$

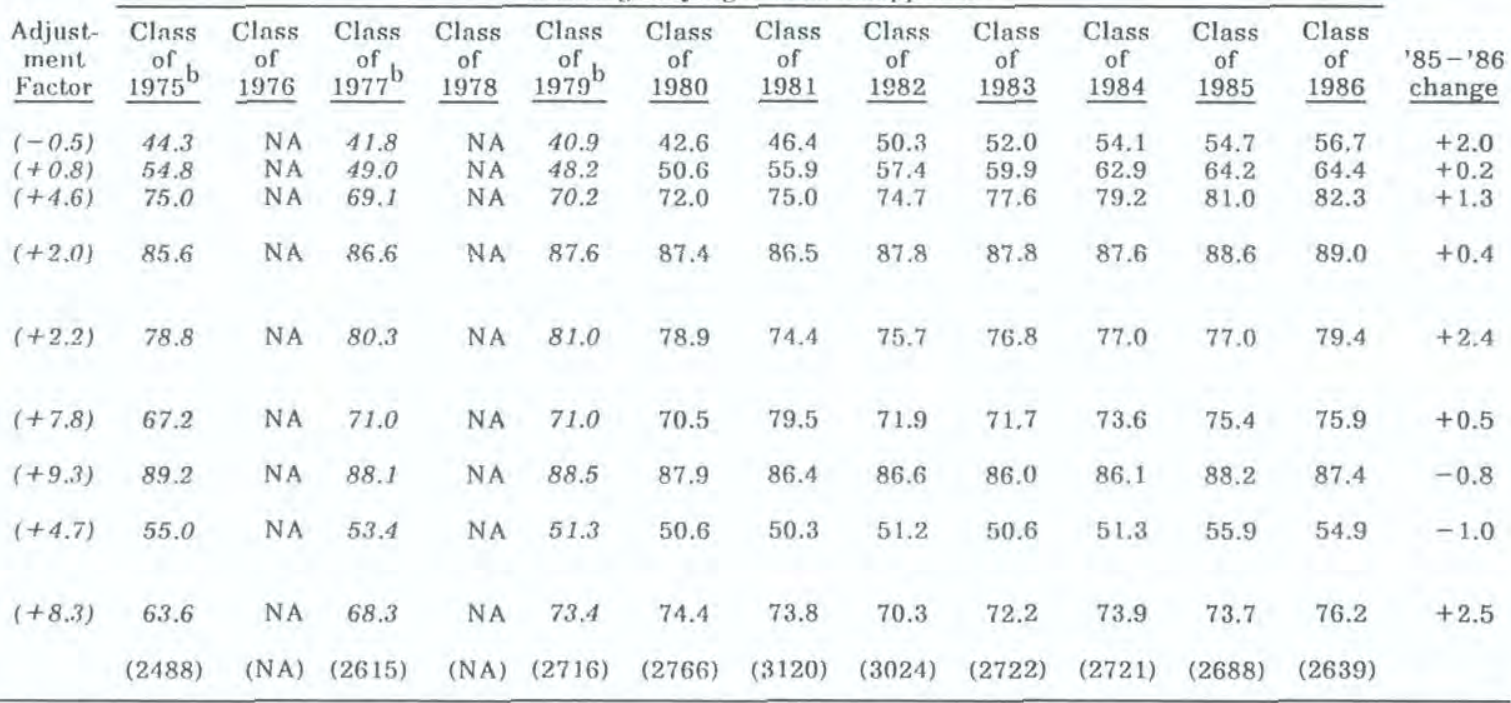

NOTE: Level of significance of difference between the two most recent classes: $\mathrm{s}=.05, \mathrm{ss}=.01, \mathrm{sss}=.001$. NA indicates data not available.

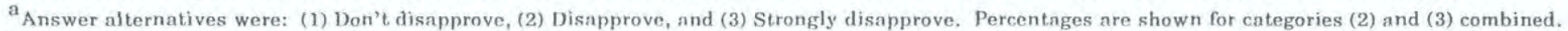

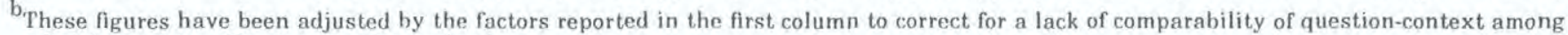
administrntions. (See text for discussion.) 
$(85 \%)$. Assuming that the students were generally correct about their parents' attitudes, these results clearly show a substantial generational difference of opinion about this drug.

- Also likely to be perceived as rating high parental disapproval (around 92\% disapproval) were occasional marijuana use, taking one or two drinks nearly every day, and pack-a-day cigarette smoking.

- Slightly lower proportions of seniors (85\%) felt their parents would disapprove of their having five or more drinks once or twice every weekend. This happened to be exactly the same percentage as said that their parents would disapprove of simply experimenting with marijuana.

- There is no reason to think that parental attitudes have softened in the period since 1979. If anything the opposite seems likely to be the case, given the rising public concern about marijuana and cocaine and the parents' movement against drugs.

\section{Current Perceptions of Friends' Attitudes}

- A parallel set of questions asked respondents to estimate their friends' attitudes about drug use (Table 19). These questions ask, "How do you think your close friends feel (or would feel) about you ...?" The highest levels of disapproval for experimenting with a drug are associated with trying LSD (89\%) and trying an amphetamine $(79 \%)$. Presumably, if heroin were on the list it would receive the highest peer disapproval; and, judging from respondents' own attitudes, experimenting with cocaine would be slightly more disapproved than experimenting with amphetamines, while experimenting with barbiturates would be still less popular.

- Even experimenting with marijuana is now "out" with most seniors' friends (57\%); and a substantial majority think their friends would disapprove if they smoked marijuana regularly $(82 \%)$.

- About three-quarters of all seniors think they would face peer disapproval if they smoked a pack or more of cigarettes daily $(76 \%)$.

- While heavy drinking on weekends is judged by only half (55\%) to be disapproved by their friends, substantially more (76\%) think consumption of one or two drinks daily would be disapproved. The great majority $(87 \%)$ would face the dis- 
approval of their friends if they engaged in heavy daily drinking.

- In sum, peer norms differ considerably for the various drugs and for varying degrees of involvement with those drugs, but overall they tend to be quite conservative. The great majority of seniors have friendship circles which do not condone use of the illicit drugs other than marijuana, and over four-fifths feel that their friends would disapprove of regular marijuana use. In fact, well over half of them now believe their friends would disapprove of their even trying marijuana.

\section{A Comparison of the Attitudes of Parents, Peers, and Respondents}

- A comparison of the perceptions of friends' disapproval with perceptions of parents' disapproval shows several interesting findings.

- First there is rather little variability among different students in their perceptions of their parents' attitudes: on any of the drug behaviors listed nearly all say their parents would disapprove. Nor is there much variability among the different drugs in perceived parental attitudes. Peer norms vary much more from drug to drug. The net effect of these facts is likely to be that peer norms have a much greater chance of explaining variability in the respondent's own individual attitudes or use than parental norms, simply because the peer norms vary more.

- Despite there being less variability in parental attitudes, the ordering of drug use behaviors is much the same for them as for peers (e.g., among the illicit drugs asked about, the highest frequencies of perceived disapproval are for trying LSD, while the lowest frequencies are for trying marijuana).

- A comparison with the seniors' own attitudes regarding drug use (see Figures 22 and 23) reveals that on the average they are much more in accord with their peers than with their parents. The differences between seniors' own disapproval ratings and those attributed to their parents tend to be large, with parents seen as more conservative overall in relation to every drug, licit or illicit. The largest difference occurred in the case of marijuana experimentation, where only $34 \%$ of seniors (in 1979) said they disapproved vs. 85\% (of 1979 seniors) who said their parents would disapprove. Despite the great increase in seniors' own disapproval (up to $55 \%$ in 
1986), it is doubtless still the most controversial of the drugusing behaviors listed here.

\section{Trends in Perceptions of Parents' and Friends' Attitudes}

- Several important changes in the perceived attitudes of others have been taking place recently-and particularly among peers. These shifts are presented graphically in Figures 22 and 23. As can be seen in those figures, adjusted (dotted) trend lines have been introduced before 1980. This was done because we discovered that the deletion in 1980 of the questions about parents' attitudes-which up until then had been located immediately ahead of the questions about friends' attitudes-removed an artifactual depression of the ratings of friends' attitudes, a phenomenon known as a question-context effect. This effect was particularly evident in the trend lines dealing with alcohol use, where otherwise smooth lines showed abrupt upward shifts in 1980. It appears that when questions about parents' attitudes were present, respondents tended to understate peer disapproval in order to emphasize the difference in attitudes between their parents and their peers. In the adjusted lines, we have attempted to correct for that artifactual depression in the 1975,1977 , and 1979 scores. ${ }^{18}$ We think the adjusted trend lines give a more accurate picture of the change taking place. For some reason, the question-context effect seems to have more influence on the questions dealing with cigarettes and alcohol than on those dealing with illicit drugs.

- For each level of marijuana use-trying once or twice, occasional use, regular use-there had been a drop in perceived disapproval for both parents and friends up until 1977 or 1978. We know from our other findings that these perceptions correctly reflected actual shifts in the attitudes of their peer groups-that is, that acceptance of marijuana was in fact increasing among seniors (see Figure 22). There is little

\footnotetext{
${ }^{18}$ The correction evolved as follows: We assumed that a more accurate estimate of the true change between 1979 and 1980 could be obtained by taking an average of the changes observed in the year prior and the year subsequent, rather than by taking the observed change (which we knew to contain the effect of a change in question context). We thus calculated an adjusted 1979-1980 change score by taking an average of one-half the 1977-1979 change score (our best estimate of the 1978-1979 change) plus the 1980-1981 change score. This estimated change score was then subtracted from the observed change score for 1979-1980, the difference being our estimate of the amount by which peer disapproval of the behavior in question was being understated because of the context in which the questions occurred prior to 1980 . The 1975, 1977, and 1979 observations were then adjusted upward by the amount of that correction factor. (Table 19 shows the correction factors in the first column.)
} 


\section{FIGURE 22a}

\section{Trends in Disapproval of Illicit Drug Use}

Seniors, Parents, and Peers

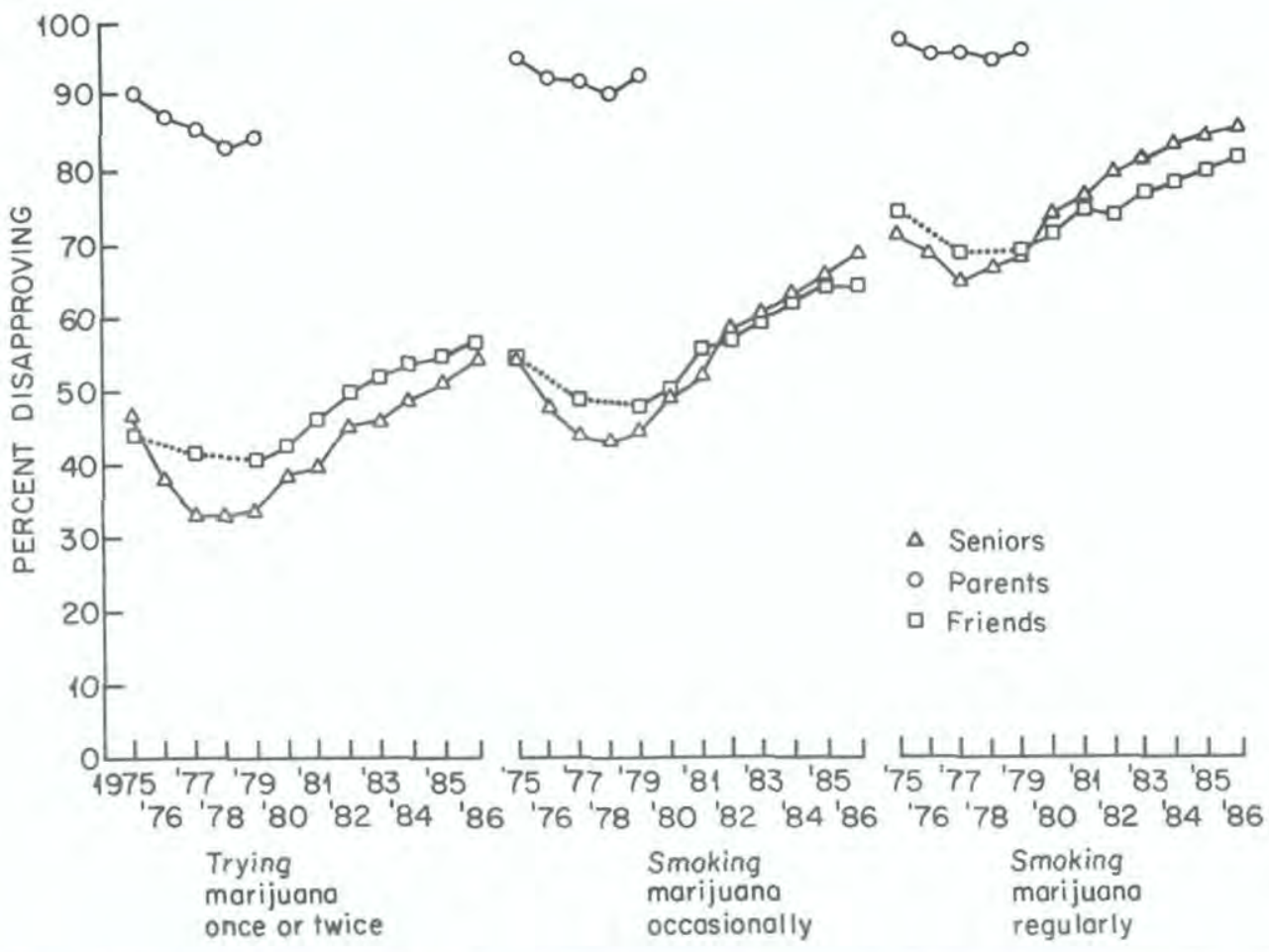

NOTE: Points connected by dotted lines have been adjusted because of lack of comparability of question-context among administrations. (See text for discussion.) 
Trends in Disapproval of Illicit Drug Use

Seniors, Parents, and Peers

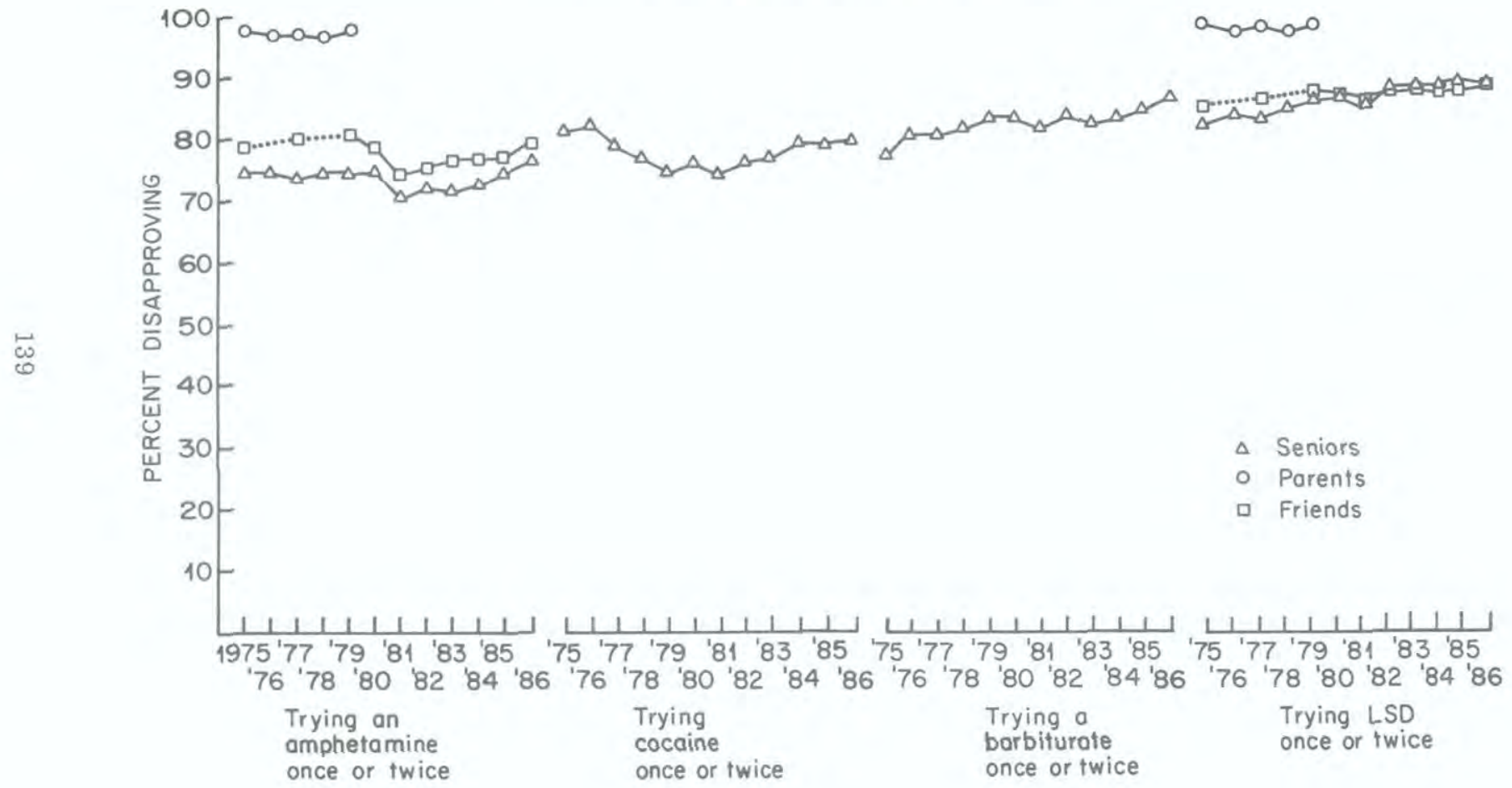

NOTE: Points connected by dotted lines have been adjusted because of lack of comparability of question-context among administrations. (See text for discussion.) 
Trends in Disapproval of Licit Drug Use

Seniors, Parents, and Peers
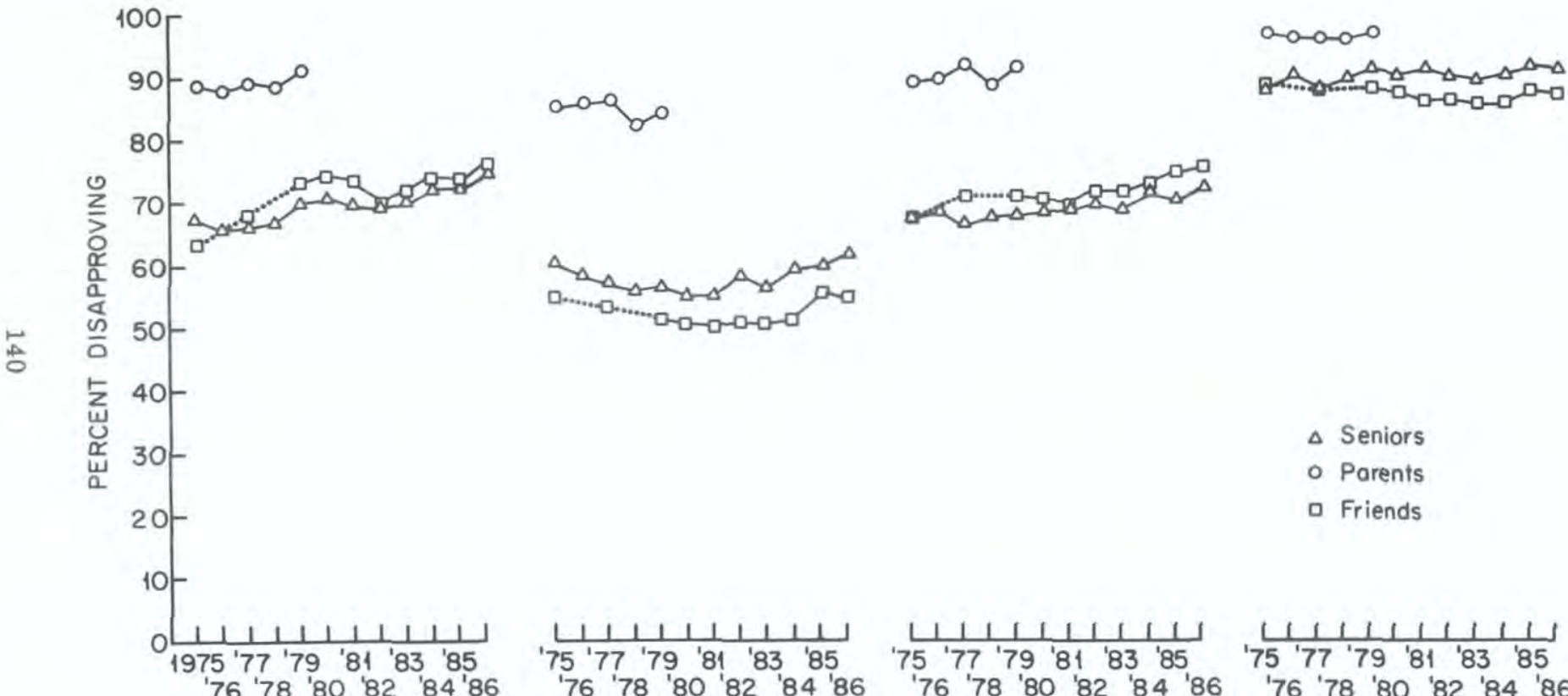
$\Delta$ Seniors
- Parents
口 Friends
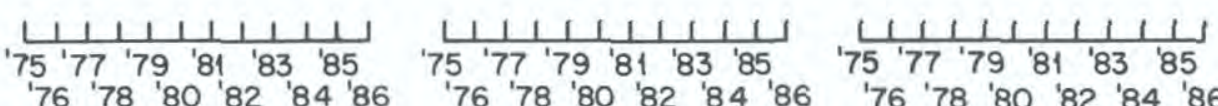

Smoking one or

more pocks of

Hoving five or

more drinks

Taking one or

two drinks nearly

'76'78'80 '82 '84 '86

cigarettes per doy

once or twice

every day

Taking four or

five drinks nearly

every doy

NOTE: Points connected by dotted lines have been adjusted because of lack of comparability of question-context among administrations. (See text for discussion.) 
reason to suppose such perceptions are less accurate in reflecting shifts in parents' attitudes. Therefore, we conclude that the social norms regarding marijuana use among adolescents had been relaxing before 1979. However, consistent with the seniors' reports about their own attitudes, there has been a sharp reversal in peer norms regarding all levels of marijuana use, and it continued in 1986.

- Until 1979 there had been relatively little change in either self-reported attitudes or perceived peer attitudes toward amphetamine use, but in 1981 both measures showed significant and parallel dips in disapproval (as use rose sharply). Since 1981 disapproval has been easing back up to the earlier levels (as use has declined).

- Peer disapproval of $L S D$ use has been inching upward since 1975.

- While perceived attitudes of friends were not asked for cocaine and barbiturates, it seems likely that the trends in such measures could have moved in parallel to the seniors' statements about their own attitudes, since such parallel movement has been shown for virtually all other drugs. (See Figure 22.) This would suggest that disapproval has risen gradually but steadily for barbiturate use since 1975 . Regarding experimenting with cocaine, seniors' disapproval dropped from 1975 to 1979 , but has been rising very gradually since.

- One of the larger changes in perceived peer norms has occurred in relation to regular cigarette smoking. The proportion of seniors saying that their friends would disapprove of them smoking a pack-a-day or more rose from $64 \%$ (adjusted version) in 1975 to $74 \%$ in 1980 . In the several years following, peer disapproval eased back a percent or two, only to begin rising again in 1984 ; by 1986 it reached $76 \%$. Overall, since 1980 peer disapproval has fluctuated within a fairly narrow range, although it now seems to be trending upward.

- For alcohol, perceived peer norms have moved pretty much in parallel with seniors' statements about their personal disapproval. Heavy daily drinking is seen by the great majority ( $87 \%$ in 1986) as disapproved by peers, with little systematic change over the decade. Weekend binge drinking showed some modest decline in peer disapproval up through 1980; it then remained level for about four years (while personal disapproval was increasing) until 1985 and 1986, where it showed evidence of a rise. (Recall that this 
form of episodic heavy drinking began to decline for the first time in 1984, continued to decline in 1985, and stabilized in 1986.)

\section{EXPOSURE TO DRUG USE BY FRIENDS AND OTHERS}

It is generally acknowledged that much of youthful drug use is initiated through a peer social-learning process; and research has shown a high correlation between an individual's illicit drug use and that of his or her friends. Such a correlation can, and probably does, reflect several different causal patterns: (a) a person with friends who use a drug will be more likely to try the drug; (b) conversely, the individual who is already using a drug will be likely to introduce friends to the experience; and (c) one who is already a user is more likely to establish friendships with others who also are users.

Given the potential importance of exposure to drug use by others, we felt it would be useful to monitor seniors' association with others taking drugs, as well as seniors' perceptions about the extent to which their friends use drugs. Two sets of questions, each covering all or nearly all of the categories of drug use treated in this report, asked seniors to indicate (a) how often during the past twelve months they were around people taking each of the drugs to get high or for "kicks," and (b) what proportion of their own friends use each of the drugs. (The questions dealing with friends' use are shown in Table 20. The data dealing with direct exposure to use may be found in Table 21.) Obviously, responses to these two questions are highly correlated with the respondents' own drug use; thus, for example, seniors who have recently used marijuana are much more likely to report that they have been around others getting high on marijuana, and that most of their friends use it.

\section{Exposure to Drug Use in 1986}

- A comparison of responses about friends' use, and about being around people in the last twelve months who were using various drugs to get high, reveals a high degree of correspondence between these two indicators of exposure. For each drug, the proportion of respondents saying "none" of their friends use it is fairly close to the proportion who say that during the last twelve months they have not been around anyone who was using that drug to get high. Similarly, the proportion saying they are "often" around people getting high on a given drug is roughly the same as the proportion reporting that "most" or "all" of their friends use that drug.

- Reports of exposure and friends' use closely parallel the figures on seniors' own use (compare Figures 2 and 24). It thus comes as no surprise that the highest levels of exposure 
FIGURE 24

Proportion of Friends Using Each Drug as Estimated by Seniors, in $\mathbf{1 9 8 6}$

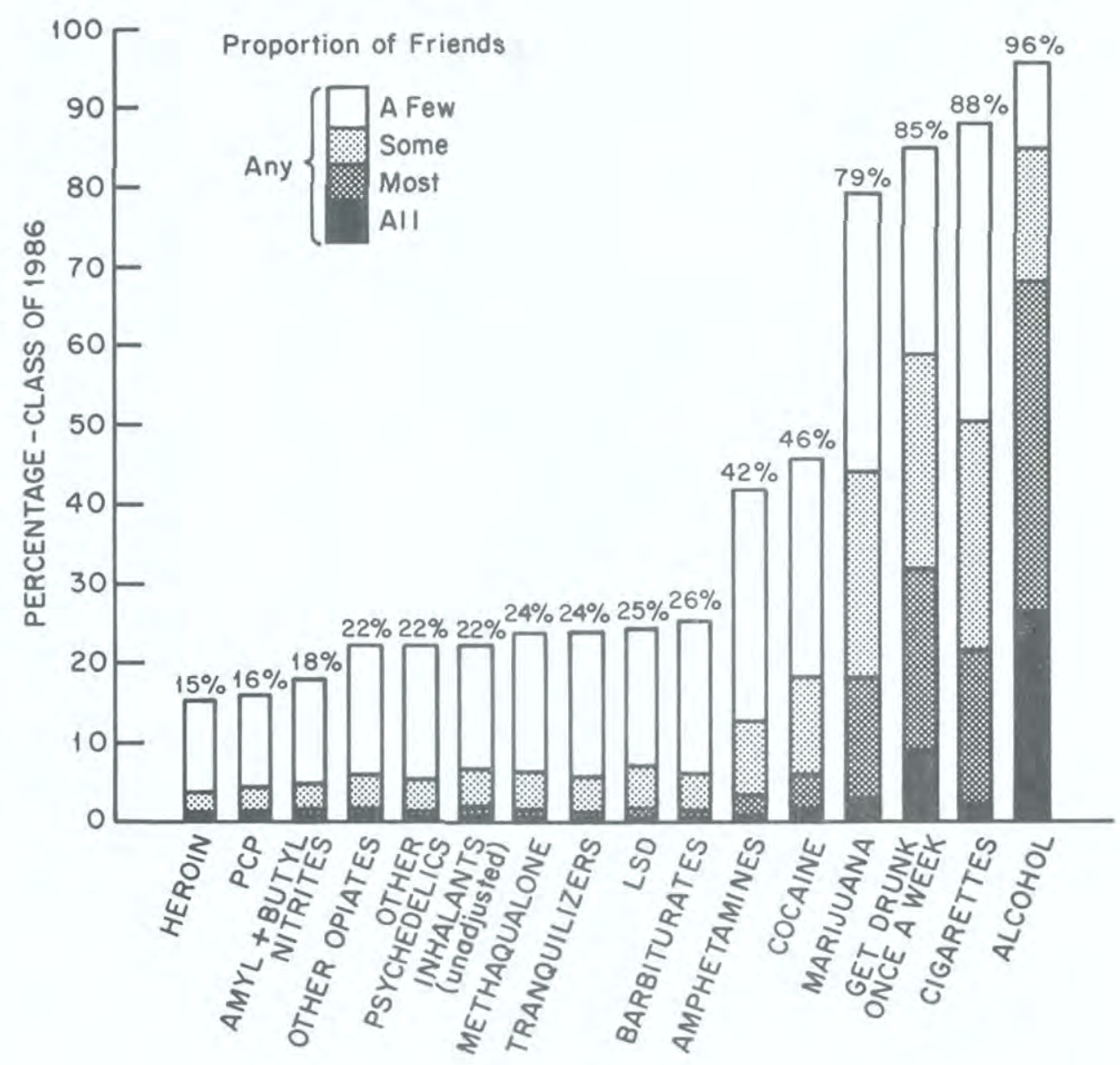


TABLE 20

\section{Trends in Proportion of Friends Using Drugs}

(Entries are percentages)

\begin{tabular}{|c|c|c|c|c|c|c|c|c|c|c|c|c|c|}
\hline $\begin{array}{l}\text { Q. Hoiv many of yout } \\
\text { friends would } \\
\text { you estimate... }\end{array}$ & $\begin{array}{c}\text { Class } \\
\text { of } \\
1975 \\
\end{array}$ & $\begin{array}{c}\text { Class } \\
\text { of } \\
1976 \\
\end{array}$ & $\begin{array}{l}\text { Class } \\
\text { of } \\
1977 \\
\end{array}$ & $\begin{array}{l}\text { Class } \\
\text { of } \\
1978 \\
\end{array}$ & $\begin{array}{c}\text { Class } \\
\text { of } \\
1979 \\
\end{array}$ & $\begin{array}{l}\text { Class } \\
\text { of } \\
1980 \\
\end{array}$ & $\begin{array}{c}\text { Class } \\
\text { of } \\
1981 \\
\end{array}$ & $\begin{array}{l}\text { Class } \\
\text { of } \\
1982 \\
\end{array}$ & $\begin{array}{c}\text { Class } \\
\text { of } \\
1983 \\
\end{array}$ & $\begin{array}{c}\text { Class } \\
\text { of } \\
1984 \\
\end{array}$ & $\begin{array}{l}\text { Class } \\
\text { of } \\
1985 \\
\end{array}$ & $\begin{array}{c}\text { Class } \\
\text { of } \\
1986 \\
\end{array}$ & $\begin{array}{l}\text { ' } 85-' 86 \\
\text { change } \\
\end{array}$ \\
\hline \multicolumn{14}{|l|}{$\begin{array}{c}\text { Smoke marijuana } \\
\% \text { saying none }\end{array}$} \\
\hline \% saying none & 17.0 & 17.1 & 14.1 & 13.9 & 12.4 & 13.6 & 17.0 & 15.6 & 19.7 & 22.3 & 20.5 & 20.8 & +0.3 \\
\hline \% saying most or all & 30.3 & 30.6 & 32.3 & 35.3 & 35.5 & 31.3 & 27.7 & 23.8 & 21.7 & 18.3 & 19.8 & 18.2 & -1.6 \\
\hline \multicolumn{14}{|l|}{$\begin{array}{l}\text { Use inhalants } \\
\text { \% saying none }\end{array}$} \\
\hline $\begin{array}{l}\text { \% saying none } \\
\% \text { saying most or all }\end{array}$ & $\begin{array}{r}75.7 \\
1.1\end{array}$ & 81.4 & $\begin{array}{r}81.1 \\
1.0\end{array}$ & 80.0 & 80.9 & 82.2 & 83.5 & 81.6 & 83.9 & 80.7 & 78.8 & 77.6 & -1.2 \\
\hline \multicolumn{14}{|l|}{$\begin{array}{l}\text { Use nitrites } \\
\text { \% saying none }\end{array}$} \\
\hline \% saying none & NA & NA & NA & NA & 78.4 & 81.0 & 82.6 & 82.5 & 85.5 & 85.0 & 84.4 & 82.0 & $-2.4 \mathrm{~s}$ \\
\hline $\begin{array}{l}\text { \% saying most or all } \\
\text { Take LSD }\end{array}$ & NA & NA & NA & NA & 1.9 & 1.3 & 1.2 & 0.9 & 0.7 & 1.2 & 1.0 & 1.2 & +0.2 \\
\hline \multicolumn{14}{|l|}{$\begin{array}{l}\text { Take LSD } \\
\text { \% saying none }\end{array}$} \\
\hline \% saying most or all & 2.7 & 2.8 & $\begin{array}{r}68.1 \\
3.0\end{array}$ & $\begin{array}{r}70.1 \\
2.0\end{array}$ & $\begin{array}{r}71.1 \\
1.9\end{array}$ & $\begin{array}{r}71.9 \\
1.8\end{array}$ & $\begin{array}{r}71.5 \\
2.2\end{array}$ & $\begin{array}{r}72.2 \\
2.4\end{array}$ & 76.0 & 76.1 & 75.6 & 75.5 & -0.1 \\
\hline \multicolumn{14}{|l|}{$\begin{array}{l}\text { Take other psychedelics } \\
\% \text { saying none }\end{array}$} \\
\hline \% saying none & 58.8 & 69.7 & 68.6 & 70.8 & 71.8 & 71.8 & 73.7 & 74,4 & 77.9 & 78.7 & 78.0 & 77.7 & -0.3 \\
\hline \% saying most or all & 4.7 & 3.0 & 2.8 & 2.0 & 2.2 & 2.2 & 2.1 & 1.9 & 1.6 & 1.9 & 1.4 & 1.3 & -0.1 \\
\hline \multicolumn{14}{|l|}{$\begin{array}{l}\text { Take PCP } \\
w_{w} \text { saying none }\end{array}$} \\
\hline o saying none & NA & NA & NA & NA & 72.2 & 77.8 & 82.8 & 82.7 & 85.8 & 85.8 & 84.1 & 83.9 & -0.2 \\
\hline \% saying most or all & NA & NA & NA & NA & 1.7 & 1.6 & 0.9 & 0.9 & 1.1 & 1.1 & 1.2 & 1.2 & 0.0 \\
\hline \multicolumn{12}{|l|}{$\begin{array}{l}\text { Take cocaine } \\
\% \text { saying none }\end{array}$} & & \\
\hline$\%$ saying most or all & 3.4 & 3.2 & 3.6 & 4.0 & 6.0 & 6.1 & 6.3 & 4.9 & $\begin{array}{r}0.4 \\
5.1\end{array}$ & $\begin{array}{r}61.1 \\
5.1\end{array}$ & $\begin{array}{r}56.2 \\
5.8\end{array}$ & $\begin{array}{r}54.4 \\
6.2\end{array}$ & $\begin{array}{l}-1.8 \\
+0.4\end{array}$ \\
\hline \multicolumn{14}{|l|}{$\begin{array}{l}\text { Take heroin } \\
\% \text { saying none }\end{array}$} \\
\hline$\%$ saying none & 84.8 & 86.4 & 87.1 & 85.7 & 87.1 & 87.0 & 87.5 & 86.8 & 88.0 & 87.0 & 85.5 & 84.7 & -0.8 \\
\hline \% saying most or all & 0.7 & 0.8 & 0.7 & 0.9 & 0.5 & 1.0 & 0.5 & 0.7 & 0.8 & 0.8 & 0.9 & 1.1 & +0.2 \\
\hline \multicolumn{14}{|l|}{$\begin{array}{l}\text { Take other narcotics } \\
\text { \% saying none }\end{array}$} \\
\hline $\begin{array}{l}\text { \% saying none } \\
\% \text { saying most or all }\end{array}$ & $\begin{array}{r}71.2 \\
2.1\end{array}$ & 75.9 & $\begin{array}{r}76.3 \\
1.7\end{array}$ & $\begin{array}{r}76.8 \\
1.4\end{array}$ & $\begin{array}{r}76.9 \\
1.5\end{array}$ & $\begin{array}{r}77.6 \\
1.7\end{array}$ & 76.9 & 76.1 & 79.2 & 78.6 & 77.2 & 78.2 & +1.0 \\
\hline$\%$ saying most or all & 2.1 & 2.2 & & & 1.5 & 1.7 & 1.5 & 1.4 & 1.4 & 1.6 & 1.4 & 1.8 & +0.4 \\
\hline
\end{tabular}

(Table continued on next page) 


\section{TABLE 20 (cont.)}

\section{Trends in Proportion of Friends Using Drugs}

(Entries are percentages)

\begin{tabular}{|c|c|c|c|c|c|c|c|c|c|c|c|c|c|}
\hline $\begin{array}{l}\text { Q. How many of your } \\
\text { friends would } \\
\text { you estimate... }\end{array}$ & $\begin{array}{c}\text { Class } \\
\text { of } \\
1975 \\
\end{array}$ & $\begin{array}{c}\text { Class } \\
\text { of } \\
1976 \\
\end{array}$ & $\begin{array}{c}\text { Class } \\
\text { of } \\
1977 \\
\end{array}$ & $\begin{array}{c}\text { Clnss } \\
\text { of } \\
1978 \\
\end{array}$ & $\begin{array}{c}\text { Class } \\
\text { of } \\
1979 \\
\end{array}$ & $\begin{array}{l}\text { Class } \\
\text { of } \\
1980 \\
\end{array}$ & $\begin{array}{c}\text { Class } \\
\text { of } \\
1981 \\
\end{array}$ & $\begin{array}{c}\text { Class } \\
\text { of } \\
1982 \\
\end{array}$ & $\begin{array}{c}\text { Class } \\
\text { of } \\
1983 \\
\end{array}$ & $\begin{array}{c}\text { Class } \\
\text { of } \\
1984 \\
\end{array}$ & $\begin{array}{c}\text { Class } \\
\text { of } \\
1985 \\
\end{array}$ & $\begin{array}{c}\text { Class } \\
\text { of } \\
1986 \\
\end{array}$ & $\begin{array}{l}\text { '85-'86 } \\
\text { change }\end{array}$ \\
\hline $\begin{array}{l}\text { Take amphetamines } \\
\text { o saying none } \\
\text { \% saying most or all }\end{array}$ & $\begin{array}{r}49.0 \\
5.9\end{array}$ & $\begin{array}{r}57.8 \\
5.6\end{array}$ & $\begin{array}{r}58.7 \\
4.1\end{array}$ & $\begin{array}{r}59.3 \\
4.7\end{array}$ & $\begin{array}{r}59.3 \\
4.3\end{array}$ & $\begin{array}{r}56.1 \\
4.8\end{array}$ & $\begin{array}{r}51.2 \\
6.4\end{array}$ & $\begin{array}{r}49.4 \\
5.4\end{array}$ & $\begin{array}{r}53.9 \\
5.1\end{array}$ & $\begin{array}{r}54.9 \\
4.5\end{array}$ & $\begin{array}{r}56.7 \\
3.4\end{array}$ & $\begin{array}{r}58.2 \\
3.4\end{array}$ & $\begin{array}{r}+1.5 \\
0.0\end{array}$ \\
\hline $\begin{array}{l}\text { Take barbiturates } \\
\% \text { saying none } \\
\% \text { saying most or all }\end{array}$ & $\begin{array}{r}55.0 \\
4.3\end{array}$ & $\begin{array}{r}63.7 \\
3.5\end{array}$ & $\begin{array}{r}65.3 \\
3.0\end{array}$ & $\begin{array}{r}67.5 \\
2.3\end{array}$ & $\begin{array}{r}\text { (59).3 } \\
2.1\end{array}$ & $\begin{array}{r}69.5 \\
2.6\end{array}$ & $\begin{array}{r}68.9 \\
2.1\end{array}$ & $\begin{array}{r}68.7 \\
1.8\end{array}$ & $\begin{array}{r}71.7 \\
1.7\end{array}$ & $\begin{array}{r}73.4 \\
1.7\end{array}$ & $\begin{array}{r}72.9 \\
1.6\end{array}$ & $\begin{array}{r}74.4 \\
1.4\end{array}$ & $\begin{array}{l}+1.5 \\
-0.2\end{array}$ \\
\hline $\begin{array}{l}\text { Take quaaludes } \\
\text { o saying none } \\
\text { \% saying most or all }\end{array}$ & $\begin{array}{r}68.3 \\
3.0\end{array}$ & $\begin{array}{r}73.0 \\
1.8\end{array}$ & $\begin{array}{r}71.7 \\
2.9\end{array}$ & $\begin{array}{r}73.0 \\
2.2\end{array}$ & $\begin{array}{r}72.3 \\
2.8\end{array}$ & $\begin{array}{r}67.5 \\
3.6\end{array}$ & $\begin{array}{r}65.0 \\
3.6\end{array}$ & $\begin{array}{r}64.5 \\
2.6\end{array}$ & $\begin{array}{r}70.3 \\
2.6\end{array}$ & $\begin{array}{r}73.9 \\
1.7\end{array}$ & $\begin{array}{r}74.0 \\
1.3\end{array}$ & $\begin{array}{r}76.5 \\
1.6\end{array}$ & $\begin{array}{l}+2.5 \\
+0.3\end{array}$ \\
\hline $\begin{array}{l}\text { Take tranquilizers } \\
\% \text { saying none } \\
\text { \% snying most or all }\end{array}$ & $\begin{array}{r}54.4 \\
3.5\end{array}$ & $\begin{array}{r}63.7 \\
3.1\end{array}$ & $\begin{array}{r}62.2 \\
2.7\end{array}$ & $\begin{array}{r}65.2 \\
1.8\end{array}$ & $\begin{array}{r}68.0 \\
2.0\end{array}$ & $\begin{array}{r}70.3 \\
1.9\end{array}$ & $\begin{array}{r}70.5 \\
1.4\end{array}$ & $\begin{array}{r}70.1 \\
1.1\end{array}$ & $\begin{array}{r}73.3 \\
1.2\end{array}$ & $\begin{array}{r}73.4 \\
1.5\end{array}$ & $\begin{array}{r}74.2 \\
1.2\end{array}$ & $\begin{array}{r}75.8 \\
1.3\end{array}$ & $\begin{array}{l}+1.6 \\
+0.1\end{array}$ \\
\hline $\begin{array}{l}\text { Drink alcoholic beverage } \\
\text { \% saying none } \\
\text { \% saying most or all }\end{array}$ & $\begin{array}{r}3.3 \\
68.4\end{array}$ & $\begin{array}{r}4.9 \\
64.7\end{array}$ & $\begin{array}{r}5.6 \\
66.2\end{array}$ & $\begin{array}{r}5.1 \\
68.9\end{array}$ & $\begin{array}{r}4.6 \\
68.5\end{array}$ & $\begin{array}{r}3.9 \\
68.9\end{array}$ & $\begin{array}{r}5.3 \\
67.7\end{array}$ & $\begin{array}{r}4.3 \\
69.7\end{array}$ & $\begin{array}{r}4.5 \\
69.0\end{array}$ & $\begin{array}{r}5.4 \\
66.6\end{array}$ & $\begin{array}{r}5.4 \\
66.0\end{array}$ & $\begin{array}{r}4.4 \\
68.0\end{array}$ & $\begin{array}{l}-1.0 \\
+2.0\end{array}$ \\
\hline $\begin{array}{l}\text { Get drunk at least once } \\
\text { a weck } \\
\text { \% saying none } \\
\text { \% saying most or all }\end{array}$ & $\begin{array}{l}17.6 \\
30.1\end{array}$ & $\begin{array}{l}19.3 \\
26.6\end{array}$ & $\begin{array}{l}19.0 \\
27.6\end{array}$ & $\begin{array}{l}18.0 \\
30.2\end{array}$ & $\begin{array}{l}16.7 \\
32.0\end{array}$ & $\begin{array}{l}16.9 \\
30.1\end{array}$ & $\begin{array}{l}18.2 \\
29.4\end{array}$ & $\begin{array}{l}16.9 \\
29.9\end{array}$ & $\begin{array}{l}16.1 \\
31.0\end{array}$ & $\begin{array}{l}18.5 \\
29.6\end{array}$ & $\begin{array}{l}17.5 \\
29.9\end{array}$ & $\begin{array}{l}15.3 \\
31.8\end{array}$ & $\begin{array}{l}-2.2 \\
+1.9\end{array}$ \\
\hline $\begin{array}{l}\text { Smoke cigarettes } \\
\% \text { saying none } \\
\% \text { saying most or all }\end{array}$ & $\begin{array}{r}4.8 \\
41.5\end{array}$ & $\begin{array}{r}6.3 \\
36.7\end{array}$ & $\begin{array}{r}6.3 \\
33.9\end{array}$ & $\begin{array}{r}6.9 \\
32.2\end{array}$ & $\begin{array}{r}7.9 \\
28.6\end{array}$ & $\begin{array}{r}9.4 \\
23.3\end{array}$ & $\begin{array}{l}11.5 \\
22.4\end{array}$ & $\begin{array}{l}11.7 \\
24.1\end{array}$ & $\begin{array}{l}13.0 \\
22.4\end{array}$ & $\begin{array}{l}14.0 \\
19.2\end{array}$ & $\begin{array}{l}13.0 \\
22.8\end{array}$ & $\begin{array}{l}12.2 \\
21.5\end{array}$ & $\begin{array}{l}-0.8 \\
-1.3\end{array}$ \\
\hline $\begin{array}{l}\text { Take any illicit drug } \\
\text { \% saying none } \\
\text { \% saying most or all }\end{array}$ & $\begin{array}{l}15.4 \\
31.6\end{array}$ & $\begin{array}{l}16.0 \\
31.7\end{array}$ & $\begin{array}{l}13.6 \\
33.0\end{array}$ & $\begin{array}{l}13.0 \\
36.2\end{array}$ & $\begin{array}{l}11.0 \\
37.0\end{array}$ & $\begin{array}{l}12.5 \\
32.5\end{array}$ & $\begin{array}{l}14.6 \\
29.8\end{array}$ & $\begin{array}{l}13.7 \\
26.5\end{array}$ & $\begin{array}{l}17.4 \\
23.8\end{array}$ & $\begin{array}{l}19.0 \\
20.9\end{array}$ & $\begin{array}{l}17.6 \\
22.7\end{array}$ & $\begin{array}{l}17.8 \\
21.5\end{array}$ & $\begin{array}{l}+0.2 \\
-1.2\end{array}$ \\
\hline $\begin{array}{l}\text { Take any illicit drug } \\
\text { other than marijuana } \\
\% \text { saying none } \\
\text { \% saying inost or all } \\
\text { Approx. } \mathrm{N}=\end{array}$ & $\begin{array}{r}38.2 \\
10.2 \\
(2640)\end{array}$ & $\begin{array}{r}47.7 \\
8.7 \\
(2697)\end{array}$ & $\begin{array}{r}46.4 \\
7.3 \\
(2788)\end{array}$ & $\begin{array}{r}46.3 \\
8.3 \\
(3247)\end{array}$ & $\begin{array}{r}38.7 \\
10.4 \\
\\
(2933)\end{array}$ & $\begin{array}{r}37.6 \\
11.1 \\
(2987)\end{array}$ & $\begin{array}{r}36.7 \\
11.9 \\
(3307)\end{array}$ & $\begin{array}{r}35.3 \\
10.9 \\
(3303)\end{array}$ & $\begin{array}{r}38.8 \\
11.0 \\
(3095)\end{array}$ & $\begin{array}{r}38.7 \\
10.3 \\
\\
(2945)\end{array}$ & $\begin{array}{r}38.2 \\
10.4 \\
(2971)\end{array}$ & $\begin{array}{r}36.7 \\
10.3 \\
(2798)\end{array}$ & $\begin{array}{l}-1.5 \\
-0.1\end{array}$ \\
\hline
\end{tabular}

NOTE: Level of significance of difference between the two most recent classes: $s=.05, \mathrm{ss}=.01, \mathrm{sss}=.001$. NA indicates data not available.

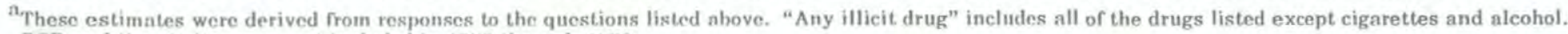
PCP and the nitrites were not included in 1975 through 1978. 
involve alcohol; a majority (58\%) say they are "often" around people using it to get high. What may come as a surprise is that fully $32 \%$ of all seniors say that most or all of their friends go so far as to get drunk at least once a week. (This is consistent, however, with the fact that $37 \%$ said they personally had taken five or more drinks in a row at least once during the prior two weeks.)

- The drug to which students are next most frequently exposed is marijuana. Only a little more than one in four (28\%) reports no exposure during the year. Some $24 \%$ are "often" around people using it to get high, and another $23 \%$ are exposed "occasionally." But fewer than one in five (18\%) now say that most or all of their friends smoke marijuana.

- After marijuana comes cocaine, with $37 \%$ of seniors reporting some exposure to use in the prior year, and $46 \%$ saying they have at least some friends who use.

- Amphetamines, the third most widely used class of illicit drugs, is also the one to which seniors are next most often exposed. Some $36.5 \%$ of all seniors have been around someone using them to get high over the past year, and $6 \%$ say they are "often" around people doing this.

- For the remaining illicit drugs there are far lower rates, with any exposure to use in the past year ranging from $20 \%$ for tranquilizers down to $6 \%$ for heroin.

- Nearly half of all seniors (45\%) report no exposure to illicit drugs other than marijuana during the prior year.

- Regarding cigarette smoking, just over one in every five seniors $(21.5 \%)$ reports that most or all of his or her friends smoke, although $88 \%$ have some friends who smoke.

\section{Recent Trends in Exposure to Drug Use}

- During the two-year interval from 1976 to 1978, seniors' reports of exposure to marijuana use increased in just about the same proportion as percentages of actual monthly use. In 1979 both exposure to use and actual use stabilized, and since 1979 both have been dropping. The proportion saying they are often around people using marijuana decreased from $39 \%$ in 1979 to $24 \%$ in 1986 - a drop of more than one-third in the past seven years.

- Cocaine had a consistent increase from 1976 to 1979 in the proportions exposed to users. From 1979 to 1984 there was 
TABLE 21

Trends in Exposure to Drug Use

(Entrios are percentages)

Q. During the LAST 12 MONTHS hou often have you been around people whe high or for "kicks"?

Marijuana

Toying not at nll

\% saying often

1.SI)

\% saying not at all

\% saying often

Other psychedelics

Th saying not at all

\% saying oflen

Cocaine

wo saying not at all

\% saying oflen

Heroin

\% saying not nt nll

$\%$ saying often

Other narrotics

\% saying not at all

os saying ofen

Amphetamines

\% saying not at all

\% saying often

Barbiturates

osaying not at all

\% saying oflen

Tranquilizers

\% saying not at. nll

\% snying often

Alcoholic beverages

\% saying not at all

\% saying often

Any illicit drug ${ }^{\mathrm{a}}$

\% saying not at all

\% saying often

Any illicit drug ${ }^{\mathrm{a}}$ other than marijuan

\% saying often

Approx. $\mathrm{N}=$ were taking each of the following to get

\% saying not at all

\begin{tabular}{|c|c|c|c|c|c|c|c|c|c|c|c|c|}
\hline $\begin{array}{c}\text { Class } \\
\text { of } \\
1975 \\
\end{array}$ & $\begin{array}{c}\text { Class } \\
\text { of } \\
1976 \\
\end{array}$ & $\begin{array}{c}\text { Class } \\
\text { of } \\
1977 \\
\end{array}$ & $\begin{array}{c}\text { Class } \\
\text { of } \\
1978 \\
\end{array}$ & $\begin{array}{c}\text { Clnss } \\
\text { of } \\
1979 \\
\end{array}$ & $\begin{array}{c}\text { Class } \\
\text { of } \\
1980 \\
\end{array}$ & $\begin{array}{c}\text { Cliss } \\
\text { of } \\
1981 \\
\end{array}$ & $\begin{array}{c}\text { Clnss } \\
\text { of } \\
1982 \\
\end{array}$ & $\begin{array}{c}\text { Class } \\
\text { of } \\
1983 \\
\end{array}$ & $\begin{array}{c}\text { Class } \\
\text { of } \\
1984 \\
\end{array}$ & $\begin{array}{c}\text { Class } \\
\text { of } \\
1985 \\
\end{array}$ & $\begin{array}{c}\text { Class } \\
\text { of } \\
1986 \\
\end{array}$ & $\begin{array}{l}\text { '85-' } 86 \\
\text { change } \\
\end{array}$ \\
\hline $\begin{array}{l}\text { NA } \\
\text { NA }\end{array}$ & $\begin{array}{l}20.5 \\
32.5\end{array}$ & $\begin{array}{l}19.0 \\
37.0\end{array}$ & $\begin{array}{l}17.3 \\
39.0\end{array}$ & $\begin{array}{l}17.0 \\
38.9\end{array}$ & $\begin{array}{l}18.0 \\
33.8\end{array}$ & $\begin{array}{l}19.8 \\
33.1\end{array}$ & $\begin{array}{l}22.1 \\
28.0\end{array}$ & $\begin{array}{l}23.8 \\
26.1\end{array}$ & $\begin{array}{l}25.6 \\
24.8\end{array}$ & $\begin{array}{l}26.5 \\
24.2\end{array}$ & $\begin{array}{l}28.0 \\
24.0\end{array}$ & $\begin{array}{l}+1.5 \\
-0.2\end{array}$ \\
\hline $\begin{array}{l}\text { NA } \\
\text { NA }\end{array}$ & $\begin{array}{r}78.8 \\
2.2\end{array}$ & $\begin{array}{r}80.0 \\
2.0\end{array}$ & $\begin{array}{r}81.9 \\
1.8\end{array}$ & $\begin{array}{r}81.9 \\
2.0\end{array}$ & $\begin{array}{r}82.8 \\
1.4\end{array}$ & $\begin{array}{r}82.6 \\
2.0\end{array}$ & $\begin{array}{r}83.9 \\
1.9\end{array}$ & $\begin{array}{r}86.2 \\
1.4\end{array}$ & $\begin{array}{r}87.5 \\
1.5\end{array}$ & $\begin{array}{r}86.8 \\
1.3\end{array}$ & $\begin{array}{r}86.9 \\
1.6\end{array}$ & $\begin{array}{l}+0.1 \\
+0.3\end{array}$ \\
\hline $\begin{array}{l}\mathrm{NA} \\
\mathrm{NA}\end{array}$ & $\begin{array}{r}76.5 \\
3.1\end{array}$ & $\begin{array}{r}76.7 \\
3.2\end{array}$ & $\begin{array}{r}76.7 \\
2.9\end{array}$ & $\begin{array}{r}77.6 \\
2.2\end{array}$ & $\begin{array}{r}79.6 \\
2.2\end{array}$ & $\begin{array}{r}82.1 \\
2.0\end{array}$ & $\begin{array}{r}83.2 \\
2.6\end{array}$ & $\begin{array}{r}86.9 \\
1.1\end{array}$ & $\begin{array}{r}87.3 \\
1.7\end{array}$ & $\begin{array}{r}87.5 \\
1.4\end{array}$ & $\begin{array}{r}88.2 \\
1.5\end{array}$ & $\begin{array}{l}+0.7 \\
+0.1\end{array}$ \\
\hline $\begin{array}{l}\text { NA } \\
\text { NA }\end{array}$ & $\begin{array}{r}77.0 \\
3.0\end{array}$ & $\begin{array}{r}73.4 \\
3.7\end{array}$ & $\begin{array}{r}69.8 \\
4.6\end{array}$ & $\begin{array}{r}64.0 \\
6.8\end{array}$ & $\begin{array}{r}62.3 \\
5.9\end{array}$ & $\begin{array}{r}63.7 \\
6.6\end{array}$ & $\begin{array}{r}65.1 \\
6.6\end{array}$ & $\begin{array}{r}66.7 \\
5.2\end{array}$ & $\begin{array}{r}64.4 \\
6.7\end{array}$ & $\begin{array}{r}61.7 \\
7.1\end{array}$ & $\begin{array}{r}62.6 \\
7.8\end{array}$ & $\begin{array}{l}+0.9 \\
+0.7\end{array}$ \\
\hline $\begin{array}{l}\text { NA } \\
\text { NA }\end{array}$ & $\begin{array}{r}91.1 \\
0.8\end{array}$ & $\begin{array}{r}90.3 \\
1.1\end{array}$ & $\begin{array}{r}91.8 \\
0.9\end{array}$ & $\begin{array}{r}92.1 \\
0.7\end{array}$ & $\begin{array}{r}92.6 \\
0.4\end{array}$ & $\begin{array}{r}93.4 \\
0.6\end{array}$ & $\begin{array}{r}92.9 \\
1.0\end{array}$ & $\begin{array}{r}91.9 \\
0.7\end{array}$ & $\begin{array}{r}94.0 \\
1.1\end{array}$ & $\begin{array}{r}94.5 \\
0.5\end{array}$ & $\begin{array}{r}94.0 \\
1.0\end{array}$ & $\begin{array}{l}-0.5 \\
+0.5\end{array}$ \\
\hline $\begin{array}{l}\text { NA } \\
\text { NA }\end{array}$ & $\begin{array}{r}81.9 \\
1.8\end{array}$ & $\begin{array}{r}81.3 \\
2.4\end{array}$ & $\begin{array}{r}81.8 \\
2.0\end{array}$ & $\begin{array}{r}82.0 \\
1.7\end{array}$ & $\begin{array}{r}80.4 \\
1.7\end{array}$ & $\begin{array}{r}82.5 \\
1.7 \\
.\end{array}$ & $\begin{array}{r}81.5 \\
2.4\end{array}$ & $\begin{array}{r}82.7 \\
2.2\end{array}$ & $\begin{array}{r}82.0 \\
2.0\end{array}$ & $\begin{array}{r}81.6 \\
1.8\end{array}$ & $\begin{array}{r}84.4 \\
2.1\end{array}$ & $\begin{array}{l}+2.8 \mathrm{~s} \\
+0.3\end{array}$ \\
\hline $\begin{array}{l}\text { NA } \\
\text { NA }\end{array}$ & $\begin{array}{r}59.6 \\
6.8\end{array}$ & $\begin{array}{r}60.3 \\
7.9\end{array}$ & $\begin{array}{r}60.9 \\
6.7\end{array}$ & $\begin{array}{r}58.1 \\
7.4\end{array}$ & $\begin{array}{r}59.2 \\
8.3\end{array}$ & $\begin{array}{l}50.5 \\
12.1\end{array}$ & $\begin{array}{l}49.8 \\
12.3\end{array}$ & $\begin{array}{l}53.9 \\
10.1\end{array}$ & $\begin{array}{r}55.0 \\
9.0\end{array}$ & $\begin{array}{r}59.0 \\
6.5\end{array}$ & $\begin{array}{r}63.5 \\
5.8\end{array}$ & $\begin{array}{l}+4.5 \mathrm{ss} \\
-0.7\end{array}$ \\
\hline $\begin{array}{l}\text { NA } \\
\text { NA }\end{array}$ & $\begin{array}{r}69.0 \\
4.5\end{array}$ & $\begin{array}{r}70.0 \\
5.0\end{array}$ & $\begin{array}{r}73.5 \\
3.1\end{array}$ & $\begin{array}{r}73.6 \\
3.3\end{array}$ & $\begin{array}{r}74.8 \\
3.4\end{array}$ & $\begin{array}{r}74.1 \\
1.0\end{array}$ & $\begin{array}{r}74.3 \\
4.3\end{array}$ & $\begin{array}{r}77.5 \\
3.0\end{array}$ & $\begin{array}{r}78.8 \\
2.7\end{array}$ & $\begin{array}{r}81.1 \\
1.7\end{array}$ & $\begin{array}{r}84.2 \\
2.1\end{array}$ & $\begin{array}{l}+3.1 \mathrm{ss} \\
+0.4\end{array}$ \\
\hline $\begin{array}{l}\text { NA } \\
\text { NA }\end{array}$ & $\begin{array}{r}67.7 \\
5.5\end{array}$ & $\begin{array}{r}666,0 \\
6.3\end{array}$ & $\begin{array}{r}67.5 \\
4.9\end{array}$ & $\begin{array}{r}67.5 \\
4.3\end{array}$ & $\begin{array}{r}70.9 \\
3.2\end{array}$ & $\begin{array}{r}71.0 \\
4.2\end{array}$ & $\begin{array}{r}73.4 \\
3.5\end{array}$ & $\begin{array}{r}76.5 \\
2.9\end{array}$ & $\begin{array}{r}76.9 \\
2.9\end{array}$ & $\begin{array}{r}76.6 \\
2.2\end{array}$ & $\begin{array}{r}80.4 \\
2.5\end{array}$ & $\begin{array}{l}+3.8 \mathrm{ss} \\
+0.3\end{array}$ \\
\hline $\begin{array}{l}\text { NA } \\
\text { NA }\end{array}$ & $\begin{array}{r}6.0 \\
57.1\end{array}$ & $\begin{array}{r}5.6 \\
60.8\end{array}$ & $\begin{array}{r}5.5 \\
60.8\end{array}$ & $\begin{array}{r}5.2 \\
61.2\end{array}$ & $\begin{array}{r}5.3 \\
60.2\end{array}$ & $\begin{array}{r}6.0 \\
61.0\end{array}$ & $\begin{array}{r}6.0 \\
59.3\end{array}$ & $\begin{array}{r}6.0 \\
60.2\end{array}$ & $\begin{array}{r}6.0 \\
58.7\end{array}$ & $\begin{array}{r}6.0 \\
59.5\end{array}$ & $\begin{array}{r}5.9 \\
58.0\end{array}$ & $\begin{array}{l}-0.1 \\
-1.5\end{array}$ \\
\hline $\begin{array}{l}\text { NA } \\
\text { NA }\end{array}$ & $\begin{array}{l}17.4 \\
34.8\end{array}$ & $\begin{array}{l}16.5 \\
39.0\end{array}$ & $\begin{array}{l}15.1 \\
40.7\end{array}$ & $\begin{array}{l}15.0 \\
10.4\end{array}$ & $\begin{array}{l}15.7 \\
36.3\end{array}$ & $\begin{array}{l}17.3 \\
36.1\end{array}$ & $\begin{array}{l}18.6 \\
31.4\end{array}$ & $\begin{array}{l}20.6 \\
29.8\end{array}$ & $\begin{array}{l}22.1 \\
28.3\end{array}$ & $\begin{array}{l}22.3 \\
27.2\end{array}$ & $\begin{array}{l}24.5 \\
26.3\end{array}$ & $\begin{array}{l}+2.2 \\
-0.9\end{array}$ \\
\hline $\begin{array}{l}\text { NA } \\
\text { NA }\end{array}$ & $\begin{array}{l}11.9 \\
11.8\end{array}$ & $\begin{array}{l}44.2 \\
13.5\end{array}$ & $\begin{array}{l}44.7 \\
12.1\end{array}$ & $\begin{array}{l}41.7 \\
13.7\end{array}$ & $\begin{array}{l}41.5 \\
14.1\end{array}$ & $\begin{array}{l}37.4 \\
17.1\end{array}$ & $\begin{array}{l}37.5 \\
16.6\end{array}$ & $\begin{array}{l}40.6 \\
14.2\end{array}$ & $\begin{array}{l}40.2 \\
14.6\end{array}$ & $\begin{array}{l}40.7 \\
12.9\end{array}$ & $\begin{array}{l}44.7 \\
12.1\end{array}$ & $\begin{array}{l}+4.0 \mathrm{~s} \\
-0.8\end{array}$ \\
\hline (NA) & (2950) & (3075) & $(3682)$ & (3253) & (3259) & (3608) & (3645) & (3334) & (3238) & (3252) & $(3078)$ & \\
\hline
\end{tabular}

NOTES: Level of significance of difference between the two most recent classes: $s=.05, \mathrm{ks}=.01$, $\mathrm{sss}=, 001$. NA indicntes data not available,

"These estimates were derived from responses to the questions listed nbove. "Any illicit drug" includes all drugs listed except alcohol. 
little change in exposure to use coinciding with a period of stability in self-reported use; but in 1985 and 1986 there was an increase in the proportion saying they were often around people using cocaine ( $8 \%$ in 1986).

- From 1979 to 1983 there had been a statistically significant decrease in exposure to others (including close friends) using psychedelics other than LSD (including PCP), which coincided with a continued decline in the self-reported use of this class of drugs. There has been little or no further change since 1983, however, in exposure to use.

- Exposure to tranquilizer use has declined gradually since 1976 , as has actual use.

- There also had been a gradual decrease in exposure to barbiturates and LSD, from 1975 through 1980. However, exposure to the use of both of these drugs then remained level for two years, as did the usage figures. Both drugs have shown further decline in use since 1981, although LSD did not show a further decline in exposure this year.

- Trend data are available only since 1979 on friends' use of $P C P$ or the nitrites. For both drugs, exposure to friends' use had dropped significantly between 1979 and 1983 . Only half as many seniors in $1983(14 \%)$ said any of their friends used PCP compared with seniors in 1979 (28\%). The corresponding drop for nitrites was from $22 \%$ to $15 \%$. Since 1983, however, there has been rather little systematic change.

- The proportion having some friends who used amphetamines rose from $41 \%$ to $51 \%$ between 1979 and 1982-paralleling the sharp increase in reported use over that period. The proportion saying they were around people using amphetamines "to get high or for kicks" also jumped substantially between 1980 and 1982 (by $9 \%$ ). ${ }^{19}$ It then fell back $14 \%$ in the last four years (as actual use has declined).

- Between 1978 and 1981 methaqualone use rose, as did the proportion of seniors saying some of their friends used. A decline in both use and exposure started in 1982, and by

${ }^{19}$ This finding was important, since it indicated that a substantial part of the increase observed in self-reported amphetamine use was due to things other than simply an increase in the use of over-the-counter diet pills or stayawake pills, which presumably are not used to get high. Obviously, more young people were using stimulants for recreational purposes. There still remained the question, of course, of whether the active ingredients in those stimulants really were amphetamines. 
1986 there were $12 \%$ fewer seniors saying they had any friends who use Quaaludes (from 35\% to $23 \%$ between 1981 and 1986).

- The proportion saying that "most or all" of their friends smoke cigarettes dropped steadily and substantially between 1976 and 1981, from $37 \%$ to $22 \%$. (During this period actual use dropped markedly, and more seniors perceived their friends as disapproving regular smoking.) After 1981, friends' use (as well as self-reported use) remained relatively stable, and in 1986 is only $1 \%$ lower than in 1981 . In 1977, the peak year, $34 \%$ said most or all of their friends smoked; in $1981,22.4 \%$, and in $1986,21.5 \%$.

- The proportion saying most or all of their friends get drunk at least once a week had been increasing steadily, between 1976 and 1979 , from $27 \%$ to $32 \%$-during a period in which the prevalence of occasional heavy drinking was rising by about the same amount. After that, there was little change in either measure until 1984, when both declined for the first time. In 1985 reported friends' use did not decline, though self-reported use did. In 1986 perceived friends' use increased but self-reported use stayed the same. But without question, what remains the most impressive fact here is that nearly a third of all high school seniors (32\% in 1986) say that most or all of their friends get drunk at least once a week!

\section{IMPLICATIONS FOR VALIDITY OF SELF-REPORTED USAGE QUESTIONS}

- We have noted a high degree of correspondence in the aggregate level data presented in this report among seniors' self-reports of their own drug use, their reports concerning friends ${ }^{4}$ use, and their own exposure to use. Drug-to-drug comparisons in any given year across these three types of measures tend to be highly parallel, as are the changes frorn year to year. ${ }^{20}$ We take this consistency as additional evidence for the validity of the self-report data, and of trends in the self-report data, since there should be less reason to distort answers on friends' use, or general exposure to use, than to distort the reporting of one's own use.

${ }^{20}$ Those minor instances of noncorrespondence may well result from the larger sampling errors in our estimates of these environmental variables, which are measured on a sample size one-fifth the size of the self-reported usage measures. 


\section{PERCEIVED AVAILABILITY OF DRUGS}

One set of questions asks for estimates of how difficult it would be to obtain each of a number of different drugs. The answers range across five categories from "probably impossible" to "very easy." While no systematic effort has been undertaken to assess directly the validity of these measures, it must be said that they do have a rather high level of face validity-particularly if it is the subjective reality of "perceived availability" which is purported to be measured. It also seems quite reasonable to us to assume that perceived availability tracks actual availability to some extent.

\section{Perceived Availability in 1986}

- There are substantial differences in the reported availability of the various drugs. In general, the more widely used drugs are reported to be available by the highest proportion of the age group, as would be expected (see Table 22 and Figure 25).

- Marijuana appears to be almost universally available to high school seniors; some $85 \%$ report that they think it would be "very easy" or "fairly easy" for them to get-34\% more than the number who report ever having used it.

- After marijuana, the students indicate that the psychotherapeutic drugs are among the most available to them: amphetamines are seen as available by $64 \%$, tranquilizers by $51 \%$, and barbiturates by $48 \%$.

- More than half of the seniors $(52 \%)$ see cocaine as readily available to them.

- LSD, other psychedelics, and opiates other than heroin are reported as available by only about one of every three or four seniors $(29 \%, 25 \%$, and $32 \%$, respectively).

- Heroin is seen by the fewest seniors (22\%) as being easy to get.

- The great majority (more than two-thirds) of "recent users" of all drugs-those who have illicitly used the drug in the past year-feel that it would be easy for them to get that same type of drug. (Data not shown here.)

\section{Trends in Perceived Availability}

- Marijuana, for the first time since the study was begun in 1975 , showed a small but statistically significant decline in 


\section{FIGURE 25}

Trends in Perceived Availability of Drugs

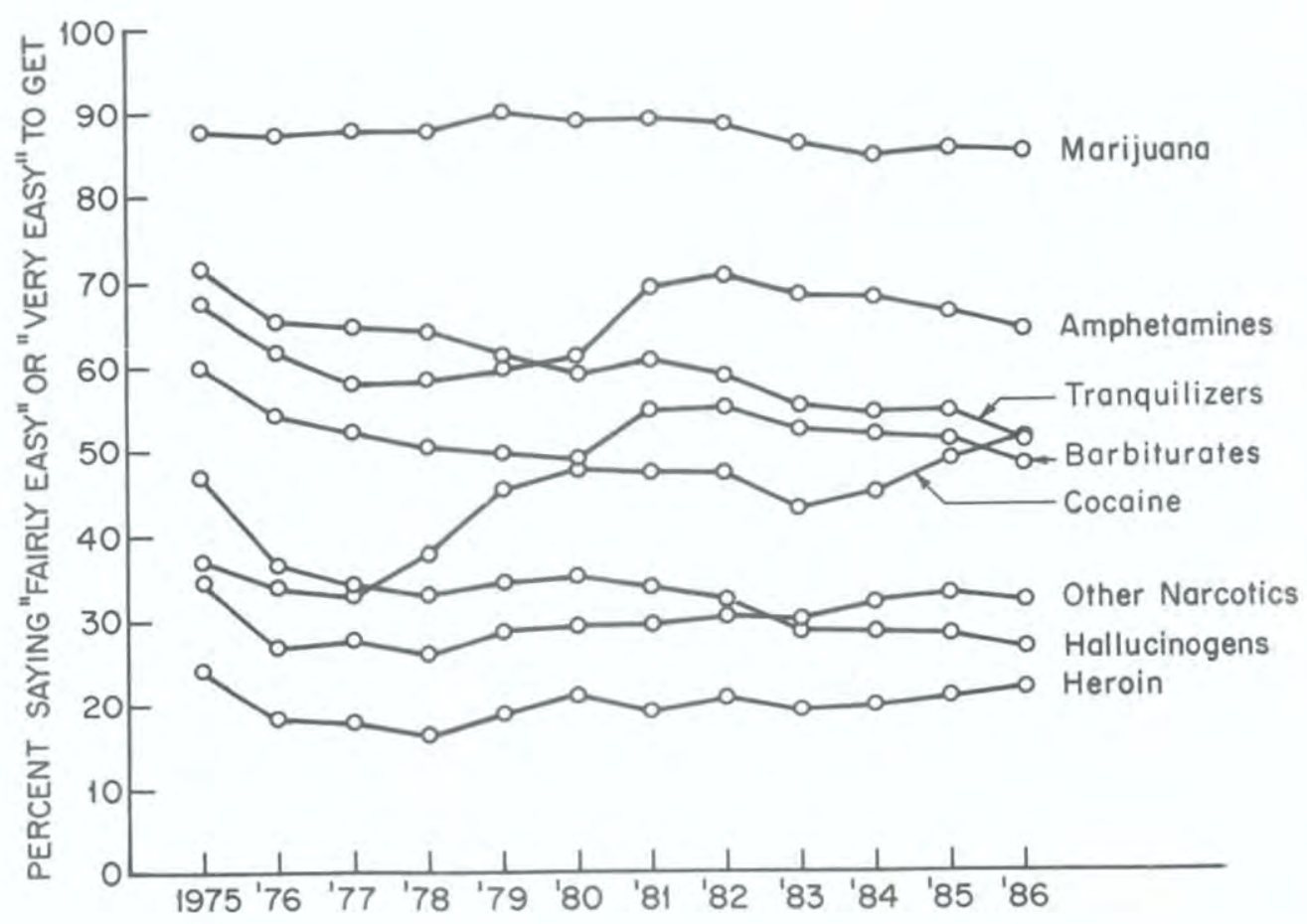


TABLE 22

\section{Trends in Reported Availability of Drugs}

\begin{tabular}{|c|c|c|c|c|c|c|c|c|c|c|c|c|c|c|}
\hline \multirow[b]{2}{*}{ Q. } & \multirow[b]{2}{*}{$\begin{array}{l}\text { How difficult do you think } \\
\text { it would be for you to } \\
\text { get each of the following } \\
\text { types of drugs, if you } \\
\text { wanted some? }\end{array}$} & \multicolumn{12}{|c|}{$\begin{array}{l}\text { Percentage saying drug would be "Fairly } \\
\text { easy" or "Very easy" for them to get }\end{array}$} & \multirow[b]{2}{*}{$\begin{array}{l}\text { '85-' } 86 \\
\text { change }\end{array}$} \\
\hline & & $\begin{array}{c}\text { Class } \\
\text { of } \\
1975 \\
\end{array}$ & $\begin{array}{c}\text { Class } \\
\text { of } \\
1976 \\
\end{array}$ & $\begin{array}{c}\text { Class } \\
\text { of } \\
1977 \\
\end{array}$ & $\begin{array}{c}\text { Class } \\
\text { of } \\
1978 \\
\end{array}$ & $\begin{array}{c}\text { Class } \\
\text { of } \\
1979 \\
\end{array}$ & $\begin{array}{c}\text { Class } \\
\text { of } \\
1980 \\
\end{array}$ & $\begin{array}{c}\text { Class } \\
\text { of } \\
1981 \\
\end{array}$ & $\begin{array}{c}\text { Class } \\
\text { of } \\
1982 \\
\end{array}$ & $\begin{array}{c}\text { Class } \\
\text { of } \\
1983 \\
\end{array}$ & $\begin{array}{c}\text { Class } \\
\text { of } \\
1984 \\
\end{array}$ & $\begin{array}{c}\text { Class } \\
\text { of } \\
1985 \\
\end{array}$ & $\begin{array}{c}\text { Class } \\
\text { of } \\
1986 \\
\end{array}$ & \\
\hline \multirow[b]{5}{*}{ jo } & Marijuana & 87.8 & 87.4 & 87.9 & 87.8 & 90.1 & 89.0 & 89.2 & 88.5 & 86.2 & 84.6 & 85.5 & 85.2 & -0.3 \\
\hline & LSD & 46.2 & 37.4 & 34.5 & 32.2 & 34.2 & 35.3 & 35.0 & 34.2 & 30.9 & 30.6 & 30.5 & 28.5 & -2.0 \\
\hline & Some other psychedelic & 47.8 & 35.7 & 33.8 & 33.8 & 34.6 & 35.0 & 32.7 & 30.6 & 26.6 & 26.6 & 26.1 & 24.9 & -1.2 \\
\hline & Cocaine & 37.0 & 34.0 & 33.0 & 37.8 & 45.5 & 47.9 & 47.5 & 47.4 & 43.1 & 45.0 & 48.9 & 51.5 & +2.6 \\
\hline & Heroin & 24.2 & 18.4 & 17.9 & 16.4 & 18.9 & 21.2 & 19.2 & 20.8 & 19.3 & 19.9 & 21.0 & 22.0 & +1.0 \\
\hline & $\begin{array}{l}\text { Some other narcotic } \\
\text { (including methadone) }\end{array}$ & 34.5 & 26.9 & 27.8 & 26.1 & 28.7 & 29.4 & 29.6 & 30.4 & 30.0 & 32.1 & 33.1 & 32.2 & -0.9 \\
\hline & Amphetamines & 67.8 & 61.8 & 58.1 & 58.5 & 59.9 & 61.3 & 69.5 & 70.8 & 68.5 & 68.2 & 66.4 & 64.3 & -2.1 \\
\hline & Barbiturates & 60.0 & 54.4 & 52.4 & 50.6 & 49.8 & 40.1 & 54.9 & 55.2 & 52.5 & 51.9 & 51.3 & 48.3 & -3.0 \\
\hline & Tranquilizers & 71.8 & 65.5 & 64.9 & (64.3 & 61.4 & 59.1 & 60.8 & 58.9 & 55.3 & 54.5 & 54.7 & 51.2 & $-3.5 s$ \\
\hline & Approx. $\mathrm{N}=$ & (2627) & $(2865)$ & $(3065)$ & (3598) & (3172) & $(3240)$ & (3578) & $(3602)$ & (3385) & (3269) & $(3274)$ & $(3077)$ & \\
\hline
\end{tabular}

NOTE: Level of significance of difference between the two most recent classes: $\mathrm{s}=.05, \mathrm{ss}=.01, \mathrm{sss}=.001$.

${ }^{a}$ Answer alternatives were: (1) Probably impossible, (2) Very diffcult, (3) Fairly difficult, (4) Fairly easy, and (5) Very easy. 
perceived availability (down $3.9 \%$ ) between 1982 and 1984, undoubtedly due to the reduced proportion of seniors who have friends who use. There has been little further change since then, and $85 \%$ of the class of 1986 think marijuana would be easy to get.

- Amphetamines showed a full $11 \%$ jump in availability between 1979 and 1982; but availability has dropped back by $6 \%$ in the four years since.

- The perceived availability of barbiturates also jumped about $6 \%$ between 1980 and 1982 , but dropped back by $7 \%$ in the subsequent four years.

- Between 1977 and 1980 there was a substantial (15\%) increase in the perceived availability of cocaine (see Figure 25 and Table 22). Among recent cocaine users there also was a substantial increase observed over that three-year interval (data not shown). Availability then levelled, and dropped some in 1983 and 1984, before rising significantly (by $4 \%$ ) in 1985 . Perceived availability rose another $2.6 \%$ in 1986, though actual use of cocaine remained the same or declined slightly.

- The availability of tranquilizers has been declining steadily since 1978.

- The perceived availability of LSD and other psychedelics dropped sharply between 1975 and 1978. LSD availability has decreased since 1978 by only $4 \%$ (from $32.2 \%$ to $28.5 \%$ ), but since 1978 the availability of other psychedelics showed a further decline of $9 \%$ by 1986 - a period during which the use of PCP dropped substantially.

- There has not been much change in the perceived availability of heroin since 1976.

- Other opiates have shown a very slight, gradual upward shift, from $27 \%$ in 1976 to $32 \%$ in 1986 .

- All these trends are similar among recent users. 


\section{YOUNG ADULTS POST-HIGH SCHOOL}




\section{PREVALENCE OF DRUG USE AMONG YOUNG ADULTS POST-HIGH SCHOOL}

As is described in the introduction to this report, the Monitoring the Future study has followed representative samples from each graduating class beginning with the class of 1976 . Two matched panels, of roughly 1200 seniors each, are selected from each graduating class-one panel being surveyed every even-numbered year after graduation, the other being surveyed every odd-numbered year. Thus, in a given year, the study encompasses one of the panels from each of the senior classes previously participating in the study. In 1986, this meant that representative samples of the classes of 1976 through 1985 were surveyed by mail.

In this section we present the results of that follow-up survey: results which should accurately characterize the approximately $85 \%$ of young adults in the class cohorts one to ten years beyond high school who are high school graduates. (They have modal ages between 19 and 28.) The high school dropout segment missing from the senior year surveys is, of course, missing from all of the follow-up segments, as well.

Figures 26 through 38 contain the 1986 prevalence data for all age groups covered, up through those who are ten years beyond high school (modal age of 28). Later figures will give the trend data for each age group, including seniors and graduates who are up to nine years past high school (modal age of 27). Age groups have been paired into twoyear intervals in both sets of figures to increase the number of cases, and thus the reliability, of each point estimate. For obvious reasons, trends on the youngest age bands can be calculated for the longest period of time. As the years pass and the earlier class cohorts get older, new age groups can be added to the figures.

\section{A NOTE ON LIFETIME PREVALENCE ESTIMATES}

In Figure 26 through 38 two different estimates of lifetime prevalence are provided-one based on the respondent's most recent statement of whether he or she ever used the drug in question (the solid line), and one based on the cumulated answers of the respondent across all previous data collections in which he or she participated (the dotted 
line). ${ }^{21}$ The former type of estimate is most commonly presented in epidemiological studies, since it can be made based on the data from a single cross-sectional survey. The latter is possible only when panel data have been gathered and a respondent can be classified as having used a drug at sometime in his or her life (based on earlier answers) even though he or she no longer indicates lifetime use in the most recent survey.

The divergence of these two lines as a function of age shows that there is more inconsistency as time passes. (Obviously there is more opportunity for inconsistency as the number of data collections increases.) Our judgement is that "the truth" lies somewhere between the two estimates, in that the lower estimate may be depressed by tendencies to forget or conceal earlier use and the upper estimate may include some earlier response errors or incorrect definitions of drugs which respondents corrected in later surveys. (It should be noted that a high proportion of those giving inconsistent answers across time had earlier reported use on only one or two occasions.) As we have reported elsewhere, cross-time stability of self-reported usage measures (which also take into account the number of occasions of self-reported use) is still very high. ${ }^{22}$

It also should be noted that the divergence between the two lifetime prevalence estimates is greatest for the psychotherapeutic drugs, (and the derivative index of "use of an illicit other than marijuana," which is heavily affected by the psychotherapeutic estimates). We believe this is due to the greater difficulty for respondents in categorizing such "pills" with a high degree of certainty-especially if they have used them only once or twice. One would expect higher inconsistency across time, when the event (in many of these cases a single event) is reported at quite different points in time with a relatively low degree of certainty. Those who have gone beyond simple experimentation with one of these drugs would undoubtedly be able to categorize them with a higher degree of certainty. Also, those who have experimented more recently (say in the past month or year) should have a higher probability of recall as well as more fresh information for accurately categorizing the drug.

We provide both estimates to make clear that a full use of respondent information provides a possible range for lifetime prevalence estimates, not a single point. However, by far the most important use of the prev-

${ }^{21}$ To be categorized as one who has used the drug based on all past answers regarding that drug, the respondent has either (a) to have reported past use in the most recent data collection and/or (b) to have reported some use in his or her lifetime on at least two earlier occasions.

${ }^{22}$ O'Malley, P.M., Bachman, J.G., \& Johnston, L.D. (1983). Reliability and consistency in self-reports of drug use. International Journal of the Addictions, 18, 805-824. 
alence data is to track trends in current (as opposed to lifetime) use; thus we are much less concerned about the nature of the variability in the lifetime estimates than we might otherwise be. The lifetime prevalence estimates are primarily of importance in showing the degsee to which a drug class has penetrated the general population.

A number of interesting findings emerge from the follow-up data. ${ }^{23}$

\section{PREVALENCE OF DRUG USE IN 1986 AMONG YOUNG ADULTS AS A FUNCTION OF AGE}

- For virtually all drugs, and for illicit drug use taken as a whole, older age groups exhibit higher levels of lifetime experience, but some of the age groups show levels of active or current use which are no higher than they are for high school seniors. For example, in 1986, among 27- and 28year-olds (who in 1986 are from the classes of 1977 and 1976), lifetime experience with any illicit drug approaches $80 \%$ versus $58 \%$ for high school seniors (who represent the class of 1986). However, those one to six years beyond high school have about the same annual and monthly prevalence rates on this index of overall illicit drug involvement as seniors, while those seven or more years beyond high school have slightly lower rates of active use. (See Figure 26.)

- A similar pattern exists for marijuana (including daily use, Figure 29), LSD (Figure 30), stimulants (Figure 33), and tranquilizers (Figure 36). That is, although lifetime prevalences increased with age, active rates of use for young adults in the first four to six years past high school are about what they are for seniors in high school. (For marijuana, the lifetime prevalence reached by respondents 9 to 10 years post-high school in 1986 is between $72 \%$ and $76 \%$.)

- It is perhaps particularly significant that daily marijuana $u$ se is not any lower among the older age groups than among high school seniors. This means that up through age 28 , at least, there is no evidence of a fall-off in active daily use as a function of age.

\footnotetext{
${ }^{23}$ In this section on post-high school drug use, we note some differences that seem to be consistently associated with age. We recognize that the separation of age effects from period or cohort effects is a difficult methodological task, and have dealt extensively with that issue elsewhere (O'Malley, P.M., Bachman, J.G., \& Johnston, L.D. (1984). Period, age, and cohort effects on substance use among American youth: 1976-1982. American Journal of Public Health, 74, 682-688). In this monograph we take a more descriptive approach, presenting the trend data along with those interpretations that we think are most reasonable.
} 
- The statistics on the use of any illicit drug other than marijuana (Figure 27) behave in a somewhat different fashion, however. Like marijuana and the any-illicit-drug-use index, lifetime rates on this index also show an appreciable rise with age, reaching $61 \%$ by age 28 .

However, among 19-24 year olds, the annual usage statistics are also slightly higher than among seniors. As the next several paragraphs illustrate, most of the drugs which constitute this category show a decline with age in annual prevalence. Thus, the one which shows an appreciable increase with age-namely, cocaine-must account for nearly all of the increase in the general category.

- Several classes of drugs show lower rates of current use among the older age groups than among seniors. $L S D$ in recent years has shown lower 30-day prevalence rates for the older ages than for seniors (Figure 30). (Annual prevalence rates also tend to be lower at present, though this has not always been true-reflecting a sharper decrease in use among the older age groups than among seniors.) We should add, however, that all of these prevalence rates are very low, and thus the differences are quite small.

- For stimulants, lifetime prevalence is again much higher among the older age groups (Figure 33)-reflecting the addition of new initiates in the early twenties. However, active use as reflected in the annual prevalence figure is somewhat lower among the older age groups at present. (Again, this is a result of a sharper decline in use in the older ages than has occurred among seniors.)

- For methaqualone, lifetime prevalence rises appreciably with age, but there is little age-related difference in annual prevalence at present among the post-high school age groups. High school seniors show a slightly higher annual prevalence than the older age groups (Figure 35 ).

- Barbiturates are similar to stimulants and methaqualone in that lifetime prevalence again rises appreciably with age, but slightly different in that active nonmedical use after high school has always been appreciably lower than during high school (Figure 34).

- Opiates other than heroin behave very similarly to barbiturates-some increase in lifetime prevalence with age, with active nonmedical use being lower in the years after high school than during high school (Figure 32). 
- Cocaine presents a somewhat unique case in that lifetime, annual, and current use all rise substantially with age, at least through age 24 (Figure 31). In 1986, lifetime prevalence by age $27-28$ was roughly $40 \%$, vs. $17 \%$ among today's high school seniors (and 10\% among the 27-28-year-old cohorts when they were seniors in the mid 1970's). Annual prevalence for 27-28-year-olds today is about $20 \%$ and 30 day prevalence around 8\%-again, appreciably higher than for the 1986 seniors. Clearly this is a drug which is used much more frequently among people in their twenties than among those in their late teens; and at the present time this fact distinguishes it from all of the other illicit drugs.

There is some evidence that active (thirty-day) cocaine use may drop off with age beyond age 24. In 1986, the thirtyday prevalence rates for those 5-6 years, 7-8 years, and 910 years beyond high school were $9.6 \%, 8.4 \%$, and $7.6 \%$ respectively.

- In the case of alcohol, lifetime prevalence varies rather little by age (obviously due to a "ceiling effect") but current use (in the past 30 days) does vary somewhat more by age, with a higher proportion of the older age groups drinking actively. Current daily drinking is also slightly higher in the older age groups (Figure 37).

Occasions of heavy drinking in the two weeks prior to the survey shows a more complex pattern (Figure 37 ), with those one to four years beyond high school showing a higher prevalence of such behaviors than seniors, but with those five or more years beyond high school dropping back to rates actually lower than those observed in senior year. We have interpreted this as a curvilinear age effect, since it seems to replicate across years and graduating classes (see footnote earlier in this section for reference).

- Cigarette smoking shows an unusual pattern in relation to age (Figure 38), in that current smoking (30-day prevalence) increases slightly with age, but heavy daily smoking increases appreciably more in proportional terms. This means that relatively few new people are recruited to smoking past high school, but many who previously were moderate smokers move into a pattern of heavier consumption during early adulthood. 


\section{SEX DIFFERENCES IN PREVALENCE AMONG YOUNG}

\section{ADULTS}

- Statistics on usage rates for young adults one to nine years beyond high school, combined, are given for the total sample and separately for males and females in Table 23.

- In general, it can be seen that most of the sex differences in drug use which pertained in high school may be found in this young adult sample as well. For example, somewhat more males than females report using any illicit drug during the prior year (46\% vs. $39 \%$ ). Males have higher annual prevalence rates in most of the illicit drugs - with the highest ratios pertaining for LSD, methaqualone, opiates other than heroin and cocaine.

Cocaine use is higher among males for cocaine generally, and for the specific form called "crack," which was used by $4.2 \%$ of males and $2.3 \%$ of females during the prior twelve months.

- Other large sex differences are to be found in daily marijuana use $(2.9 \%$ for females vs. $5.3 \%$ for males in 1986), daily alcohol use (3.4\% vs. $8.9 \%$ ), and occasions of drinking five or more drinks in a row in the prior two weeks $(26 \%$ vs. $50 \%)$. The sex difference in occasions of heavy drinking is greater than in high school.

- The use of stimulants, which was slightly higher among females in high school, is slightly higher among males in this post-high school period.

- For cigarettes, smoking at the rate of half-a-pack per day is almost identical for males and females (at 20\%), while smoking less heavily is slightly more prevalent among females as is true in high school. 
TABLE 23

Prevalence of Use of Thirteen Types of Drugs, 1986

Among Follow-Up Respondents 1-9 Years Beyond High School by Sex

Approx. Wtd. $\mathrm{N}=$

Marijuana

Annual

Thirty-Day

Daily

LSD

Annual

Thirty-Day

Cocaine

Annual

Thirty-Day

"Crack"b

Annual

Heroin

Annual

Thirty-Day

Other Opiates ${ }^{a}$

Annual

Thirty-Day

Stimulants, Adjusted ${ }^{\mathrm{a}, \mathrm{c}}$

Annual

Thirty-Day

Sedatives ${ }^{a}$

Annual

Thirty-Day

Barbiturates $^{a}$

Annual

Thirty-Day

Methaqualone $e^{a}$

Annual

Thirty-Day

Tranquilizers ${ }^{\mathrm{a}}$

Annual

Thirty-Day

Alcohol

Annual

Thirty-Day

Daily

$5+$ drinks in a row

in past two weeks

\section{Cigarettes}

Thirty-Day

Daily (Any)

1/2 pack or more per day

$\begin{array}{lll}\text { Males } & \text { Females } & \text { Total } \\ (2800) & (3400) & (6200)\end{array}$

40.5

26.0

5.3

33.9

18.7

36.9

22.0

4.0

4.5

1.9

3.1

1.5

0.4

0.9

23.3

10.3

16.4

6.6

19.6

8.3

4.2

2.3

3.2

0.1

0.2

0.2

0.0

0.0

0.1

3.8

2.6

0.9

3.2

1.0

11.3

10.3

10.8

4.0

4.1

4.1

3.4

2.8

3.1

0.9

0.8

0.9

2.6

0.7

2.2

2.4

0.7

1.6

0.3

1.0

1.3

0.2

0.2

5.6

2.0

4.9

5.2

1.5

1.7

90.5

81.4

8.9

87.4

70.4

88.8

75.4

3.4

5.9

49.9

25.5

36.7

30.2

23.8

31.3

25.7

19.9

30.8

24.9

19.8

19.9

aOnly drug use which was not under a doctor's orders is included here.

${ }^{6}$ This drug was asked about in only one of the five questionnaire forms. $N$ is one-fifth of $\mathrm{N}$ indicated.

${ }^{c}$ Based on the data from the revised question, which attempts to exclude the inappropriate reporting of non-prescription stimulants. 
FIGURE 26

\section{Any Illicit Drug: Lifetime, Annual, and Thirty-Day Prevalence Among Young Adults, 1986 by Age Group}

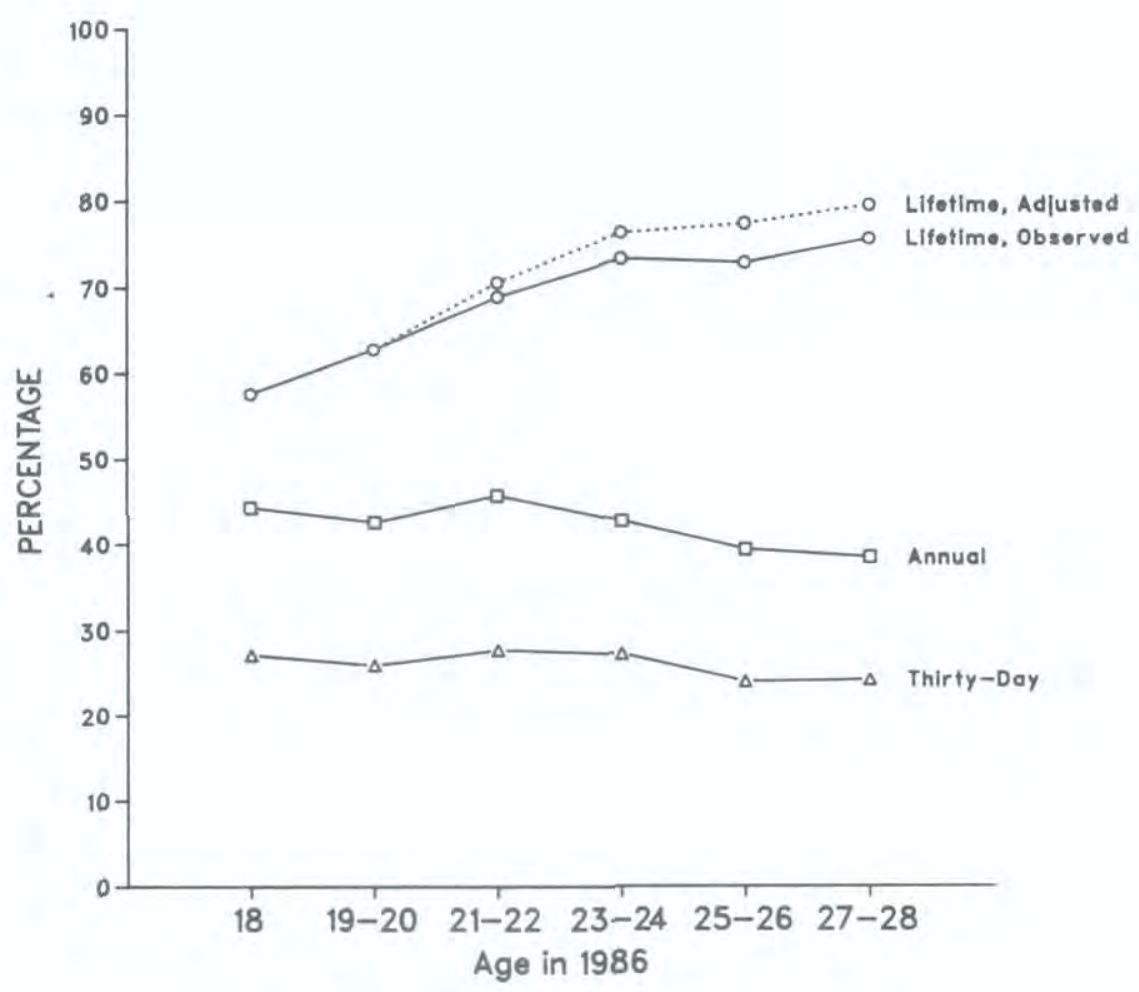

NOTE: Lifetime prevalence estimates were adjusted for inconsistency in selfreports of drug use over time. See text for discussion. 


\section{FIGURE 27}

Any Illicit Drug Other than Marijuana: Lifetime, Annual, and Thirty-Day Prevalence Among Young Adults, 1986 by Age Group

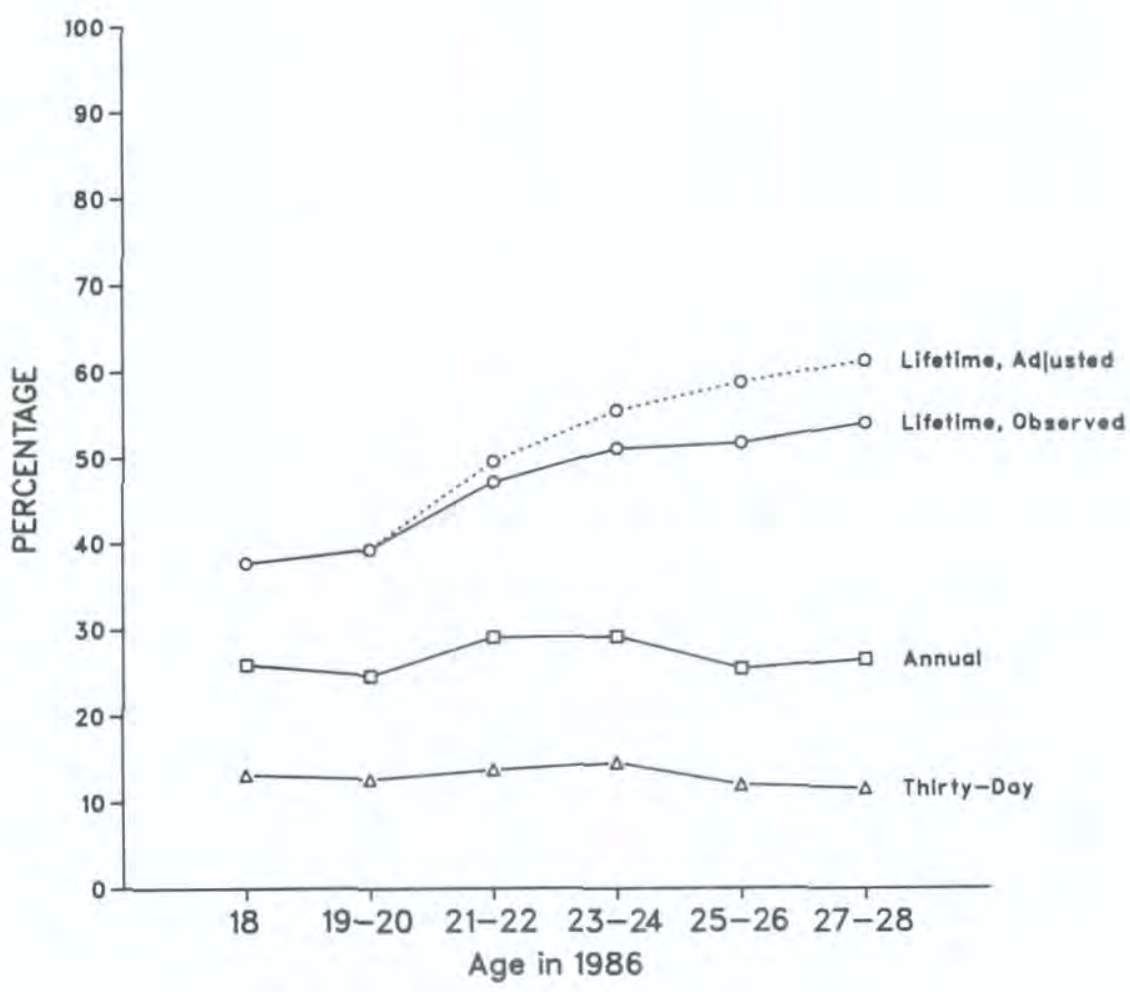

NOTE: Lifetime prevalence estimates were adjusted for inconsistency in selfreports of drug use over time. See text for discussion. 


\section{FIGURE 28}

Any Illicit Drug Other than Marijuana or Stimulants: Lifetime, Annual, and Thirty-Day Prevalence Among Young Adults, 1986 by Age Group

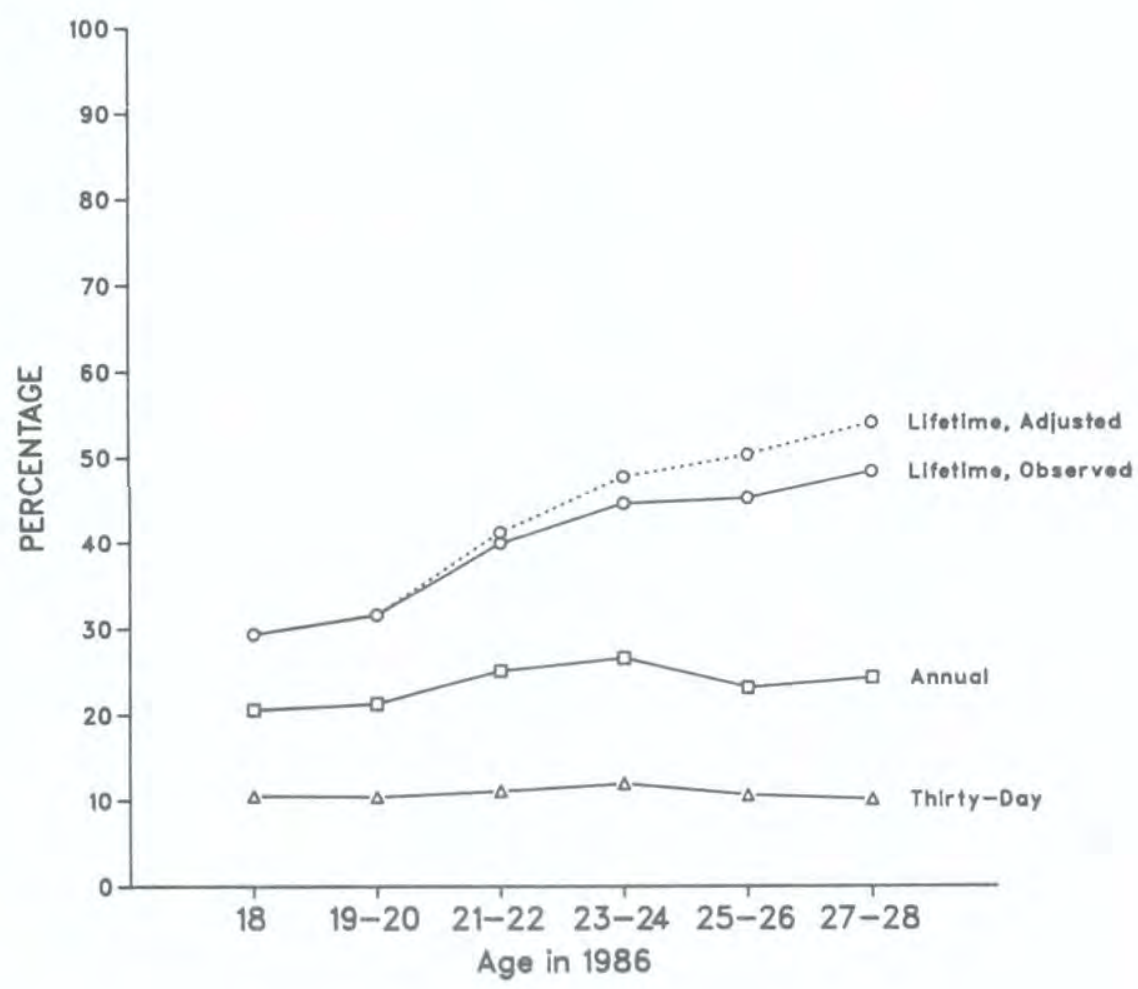

NOTE: Lifetime prevalence estimates were adjusted for inconsistency in selfreports of drug use over time. See text for discussion. 
FIGURE 29

\section{Marijuana: Lifetime, Annual, Thirty-Day, and Daily \\ Prevalence Among Young Adults, 1986 \\ by Age Group}

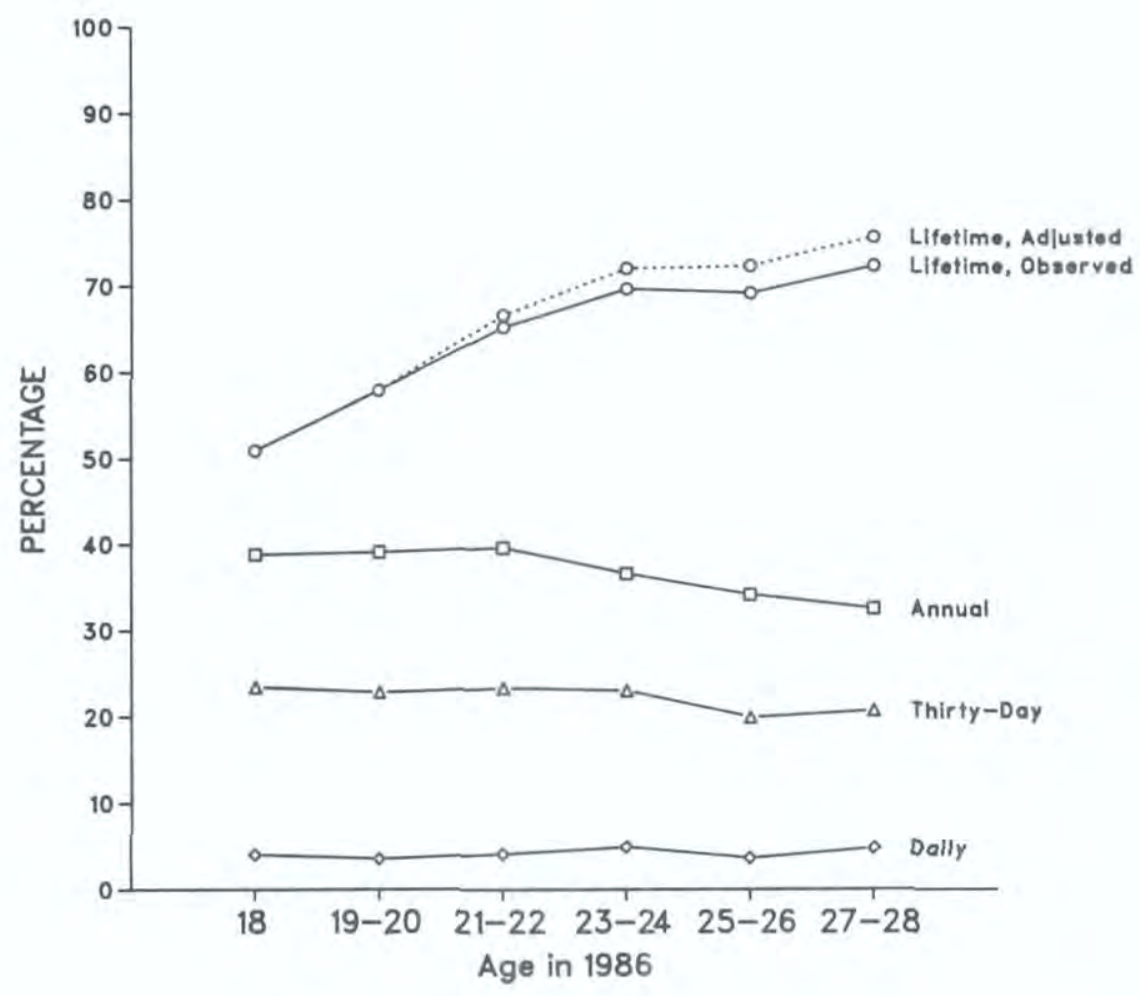

NOTE: Lifetime prevalence estimates were adjusted for inconsistency in selfreports of drug use over time. See text for discussion. 


\section{FIGURE 30}

LSD: Lifetime, Annual, and Thirty-Day Prevalence Among Young Adults, 1986 by Age Group

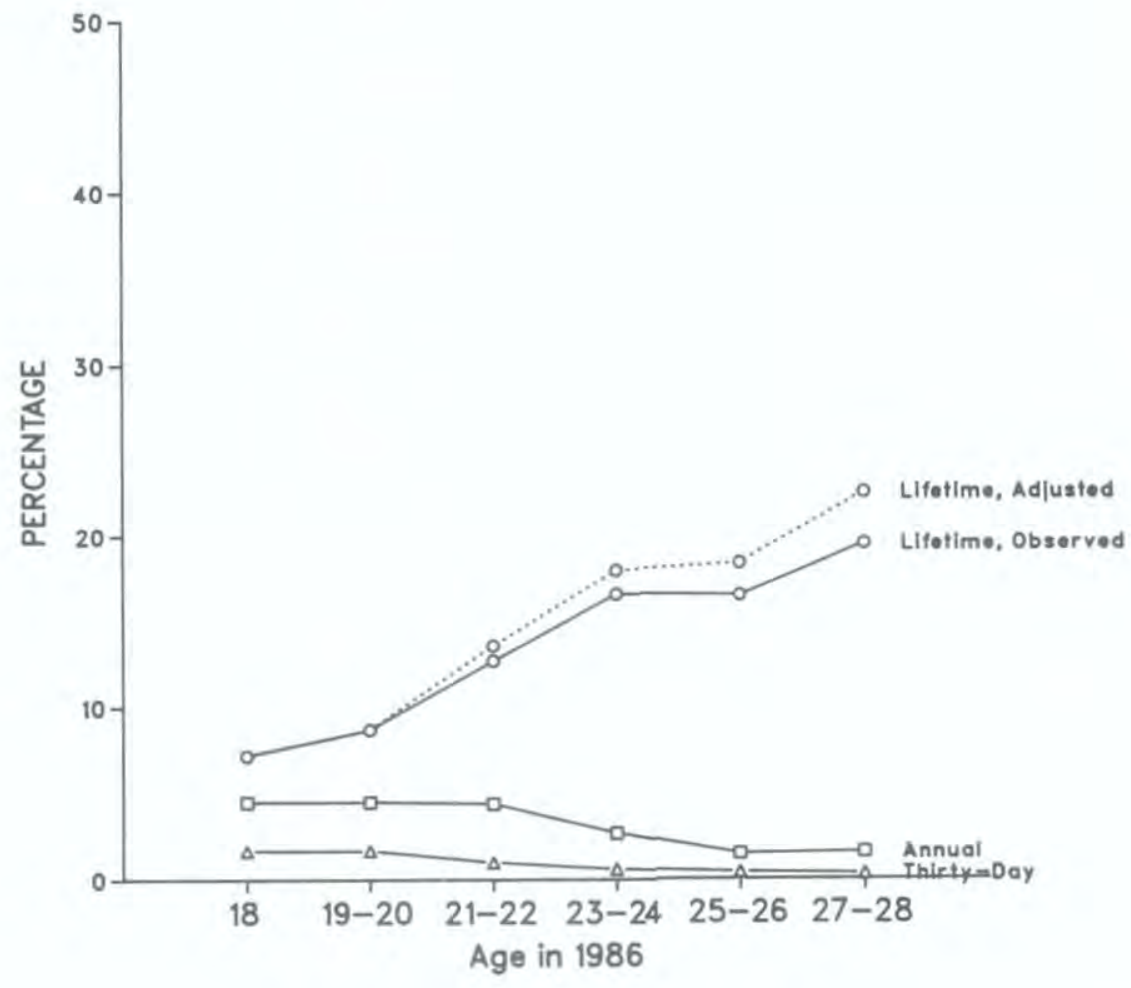

NOTE: Lifetime prevalence estimates were adjusted for inconsistency in selfreports of drug use over time. See text for discussion. 


\section{FIGURE 31}

Cocaine: Lifetime, Annual, and Thirty-Day

Prevalence Among Young Adults, 1986

by Age Group

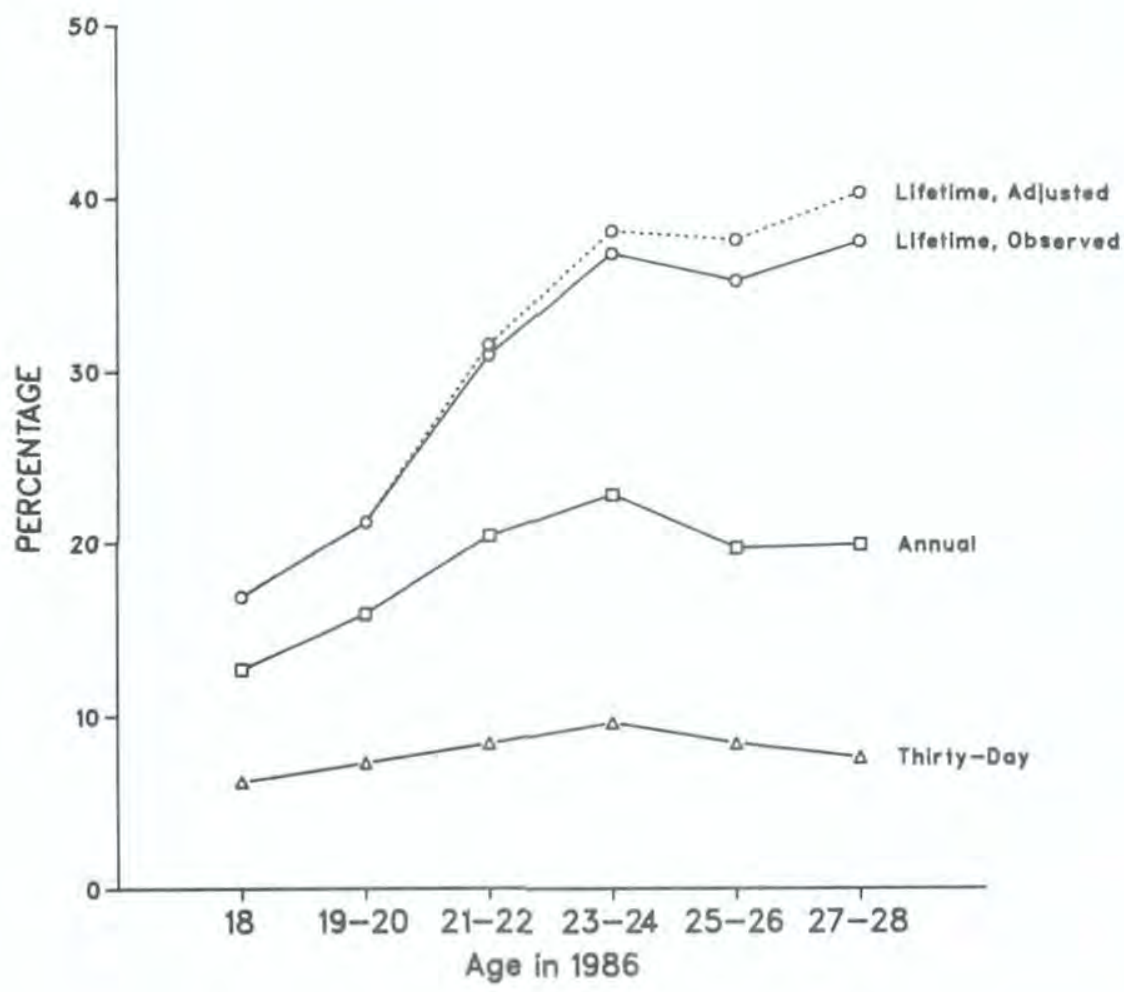

NOTE: Lifetime prevalence estimates were adjusted for inconsistency in selfreports of drug use over time. See text for discussion. 


\section{FIGURE 32}

Other Opiates: Lifetime, Annual, and Thirty-Day

Prevalence Among Young Adults, 1986

by Age Group

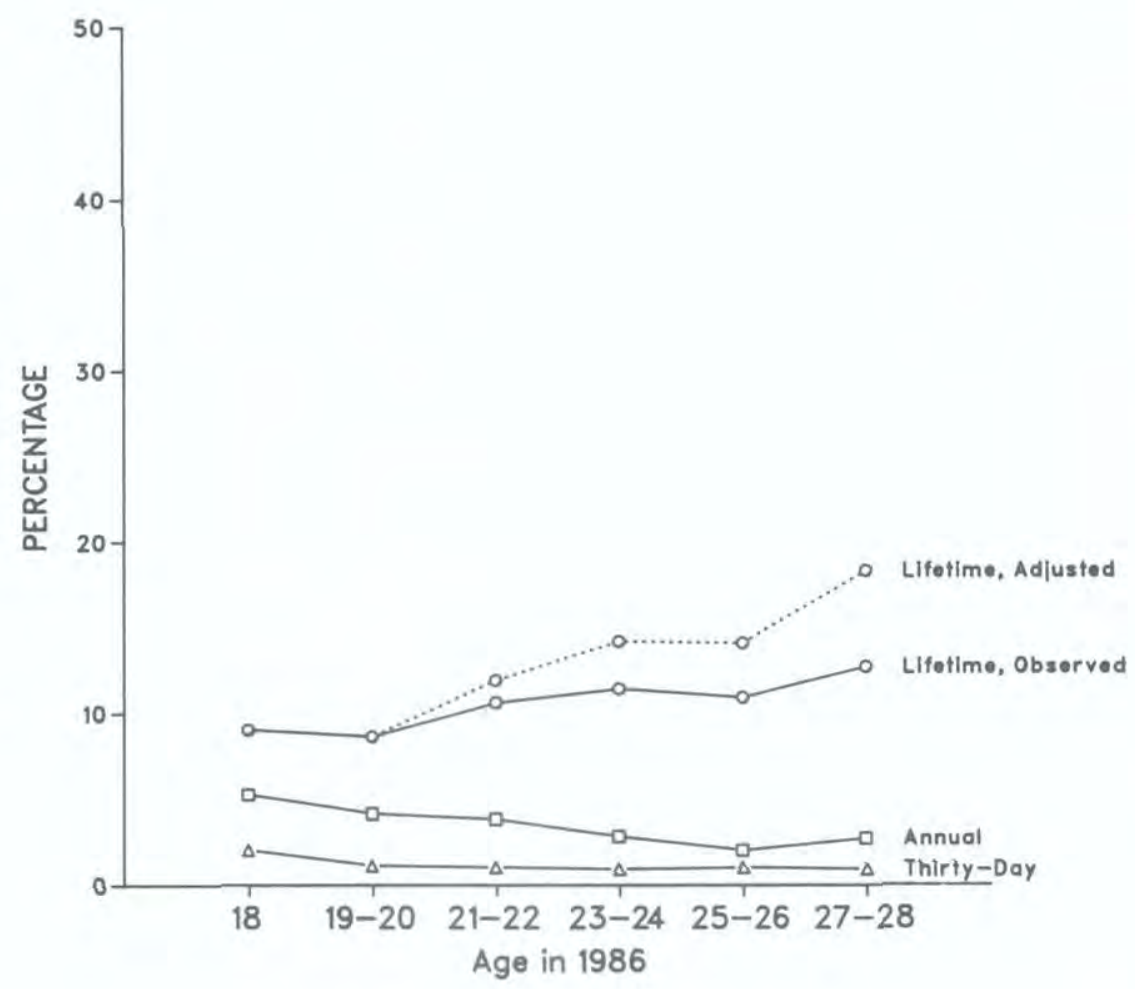

NOTE: Lifetime prevalence estimates were adjusted for inconsistency in selfreports of drug use over time. See text for discussion. 


\section{FIGURE 33}

\section{Stimulants: Lifetime, Annual, and Thirty-Day Prevalence Among Young Adults, 1986 by Age Group}

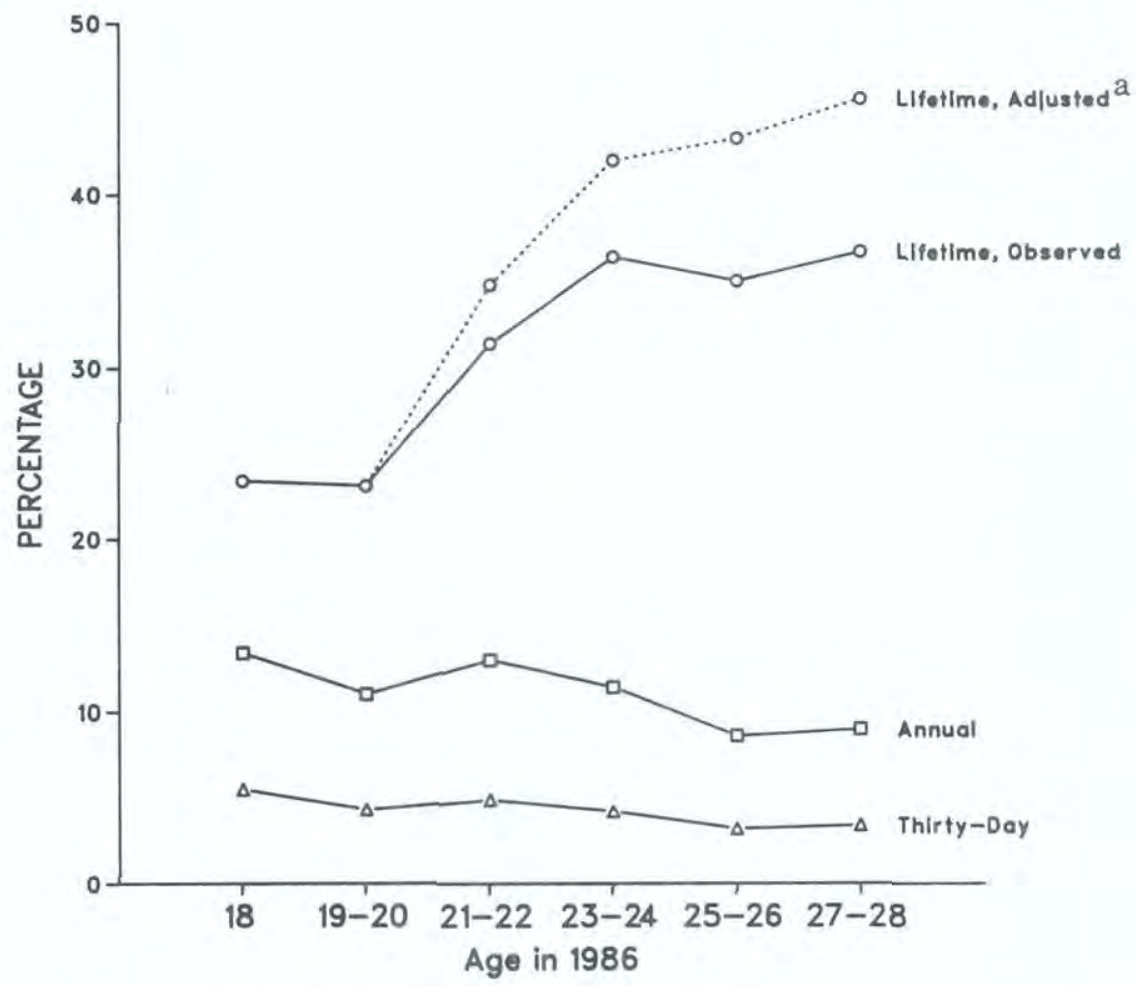

NOTE: Lifetime prevalence estimates were adjusted for inconsistency in selfreports of drug use over time. See text for discussion.

aThe divergence between the two lifetime prevalence estimates is due in part to the change in question wording initiated in 1982/1983, which clarified the instruction to omit non-prescription stimulants. 
FIGURE 34

\section{Barbiturates: Lifetime, Annual, and Thirty-Day \\ Prevalence Among Young Adults, 1986 by Age Group}

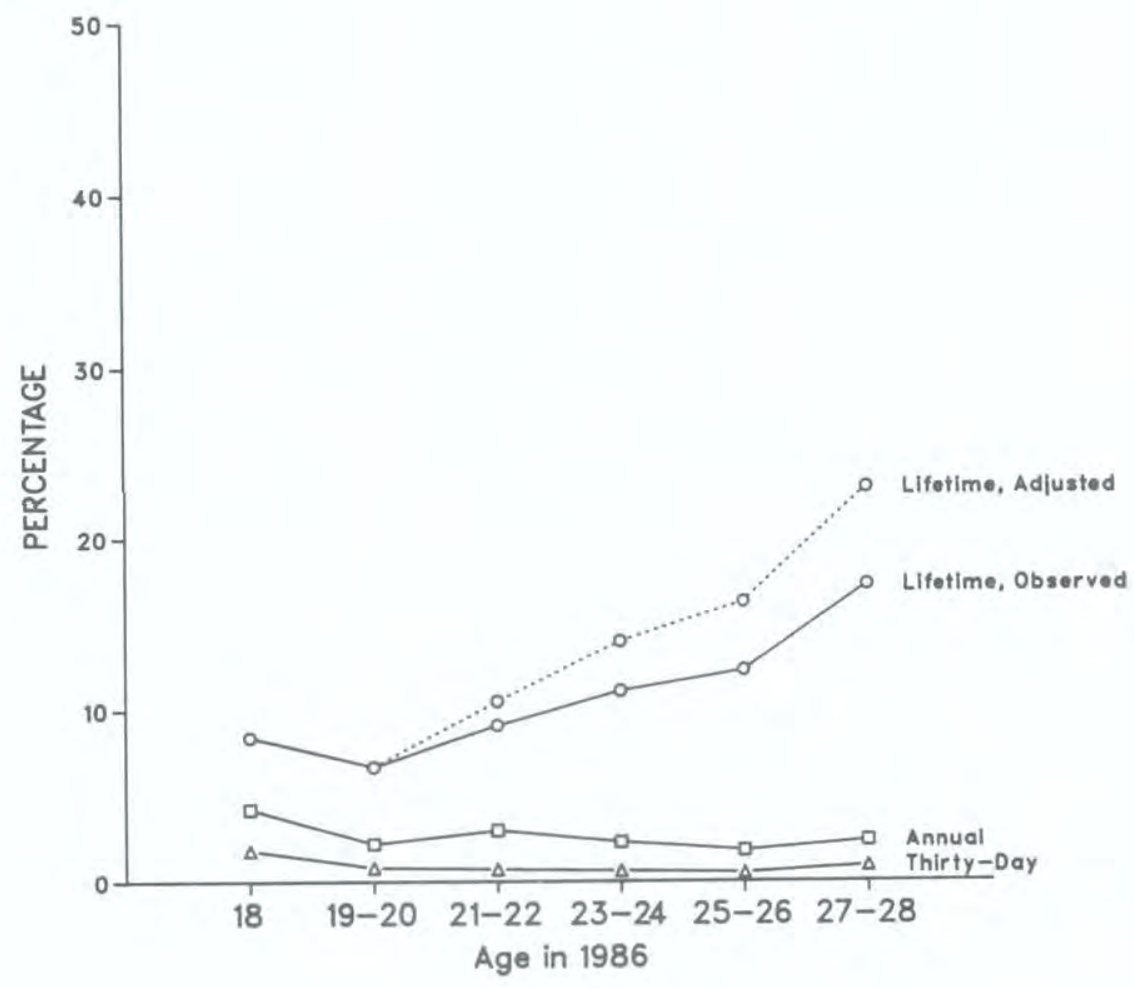

NOTE: Lifetime prevalence estimates were adjusted for inconsistency in selfreports of drug use over time. See text for discussion. 


\section{FIGURE 35}

\section{Methaqualone: Lifetime, Annual, and Thirty-Day \\ Prevalence Among Young Adults, 1986 \\ by Age Group}

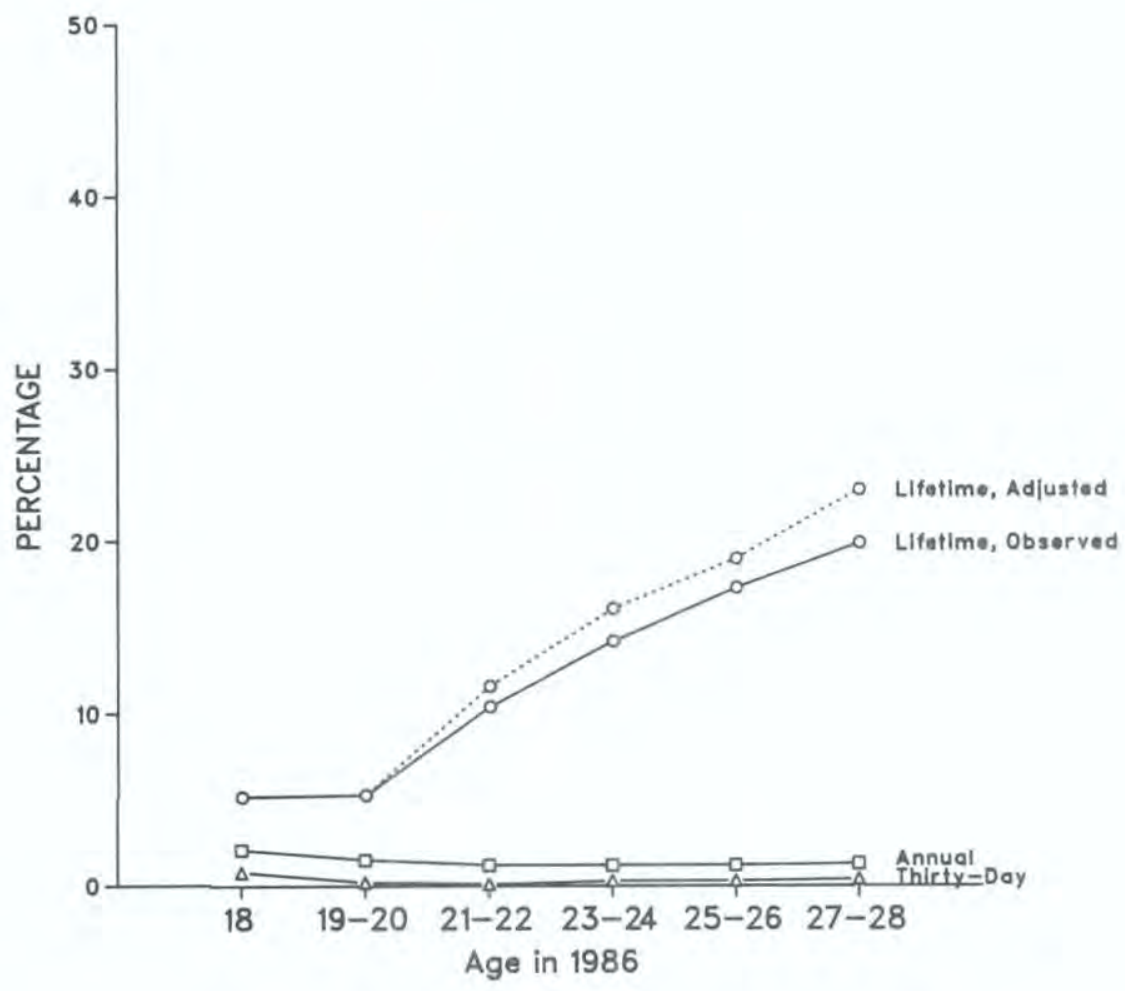

NOTE: Lifetime prevalence estimates were adjusted for inconsistency in selfreports of drug use over time. See text for discussion. 


\section{FIGURE 36}

Tranquilizers: Lifetime, Annual, and Thirty-Day

Prevalence Among Young Adults, 1986

by Age Group

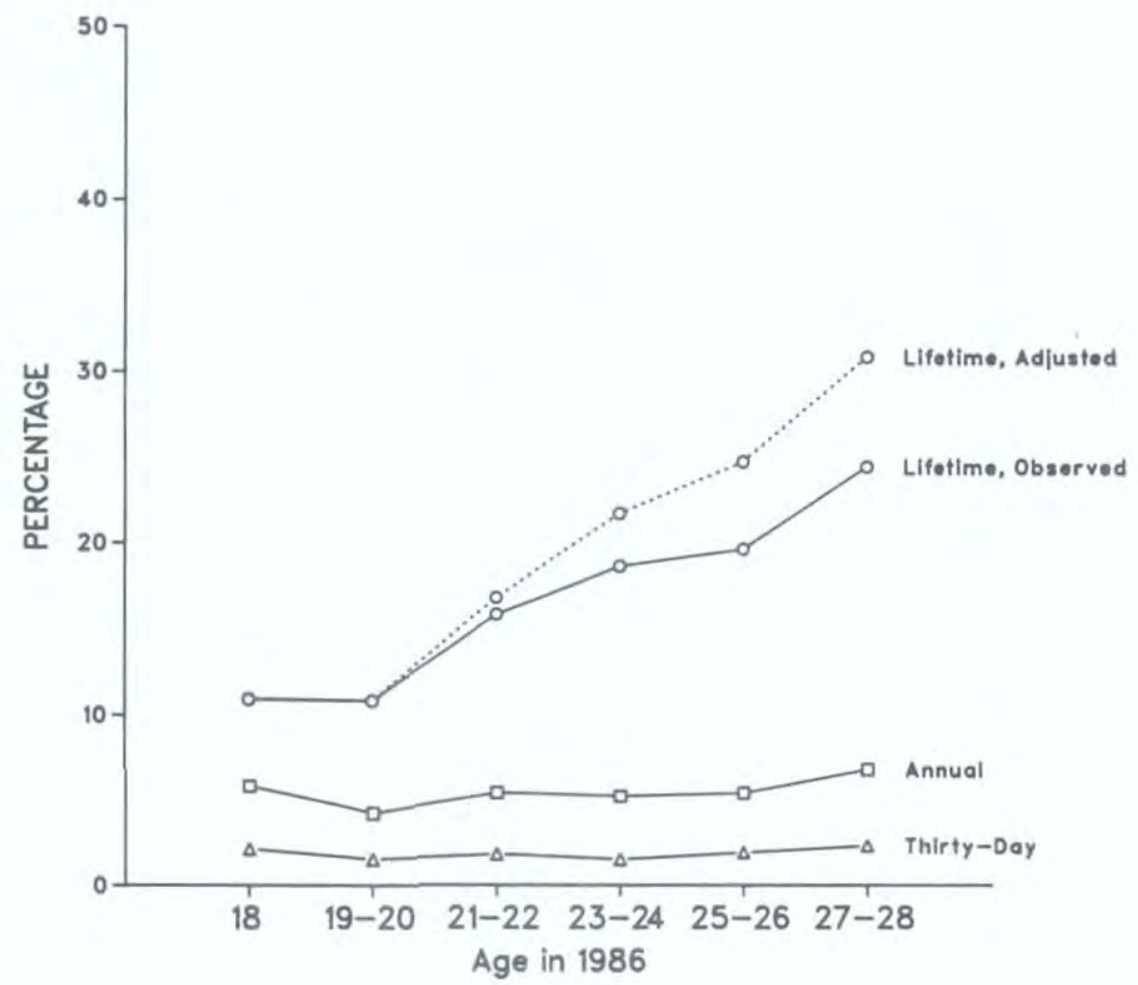

NOTE: Lifetime prevalence estimates were adjusted for inconsistency in selfreports of drug use over time. See text for discussion. 


\section{FIGURE 37}

\section{Alcohol: Various Prevalence Rates Among Young Adults, 1986}

by Age Group

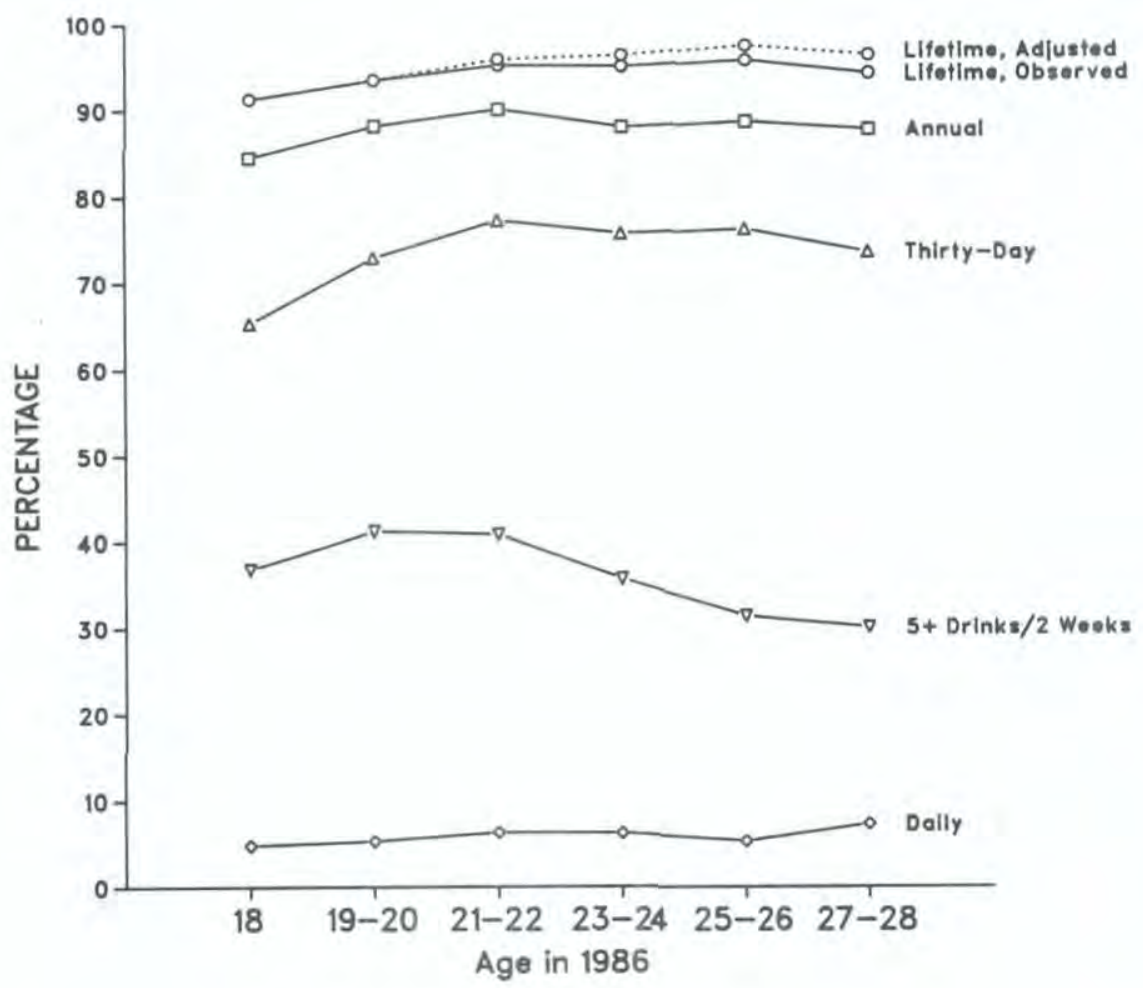

NOTE: Lifetime prevalence estimates were adjusted for inconsistency in selfreports of drug use over time. See text for discussion. 


\section{FIGURE 38}

Cigarettes: Thirty-Day, Daily, and Half-Pack

Prevalence Among Young Adults, 1986

by Age Group

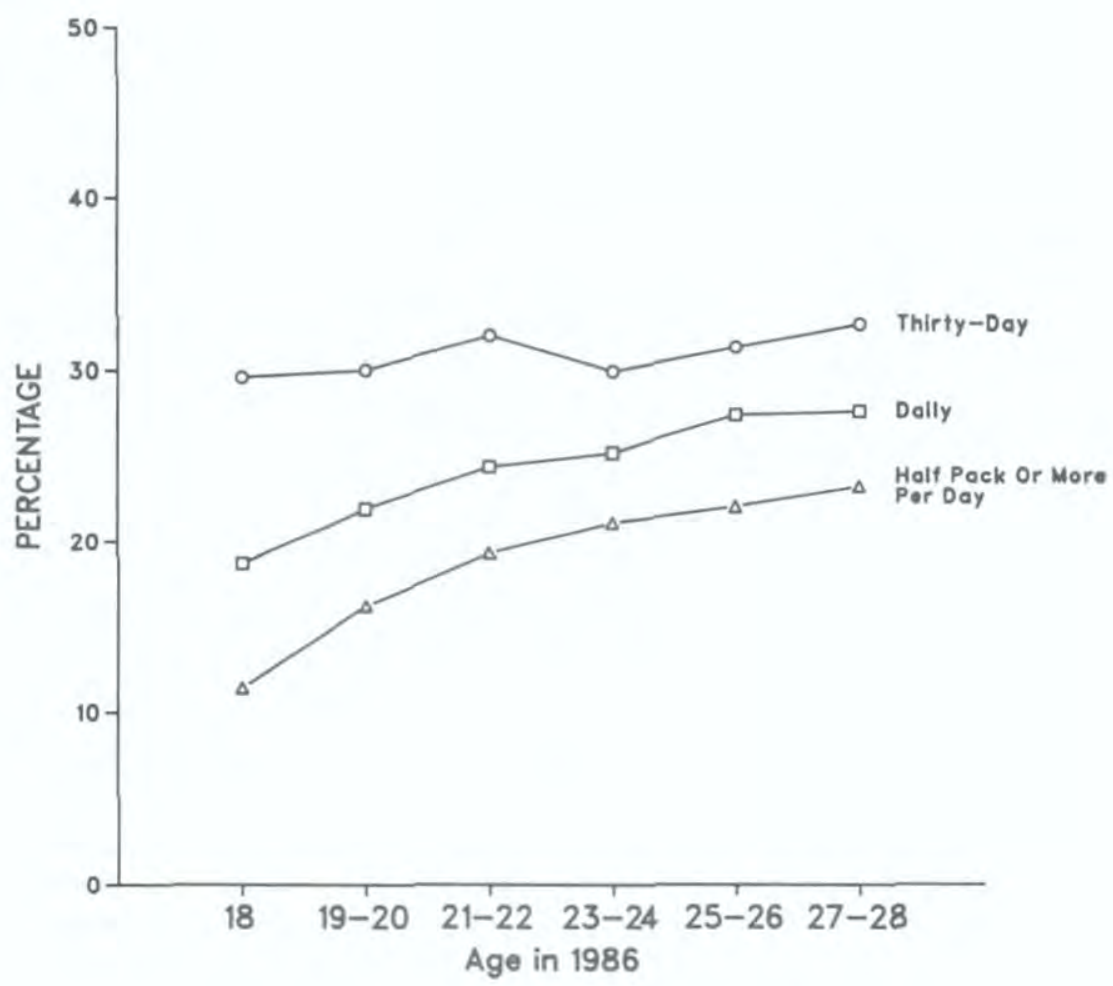

NOTE: Lifetime prevalence is not asked in the follow-up surveys. 


\section{TRENDS IN DRUG USE AMONG YOUNG ADULTS POST-HIGH SCHOOL}

Trends in the use of the various licit and illicit drugs are presented in Figures 39 through 51 for all high school graduates from one up to eight years beyond high school. Each data point in these figures, which represents two adjacent class cohorts, is based on approximately 1200 weighted data cases. (Actual N's are somewhat larger.)

\section{TRENDS IN PREVALENCE THROUGH 1986: YOUNG ADULTS}

- For most drugs, the trends in use among the older age groups have paralleled the changes among seniors discussed earlier in this monograph. This means that many of the changes observed have been secular trends-that is, they are observable across the various age groups. This has generally been true for the recent downward trends in the lifetime, annual, and 30-day prevalence measures for the use of any illicit drug, marijuana, LSD, methaqualone, stimulants, barbiturates, tranquilizers, and opiates other than heroin.

- Several of these drug classes have actually exhibited a faster decline in use during recent years among the older age group than among the high school seniors. These include $L S D$, stimulants, and methaqualone.

- The alcohol statistics for the older age group also generally have tracked those reported for seniors (meaning a very gradual increase in the late 70's and then a fairly level period through 1983), with one important exception. The slight decline observed among seniors between 1983 and 1985particularly in 30-day prevalence and in occasions of heavy drinking during the prior two weeks-is not observable among those in their early to mid-twenties. Whether these differential trends may be due to the effects of changes in the drinking age laws in many states, which would tend to impact only specific age groups, remains to be determined. (The authors have begun an investigation of that possibility under a separate grant from the National Institute on Alcohol Abuse and Alcoholism.) 
- The prevalence statistics for cigarette smoking do not tend to show parallel trends across age groups. While the curves are of the same general shape for each age group, each curve tends to be displaced to the right of the one for the immediately preceding age group (which was two years younger). This pattern is very similar to the one described earlier for lifetime smoking rates for various grade levels below senior year. This is the classic pattern exhibited when there is a "cohort effect" present, meaning that a class cohort tends to be different from other cohorts in a consistent way across the life span. This is how we interpret the cigarette data (O'Malley et al., 1984, referenced on page 158), and we believe that the cohort differences tend to remain throughout the lifespan due to the highly dependence-producing nature of nicotine. None of the other drugs studied here shows such a clear pattern of enduring cohort differences, despite wide variations in their use by different cohorts at a given age.

- Looking specifically at the trends from 1985 to 1986 , a year in which the high school data suggest a resumption in the decline of most types of drug use, we find that the data from these young adults tend to produce a similar finding. Tables 24 through 27 present the trends in prevalence for 19851986 for all respondents one to nine years beyond high school combined. They show that in 1986 there were significant declines in the proportion of young adults reporting the use in the past year of any illicit drug, and any illicit drug other than marijuana. (Any illicit drug other than marijuana or stimulants showed a nonsignificant decline.) The annual prevalence of marijuana, specifically, also declined significantly (Table 24).

- Also parallel to the high school results are the findings that stimulants and methaqualone both showed further significant declines in 1986.

- Another class of drugs showing a small but statistically significant $(0.7 \%)$ decrease in annual prevalence among seniors in 1986-opiates other than heroin-also showed a smaller $(0.3 \%)$, not statistically significant, decrease among the young adult samples.

- The data from young adults also showed no significant change in 1986 in the annual prevalence rates of tranquilizers and barbiturates, as was true among seniors. Annual prevalence for heroin remained stable for both groups. 
- Cocaine use remained quite stable among both high school seniors and the young adult samples in 1986, following increases in both groups in 1985 .

- Cigarette smoking showed a significant decrease among young adults in 1986, reflecting the cohort effect seen at an earlier point among high school seniors.

- In sum, these various samples of high school seniors and young adults show longer-term trends in substance use, as well as near-term trends, which tend to be highly parallel. Although divergent trends would not necessarily demonstrate a lack of validity in either set of data (because such a divergence would not be unreasonable to expect in reality), we believe that the high degree of convergence provides an important new source of validation of the trends which have been reported among the seniors. In fact, each of these sets of data helps to validate the "trend story" reported by the other.

\section{SEX DIFFERENCES IN TRENDS}

- Table 27 gives one-year trends in illicit drug use indexes for 19- to 27-year-old males and females, separately. They are very similar. In general, the longer-term trends in use also have been very similar for the two sexes (data not shown). The same is true for most of the individual drug classes.

- However, methaqualone use has declined much more among males (who started from a distinctly higher level), and both sexes now show similar (very low) rates of use. As mentioned earlier, this may be due in part to the fact that this substance is no longer manufactured or distributed legally in the United States. 


\section{TABLE 24}

\section{Trends in Annual Prevalence of Thirteen Types of Drugs}

Among Follow-Up Respondents 1-9 Years Beyond High School

Percent who used in last twelve months

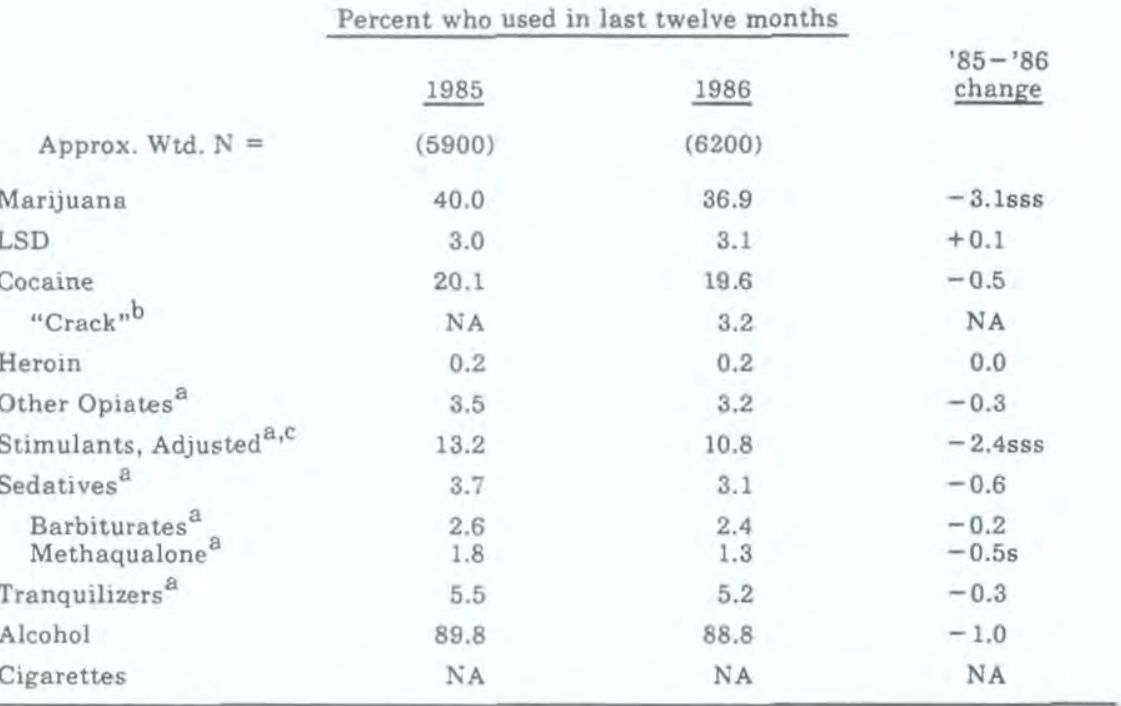

NOTES: Level of significance of difference between the two most recent years: $\mathrm{s}=.05, \mathrm{ss}=.01, \mathrm{sss}=.001$. NA indicates data not available.

anly drug use which was not under a doctor's orders is included here.

${ }^{b}$ This drug was asked about in only one of the five questionnaire forms. $\mathrm{N}$ is one-fifth of $\mathrm{N}$ indicated.

${ }^{\mathrm{c}}$ Based on the data from the revised question, which attempts to exclude the inappropriate reporting of non-prescription stimulants. 
TABLE 25

\section{Trends in Thirty-Day Prevalence of Twelve Types of Drugs} Among Follow-Up Respondents 1-9 Years Beyond High School

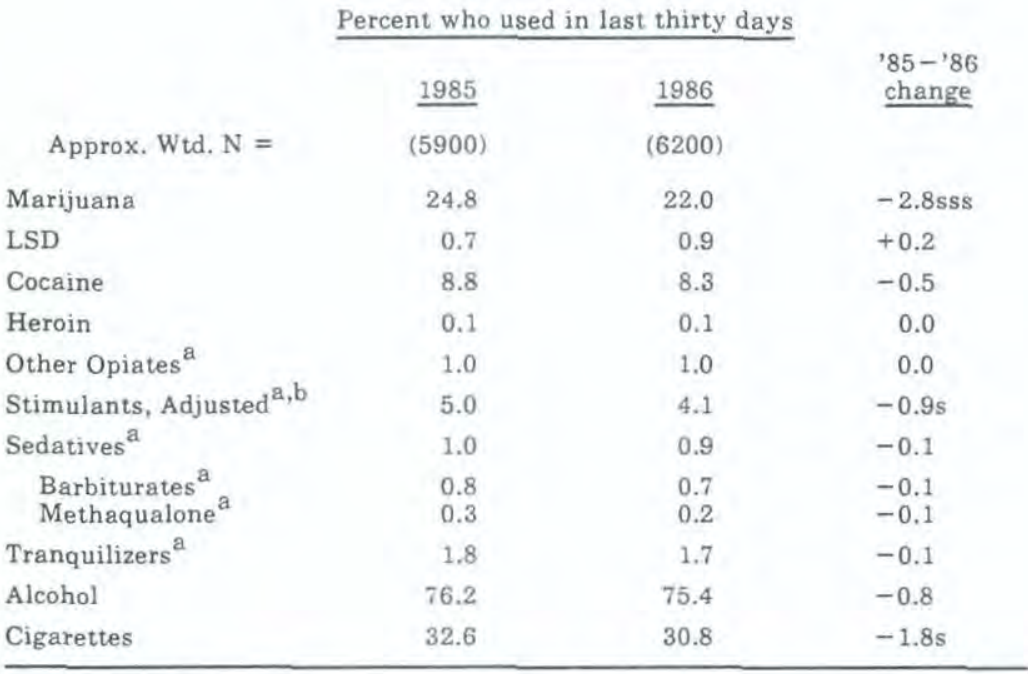

NOTES: Level of significance of difference between the two most recent years: $\mathrm{s}=.05, \mathrm{ss}=.01$, sss $=.001$.

a Only drug use which was not under a doctor's orders is included here.

${ }^{b}$ Based on the data from the revised question, which attempts to exclude the inappropriate reporting of non-prescription stimulants. 
TABLE 26

\section{Trends in Thirty-Day Prevalence of Daily Use of Twelve Types of Drugs}

Among Follow-Up Respondents 1-9 Years Beyond High School

\begin{tabular}{|c|c|c|c|}
\hline & $\begin{array}{l}\text { Percent } \\
\text { in last t }\end{array}$ & $\begin{array}{l}\text { ng daily } \\
\text { ty days } \\
\end{array}$ & \\
\hline & $\underline{1985}$ & $\underline{1986}$ & $\begin{array}{l}\begin{array}{l}85-' 86 \\
\text { change }\end{array} \\
\end{array}$ \\
\hline Approx. Wtd. $N=$ & $(5900)$ & $(6200)$ & \\
\hline Marijuana & 5.5 & 4.0 & $-1.5 \mathrm{sss}$ \\
\hline$S D$ & 0.0 & 0.0 & 0.0 \\
\hline Cocaine & 0.2 & 0.2 & 0.0 \\
\hline Heroin & 0.0 & 0.0 & 0.0 \\
\hline ther Opiates ${ }^{\mathrm{a}}$ & 0.0 & 0.0 & 0.0 \\
\hline timulants, Adjusted $^{\mathrm{a}, \mathrm{b}}$ & 0.1 & 0.2 & +0.0 \\
\hline edatives $^{a}$ & 0.0 & 0.0 & 0.0 \\
\hline $\begin{array}{l}\text { Barbiturates }{ }^{a} \\
\text { Methaqualone }^{\text {a }}\end{array}$ & $\begin{array}{l}0.0 \\
0.0\end{array}$ & $\begin{array}{l}0.0 \\
0.0\end{array}$ & $\begin{array}{l}0.0 \\
0.0\end{array}$ \\
\hline ranquilizers ${ }^{a}$ & 0.0 & 0.0 & 0.0 \\
\hline Alcohol & 6.7 & 5.9 & -0.8 \\
\hline $\begin{array}{l}\text { Five or more drinks in a row } \\
\text { in last two weeks }\end{array}$ & 37.4 & 36.7 & -0.7 \\
\hline igarettes & 26.1 & 24.9 & -1.2 \\
\hline Half pack or more per day in last thirty days & 21.1 & 19.9 & -1.2 \\
\hline
\end{tabular}

NOTES: Level of significance of difference between the two most recent years: $\mathrm{s}=.05, \mathrm{ss}=.01, \mathrm{sss}=.001$.

${ }^{a}$ Only drug use which was not under a doctor's orders is included here.

${ }^{b}$ Based on the data from the revised question, which attempts to exclude the inappropriate reporting of non-prescription stimulants. 


\section{TABLE 27}

\section{Trends in Annual and Thirty-Day Prevalence of An Illicit Drug Use Index Among Follow-Up Respondents 1-9 Years Beyond High School by Sex}

\begin{tabular}{|c|c|c|c|}
\hline & $\underline{1985}$ & $\underline{1986}$ & $\begin{array}{l}\text { '85-'86 } \\
\text { change } \\
\end{array}$ \\
\hline & $\begin{array}{r}\text { Perc } \\
\text { use in } 18\end{array}$ & $\begin{array}{l}\text { ng } \\
\text { nonths }\end{array}$ & \\
\hline Any Illicit Drug & 45.6 & 42.2 & $-3.4 \mathrm{sss}$ \\
\hline $\begin{array}{l}\text { Males } \\
\text { Females }\end{array}$ & $\begin{array}{l}49.4 \\
42.7\end{array}$ & $\begin{array}{l}45.7 \\
39.2\end{array}$ & $\begin{array}{l}-3.7 \mathrm{ss} \\
-3.5 \mathrm{ss}\end{array}$ \\
\hline Any Illicit Drug Other than Marijuana & 29.8 & 26.9 & $-2.9 \mathrm{sss}$ \\
\hline $\begin{array}{l}\text { Males } \\
\text { Females }\end{array}$ & $\begin{array}{l}33.2 \\
27.0\end{array}$ & $\begin{array}{l}30.3 \\
23.9\end{array}$ & $\begin{array}{l}-2.9 \mathrm{~s} \\
-3.1 \mathrm{ss}\end{array}$ \\
\hline $\begin{array}{l}\text { Any Illicit Drug Other than } \\
\text { Marijuana or Stimulants }\end{array}$ & 25.3 & 23.9 & -1.4 \\
\hline $\begin{array}{l}\text { Males } \\
\text { Females }\end{array}$ & $\begin{array}{l}29.1 \\
22.2\end{array}$ & $\begin{array}{l}27.8 \\
20.6\end{array}$ & $\begin{array}{l}-1.3 \\
-1.6\end{array}$ \\
\hline & $\begin{array}{r}\text { Perc } \\
\text { use in }\end{array}$ & $\begin{array}{l}\text { ng } \\
\text { days }\end{array}$ & \\
\hline Any Illicit Drug & 28.5 & 25.9 & $-2.6 \mathrm{ss}$ \\
\hline $\begin{array}{l}\text { Males } \\
\text { Females }\end{array}$ & $\begin{array}{l}32.8 \\
25.2\end{array}$ & $\begin{array}{l}30.0 \\
22.4\end{array}$ & $\begin{array}{l}-2.8 \mathrm{~s} \\
-2.8 \mathrm{ss}\end{array}$ \\
\hline Any Illicit Drug Other than Marijuana & 14.8 & 13.1 & $-1.7 \mathrm{ss}$ \\
\hline $\begin{array}{l}\text { Males } \\
\text { Females }\end{array}$ & $\begin{array}{l}17.0 \\
13.0\end{array}$ & $\begin{array}{l}15.3 \\
11.1\end{array}$ & $\begin{array}{l}-1.7 \\
-1.9 \mathrm{~s}\end{array}$ \\
\hline $\begin{array}{l}\text { Any Illicit Drug Other than } \\
\text { Marijuana or Stimulants }\end{array}$ & 11.8 & 10.9 & -0.9 \\
\hline $\begin{array}{l}\text { Males } \\
\text { Females }\end{array}$ & $\begin{array}{l}14.0 \\
10.0\end{array}$ & $\begin{array}{r}13.4 \\
8.7\end{array}$ & $\begin{array}{l}-0.6 \\
-1.3\end{array}$ \\
\hline
\end{tabular}

Approximate Weighted N's
All Respondents $=$
(5900)
(6200)
Males
$(2600)$
(2800)
Females
(3300)
(3400)

NOTES: Level of significance of difference between the two most recent years: $\mathrm{s}=.05, \mathrm{ss}=.01, \mathrm{sss}=.001$. 
FIGURE 39

Any Illicit Drug: Trends in Annual Prevalence Among Young Adults by Age Group

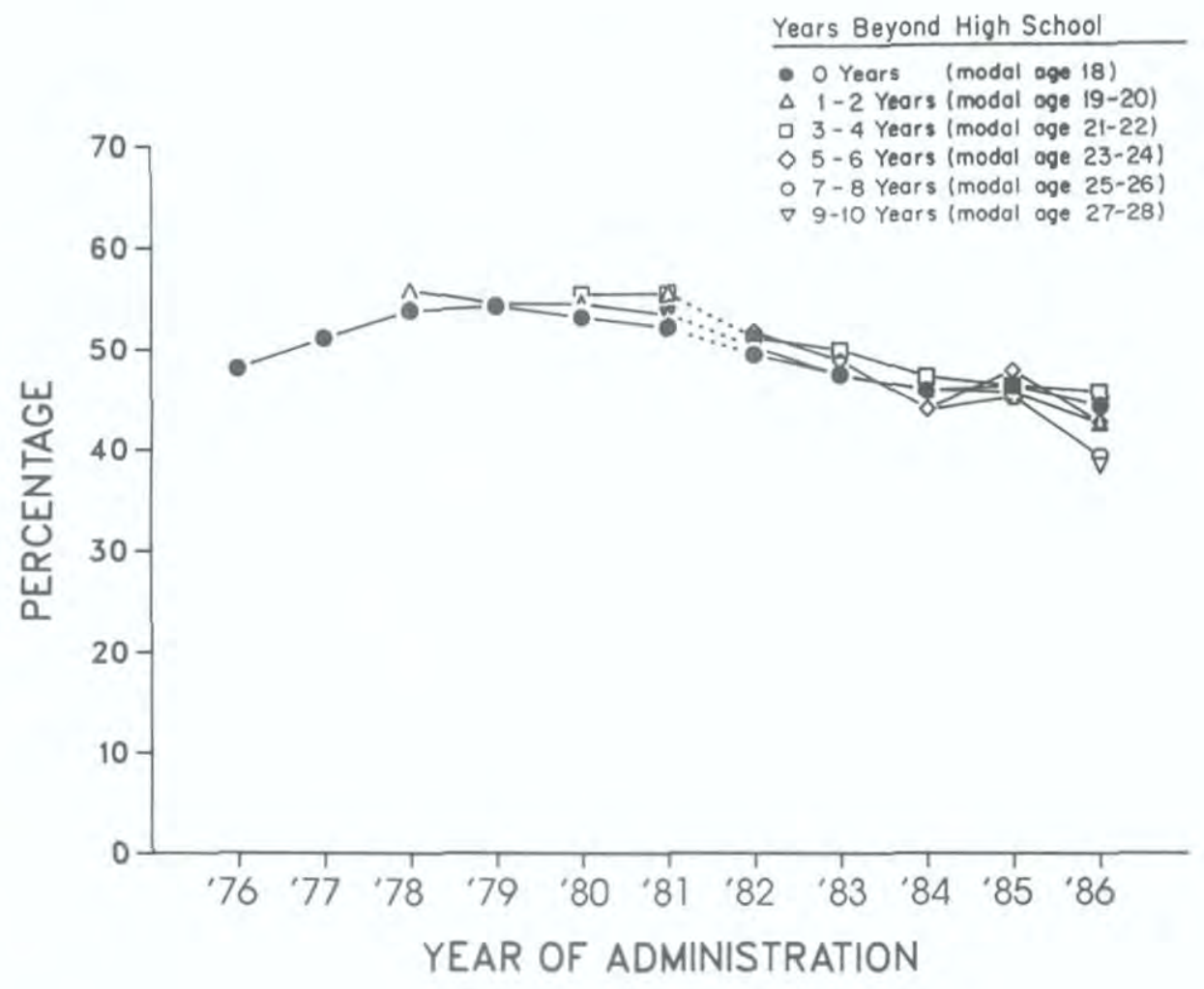

NOTE: The dotted lines between 1981 and 1982 denote the change in the amphetamine question. 


\section{FIGURE 40}

\section{Any Ilicit Drug Other than Marijuana: Trends in Annual Prevalence Among Young Adults \\ By Age Group}

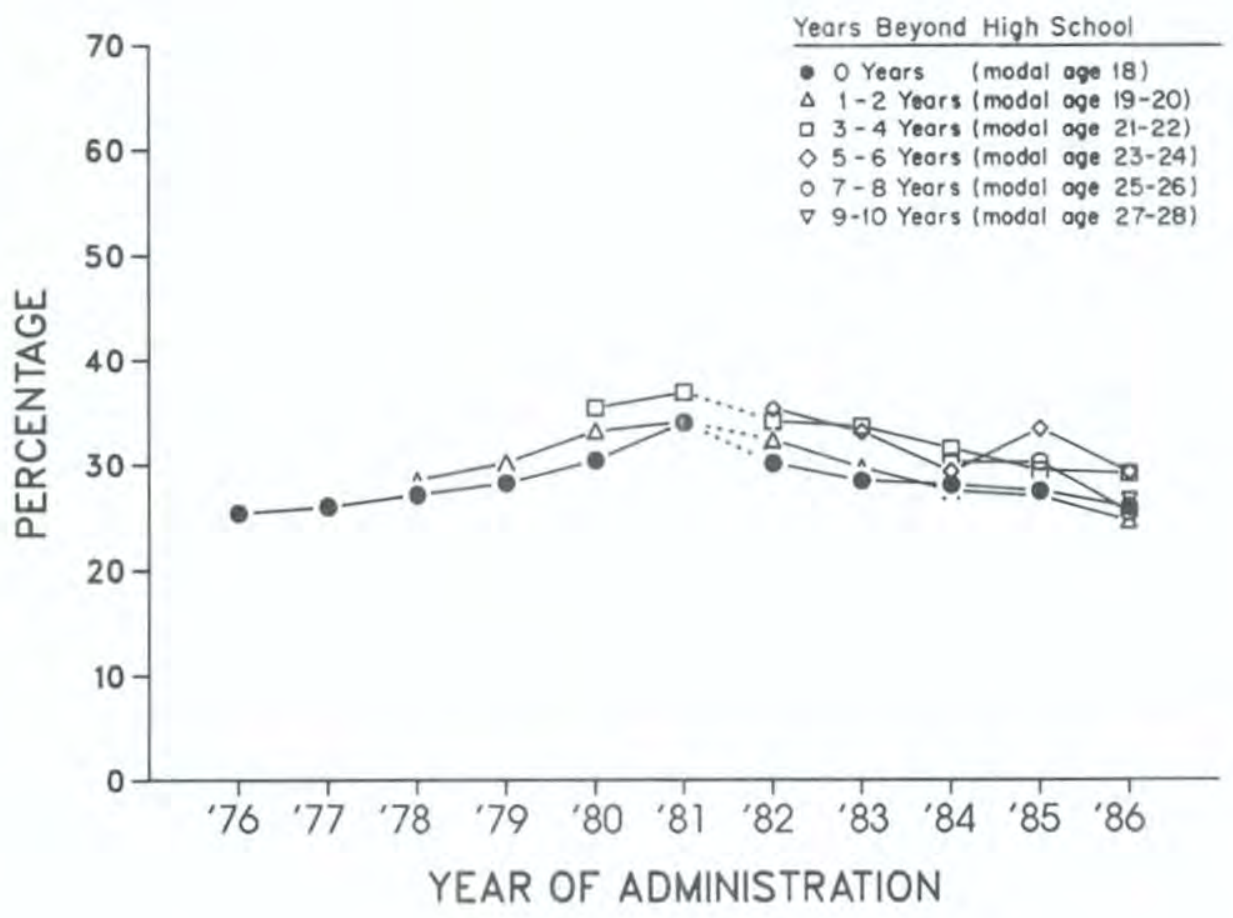

NOTE: The dotted lines between 1981 and 1982 denote the change in the amphetamine question. 


\section{FIGURE 41}

Any Illicit Drug Other than Marijuana or Stimulants:

Trends in Annual Prevalence Among Young Adults by Age Group

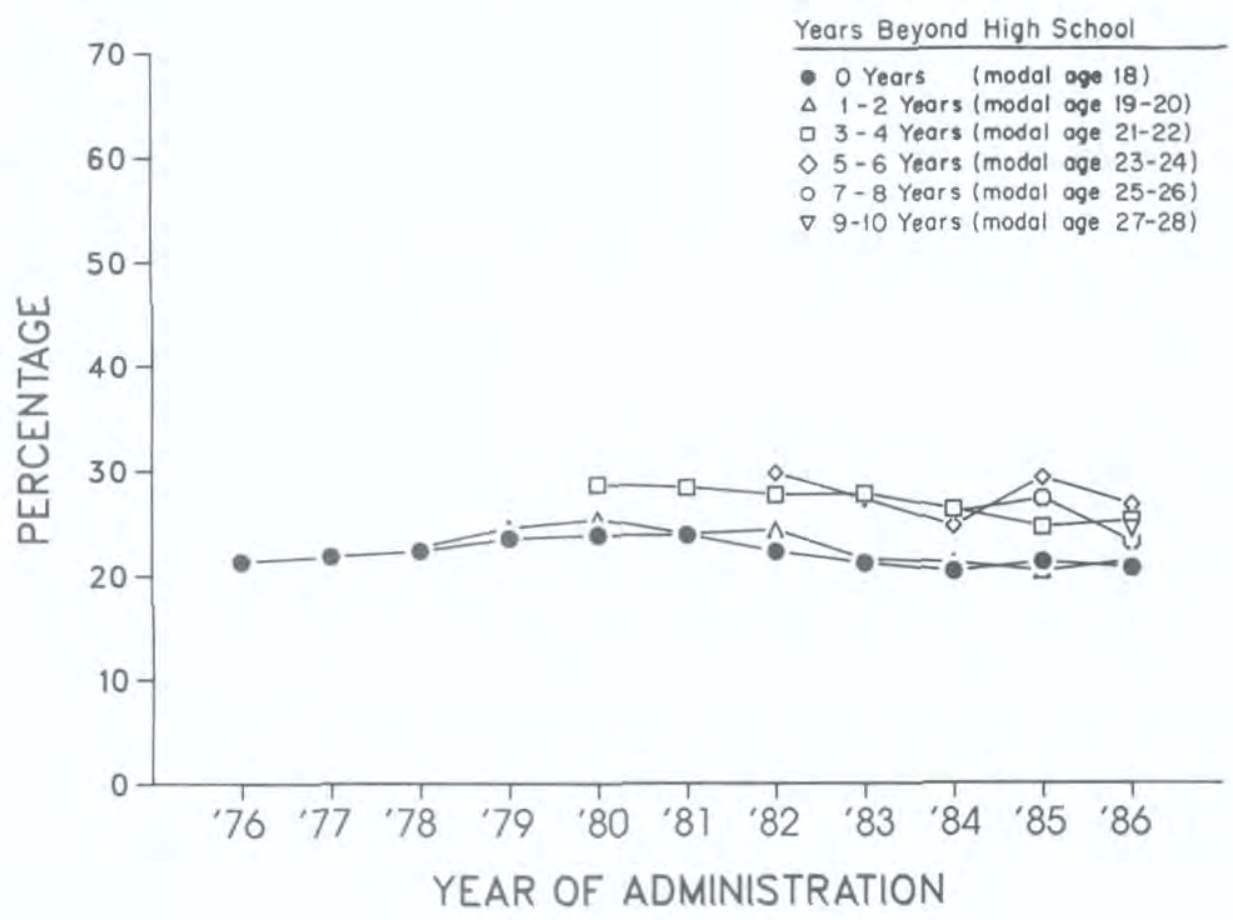




\section{FIGURE $42 \mathrm{a}$}

Marijuana: Trends in Annual Prevalence Among Young Adults by Age Group

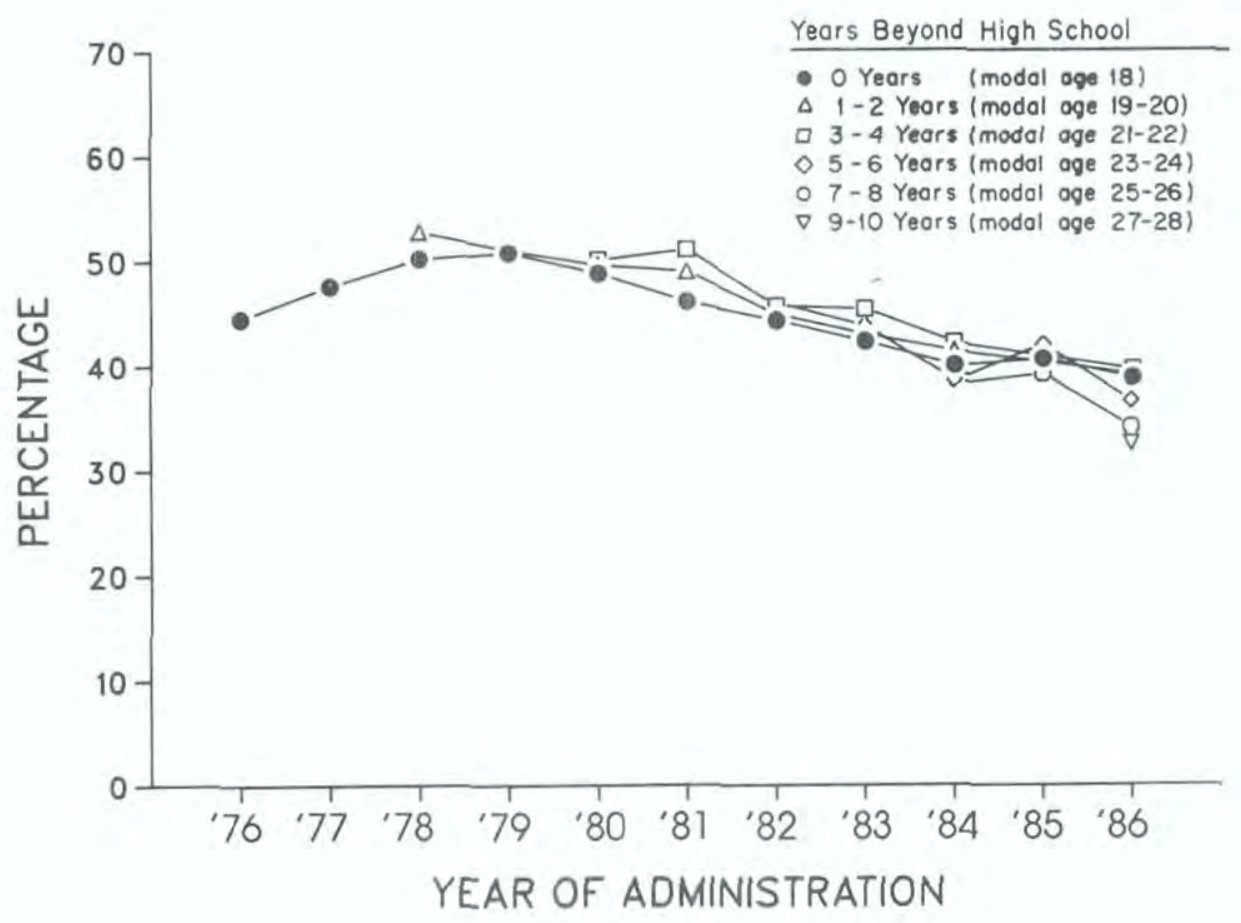




\section{FIGURE $42 \mathrm{~b}$}

Marijuana: Trends in Thirty-Day Prevalence Among Young Adults by Age Group



Marijuana: Trends in Thirty-Day Prevalence of

Daily Use Among Young Adults

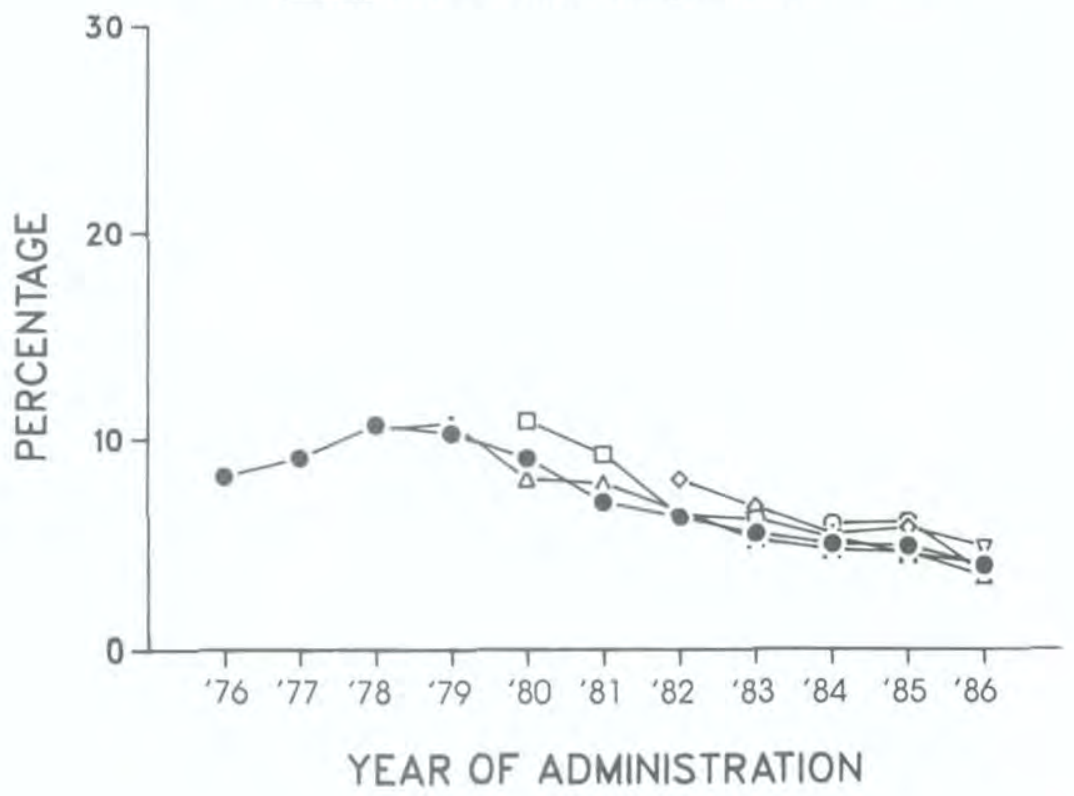


FIGURE 43

LSD: Trends in Annual Prevalence Among Young Adults by Age Group

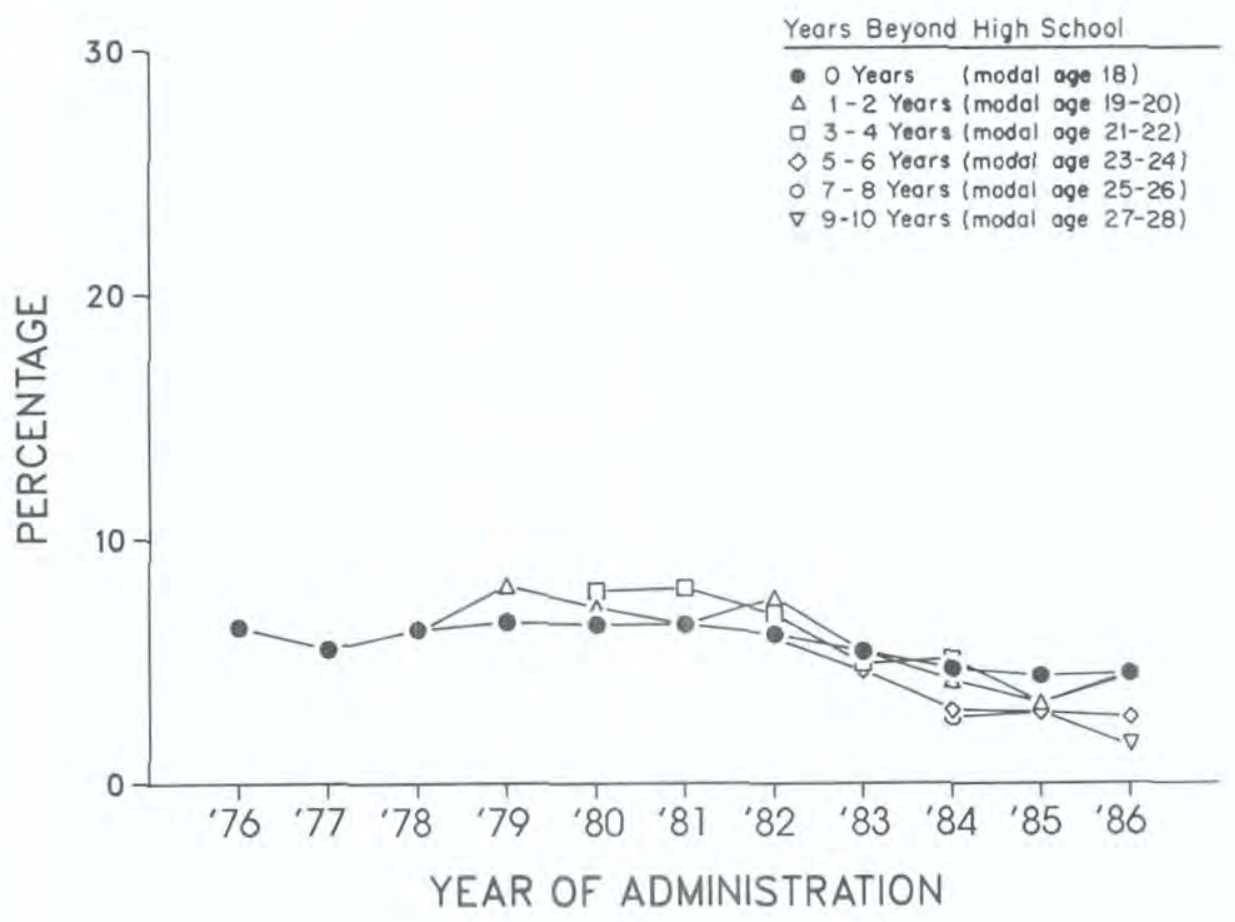


FIGURE 44

Cocaine: Trends in Annual Prevalence Among Young Adults by Age Group

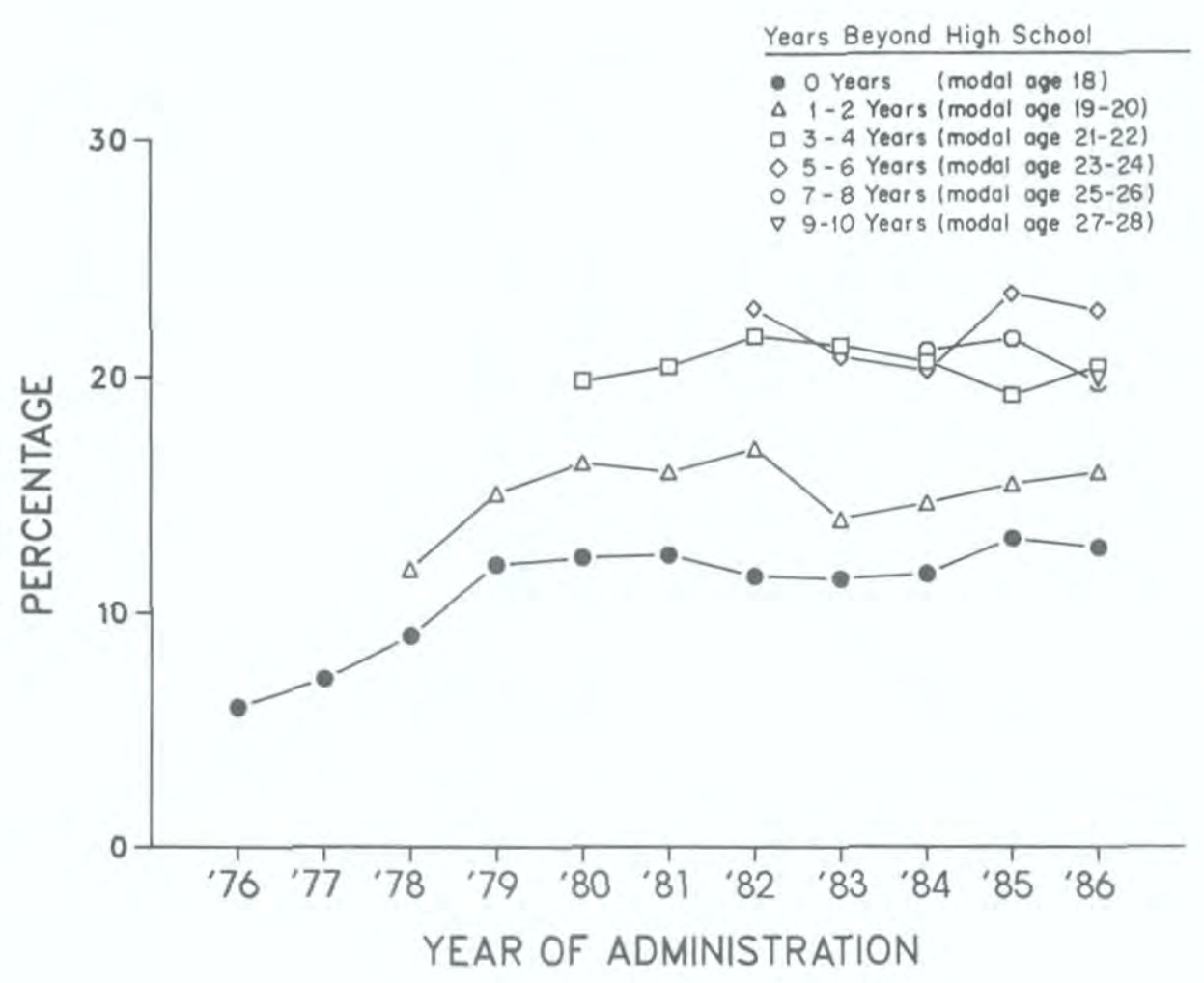




\section{FIGURE 45}

Other Opiates: Trends in Annual Prevalence Among Young Adults by Age Group

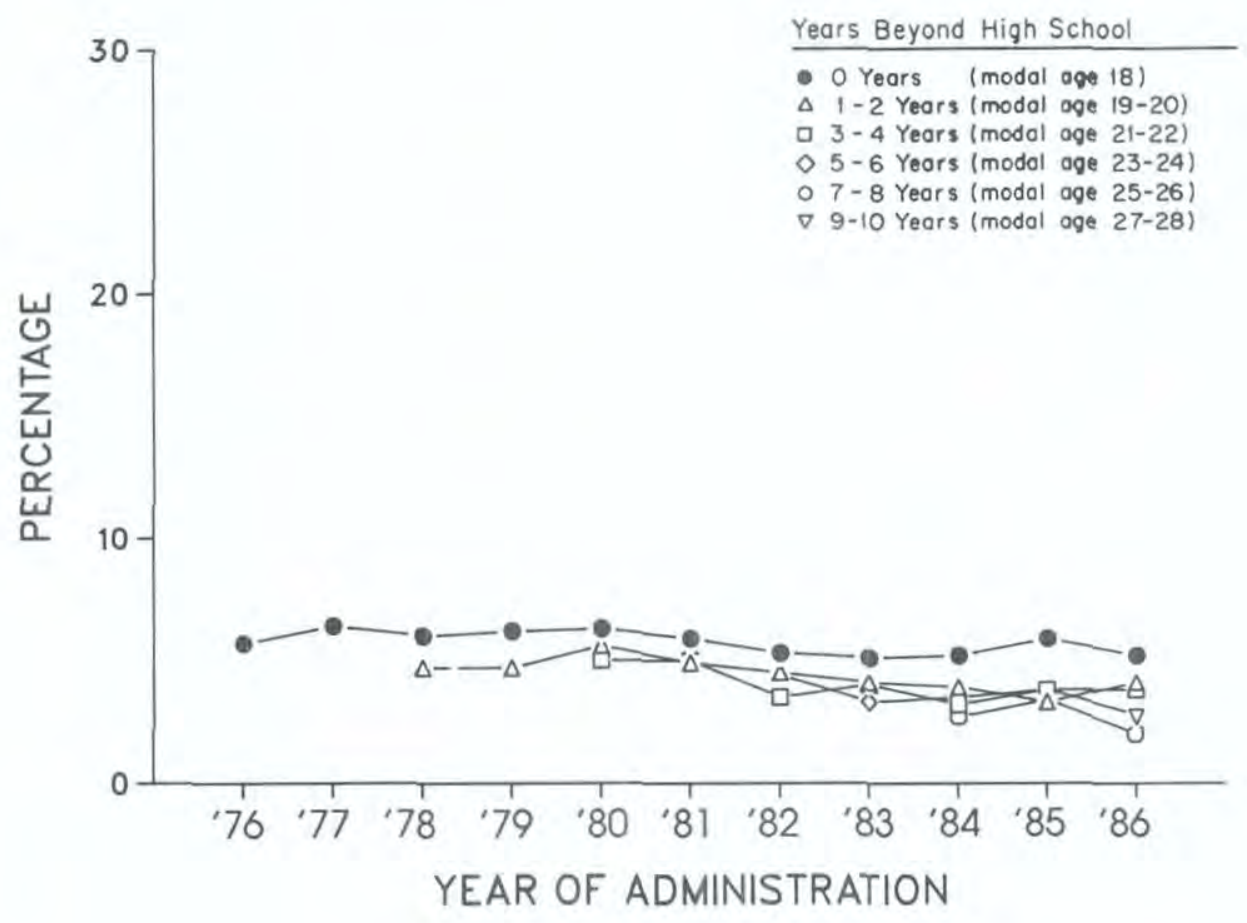


FIGURE 46

Stimulants: Trends in Annual Prevalence Among Young Adults by Age Group

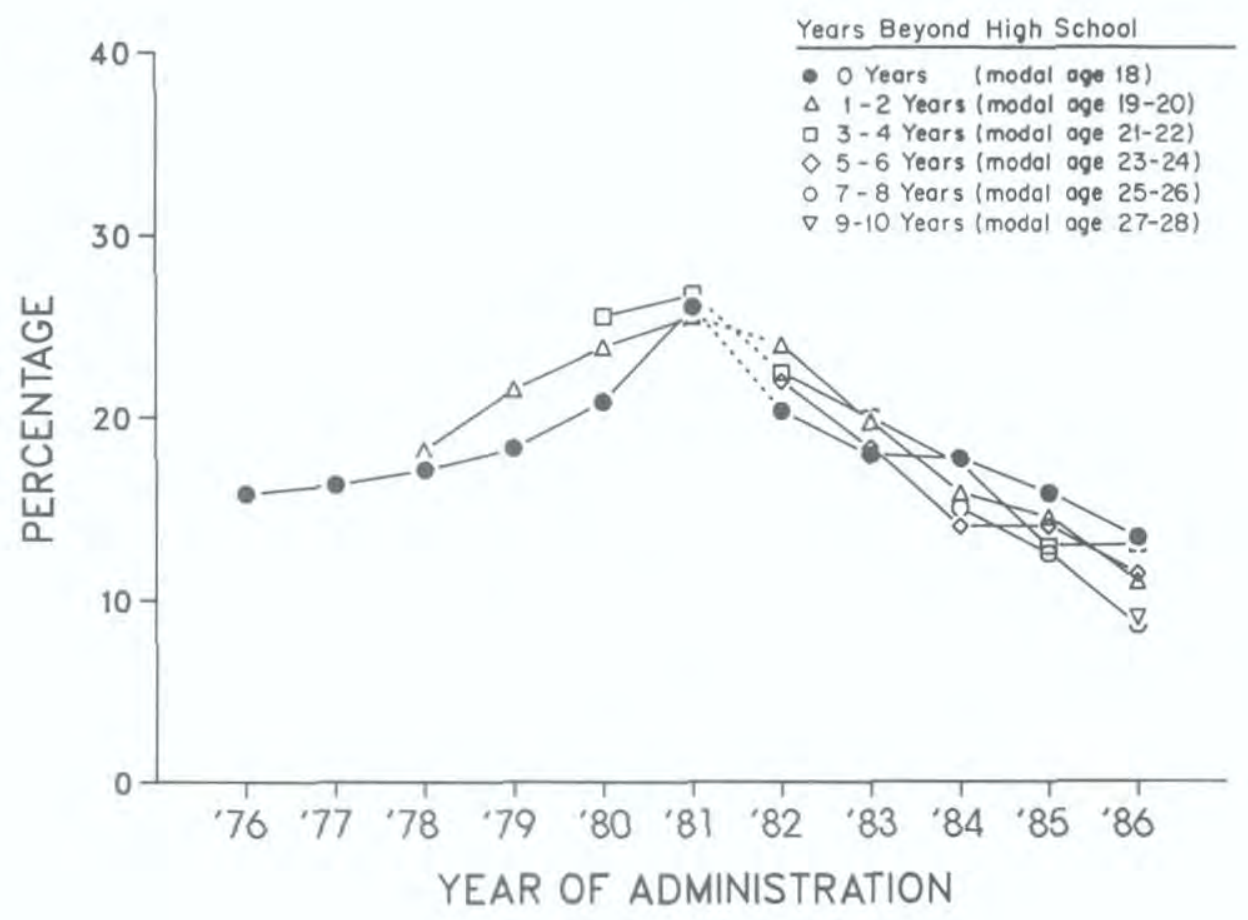

NOTE: The dotted lines between 1981 and 1982 denote the change in the amphetamine question. 
FIGURE 47

Barbiturates: Trends in Annual Prevalence Among Young Adults by Age Group

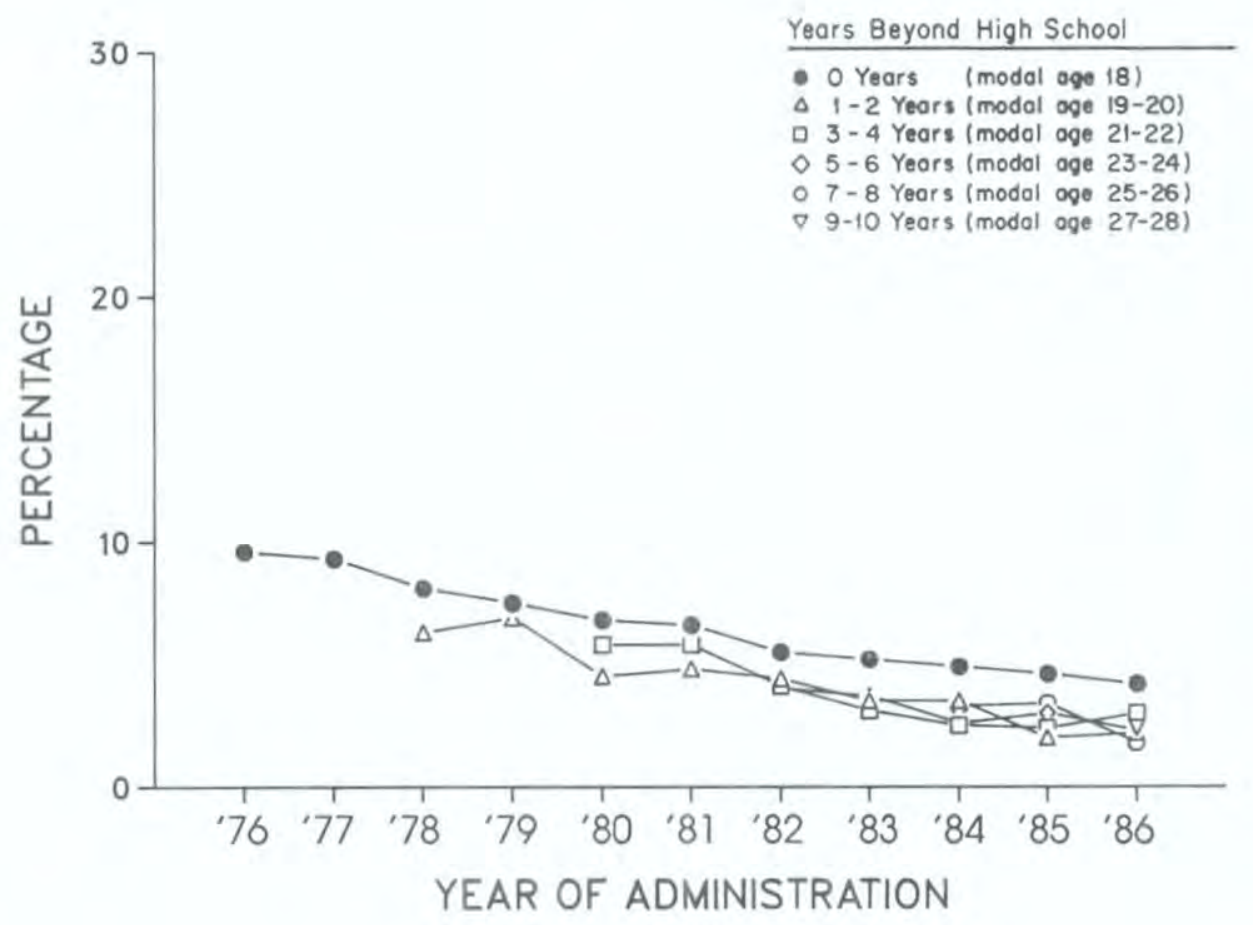




\section{FIGURE 48}

Methaqualone: Trends in Annual Prevalence Among Young Adults by Age Group

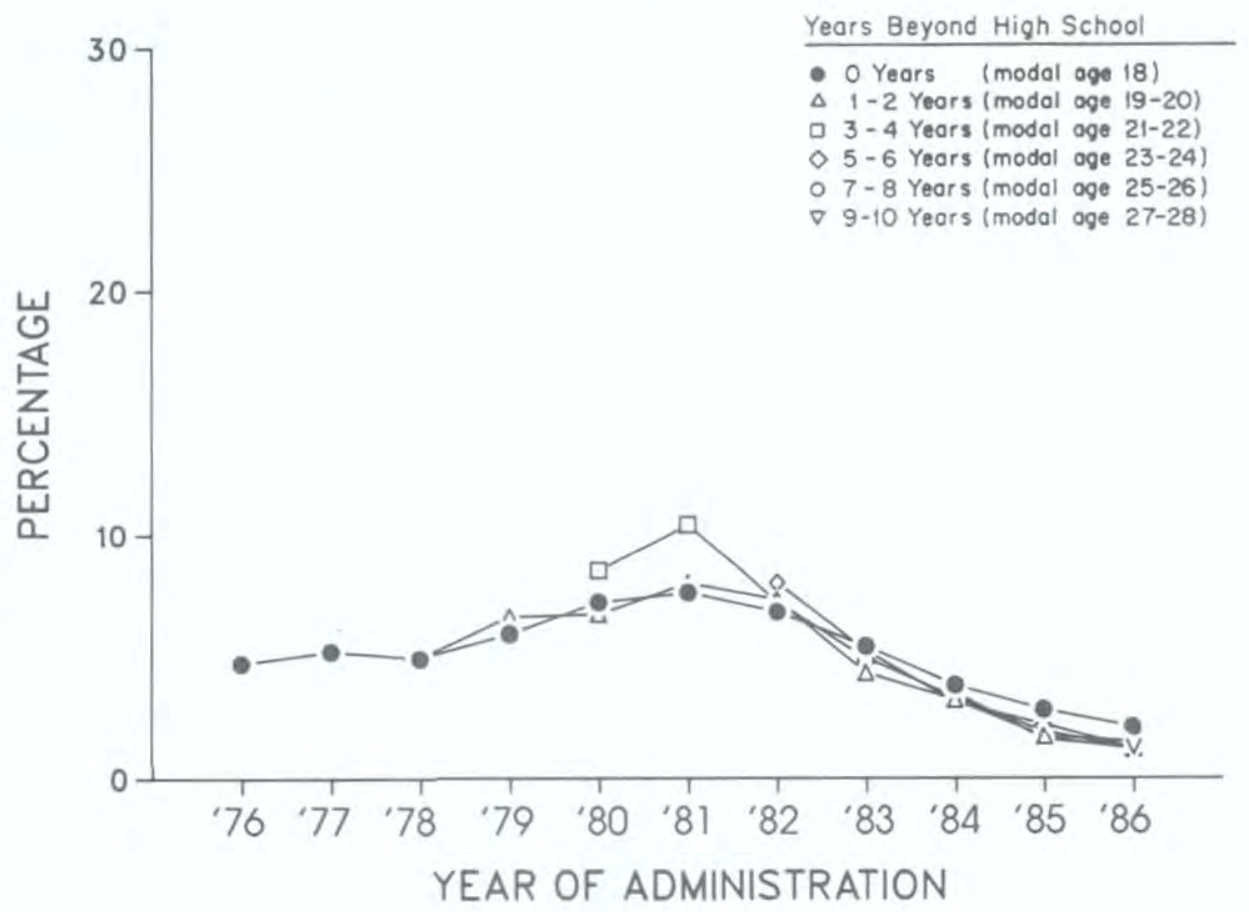


Tranquilizers: Trends in Annual Prevalence Among Young Adults by Age Group

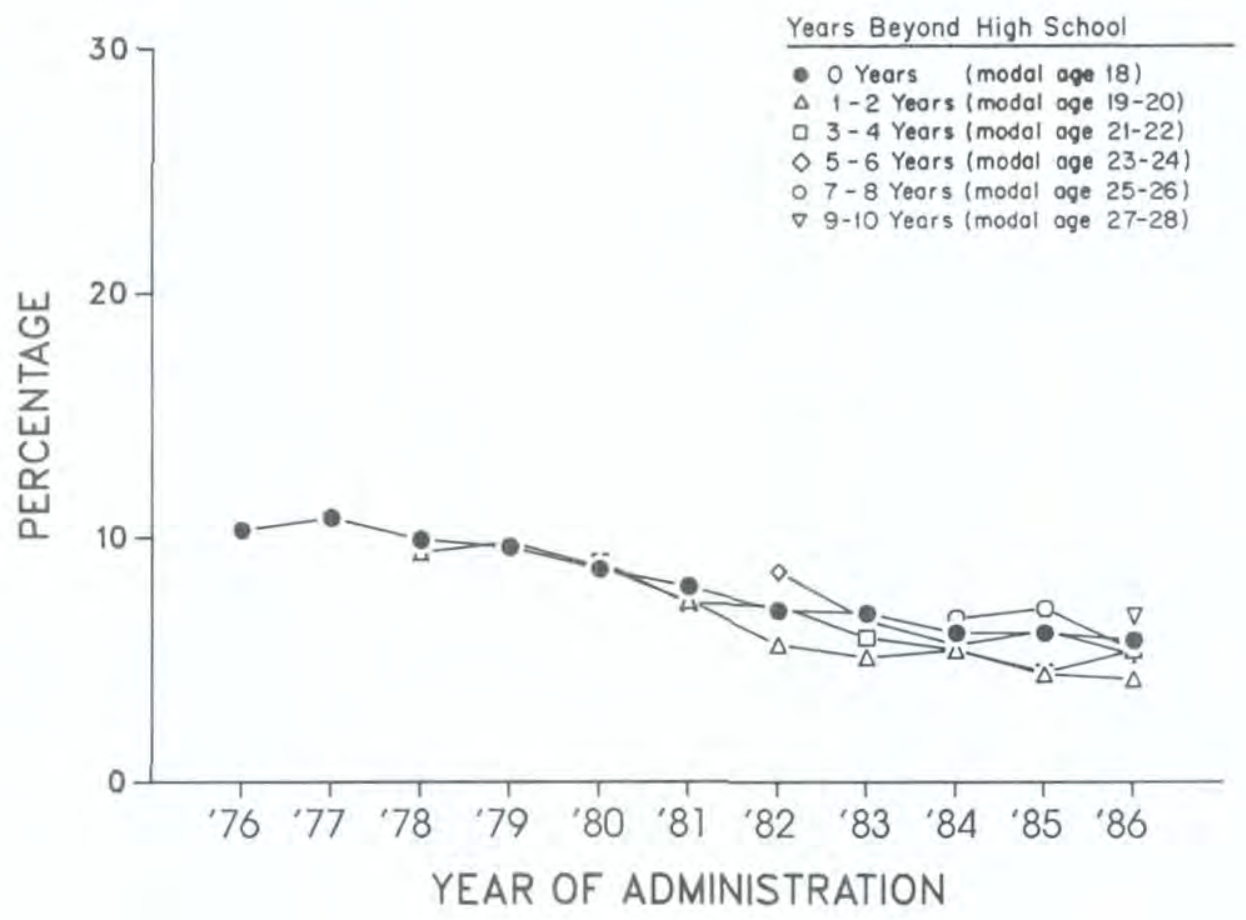


FIGURE 50a

Alcohol: Trends in Annual Prevalence Among Young Adults

by Age Group

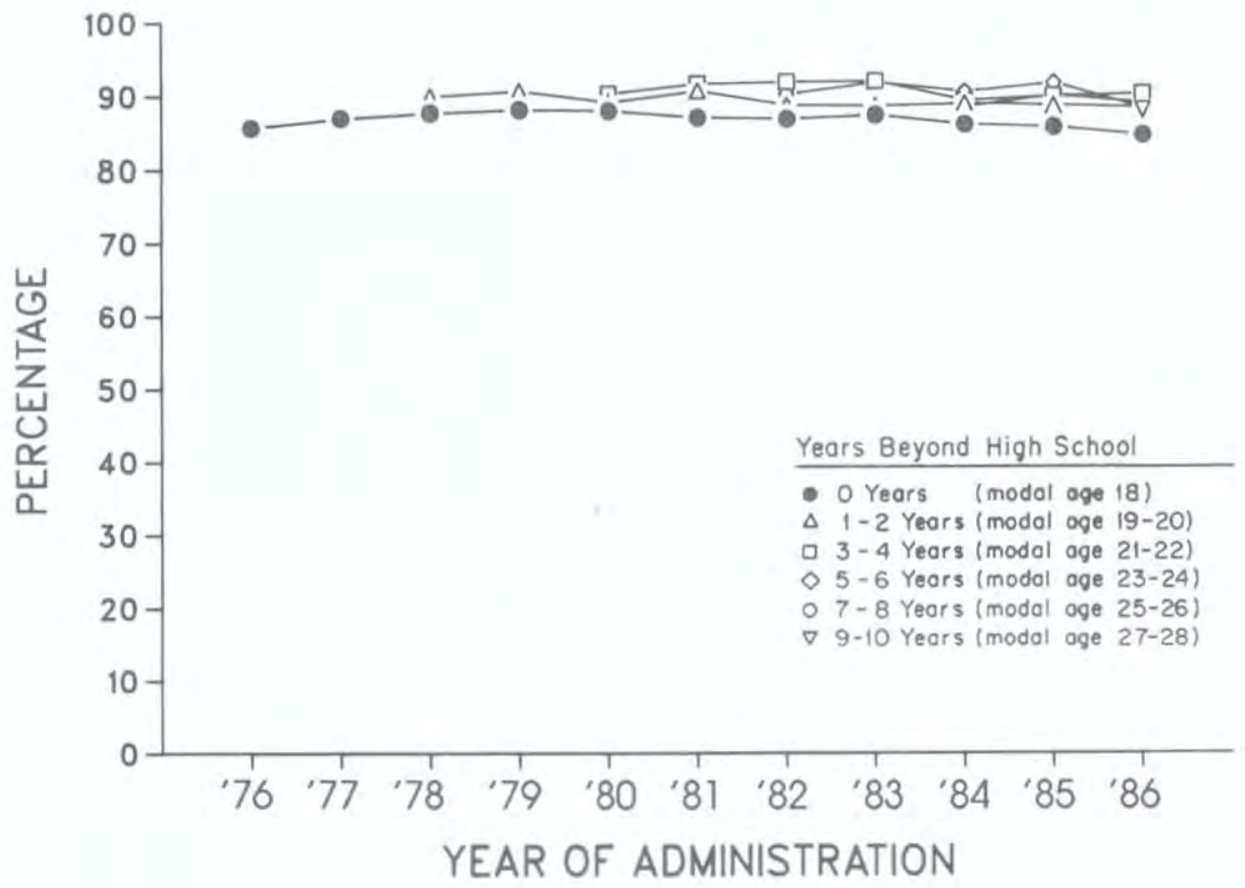


Alcohol: Trends in Thirty-Day Prevalence Among Young Adults by Age Group

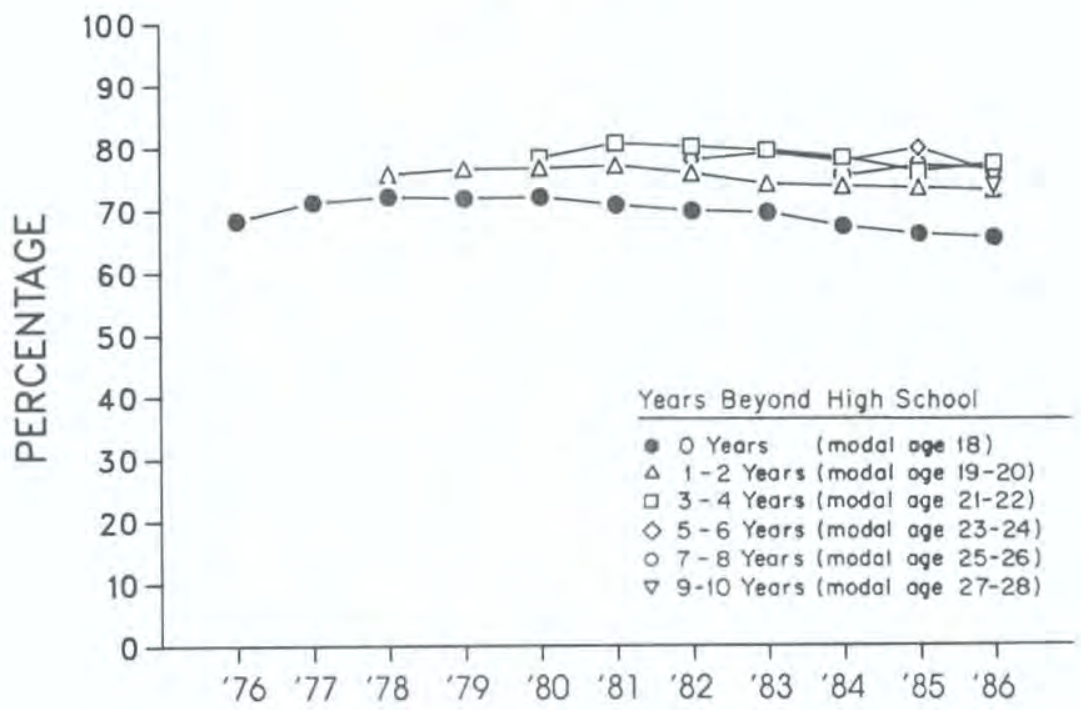

Alcohol: Trends in Thirty-Day Prevalence of Daily Use Among Young Adults

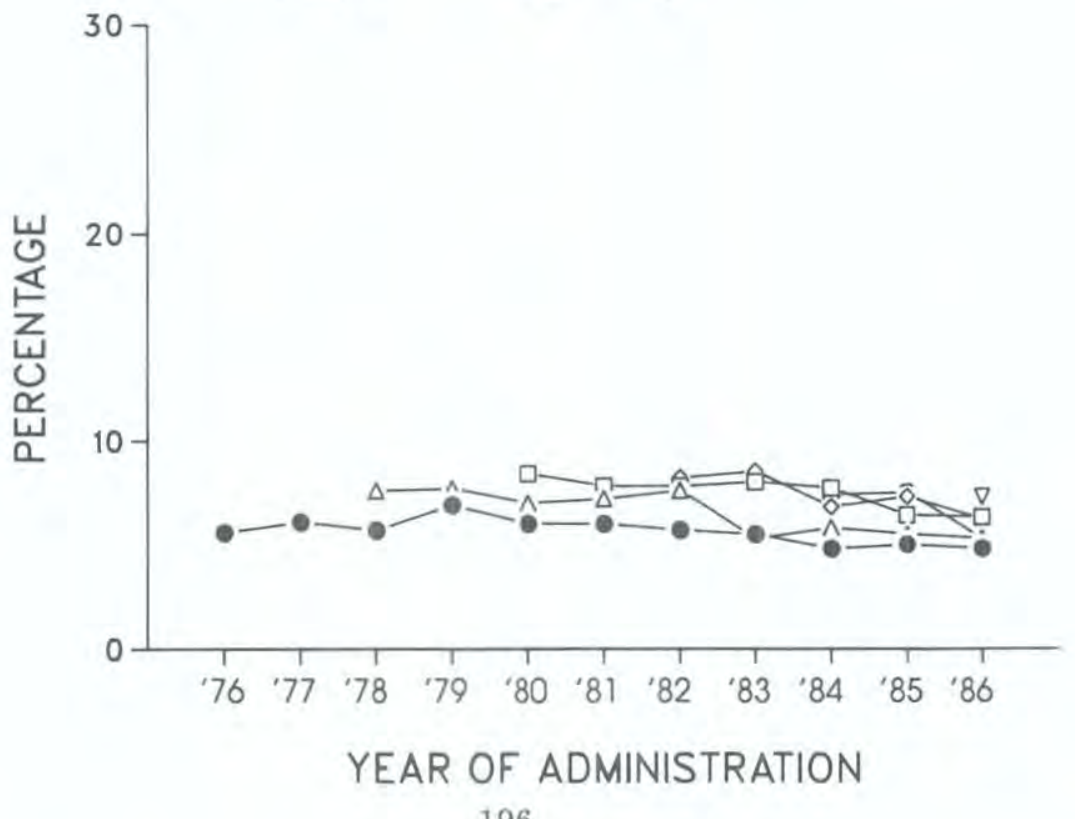




\section{FIGURE 50c}

Alcohol: Trends in Two-Week Prevalence of Five or More Drinks in a Row Among Young Adults

by Age Group

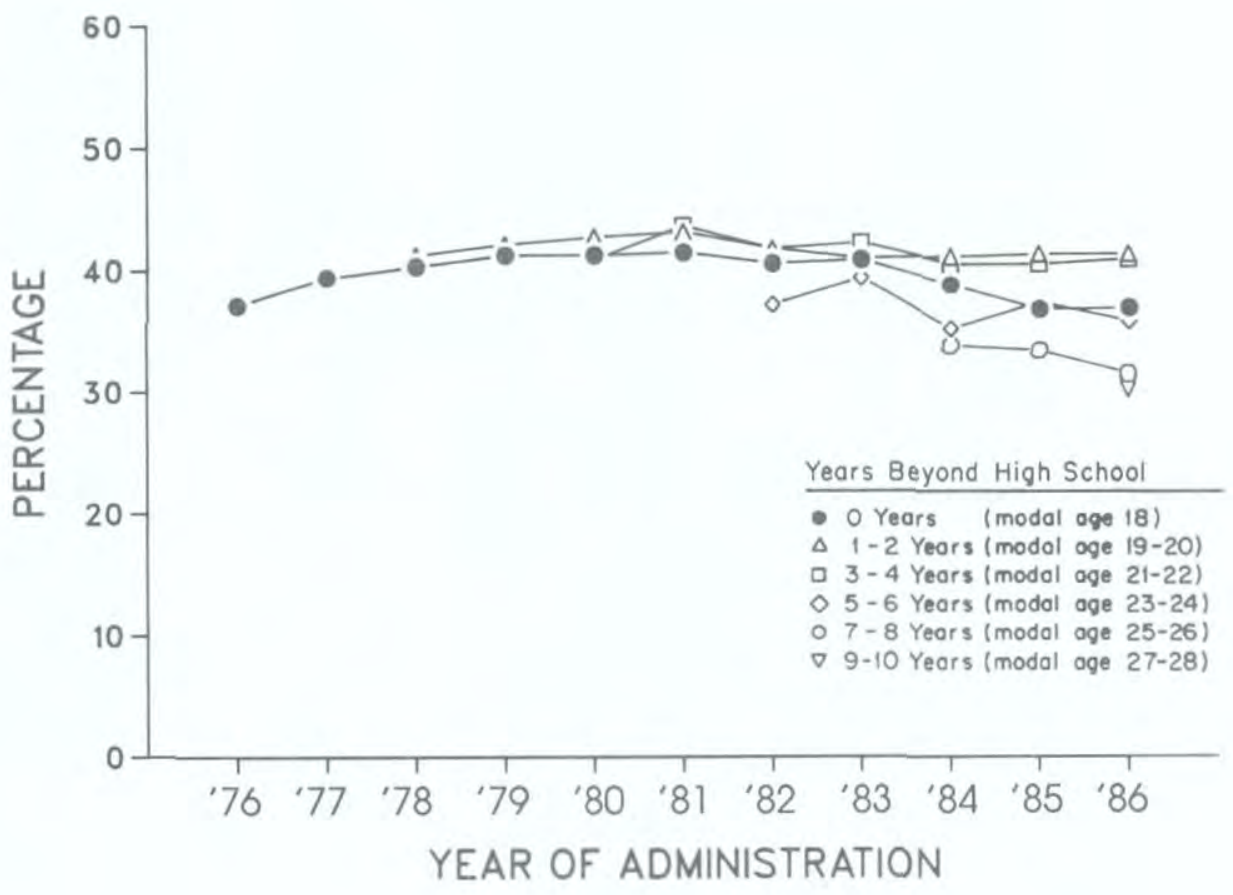




\section{FIGURE 51a}

Cigarettes: Trends in Thirty-Day Prevalence Among Young Adults by Age Group

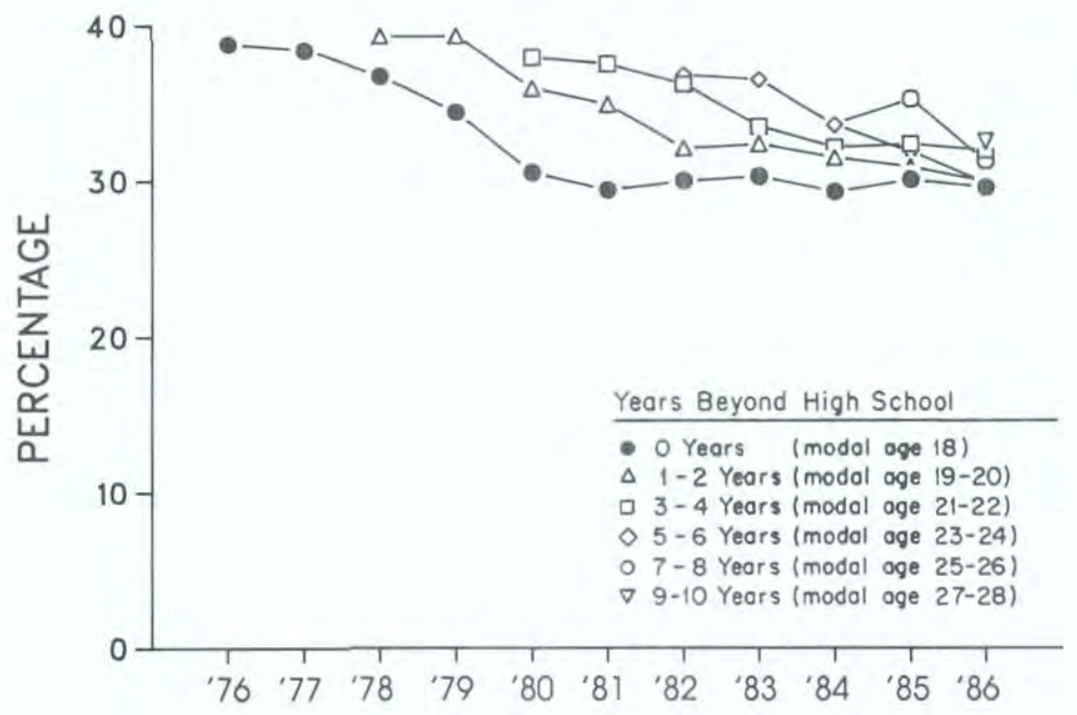

Cigarettes: Trends in Thirty-Day Prevalence of Daily Use Among Young Adults

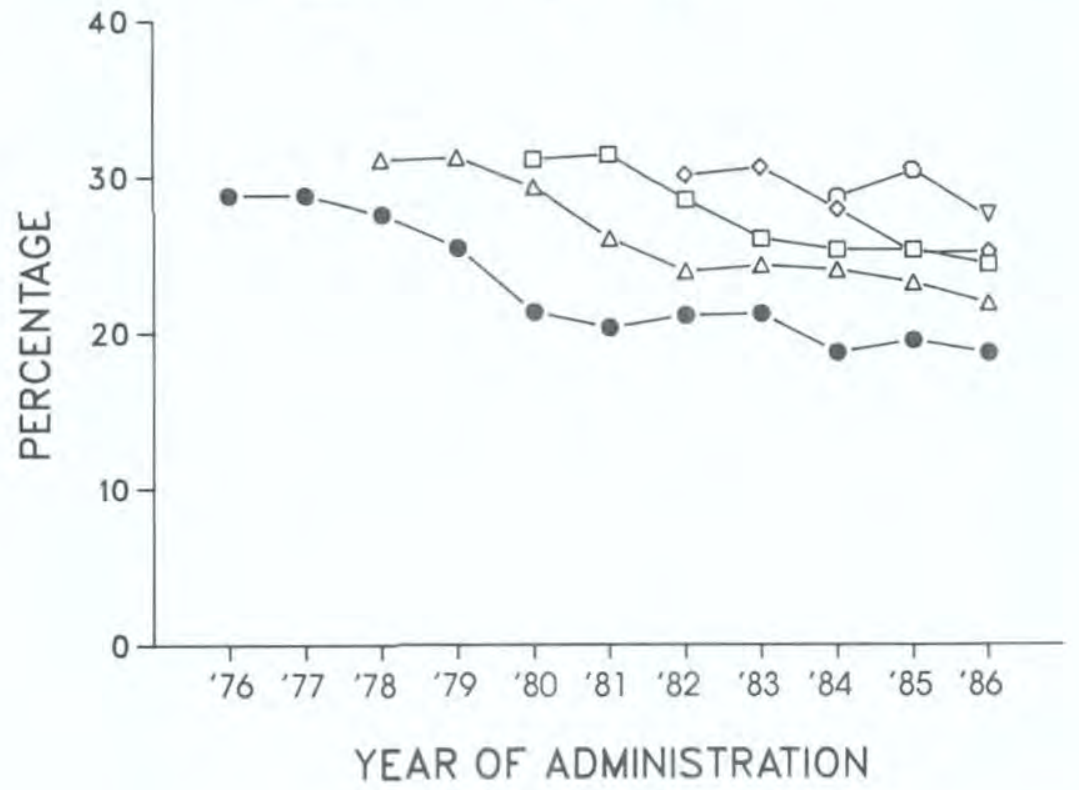




\section{FIGURE $51 \mathrm{~b}$}

Cigarettes: Trends in Thirty-Day Use of Half-Pack a Day or More Among Young Adults

by Age Group

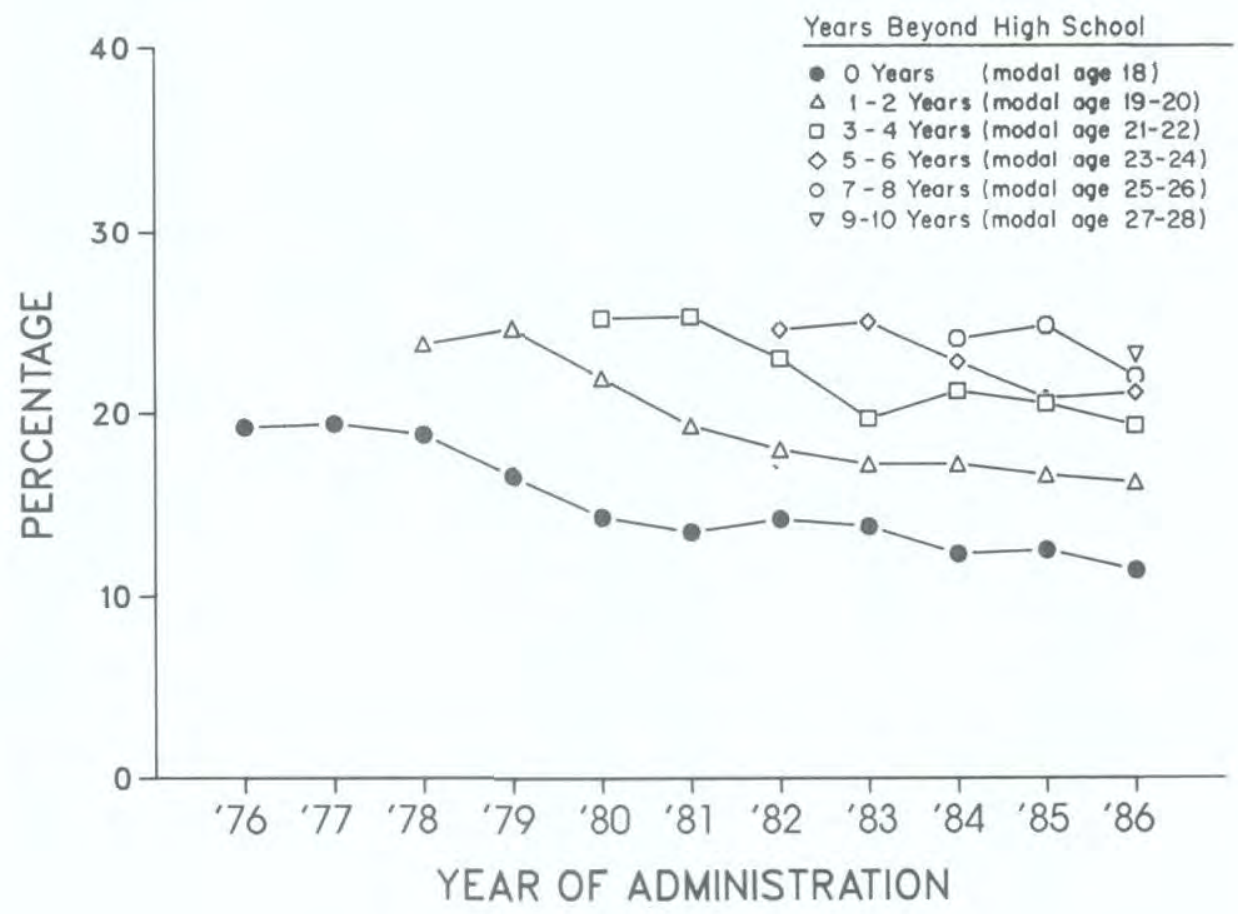




\section{COLLEGE STUDENTS}




\section{PREVALENCE OF DRUG USE AMONG COLLEGE STUDENTS}

The follow-up design of the Monitoring the Future project is capable of generating an excellent national sample of college students-better in many ways than a design which first samples colleges and then samples students within them, because in the present sample the students are not clustered in a limited number of colleges. Given the much greater diversity in post-secondary institutions than in high schools, the use of a clustered sample would place far greater limitations on sample accuracy at the college level than at the high school level. Further, the absence of dropouts in the high school senior sample should have practically no effect on the college sample, since very few of the dropouts would go on to college.

Perhaps the major limitation of the present design is that it must delimit the college sample to a certain age level. For trend estimation purposes, we have decided to limit the age band to the most typical one for college attendance, i.e., one to four years past high school, which corresponds to the modal ages of 19 to 22 years old. According to statistics from the United States Bureau of the Census, ${ }^{24}$ this age should encompass about $85 \%$ of all students enrolled in college full-time in 1980. While extending the age band to be covered by an additional two years would cover $92 \%$ of all enrolled college students, it would also reduce by two years the interval over which we could report trend data. The differences which would result in the 1985 prevalence estimates under the two definitions are extremely small, however. The annual prevalence of all drugs except cocaine would shift only about one- or two-tenths of a percent, based on comparisons made in 1985. Cocaine, which has the greatest amount of change with age, would have an annual prevalence rate only $0.8 \%$ higher if the six-year age span were covered rather than the four year age span. Thus, for purposes of estimating all prevalence rates except lifetime prevalence, the four-year and six-year intervals are nearly interchangeable.

On the positive side, controlling the age band (either one to four or one to six years after high school) may be desirable for trend estimation purposes, in the event that the age composition of college students should change much with time. Otherwise college students charac-

${ }^{24}$ U.S. Bureau of the Census. Current population reports: Population characteristics, Series P-20, No, 400. Washington, DC: U.S. Government Printing Office, 1982. 
terized in one year would represent a noncomparable segment of the population when compared to college students surveyed in another year.

College students are here defined as those follow-up respondents one to four years past high school who say they were registered as full-time students at the beginning of March in the year in question and who say they are enrolled in a two- or four-year college. Thus, the definition encompasses only those who are one to four years past high school and are active full-time undergraduate college students in the year in question. It excludes those who may previously have been college students or may have already completed college.

Prevalence rates for college students are provided in Tables 28 to 31 . They are also included in the figures providing trends in annual prevalence (see Figures 52 through 64 ) along with the prevalence rates for all remaining respondents one to four years past high school, other than the college students. Having statistics for both groups makes it possible to see whether college students are above or below their age peers in terms of their usage rates. (The college-enrolled sample constitutes about $40 \%$ of the entire follow-up sample one to four years past high school.) Any difference between the two groups would likely be enlarged if data from the missing high school dropout segment were available. Therefore, any differences observed here are only an indication of the direction and relative size of differences between the college and non-college-enrolled populations, not an absolute estimate of them.

The findings are presented below.

\section{PREVALENCE OF DRUG USE IN 1986: COLLEGE STUDENTS}

- There is very little difference between those enrolled in college versus high school graduates of the same age (i.e., one to four years past high school) not enrolled in college, in their annual prevalence of any illicit drug use, use of any illicit drug other than marijuana, or use of any illicit drug other than marijuana or stimulants (Figures 52-54).

- College students are also average for their age group in their annual and monthly prevalence rates for marijuana use. However, their rate of current daily marijuana use is only $2.1 \%$ versus $5.0 \%$ for their age peers. Recall that a similar large difference in daily use was observable in high school between the college-bound and those not bound for college.

- College students have slightly below average rates for their age group of cocaine use in general, and methaqualone use in 1986, though in the past the differences tended to be somewhat larger. Annual use of "crack" cocaine, first asked 
about in 1986, is distinctly lower among college students than among their noncollege-age peers, $1.3 \%$ vs. $4.3 \%$, respectively.

- Stimulants show the next largest difference in annual prevalence among the illicit drugs, $10.3 \%$ for college students versus $13.0 \%$ for those not in college.

- College students are very slightly below their noncollege age peers in annual usage rates for $L S D$ (3.9\% vs. $4.9 \%)$, barbiturates $(2.1 \%$ vs. $2.9 \%)$, and tranquilizers $(4.4 \%$ vs. $5.1 \%)$.

- There is essentially no difference between the two groups in their use of opiates other than heroin (both are at about $4 \%$ annual prevalence).

- Although both groups give very low levels of self-reported heroin use, since 1981 annual prevalence has consistently been lower among the college enrolled than among their age peers not in college.

- Regarding alcohol use, today's college students have slightly higher annual prevalence compared to their age peers (92\% vs. $87 \%$ ), a higher monthly prevalence $(80 \%$ vs. $72 \%)$, and a slightly below average daily prevalence $(4.6 \%$ vs. $6.6 \%)$. The most important difference, however, lies in the prevalence of occasions of heavy drinking (five or more drinks in a row in the past two weeks), which is $45 \%$ among college students, versus $38 \%$ among their age-peers.

- By far the largest difference between college students and others their age occurs for cigarette smoking. For example, their prevalence of daily smoking is only $13 \%$ vs. $30 \%$ for all high school graduates that age who are not in college. Smok. ing at the rate of half-a-pack a day stands at $8.3 \%$ vs. $24.2 \%$ for these two groups, respectively. Recall that the high school senior data show the college-bound to have much lower smoking rates in high school than the noncollege-bound: thus most or all of the differences observed at college age actually preceded college attendance.

\section{SEX DIFFERENCES IN PREVALENCE AMONG COLLEGE STUDENTS}

While tabular data are not provided for male and female college students separately (except for Table 31, giving differences on the illicit 
drug use indexes), sex differences are plotted in Figures 52 through 64 for the various drugs.

- It may be seen that most of the sex differences among college students replicate those discussed earlier for all young adults (one to nine years past high school), which in turn replicated sex differences in high schocl for the most part. That means that among college students, males have higher annual prevalence rates for most drugs, with the largest proportional differences for marijuana (45\% vs. $38 \%)$, LSD $(5.2 \%$ vs. $2.7 \%)$, cocaine (21\% vs. $14 \%)$, opiates other than heroin $(4.7 \%$ vs. $3.4 \%)$, and barbiturates (3.0\% vs. $1.2 \%$ ). (However, for barbiturates there has not been a consistent sex difference in recent years.)

- There has been no consistent sex difference for tranquilizers over the years and only a very slight one for stimulant use in recent years (the 1986 annual prevalence for college males is $11 \%$ vs. $10 \%$ for females).

- Males also have higher prevalence rates on methaqualone $(1.7 \%$ vs. $0.8 \%$ ), but both sexes are so close to zero that the absolute differences are now negligible.

- As is true for the entire young adult sample, substantial sex differences are to be found in daily marijuana use $(2.8 \%$ for males vs. $1.5 \%$ for females), daily alcohol use $(6.4 \%$ vs. $3.1 \%$ ), and occasions of drinking five or more drinks in $\boldsymbol{a}$ row in the prior two weeks (58\% vs. $34 \%$ ).

- The one drug-using behavior which shows a sex difference appreciably different from those observed in the sample of all young adults involves cigarette smoking. While there consistently has been little or no sex difference in smoking rates among their age-peers not in college, among college students there has been a consistent and appreciable sex difference, with college women more likely to smoke. The half-a-pack per day rate in 1986 is considerably lower for college men than women ( $7 \%$ vs. $10 \%$, respectively), as is the daily figure $(10 \%$ vs. $15 \%)$ and the monthly prevalence figure $(20 \%$ vs. $24 \%$ ). For whatever reason, college women are quite a bit more likely to be cigarette smokers than their male counterparts. 
TABLE 28

\section{Trends in Annual Prevalence of Thirteen Types of Drugs Among College Students 1-4 Years Beyond High School}

\begin{tabular}{|c|c|c|c|c|c|c|c|c|}
\hline & \multicolumn{7}{|c|}{ Percent who used in last twelve months } & \multirow{2}{*}{$\begin{array}{l}\text { '85-'86 } \\
\text { change } \\
\end{array}$} \\
\hline & $\underline{1980}$ & $\underline{1981}$ & $\underline{1982}$ & $\underline{1983}$ & $\underline{1984}$ & $\underline{1985}$ & $\underline{1986}$ & \\
\hline Approx. Wtd. $\mathrm{N}=$ & $(1040)$ & $(1130)$ & (1150) & (1170) & (1110) & $(1080)$ & (1190) & \\
\hline Marijuana & 51.2 & 51.3 & 44.7 & 45.2 & 40.7 & 41.7 & 40.9 & -0.8 \\
\hline LSD & 6.1 & 4.6 & 6.3 & 4.2 & 3.7 & 2.2 & 3.9 & $+1.7 \mathrm{~s}$ \\
\hline Cocaine & 16.9 & 15.9 & 17.2 & 17.2 & 16.4 & 17.3 & 17.1 & -0.2 \\
\hline "Crack"b & NA & NA & NA & NA & NA & NA & 1.3 & NA \\
\hline Heroin & 0.4 & 0.2 & 0.1 & 0.0 & 0.1 & 0.2 & 0.1 & -0.1 \\
\hline Other Opiates ${ }^{8}$ & 5.1 & 4,4 & 3.8 & 3.8 & 3.8 & 2.4 & 4.0 & $+1.6 \mathrm{~s}$ \\
\hline $\begin{array}{l}\text { Stimulants }{ }^{\mathrm{a}} \\
\text { Stimulants, Adjusted }{ }^{\mathrm{a}, \mathrm{c}}\end{array}$ & $\begin{array}{r}22.4 \\
\mathrm{NA}\end{array}$ & $\begin{array}{r}22.2 \\
\mathrm{NA}\end{array}$ & $\begin{array}{r}\text { NA } \\
21.1\end{array}$ & $\begin{array}{r}\text { NA } \\
17.3\end{array}$ & $\begin{array}{r}\text { NA } \\
15.8\end{array}$ & $\begin{array}{r}\text { NA } \\
11.9\end{array}$ & $\begin{array}{r}\text { NA } \\
10.3\end{array}$ & $\begin{array}{r}\text { NA } \\
-1.6\end{array}$ \\
\hline Sedatives $^{\mathrm{a}}$ & 8.3 & 7.9 & 8.0 & 4.5 & 3.4 & 2.5 & 2.6 & +0.1 \\
\hline $\begin{array}{l}\text { Barbiturates }{ }^{\mathrm{a}} \\
\text { Methaqualone }^{\mathrm{a}}\end{array}$ & $\begin{array}{l}2.9 \\
7.2\end{array}$ & $\begin{array}{l}2.8 \\
6.5\end{array}$ & $\begin{array}{l}3.2 \\
6.6\end{array}$ & $\begin{array}{l}2.2 \\
3.1\end{array}$ & $\begin{array}{l}1.9 \\
2.5\end{array}$ & $\begin{array}{l}1.3 \\
1.4\end{array}$ & $\begin{array}{l}2.1 \\
1.2\end{array}$ & $\begin{array}{l}+0.8 \\
-0.2\end{array}$ \\
\hline Tranquilizers $^{a}$ & 6.9 & 4.8 & 4.7 & 4.6 & 3.5 & 3.5 & 4.4 & +0.9 \\
\hline Alcohol & 90.5 & 92.5 & 92.2 & 91.6 & 90.0 & 92.0 & 91.5 & -0.5 \\
\hline Cigarettes & NA & NA & NA & NA & $\mathrm{NA}$ & $\mathrm{NA}$ & NA & NA \\
\hline
\end{tabular}

NOTES: Level of significance of difference between the two most recent years: $\mathrm{s}=.05, \mathrm{ss}=.01, \mathrm{sss}=.001$.

NA indicates data not available.

${ }^{a}$ Only drug use which was not under a doctor's orders is included here.

${ }^{b}$ This drug was asked about in only one of the five questionnaire forms. $\mathrm{N}$ is one-fifth of $\mathrm{N}$ indicated.

${ }^{c}$ Based on the data from the revised question, which attempts to exclude the inappropriate reporting of non-prescription stimulants. 
TABLE 29

\section{Trends in Thirty-Day Prevalence of Twelve Types of Drugs Among College Students 1-4 Years Beyond High School}

\begin{tabular}{|c|c|c|c|c|c|c|c|c|}
\hline & & $\mathrm{Pe}$ & t who 1 & sed in $1 \mathrm{~s}$ & st thirty & days & & \\
\hline & $\underline{1980}$ & $\underline{1981}$ & $\underline{1982}$ & $\underline{1983}$ & $\underline{1984}$ & $\underline{1985}$ & $\underline{1986}$ & change \\
\hline pprox. Wtd. $\mathrm{N}=$ & $(1040)$ & $(1130)$ & $(1150)$ & $(1170)$ & $(1110)$ & $(1080)$ & (1190) & \\
\hline juana & 34.0 & 33.2 & 26.8 & 26.2 & 23.0 & 23.6 & 22.3 & -1.3 \\
\hline & 1.3 & 1.4 & 1.7 & 0.9 & 0.8 & 0.7 & 1.4 & +0.7 \\
\hline ine & 6.9 & 7.3 & 7.9 & 6.4 & 7.6 & 6.9 & 7.0 & +0.1 \\
\hline & 0.3 & 0.0 & 0.0 & 0.0 & 0.0 & 0.0 & 0.0 & 0.0 \\
\hline Opiates ${ }^{a}$ & 1.8 & 1.1 & 1.0 & 1.1 & 1.4 & 0.7 & 0.7 & 0.0 \\
\hline $\begin{array}{l}\text { ulants }^{\mathrm{a}} \\
\text { ulants, Adjusted } \\
\text { a,b }\end{array}$ & $\begin{array}{r}13.4 \\
\mathrm{NA}\end{array}$ & $\stackrel{12.3}{\mathrm{NA}}$ & $\begin{array}{l}\text { NA } \\
9.9\end{array}$ & $\begin{array}{l}\text { NA } \\
7.0\end{array}$ & $\begin{array}{l}\text { NA } \\
5.5\end{array}$ & $\begin{array}{l}\mathrm{NA} \\
4.2\end{array}$ & $\begin{array}{c}\text { NA } \\
3.7\end{array}$ & $\begin{array}{r}\text { NA } \\
-0.5\end{array}$ \\
\hline latives ${ }^{a}$ & 3.7 & 3.4 & 2.5 & 1.1 & 1.0 & 0.7 & 0.6 & -0.1 \\
\hline $\begin{array}{l}\text { Barbiturates }{ }^{\mathrm{a}} \\
\text { Methaqualone }^{\mathrm{a}}\end{array}$ & $\begin{array}{l}0.9 \\
3.1\end{array}$ & $\begin{array}{l}0.8 \\
3.0\end{array}$ & $\begin{array}{l}0.9 \\
1.9\end{array}$ & $\begin{array}{l}0.5 \\
0.7\end{array}$ & $\begin{array}{l}0.7 \\
0.5\end{array}$ & $\begin{array}{l}0.4 \\
0.2\end{array}$ & $\begin{array}{l}0.5 \\
0.1\end{array}$ & $\begin{array}{l}+0.1 \\
-0.1\end{array}$ \\
\hline quilizers $^{\mathrm{a}}$ & 2.0 & 1.4 & 1.4 & 1.1 & 1.2 & 1.4 & 1.8 & +0.4 \\
\hline 101 & 81.8 & 81.9 & 82.8 & 80.3 & 79.1 & 80.3 & 79.7 & -0.6 \\
\hline rettes & 25.8 & 25.9 & 24.4 & 24.7 & 21.5 & 22.4 & 22.4 & 0.0 \\
\hline
\end{tabular}

NOTES: Level of significance of difference between the two most recent years: $\mathrm{s}=.05 . \mathrm{ss}=.01$, sss $=.001$. NA indicates data not available.

anly drug use which was not under a doctor's orders is included here.

${ }^{b}$ Based on the data from the revised question, which attempts to exclude the inappropriate reporting of non-prescription stimulants. 
TABLE 30

\section{Trends in Thirty-Day Prevalence of Daily Use of Twelve Types of Drugs} Among College Students 1-4 Years Beyond High School

\begin{tabular}{|c|c|c|c|c|c|c|c|c|}
\hline & \multicolumn{7}{|c|}{ Percent who used daily in last thirty days } & \multirow{2}{*}{$\begin{array}{l}\text { '85-' } 86 \\
\text { change } \\
\end{array}$} \\
\hline & $\underline{1980}$ & $\underline{1981}$ & $\underline{1982}$ & $\underline{1983}$ & $\underline{1984}$ & $\underline{1985}$ & $\underline{1986}$ & \\
\hline Approx. Wtd. $\mathrm{N}=$ & $(1040)$ & $(1130)$ & (1150) & (1170) & (1110) & $(1080)$ & $(1190)$ & \\
\hline arijuana & 7.2 & 5.6 & 4.2 & 3.8 & 3.6 & 3.1 & 2.1 & -1.0 \\
\hline $\mathrm{D}$ & 0.0 & 0.0 & 0.0 & 0.0 & 0.0 & 0.0 & 0.0 & 0.0 \\
\hline caine & 0.2 & 0.0 & 0.3 & 0.1 & 0.4 & 0.1 & 0.1 & 0.0 \\
\hline roin & 0.0 & 0.0 & 0.0 & 0.0 & 0.0 & 0.0 & 0.0 & 0.0 \\
\hline her Opiates ${ }^{\mathrm{a}}$ & 0.0 & 0.1 & 0.0 & 0.1 & 0.1 & 0.0 & 0.0 & 0.0 \\
\hline $\begin{array}{l}\text { imulants }{ }^{a} \\
\text { imulants, Adjusted }\end{array}$ & $\begin{array}{l}0.5 \\
\mathrm{NA}\end{array}$ & $\begin{array}{l}0.4 \\
\text { NA }\end{array}$ & $\begin{array}{l}\text { NA } \\
0.3\end{array}$ & $\begin{array}{l}\text { NA } \\
0.2\end{array}$ & $\begin{array}{l}\mathrm{NA} \\
0.2\end{array}$ & $\begin{array}{l}\text { NA } \\
0.0\end{array}$ & $\begin{array}{l}\text { NA } \\
0.1\end{array}$ & $\begin{array}{r}\text { NA } \\
+0.1\end{array}$ \\
\hline datives $^{\mathrm{a}}$ & 0.0 & 0.0 & 0.1 & 0.0 & 0.1 & 0.0 & 0.0 & 0.0 \\
\hline $\begin{array}{l}\text { Barbiturates }{ }^{a} \\
\text { Methaqualone }\end{array}$ & $\begin{array}{l}0.0 \\
0.0\end{array}$ & $\begin{array}{l}0.0 \\
0.0\end{array}$ & $\begin{array}{l}0.1 \\
0.0\end{array}$ & $\begin{array}{l}0.0 \\
0.0\end{array}$ & $\begin{array}{l}0.1 \\
0.0\end{array}$ & $\begin{array}{l}0.0 \\
0.0\end{array}$ & $\begin{array}{l}0.0 \\
0.0\end{array}$ & $\begin{array}{l}0.0 \\
0.0\end{array}$ \\
\hline anquilizers ${ }^{a}$ & 0.0 & 0.0 & 0.1 & 0.0 & 0.1 & 0.0 & 0.0 & 0.0 \\
\hline cohol & 6.5 & 5.4 & 6.1 & 6.1 & 6.6 & 5.0 & 4.6 & -0.4 \\
\hline $\begin{array}{l}\text { Five or more drinks in a row } \\
\text { in last two weeks }\end{array}$ & 43.9 & 43.6 & 44.0 & 43.1 & 45.5 & 44.6 & 45.0 & +0.4 \\
\hline garettes & 18.3 & 17.1 & 16.2 & 15.3 & 14.8 & 14.3 & 12.7 & -1.6 \\
\hline $\begin{array}{l}\text { Half pack or more per day } \\
\text { in last thirty days }\end{array}$ & 12.7 & 11.9 & 10.5 & 9.6 & 10.2 & 9.4 & 8.3 & -1.1 \\
\hline
\end{tabular}

NOTES: Level of significance of difference between the two most recent years:

$$
\mathrm{s}=.05, \mathrm{ss}=.01, \mathrm{sss}=.001 \text {. }
$$

NA indicated data not available.



based on the data from the revised question, which attempts to exclude the inappropriate reporting of nonprescription stimulants. 


\section{TABLE 31}

\section{Trends in Annual and Thirty-Day Prevalence of An Illicit Drug Use Index}

Among College Students 1-4 Years Beyond High School

by Sex

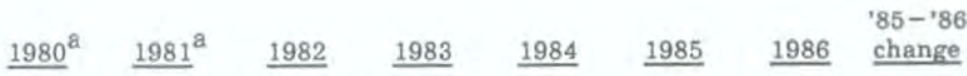

Percent reporting use in last twelve months

\begin{tabular}{|c|c|c|c|c|c|c|c|}
\hline Any Illicit Drug & 56.2 & 55.0 & 49.5 & 49.9 & 45.1 & 46.3 & 45.0 \\
\hline $\begin{array}{l}\text { Males } \\
\text { Females }\end{array}$ & $\begin{array}{l}58.9 \\
53.3\end{array}$ & $\begin{array}{l}56.2 \\
54.0\end{array}$ & $\begin{array}{l}54.6 \\
44.9\end{array}$ & $\begin{array}{l}53.4 \\
46.7\end{array}$ & $\begin{array}{l}48.4 \\
41.9\end{array}$ & $\begin{array}{l}50.9 \\
42.7\end{array}$ & $\begin{array}{l}49.8 \\
41.0\end{array}$ \\
\hline $\begin{array}{l}\text { Any Illicit Drug Other than } \\
\text { Marijuana }\end{array}$ & 32.3 & 31.8 & 30.0 & 29.9 & 27.2 & 26.7 & 25.0 \\
\hline $\begin{array}{l}\text { Males } \\
\text { Females }\end{array}$ & $\begin{array}{l}33.7 \\
31.1\end{array}$ & $\begin{array}{l}32.8 \\
30.9\end{array}$ & $\begin{array}{l}33.4 \\
26.9\end{array}$ & $\begin{array}{l}33.5 \\
26.7\end{array}$ & $\begin{array}{l}29.2 \\
25.2\end{array}$ & $\begin{array}{l}29.7 \\
24.4\end{array}$ & $\begin{array}{l}28.6 \\
22.0\end{array}$ \\
\hline $\begin{array}{l}\text { Any Illicit Drug Other than } \\
\text { Marijuana or Stimulants }\end{array}$ & 25.2 & 22.6 & 22.3 & 23.6 & 21.1 & 21.4 & 21.6 \\
\hline $\begin{array}{l}\text { Males } \\
\text { Females }\end{array}$ & $\begin{array}{l}28.4 \\
22.1\end{array}$ & $\begin{array}{l}25.7 \\
19.8\end{array}$ & $\begin{array}{l}25.7 \\
19.3\end{array}$ & $\begin{array}{l}26.6 \\
21.1\end{array}$ & $\begin{array}{l}25.3 \\
17.0\end{array}$ & $\begin{array}{l}24.4 \\
19.0\end{array}$ & $\begin{array}{l}25.8 \\
18.0\end{array}$ \\
\hline
\end{tabular}

Percent reporting use in last thirty days

Any Illicit Drug
Males
Females
Any Illicit Drug Other than
Marijuana
Males
Females

Any Illicit Drug Other than Marijuana or Stimulants

Males

Females

\section{$38.4 \quad 37.6$}

42.9

34.0

40.6

34.8

$20.7 \quad 18.6$

22.9

18.6

18.6

18.5

$12.6 \quad 11.5$

13.3

15.2

10.1

13.3
9.9

31.3

29.4

27.0

26.1

25.9

33.8

30.4

29.9

23.2

31.0

21.6

$-0.2$

25.6

25.5

23.7

11.8

11.6

$-0.2$

20.2

16.0

16.1

12.7

14.4

11.6

11.2

$14.2 \quad 12.2$

10.7
13.5
8.0

9.1

$9.7+0.6$

13.1

12.1

7.8

10.6

$12.7+2.1$

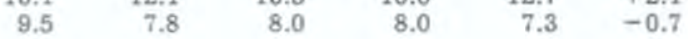

Approximate Weighted N's

$\begin{array}{lrrrrrrr}\text { All Respondents }= & (1040) & (1130) & (1150) & (1170) & (1110) & (1080) & (1190) \\ \text { Males } & (520) & (530) & (550) & (550) & (540) & (490) & (540) \\ \text { Females } & (520) & (600) & (610) & (620) & (570) & (600) & (650)\end{array}$

NOTES: Level of significance of difference between the two most recent years:

$$
\mathbf{s}=.05, \mathrm{ss}=.01, \mathrm{sss}=.001 .
$$

${ }^{\text {a }}$ Revised questions about stimulant use were introduced in 1982 to exclude more completely the inappropriate reporting of nonprescription stimulants. The data in italics are therefore not strictly comparable to the other data. 
Any Illicit Drug: Trends in Annual Prevalence Among College Students Vs. Others ${ }^{a}$ 1-4 Years Beyond High School



Any Illicit Drug: Trends in Annual Prevalence Among Male and Female College Students

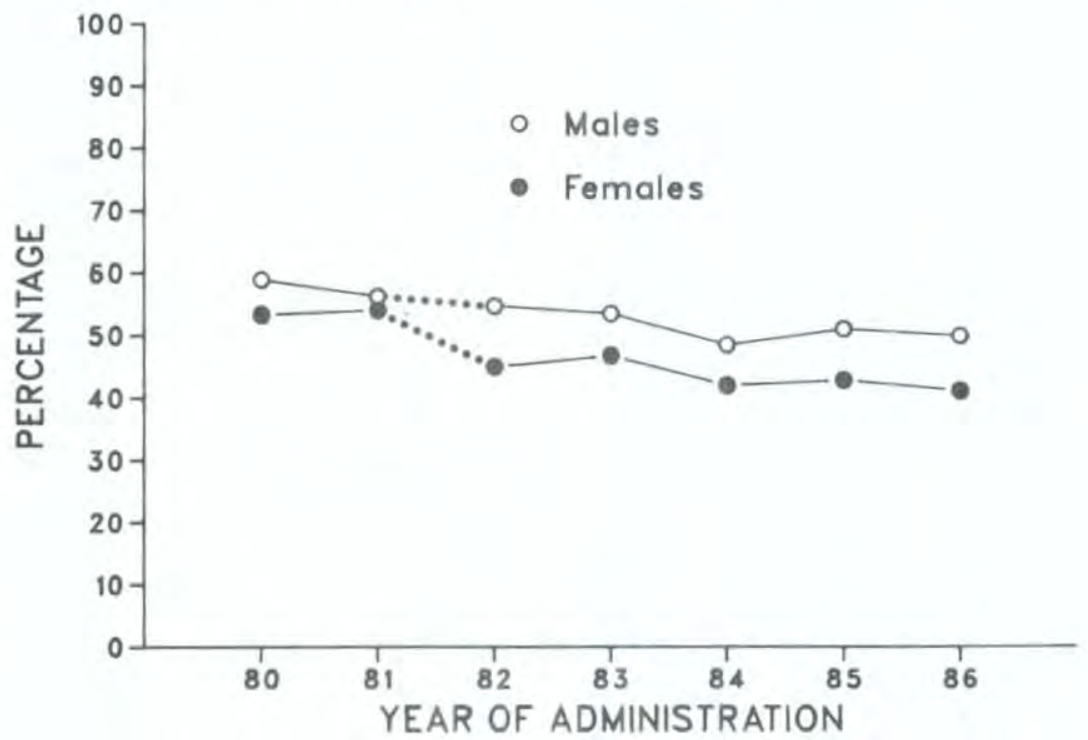

NOTE: The dotted lines between 1981 and 1982 denote the change in the amphetamine question.

a"Others" refers to high school graduates 1-4 years beyond high school not currently enrolled full-time in college. 
Any Illicit Drug Other than Marijuana: Trends in Annual

Prevalence Among College Students Vs. Others

1-4 Years Beyond High School

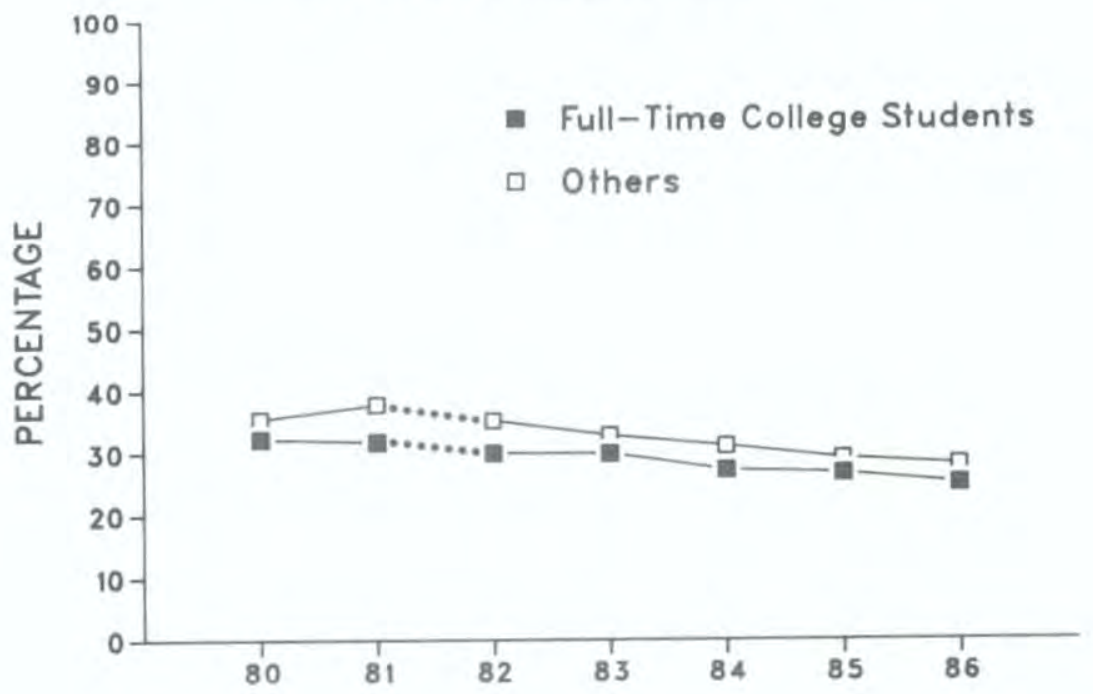

Any Illicit Drug Other than Marijuana: Trends in Annual Prevalence Among Male and Female College Students

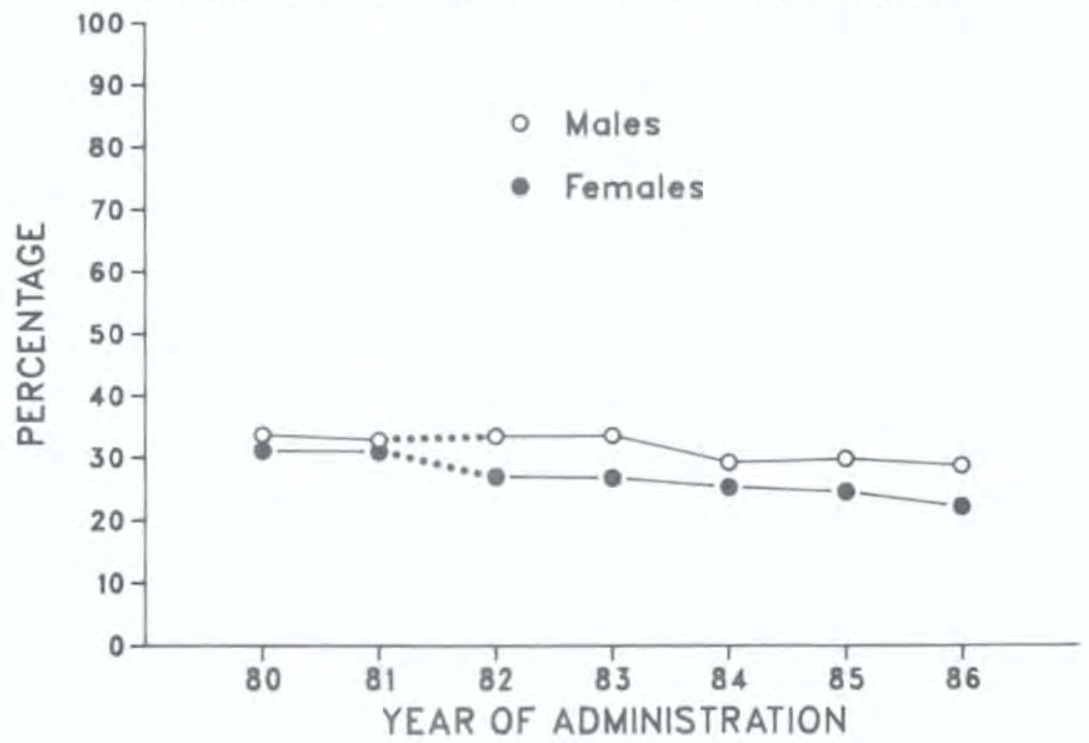

NOTE: The dotted lines between 1981 and 1982 denote the change in the amphetamine question. 
FIGURE 54

Any Illicit Drug Other than Marijuana or Stimulants: Trends in Annual Prevalence Among College Students Vs. Others 1-4 Years Beyond High School

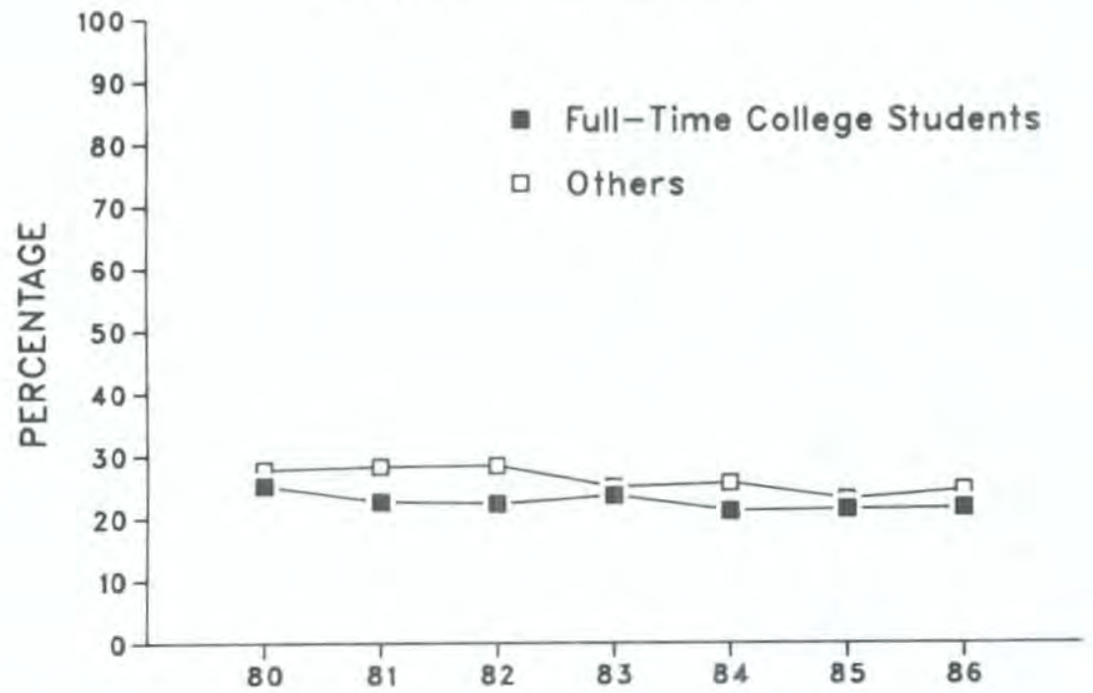

Any Illicit Drug Other than Marijuana or Stimulants: Trends in Annual Prevalence Among Male and Female College Students

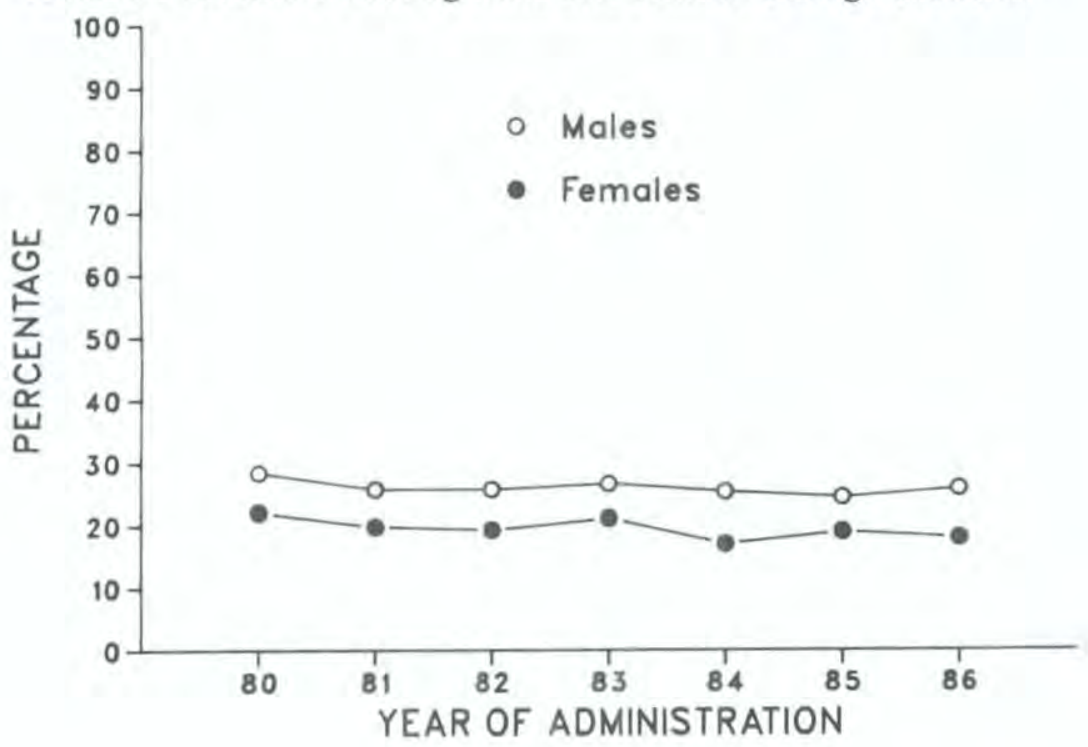




\section{FIGURE 55a}

Marijuana: Trends in Annual Prevalence Among College Students Vs. Others 1-4 Years Beyond High School

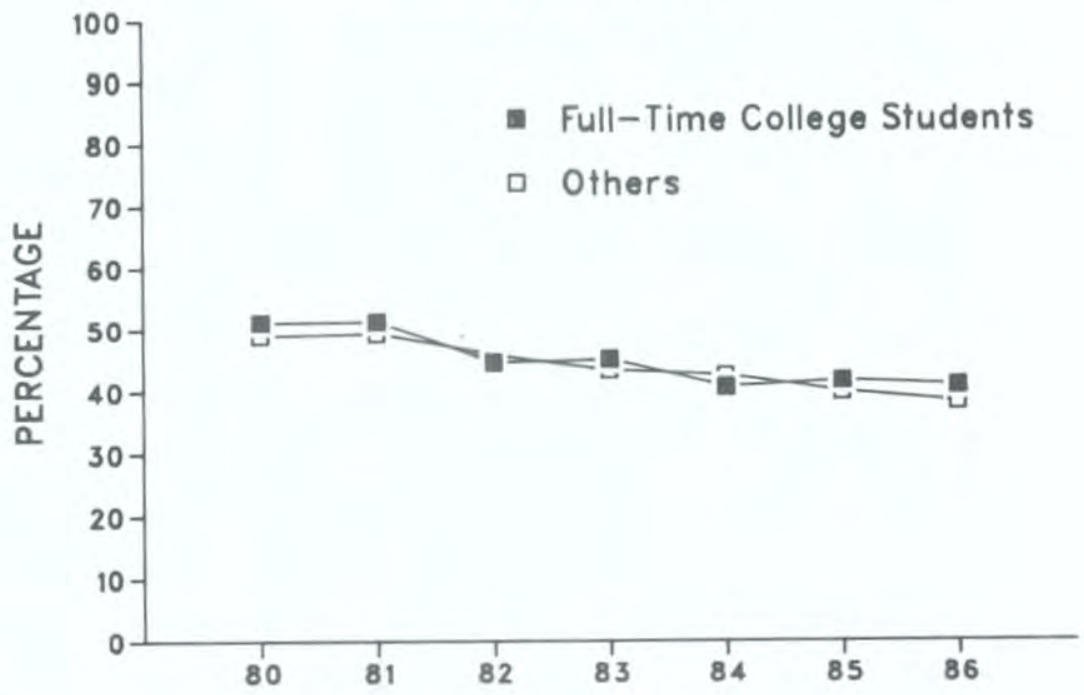

Marijuana: Trends in Annual Prevalence Among Male and Female College Students

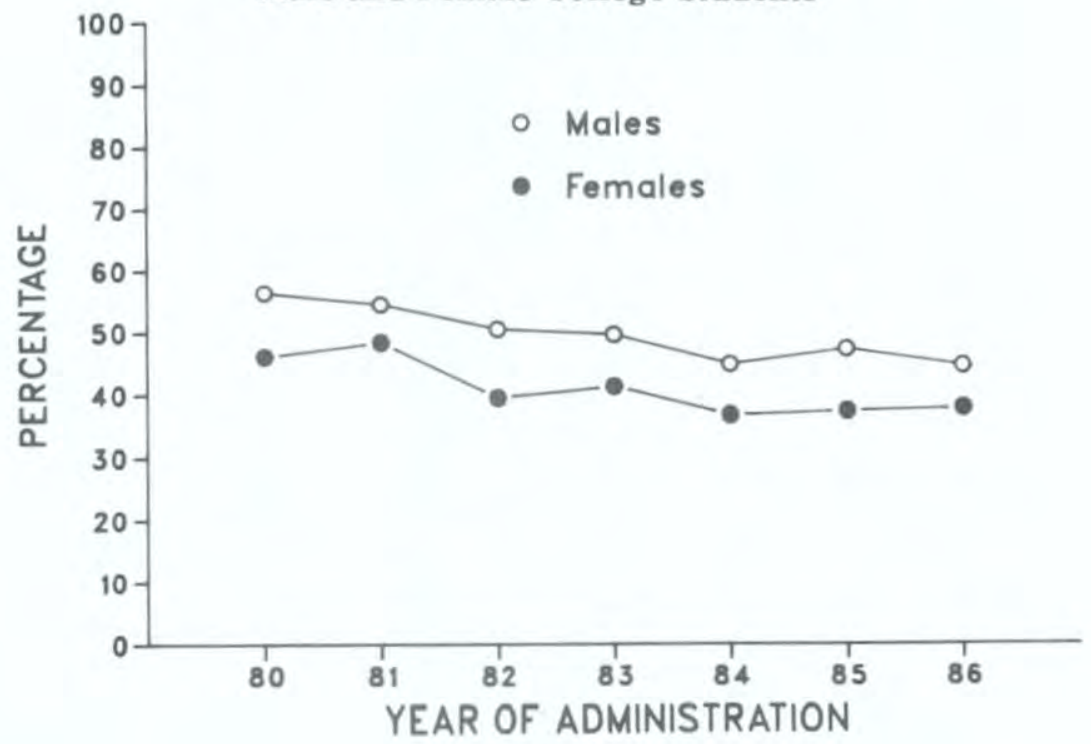




\section{FIGURE 55b}

Marijuana: Trends in Thirty-Day Prevalence of Daily Use Among College Students Vs. Others 1-4 Years Beyond High School

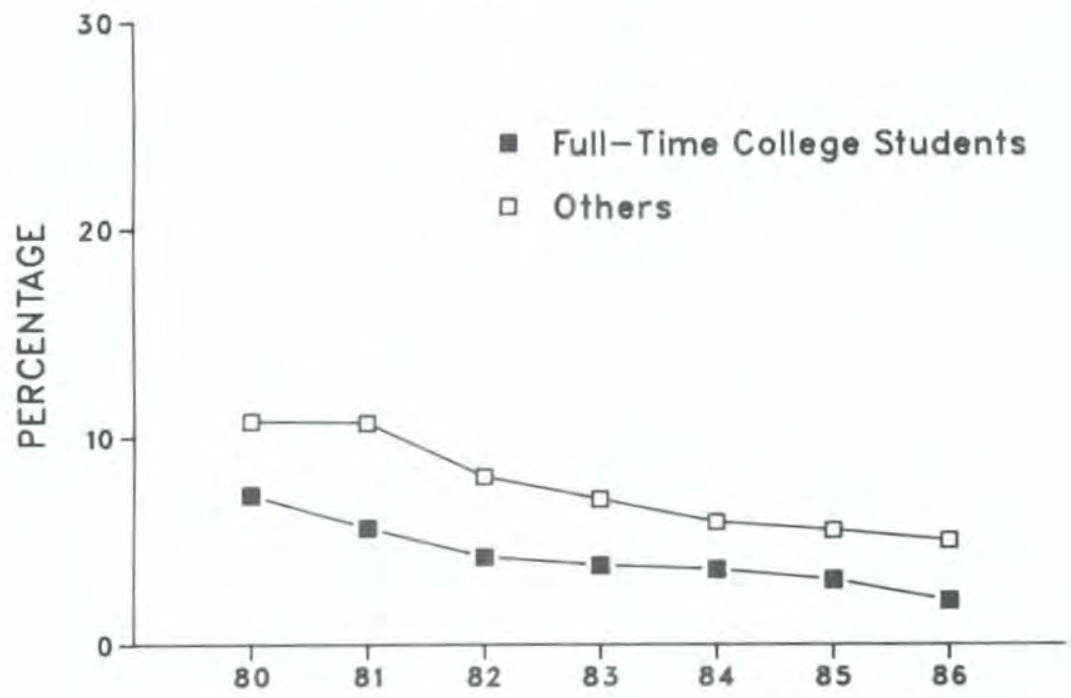

Marijuana: Trends in Thirty-Day Prevalence of Daily Use Among Male and Female College Students

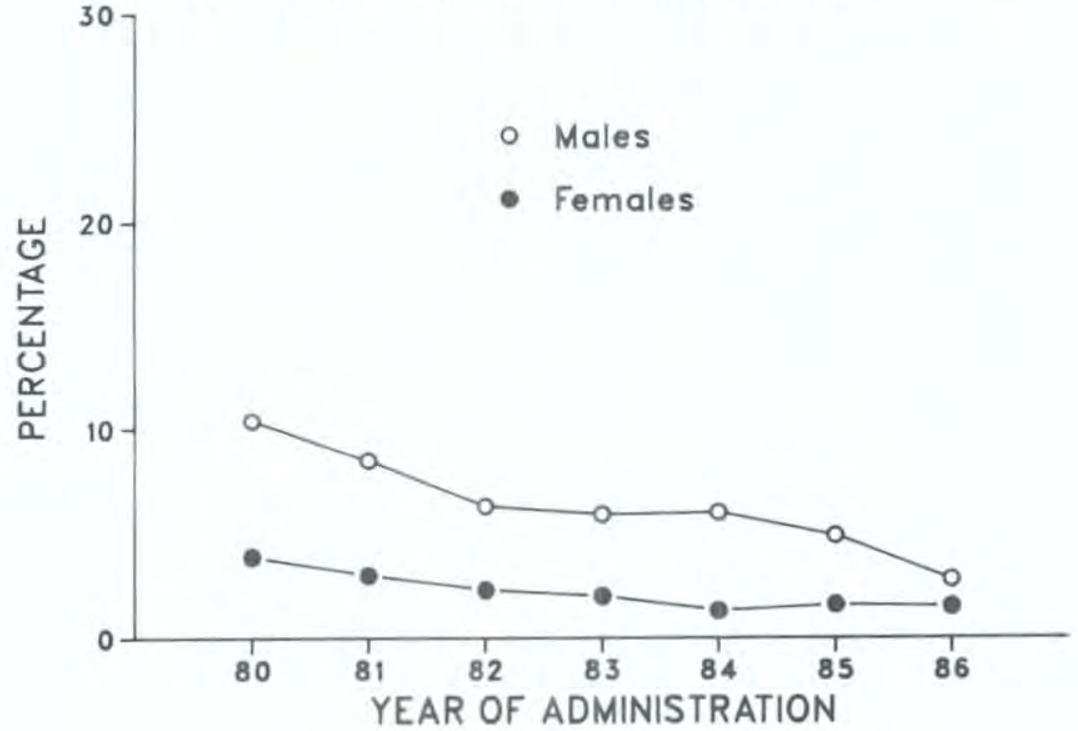


LSD: Trends in Annual Prevalence Among College Students Vs. Others 1-4 Years Beyond High School

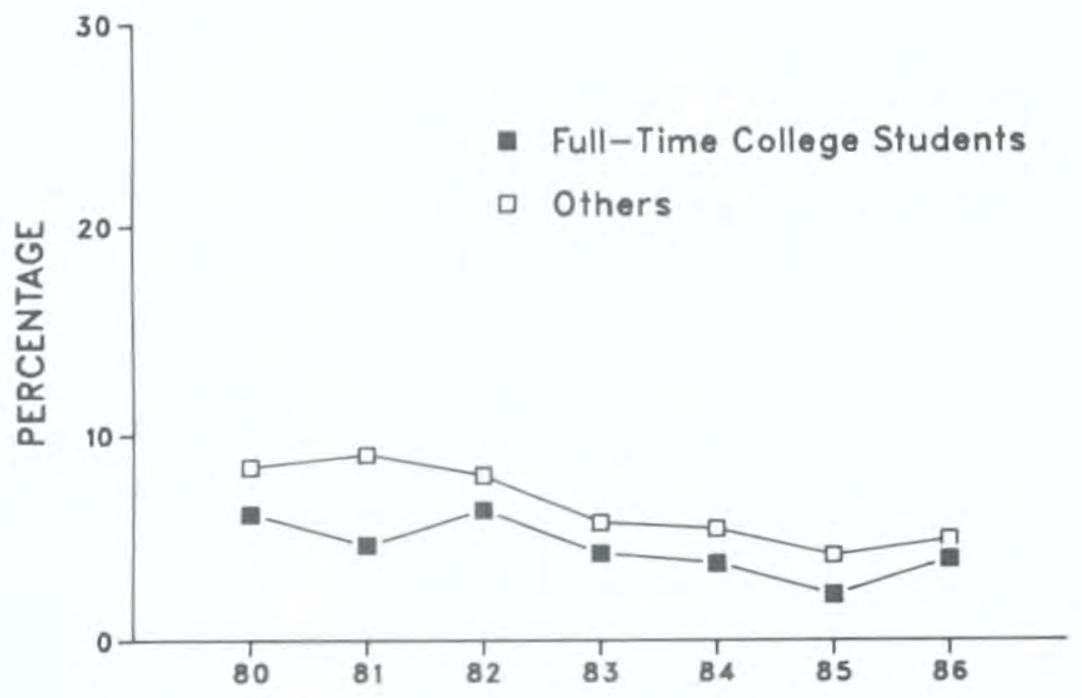

LSD: Trends in Annual Prevalence Among Male and Female College Students

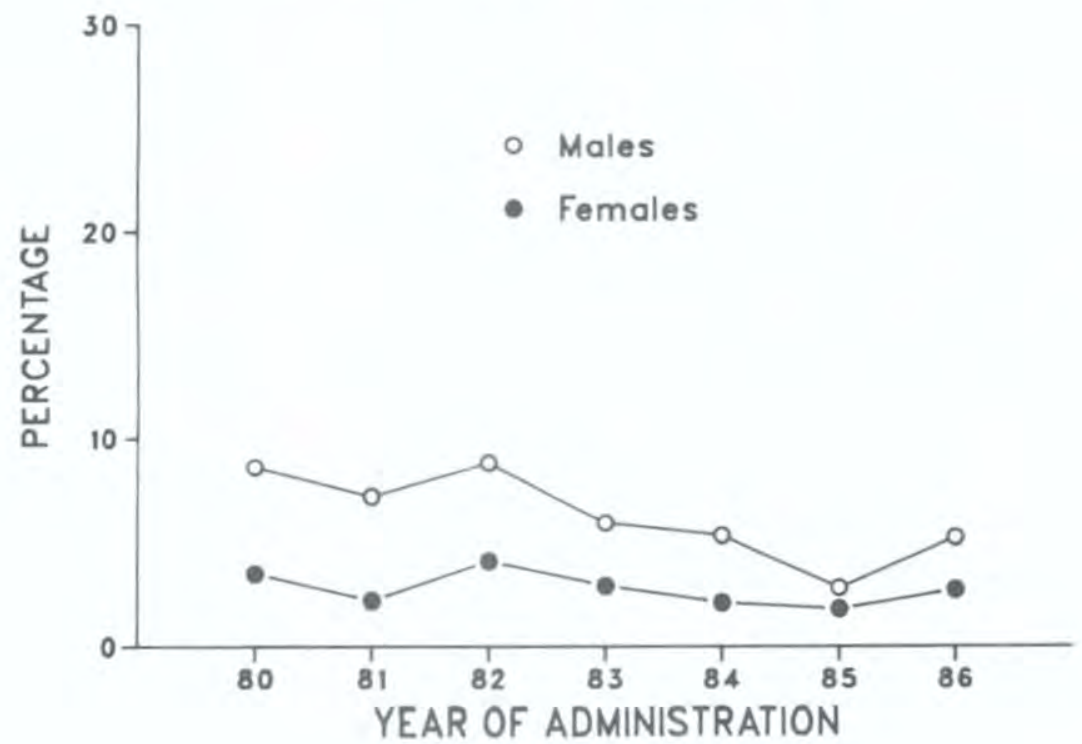


Cocaine: Trends in Annual Prevalence Among College Students Vs. Others 1-4 Years Beyond High School

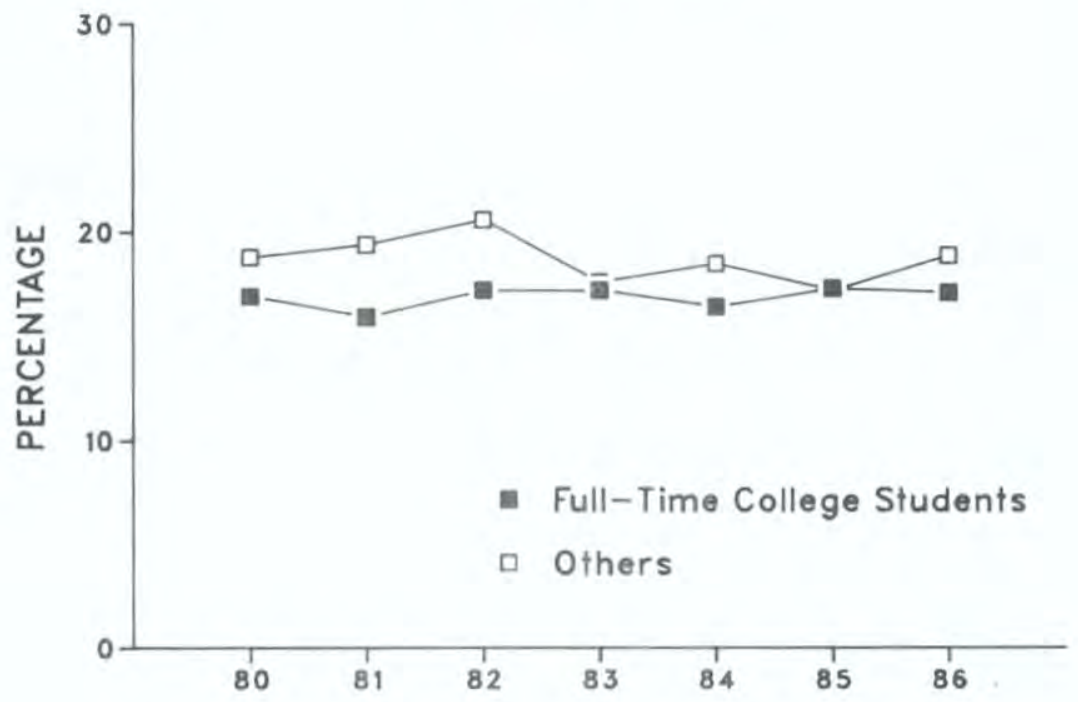

Cocaine: Trends in Annual Prevalence Among Male and Female College Students

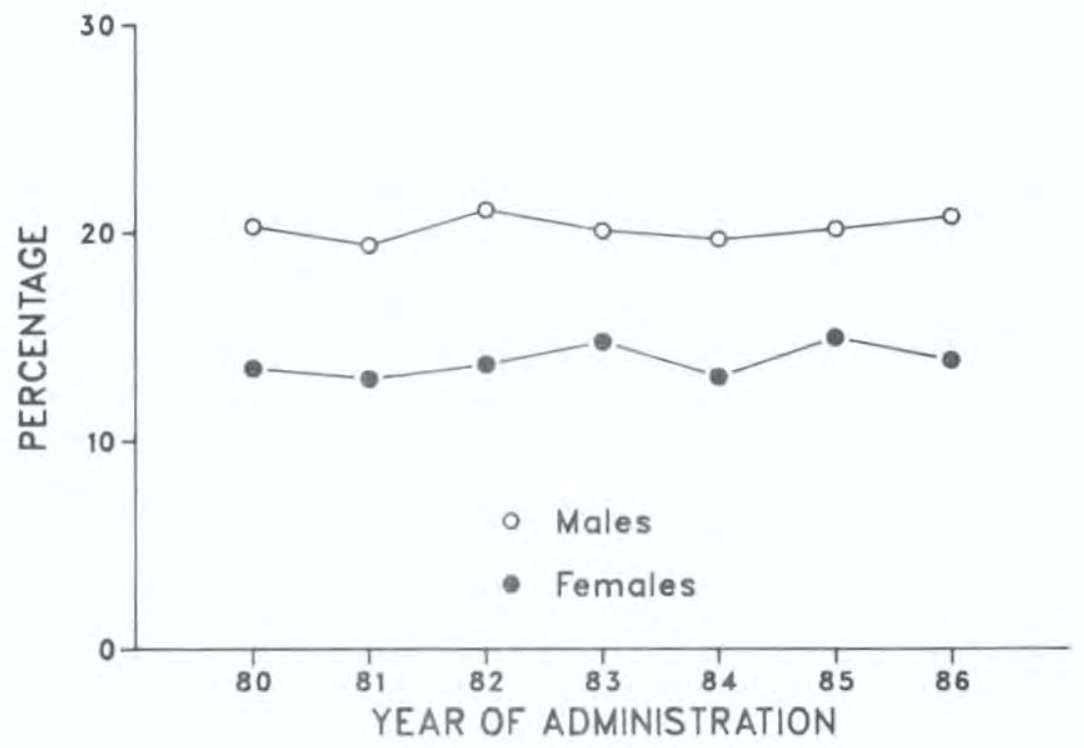


FIGURE 58

Other Opiates: Trends in Annual Prevalence Among College Students Vs. Others 1-4 Years Beyond High School

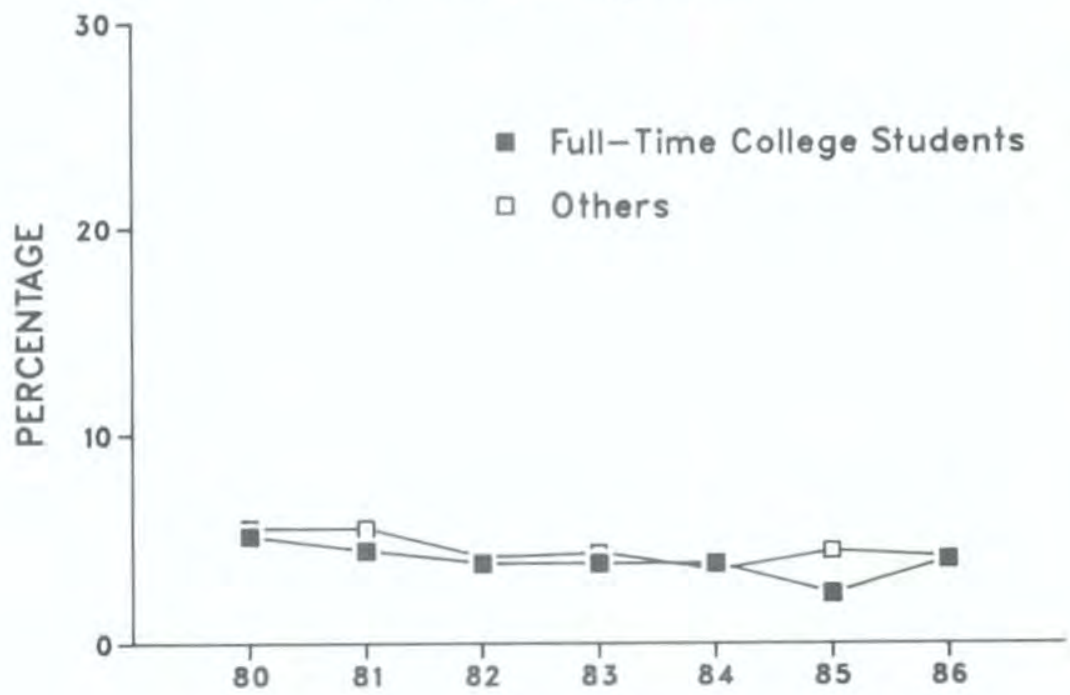

Other Opiates: Trends in Annual Prevalence Among Male and Female College Students

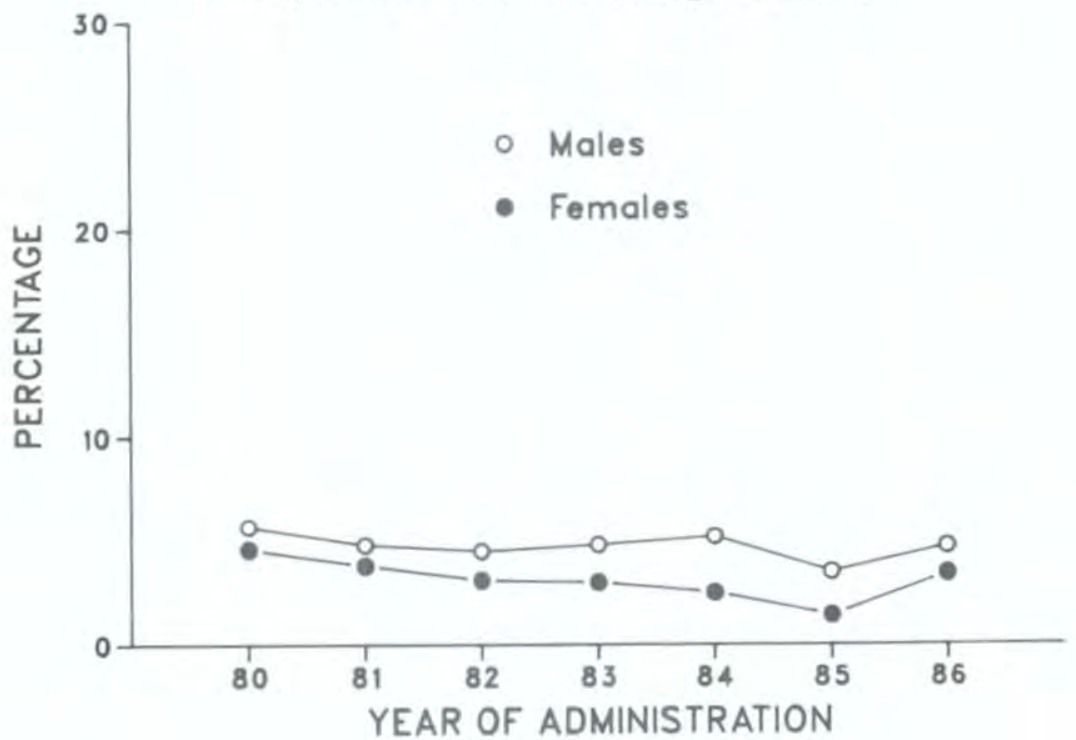


Stimulants: Trends in Annual Prevalence Among College Students Vs. Others 1-4 Years Beyond High School

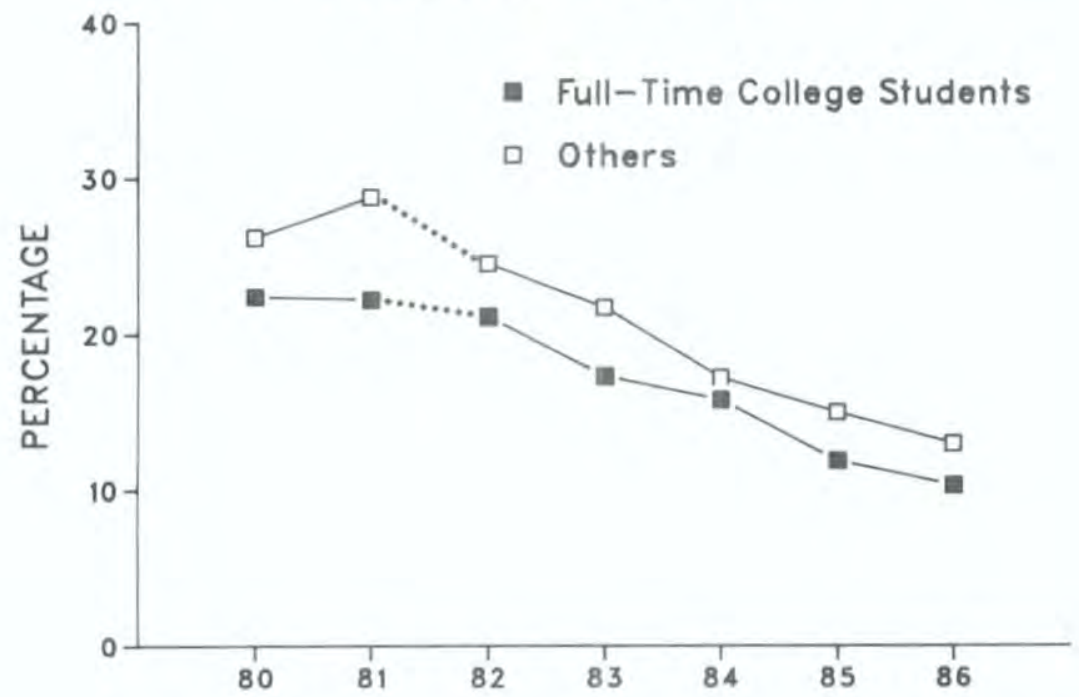

Stimulants: Trends in Annual Prevalence Among Male and Female College Students

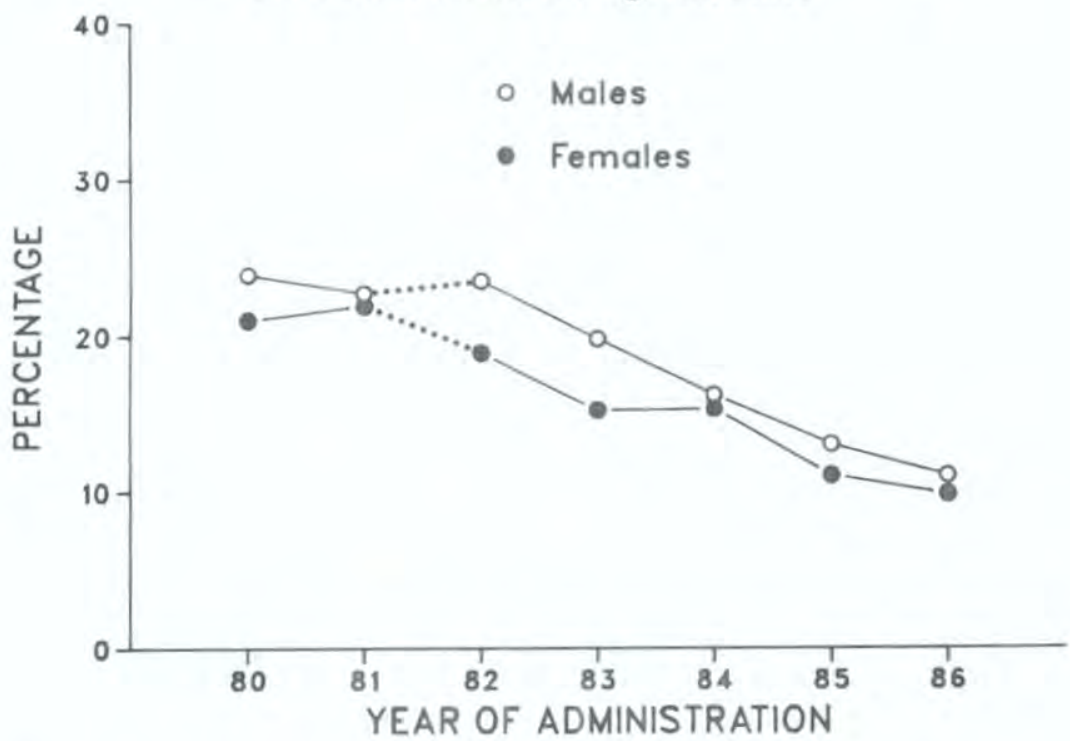

NOTE: The dotted lines between 1981 and 1982 denote the change in the amphetamine question. 


\section{FIGURE 60}

Barbiturates: Trends in Annual Prevalence Among College Students Vs. Others

1-4 Years Beyond High School

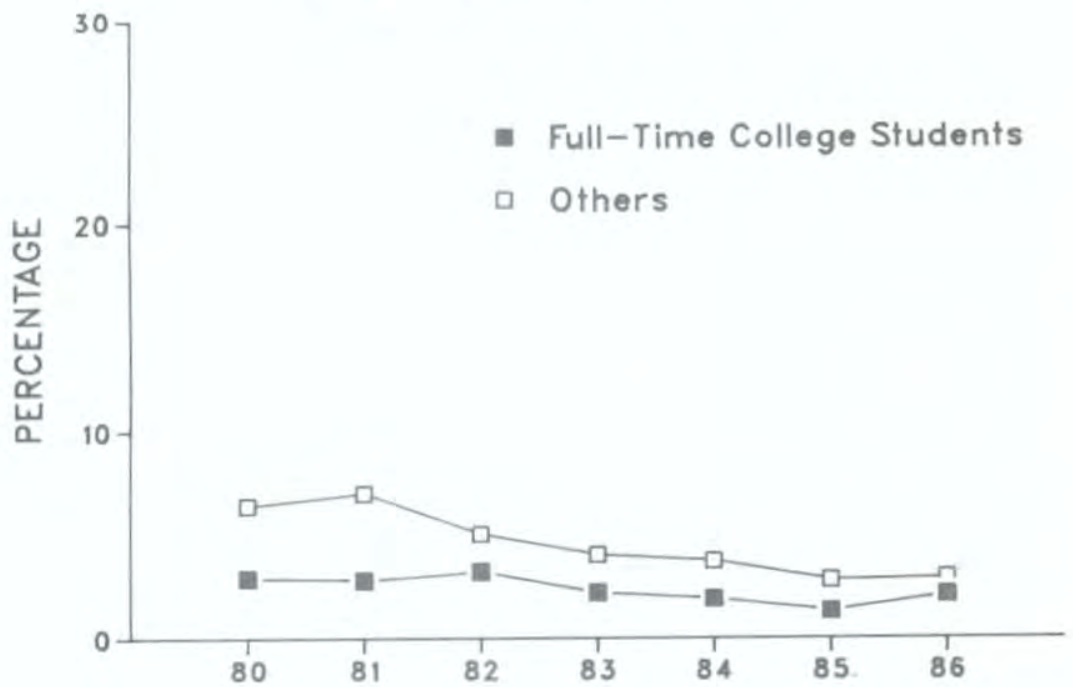

Barbiturates: Trends in Annual Prevalence Among Male and Female College Students

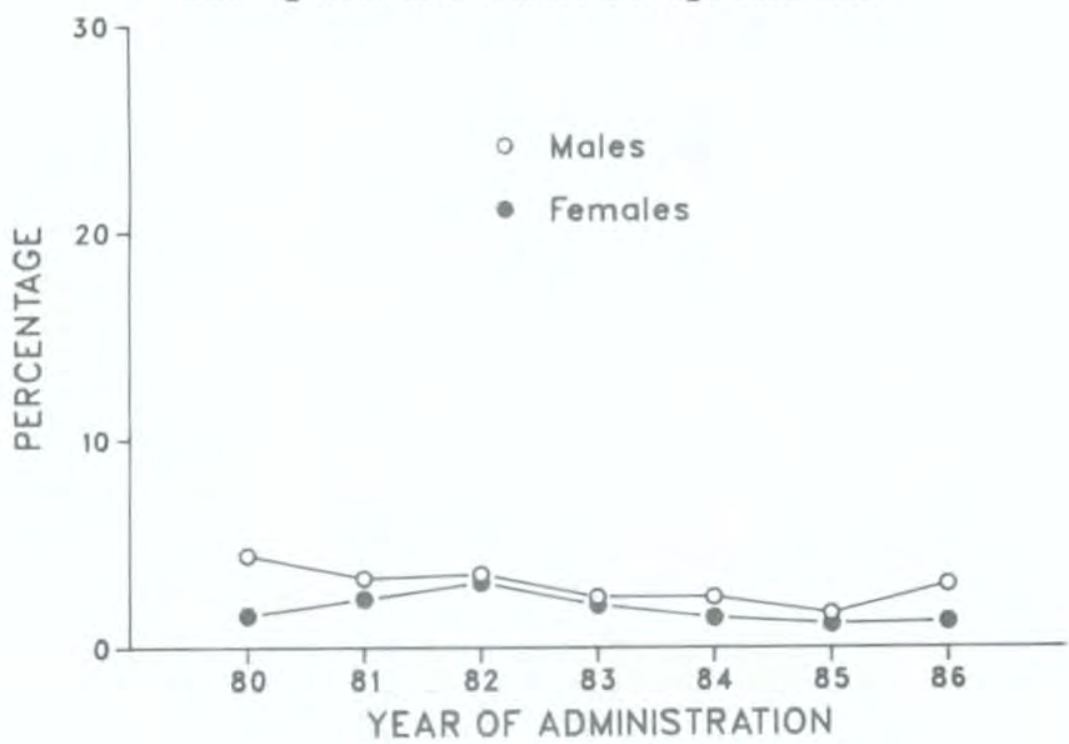


FIGURE 61

Methaqualone: Trends in Annual Prevalence Among College Students Vs. Others

1-4 Years Beyond High School

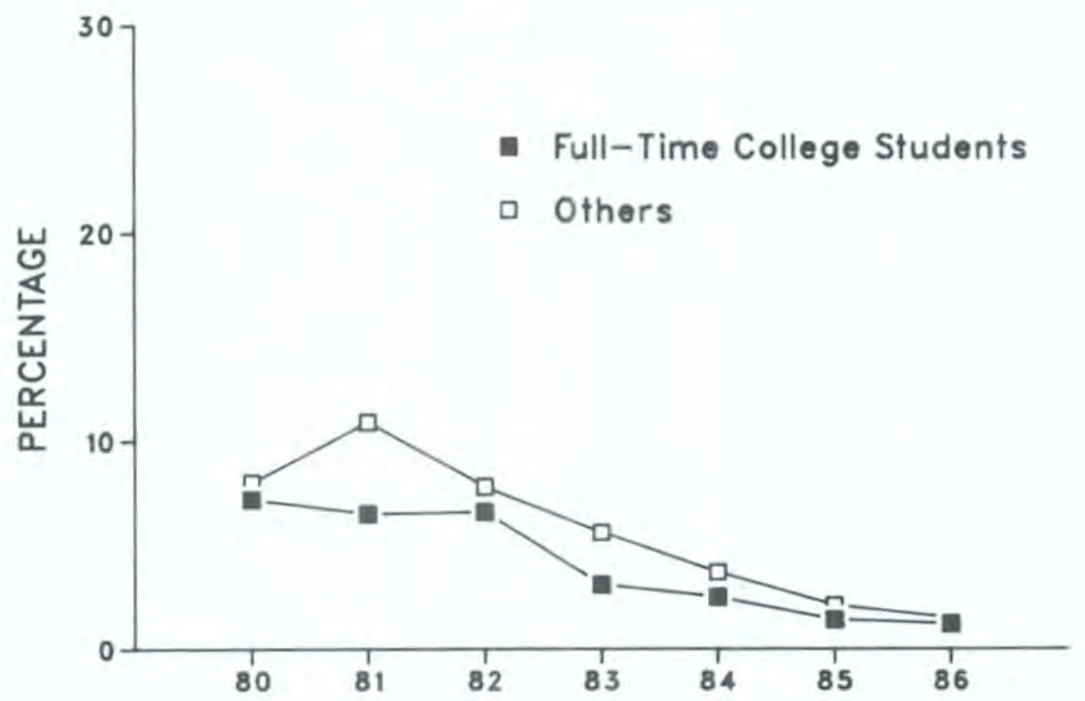

Methaqualone: Trends in Annual Prevalence Among Male and Female College Students

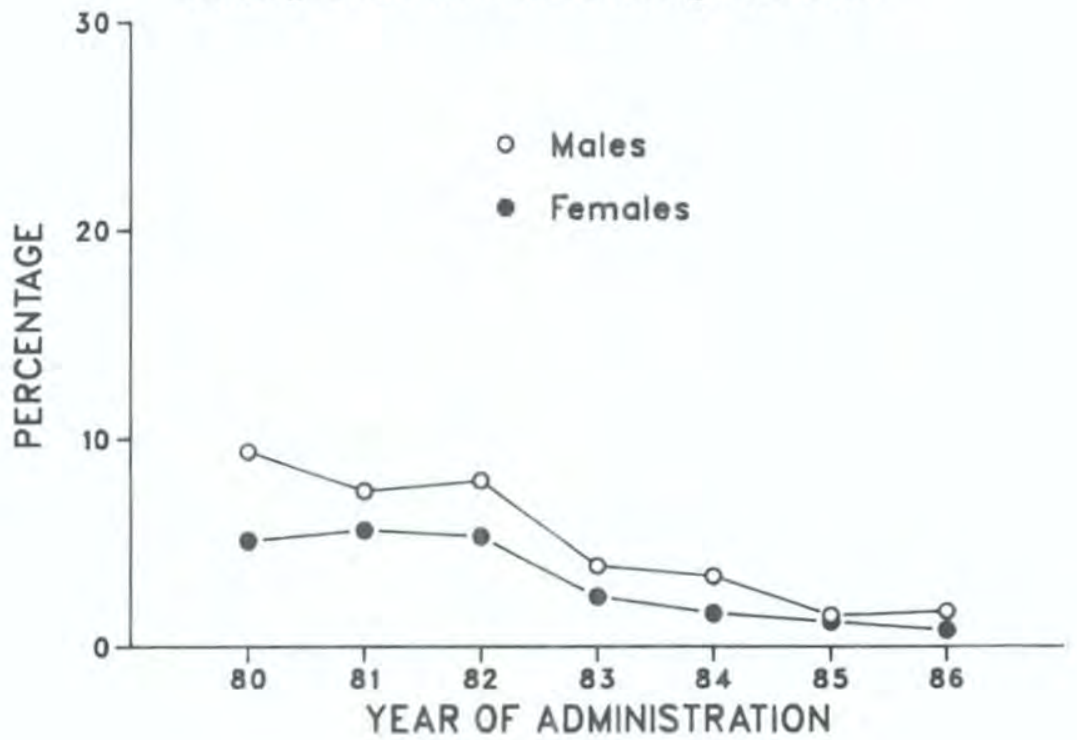




\section{FIGURE 62}

Tranquilizers: Trends in Annual Prevalence Among College Students Vs. Others

1-4 Years Beyond High School

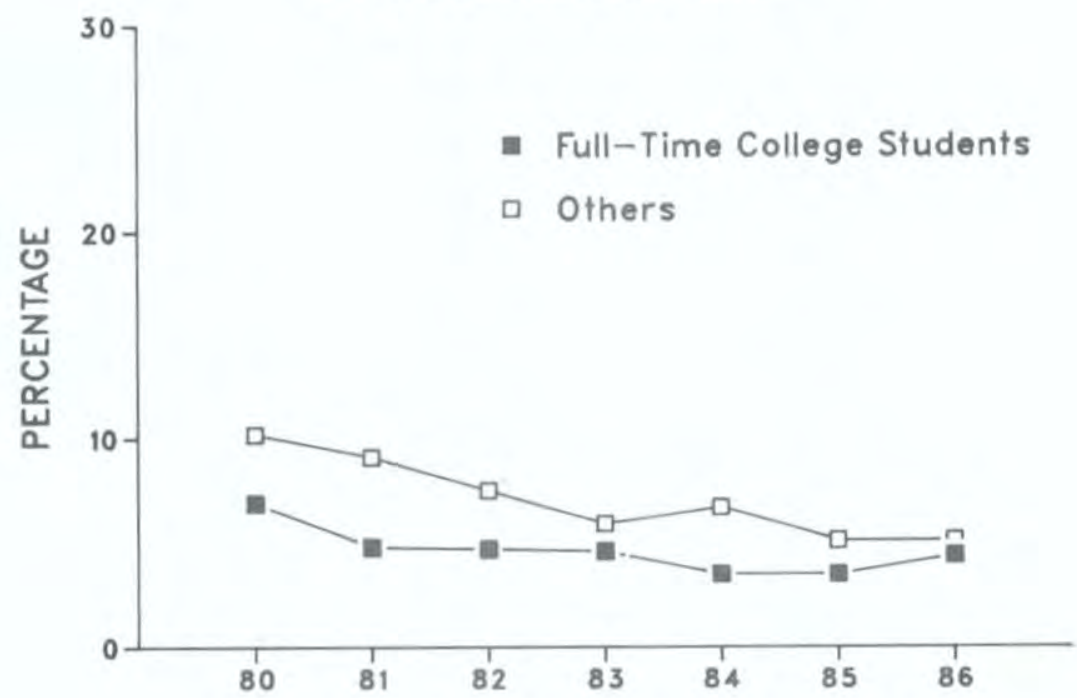

Tranquilizers: Trends in Annual Prevalence Among Male and Female College Students

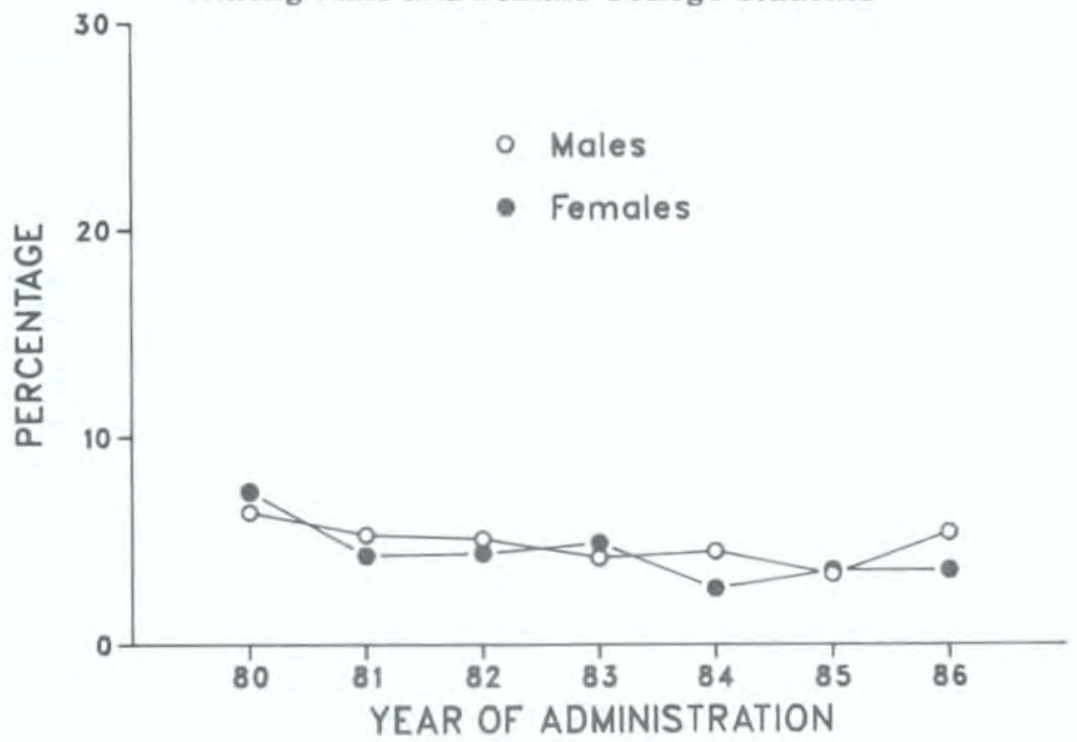


Alcohol: Trends in Annual Prevalence Among College Students Vs. Others 1-4 Years Beyond High School

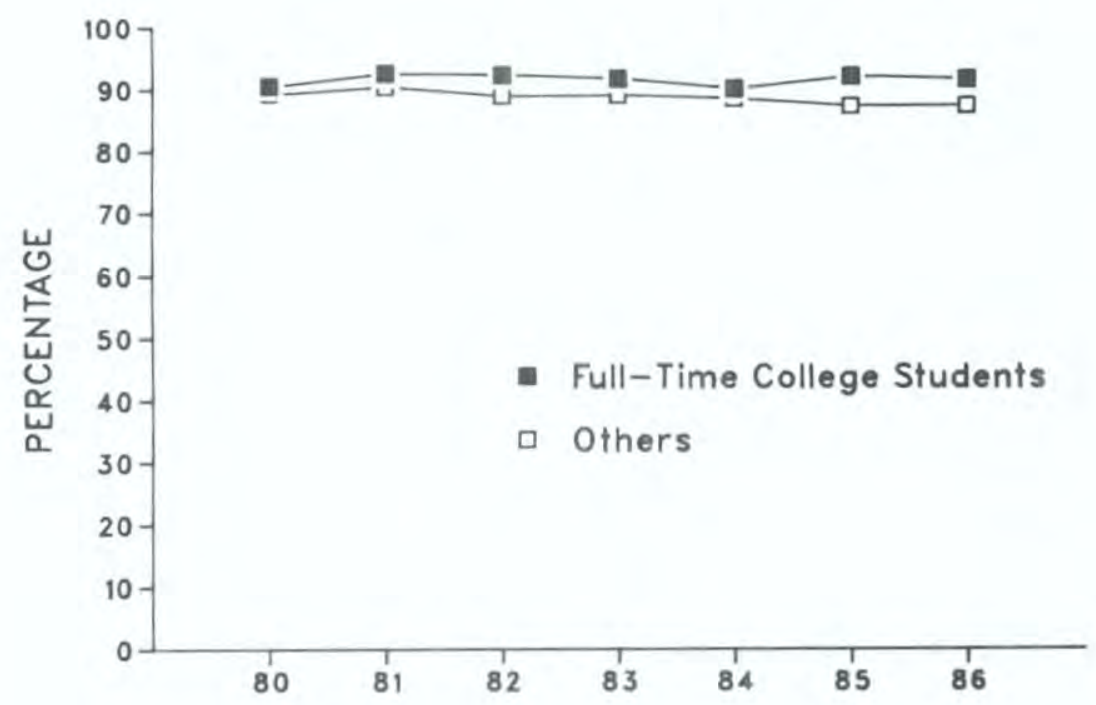

Alcohol: Trends in Annual Prevalence Among Male and Female College Students

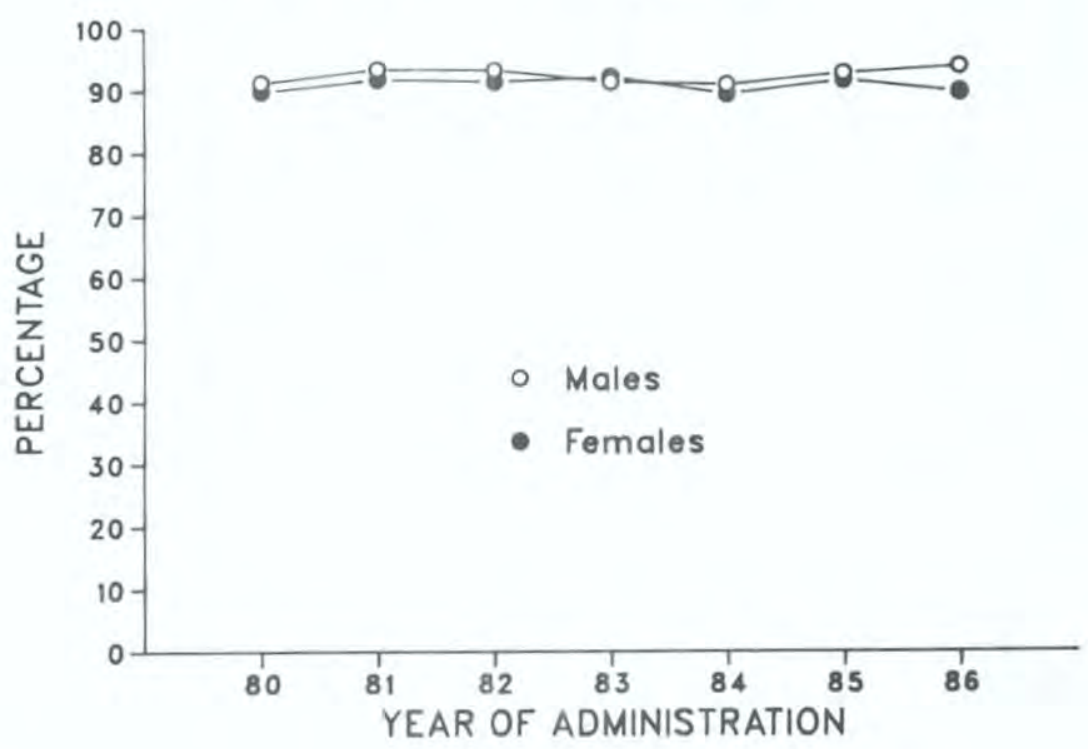




\section{FIGURE 63b}

Alcohol: Trends in Thirty-Day Prevalence of Daily Use Among College Students Vs. Others 1-4 Years Beyond High School

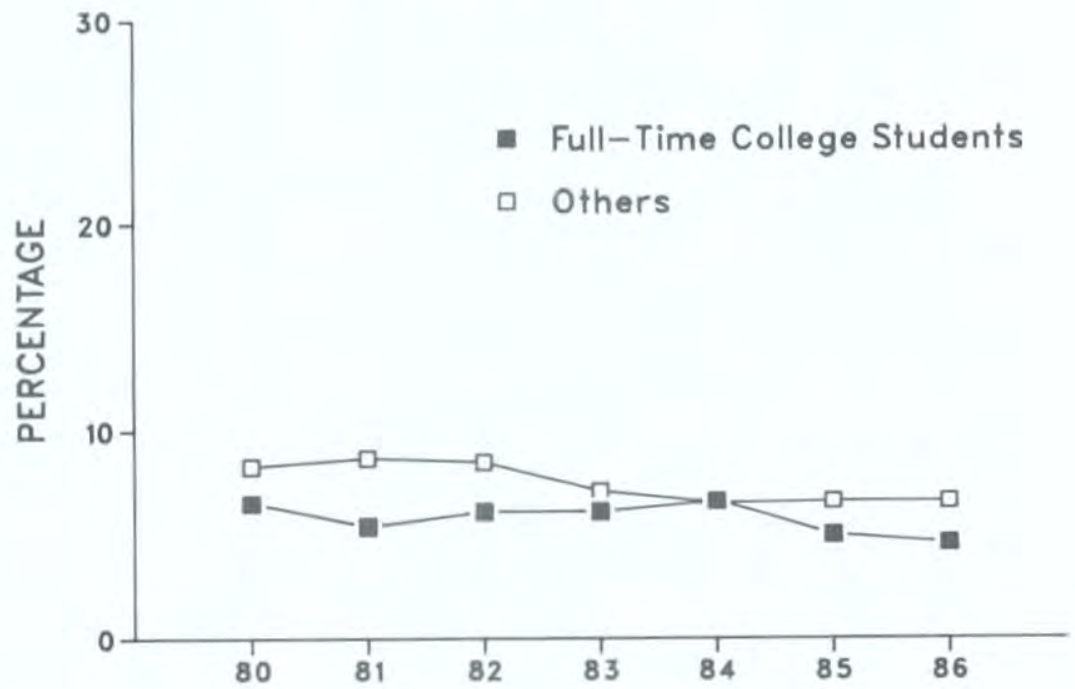

Alcohol: Trends in Thirty-Day Prevalence of Daily Use Among Male and Female College Students

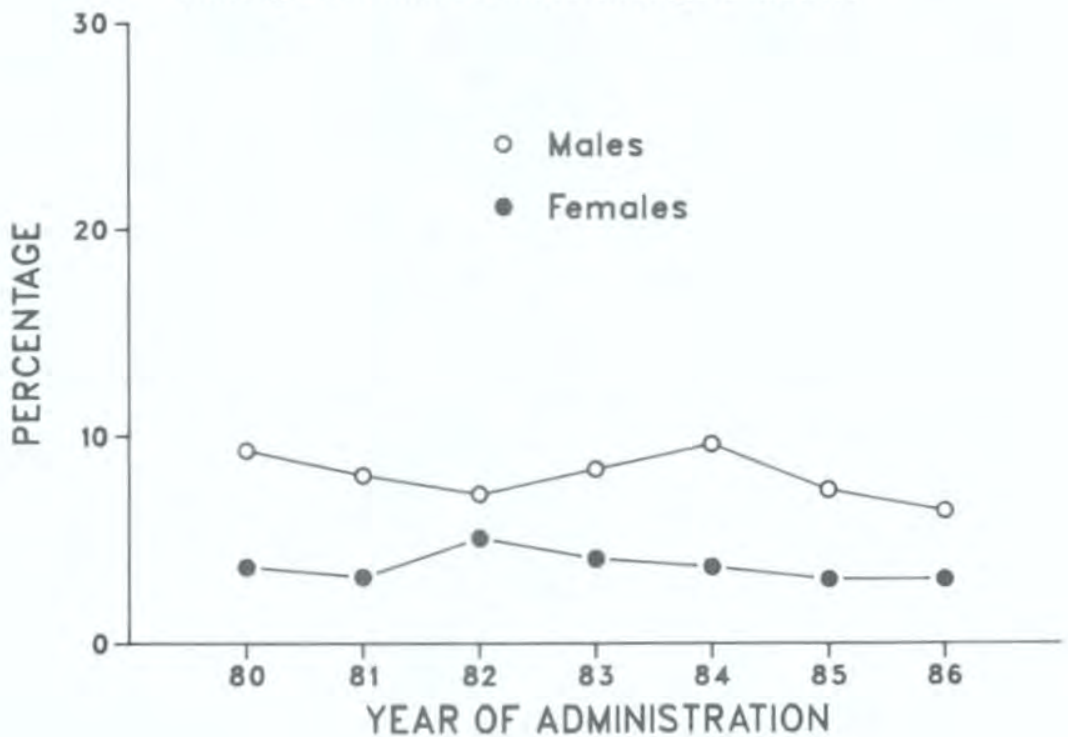




\section{FIGURE 63c}

Alcohol: Trends in Two Week Prevalence of 5 or More Drinks in a Row Among College Students Vs. Others 1-4 Years Beyond High School

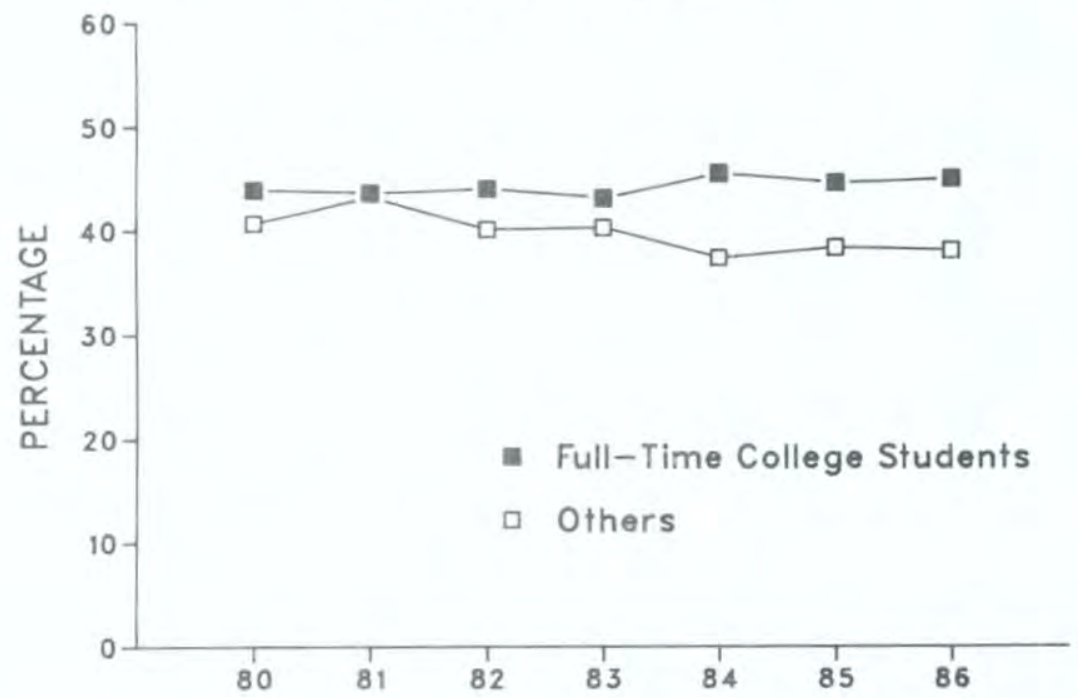

Alcohol: Trends in Two Week Prevalence of 5 or More Drinks in a Row Among Male and Female College Students

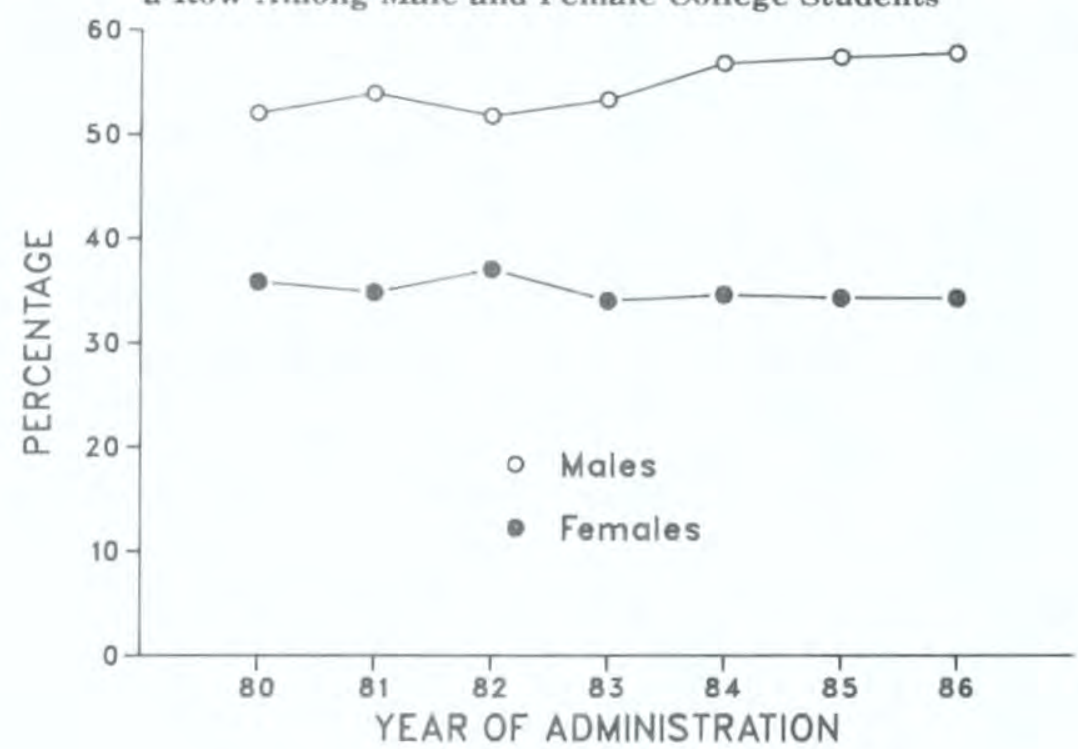


FIGURE 64a

Cigarettes: Trends in Thirty-Day Prevalence Among College Students Vs. Others

1-4 Years Beyond High School

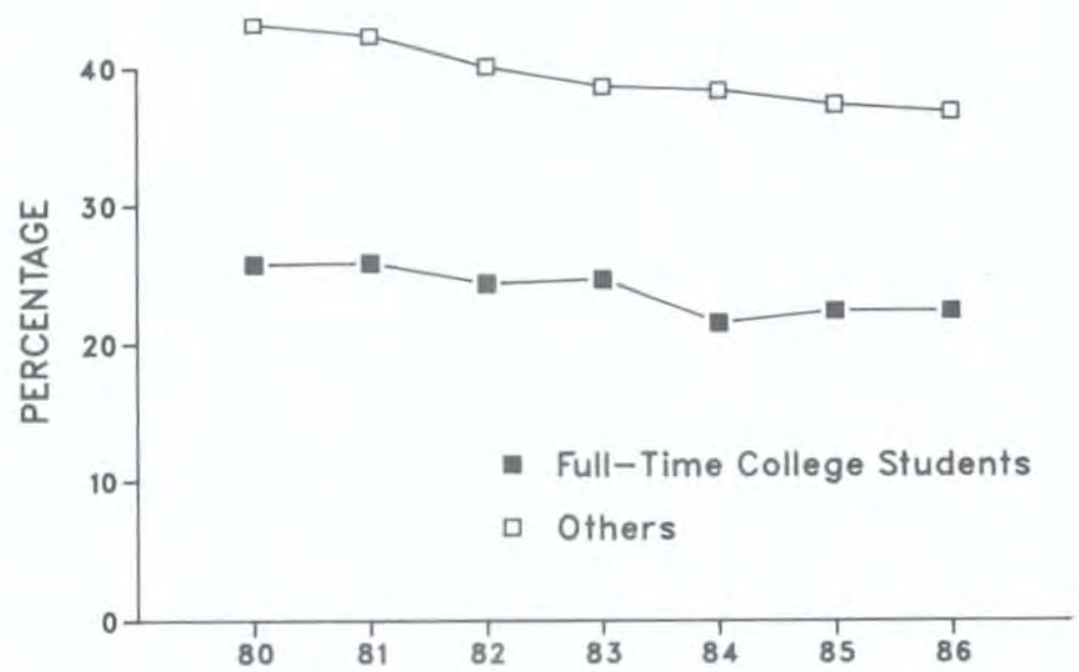

Cigarettes: Trends in Thirty-Day Prevalence

Among Male and Female College Students

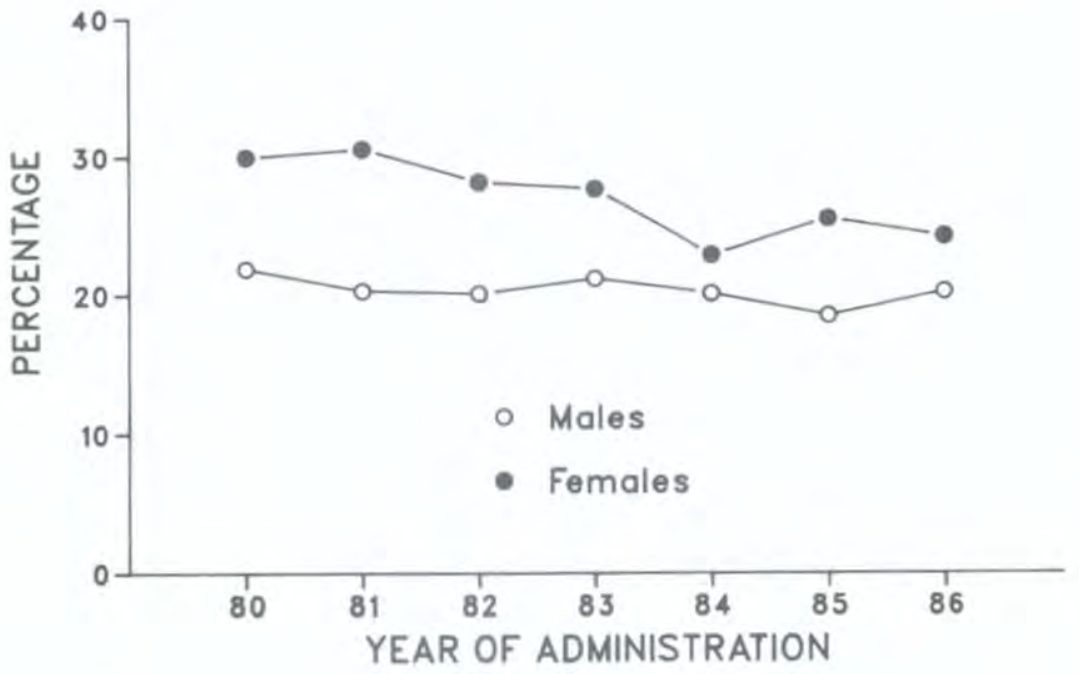




\section{FIGURE $64 \mathrm{~b}$}

Cigarettes: Trends in Thirty-Day Prevalence of Daily Use Among College Students Vs. Others

1-4 Years Beyond High School

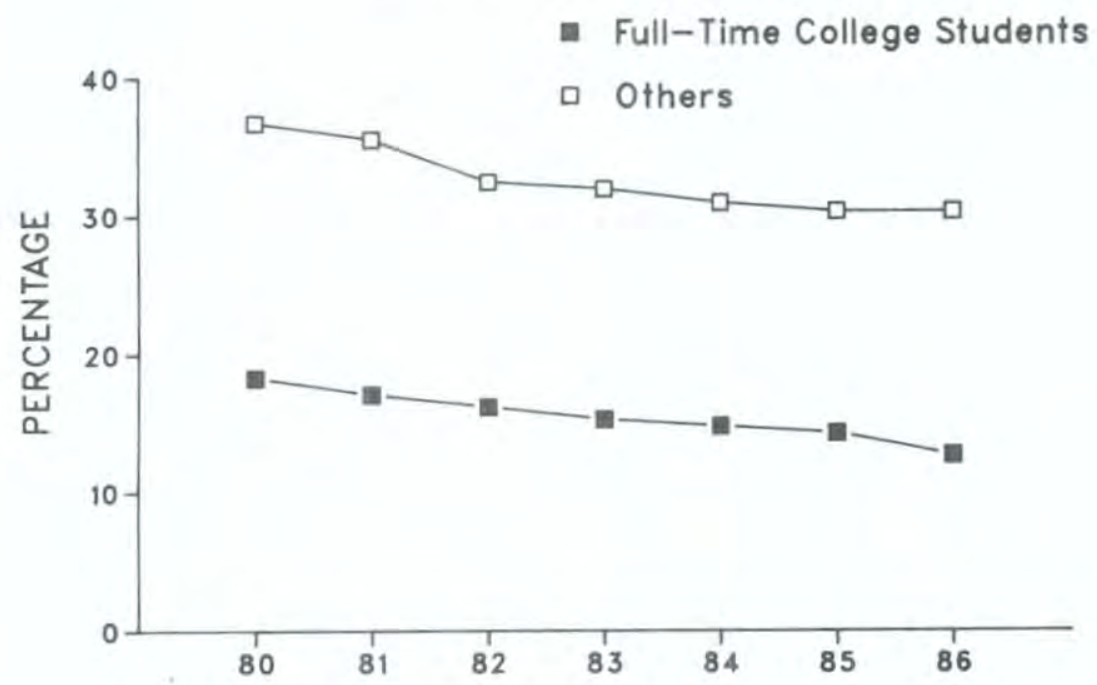

Cigarettes: Trends in Thirty-Day Prevalence of Daily Use Among Male and Female College Students

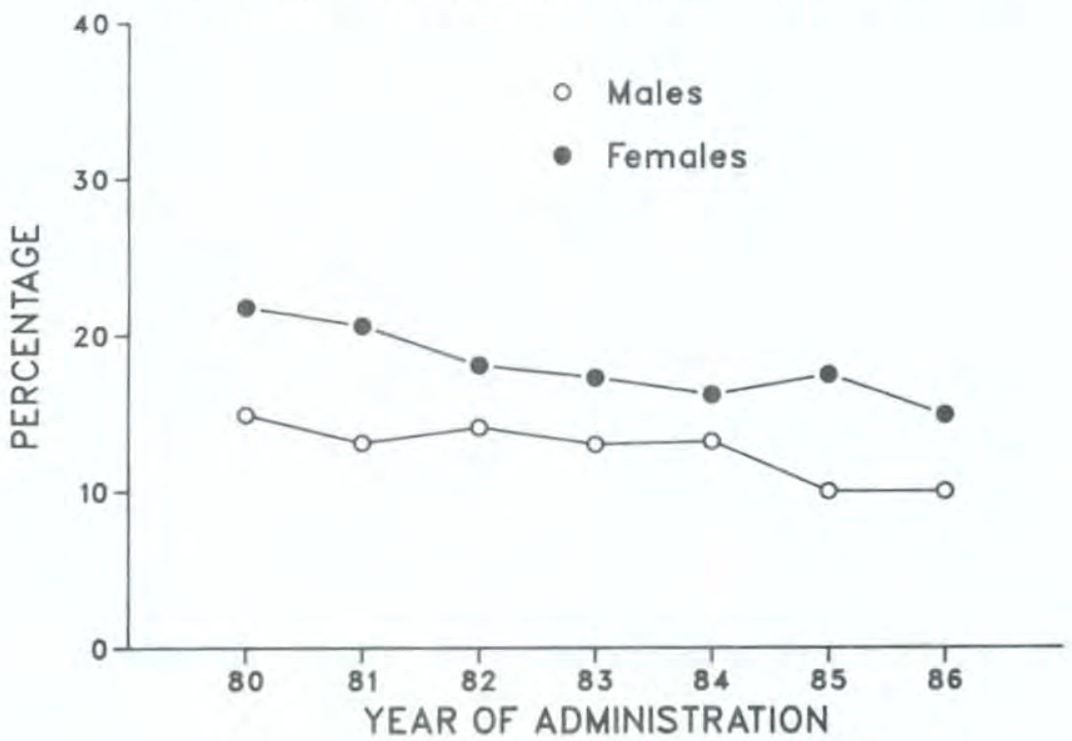




\section{FIGURE 64c}

Cigarettes: Trends in Thirty-Day Use of Half-Pack a Day or More Among College Students Vs. Others 1-4 Years Beyond High School

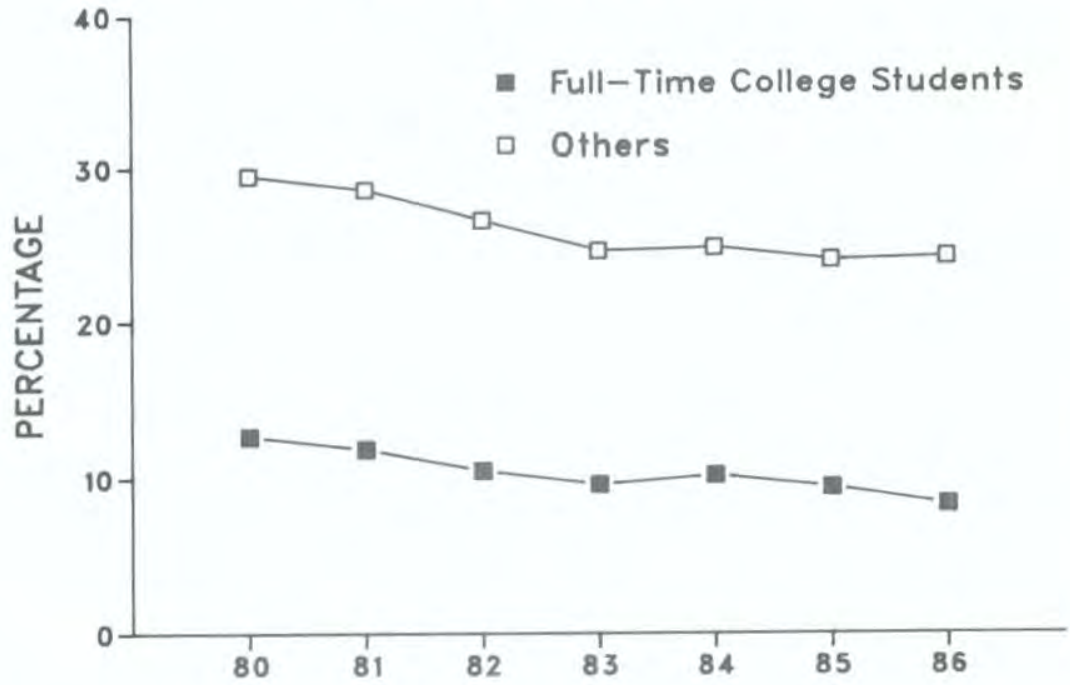

Cigarettes: Trends in Thirty-Day Use of Half-Pack a Day or More Among Male and Female College Students

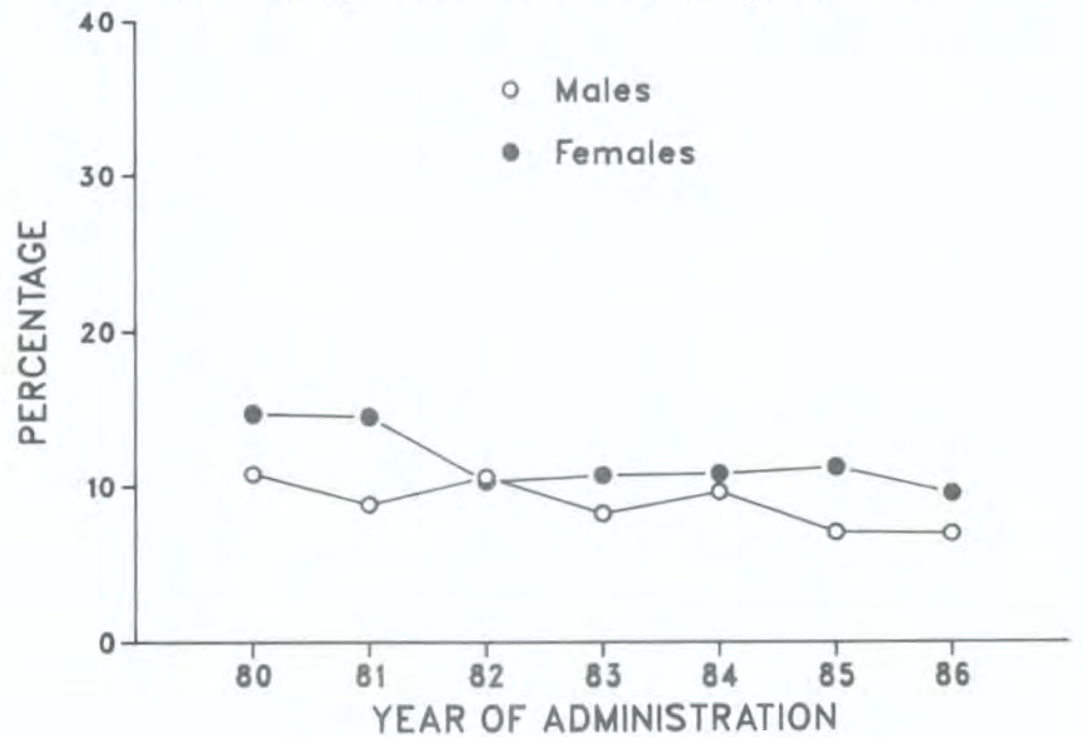




\section{TRENDS IN DRUG USE AMONG COLLEGE STUDENTS}

Since the drug-using behaviors of American college students in the late 1960 's and early 1970's represented the beginning of what was to become an epidemic of certain types of drug use in the general population-especially with regard to the use of marijuana and LSD-it is interesting and important to note what has happened to those behaviors among college students in recent years.

In this section we continue to use the definition of college students as high school graduates one to four years past high school who are enrolled full time in a two-year or four-year college at the beginning of March in the year in question. For comparison purposes we also provide trend data on the remaining respondents who are also one to four years past high school. (See Figures 52 through 64.) Because the rate of college enrollment declines steadily with number of years beyond high school, the comparison group is slightly older on the average than the college-enrolled group. However, this should influence the comparisons of the college-enrolled with the other group rather little, since few of the drugs show an age effect in their usage rates at this age.

It should also be remembered that the difference between the enrolled and other group shows the degree to which college students are above or below average for other high school graduates in this age band. Were we able to include the high school dropout segment in the "other" calculation, any differences with the college-enrolled would probably be accentuated.

For each year there are approximately 1100 respondents constituting the college student sample (see Table 31 for N's per year) and roughly 2800 respondents constituting the total age group one to four years past high school. Comparisons of the trends in these two groups are given below. Please note that all tables and figures to which we refer in this section are contained in the section immediately preceding.

\section{TRENDS IN PREVALENCE 1980-1986: COLLEGE STUDENTS}

- The proportion of college students using any illicit drug in the prior year dropped steadily from 1980 to 1984 (from 56\% to $45 \%$ ), but but has changed rather little since. (See Table 31 and Figure 52.) Marijuana use has shown a similar pat- 
tern, and in both cases the trend curves have been almost identical for both college students and those not enrolled in college.

- Use of any illicits other than marijuana has declined more steadily between 1980 and 1986 (with annual prevalence among college students dropping gradually from $32 \%$ to $25 \%$ ). Again, this parallels the trend for the age group as a whole.

- Also, for most individual classes of drugs, the trends since 1980 among those enrolled in college tend to parallel those for that age group as a whole, as well as the trends observed among seniors. That means that for most drugs there has been a decline in use over that time interval.

- In particular, daily marijuana use among college students fell significantly between 1980 and 1986 , from $7.2 \%$ to $2.1 \%$, as it did for the age group as a whole and as it did among high school seniors. In essence, the proportion of American college students who are actively smoking marijuana on a daily basis has dropped by more than two-thirds since 1980 .

- Among the other drugs, one of the largest declines observed among college students is for $L S D$, with annual prevalence falling from $6.3 \%$ in 1982 to $2.2 \%$ in 1985 . However, this figure rose to $3.9 \%$ in 1986 , a statistically significant increase which is not paralleled in our data for high school seniors. Those young adults not in college full-time also showed an increase, although it was smaller than that of their peers and not statistically significant.

- An appreciable decline also occurred for stimulant use, for which annual prevalence dropped from $21 \%$ in 1982 to $10 \%$ in 1986. Proportionately this also is a larger drop than among seniors, but is fairly parallel to the overall change among all young people of college age.

- Methaqualone showed a dramatic drop among college students, going from an annual prevalence of $7.2 \%$ in 1980 to $1.2 \%$ in 1986 . Again, this drop has been greater than among high school students, though only slightly greater, and parallels the changes in this age group as a whole.

- Barbiturate use was already quite low among college students in 1980 (at $2.9 \%$ annual prevalence) but it fell more than half to $1.3 \%$ by 1985 . This proportional decline was, once again, more sharp than among high school students, but this time a little less sharp than among the young adult 
sample taken as a whole. In 1986, however, annual prevalence among college students has risen (nonsignificantly) to $2 \%$, while use by high school seniors continued to decline.

- The annual prevalence of tranquilizer use dropped by half in the period $1980-1984$, from $6.9 \%$ to $3.5 \%$. No further decline was observed in 1985 , and a slight increase (to $4.4 \%$ ) occurred in 1986. (There was no increase among young adults generally, nor among high school seniors.)

- After dropping slightly between 1980 and 1982 (annual prevalence fell from $5.1 \%$ to $3.8 \%$ ) the use of opiates other than heroin has held fairly steady (4.0\% in 1986). This trend parallels quite closely what has been happening for the age group as a whole.

- Like the high school seniors, college students showed a relatively stable pattern of cocaine use between 1980 and 1986 , with annual prevalence rate hovering between $16 \%$ and $17 \%$. (It was $17 \%$ in 1986, while for those not in college it was 19\%.) The differences between the two groups have narrowed in recent years.

- It is in regard to alcohol use that college students appear to be showing shifts in use which are different from those observed either among their total age group or among high school seniors. Up to 1986, both of the latter groups had shown some drop in the frequency of having five or more drinks in a row during the two-week interval preceding the survey, but college students did not show this decline. Indeed, they reported their highest rate in 1984, and remained at this rate $(45 \%)$ through 1986 . Thus it is clear that more college students report occasions of heavy drinking than other young adults, and that pattern of drinking had been on the increase among college students at the same time it was showing some fall-off among their age mates and among high school students. Both of the latter groups' declines, however, appear to have stopped in 1986 , so the divergence did not continue in 1986.

College students also have a thirty-day prevalence of alcohol consumption which is higher than their peers ( $80 \%$ vs. $72 \%$ ). The difference has changed rather little since 1980 .

On the other hand, college students generally have had slightly lower rates of daily drinking than their age group taken as a whole, and this fact has changed rather little in the past six years, insofar as both have shown some decline in daily use. In 1986 daily drinking among college students 
stands at $4.6 \%$, compared with $5.8 \%$ for their age group and $4.8 \%$ for high school seniors.

- Cigarette smoking among American college students has declined modestly in the period 1980-1985. Thirty-day prevalence fell from $25.8 \%$ to $21.5 \%$ between 1980 and 1984 , then rose slightly (to $22.4 \%$ ) in 1985 , where it stayed in 1986. (Smoking rates among seniors also were unchanged in 1986.) The daily smoking rate fell from $18.3 \%$ in 1980 to $14.3 \%$ in 1985 , though the rate of decline decelerated after 1983. In 1986, this figure again declined to $12.7 \%$. While the rates of smoking are dramatically lower than among those not in college, the trends are highly parallel (Figure $64 b)$.

Among seniors, however, the trend line for daily use of cigarettes during the 1980-1986 interval has been much less steep. This divergence of trends between high school and college-age graduates has resulted in much less difference in daily usage rates in 1986 between high school seniors (19\%) and college-age graduates (23\%) than there was in $1980(21 \%$ vs. $30 \%)$.

- In sum, the trends in substance use among American college students appear to parallel closely those occurring among their age group as a whole, though there are some important differences in absolute levels. The major exception occurs for occasions of heavy drinking, which until this year appeared to be falling among those not enrolled full-time in college (as well as among high school seniors) but, if anything, was rising among college students.

The trends among college students are highly parallel for the most part, to the trends among high school seniors, although declines in many drugs over the last half-decade (1980-1986) have been proportionately larger among college students (and for that matter among all young adults of college age). This year, the biggest exception to this similarity in trends is $L S D$, which rose significantly among the college students, but remained level for the high school seniors.

\section{SEX DIFFERENCES IN TRENDS AMONG COLLEGE STUDENTS}

One trend which is not obvious from the figures included here is the fact that the proportion of college students who are female has been rising slowly. Females constituted $50 \%$ of our 1980 sample of college students, but $55 \%$ of our 1986 sample. Given that there exist substantial 
sex differences in the use of some drugs, we are concerned that over a longer time apparent trends in the levels of drug use among college students might actually be attributable to changes in the sex composition of that population. For that reason, in particular, we present separate trend lines for the male and female components of the college student population. Differences in the trends observed for these two groups are illustrated in Figures 52 through 64, and are discussed below:

- In general, trends in the use of the various drugs, and in the overall drug use indexes, have been highly parallel for male and female college students, as an examination of the relevant figures will show. The most noteworthy exceptions are mentioned below.

- Certain drug use measures have shown a convergence of usage levels between the sexes, mainly because they are converging toward zero. Daily marijuana use is one such example, with the male-female ratio dropping from 3 to 1 in 1980 to 2 to 1 in 1986.

- Methaqualone also showed a convergence in use, with males declining more, and $L S D$ had been showing such a convergence until 1986, when use among college males bounced up again.

- Stimulant use also shows some convergence between 1982 (when the revised questions were first introduced) and 1986. While use by both sexes is dropping, males (who have consistently been higher) have dropped more.

- Regarding alcohol use, annual prevalence has remained virtually identical for the two sexes throughout the period. However, there had been some evidence of a divergence in thirty-day prevalence between 1982 and 1984, with females dropping and males rising overall, but more recently they have been converging again. Roughly the same has been true for daily prevalence. Perhaps most important, however, has been the divergence in occasions of heavy drinking. We can see in Figure 63c that college males account for the overall difference in trends between college students and their larger age group. Between 1982 and 1986 the prevalence of such heavy drinking has risen from $52 \%$ to $58 \%$ among college males, whereas among college females it has dropped from $37 \%$ to $34 \%$.

- Since 1980 cigarette smoking has consistently been higher among females than males in college. 
OTHER FINDINGS FROM THE STUDY 


\section{OTHER FINDINGS FROM THE STUDY}

Each year this section presents additional recent findings from the Monitoring the Future study. Some of these have been published elsewhere; however, the first two analyses included here-on the use of nonprescription stimulants and daily marijuana use-are not reported elsewhere.

\section{THE USE OF NONPRESCRIPTION STIMULANTS}

As is discussed in other chapters of this report, between 1979 and 1981 we observed a substantial increase in reported stimulant use by high school students. We had reason to believe that a fair part of that increase was attributable to nonprescription stimulants of two general types- "look-alike" drugs (pseudo-amphetamines, usually sold by mail order, which look like, and often have names that sound like, real amphetamines) and over-the-counter stimulants (primarily diet pills and stay-awake pills). These drugs usually contain caffeine, ephedrine, and/ or phenylpropanolamine as their active ingredients.

Beginning with the 1982 survey we introduced new questions on some questionnaire forms in order to more accurately assess the use of amphetamines as well as to assess the use of the "look-alikes," diet pills, and stay-awake pills of the nonprescription variety. For example, on one of the five questionnaire forms respondents were asked to indicate on how many occasions (if any) they had taken nonprescription diet pills such as Dietac"m, Dexatrim"s, and Prolamine"m (a) in their lifetime, (b) in the prior twelve months, and (c) in the prior thirty days. (These correspond to the standard usage questions asked for all drugs.) Similar questions were asked about nonprescription stay-awake pills (such as No-Doz", Vivarin", Wake", and Caffedrine ${ }^{m}$ ) and the "lookalike" stimulants. (The latter were described at some length in the actual question.)

On three of the five questionnaire forms in 1982 and 1983 (and in all questionnaire forms thereafter) respondents were also asked about their use of prescription amphetamines, with very explicit instructions to exclude the use of over-the-counter and "look-alike" drugs. These questions yielded the data described in this volume as "stimulants, adjusted." Here we will refer to them as "amphetamines, adjusted," to distinguish them more clearly from the nonamphetamine stimulants. 
TABLE 32

Various Stimulants: Trends in Lifetime, Annual, and Thirty-day Prevalence by Sex

(Entries are percentages)

\section{Diet Pills}

Class Class Class Class Class

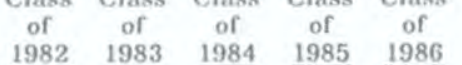

Lifetime Prevalnence

Total
U Males
or
Females

Annual Prevalence

$$
\text { Total }
$$

Males

Males
Females

$\begin{array}{rrrrrr}29.6 & 31.4 & 29.7 & 28.7 & 26.6 & -2.1 \\ 16.5 & 17.4 & 14.8 & 14.8 & 13.1 & -1.7 \\ 42.2 & 44.8 & 43.1 & 41.5 & 39.7 & -1.8 \\ & & & & & \\ & & & & & \\ 20.5 & 20.5 & 18.8 & 16.9 & 15.3 & -1.6 \\ 10.7 & 10.6 & 9.2 & 9.0 & 6.9 & -2.1 \\ 29.5 & 30.0 & 27.5 & 24.4 & 23.2 & -1.2\end{array}$

Thirty-Day Prevalence

$\begin{array}{lrrrrrr}\text { Total } & 9.8 & 9.5 & 9.9 & 7.3 & 6.5 & -0.8 \\ & & & & & & \\ \text { Males } & 5.0 & 4.0 & 4.8 & 3.7 & 3.2 & -0.5 \\ \text { Females } & 14.0 & 13.7 & 14.2 & 10.7 & 9.6 & -1.1\end{array}$

$\begin{array}{lllll}5.5 & 5.3 & 5.8 & 7.2 & 9.6+2.4 \mathrm{ss} \\ 6.0 & 5.5 & 6.2 & 7.7 & 9.5+1.8 \\ 4.7 & 4.5 & 5.5 & 6.7 & 9.3+2.6 \mathrm{~s}\end{array}$

$\underline{\text { Stay-A wake Pills }}$

Class Class Class Class Class

of of of

$\begin{array}{llll}1983 & 1984 & 1985 & 1986\end{array}$

$85-' 86$
change

$\underline{1982}$

$\begin{array}{lllll}19.1 & 20.4 & 22.7 & 26.3 & 31.5+5.2 \mathrm{sss} \\ 20.2 & 22.3 & 23.2 & 28.0 & 32.0+4.0 \\ 16.9 & 18.2 & 21.7 & 24.9 & 31.3+6.4 \mathrm{ss}\end{array}$

$\begin{array}{lllll}16.9 & 18.2 & 23.2 & 28.0 & 32.0+4.0 \\ & & 21.7 & 24.9 & 31.3+6.4 \mathrm{ss}\end{array}$

(1)

NOTE: Level of significance of difference between the two most recent clnsses: $\mathrm{s}=.05, \mathrm{ss}=.01, \mathrm{sss}=.001$.

Look-Alikes

Class Class Class Class Class

of of of of of '85-'86

$\underline{1982} \underline{1983} \quad \underline{1984} \quad \underline{1985} \underline{1986} \quad \underline{\text { change }}$ 
TABLE 33

Percent of Respondents in Each Category of an Illicit Drug Use Index

Who Have Tried Various Over-the-Counter Stimulants, Class of 1986

\begin{tabular}{lrcc} 
& \multicolumn{2}{c}{ Lifetime Illicit Drug Use } \\
\cline { 3 - 4 } Lifetime use of... & $\underline{\text { No Use }}$ & $\begin{array}{c}\text { Marijuana } \\
\text { Only }\end{array}$ & $\begin{array}{c}\text { Other } \\
\text { Illicit Drugs }\end{array}$ \\
Diet Pills & $14.4^{\mathrm{a}}$ & 22.2 & 45.5 \\
Stay-Awake Pills & 13.5 & 34.7 & 54.1 \\
"Look-Alikes" & 1.1 & 5.8 & 32.9 \\
Approx. N = & $(1238)$ & $(593)$ & $(1024)$ \\
\hline
\end{tabular}

This means that, of those who have never used an illicit drug, $14.4 \%$ have used a diet pill at least once. 


\section{Prevalence of Use in 1986}

- Table 32 gives the prevalence levels for these various classes of stimulants. As can be seen, a substantial proportion of students $(27 \%)$ have used over-the-counter diet pills and $7 \%$ have used them in just the past month. Some $0.4 \%$ are using them daily.

- Very similar proportions are using actual amphetamines (adjusted): $23 \%$ lifetime, $6 \%$ monthly, and $0.3 \%$ daily prevalence.

- Only about half as many students are knowingly using the "look-alikes" as are using diet pills or amphetamines (adjusted): $13 \%$ lifetime, $3 \%$ monthly, and $0.3 \%$ daily prevalence. Of course, it is probable that some proportion of those who think they are getting real amphetamines have actually been sold "look-alikes," which are far cheaper for drug dealers to purchase.

- This year, stay-awake pills are the most widely used stimulant: $32 \%$ lifetime, $10 \%$ monthly, and $0.4 \%$ daily prevalence,

- In 1983 , the newly revised question on amphetamine use yielded prevalence estimates which were about one-quarter to one-third lower than the original version of the question, indicating that some distortion in the unadjusted estimates was occurring as a result of the inclusion of some nonprescription stimulant use.

\section{Subgroup Differences}

- Figure 65 shows the prevalence figures for these drug classes for males and females separately. It can be seen that the use of diet pills is dramatically higher among females than among males. In fact, the absolute prevalence levels for females are impressively high, with some $40 \%$ reporting some experience with them and $10 \%$ - or one in every ten females-reporting use in just the last month. For all other stimulants the prevalence rates for both sexes are fairly close.

- A similar comparison for those planning four years of college (referred to here as the "college-bound") and those who are not shows some differences as well (data not shown). As is true for the controlled substances, use of the "look-alikes" is lower among the college-bound $(5 \%$ annual prevalence vs. $10 \%$ among the noncollege-bound). 
This year's results show virtually no difference between these two groups in their use of diet pills; and use of stay-awake pills is actually higher for the college-bound-annual prevalence is $25 \%$ vs. $18 \%$ for the noncollege-bound.

- There are no dramatic regional differences in the use of diet pills or "look-alikes." The West and the North Central, however, tend to be somewhat higher in the use of stayawake pills (annual prevalence of $26 \%$ and $25 \%$ ) than the Northeast and South (both at 20\%).

- There generally have not been systematic differences in use of nonprescription stimulants associated with population density.

- The use of all of the nonprescription stimulants (i.e., diet pills, stay-awake pills, and "look-alikes") is substantially higher among those who have had experience with the use of illicit drugs than among those who have not, and highest among those who have become most involved with illicit drugs (see Table 33). For example, 1\% of those who have abstained from any illicit drug use report ever using a "lookalike" stimulant, compared to $6 \%$ of those who have used only marijuana and $33.0 \%$ of those who have used some illicit drug other than marijuana.

\section{Trends in Use}

- Because these questions were new in 1982 , trends can be directly assessed only since then.

- However, it is worth noting that the adjusted 1982 figures for amphetamines are higher than the unadjusted figures for all years prior to 1980. (See Tables 7 through 10.) This suggests that there was indeed an increase in amphetamine use between 1979 and 1982 - or at least an increase in what, to the best of the respondent's knowledge, were amphetamines.

- In recent years, there have been increased legislative and law enforcement efforts to curb the manufacture and distribution of "look-alike" pills. Perhaps as a result, the use of these pills decreased from 1982 to 1986; for example, annual prevalence went from $10.8 \%$ to $6.9 \%$.

- Use of diet pills decreased slightly between 1983 and 1986. Annual prevalence fell over that interval from $20.5 \%$ to $15.3 \%$. 


\section{FIGURE 65}

Prevalence and Recency of Use, by Sex

Amphetamines and Non-Prescription Stimulants, Class of 1986

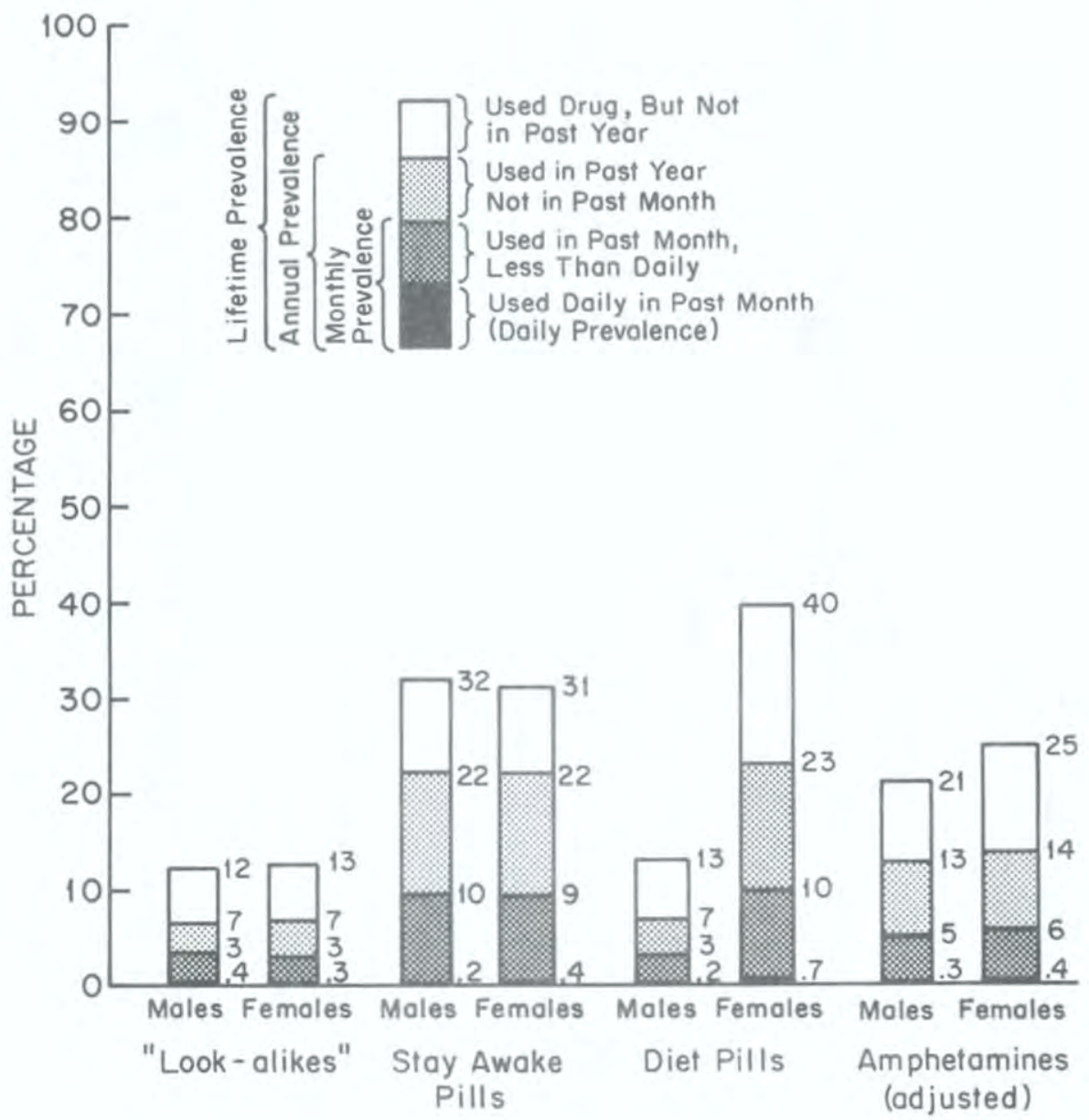


- Only the use of stay-awake pills has increased significantly in recent years, particularly in 1985 and 1986, with annual prevalence increasing from $12 \%$ in 1982 to $14 \%$ in 1984 to $22 \%$ in 1986.

- All subgroups (defined by sex, college plans, region of the country, and population size) have shown similarly large increases over this interval in their use of stay-awake pills. However, the increase among the college-bound has been even greater than among the noncollege-bound, reversing their relative positions. For example, in 1982 the collegebound had a slightly lower annual prevalence (at $10 \%$ vs. $11 \%$ ) whereas in 1986 they have a somewhat higher annual prevalence ( $25 \%$ vs. $18 \%$ ).

- Subgroup differences in trends for the diet pills and the look-alikes for the most part reflect the overall trends.

\section{THE USE OF MARIJUANA ON A DAILY BASIS}

In past reports in this series, we summarized a number of findings regarding daily marijuana users, including what kind of people they are, how use changes after high school for different subgroups, and what daily users see to be the negative consequences of their use. ${ }^{25}$ In 1982 a special question segment was introduced into the study in one of the five questionnaire forms in order to secure more detailed measurement of individual patterns of daily use. More specifically, respondents were asked (a) whether if at any time during their lives they had ever used marijuana on a daily or near-daily basis for at least a month and, if so, (b) how recently they had done that, (c) when they first had done it, and (d) how many total months they had smoked marijuana daily, cumulating over their whole lifetime. The results of our analyses of these questions follow.

\section{Lifetime Prevalence of Daily Use}

- Current daily use, defined as use on twenty or more occasions in the past thirty days, has been fluctuating widely over the past eight years, as we know from the trend data presented earlier in this report. It rose from $6.0 \%$ among

${ }^{25}$ For the original reports see the following, which are available from the author: Johnston, L.D. (1981). Frequent marijuana use: Correlates, possible effects, and reasons for using and quitting. In R. DeSilva, R. Dupont, \& G. Russell (Eds.), Treating the marijuana dependent person, New York: The American Council on Marijuana. Also see Johnston, L.D. (1982). A review and analysis of recent changes in marijuana use by American young people. In Marijuana: The national impact on education, New York: The American Council on Marijuana. 
seniors in 1975 to $10.7 \%$ in 1978 , then down to $4.0 \%$ in 1986.

- Since 1982 , we have found the lifetime prevalence of daily use for a month or more to be far higher than current daily use-e.g., at $14.9 \%$ or one in every seven seniors in 1986 vs. $4.0 \%$ for current daily use. In other words, the proportion who describe themselves as having been daily or near-daily users at some time in their lives is over three times as high as the number who describe themselves as current daily users. However, we believe it very likely that this ratio has changed dramatically over the life of the study as a result of the large secular trends in daily use. Therefore, it would be inaccurate to extrapolate to the class of 1978, for example, and deduce that their lifetime prevalence of daily use was three times their $10.7 \%$ current use figure. (An investigation of data from a follow-up panel of the class of 1978 confirms this assertion.)

- Utilizing data collected in 1986 from follow-up panels from the earlier graduating classes of 1976 through 1985, we find that the lifetime prevalence of daily marijuana use for these recent graduates (ranging in age from about 19 to 28 ) is $20 \%$. Approximately one-fourth of the older portion of that groupgraduates from the classes of 1976 through 1980-indicate having been daily marijuana users for a month or more at some time in their lives.

\section{Grade of First Daily Use}

- Of those 1986 seniors who were daily users at some time, over half $(57 \%$, or nearly $9 \%$ of all seniors) began that pattern of use before tenth grade. However, the secular trends in daily use must be recalled. Active daily use reached its peak among seniors in 1978 , when this 1986 graduating class was in fourth grade. Thus we are confident that different graduating classes show different age-associated patterns.

- Nearly all who were to become daily users by the end of high school had done so by the end of grade ten (79\% of the eventual daily users). The percentages of all seniors who started daily marijuana use in each grade level is presented in Table 34.

\section{Recency of Daily Use}

- Two-thirds $(68 \%)$ of those who report ever having been daily marijuana users (for at least a one-month interval) have smoked that frequently in the past year-and-a-half, while 
TABLE 34

Daily Marijuana Use: Responses to Selected Questions by Subgroups, 1986 Seniors

Q. Thinking back over your whole

life, has there ever been a

period when you used marijuana

or hashish on a daily, or almost

daily, basis for at least a month?

No

Yes

Q. How old were you when you first sinoked marijuana or hashish that frequently?

Grade 6 or earlier

Grade 7 or 8

Grade 9 (Freshman)

Grade 10 (Sophomore)

Grade 11 (Junior)

Grade 12 (Senior)

is Never used daily

Q. How recently did you use marijuana

or hashish on a daily, or almost

daily, basis for at least a month?

During the past month

2 months ago

3 to 9 months ago

About 1 year ngo

About 2 years ago

3 or more years ago

Never used daily

Q. Over your whole lifetime, during how

many months have you used marijuann

or hashish on a daily or near-daily basis?

Less than 3 months

3 to 9 months

About 1 year

About 1 and $1 / 2$ years

About 2 years

About 3 to 5 years

6 or more years

Never used daily

$$
\mathrm{N}=
$$

$\mathrm{N}=$
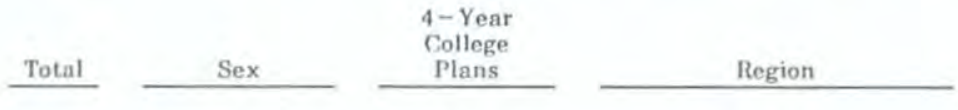

Population
Density

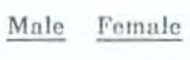

85.1

14.9

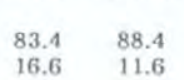

No

82.8
17.2

\section{$\underline{Y e s}$}

89.0

\section{North North}

East Central South West

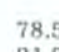

78.5
21.5

$$
\begin{aligned}
& 88.7 \\
& 11.3
\end{aligned}
$$$$
88.7
$$

18.3

Large Other NonSMSA SMSA SMSA

$83.0 \quad 85.1 \quad 86.8$

$\begin{array}{lll}17.0 & 14.9 & 13.2\end{array}$

$\begin{array}{ll}1.2 & 0.5 \\ 5.7 & 2.3 \\ 3.7 & 2.3 \\ 3.1 & 3.0 \\ 2.6 & 1.6 \\ 0.8 & 1.2\end{array}$

$82.8 \quad 89.0$

0.9
4.4
5.0
6.2
3.5
1.5

78.5

1.0
3.3
3.0
1.6
1.6
0.9

88.7

$\begin{array}{ll}0.9 & 1.5 \\ 3.3 & 5.4 \\ 2.2 & 4.3 \\ 2.6 & 3.5 \\ 1.5 & 2.6 \\ 0.9 & 1.0\end{array}$

$88.7 \quad 81.7$

$\begin{array}{lll}1.3 & 1.1 & 0.7 \\ 3.9 & 3.8 & 4.1 \\ 4.4 & 3.4 & 2.8 \\ 3.9 & 3.2 & 3.1 \\ 2.8 & 2.4 & 1.4 \\ 0.8 & 1.0 & 1.1\end{array}$

$83.0 \quad 85.1 \quad 86.8$

4.9
3.1
1.7
1.3
1.6
1.7
0.7
85.1

3.4
1.7
3.1
1.9
2.3
2.5

$\begin{array}{ll}4.4 & 1.8 \\ 1.8 & 1.2 \\ 3.3 & 2.7 \\ 2.2 & 1.5 \\ 2.1 & 2.3 \\ 2.8 & 2.1\end{array}$

$\begin{array}{rr}4.7 & 1.8 \\ 2.4 & 1.0 \\ 3.4 & 2.5 \\ 2.0 & 1.8 \\ 2.1 & 1.8 \\ 2.6 & 1.9 \\ 82.8 & 89.0\end{array}$

5.6
2.1
4.8
3.0
3.1
3.0

$\begin{array}{rrr}2.4 & 2.7 & 3.2 \\ 0.7 & 2.1 & 2.2 \\ 1.9 & 2.3 & 3.9 \\ 1.6 & 1.0 & 2.6 \\ 1.9 & 1.8 & 2.5 \\ 2.7 & 1.4 & 3.8 \\ 88.7 & 88.7 & 81.7\end{array}$

$\begin{array}{rrr}3.2 & 3.3 & 3.6 \\ 1.9 & 1.7 & 1.4 \\ 2.7 & 2.8 & 3.9 \\ 2.9 & 2.3 & 0.6 \\ 3.7 & 2.1 & 1.5 \\ 2.7 & 2.7 & 2.3 \\ 83.0 & 85.1 & 86.8\end{array}$

NOTE: Entries are percentages which sum vertically to $100 \%$. 
one-third $(32 \%)$ of them say they last used that frequently "about two years ago" or longer. On the other hand, only $23 \%$ of all such users (or $3.4 \%$ of the entire sample) say they have used daily or almost daily in the past month (the period for which we define current daily users). The fact that only $3.4 \%$ of the entire sample report themselves to be current daily users, versus the $4,0 \%$ estimate given earlier in this report, suggests that some students have a more stringent definition of "daily or near-daily use" than the operational one used in this report (i.e., use on twenty or more occasions during the past month).

\section{Duration of Daily Use}

- It seems likely that the most serious long-term health consequences associated with marijuana use will be directly related to the duration of heavy use. Thus a question was introduced which asks the cumulative number of months the student has smoked marijuana daily or nearly daily. While hardly an adequate measure of the many different possible cross-time patterns of use-a number of which may eventually prove to be important to distinguish-it does provide a gross measure of the total length of exposure to heavy use.

- Table 34 gives the distribution of answers to this question. It shows that almost two-thirds $(65 \%)$ of those with daily use experience have used "about one year" or less cumulativelyat least by the end of twelfth grade. In fact, a third $(33 \%)$ have used less than three months cumulatively.

- On the other hand, over one-fourth $(27 \%$, or $4 \%$ of all seniors) have used "about two years" or more cumulatively on a daily or near-daily basis.

\section{Subgroup Differences}

- There is some sex difference in the proportion having ever been a daily user-17\% for males and $12 \%$ for females. Furthermore, the cumulative duration of daily use is distinctly longer for the males. These two sex differences combine to account for the large male-female difference in current daily use. There is also some difference in their age at onset, with the males tending to start earlier on the average.

- Whether or not the student has college plans is strongly related to lifetime prevalence of daily marijuana use, as well as to current prevalence. Of those planning four years of college, $11 \%$ had used daily compared with $17 \%$ of those without such plans. And the college-bound users show a distinctly 
shorter cumulative duration of use, with a lower proportion of them still using daily. Nevertheless, among those in each group who did use daily, the age-at-onset pattern is fairly similar.

- There are some large regional differences in lifetime prevalence of daily use, all consistent with those found for current daily use. The Northeast is highest, with $22 \%$ having used daily at some time, the West is next at $18 \%$, and the North Central and South tie for lowest at $11 \%$.

- The subgroup differences associated with urbanicity are likewise similar to those found for current daily use. Lifetime prevalence of daily marijuana use is $17 \%$ in the large cities, $15 \%$ in the smaller cities, and $13 \%$ in the nonurban areas.

\section{Trends in the Use of Marijuana on a Daily Basis}

- Table 35 presents trend data on lifetime daily use. Compared to the class of 1982, significantly fewer seniors in the class of 1984 had described themselves as having been daily or nearly daily users of marijuana at some time in their lives (21\% vs. $16 \%)$; in 1985 the proportion was essentially unchanged $(16 \%)$. It dropped very slightly in 1986 , to $15 \%$.

- Between 1982 and 1986, the decline in lifetime daily use was stronger among females (from $18 \%$ in 1982 to $12 \%$ in 1986) than among males (20\% to $17 \%)$.

- Both the college-bound and noncollege-bound groups declined in lifetime daily use between 1982 and 1985 by about $3 \%$ each. In 1986, the noncollege-bound declined another $2.4 \%$, while the college-bound remained constant.

- Lifetime prevalence of daily use has dropped in all four regions of the country since 1982 . The decline has been greatest in the North Central and least in the West.

- All three population density levels have shown declines in lifetime daily use.

- The trends in daily use of marijuana at earlier grade levels parallel fairly closely the trends in lifetime prevalence. 
TABLE 35

\section{Trends in Daily Use of Marijuana in Lifetime} by Subgroups

\begin{tabular}{|c|c|c|c|c|c|c|c|c|c|c|c|c|}
\hline & \multicolumn{5}{|c|}{ Percentage ever used } & \multicolumn{6}{|c|}{$\begin{array}{l}\text { Percentage reporting first use } \\
\text { prior to tenth grade }\end{array}$} & \multirow[b]{2}{*}{$\begin{array}{l}\text { '85-'86 } \\
\text { change } \\
\end{array}$} \\
\hline & $\begin{array}{c}\text { Class } \\
\text { of } \\
1982 \\
\end{array}$ & $\begin{array}{c}\text { Class } \\
\text { of } \\
1983 \\
\end{array}$ & $\begin{array}{c}\text { Class } \\
\text { of } \\
1984 \\
\end{array}$ & $\begin{array}{c}\text { Class } \\
\text { of } \\
1985 \\
\end{array}$ & $\begin{array}{c}\text { Class } \\
\text { of } \\
1986 \\
\end{array}$ & $\begin{array}{l}\text { '85-'86 } \\
\text { change }\end{array}$ & $\begin{array}{c}\text { Class } \\
\text { of } \\
1982 \\
\end{array}$ & $\begin{array}{c}\text { Class } \\
\text { of } \\
1983 \\
\end{array}$ & $\begin{array}{c}\text { Class } \\
\text { of } \\
1984 \\
\end{array}$ & $\begin{array}{c}\text { Class } \\
\text { of } \\
1985 \\
\end{array}$ & $\begin{array}{c}\text { Class } \\
\text { of } \\
1986 \\
\end{array}$ & \\
\hline All seniors & 20.5 & 16.8 & 16.3 & 15.6 & 14.9 & -0.7 & 13.1 & 11.1 & 10.9 & 8.8 & 8.5 & -0.3 \\
\hline \multicolumn{13}{|l|}{ Sex: } \\
\hline Male & 20.1 & 18.1 & 17.2 & 17.7 & 16.6 & $-1,1$ & 12.9 & 12.1 & 11.8 & 9.8 & 8.7 & -1.1 \\
\hline Female & 18.0 & 13.5 & 12.9 & 12.0 & 11.6 & -0.4 & 11.5 & 8.3 & 8,0 & 6.5 & 6.6 & +0.1 \\
\hline \multicolumn{13}{|l|}{ College Plans: } \\
\hline None or under 4 yrs & 22.5 & 20.3 & 18.9 & 19.6 & 17.2 & -2.4 & 14.2 & 13.5 & 12.3 & 11.8 & 10.7 & -1.1 \\
\hline Complete 4 yrs & 13.8 & 10.5 & 10.7 & 10.6 & 11.0 & +0.4 & 8.2 & 6.5 & 6.6 & 5.5 & 5.2 & -0.3 \\
\hline \multicolumn{13}{|l|}{ Region: } \\
\hline Northenst & 25.1 & 20.4 & 24.1 & 20.9 & 21.5 & +0.6 & 17.3 & 11.9 & 17.2 & 12.9 & 10.3 & -2.6 \\
\hline North Central & 21.1 & 15.9 & 12.8 & 16.3 & 11.3 & $-5.0 \mathrm{~s}$ & 13.3 & 12.4 & 8.4 & 9.1 & 7.3 & -1.8 \\
\hline South & 15.7 & 12.7 & 14.0 & 8.9 & 11.3 & +2.4 & 9.3 & 8.3 & 8.5 & 5.0 & 6.4 & +1.4 \\
\hline West & 20.8 & 21.4 & 17.6 & 18.5 & 18.3 & -0.2 & 12.6 & 13.9 & 12.1 & 8.9 & 11.2 & +2.3 \\
\hline \multicolumn{13}{|l|}{ Population Density: } \\
\hline Large SMSA & 23.8 & 20.0 & 19.4 & 18.1 & 17.0 & -1.1 & 15.6 & 13.7 & 12.4 & 12.0 & 9.6 & -2.4 \\
\hline Other SMSA & 20.3 & 18.2 & 16.6 & 16.0 & 14.9 & -1.1 & 12.5 & 12.0 & 11.5 & 8.3 & 8.4 & +0.1 \\
\hline Non-SMSA & 17.9 & 12.6 & 13.2 & 12.8 & 13.2 & +0.4 & 11.7 & 8.2 & 8.5 & 6.6 & 7.6 & +1.0 \\
\hline
\end{tabular}

NOTES: Level of significance of difference between the two most recent classes: $\mathrm{s}=.05, \mathrm{ss}=.01, \mathrm{sss}=.001$. 


\section{MEDICALLY SUPERVISED USE OF PSYCHOTHERAPEUTIC DRUGS}

The psychotherapeutic use of drugs was the subject of a recent article in the Journal of Adolescent Health Care. ${ }^{26}$ All five forms of the study's questionnaires contain questions about the use without medical supervision of four major classes of psychotherapeutic drugs: amphetamines, barbiturates, minor tranquilizers, and opiates other than heroin. Questions on the medically supervised use of these psychotherapeutics are also included in the study, but they are in only one of the five forms, and they deal only with lifetime prevalence. Those who report having used any of these drugs under medical supervision are further distinguished into those who said that their first use of the class of drugs was under medical supervision and those who said that they first used the class of drugs on their own.

- Of the four drug classes, the highest reported prevalence was for opiates and opiate-type drugs, which include analgesics, cough suppressants, and anti-diarrheal medications. Nearly one in five seniors in the class of 1985 (18\%) reported having used one or more drugs in this class under medical supervision. Minor tranquilizers were next most prevalent, with nearly one in eight seniors $(12 \%)$ reporting their use under medical supervision. About one in thirteen seniors (8\%) reported having received a prescription for amphetamines, whereas only about one in twenty $(5 \%)$ indicated use of barbiturates under medical supervision. The great majority of seniors who received these drugs under prescription had not used them previously on their own; this fact suggests that, in general, these youngsters were not manipulating their physicians to secure psychotherapeutics for recreational use.

- There was rather little difference between the sexes in lifetime prevalence rates. Females were slightly more likely than males to receive opiates from physicians $(19.7 \%$ versus $16.9 \%$ ), whereas males were slightly more likely than females to receive barbiturates $(5.8 \%$ versus $4.5 \%)$. Although these two sex differences were very small, they were replicated in virtually every year of the study since 1976. Minor tranquilizers also showed a small sex difference $(13.1 \%$ for females, $10.5 \%$ for males), but this difference had not been consistently present during the 1980's. (Rates for males were slightly higher than for females in three of the years from 1981 to 1985.$)$

${ }^{26}$ Johnston, L.D., O'Malley, P.M., \& Bachman, J.G. (1987). Psychotherapeutic, licit, and illicit use of drugs among adolescents: An epidemiological perspective. Journal of Adolescent Health Care, 8, 36-51. 
- Over the decade encompassed by this study (1975-1985), some important shifts occurred in physicians' prescribing practices. (Of course, the actual changes in practice likely began earlier than the senior surveys would indicate, because the prevalence figures reflect a cumulative impact across the respondents' lifetimes.) The proportion of seniors who reported having had amphetamines prescribed for them fell by about half, from $15 \%$ to $7 \%$, between 1976 and 1982 ; between 1982 and 1985 , the proportion was relatively stable. Similarly, the proportion receiving prescriptions for barbiturates fell from $13 \%$ in 1976 to $7 \%$ in 1982, and was relatively stable after 1982 . The proportion receiving prescriptions for minor tranquilizers also fell during the same general period, from a high of $18 \%$ in 1977 to $12 \%$ in the class of 1982; and again, use has been fairly stable since 1982. Only in the case of opiates and opiate-type drugs was there no appreciable change over the decade. Lifetime prevalence ranged between $16 \%$ and $19 \%$ throughout the interval with, if there were any trend, prevalences being slightly higher after 1980 compared to before 1980 .

- One natural question is whether the decline in the prescription of psychotherapeutic drugs to American young people could be causally connected with the decline in their nonmedical use of these and other abusable drugs, a decline that was described earlier in this monograph. Certainly there are some notable parallels in the trends for the medical and nonmedical use of most of the psychotherapeutic drugs. For example, there were appreciable declines over much of the past decade in both medical and nonmedical use of barbiturates and minor tranquilizers. Opiates similarly showed parallel trends in both medical and nonmedical use, though in this case the trend was basically one of very little change. The parallelism is least evident for amphetamines, where most of the decline in medical use occurred prior to 1982 , whereas most of the decline in nonmedical use occurred after 1982. However, such a temporal sequence is certainly consistent with there being a causal connection between medical use and subsequent nonmedical use.

- Parallel trends at the aggregate level do not, of course, constitute sufficient evidence of any causal relationship. Another piece of relevant evidence might derive from an individual level of analysis, where it can be asked whether young people who are introduced to the use of a psychotherapeutic drug by a physician are subsequently any more likely to use that drug without medical supervision or to be more involved in illicit drug use generally. (As discussed in the journal article, results from such analyses can be far from conclusive; there 
could still be a variety of alternative explanations for any observed associations. Nevertheless, the presence of an association is at least consistent with the hypothesis, and the lack of an association would argue against medical use playing any causal role in illicit or nonmedical use.)

- Data presented in the article demonstrated that, indeed, those seniors who reported receiving psychotherapeutic drugs from doctors (prior to having taken them on their own) end up with rates of illicit drug use that exceed those for seniors who never were prescribed such drugs. Thus, it seems possible that physicians who reduced their prescriptions of minor tranquilizers, barbiturates, or amphetarnines in order to reduce the likelihood of their stimulating the abuse of these or other psychoactive substances may well have been justified in their rationale for doing so. Although the data were clearly not definitive about any causal connection, the data were consistent with such a connection. (See the journal article for more discussion.)

\section{SOME CORRELATES OF CIGARETTE SMOKING}

During the past year some original findings from the study were presented to the House Subcommittee on Health and the Environment at hearings on cigarette advertising. ${ }^{27}$ Listed here are a few of the findings presented. They are based on analyses of data from the Class of 1985 .

- The initiation of daily smoking-not just the first few cigarettes, but daily smoking-is highest in junior high school-that is, among children who are twelve to fourteen years old. Of those who report daily smoking by senior year, $57 \%$ began daily smoking by age fourteen (ninth grade).

- Evidence of the addictive nature of smoking is found in the strong "cohort effect" discussed earlier in this monographthat is, if a class cohort shows a high rate of smoking at an early age relative to other cohorts, it will show a high rate of smoking throughout the life cycle. Evidence also comes from seniors who are smoking half-a-pack-a-day or more by senior year, $53 \%$ of whom say they already have tried to quit smok-

${ }^{27}$ Johnston, Lloyd D. (1986), Prepared testimony regarding cigarette advertising and its likely impact on youth, delivered before the Subcommittee on Health and the Environment, of the House Committee on Energy and Commerce. Published in the report of the Hearings of July 18 and August 1, 1986, Advertising of Tobacco Products, Washington, D.C.: U.S. Government Printing Office (Serial No. 99-167), 860-886. 
ing and were unable to do so. About half $(47 \%)$ of all daily smoking seniors say they would like to quit at present.

- The very strong negative relationship between smoking and academic performance was reported to the Subcommittee. Of those seniors with an A average in senior year, only $7 \%$ are current daily smokers: of those with a D average, $47 \%$ are. (See Table 36.) Note was made of the fact that those most likely to smoke are the hardest to reach through formal school curricula, and through written communications such as warning labels. The corollary is that they are also the ones most susceptible to the visual imagery contained in many cigarette ads. (Examples are given in the testimony.)

- The dramatic association between smoking and illicit drug use was documented. Of the pack-a-day smokers, 95\% have used an illicit drug and $81 \%$ have used an illicit drug other than marijuana. Two-thirds (67\%) of the pack-a-day smokers were actively using illicit drugs (in the prior thirty days) versus only $10 \%$ of those who never smoked. (See Tables 3740.) Current marijuana use was eight times as high among pack-a-day smokers as nonsmokers, and daily marijuana use 20 times as high. Daily use of some illicit drug other than marijuana is 13 times as high.

The relationship between smoking and alcohol use is also very strong. Pack-a-day smokers ars 11 times as likely to be current daily drinkers as those who never smoked $(18.4 \%$ vs. $1.7 \%$ ). They are also four times as likely to report an occasion of heavy drinking (67.9\% vs. $17.2 \%)$.

- Clearly the use of illicit drugs and alcohol bears a strong relationship both to the amount that young people have smoked and how much they smoke at present. Two hypotheses are offered, but not tested, as to how cigarettes may play a causal role in the development of the other drug using behaviors known to develop later in the temporal sequence. One is that the experience of smoking can teach youngsters to use a psychoactive drug to influence mood and alertness, as nicotine does, and then reinforce that behavior. The second is that smoking cigarettes prepares young people for the relevant mode of ingestion for one of the next drugs in the sequence-namely marijuana. It is pointed out that drawing a foreign substance into the lungs is not a normal behavior for humans or other animals: it is a behavior which has to be learned and rewarded enough to overcome the aversive experiences which usually result. 


\section{OTHER DATA ON CORRELATES AND TRENDS}

Hundreds of correlates of drug use, without accompanying interpretation, may be found in the series of annual volumes from the study entitled Monitoring the Future: Questionnaire Responses from the Nation's High School Seniors. ${ }^{28}$ For each year since 1975, a separate hardbound volume presents univariate and selected bivariate distributions on all questions contained in the study. Many variables dealing explicitly with drugs-variables not discussed here-are contained in that series; and bivariate tables are provided for all questions each year distributed against an index of lifetime illicit drug involvement. A special cross-time reference index is contained in each volume to facilitate locating the same question across different years. One can thus derive trend data on some 1500 to 2000 variables for the entire sample or for important subgroups (based on sex, race, region, college plans, or drug involvement).

${ }^{28}$ This series is available from the Publications Division, Institute for Social Research, The University of Michigan, Ann Arbor, Michigan 48109. 


\section{TABLE 36}

\section{Smoking as Related to Average Academic Grades in High School, Class of 1985}

(Entries are percentages)

\begin{tabular}{|c|c|c|c|c|}
\hline Average Grade & $\begin{array}{c}\text { So Who } \\
\text { Never Smoked } \\
\end{array}$ & 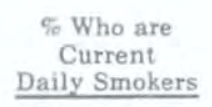 & $\begin{array}{l}\text { So Smoking } \\
\text { Pack-a-Day } \\
\text { or More }\end{array}$ & $\begin{array}{c}\begin{array}{c}\text { Number of } \\
\text { Respondents }\end{array} \\
\end{array}$ \\
\hline A & $52.8^{\mathrm{a}}$ & 7.1 & 1.9 & 1375 \\
\hline A- & 41.2 & 10.6 & 2.5 & 1668 \\
\hline $\mathrm{B}+$ & 35.6 & 14.8 & 4.2 & 2625 \\
\hline B & 30.7 & 18.3 & 5.4 & 3237 \\
\hline B- & 27.0 & 20.6 & 7.5 & 2380 \\
\hline $\mathrm{C}+$ & 22.9 & 24.3 & 9.1 & 2151 \\
\hline $\mathrm{C}$ & 22.8 & 28.0 & 10.0 & 1507 \\
\hline c. & 18.2 & 35.9 & 14.2 & 588 \\
\hline D & 13.5 & 46.9 & 23.3 & 188 \\
\hline
\end{tabular}

${ }^{\mathrm{a}}$ This means that 52.8 percent of students who had an A average never smoked. 
TABLE 37

\section{Lifetime Use of Drugs by Five Categories of Smoking Behavior, Class of 1985}

(Entries are percentages)

\begin{tabular}{|c|c|c|c|c|c|}
\hline & $\begin{array}{c}\text { Never } \\
\text { Smoked } \\
\end{array}$ & $\begin{array}{c}\begin{array}{c}\text { Smoked } \\
1-2 \text { Times } \\
\text { in } \\
\text { Lifetime }\end{array} \\
\end{array}$ & $\begin{array}{c}\text { Smoked } \\
\text { 3+ Times, } \\
\text { Not Current } \\
\text { Daily }\end{array}$ & $\begin{array}{c}\text { Current } \\
\text { Daily } \\
\text { Smoking, } \\
\text { Less than } \\
\text { Pack-a-Day } \\
\end{array}$ & $\begin{array}{c}\text { Current } \\
\text { Daily Use } \\
\text { Pack-a-Day } \\
\text { or More }\end{array}$ \\
\hline Any Illicit Drug & $26.8^{\mathrm{a}}$ & 62.5 & 81.0 & 89.5 & 95.3 \\
\hline $\begin{array}{l}\text { Any Illicit Drug Other } \\
\text { than Marijuana }\end{array}$ & 16.1 & 33.7 & 53.0 & 69.0 & 80.7 \\
\hline Marijuana & 19.5 & 54.5 & 75.2 & 86.8 & 93.8 \\
\hline Inhalants (unadjusted) ${ }^{b}$ & 6.4 & 12.8 & 21.5 & 25.3 & 33.6 \\
\hline Hallucinogens (unadjusted) & 2.3 & 5.2 & 12.6 & 23.2 & 39.9 \\
\hline $\begin{array}{l}\mathrm{LSD} \\
\mathrm{PCP}^{\mathrm{C}}\end{array}$ & $\begin{array}{l}1.6 \\
0.9\end{array}$ & $\begin{array}{l}3.6 \\
2.3\end{array}$ & $\begin{array}{l}8.3 \\
6.1\end{array}$ & $\begin{array}{r}17.6 \\
9.8\end{array}$ & $\begin{array}{l}32.8 \\
21.6\end{array}$ \\
\hline Cocaine & 5.0 & 10.8 & 23.5 & 37.7 & 49.3 \\
\hline Heroin & 0.4 & 0.4 & 1.3 & 2.7 & 5.5 \\
\hline Other Opiates & 3.3 & 6.2 & 13.7 & 18.6 & 35.5 \\
\hline Stimulants (adjusted) & 8.4 & 20.0 & 36.3 & 51.0 & 62.7 \\
\hline Sedatives & 3.2 & 6.9 & 14.9 & 25.4 & 40.6 \\
\hline $\begin{array}{l}\text { Barbiturates } \\
\text { Methaqualone }\end{array}$ & $\begin{array}{l}2.5 \\
1.4\end{array}$ & $\begin{array}{l}5.2 \\
3.4\end{array}$ & $\begin{array}{r}11.5 \\
8.2\end{array}$ & $\begin{array}{l}19.1 \\
15.3\end{array}$ & $\begin{array}{l}34.7 \\
26.0\end{array}$ \\
\hline Tranquilizers & 4.4 & 7.9 & 15.9 & 22.1 & 34.9 \\
\hline Alcohol & 78.8 & 97.3 & 99.0 & 98.9 & 99.3 \\
\hline Approx. $\mathrm{N}=$ & 5040 & 4790 & 3210 & 2070 & 1070 \\
\hline
\end{tabular}

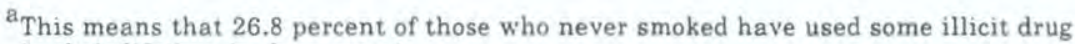
in their lifetime (at least once).

${ }^{b}$ Asked in four of the five questionnaire forms. $\mathrm{N}$ is four-fifths of $\mathrm{N}$ indicated.

${ }^{c}$ Asked in one of the five questionnaire forms. $\mathrm{N}$ is one-fifth of $\mathrm{N}$ indicated. 
TABLE 38

\section{Annual Use of Drugs by Five Categories of Smoking Behavior, Class of $\mathbf{1 9 8 5}$}

(Entries are percentages)

\begin{tabular}{|c|c|c|c|c|c|}
\hline & $\begin{array}{c}\text { Never } \\
\text { Smoked } \\
\end{array}$ & $\begin{array}{c}\text { Smoked } \\
1-2 \text { Times } \\
\text { in } \\
\text { Lifetime }\end{array}$ & $\begin{array}{c}\text { Smoked } \\
3+\text { Times, } \\
\text { Not Current } \\
\text { Daily }\end{array}$ & $\begin{array}{c}\text { Current } \\
\text { Daily } \\
\text { Smoking, } \\
\text { Less than } \\
\text { Pack-a-Day } \\
\end{array}$ & $\begin{array}{c}\text { Current } \\
\text { Daily Use } \\
\text { Pack-a-Day } \\
\text { or More }\end{array}$ \\
\hline Any Illicit Drug & 17.3 & 42.4 & 65.9 & 78.6 & 83.6 \\
\hline $\begin{array}{l}\text { Any Illicit Drug Other } \\
\text { than Marijuana }\end{array}$ & 9.5 & 20.7 & 37.0 & 53.9 & 63.9 \\
\hline Marijuana & 12.7 & 36.0 & 58.9 & 74.0 & 78.5 \\
\hline Inhalants (unadjusted) ${ }^{a}$ & 2.4 & 4.5 & 8.3 & 8.7 & 14.1 \\
\hline Hallucinogens (unadjusted) & 1.5 & 2.8 & 7.2 & 15.3 & 25.3 \\
\hline $\begin{array}{l}\mathrm{LSD}_{\mathrm{b}} \\
\mathrm{PCP}^{\mathrm{b}}\end{array}$ & $\begin{array}{l}0.9 \\
0.7\end{array}$ & $\begin{array}{l}1.9 \\
1.1\end{array}$ & $\begin{array}{l}4.6 \\
3.6\end{array}$ & $\begin{array}{r}11.3 \\
7.0\end{array}$ & $\begin{array}{l}18.6 \\
12.0\end{array}$ \\
\hline Cocaine & 3.8 & 7.9 & 16.8 & 29.1 & 40.1 \\
\hline Heroin & 0.3 & 0.2 & 0.5 & 1.4 & 2.7 \\
\hline Other Opiates & 1.6 & 3.7 & 7.8 & 11.2 & 21.0 \\
\hline Stimulants (adjusted) & 4.3 & 10.5 & 21.2 & 35.6 & 41.6 \\
\hline Sedatives & 1.7 & 2.8 & 6.9 & 12.4 & 23.7 \\
\hline $\begin{array}{l}\text { Barbiturates } \\
\text { Methaqualone }\end{array}$ & $\begin{array}{l}1.4 \\
0.7\end{array}$ & $\begin{array}{l}2.1 \\
1.2\end{array}$ & $\begin{array}{l}5.3 \\
3.0\end{array}$ & $\begin{array}{l}9.5 \\
6.5\end{array}$ & $\begin{array}{l}20.0 \\
13.2\end{array}$ \\
\hline Tranquilizers & 2.1 & 3.5 & 8.0 & 11.6 & 21.1 \\
\hline Alcohol & 68.7 & 88.7 & 96.5 & 97.4 & 96.6 \\
\hline Approx. $\mathrm{N}=$ & 5040 & 4790 & 3210 & 2070 & 1070 \\
\hline
\end{tabular}

${ }^{a}$ Asked in four of the five questionnaire forms. $\mathrm{N}$ is four-fifths of $\mathrm{N}$ indicated.

${ }^{\mathrm{b}}$ Asked in one of the five questionnaire forms. $\mathrm{N}$ is one-fifth of $\mathrm{N}$ indicated. 
TABLE 39

\section{Thirty-Day Use of Drugs by Five Categories of Smoking Behavior, Class of 1985}

(Entries are percentages)

\begin{tabular}{|c|c|c|c|c|c|}
\hline & $\begin{array}{l}\text { Never } \\
\text { Smoked } \\
\end{array}$ & $\begin{array}{c}\text { Smoked } \\
\text { 1-2 Times } \\
\text { in } \\
\text { Lifetime } \\
\end{array}$ & $\begin{array}{c}\text { Smoked } \\
3+\text { Times, } \\
\text { Not Current } \\
\text { Daily }\end{array}$ & $\begin{array}{c}\text { Current } \\
\text { Daily } \\
\text { Smoking, } \\
\text { Less than } \\
\text { Pack-a-Day } \\
\end{array}$ & $\begin{array}{c}\text { Current } \\
\text { Daily Use } \\
\text { Pack-a-Day } \\
\text { or More }\end{array}$ \\
\hline Any Illicit Drug & 9.7 & 22.6 & 40.6 & 60.6 & 66.8 \\
\hline $\begin{array}{l}\text { Any Illicit Drug Other } \\
\text { than Marijuana }\end{array}$ & 4.6 & 8.6 & 18.9 & 32.9 & 46.4 \\
\hline Marijuana & 7.2 & 19.4 & 35.2 & 55.3 & 59.0 \\
\hline Inhalants (unadjusted) ${ }^{a}$ & 1.0 & 1.7 & 2,7 & 3,4 & 6.9 \\
\hline Hallucinogens (unadjusted) & 0.7 & 1.1 & 2.9 & 5.2 & 10.7 \\
\hline $\begin{array}{l}\mathrm{LSD}_{\mathrm{b}} \\
\mathrm{PCP}^{\mathrm{b}}\end{array}$ & $\begin{array}{l}0.4 \\
0.4\end{array}$ & $\begin{array}{l}0.6 \\
0.7\end{array}$ & $\begin{array}{l}1.8 \\
1.7\end{array}$ & $\begin{array}{l}3.4 \\
3.9\end{array}$ & $\begin{array}{l}7.9 \\
6.5\end{array}$ \\
\hline Cocaine & 1.8 & 3.1 & 8.1 & 15.8 & 25.3 \\
\hline Heroin & 0.1 & 0.1 & 0.3 & 0.4 & 0.9 \\
\hline Other Opiates & 0.6 & 1.6 & 3.1 & 3.7 & 9.1 \\
\hline Stimulants (adjusted) & 2.1 & 3.4 & 8.3 & 17.0 & 22.0 \\
\hline Sedatives & 0.7 & 0.9 & 2.9 & 4.8 & 11.5 \\
\hline $\begin{array}{l}\text { Barbiturates } \\
\text { Methaqualone }\end{array}$ & $\begin{array}{l}0.6 \\
0.3\end{array}$ & $\begin{array}{l}0.8 \\
0.3\end{array}$ & $\begin{array}{l}2.6 \\
0.7\end{array}$ & $\begin{array}{l}3.9 \\
2.2\end{array}$ & $\begin{array}{l}8.8 \\
6.1\end{array}$ \\
\hline Tranquilizers & 0.6 & 0.9 & 2.7 & 3.8 & 9.6 \\
\hline Alcohol & 42.4 & 66.3 & 82.2 & 86.1 & 87.4 \\
\hline Approx. $\mathrm{N}=$ & 5040 & 4790 & 3210 & 2070 & 1070 \\
\hline
\end{tabular}


TABLE 40

\section{Daily Use of Drugs During the Last Thirty Days by Five Categories of Smoking Behavior, Class of 1985}

(Entries are percentages)

\begin{tabular}{|c|c|c|c|c|c|}
\hline & $\begin{array}{c}\text { Never } \\
\text { Smoked } \\
\end{array}$ & $\begin{array}{c}\text { Smoked } \\
1-2 \text { Times } \\
\text { in } \\
\text { Lifetime }\end{array}$ & $\begin{array}{c}\text { Smoked } \\
3+\text { Times, } \\
\text { Not Current } \\
\text { Daily }\end{array}$ & $\begin{array}{c}\text { Current } \\
\text { Daily } \\
\text { Smoking, } \\
\text { Less than } \\
\text { Pack-a-Day } \\
\end{array}$ & $\begin{array}{c}\text { Current } \\
\text { Daily Use } \\
\text { Pack-a-Day } \\
\text { or More }\end{array}$ \\
\hline Any Illicit Drug & 1.2 & 1.7 & 4.9 & 13.6 & 26.0 \\
\hline $\begin{array}{l}\text { Any Illicit Drug Other } \\
\text { than Marijuana }\end{array}$ & 0.4 & 0.3 & 1.0 & 2.3 & 5.1 \\
\hline Marijuana & 1.1 & 1.7 & 4.8 & 12.5 & 23.0 \\
\hline Inhalants (unadjusted) ${ }^{a}$ & 0.1 & 0.2 & 0.1 & 0.5 & 0.4 \\
\hline Hallucinogens (unadjusted) & 0.1 & 0.0 & 0.0 & 0.1 & 0.5 \\
\hline $\begin{array}{l}\mathrm{LSD}_{\mathrm{b}} \\
\mathrm{PCP}^{\mathrm{b}}\end{array}$ & $\begin{array}{l}0.1 \\
0.3\end{array}$ & $\begin{array}{l}0.0 \\
0.0\end{array}$ & $\begin{array}{l}0.0 \\
0.0\end{array}$ & $\begin{array}{l}0.1 \\
1.2\end{array}$ & $\begin{array}{l}0.3 \\
1.0\end{array}$ \\
\hline Cocaine & 0.1 & 0.2 & 0.3 & 0.8 & 1.6 \\
\hline Heroin & 0.0 & 0.0 & 0.0 & 0.0 & 0.0 \\
\hline Other Opiates & 0.0 & 0.0 & 0.3 & 0.2 & 0.2 \\
\hline Stimulants (adjusted) & 0.1 & 0.1 & 0.3 & 1.2 & 2.1 \\
\hline Sedatives & 0.0 & 0.0 & 0.1 & 0.1 & 0.6 \\
\hline $\begin{array}{l}\text { Barbiturates } \\
\text { Methaqualone }\end{array}$ & $\begin{array}{l}0.0 \\
0.0\end{array}$ & $\begin{array}{l}0.0 \\
0.0\end{array}$ & $\begin{array}{l}0.1 \\
0.0\end{array}$ & $\begin{array}{l}0.1 \\
0.0\end{array}$ & $\begin{array}{l}0.4 \\
0.3\end{array}$ \\
\hline Tranquilizers & 0.0 & 0.0 & 0.0 & 0.0 & 0.3 \\
\hline Alcohol, Daily & 1,7 & 3.3 & 5.7 & 9.1 & 18.4 \\
\hline $\begin{array}{l}5+\text { Drinks in a Row } \\
\text { in Last Two Weeks }\end{array}$ & 17.2 & 32.8 & 47.4 & 61.4 & 67.9 \\
\hline Approx. $\mathrm{N}=$ & 5040 & 4790 & 3210 & 2070 & 1070 \\
\hline
\end{tabular}

${ }^{\text {a }}$ Asked in four of the five questionnaire forms. $\mathrm{N}$ is four-fifths of $\mathrm{N}$ indicated.

${ }^{b}$ Asked in one of the five questionnaire forms. $\mathrm{N}$ is one-fifth of $\mathrm{N}$ indicated. 
APPENDIX 


\section{ESTIMATES ADJUSTED FOR ABSENTEES AND DROPOUTS}

One question which has arisen over the years in regard to this study has concerned the degree to which the prevalence and trend estimates derived from high school seniors are an accurate reflection of the reality which pertains for all young people who would be in the same class or age cohort, including those who have dropped out of school by senior year. In 1985 we published an extensive chapter on this topic in a volume in the NIDA Research Monograph series. ${ }^{29}$ We will attempt in this Appendix to summarize the main points relevant to this issue of sample coverage.

First, it should be noted that two segments of the entire class/age cohort are missing from the data collected each year from seniors: those who are still enrolled in school but who are absent the day of data collection (the "absentees") and those who have formally left school (the dropouts). The "absentees" constitute virtually all of the nonrespondents shown in the response rate table given in the Introduction to this volume (since refusal rates are negligible) or about $18 \%$ of all seniors (or $15 \%$ of the class/age cohort). Based on our review of available Census data the dropouts account for approximately $15 \%$ of the class/age cohort.

The methods we used to estimate the prevalence rates for these two missing segments are summarized briefly here. Then, the effects of adding in these two segments to the calculation of the overall prevalence rates for two drug classes are presented along with the impact on the trend estimates. Two illicit drugs have been chosen for illustrative purposes: marijuana, the most prevalent of the illicit drugs, and cocaine, one of the more dangerous and less prevalent drugs. Estimates for high school seniors are presented for both lifetime and 30-day prevalence for each drug.

${ }^{29}$ Johnston, L.D., \& O'Malley, P.M. (1985). Issues of validity and population coverage in student surveys of drug use. In B.A. Rouse, N.J. Kozel, \& L.G. Richards (Eds.), Self-report methods of estimating drug use: Meeting current challenges to validity (NIDA Research Monograph No. 57; (ADM) 851402). Washington, DC: U.S. Government Printing Office. 


\section{THE EFFECTS OF MISSING ABSENTEES}

To be able to assess the effects on the estimates of drug use of missing the absentees, we included a question in the study which asks students how many days of school they had missed in the previous four weeks. Using this variable, we can place individuals into different strata as a function of how often they tend to be absent. For example, all students who had been absent $50 \%$ of the time could form one stratum. Assuming that absence on the day of the administration is a fairly random event, we can use the respondents in this stratum to represent all students in their stratum, including the ones who happen to be absent that particular day. By giving them a double weight, they can be used to represent both themselves and the other $50 \%$ of their stratum who were absent that day. Those who say they were in school only onethird of the time would get a weight of three to represent the two-thirds in their stratum who were not there, and so forth. Using this method, we found that absentees as a group have appreciably higher than average usage levels for all licit and illicit drugs. However, looking at 1983 data, we found that their omission did not depress any of the prevalence estimates in any of the drugs by more than $2.7 \%$, due to the fact that they represent such a small proportion of the total target sample. Considering that a substantial proportion of those who are absent likely are absent for reasons unrelated to drug use-such as illness and participation in extracurricular activities-it may be surprising to see even these differences. In any case, from the point of view of instructing policy or public perceptions, the small "corrections" would appear to be of little or no significance. (The correction across all 13 drugs in lifetime prevalence averaged only $1.4 \%$.) Further, such corrections should have virtually no effect on cross-time trend estimates unless the rate of absenteeism were changing appreciably; and we find no evidence in our data that it is. Put another way, the presence of a fairly slight underestimate which is constant across time should not influence trend results. Should absentee rates start changing, then it could be argued more convincingly that such corrections should be presented routinely.

\section{THE EFFECTS OF MISSING DROPOUTS}

Unfortunately, we cannot derive corrections from data gathered from seniors to impute directly the prevalence rates for dropouts, as we did for absentees, since we have no completely appropriate stratum from which we have "sampled," We do know from our own previous research, as well as the work of others, that dropouts have prevalence rates for all classes of drugs substantially higher than the in-school students. In fact, the dropouts may be fairly similar to the absentees.

We have consistently estimated the proportion who fail to complete high school to be approximately $15 \%$; Figure A-1 displays the completion 


\section{FIGURE A-1}

High School Completion by Persons 20-24 Years Old, 1972-1986

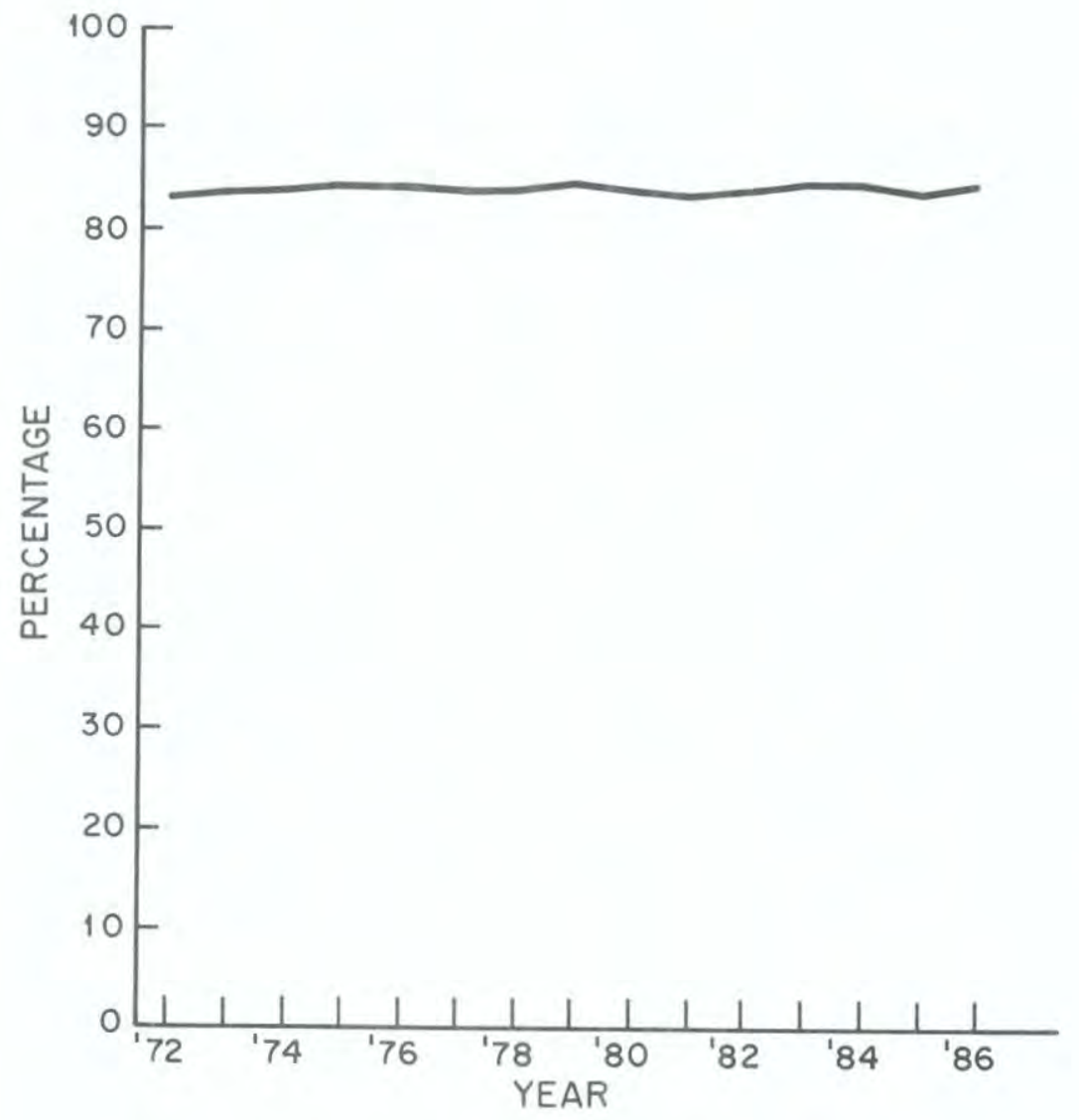

Source: U.S. Bureau of the Census, Current Populations Surveys, published and unpublished data; and 1980 Census. 
rate for the years 1972 through 1986 based on Census data. As the figure indicates, completion rates (and the complement, dropout rates) have been quite constant over this interval for persons 20-24 years old. $^{30}$ (Younger age brackets are more difficult to use because they include some who are still enrolled in high school.) Monitoring the Future probably covers some small proportion of the $15 \%$, in fact, since the survey of seniors takes place a few months before graduation, and not everyone will graduate. On the other hand, perhaps $1 \%$ to $2 \%$ of the age group which Census shows as having a diploma get it through a General Equivalency Degree and thus would not be covered in Monitoring the Future. (Elliot and Voss report this result for less than 2\% of their sample in their follow-up study of 2617 ninth graders in California who were followed through their high school years. $)^{31}$ So these two factors probably cancel each other out. Thus, we use $15 \%$ as our estimate of the proportion of a class cohort not covered.

Extrapolating to dropouts from absentees. To estimate the drug usage prevalence rates for this group we have used two quite different approaches. The first was based on extrapolations from seniors participating in this study. Using this method we developed estimates under three different assumptions: that the difference between dropouts and the participating seniors in the study was equivalent to (a) the difference between absentees and the participating seniors, (b) one and one-half times that difference, and (c) twice that difference. The last assumption we would consider a rather extreme one.

The second general method involved using the best recent national data on drug use among dropouts-namely the National Household Surveys on Drug Abuse. ${ }^{32}$ While these surveys have rather small samples of dropouts in the relevant age range in any given year, they should at least provide unbiased estimates for dropouts still in the household population.

Using the first method of estimation, we found that, under the assumption that dropouts are just like absentees, no prevalence rate was changed by more than $5 \%$ over the estimate based on 1983 seniors only, even with the simultaneous correction for both absentees and

${ }^{30}$ U.S. Bureau of the Census (various years). Current population reports, Series P-20, various numbers. Washington, DC: L.S. Government Printing Office.

${ }^{31}$ Elliott, D., \& Voss, H.L. (1974). Delinquency and dropout. Lexington, MA: D.C. Heath-Lexington Books.

${ }^{32}$ Fishburne, P.M., Abeison, H.I., \& Cisin, I. (1980). National survey on drug abuse: Main findings, 1979 (NIDA (ADM) 80-976). Washington, DC: U.S. Government Printing Office. Also see Miller, J.D., et al., (1983). National survey on drug abuse: Main findings, 1982 (NIDA (ADM) 83-1263). Washington, DC: U.S. Government Printing Office. 
dropouts. (The method for calculating prevalence rates for the absentees is the one described in the previous section.) The largest correction in 1983 involved marijuana, with lifetime prevalence rising from just under $60 \%$ to $64 \%$. Even under the most extreme assumption-which results in exceptionally high prevalence rates for dropouts on all drugs, for example $90 \%$ lifetime prevalence for marijuana, the overall correction in any of the prevalence figures for any drug remains less than $7.5 \%$. Again, marijuana shows the biggest correction $(7.5 \%$ in annual prevalence, raising it from $46 \%$ uncorrected to $54 \%$ with corrections for both absentees and dropouts). As we would have expected, the biggest proportional change occurs for heroin, since it represents the most deviant end of the drug-using spectrum and thus would be most associated with truancy and dropping out.

Extrapolating from the household surveys. The second method of estimating drug use among dropouts was by comparing the household survey data on dropouts with the data from those remaining in school. We conducted secondary analyses of the archived data from the 1977 and 1979 National Household Surveys. Analyses were restricted to the age range 17 to 19 years old, since about $95 \%$ of the Monitoring the Future respondents fall in this range. Of course, the numbers of cases are small. In the 1977 survey there were only 46 dropouts and 175 enrolled seniors in this age group. In the 1979 survey 92 dropouts and 266 seniors were included.

For marijuana, the estimated differences from the household survey data came out at a level which was at or below the least extreme assumption made in the previous method (where dropouts are assumed to have the same drug use levels as absentees). While this may have been comforting to the authors of the present report, we must admit that we believe the household sample underrepresents the more drugprone dropouts to some degree. Those without permanent residence and those in the prison population, to take two examples, would be excluded from the sample coverage in a household survey. Thus we concluded that estimates closer to those made under the second assumption in the previous method may be closer to reality-that is, that dropouts are likely to deviate from participating seniors by one and onehalf times the amount that absentees deviate from them.

Again, we emphasize that there are a number of reasons for dropping out, many of which bear no relationship to drug use, including economic hardship in the family and certain learning disabilities and health problems. At the national level, the extreme groups such as those in jail or without a permanent place of residence are undoubtedly very small as a proportion of the total age group and probably even as a proportion of all dropouts. Thus, regardless of their prevalence rates, they would be unable to move the prevalence estimates by a very large proportion except in the case of the most rare events-in particular, heroin use. We do believe that in the case of heroin use-particularly 
regular use-we are very likely unable to get a very accurate estimate even with the corrections used in this paper. For the remaining drugs, we conclude that our estimates based on participating seniors, though somewhat low, are not bad approximations for the age group as a whole.

Effects of omitting dropouts in trend estimates. Whether the omission of dropouts affects the estimates of trends in prevalence rates is a separate question, however, from the degree to which it affects absolute estimates at a given point in time. The relevant issues parallel those discussed earlier regarding the possible effects on trends of omitting the absentees. Most important is the question of whether the rate of dropping out has been changing in the country, since a substantial change would mean that seniors studied in different years would represent noncomparable segments of the whole class/age cohort. Fortunately for the purposes of this study, at least, the official government data provided in Figure A-1 indicate a very stable rate of dropping out from 1972 to 1985.

Given that there appears to be no sound evidence of a change in the dropout rate, the only reason that trend data from seniors would deviate from trends for the entire class cohort (including dropouts) would be if the constant proportion who have been dropouts for some reason showed trends contrary to those observed among seniors; and even then, because of their small numbers, they would have to show dramatically different trends to be able to change the trend "story" very much for the age group as a whole. There has been no hypothesis offered for such a differential shift among dropouts which these authors, at least, find very convincing.

The one hypothesis which is occasionally heard is that more youngsters are being expelled from school, or voluntarily leaving school, because of their drug use; and that this explains the recent downturn in the use of many drugs being reported by the study. However, it is hard to reconcile this hypothesis with the virtually flat dropout rates over the fourteen-year period displayed in Figure A-1, unless one posits a perfectly offsetting tendency for more completion among those who are less drug prone-hardly a very parsimonious set of explanations. Further, the reported prevalence of some drugs has remained remarkably stable throughout the life of the study (e.g., alcohol and opiates other than heroin) and the prevalence of some has risen (cocaine and, until fairly recently, amphetamines). These facts are not very consistent with the hypothesis that there has been a recent increased rate of departure by the most drug prone. Certainly more youngsters leaving school in the 80 's have drug problems than was true in the 60's. (So do more of those who stay in.) However, they still seem likely to be very much the same segment of the population, given the degree of association that exists between drug use and deviance and problem behaviors of various sorts. 


\section{FIGURE A-2}

Estimates of Prevalence and Trends for the Entire Age/Class Cohort, Adjusting for Absentees and Dropouts

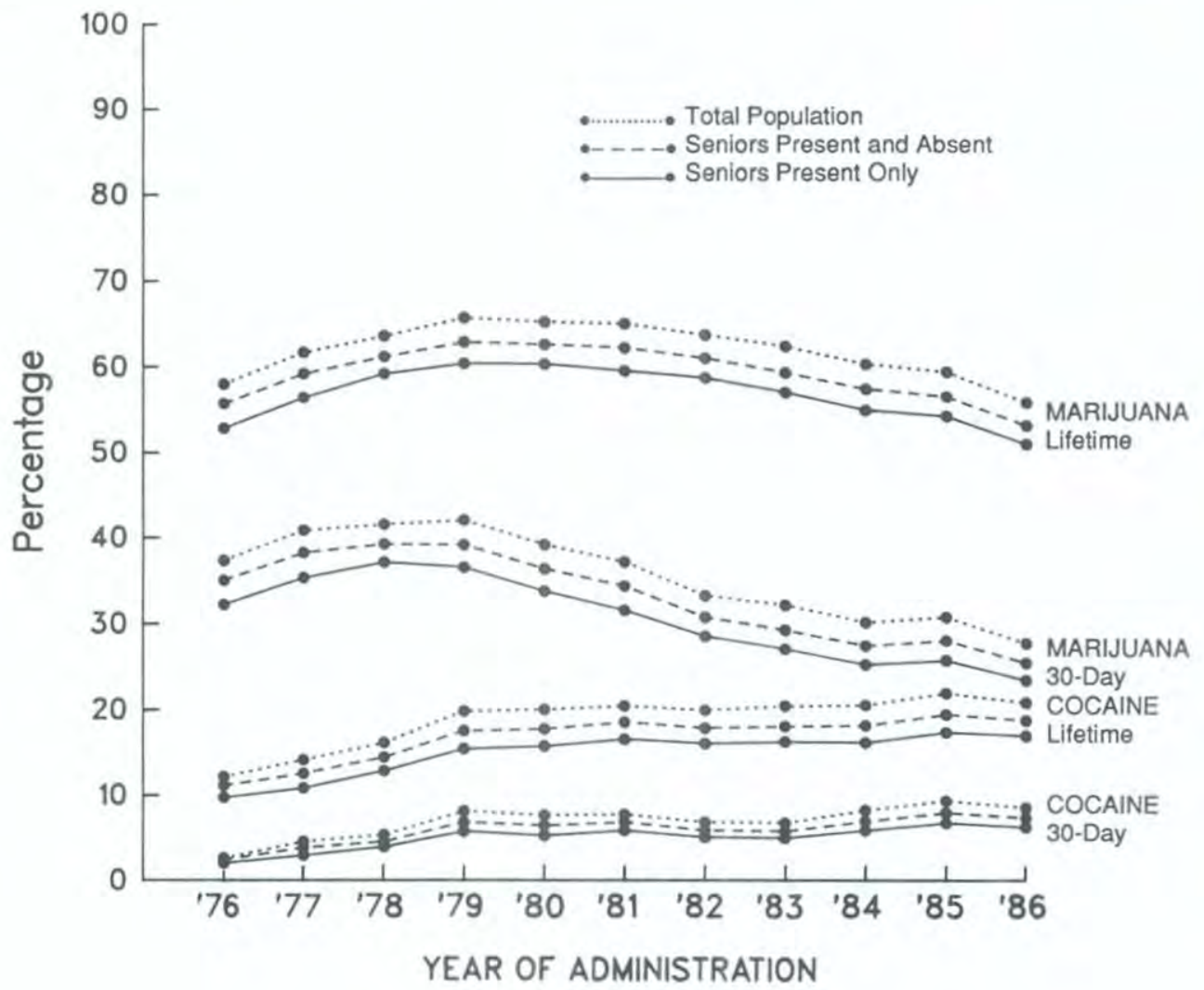




\title{
SUMMARY AND CONCLUSIONS
}

In sum, while we believe there is some underestimation of the prevalence of drug use in the cohort at large as a result of the dropouts being omitted from the universe of the study, we think the degree of underestimation is rather limited for all drugs (with the possible exception of heroin) and, more importantly, that trend estimates have been rather little affected. Short of having good trend data gathered directly from dropouts-a very expensive research undertaking-we cannot close the case definitively. Nevertheless, we think the available evidence argues strongly against alternative hypotheses-a conclusion which was also reached by the members of the NIDA technical review on this subject held in $1982 .^{33}$

\begin{abstract}
... the analyses provided in this report show that failure to include these two groups (absentees and dropouts) does not substantially affect the estimates of the incidence and prevalence of drug use.
\end{abstract}

\section{EXAMPLES OF REVISED ESTIMATES FOR TWO DRUGS}

Figure A-2 provides the prevalence and trend estimates of marijuana and cocaine, for both the lifetime and thirty-day prevalence periods, showing (a) the original estimates based on participating seniors only; (b) the empirically derived, revised estimates based on all seniors, including the absentees; and (c) estimates for the entire class/age cohort. The last estimate was developed using the assumption judged to be most reasonable above-namely that the dropouts differ from participating seniors by one and one-half times the amount that the absentees do. Estimates were calculated separately for each year, thus taking into account any differences from year to year in the participation or absentee rate. The dropout rate was taken as a constant $15 \%$ of the age group across all years.

As Figure A-2 illustrates, any difference in the slopes of the trend lines between the original and revised estimates is extremely, almost infinitesimally, small. The prevalence estimates are higher, of course, but not dramatically so, and certainly not enough so to have any serious policy-implication effects in the interpretation of the data.

${ }^{33}$ Clayton, R.R., \& Voss, H.L. (1982). Technical review on drug abuse and dropouts. Rockville, MD: National Institute on Drug Abuse. 
DHHS Publication No. (ADM)87-1535 Printed 1987 